Physics Division Physics Division Physics Division Physics Division hysics Division Phyrics Division 10utsics Division Eustc Oivision Physics Division Physics Division Physics Division phrsics Division Pastcs bivision Dajsics DNision Dhrics Division Bursios bivision. Physics Division $40 / 941979501$ Physics Division Physios Division Physics Division Physics Division Puysicspivision Physics Division Physics Division Physics Ditstor Physios Dins 10 . Phisics bivistoPhysosulystor Why os buston PInstos aviso: H. H. Busciospysion Physics Division Physies Division

Physics Division Physics Division Annual Repolt: January - December

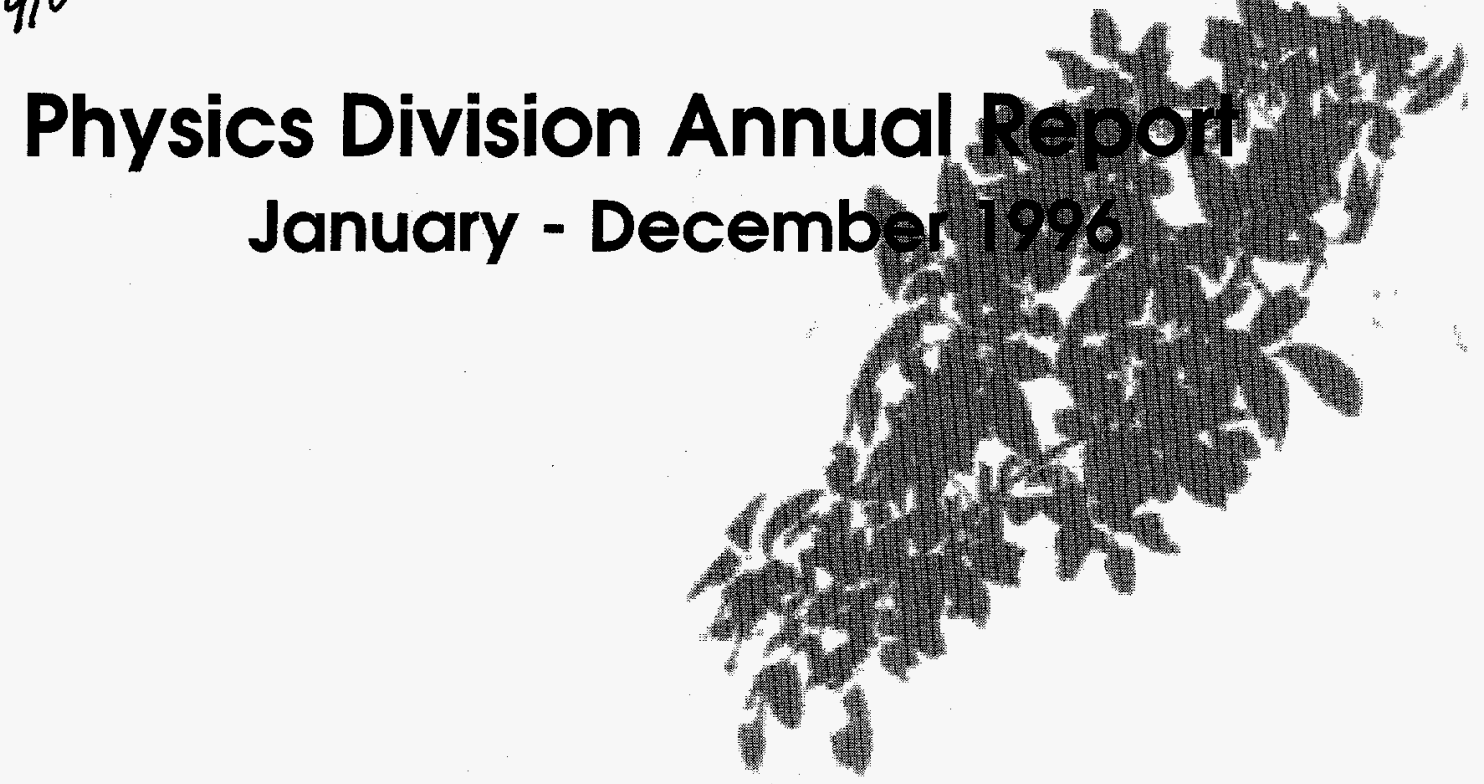
.
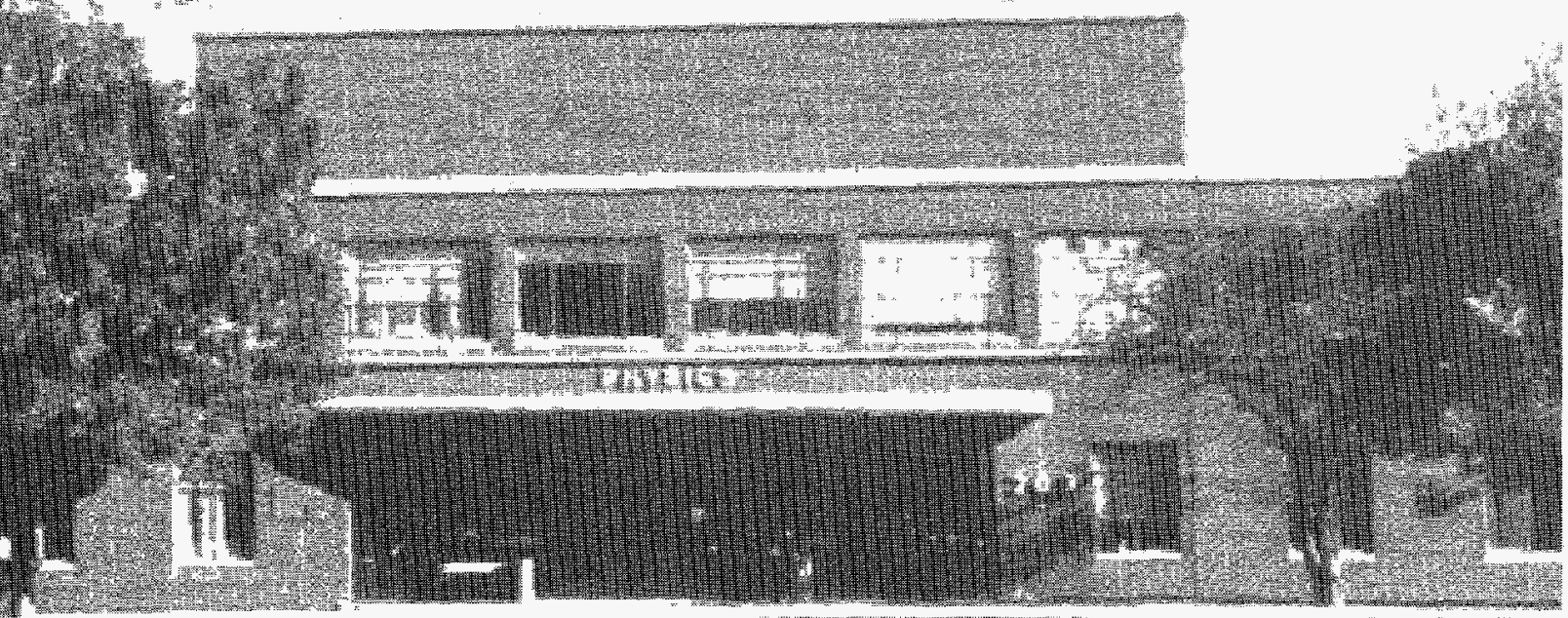

Physics División

Physics Division

Physics Division

Physics Division

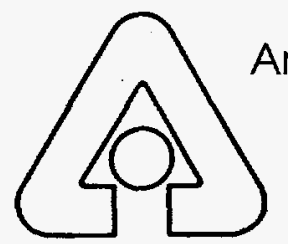

Argonne National Laboratory, Argonne, Illinois 60439

operated by The University of Chicago

for the United States Department of Energy under Contract W-31-109-Eng-38

Physics Division

Physics Division 
Argonne National Laboratory, with facilities in the states of Illinois and Idaho, is owned by the United States government, and operated by The University of Chicago under the provisions of a contract with the Department of Energy.

\section{DISCLAIMER}

This report was prepared as an account of work sponsored by an agency of the United States Government. Neither the United States Government nor any agency thereof, nor any of their employees, makes any warranty, express or implied, or assumes any legal liability or responsibility for the accuracy, completeness, or usefulness of any information, apparatus, product, or process disclosed, or represents that its use would not infringe privately owned rights. Reference herein to any specific commercial product, process, or service by trade name, trademark, manufacturer, or otherwise, does not necessarily constitute or imply its endorsement, recommendation, or favoring by the United States Government or any agency thereof. The views and opinions of authors expressed herein do not necessarily state or reflect those of the United States Government or any agency thereof.

Reproduced from the best available copy.

Available to DOE and DOE contractors from the

Office of Scientific and Technical Information

P.O. Box 62

Oak Ridge, TN 37831

Prices available from (423) 576-8401

Available to the public from the

National Technical Information Service

U.S. Department of Commerce

5285 Port Royal Road

Springfield, VA 22161 
Distribution Category:

General Physics (UC-410)

\author{
ANL-97/14 \\ ARGONNE NATIONAL LABORATORY \\ 9700 S. Cass Avenue \\ Argonne, Illinois 60439-4801
}

\title{
PHYSICS DIVISION ANNUAL REPORT
}

\author{
January - December 1996
}

Walter F. Henning

Director

August 1997

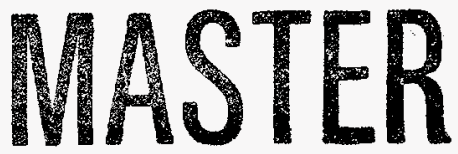

Preceding Annual Reports

ANL-94/31 1993-1994

ANL-95/14 1994-1995

ANL-96/15 1995-1996

DISTRIBUTION OF THIS DOCUMENT IS UNLIMITED 
Edited by Karen J. Thayer 


\section{DISCLAMIXR}

Portions of this document may be illegible in electronic image products. Images are produced from the best avallable original docoment. 


\section{FOREWORD}

The past year has seen several of the Physics Division's new research projects reach major milestones with first successful experiments and results:

- the atomic physics station in the Basic Energy Sciences Research Center at the Argonne Advanced Photon Source was used in first high-energy, high-brilliance $\mathrm{x}$-ray studies in atomic and molecular physics;

- the Short Orbit Spectrometer in Hall C at the Thomas Jefferson National Accelerator (TJNAF) Facility that the Argonne medium energy nuclear physics group was responsible for, was used extensively in the first round of experiments at TJNAF;

- at ATLAS, several new beams of radioactive isotopes were developed and used in studies of nuclear physics and nuclear astrophysics;

- the new ECR ion source at ATLAS was completed and first commissioning tests indicate excellent performance characteristics.

- Quantum Monte Carlo calculations of mass-8 nuclei were performed for the first time with realistic nucleon-nucleon interactions using state-of-the-art computers, including Argonne's massively parallel IBM SP.

At the same time other future projects are well under way: preparations for the move of Gammasphere to ATLAS in September 1997 have progressed as planned. The Canadian Penning Trap Collaboration has decided to mount its high-mass resolution trap at ATLAS and is planning a three-year research program. R\&D for the proposal for a full-powered ISOL facility based on ATLAS is progressing, with accelerator development and the re-instatement of the operation of the Division's Dynamitron accelerator for isotope production and ion-source tests with radioactive beams. The medium energy group is leading the effort in the HERMES experiment at DESY to install a novel ring-imaging Cerenkov counter for kaon identification. The high-luminosity, laserpolarized hydrogen gas target was installed at the Indiana Cooler Ring for in-beam tests and experiments.

These new efforts are imbedded in, or flowing from, the vibrant ongoing research program described in some detail in this report: nuclear structure and reactions with heavy ions; measurements of reactions of astrophysical interest; studies of nucleon and sub-nucleon structures using leptonic probes at intermediate and high energies; atomic and molecular structure with highenergy x-rays. The experimental efforts are being complemented with efforts in theory, from QCD to nucleon-meson systems to structure and reactions of nuclei. Finally, the operation of ATLAS as a national users facility has achieved a new milestone, with 5800 hours beam on target for experiments during the past fiscal year!

As in past years, the staff of the Physics Division has had the pleasure to work with visitors and students who have been essential to the successful research program and to the vitality of the Division.

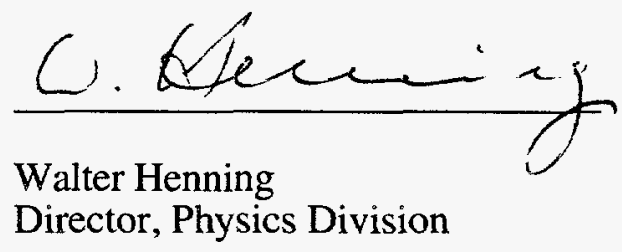




\section{TABLE OF CONTENTS}

$\underline{\text { Page }}$

\section{HEAVY-ION NUCLEAR PHYSICS RESEARCH $\ldots \ldots 1$}

\section{A. EXPERIMENTS WITH SECONDARY BEAMS AND STRUCTURE OF NUCLEI AT THE LIMITS OF STABILITY

a.1. Study of the Single Particle Structure Around the Closed-Shell Nucleus

${ }^{56} \mathrm{Ni}$ Using a Radioactive $56 \mathrm{Ni}$ Beam

a.2. Search for the Optimum One-Neutron Transfer Reaction for Experiments

with ${ }^{56} \mathrm{Ni}$ Beams

a.3. Exploring the ${ }^{18} \mathrm{~F}(\mathrm{p}, \gamma){ }^{19} \mathrm{Ne}$ Gateway to the Formation of Heavy

Elements in Hot Stars ................................................ 8

a.4. Astrophysical Reaction Rate for the ${ }^{18} \mathrm{~F}(\mathrm{p}, \alpha){ }^{15} \mathrm{O}$ Reaction ................. 10

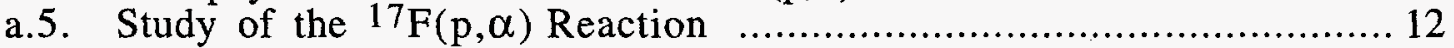

a.6. The Use of the Fragment Mass Analyzer for the Study of Radiative Capture

Reactions with Radioactive Ion Beams .................................... 13

a.7. In-Beam Studies of Very Neutron-Deficient Even-Even Os and Pt

Isotopes using Recoil-Decay Tagging . ............................... 14

a.8. Study of $176-179 \mathrm{Hg}$ Using the Recoil Decay Tagging Method................... 15

a.9. Study of ${ }^{177}$ Au Using the Recoil Decay Tagging Method .................... 17

a.10. First Observation of Excited States in $118,117 \mathrm{Cs}$ and $119,118 \mathrm{Ba} \ldots \ldots \ldots \ldots \ldots \ldots . .17$

a.11. Spectroscopy of ${ }^{192}$ Po with the RDT Technique.............................. 18

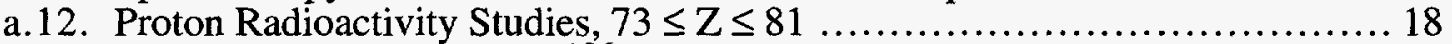

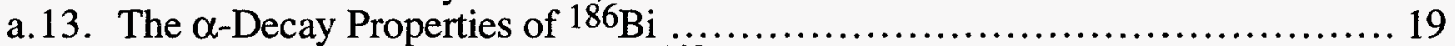

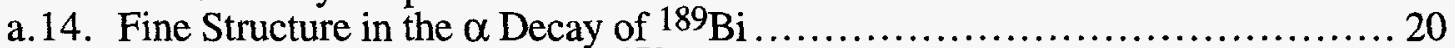

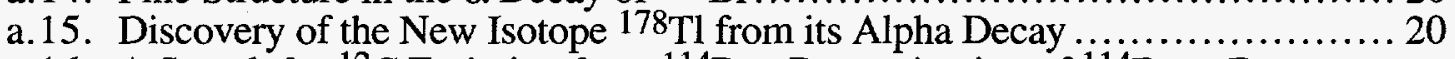

a.16. A Search for ${ }^{12} \mathrm{C}$ Emission from ${ }^{114} \mathrm{Ba}$ : Determination of ${ }^{114} \mathrm{Ba} \alpha$-Decay,

Half-Life, and Mass ................................................. 21

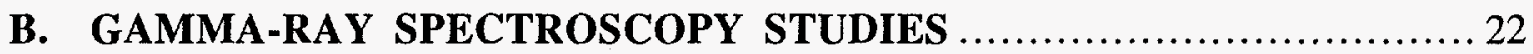

b.1. Spins, Parity, Excitation Energies and Structure of an Excited

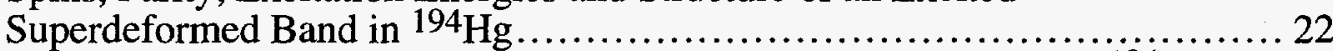

b.2. Spins, Parity and Excitation Energies of a Superdeformed Band in ${ }^{194} \mathrm{~Pb}$.... 24

b.3. Search for Transitions Linking Superdeformed and Normal Deformed

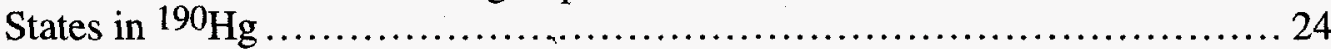

b.4. Search for One-Step Decay Gamma Rays Connecting Superdeformed and Normal States in $192 \mathrm{Hg}$

b.5. Search for One-Step Decays from Superdeformed Bands in ${ }^{193} \mathrm{Hg}$ to

the Yrast Line ...................................................... 25

b.6. Fluctuations in the Strengths of Primary Transitions from Decay Out of a Superdeformed Band ..........................................................................26

b.7. The Analysis of the Quasicontinuum of Gamma Rays in Coincidence with the One-Step Decay-Out Lines in ${ }^{194} \mathrm{Hg}$............................................226

b.8. Determination of the Spin and the Excitation Energy of the Yrast SD Band in ${ }^{194} \mathrm{Hg}$ from Analysis of the Quasicontinuum Gamma Rays 
b.9. Quasicontinuum Spectrum of Gamma Rays Which Depopulate SD

States in ${ }^{194} \mathrm{~Pb}$

b.10. Similarities in the Decay Spectra from Superdeformed and

Neutron-Capture States

b.11. Differential Quadrupole Moments of Identical Superdeformed

Bands in $192,194 \mathrm{Hg}$.

b.12. Relative Quadrupole Moments of $192,193 \mathrm{Hg}$ Superdeformed Bands.

b.13. First Measurement of Dipole Transition Rates in the Superdeformed Well...........30

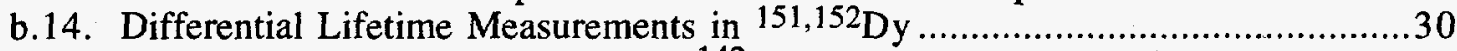

b.15. DSAM Lifetime Measurements of $142 \mathrm{Sm}$ Superdeformed Bands ............. 33

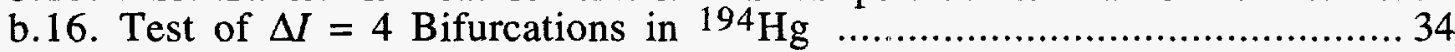

b.17. $\Delta I=4$ Bifurcation in Identical Superdeformed Bands .................... 35

b.18. Superdeformation and Normal Deformation Studies in $191 \mathrm{Tl}$..................... 35

b.19. Superdeformation in 192 Tl: Are Alignments Additive? .................... 36

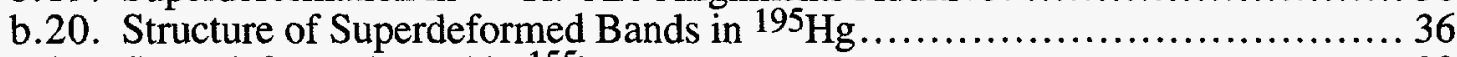

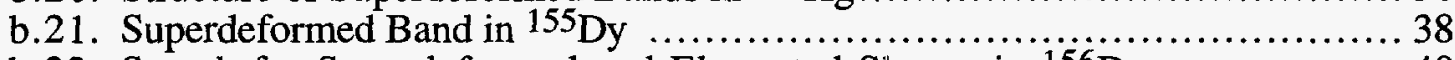

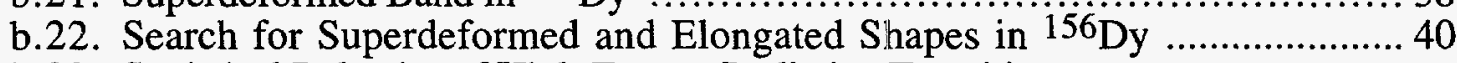

b.23. Statistical Behavior of High-Energy Radiative Transitions to

Low-Energy Rotational States

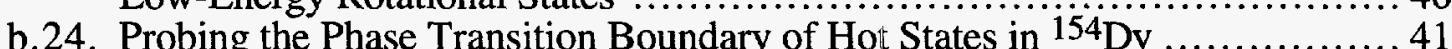

b.25. Spectroscopy at I $50 \hbar$ in Normal Deformed Dysprosium Nuclei ........... 42

b.26. Measurement of Absolute BE(2)s in the A 130 Even-Even Xe Nuclei ....... 43

b. 27. Search for the Two-Phonon Octupole Vibrational States in ${ }^{208} \mathrm{~Pb} \ldots \ldots \ldots \ldots \ldots 43$

b. 28. Coulomb Excitation of Actinides $246,248 \mathrm{Cm}$ and $240 \mathrm{Pu} . \ldots \ldots \ldots \ldots \ldots \ldots . . . \ldots 44$

b. 29. Level Structures at High Spins In Nuclei Near and at $\mathrm{N}=52 \ldots \ldots \ldots \ldots \ldots \ldots . \ldots 4$

b.30. Spin-Dependent Triaxial Deformation in Neutron-Rich Mo Isotopes .......... 46

b.31. Yrast Excitations around Doubly Magic ${ }^{132} \mathrm{Sn}$ Nucleus from Fission Product $\gamma$-Ray Studies ............................................. 47

b.32. Octupole Correlations in Neutron-Rich Odd-A La Isotopes ..................... 48

b.33. Parities and Multipolarities of Gamma Rays in Neutron-Rich

Odd-Mass Ba Isotopes

b.34. Octupole Correlations in Neutron-Rich, Even-Even Ba Isotopes ................ 50

b.35. Single Particle States in the Heaviest Elements and the Stability of the Superheavy Elements

b.36. Conference on Nuclear Structure at the Limits .............................. 51

C. REACTION STUDIES ................................................. 53

c.1. Multi-Neutron Transfer in the ${ }^{58} \mathrm{Ni}+{ }^{124} \mathrm{Sn}$ Reaction at Sub-Barrier

Energies ..................................................... 53

c.2. Measurement of the Fusion Excitation Function in the System

${ }^{78} \mathrm{Kr}+{ }^{100} \mathrm{Mo}$ at Low Energies Using a New Technique ................... 54

c.3. A New Method for the Measurement of the Fusion Excitation

Functions at Sub-Barrier Energies Using Fusion-Recoil Velocity

Distributions at Zero Degrees .......................................... 55

c.4. Spin Distributions for ${ }^{64} \mathrm{Ni}+{ }^{100} \mathrm{Mo}$ with the Argonne-Notre Dame

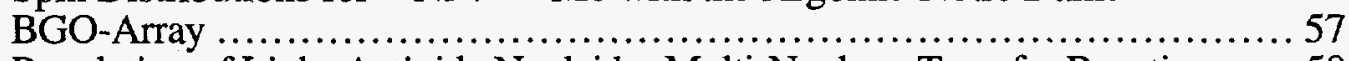

c.5. Population of Light-Actinide Nuclei by Multi-Nucleon Transfer Reactions .... 58

c.6. Angular Correlation Measurements for ${ }^{12} \mathrm{C}\left({ }^{12} \mathrm{C},{ }^{12} \mathrm{C}\right){ }^{12} \mathrm{C}\left(3^{-}\right)$Scattering ...... 59

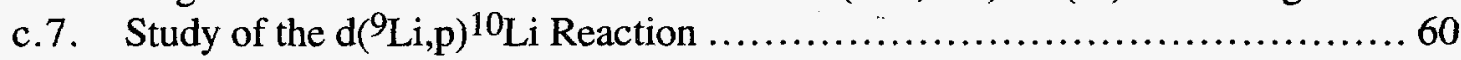


c.8. Study of the ${ }^{9,11} \mathrm{Li}+{ }^{12} \mathrm{C}$ Elastic Scattering using the S800 Spectrometer..... 61

c.9. Survival Probability of Target-Like Recoils in Deep-Inelastic Reactions........ 61

c.10. Evaporation Residue Cross Sections in the 216-218Th System ................ 62

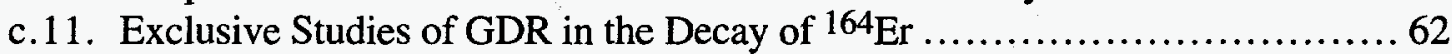

c. 12. Studies of $\mathrm{Au}+\mathrm{Au}$ Collisions at 6,8 , and $10.8 \mathrm{AGeV}$ at the AGS ...........6 63

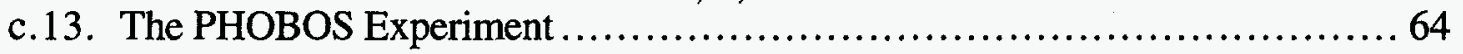

D. SUPERCRITICAL FIELDS AND OTHER TOPICS $\ldots \ldots \ldots \ldots \ldots \ldots \ldots \ldots 7$

d.1. Search for Monoenergetic Positron Production in ${ }^{238} \mathrm{U}+{ }^{181} \mathrm{Ta}$ and

$238 \mathrm{U}+{ }^{232} \mathrm{Th}$ Collisions

d.2. Dynamic Positron Production in ${ }^{238} \mathrm{U}+{ }^{181} \mathrm{Ta}$ and ${ }^{238} \mathrm{U}+{ }^{232} \mathrm{Th}$ Collisions ......68

d.3. Dynamic Positron Production in $\mathrm{Pb}+\mathrm{Pb}$ Collisions .......................... 69

d.4. Positron-Electron Angular Correlations in Internal Pair Conversion ........... 70

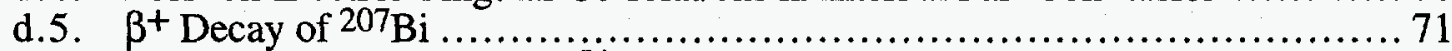

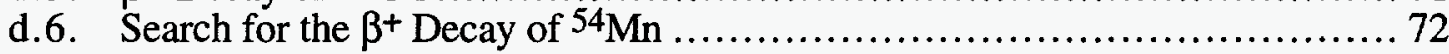

d.7. Transitions in Cold Crystalline Plasmas to Infinite Matter Behavior.............. 73

d.8. Behavior of Bunched Beams in a Storage Ring and in Simulations ........... 74

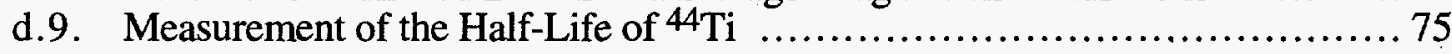

E. EQUIPMENT DEVELOPMENT AT THE ATLAS FACILITY ............. 76

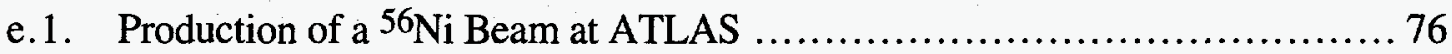

e.2. Production Rate and Transport Efficiency Calculations for Secondary Beams. 77

e.3. Development of a Gas Target for In-Flight Production of Exotic Beams ..... 77

e.4. Development of Beam Diagnostic Devices for Low Intensity Beams............ 78

e.5. A New Large Area Focal Plane Detector for the ATLAS Split Pole

Spectrograph................................................... 79

e.6. Construction of a Large Area Si-Detector Array for Measuring (d,p)

Reactions in Inverse Kinematics ..................................... 80

e.7. The Move of Gammasphere from LBNL to ANL.............................. 80

e.8. Status of the Argonne-Notre Dame BGO Gamma-Ray Facility at ATLAS ..... 81

e.9. Development of Fast, Position-Sensitive Micro-Channel Plate

Detectors for the FMA .................................................. 81

e.10. Coulomb Excitation of Radioactive Beams Prepared by the FMA ........... 82

e.11. Studies of Proton Dripline Nuclei from $A=50$ to 100 Using Neutron

Detectors, Microball and Gammasphere ............................... 83

e.12. Temperature Dependence of the Scintillation Properties of $\mathrm{BaF}_{2} \quad \ldots \ldots \ldots \ldots \ldots 84$

e.13. Rotating Target Wheel for the FMA Gamma-Ray Chamber .................. 84

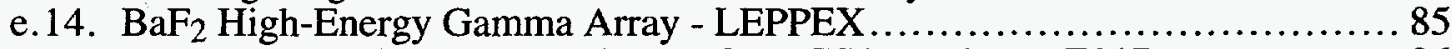

e.15. A Beam Vertexing Detector System for AGS Experiment E917 ............ 86

e.16. Nuclear Target Development ........................................... 87

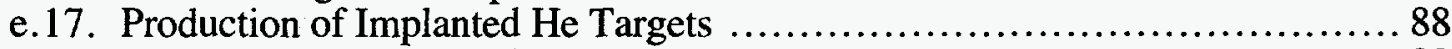

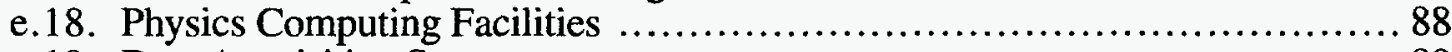

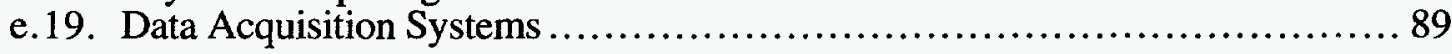


F. ASSISTANCE TO OUTSIDE USERS OF ATLAS .................... 90

a. Experiments Involving Outside Users........................................... 91

b. Outside Users of ATLAS and of ATLAS Technology -$10 / 1 / 95$ - 9/30/96........................................................... 95

c. Summaries of the Continuing User Programs for FY $1996 \ldots \ldots \ldots \ldots \ldots \ldots . . .97$

c.a.1. Nuclear Physics ............................................... 97

c.a.2. Atomic Physics.............................................................. 97

d. ATLAS - Technology Transfer .................................... 99

\section{OPERATION AND DEVELOPMENT}

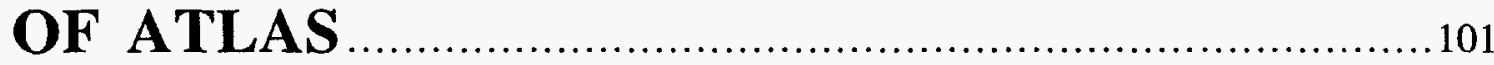

A. OPERATION OF THE ACCELERATOR .............................. 103

a. First Operation of the new 14-GHz ECR Ion Source...............................103

b. Radioactive Beams via Reactions in the Beam Line.................................104

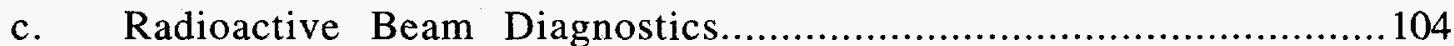

d. ATLAS Control System.............................................. 105

e. ATLAS Cryogenic System …..................................... 105

B. ACCELERATOR PHYSICS AND LINAC DEVELOPMENT ..............105

a. Superconducting Accelerating Structures for Low-Beta Heavy-lons...........106

b. Development of a New Type of Beam Chopper for ATLAS ................107

C. ACCELERATOR PHYSICS RELATED TO A FUTURE ADVANCED

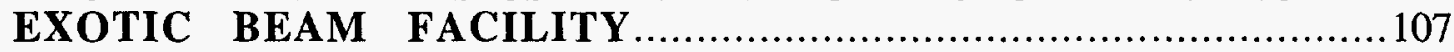

a. Acceleration of Very Low Velocity and q/m Radioactive Ions....................107

b. Superconducting Driver Linac Studies ................................... 108

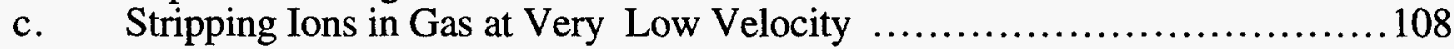

d. Ion Sources and Targetry for Use in an Advanced Radioactive Beam Facility 109

e. Ion Optics of Energy-Spread-Compensated Isobar Separators.................109

\section{MEDIUM-ENERGY NUCLEAR PHYSICS

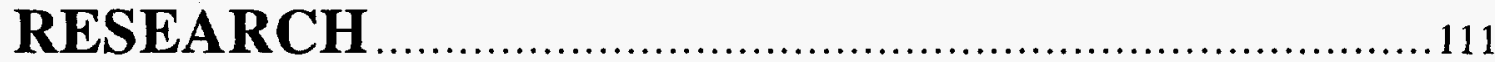

A. SUBNUCLEONIC EFFECTS IN NUCLEII .........................113

a.1. The Energy Dependence of Nucleon Propagation in Nuclei as

Measured in the $(e, e$ 'p) Reaction ....................................113

a.2. Measurements of Deuteron Photodisintegration up to $4.0 \mathrm{GeV}$.........................11 14

a.3. Electroproduction of Kaons and Light Hypernuclei ..............................115

a.4. Momentum Transfer Dependence of $\mathrm{H}\left(\mathrm{e}, \mathrm{e}^{\prime} \mathrm{K}^{+}\right) \mathrm{Y}$ Reactions .................116

a.5. A Study of Longitudinal Charged-Pion Electroproduction in

D, ${ }^{3} \mathrm{He}$, and ${ }^{4} \mathrm{He}$ 
a.6. Precise Measurements of the Inclusive Spin-dependent Quasi-elastic

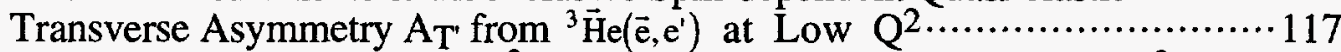

a.7. Measurement of the Neutron $\left({ }^{3} \mathrm{He}\right)$ Spin Structure Function at Low $Q^{2}$ : A Connection Between the Björken and Gerasimov-Drell-Hearn Sum Rules 118

a.8. The Fundamental $\gamma \mathrm{n} \rightarrow \pi^{-}$p Process in ${ }^{2} \mathrm{H},{ }^{4} \mathrm{He}$, and ${ }^{12} \mathrm{C}$ in the 1.2 - $6.0 \mathrm{GeV}$ Region

a.9. Measurement of Proton Polarization in the $d(\gamma, p) n$ Reaction .................119

a.10. Short-Orbit Spectrometer for Hall C at CEBAF .......................... 120

a.11. ${ }^{3} \mathrm{He}$ Target for $\mathrm{Hall} \mathrm{C}$ at CEBAF.......................................... 120

a.12. Laser-Driven Polarized Hydrogen and Deuterium Internal Target ...............120

a.13. Test of the Laser Driven Polarized Target at IUCF and Measurement of the $\vec{d}(\vec{p}, p p) n$ Reaction.

a.14. Polarized Tritium Target Development ............................................121

a.15. HERMES, Measurements of Spin-Structure Functions and Semi-Inclusive Asymmetries for the Proton and Neutron at HERA . ..........122

a.16. A Dual Radiator Ring Imaging Cerenkov Counter for HERMES ............... 124

a.17. Nuclear Transparency and Longitudinal Asymmetry in $A\left(e, e^{\prime} \rho^{0}\right)$ at High Energies ...................................................... 125

a. 18. Deep-Inelastic Muon Scattering from Nuclei with Hadron Detection.......... 126

a.19. Measurement of $\bar{d} / \bar{u}$ in the Nucleon ..................................... 127

\section{THEORETICAL PHYSICS}

\section{A. NUCLEAR DYNAMICS WITH SUBNUCLEONIC DEGREES OF} FREEDOM

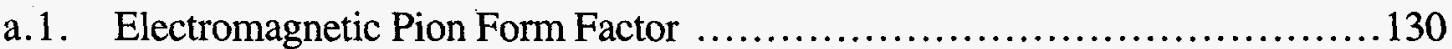

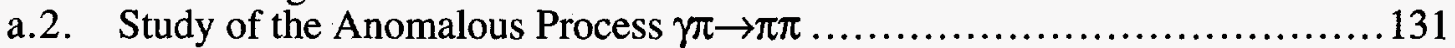

a.3. Electromagnetic Charged and Neutral Kaon Form Factors..............................131

a.4. Quark and Diquark Confinement beyond Rainbow/Ladder

Approximation

a.5. Renormalization and Chiral Symmetry Breaking in Quenched QED in

Arbitrary Covariant Gauge .............................................. 132

a.6. Dynamical Chiral Symmetry Breaking and Confinement at Finite

Temperature....................................................... 132

a.7. Bethe-Salpeter Equation Studies of SUf(3) Mesons.......................... 133

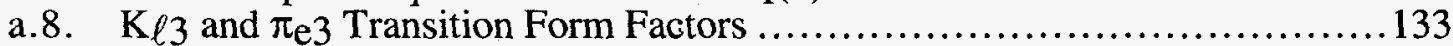

a.9. Electromagnetic Nucleon Form Factors ............................... 134

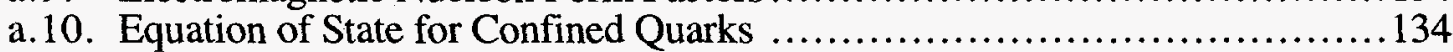

a.11. Thermodynamics and Bound State Properties at Finite Temperature ...........135

a.12. Bethe-Salpeter Equation Studies of Light Quark Mesons ................... 135

a.13. Nucleons in a Nuclear Medium.............................................. 135

a.14. The Strong Running Coupling from an Approximate Gluon DysonSchwinger Equation .............................................. 136

a.15. A Dynamical $\eta '-$ Mass from an Infrared Enhanced Gluon Exchange............137

a.16. Production of Mesons and Nucleon Resonances at GeV Energies............... 137

a. 17. Exclusive Diffractive Processes and the Quark Substructure

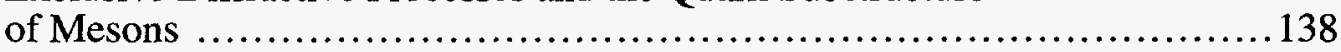

a.18. The E2/M1 Ratio of the $\Delta \leftrightarrow \gamma \mathrm{N}$ Transition Within the Chiral Constituent Quark Model. 
a.19. Phenomenological Coupled-Channel Model for $\pi N$ and $\gamma \mathrm{N}$ Reactions With $\mathrm{N}^{*}$ Excitations

a.20. The $\Delta$ Dynamics in ${ }^{3} \mathrm{He}\left(\mathrm{e}, \mathrm{e}^{\mathrm{e}} \Delta^{++}\right)$Reaction .............................139

a.21. Chiral Perturbation Theory and the $\mathrm{pp} \rightarrow \mathrm{pp} \pi^{0}$ Reaction Near Threshold .... 140

a.22. Neutral Pion Photoproduction on Deuterium in Baryon Chiral Perturbation

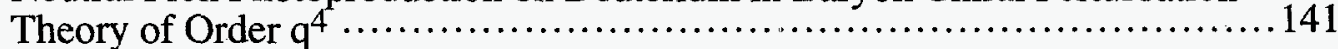

a.23. Chiral Transparency ................................................... 141

a.24. Near Threshold $\eta$ Production in Proton-Proton Collisions ................. 141

a.25. Pion Absorption Cross Sections for ${ }^{2} \mathrm{H}$ and ${ }^{3} \mathrm{He}$ in the $\Delta$-Isobar Region ..... 142

a.26. Medium Effects on Binary Collisions with the $\Delta$ Resonance............... 142

a.27. The $\pi \mathrm{N}$ and $\gamma \mathrm{N}$ Reactions Within the Chiral Constituent Quark Model ........ 142

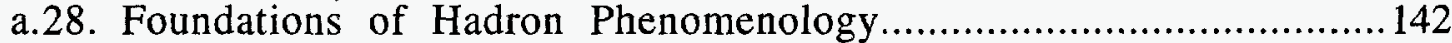

a.29. Electromagnetic Currents of the Nucleon Resonances ...................... 143

a.30. Current Matrices, Covariant Current Kernels, and Form Factors ............ 144

B. NUCLEAR FORCES AND NUCLEAR SYSTEMS ...................145

b.1. Variational Monte Carlo Calculations of Light p-shell Nuclei ................... 146

b.2. Green's Function Monte Carlo Studies of p-Shell Nuclei ..................... 147

b.3. Charge-independence Breaking in Light Nuclei ........................... 150

b.4. Microscopic Calculation of Elastic and Transition Form Factors of Lithium .. 151

b.5. Short-Range Structure of the Deuteron and Other Nuclei .................... 151

b.6. Three-body Correlations in Nuclear and Neutron Matter ................... 152

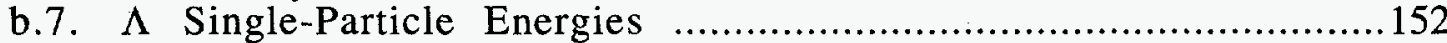

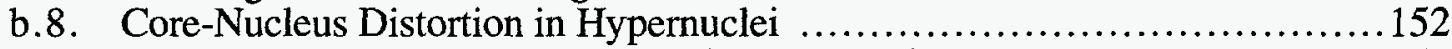

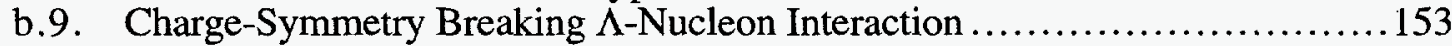

b. 10. Suppression of the $\Lambda-\Sigma$ Coupling in Nuclear Matter ....................... 153

b.11. Nuclear Core Dynamics and Rearrangement Energy for Hypernuclei ........ 153

C. NUCLEAR STRUCTURE AND HEAVY-ION REACTIONS ..........154

c. 1. Momentum Distributions of ${ }^{7}$ Be Fragments in Breakup Reactions of ${ }^{8} \mathrm{~B} \ldots \ldots 155$

c.2. Momentum Distributions in Nuclear Induced Breakup Reactions ............. 155

c.3. Pairing Gap in the Inner Crust of Neutron Stars ......................... 156

c. 4. Coupled Channels Analysis of High Precision Fusion Data ................ 156

c.5. Single Particle States in the Heaviest Elements............................... 157

c.6. Nuclear Energy Surfaces in the A 180 Mass Region............................ 158

c. 7 . Very Extended Shapes in Nuclei ....................................... 159

c.8. Many Body Wave Functions .............................................. 160

c.9. Nuclear Structure near the Proton Drip Line ................................ 160

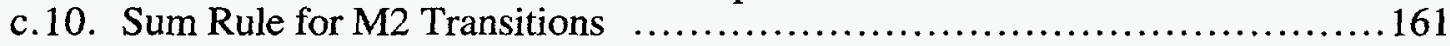

\section{ATOMIC THEORY AND FUNDAMENTAL QUANTUM} MECHANICS

d.1. Interactions of High-Energy Photons with Matter ........................ 161

d.2. Interactions of Fast Charged Particles with Matter.......................... 162

d.3. The Electron .......................................................... 162

d.4. Tight Binding Hamiltonians and Quantum Turing Machines ................. 162 
e.1. Theory Workshop on QCD-based Studies of Hadron Spectroscopy and Interactions.

e.2. Ninth Annual Midwest Nuclear Theory Get-Together.

\section{ATOMIC AND MOLECULAR PHYSICS RESEARCH}

a. Experimental Separation of Compton and Rayleigh X-Ray Scattering from

b. Argon KM Photoelectron Satellites

c. Non-Dipole Resonant X-Ray Raman Spectroscopy: Polarized Inelastic

Scattering at the K-Edge of $\mathrm{Cl}_{2}$

d. Xenon Spectator and Diagram $\mathrm{L}_{3}-\mathrm{M}_{4,5} \mathrm{M}_{4,5}$ Auger Intensities Near

the L3 Threshold ....

e. Angular Correlation Between $\mathrm{K} \alpha$ Photons and $\mathrm{L}_{2,3}-\mathrm{M}_{2,3} \mathrm{M}_{2,3}$ Auger

Electrons Following Argon 1s Photoionization

f. Electron-Recoil Ion Coincidence Study of Helium and Neon Double

Photoionization at Low Photon Energies.

g. Laser-Cooled and Trapped Li Atoms ....................................... 173

h. Two-Photon Decay in H- and He-Like Nickel ........................... 174

i. E1-M1 Damping Interference in the Electric Field Quenching of Metastable $\mathrm{Ar} 17+$ Ions.

j. Extreme-Ultraviolet Wavelength and Lifetime Measurements in Highly-

Ionized Krypton...

k. The Coulomb Explosion of $173-\mathrm{MeV} \mathrm{HeH}^{+}$Ions Traversing

Carbon Foils

\section{OTHER EDUCATIONAL ACTIVITIES IN THE PHYSICS DIVISION}

a. Enhancement of Minority Involvement in DOE Nuclear Physics Programs ... 179

b. Nuclear Physics Award for Faculty in Undergraduate Institutions ............. 179

c. Scientific Support of SciTech Museum Exhibits and Outreach Programs...... 180

d. The "Museum in a School" Program .......................................... 180

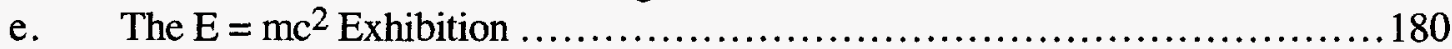

Staff List.

Publications 


\section{HEAVY-ION NUCLEAR PHYSICS RESEARCH}

\section{OVERVIEW}

The heavy-ion program in the Argonne Physics Division spans a broad range of activities from the exploration of the multiple facets of nuclear structure at the limits of the valley of stability to the delineation of the properties of superdeformed nuclei; from the characterization of reactions in the vicinity of the Coulomb barrier to investigations of the effects of the strong fields generated in collisions between very heavy nuclei; from the measurement of cross sections of astrophysics interest to the development of the instrumentation and techniques required to perform experiments with radioactive beams of small intensity. This is a versatile program with involvement of many university groups and outside institutions, characterized by extensive collaborations between in-house and outside researchers. Most of these studies are carried out at the ATLAS accelerator, but parts of the program take advantage of world-class instrumentation available elsewhere.

Some of the main goals of the program can be summarized as follows:

- Study the evolution of nuclear structure with spin, excitation energy and mass. In particular, understand the physics of superdeformed nuclei by comprehensive measurements of spins, parity, excitation energies, state lifetimes, alignments, and limits of regions of superdeformation with the highest detection sensitivity (Gammasphere).

- Determine the properties of nuclei at the limits of proton-stability by measuring (1) groundstate properties, (2) particle decay modes (proton, alpha and beta decay) and (3) gamma decay of excited states taking advantage of the selectivity offered by the Fragment Mass Analyzer (FMA).

- Produce and study isotopes of very heavy elements utilizing the exceptional separation capabilities of the FMA and the intense beams available at ATLAS with the ECR sources.

- Develop and utilize short-lived nuclear beams (1) to measure reactions of astrophysics interest (for example, $17,18 \mathrm{~F}$ for the hot $\mathrm{CNO}$ cycle) and (2) to study the properties of proton-rich nuclei (for example, single particle states near the unstable doubly-magic nucleus $56 \mathrm{Ni}$ ). 
- Study the effects of strong fields produced in near-barrier collisions of very heavy-ions by measuring electron and positron spectra.

- Establish the degrees of freedom important for the description of nuclear reactions between complex nuclei in the vicinity of the Coulomb barrier by measuring (1) cross sections of all competing channels, (2) fission time scales and (3) correlations between fragments.

- Pursue unique applications with energetic heavy-ion beams such as defects in high Tc superconductors, and accelerator mass spectrometry of heavy (transactinide) radioisotopes.

- Understand the behavior of nuclear matter at high density by exploring collisions between relativistic heavy ions.

Substantial progress has been made during the last year in many of the areas listed above. Among the most important are:

- For the first time, the spins, parity and excitation energy of an excited superdeformed (SD) band (in ${ }^{194} \mathrm{Hg}$ ) have been determined from one-step decay transitions to the low-lying yrast states. Two important results are: (1) the band is "identical" to the SD band of ${ }^{192} \mathrm{Hg}$, yet the spins between the two bands differ by one unit and the parities are opposite, (2) excited SD bands of ${ }^{194} \mathrm{Hg}$ most likely correspond to octupole vibrations and not to quasiparticle excitations.

- Differential lifetime measurements of unprecedented accuracy have been performed in the SD bands of $151,152,154,155 \mathrm{Dy}$ and $192,194 \mathrm{Hg}$. The results show that identical bands are characterized by identical quadrupole moments; i.e. changes in mass are compensated by shell correction effects (i.e. changes in alignment and/or pairing).

- Lifetime measurements show that quadrupole moments of SD bands near A = 150 depend strongly on the intrinsic quasiparticle configurations. Little dependence has been found for SD bands near $A=190$.

- Experiments on ${ }^{192} \mathrm{Tl}$ show for the first time that alignments observed in twoquasiparticle SD excitations can be completely accounted for by the additivity of their one-quasiparticle constituents.

- The number of hours for experiments with exotic beams has risen from 168 in 1995 to 672 in 1996. Several new, high efficiency detectors have been developed for physics with these low intensity beams.

- Besides the $18 \mathrm{~F}$ beam $\left(\mathrm{t}_{1 / 2}=1.8 \mathrm{~h}\right)$ developed last year, two new beams of short-lived nuclei have been developed and are now available with intensities sufficient for experiments $\left(>10^{5 / \mathrm{s}}\right):{ }^{17} \mathrm{~F}(\mathrm{t} 1 / 2=64.5 \mathrm{~s})$ and ${ }^{56} \mathrm{Ni}\left(\mathrm{t}_{1} / 2=5.9 \mathrm{~d}\right)$. The production techniques for these two beams are different: ${ }^{17} \mathrm{~F}$ is produced in flight at ATLAS with the ${ }^{16} \mathrm{O}(\mathrm{d}, \mathrm{n})$ or ${ }^{17} \mathrm{O}(\mathrm{p}, \mathrm{n})$ reactions in a gas cell, while ${ }^{56 \mathrm{Ni}}$ is produced in an irradiation at the Argonne Intense Pulsed Neutron Source (IPNS) with the ${ }^{58} \mathrm{Ni}(\mathrm{p}, \mathrm{p} 2 \mathrm{n})$ reaction. 
- First measurements of the ${ }^{18} \mathrm{~F}(\mathrm{p}, \gamma)$ and ${ }^{17} \mathrm{~F}(\mathrm{p}, \alpha)$ reactions at energies of importance to astrophysics (break-out from the hot $\mathrm{CNO}$ cycle) have been performed. A new resonance was found for the ${ }^{18} \mathrm{~F}(\mathrm{p}, \alpha)$ reaction which dominates this reaction at energies corresponding to temperatures found in novae.

- Results from the APEX experiment demonstrate the absence of sharp structures in positron energy, and positron-electron sum- energy spectra in $U+T h$ collisions.

- APEX measurements with radioactive sources have confirmed the initially surprising theoretical prediction of marked differences in the angular correlations of electron-positron pairs associated with the internal pair conversion of electric and magnetic dipole transitions in high- $Z$ nuclei.

- The new proton radioactivity ${ }^{177} \mathrm{Tl}$ has been discovered, bringing to eight the number of proton-emitters discovered at ATLAS and completing the series of proton emitters for $Z=69-83$. A spherical shell model calculation of the spectroscopic factors for proton decay of all elements with $64<Z<82$ agrees remarkably well with the data.

- Ground state mass excesses for ten nuclei at and beyond the proton drip line have been obtained. The results underline the deficiencies of a number of mass predictions. The new isotopes $172,173,174 \mathrm{Hg}$ and ${ }^{178} \mathrm{Tl}$ have been discovered.

- Measurements with the recoil-decay tagging technique (RDT) at the FMA show that ${ }^{178} \mathrm{Hg}$ is the lightest $\mathrm{Hg}$ isotope to exhibit coexistence between an oblate groundstate and a prolate excited structure. Also, in ${ }^{176} \mathrm{Hg}$ the groundstate evolves from a oblate towards a spherical shape.

- The RDT method was used to perform gamma-ray spectroscopy beyond the proton drip line in ${ }^{147} \mathrm{Tm},{ }^{157} \mathrm{Ta}$ and ${ }^{161} \mathrm{Re}$.

- Strong hindrance of fission has been found for target-like ejectiles in deep inelastic collisions, in qualitative agreement with observations based on prescission neutron emission, and the emission of high-energy (GDR) gamma rays in similar systems.

- First experiments with the LEPPEX $\mathrm{BaF}_{2}$ array have been performed. The GDR gamma rays measured in coincidence with evaporation residues at the FMA show that marked differences exist between the shape of the resonance in inclusive spectra and in spectra gated on a specific residue mass.

- Multiple particle correlation techniques for studying reaction mechanisms have been demonstrated to make definite spin assignments to resonances observed in the ${ }^{12} \mathrm{C}+{ }^{12} \mathrm{C}\left(3^{-}\right)$system.

- The fusion excitation function in the ${ }^{78} \mathrm{Kr}+{ }^{100} \mathrm{Mo}$ system has been extended into the micro-barn region below the barrier by using a new time-of-flight technique at the FMA. 
- The prototype Silicon pixel detectors for the PHOBOS experiment at RHIC have been successfully tested, and the design of the multiplicity and vertex detector is complete. PHOBOS has entered the construction phase.

- As part of the E917 experiment at the AGS, a new beam-vertexing detector based on scintillating fiber ribbons was designed, constructed, tested and successfully used in the 1996/1997 Au run. A resolution of 300 micro-meters was obtained, which provides the unambiguous tracking of the beam, the determination of the reaction plane and a basis for the measurement of flow.

- Simulations are being carried out to study in detail the nature of the transition of the ordered state of confined ions from a multi-layered shell structure to the body-centered cubic order of infinite ionic matter.

- The way in which transverse excitations can couple into longitudinal ones in bunched cold ion beams has been investigated.

- Site preparations for the installation of Gammasphere at ATLAS are well underway.

\section{A. EXPERIMENTS WITH SECONDARY BEAMS AND STRUCTURE OF NUCLEI AT THE LIMITS OF STABILITY}

The study of the properties of nuclei at the very limits of stability is currently a subject of renewed interest, as can be seen from the conclusions expressed in the recent Long Range Plan for Nuclear Science prepared by the Nuclear Science Advisory Committee (NSAC). Theoretical interest in nuclei far from stability was also stimulated by the conceptual developments of future Radioactive Beam Facilities. With the facilities available at ATLAS, it is possible to address some of the physics issues in a major way either by accelerating beams of short-lived nuclei or by exploiting the capabilities of the Fragment Mass Analyzer (FMA).

The efforts towards the acceleration of beams of short-lived nuclei have considerably expanded. The number of hours for experiments with beams of short-lived nuclei has risen from 168 in 1995 to 672 in 1996 . Besides the ${ }^{18} \mathrm{~F}$ beam $\left(\mathrm{t}_{1 / 2}=1.8 \mathrm{~h}\right)$ developed last year, two new beams of short-lived nuclei have been developed and are now available with intensities sufficient for experiments $(\mathrm{I}>105 / \mathrm{s}):{ }^{17} \mathrm{~F}\left(\mathrm{t}_{1 / 2}=64.5 \mathrm{~s}\right)$ and ${ }^{56} \mathrm{Ni}\left(\mathrm{t}_{1 / 2}=5.9 \mathrm{~d}\right)$. These beams have been used in measurements of interest either in nuclear astrophysics or in nuclear structure.

The FMA has emerged as a unique and powerful tool for selecting nuclei at the very limits of stability. In fact, the proton drip line has been reached and even passed as demonstrated in measurements of groundstate proton decay. For example, the new proton radioactivity $177 \mathrm{Tl}$ has been discovered, bringing to eight the number of proton-emitters discovered at ATLAS and completing the series of proton emitters for $Z=69-83$. The gamma decay of nuclei near the drip line is also studied with the recoil-decay tagging (RDT) technique where prompt gamma rays are associated with a particular isotope through a correlation with the characteristic alpha decay. 


\section{a.1. Study of the Single Particle Structure Around the Closed-Shell Nucleus 56 Ni Using}

a Radioactive 56 Ni Beam (K. E. Rehm, I. Ahmad, F. Borasi, C. N. Davids, S. Fischer, J. P. Greene, G. Hackman, B. Harss, D. Henderson, W. Henning, R. V. F. Janssens, C. L. Jiang, V. Nanal, J. Nolen, R. C. Pardo, P. Reiter, J. P. Schiffer, D. Seweryniak, A. H. Wuosmaa, M. Paul, * J. Görres, $\dagger$ R. E. Segel,$\ddagger$ and M. Wiescher, $\dagger)$

The doubly closed shell nucleus $56 \mathrm{Ni}$ is an important test case in many areas of nuclear physics since it is probably the heaviest $\mathrm{N}=\mathrm{Z}$ nucleus for which a beam with sufficient intensity can be produced. With the excellent beam properties of ATLAS, high resolution studies of single particle states, populated in fewnucleon transfer reactions, should become feasible. In the area of astrophysics, ${ }^{56} \mathrm{Ni}$ is an interesting nucleus since its high binding energy accounts for the abundance of ${ }^{56} \mathrm{Fe}$ in the universe. Furthermore, the small Q-value for the ${ }^{56} \mathrm{Ni}(\mathrm{p}, \gamma){ }^{57} \mathrm{Cu}$ reaction provides a bottle neck in the rp-process.

We have started a program to measure the spectroscopic factors for one-particle transfer reactions on $56 \mathrm{Ni}$. The technique used to produce the ${ }^{56} \mathrm{Ni}$ beam is described in a separate contribution. In a series of test experiments with stable $58 \mathrm{Ni}$ beams we have first measured the spectroscopic factors for the ${ }^{12} \mathrm{C}\left({ }^{58} \mathrm{Ni},{ }^{11} \mathrm{C}\right){ }^{59} \mathrm{Cu}$ and ${ }^{12} \mathrm{C}\left({ }^{58} \mathrm{Ni},{ }^{11} \mathrm{C}\right){ }^{59} \mathrm{Ni}$ reactions. The $\mathrm{Q}$-values for the ${ }^{56} \mathrm{Ni}$ induced reactions, however, are quite negative and result in very small cross sections for one-particle transfer reactions. Since a considerable increase in yield can be obtained by using a deuteron target, we have investigated the possibility to measure the $\mathrm{d}\left({ }^{56} \mathrm{Ni}, \mathrm{p}\right){ }^{57} \mathrm{Ni}$ reaction.

In several short test runs with stable beams of ${ }^{58} \mathrm{Ni}$ and ${ }^{28} \mathrm{Si}$ we have tested the FMA for the measurement of one-particle transfer reactions in inverse kinematics, e.g. $\mathrm{d}\left({ }^{28} \mathrm{Si},{ }^{29} \mathrm{Si}\right)$ p, by detecting ${ }^{29} \mathrm{Si}$ ions at forward angles in the FMA in coincidence with protons detected in a $x-y$ position-sensitive Si strip detector located at backward angles in the scattering chamber of the FMA. The angular distribution for the $(d, p)$ reaction is obtained from the proton angles. The coincidence with the heavy beam-like particles in the FMA is needed in order to separate reactions induced by the various isobars $\left({ }^{56} \mathrm{Co}, 56 \mathrm{Fe}\right)$ that are present in the radioactive $56 \mathrm{Ni}$ beams.

Since the FMA can only bend particles with an electric rigidity $\mathrm{E} / \mathrm{q}<10$, the energy of the outgoing Ni-like particles needs to be reduced to about $150 \mathrm{MeV}$. To minimize background reactions, a $7 \mathrm{mg} / \mathrm{cm}^{2}$ stack of $\mathrm{Au}$ foils was chosen to reduce the energy. The Coulomb barrier for the $\mathrm{Ni}+\mathrm{Au}$ system (in the laboratory system) is $-290 \mathrm{MeV}$ so that reactions with the $\mathrm{Au}$ are expected to be negligible at $\mathrm{E}_{\mathrm{lab}}=250 \mathrm{MeV}$. The Au foil stack also proved to be useful in connection with the Z-identification for the beam-like particles, as discussed below.

A general problem in experiments with radioactive beams is the presence of contaminating isobars in the ion beam that can also initiate reactions with the target. For a ${ }^{56} \mathrm{Ni}$ beam, these isobars are ${ }^{56} \mathrm{Co}\left(\mathrm{T}_{1 / 2}\right.$

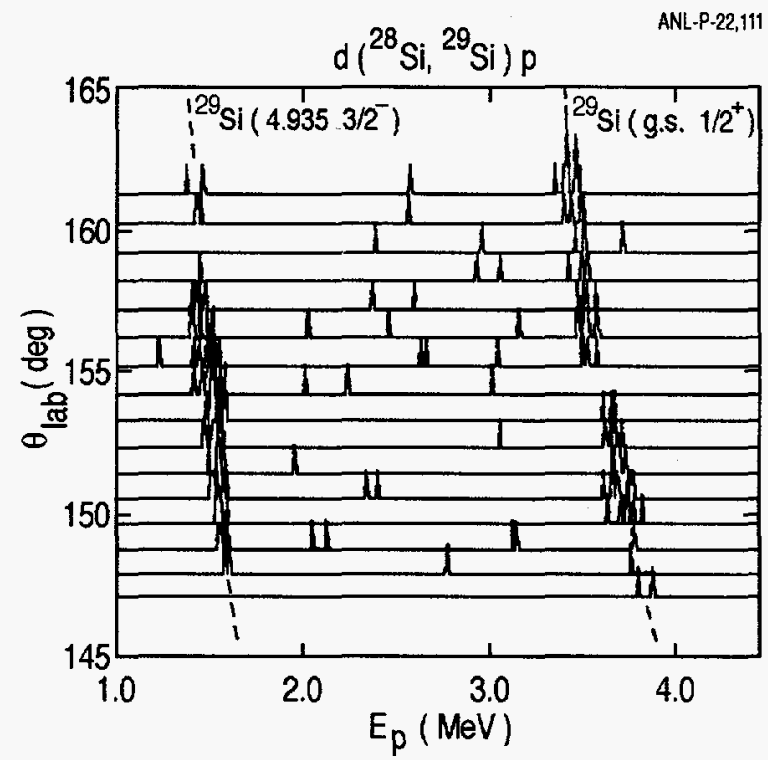

Fig. I-1. Proton energy vs. angle obtained in the reaction d $\left({ }^{28}{ }_{S i},{ }^{29}\right.$ Si $) p$. The lines indicate kinematic curves expected for the population of the $1 / 2+$ groundstate and the $3 / 2+$ excited state.

$=79 \mathrm{~d}$ ) and ${ }^{56} \mathrm{Fe}$ (stable). To identify the reaction products ${ }^{57} \mathrm{Ni},{ }^{57} \mathrm{Co}$ and ${ }^{57} \mathrm{Fe}$ produced in inverse $(d, p)$ reactions, the nuclear charge of the outgoing particles has to be measured. To achieve this we used the $7 \mathrm{mg} / \mathrm{cm}^{2}$ thick Au foil stack as a passive absorber which slows down the $\mathrm{Ni}, \mathrm{Co}$, and $\mathrm{Fe}$ products

\footnotetext{
*Hebrew University, Jerusalem, Israel, $\nmid$ University of Notre Dame, $\ddagger$ Northwestern University
} 
$(E=250 \mathrm{MeV}$ ) to energies of $\sim 142,150$ and $158 \mathrm{MeV}$, respectively. A measurement of the residual energy in a Si detector mounted in an ionization chamber behind the focal plane can then be used together with the energy loss and the time-of-flight signal to identify the different elements. This technique was tested with stable particles using a mixed beam of $58 \mathrm{Ni} / 58 \mathrm{Fe}$ which was accelerated to $250 \mathrm{MeV}$ through ATLAS and transported to the FMA where it produced mass 59 particles via inverse $(d, p)$ reactions on a $C D_{2}$ target. Figure I-1 shows a two-dimensional spectrum of proton energy vs. angle obtained in a short test run for the reaction $\mathrm{d}\left({ }^{28} \mathrm{Si},{ }^{29} \mathrm{Si}\right)$. The protons were measured in coincidence with ${ }^{29} \mathrm{Si}$ particles detected in the focal plane of the FMA at $\mathrm{E}_{1 \mathrm{ab}}=125 \mathrm{MeV}$ using a
$120 \mu \mathrm{g} / \mathrm{cm}^{2} \mathrm{CD}_{2}$ target. The lines indicate the kinematic curves expected for population of the $\left(1 / 2^{+}\right)$ (g.s.) and $3 / 2^{+-}\left(E_{X}=1.27 \mathrm{MeV}\right)$ state, respectively. These data were obtained with a total accumulated charge of only $18 \mathrm{pnC}$ and one Si detector covering a solid angle of $0.25 \mathrm{sr}$. Figure $\mathrm{I}-2$ gives a $\Delta \mathrm{E}-\mathrm{E}$ spectrum measured in the ionization chamber for a mixed beam of ${ }^{56} \mathrm{Fe},{ }^{56} \mathrm{Co}$ and ${ }^{56} \mathrm{Ni}$ showing the $\mathrm{Z}$ separation that can be achieved by this technique.

Together with a new large-area Si-detector array (see contribution e.6) this system provides the parameters necessary for a measurement of $(d, p)$ reactions in inverse kinematics using low intensity radioactive ion beams. First experiments with ${ }^{56} \mathrm{Ni}$ beams have begun.

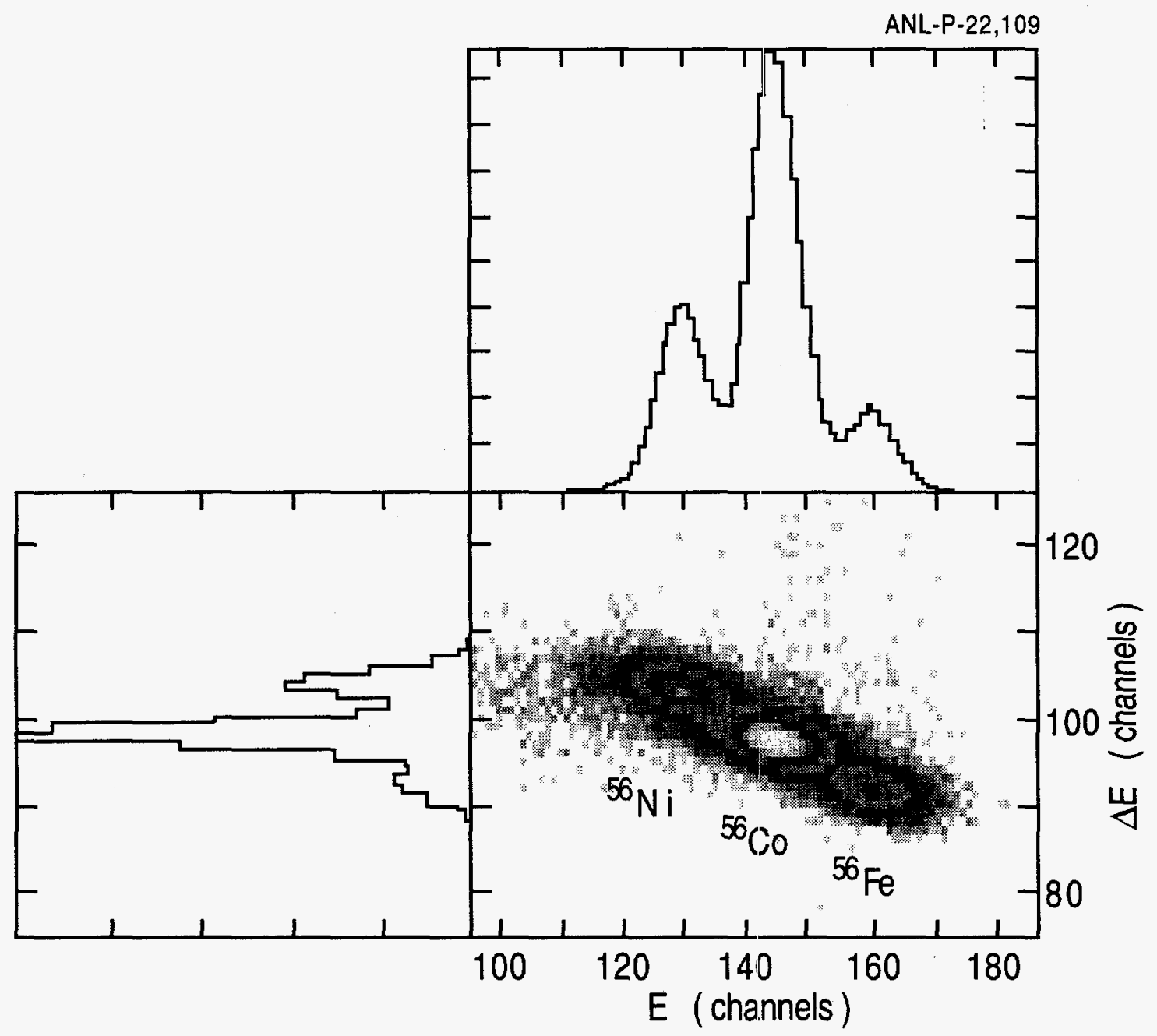

Fig. I-2. Z separation achieved with the technique described in the text. 


\section{a.2. Search for the Optimum One-Neutron Transfer Reaction for Experiments with ${ }^{56} \mathrm{Ni}$ Beams (D. Seweryniak, K. E. Rehm, C. L. Jiang, D. Ackermann, D. Blumenthal, W. Henning, R. V. F. Janssens, J. Nolen, R. C. Pardo, J. P. Schiffer, A. H. Wuosmaa, M. Paul, * J. Görres, $\dagger$ R. E. Segel, $\ddagger$ and M. Wiescher, $\dagger$ )}

For our studies of spectroscopic factors around the doubly closed shell nucleus ${ }^{56} \mathrm{Ni}$ various possible target nuclei ranging from ${ }^{2} \mathrm{H}$ to ${ }^{12} \mathrm{C}$ have been considered. The one-particle transfer reactions ${ }^{12} \mathrm{C}\left({ }^{56} \mathrm{Ni},{ }^{57} \mathrm{Ni}\right){ }^{11} \mathrm{C}$ or ${ }^{12} \mathrm{C}\left({ }^{56} \mathrm{Ni},{ }^{57} \mathrm{Cu}\right){ }^{11} \mathrm{~B}$ have the advantage that with ${ }^{12} \mathrm{C}$ a stable, monoisotopic and homogenous target is available. The angular distributions at bombarding energies of 5-7 MeV/u are bell-shaped, and independent of the transferred angular momentum. The peak of the angular distribution for the projectile-like products occurs at angles which are sufficiently large that the particles of interest can be cleanly separated from the incident beam. The cross sections are well described by DWBA calculations if the normalization constant has been determined. This can achieved, e.g. by a comparison of light- and heavy-ion induced reactions performed at similar energies on a neighboring stable nucleus.

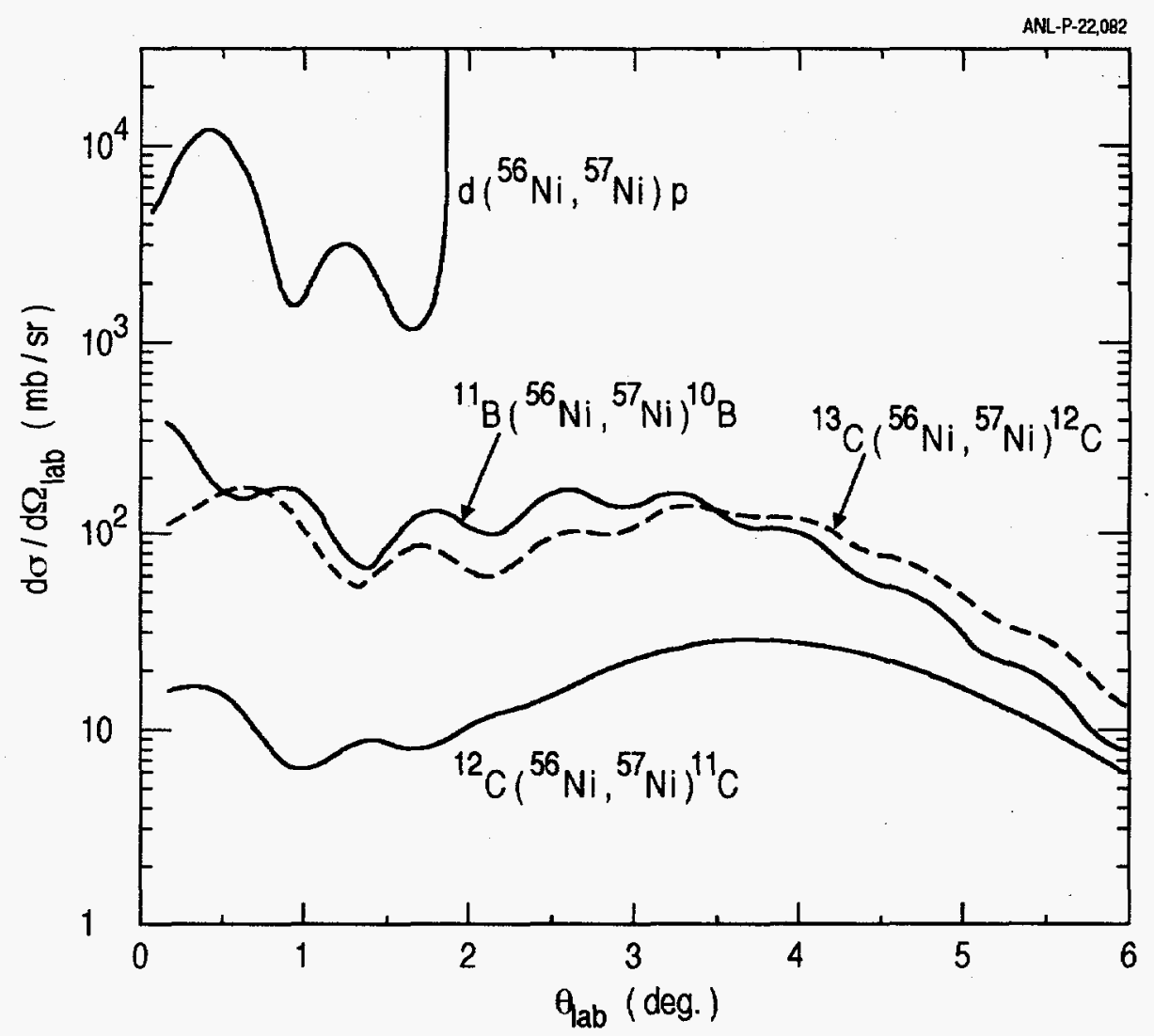

Fig. 1-3. Calculated angular distributions for one-neutron transfer reactions.

The inverse $(\mathrm{d}, \mathrm{p})$ reaction $\mathrm{d}\left({ }^{56} \mathrm{Ni},{ }^{57} \mathrm{Ni}\right) \mathrm{p}$ reaction, on the other hand, shows $\ell$-dependent angular distributions which are theoretically well understood. The heavy reaction products, however, are emitted at very small angles where it is difficult to separate them from the incident beam.

We have investigated the advantages and disadvantages of both reactions by studying one-nucleon transfer reactions with stable ${ }^{58} \mathrm{Ni}$ beams using the split-pole spectrograph $\left[{ }^{12} \mathrm{C}\left({ }^{58} \mathrm{Ni},{ }^{59} \mathrm{Ni}\right){ }^{11} \mathrm{C}\right]$ and the FMA $\left[\mathrm{d}\left({ }^{58} \mathrm{Ni}, 59 \mathrm{Ni}\right) \mathrm{p}\right]$, respectively.

In the spectrograph experiment the $\mathrm{Ni}$-like particles emitted at $\theta=2.5^{\circ}-5^{\circ}$ were identified in the focal plane with a hybrid position-sensitive detector which allowed single mass and $\mathrm{Z}$-separation in the mass 60 region. The coincident $\mathrm{C}$-like particles were detected in a large area $\left(25 \mathrm{~cm}^{2}\right), 50$-strip $\mathrm{Si}$ detector mounted at the 
appropriate angle. From the energy-angle correlation measured with the Si-detector a $\mathrm{Q}$-value resolution of $500 \mathrm{keV}$ has been achieved.

The normalization constants for the one-neutron and one-proton transfer reactions ${ }^{58} \mathrm{Ni}\left({ }^{12} \mathrm{C},{ }^{11} \mathrm{C}\right){ }^{59} \mathrm{Ni}$ and ${ }^{58} \mathrm{Ni}\left({ }^{12} \mathrm{C},{ }^{11} \mathrm{~B}\right){ }^{59} \mathrm{Cu}$ were measured with a ${ }^{12} \mathrm{C}$ beam of $76.67 \mathrm{MeV}$ bombarding a $76 \mu \mathrm{g} / \mathrm{cm}^{2}{ }^{58} \mathrm{Ni}$ target mounted in the scattering chamber of the split-pole spectrograph. With these results it is possible to calculate the angular distributions (in the laboratory system) for the one-neutron transfer reactions ${ }^{12} \mathrm{C}\left({ }^{56} \mathrm{Ni},{ }^{57} \mathrm{Ni}\right){ }^{11} \mathrm{C}$ and $\mathrm{d}\left({ }^{56} \mathrm{Ni},{ }^{57} \mathrm{Ni}\right) \mathrm{p}$ expected for a $56 \mathrm{Ni}$ beam. They are shown in Figure I-3 for the g.s. transition $\left(\mathrm{p}_{3} / 2\right)$ together with similar one-neutron transfer reactions on ${ }^{11} \mathrm{~B}$ and ${ }^{13} \mathrm{C}$ at a bombarding energy of $360 \mathrm{MeV}$. The small cross section for the $12 \mathrm{C}+56 \mathrm{Ni}$ system of $30 \mathrm{mb} / \mathrm{sr}$ is caused by the unfavorable $\mathrm{Q}$-matching $(\mathrm{Qgs}=-8.45 \mathrm{MeV})$ for this reaction. Choosing better matched reactions $\left({ }^{11} \mathrm{~B}+\right.$ $56 \mathrm{Ni}$ or ${ }^{13} \mathrm{C}+{ }^{56} \mathrm{Ni}$ ) gives about one order of magnitude increased yields, but it is clear from Figure I-3 that due to the favorable Jakobian the $\mathrm{d}\left({ }^{56} \mathrm{Ni},{ }^{57} \mathrm{Ni}\right) \mathrm{p}$ reaction is the best candidate for experiments with low intensity beams.

These experiments require the use of the Fragment Mass Analyzer: and are described in contribution a.1.

\section{a.3. Exploring the ${ }^{18} \mathrm{~F}(\mathrm{p}, \gamma){ }^{19} \mathrm{Ne}$ Gateway to the Formation of Heavy Elements in Hot}

Stars (K. E. Rehm, C. L. Jiang, D. Blumenthal, C. N. Davids, P. Decrock, S. M. Fischer, D. Henderson, C. J. Lister, J. A. Nolen, R. C. Pardo, J. P. Schiffer, D. Seweryniak, M. Paul, ${ }^{*}$ L. A. Daniel, $\dagger$ J. Nickles, $\dagger$ and R. E. Segel $\ddagger$ )

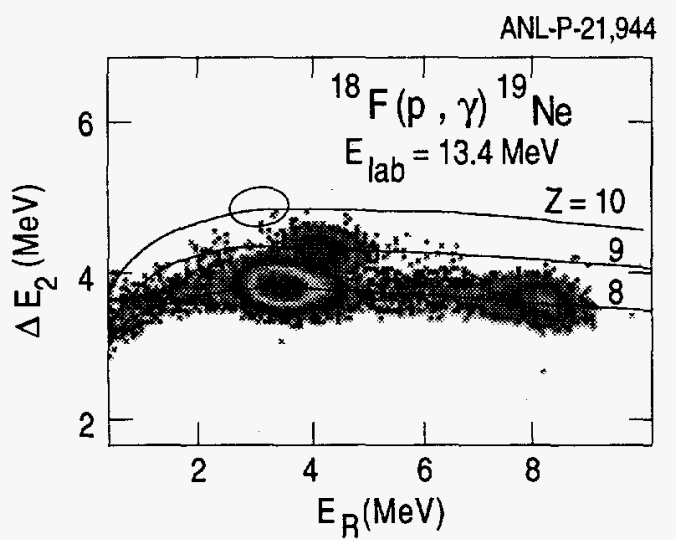

The isotope ${ }^{18} \mathrm{~F}\left(\mathrm{t}_{1 / 2}=110 \mathrm{~min}\right)$ plays an important role in our understanding of explosive nucleosynthesis. $18 \mathrm{~F}$ is produced in the hot CNO cycle and is the stepping stone to the formation of ${ }^{19} \mathrm{Ne}$ which in turn is the gateway to the rp process, where in a series of $(p, \gamma)$ reactions, nuclei up to $56 \mathrm{Ni}$ and beyond are produced. In a hot stellar environment ${ }^{19} \mathrm{Ne}$ can be generated either via the ${ }^{18} \mathrm{~F}(\mathrm{p}, \gamma){ }^{19} \mathrm{Ne}$ reaction or through radiative capture of ${ }^{4} \mathrm{He}$ on ${ }^{15} \mathrm{O}$, with ${ }^{15} \mathrm{O}$ produced via the ${ }^{18} \mathrm{~F}(\mathrm{p}, \alpha)$ reaction. These reactions occur at various sites in the universe including novae, type II supernova explosions, and x-ray bursts. Following our
Fig. 1-4. $\Delta E-E_{\text {res }}$ spectrum measured in the ionization chamber behind the focal plane of the FMA with a $18 \mathrm{~F}$ beam containing a considerable $18 \mathrm{O}$ contamination. The area where ${ }^{19} \mathrm{Ne}$ events from the ${ }^{18} F(p, \gamma)^{19}$ Ne reaction are expected is encircled.

${ }^{18} \mathrm{~F}(\mathrm{p}, \alpha){ }^{15} \mathrm{O}$ studies ${ }^{1}$ which identified a new $3 / 2^{+}$state in ${ }^{19} \mathrm{Ne}$ at $\mathbf{E}_{\mathbf{X}} \sim 7 \mathrm{MeV}$, we have investigated the influence of this state on the ${ }^{18} \mathrm{~F}(\mathrm{p}, \gamma)$ yield.

The experiment was performed at the ATLAS accelerator system of Argonne National Laboratory using a two-accelerator method for generating the radioactive ${ }^{18} \mathrm{~F}$ ion beam. The ${ }^{18} \mathrm{~F}$ material was produced at the cyclotron of the University of Wisconsin via the ${ }^{18} \mathrm{O}(\mathrm{p}, \mathrm{n})^{18} \mathrm{~F}$ reaction, flown to Argonne National Laboratory, and installed in the negative ion source of the ATLAS tandem injector.

\footnotetext{
*Hebrew University, Jerusalem, Israel, $\uparrow$ University of Wisconsin, $\ddagger$ Northwestern University

${ }^{1}$ K. E. Rehm et al., Phys. Rev. C 52, R460 (1995), ${ }^{2}$ P. Decrock et al., Phys. Rev. Lett. 67,808 (1991).
} 


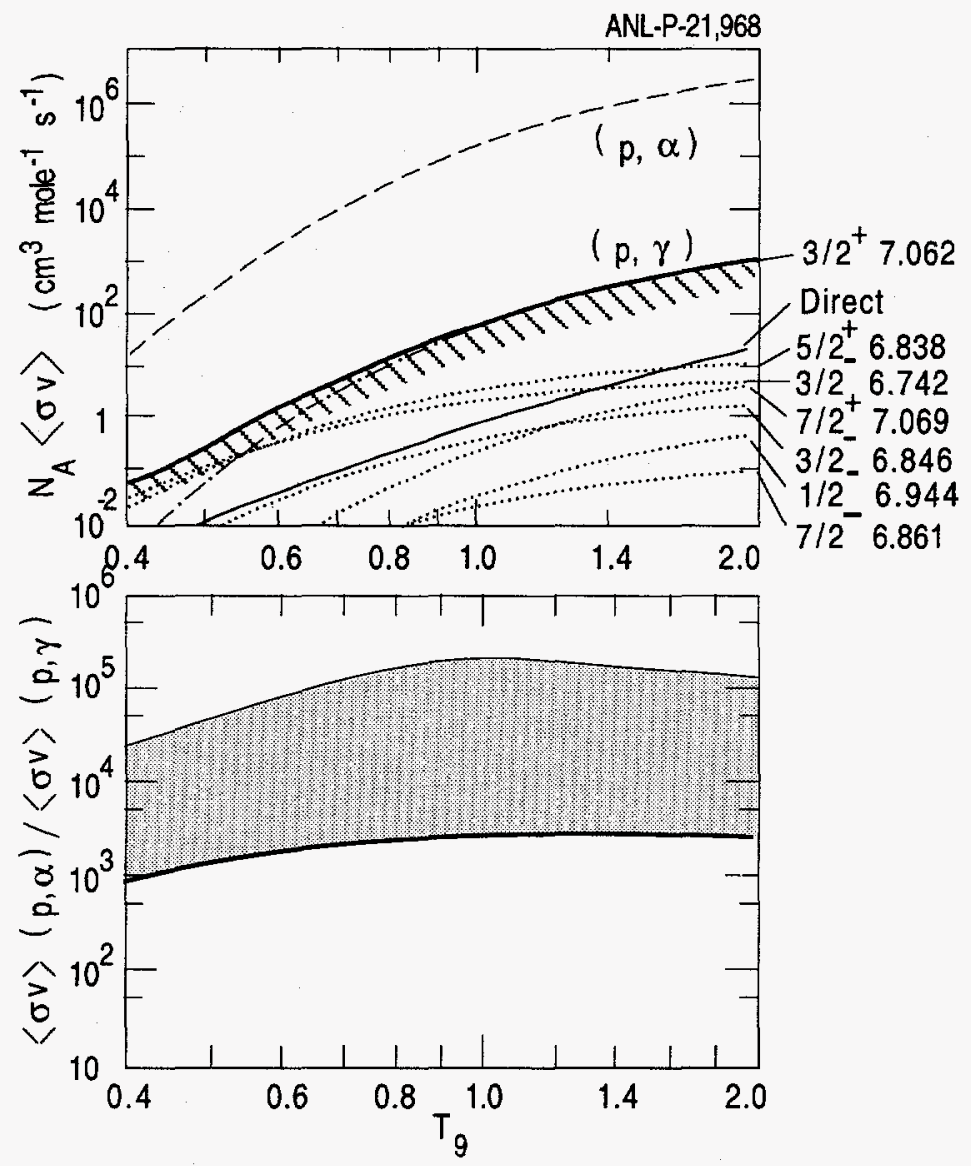

Fig. I-5 (a) Limits to the astrophysical reaction rate calculated from the resonance strength determined in this experiment (thick solid line). The dashed line is the reaction rate measured for the $18 F(p, \alpha){ }^{15} O$ reaction. The thick solid line represents a lower limit for the reaction rate given by the direct capture process. (b) Upper and lower limits for the ratio of the reaction rates between the ${ }^{18} F(p, \alpha){ }^{15} O$ and the ${ }^{18} F(p, \gamma)^{19}$ Ne reactions.

To improve the detection efficiency the Fragment Mass Analyzer was used for detecting the ${ }^{19} \mathrm{Ne}$ reaction products produced in the $\mathrm{p}\left({ }^{18} \mathrm{~F},{ }^{19} \mathrm{Ne}\right) \gamma$ reaction. The runs with ${ }^{18} \mathrm{~F}$ beams were performed at a bombarding energy (at the middle of the target) of $E_{\mathrm{cm}}=670 \mathrm{keV}$, i.e., slightly above the s-wave resonance found in the ${ }^{18} \mathrm{~F}(\mathrm{p}, \alpha){ }^{15} \mathrm{O}$ reaction.

Five runs with different samples of ${ }^{18} \mathrm{~F}$, each of about $300 \mathrm{mCi}$ activity, were accumulated. The data from these runs were summed and the corresponding $\Delta \mathrm{E}$ $\mathrm{E}_{\text {res }}$ spectrum is shown in Figure I-4. The region where events from the ${ }^{18} \mathrm{~F}(\mathrm{p}, \gamma){ }^{19} \mathrm{Ne}^{7+}$ reaction are expected is marked in the figure. There are three events that fall within this region, all of which are located close to the borderline between the $Z=9$ and 10 events. These three events have been further analyzed using the additional energy loss signal $\Delta E_{1}$ from the focal plane detector. The three events have energy loss values very close to the ones observed for ${ }^{19} \mathrm{~F}$ ions and we conclude that probably all three of these events are ${ }^{19} \mathrm{~F}$ ions. The cross section corresponding to a single ${ }^{19} \mathrm{Ne}$ event is calculated to $42 \mu \mathrm{b}$. Taking $42 \mu \mathrm{b}$ and the widths $\Gamma_{\mathrm{p}}$ and $\Gamma_{\mathrm{t}}$ for the $3 / 2^{+}$resonance as given in Ref. 1, a limit for the resonance strength $\omega \gamma \leq 740 \mathrm{meV}$ and the gamma width $\Gamma_{\gamma} \leq 3 \mathrm{eV}$ can be calculated. This upper limit for $\Gamma \gamma$ is comparable to the width obtained for the s-wave resonance populated in the ${ }^{13} \mathrm{~N}(\mathrm{p}, \gamma){ }^{14} \mathrm{O}$ reaction. 2

Such a limit for the radiative strength of the $3 / 2^{+}$ resonance allows one to reach some conclusions about the production of ${ }^{19} \mathrm{Ne}$ in the hot $\mathrm{CNO}$ cycle. The upper limit of the astrophysical reaction rate for the ${ }^{18} \mathrm{~F}(\mathrm{p}, \gamma){ }^{19} \mathrm{Ne}$ reaction populating the $3 / 2^{+}$state in ${ }^{19} \mathrm{Ne}$ as function of $\mathrm{T} 9$ is shown as the dash-dot line in 
Figure I-5a. Also included (dashed line) is the rate for the ${ }^{18} \mathrm{~F}(\mathrm{p}, \alpha){ }^{15} \mathrm{O}$ reaction which is larger than the corresponding $(\mathrm{p}, \gamma)$ rate by at least a factor of $10^{3}$. Estimated upper limits for other states located in this excitation energy region are shown as dotted lines in Figure I-5a. The upper limit for the total $(\mathrm{p}, \gamma)$ reaction rate is indicated by the thick solid line. A rough estimate for a lower limit for the $(p, \gamma)$ reaction rate may be provided from a calculation of direct proton capture, which is shown as a thin solid line in Figure I-5a.

The controlling factor for the breakout from the hot CNO cycle to the rp-process is the ratio of the reaction rates $R\left[{ }^{18} \mathrm{~F}(\mathrm{p}, \boldsymbol{\alpha})\right] / \mathrm{R}\left[{ }^{18} \mathrm{~F}(\mathrm{p}, \gamma)\right]$. The region allowed for this ratio by the present measurement and the direct capture calculation is given by the two solid lines in Figure I-5b. Due to the large value of the ${ }^{18} \mathrm{~F}(\mathrm{p}, \alpha)^{15} \mathrm{O}$ reaction rate which above $\mathrm{T}_{9}>0.5$ is dominated by the recently discovered $3 / 2^{+}$resonance, and the lack of a strong enhancement of proton capture from this resonance, the ratio of the reaction rates is larger than $\sim 1000$ in this temperature range. This would imply that at these temperatures, the production of ${ }^{19} \mathrm{Ne}$ through the ${ }^{18} \mathrm{~F}(\mathrm{p}, \gamma)$ reaction is probably negligible and the mechanism for generating this isotope is most likely the ${ }^{15} \mathrm{O}(\alpha, \gamma)$ reaction, involving the more abundant ${ }^{15} \mathrm{O}$ material, although so far no direct measurements of this reaction have been performed.

a.4. Astrophysical Reaction Rate for the ${ }^{18} \mathbf{F}(p, \alpha){ }^{15}$ O Reaction (K. E. Rehm, C. L. Jiang, D. J. Blumenthal, S. M. Fischer, J. Gehring, D. Henderson, J. Nolen, R. C. Pardo, J. P. Schiffer, D. Seweryniak, M. Paul, * A. D. Roberts, $\dagger$ J. Nickles, $\dagger$ and R. E. Segel $\ddagger)$

The synthesis of heavier elements in explosive nucleosynthesis in a proton-rich environment is believed to proceed through the nuclide ${ }^{19} \mathrm{Ne}$ which is produced either directly via the ${ }^{15} \mathrm{O}(\alpha, \gamma){ }^{19} \mathrm{Ne}$ reaction or via the ${ }^{14} \mathrm{O}(\alpha, \mathrm{p}){ }^{17} \mathrm{~F}$ reaction followed by the sequence ${ }^{17} \mathrm{~F}(\mathbf{p}, \gamma){ }^{18} \mathrm{Ne}\left(\beta^{+}\right){ }^{18} \mathrm{~F}(\mathrm{p}, \gamma){ }^{19} \mathrm{Ne} .{ }^{19} \mathrm{Ne}$ is then the starting point for the rp-process producing nuclei up to $56 \mathrm{Ni}$ and beyond. In the reaction chain starting with proton capture by ${ }^{17} \mathrm{~F}$, however, there is a competing reaction, ${ }^{18} \mathrm{~F}(\mathrm{p}, \alpha){ }^{15} \mathrm{O}$, which recycles material back into the CNO cycle. How ${ }^{18} \mathrm{~F}$ interacts with protons at low energies is, therefore, of key importance for controlling the breakout from the "hot CNO cycle" into the rp-process.

During the last year two studies of the ${ }^{18} \mathrm{~F}(\mathrm{p}, \alpha)^{15} \mathrm{O}$ reaction have been reported. 1,2 Both experiments identified a $3 / 2^{+}$state at an energy about $650 \mathrm{keV}$ above threshold. The experiment at ATLAS was performed using a two-stage method for producing the ${ }^{18} \mathrm{~F}$ beam which is described in more detail in Ref. 3 .

The $3 / 2^{+}$state in ${ }^{19} \mathrm{Ne}$, formed by $\ell=0$ protons, has a strong influence on the astrophysical reaction rate for the ${ }^{18} \mathrm{~F}(\mathrm{p}, \boldsymbol{\alpha})$ reaction. In order to predict the total reaction rate, the contributions from other states located in this excitation energy region must be considered. Such calculations are complicated by the fact that not all analog states of ${ }^{19} \mathrm{~F}$ have been located in the mirror nucleus ${ }^{19} \mathrm{Ne}$. There are seven states known in ${ }^{19} \mathrm{~F}$ between an excitation energy of $6.75 \mathrm{MeV}$ and 7.25 $\mathrm{MeV}$. They are summarized together with their spin values in Table 1.

\footnotetext{
*Hebrew University, Jerusalem, Israel, †University of Wisconsin, $\ddagger$ Northwestern University

${ }^{1}$ K. E. Rehm et al., Phys. Rev. C $\underline{52}$, R460 (1995).

${ }^{2}$ R. Coszach et al., Phys. Lett. B $\underline{353}, 184$ (1995).

${ }^{3}$ A. Roberts et al., Nucl. Instrum. Methods B103, 523 (1995).
} 
Table 1. Energies and widths for various states induced in the reaction rate calculations

\begin{tabular}{cccccc}
$\mathrm{J}^{\pi}$ & $\begin{array}{c}\mathrm{E}\left({ }^{19} \mathrm{~F}\right) \\
(\mathrm{MeV})\end{array}$ & $\begin{array}{c}\mathrm{E}_{\mathbf{x}}\left({ }^{19} \mathrm{Ne}\right) \\
(\mathrm{MeV})\end{array}$ & $\begin{array}{c}\mathrm{E}_{\mathrm{cm}} \\
(\mathrm{keV})\end{array}$ & $\begin{array}{c}\Gamma_{\mathrm{p}} \\
(\mathrm{keV})\end{array}$ & $\begin{array}{c}\Gamma_{\alpha} \\
(\mathrm{keV})\end{array}$ \\
\hline $32^{+}$ & 7.10 & & & & \\
$72^{+}$ & 7.114 & 7.063 & 652 & $5.0 \pm 1.6$ & $8.6 \pm 2.5$ \\
$12^{-}$ & 6.989 & 7.069 & 658 & $3.3 \times 10^{-2}$ & 34 \\
$72^{-}$ & 6.927 & 6.944 & 633 & $2.3 \times 10^{-2}$ & 53 \\
$32^{-}$ & 6.891 & 6.861 & 450 & $1.8 \times 10^{-5}$ & 2.5 \\
$5 / 2^{+}$ & 6.838 & 6.846 & 435 & $6.5 \times 10^{-3}$ & 29 \\
$32^{-}$ & 6.787 & 6.793 & 382 & $8.3 \times 10^{-4}$ & 1.2 \\
& & 6.742 & 329 & $9 \times 10^{-4}$ & 2.6
\end{tabular}

Their proton widths were estimated from their singleparticle values by assuming $\Gamma_{\mathrm{p}}=0.01 * \Gamma_{\mathrm{sp}}$ for negative-parity states and $\Gamma_{\mathrm{p}}=0.01 * \Gamma_{\mathrm{sp}}$ for positive parity states. The alpha widths $\Gamma_{\alpha}$ were scaled by penetrabilities from the experimental values of mirror states in ${ }^{19} \mathrm{~F}$.

The reaction rate from an isolated resonance at $E=E_{O}$ was then calculated from the integral

$$
\mathrm{N}_{\mathrm{A}}<\sigma v>=\mathrm{N}_{\mathrm{A}}\left[8 /\left(\pi \mu(\mathrm{kT})^{3}\right)\right] \int_{0}^{1 / 2} \sigma(\mathrm{E}) \exp (-\mathrm{E} / \mathrm{kT}) \mathrm{EdE}
$$

where

$$
\sigma(E)=\pi \pi^{2} \omega \frac{\Gamma_{p}(E) \Gamma_{\alpha}}{\left(E-E_{o}\right)^{2}+\Gamma_{t}^{2} / 4}
$$

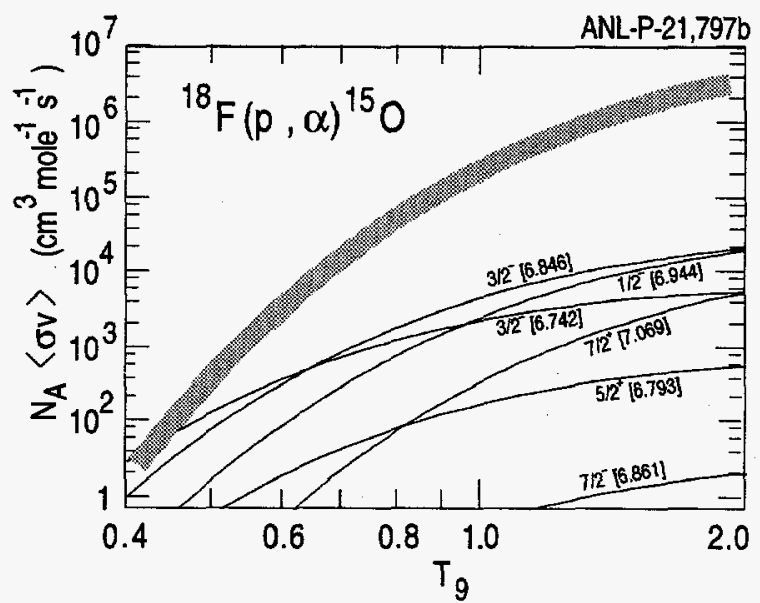

Fig. I-6. Reaction rate for the $18 F(p . \alpha)^{15} 0$ reaction.
$\mathrm{N}_{\mathrm{A}}$ is Avogadro's number, $\mu$ the reduced mass, $\omega$ the spin statistical factor associated with the resonance at a c.m. energy $E_{0}, \Gamma_{t}=\Gamma_{p}+\Gamma_{\alpha}$, and $\lambda$ the reduced de Broglie wave length, respectively. The reaction rate from equation (1) is plotted as a function of $\mathrm{T}_{9}$ in Figure I-6 for each resonance given in Table 1.

One can clearly see that the reaction rate above $\mathrm{T}_{9}=$ 0.5 is dominated by the new $\ell_{\mathrm{p}}=0,3 / 2^{+}$state at 7.063 $\mathrm{MeV}$. Only at temperatures $\mathrm{T}_{9}<0.5$ do contributions from other states start to be significant with the $3 / 2^{-}$ level at $6.742 \mathrm{MeV}$ excitation energy being the most important state. 


\section{a.5. Study of the ${ }^{17} \mathbf{F}(p, \alpha)$ Reaction (B. Harss, K. E. Rehm, R. C. Pardo, C. L. Jiang,}

F. Borasi, P. Decrock, S. M. Fischer, J. P. Greene, D. Nisius, J. A. Nolen, J. P. Schiffer, D. Seweryniak, J. C. Blackmon,* M. Paul, † R. E. Segel, $\ddagger$ and T. F. Wang§)

Classical novae are powered by the explosive burning of hydrogen on the surface of accreting white dwarf stars through the hot $\mathrm{CNO}$ cycle. If the temperature and densities in these systems are high enough, the $\beta$ decays of ${ }^{14} \mathrm{O}$ and ${ }^{15} \mathrm{O}$, which limit the energy production in the CNO cycle, are bypassed by the

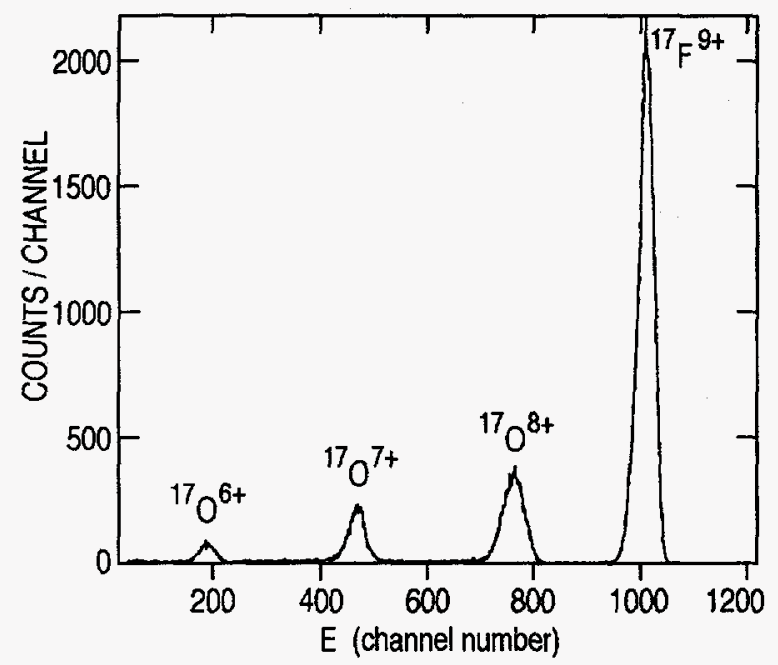

Fig. 1-7. Energy spectrum of the secondary particles measured in the focal plane of the split-pole spectrograph.

${ }^{14} \mathrm{O}(\alpha, \mathrm{p}){ }^{17} \mathrm{~F}$ and ${ }^{15} \mathrm{O}(\alpha, \gamma){ }^{19} \mathrm{Ne}$ reactions, increasing the energy generation by a factor of $\sim 100$. The latter reaction is also the first step to the rp process which leads to the nucleosynthesis of heavier elements. Direct measurements of these reactions are difficult since, in addition to intense radioactive ion beams of $14,15 \mathrm{O}$, they also require implanted ${ }^{4} \mathrm{He}$ targets. Another way of obtaining the astrophysical reaction rate is a measurement of the time-inversed ${ }^{17} \mathrm{~F}(\mathrm{p}, \alpha){ }^{14} \mathrm{O}$ reaction and using detailed balance to calculate the $(\alpha, p)$ rate. We have started a program to measure the ${ }^{17} \mathrm{~F}(\mathrm{p}, \boldsymbol{\alpha}){ }^{14} \mathrm{O}$ reaction populating states in ${ }^{18} \mathrm{Ne}$ at $\mathrm{E}_{\mathrm{X}} \approx$ $7 \mathrm{MeV}$ excitation energy using the newly developed ${ }^{17} \mathrm{~F}$ beam at ATLAS.
The ${ }^{17} \mathrm{~F}$ ions were produced in an inverse $(p, n)$ reaction, $\mathrm{p}\left({ }^{17} \mathrm{O},{ }^{17} \mathrm{~F}\right) \mathrm{n}$, by bombarding a $7.5 \mathrm{~cm}$ long gas cell, filled with hydrogen at pressures of 300-500 Torr with $200-300 \mathrm{pnA}$ of ${ }^{17} \mathrm{O}$. Details of the gas cell are given in a separate contribution.

By selecting the ${ }^{17} F^{9+}$ ions with a bending magnet after the gas cell, a good suppression of the primary ${ }^{17} \mathrm{O}$ beam has been achieved. Figure I-7 shows an energy spectrum of the particles detected after the bending magnet in the focal plane of the split pole spectrograph. The energy resolution $\Delta \mathrm{E} / \mathrm{E}$ of the secondary ${ }^{17} \mathrm{~F}$ beam was measured to be less than $\pm 8 \mathrm{x}$ $10^{-3}$. At a primary ${ }^{17} \mathrm{O}$ intensity of $250 \mathrm{pnA},{ }^{17} \mathrm{~F}$ rates up to $5 \times 10^{5 \cdot 17} \mathrm{~F} / \mathrm{sec}$ have been obtained in the scattering chamber of the spectrograph.

The $\mathrm{p}\left({ }^{17} \mathrm{~F},{ }^{14} \mathrm{O}\right) \alpha$ reaction was measured at the split pole spectrograph by using a $100 \mu \mathrm{g} / \mathrm{cm}^{2}$ thick $\mathrm{CH}_{2}$ target and by detecting the ${ }^{14} \mathrm{O}$ particles emitted at $\theta_{\text {lab }}$ $=3.6^{\circ}-6.4^{\circ}$ in the focal plane detector which provides single mass and Z-resolution. To reduce contributions from background reactions, the outgoing $\alpha$ particles were detected in coincidence using a large-area position-sensitive $\mathrm{Si}$-detector covering the angle range from $\theta=6.5^{\circ}-40^{\circ}$. Excitation functions around two possible resonances in ${ }^{18} \mathrm{Ne}$ at $E_{X}=7.05$ and $7.35 \mathrm{MeV}$ have been measured. A preliminary analysis of the data indicates that the contributions from direct processes off resonance are less than $0.1 \mathrm{mb} / \mathrm{sr}$ while an increased ${ }^{14} \mathrm{O}$ yield of $\sim 1 \mathrm{mb} / \mathrm{sr}$ is observed in the excitation energy region $\mathrm{E}_{\mathrm{X}}\left({ }^{18} \mathrm{Ne}\right)=7.4 \mathrm{MeV}$.

To increase the ${ }^{17} \mathrm{~F}$ rate we are planning to move the gas cell in front of a superconducting solenoid-buncher combination. This arrangement should result in an improved beam quality and a higher capture rate for the secondary beam.

*University of North Carolina, †Hebrew University, Jerusalem, Israel, $¥$ Northwestern University, §Lawrence Livermore National Laboratory 


\section{a.6. The Use of the Fragment Mass Analyzer for the Study of Radiative Capture Reactions with Radioactive Ion Beams (D. Seweryniak, K. E. Rehm, C. L. Jiang, D. Blumenthal, C. N. Davids, P. Decrock, S. M. Fischer, D. Henderson, C. J. Lister, J. A. Nolen, R. C. Pardo, J. P. Schiffer, M. Paul,* and R. E. Segel $\ddagger$ )}

Radiative capture reactions play an important role in the synthesis of the elements in stars. In the CNO cycle,

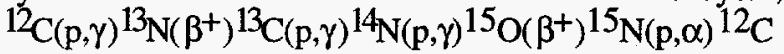
the three $(\mathrm{p}, \gamma)$ reactions contribute more than $60 \%$ to the total energy production. At higher temperatures $(\mathrm{p}, \gamma)$ and $(\alpha, \gamma)$ reactions e.g. ${ }^{19} \mathrm{Ne}(\mathrm{p}, \gamma)^{20} \mathrm{Na}$ or ${ }^{15} \mathrm{O}(\alpha, \gamma){ }^{19} \mathrm{Ne}$ provide a path for the production of heavier elements in the so-called rapid-proton (rp) process, which occurs in novae and supernova explosions and in $\mathrm{x}$-ray bursts.

Because of the relatively small cross sections (typically of the order of tens of $\mu b$ ) and the low $\gamma$-detection efficiency $(<1 \%)$, measurements of $(p, \gamma)$ cross sections usually require high beam intensities. In order to compensate for the low intensities available with today's radioactive ion beams, new techniques with considerably higher detection efficiencies are needed.

To improve the detection efficiency for radiative capture reactions, we have used the fragment mass analyzer (FMA) which, due to its excellent mass resolution can separate the $(p, \gamma)$ reaction products from the incident beam particles. Since the electric rigidity of the $(p, \gamma)$ reaction products is lower than that of the beam particles by about $5 \%$, the primary beam is stopped at a collimator located after the first electric dipole. The subsequent magnetic and electric dipoles provide for additional suppression of scattered beam particles and for the transport of the reaction products to the focal plane of the FMA where the ions are dispersed by $\mathrm{m} / \mathrm{q}$ and detected in a position-sensitive parallel-grid avalanche counter. This detector is followed by a largevolume ionization chamber for $\mathrm{Z}$ identification. With this arrangement, a suppression factor for the incident beam relative to the $(\mathrm{p}, \gamma)$ reaction products of $10^{12}$ is achieved.

The efficiency of the FMA and its detector system for radiative capture reactions was determined by measuring an excitation function for the $\mathrm{p}\left({ }^{18} \mathrm{O},{ }^{19} \mathrm{~F}\right) \gamma$ reaction around the previously known $\ell=0$ resonance in ${ }^{19} \mathrm{~F}$ at $\mathrm{E}_{\mathrm{cm}}=799 \mathrm{keV}$.

Figure $\mathrm{I}-8$ shows a $\Delta \mathrm{E}-\mathrm{E}_{\text {res }}$ spectrum measured for this calibration run in the ionization chamber at an ${ }^{18} \mathrm{O}$ energy of $16 \mathrm{MeV}$. The field settings of the FMA were chosen to detect the ${ }^{19} \mathrm{~F}^{7+}$ reaction products originating from the ${ }^{18} \mathrm{O}(\mathrm{p}, \gamma)$ reaction.

The overall efficiency for detecting the ${ }^{19} \mathrm{~F}$ particles is $30 \pm 3 \%$ i.e. considerably higher than obtained in typical $(\mathrm{p}, \gamma)$ experiments using $\gamma$-ray detection techniques. The fraction of the ${ }^{19} \mathrm{~F}$ ions in the $7^{+}$ charge state $\left(\mathrm{f}_{\mathrm{q}}=55 \%\right)$ was determined by measuring a charge state distribution of ${ }^{19} \mathrm{~F}$ at the resonance energy. The remaining part of the overall efficiency is due to the acceptance limitations imposed by a $1.7 \mathrm{~cm}$ wide collimator that was installed after the first electric dipole and to small angle scattering of the particles in the focal plane detector.

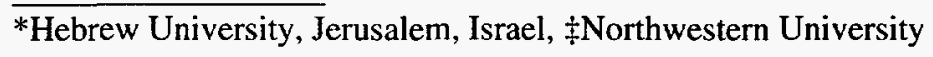

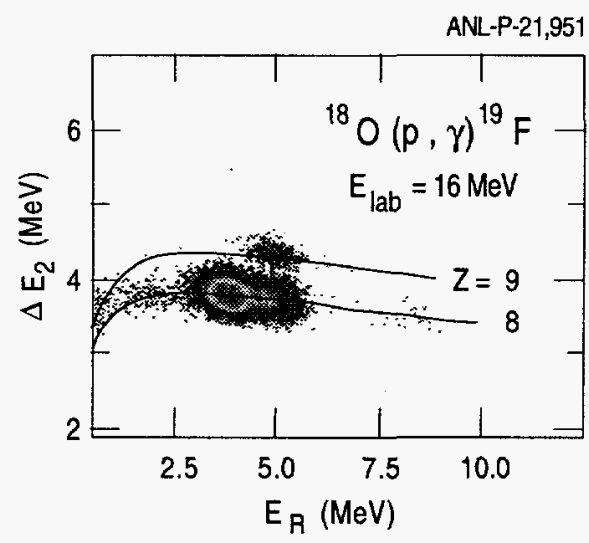

Fig. I-8. $\Delta E-E_{\text {res }}$ spectrum measured in the focal plane of the FMA for the ${ }^{18} O(p, \gamma){ }^{19} \mathrm{~F}$ reaction. The solid lines are the result of simulations of $\Delta E v s E_{\text {res }}$ for different ions. 


\section{a.7. In-Beam Studies of Very Neutron-Deficient Even-Even Os and Pt Isotopes using Recoil-Decay Tagging (D. Seweryniak, D. Ackermann, H. Amro, L. T. Brown, M. P. Carpenter, C. N. Davids, S. M. Fischer, G. Hackman, R. V. F. Janssens, D. Nisius, P. Reiter, L. Conticchio, W. B. Walters*, S. Hamada $\dagger$, and P. J. Woods $\ddagger$ )}

Conventional in-beam spectroscopic studies of neutrondeficient, $A>150$, nuclei far from the line of stability are hampered by the competition from fission, leading to low fusion cross sections, and by high fragmentation of fusion-evaporation reaction channels. The RecoilDecay Tagging (RDT) method ${ }^{1}$, however, has significantly improved our ability to observe excited states in heavy nuclei at the proton drip-line using inbeam $\gamma$-ray spectroscopic methods. RDT has been successfully applied at ANL to observe for the first time $\gamma$-ray transitions in several nuclei in the $\mathrm{N}>82, \mathrm{Z}<82$ corner of the chart of nuclides, such as $155 \mathrm{Lu}, 156 \mathrm{Hf}$, ${ }^{157} \mathrm{Ta},{ }^{160} \mathrm{~W},{ }^{176} \mathrm{Hg}$ and ${ }^{178} \mathrm{Hg}$, to name a few. Also, several new proton emitters in this region have recently been found at ATLAS ${ }^{2}$. One possible explanation for small proton-decay spectroscopic factors deduced for the heavy proton emitters $165,166,167 \mathrm{Ir}$ and ${ }^{171} \mathrm{Au}$ is the presence of deformation. However, no spectroscopic information about excited states of the proton emitters and their daughter nuclei was available prior to this work. The lightest known even-even Os and $\mathrm{Pt}$ isotopes are ${ }^{170} \mathrm{Os}$ and ${ }^{174} \mathrm{Pt}$, respectively. In order to verify this hypothesis, we proposed to study excited states in the very neutron-deficient, even-even nuclei $170,172 \mathrm{Pt}$ and $164,166,168 \mathrm{Os}$ using RDT. Inbeam $\gamma$ rays were detected in the ANL Notre-Dame Gedetector array. Reaction channel selection was obtained using the FMA with a Double-Sided Silicon Strip Detector placed behind the focal plane.
A seven-day long experiment was performed using a ${ }^{78} \mathrm{Kr}$ beam from ATLAS. Beam energies and targets used to produce individual nuclei are listed in Table 1. Gamma-ray transitions correlated with $\alpha$ decays of each of the nuclei of interest, namely $164,166,168 \mathrm{Os}$ and $170,172 \mathrm{Pt}$, were found. For $166,168 \mathrm{Os}$ sufficient data were collected to allow studies of $\gamma-\gamma$ coincidences, whereas for lighter isotopes only singles $\gamma$-ray spectra were extracted. Figure I-9 shows $\gamma$-ray spectra for ${ }^{168}$ Os which illustrate the very good reaction channel selection obtained with RDT. Preliminary results indicate that the observed light isotopes of $\mathrm{Os}$ and $\mathrm{Pt}$ seem to be more spherical in character than their heavier neighbors as indicated by the higher excitation energies of the observed first $2^{+}$states. Data analysis is still in progress.

Table 1: Reactions used to study individual Os and $\mathrm{Pt}$ isotopes.

$\begin{array}{lll}\text { Beam } & \begin{array}{l}\text { Energy } \\ {[\mathrm{MeV}]}\end{array} & \text { Target Recoil }\end{array}$

\begin{tabular}{llll}
\hline $78_{\mathrm{Kr}}$ & 385 & $90 \mathrm{Zr}$ & $1640 \mathrm{~s}(4 \mathrm{n})$ \\
$78_{\mathrm{Kr}}$ & 385 & $92_{\mathrm{Mo}}$ & 166 Os $(2 \mathrm{p} 2 \mathrm{n})$ \\
$78_{\mathrm{Kr}}$ & 380 & ${ }^{94} \mathrm{Mo}$ & $168 \mathrm{Os}(2 \mathrm{p} 2 \mathrm{n})$ \\
$78_{\mathrm{Kr}}$ & 385 & $96 \mathrm{Ru}$ & $170 \mathrm{Pt}(2 \mathrm{p} 2 \mathrm{n})$ \\
$78_{\mathrm{Kr}}$ & 345 & $96 \mathrm{Mo}$ & $172 \mathrm{Pt}(2 \mathrm{n})$
\end{tabular}

*University of Maryland, †JAERI, Ibaraki, Japan, łUniversity of Edinburgh, United Kingdom

${ }^{1}$ D. Seweryniak et al., submitted to Phys. Rev. $C$ and references therein.

${ }^{2}$ C. N. Davids et al., accepted for publication in Phys. Rev. C and references therein. 
Fig. I-9. Gamma-ray spectra obtained for ${ }^{168}$ Os; a) $\gamma$ rays detected in coincidence with recoils $b$ ) $\gamma$ rays detected in coincidence with $M=168$ residues; c) $y$ rays correlated with the $\alpha$ decay of the ground state of 168 Os.

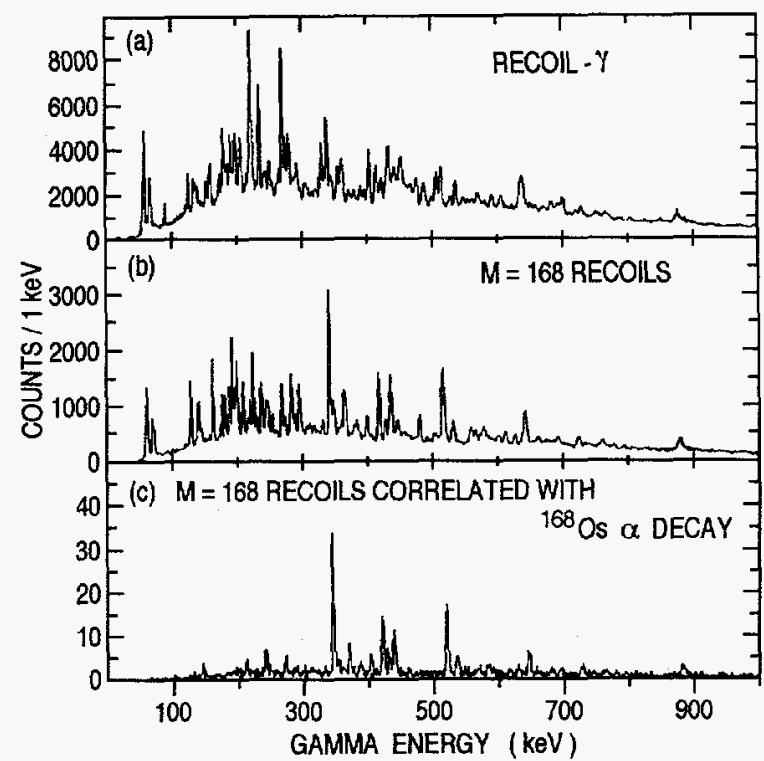

a.8. Study of $176-179 \mathrm{Hg}$ Using the Recoil Decay Tagging Method (M. P. Carpenter, R. V. F. Janssens, D. J. Blumenthal, D. Seweryniak, D. Ackermann, I. Ahmad, C. N. Davids, S. M. Fischer, G. Hackman, T. L. Khoo, T. Lauritsen, C. J. Lister, D. Nisius, T. Brown, H. Amro, J. Schwartz, P. J. Woods, * J. H. Hamilton, $\dagger$

A. V. Ramayya, $\dagger$ W. Reviol, $\ddagger$ J. Simpson, $\S$ and J. Wauters $\ddagger$ )

In light $\mathrm{Hg}$ isotopes $(100>\mathrm{N} \mathrm{108)}$, shape co-existence has been established close to the ground state, where rotational bands built on collective oblate and prolate shapes are observed. From the level systematics in the even-even $\mathrm{Hg}$ isotopes and, in particular, from data on ${ }^{180} \mathrm{Hg}^{1}$, the lightest $\mathrm{Hg}$ nucleus for which excited states are known, it has been concluded that the excited prolate minimum lies lowest in energy relative to the oblate ground state for neutron number $N=102$, i.e. in ${ }^{182} \mathrm{Hg}$. Recent Nilsson-Strutinsky calculations ${ }^{2}$ indicate that for neutron number $\mathrm{N}<100$ the oblate ground state evolves steadily as a function of decreasing $\mathrm{N}$ towards a spherical shape, while the prolate minimum disappears and gives way to a new minimum at large deformation $\left(\beta_{2}=0.5-0.56\right)$ which is, however, located between 3.5 and $5 \mathrm{MeV}$ above the ground state. It is clearly of interest to pursue the exploration of the structure of the $\mathrm{Hg}$ isotopes towards lower neutron numbers. However, this task becomes increasingly difficult as one approaches the proton drip line. The heavy-ion induced fusion reactions required to produce the nuclei of interest are dominated by the fission process which results in a very large, unwanted background in the $\gamma$-ray spectra.

In an attempt to study this shape-coexistence phenomenon all the way to the proton-drip line, we have identified for the first time $\gamma$-ray transitions in $176-$ ${ }^{179} \mathrm{Hg}$ using the AYE-Ball Compton Suppressed Ge array which was setup at the target position of the Fragment Mass Analyzer (FMA). Unambiguous assignment of $\gamma$ transitions to a particular nuclide was accomplished by utilizing the Recoil Decay Tagging (RDT) method, a technique which correlates $\gamma$ rays emitted from excited residues with the charged particle radioactivity of their decay ${ }^{3}$. The gamma-ray spectra correlated with the alpha decay of $178 \mathrm{Hg}$ and $176 \mathrm{Hg}$ are shown in Figure I-10(a) and (b), respectively. The

\footnotetext{
*University of Edinburgh, United Kingdom, †Vanderbilt University, $¥$ University of Tennessee, §Daresbury Laboratory, United Kingdom

${ }^{1}$ G. Dracoulis et al., Phys. Lett. B208, 365 (1988), ${ }^{2}$ W. Nazarewicz, Phys. Lett. B305, 195 (1993), ${ }^{3}$ E. S. Paul et al., Phys. Rev. C 51, 78 (1995), ${ }^{4}$ R. S. Simon et al., Z. Phys. A325, 197 (1986) ${ }^{5}$ G. Dracoulis, Phys. Rev. C 49,3324 (1994).
} 


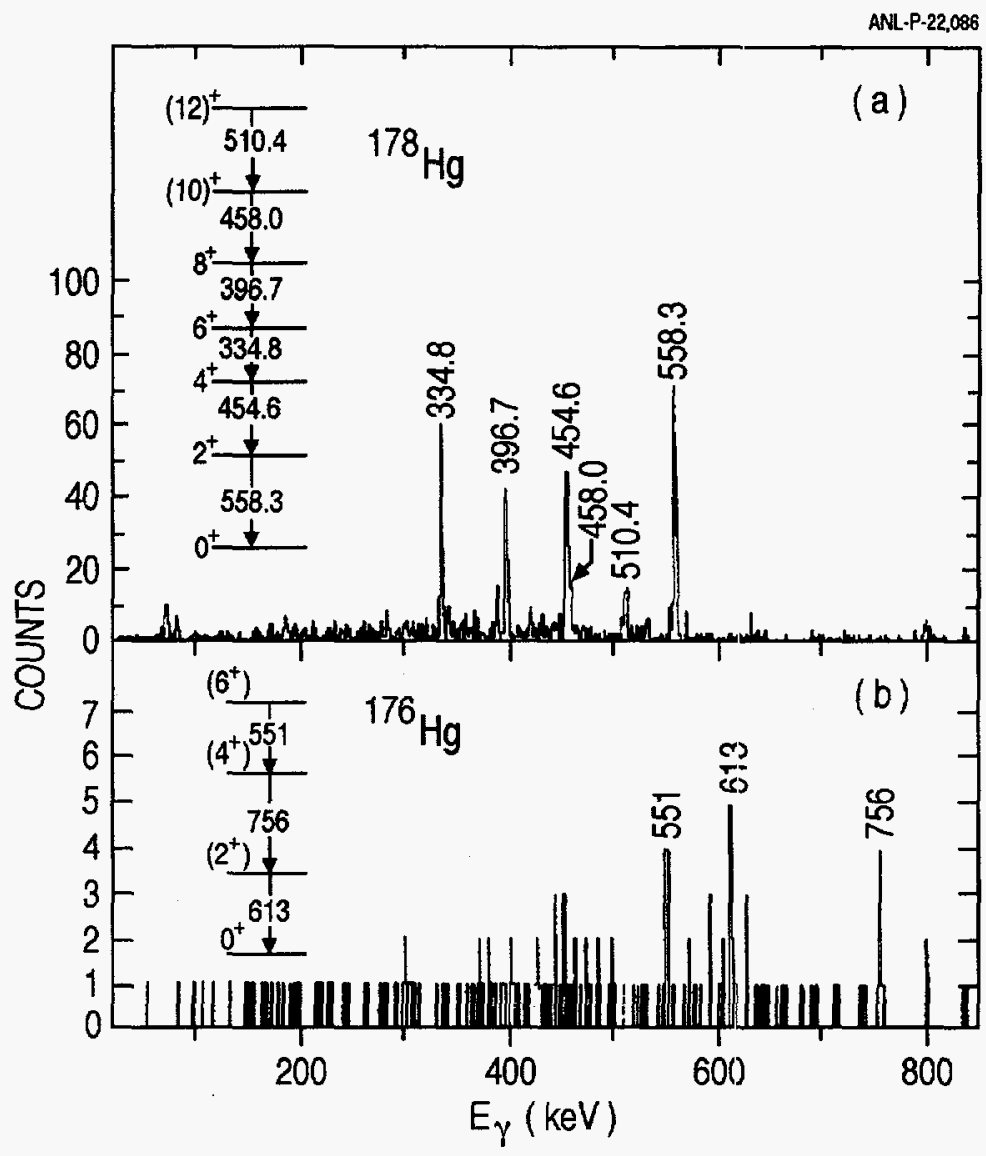

Fig. I-10. (a) $\gamma$ rays correlated with the ${ }^{178} \mathrm{Hg} \alpha$ decay (the proposed $178 \mathrm{Hg}$ level scheme is given in the inset). (b) $\gamma$ rays correlated with the ${ }^{176} \mathrm{Hg} \alpha$ line. The second inset shows the level scheme for ${ }^{176} \mathrm{Hg}$ deduced from the present measurements.

deduced level structure for both nuclei are given as insets to the figure. Transitions have also been assigned to $177,179 \mathrm{Hg}$, however, due to limited statistics, no level structures have been proposed.

For the even-even isotopes it is important to note that the three ${ }^{176} \mathrm{Hg}$ transitions have energies $>550 \mathrm{keV}$, in contrast with the ${ }^{178} \mathrm{Hg}$ spectrum where all transitions placed in the level structure have $E_{\gamma}<560 \mathrm{keV}$. This difference is understood in the following way. For ${ }^{178} \mathrm{Hg}$, the prolate band crosses the ground state band and becomes yrast by $\mathrm{I}=6^{+}$. From a two-band mixing calculation to the data, where both the ground state band and prolate band are described by a VMI parameterization, we estimate that the unperturbed prolate band head lies at an excitation energy of 710 $\mathrm{keV}$. This represents a substantial increase over what is observed for ${ }^{180} \mathrm{Hg}$ and ${ }^{182} \mathrm{Hg}$ where the unperturbed excitation energy of the prolate band is estimated to be
$387 \mathrm{keV}$ and $264 \mathrm{keV}$, respectively ${ }^{4}$. The increase of the $2^{+}$level in ${ }^{178} \mathrm{Hg}$ to $551 \mathrm{keV}$ relative to the heavier $\mathrm{Hg}$ isotopes also indicates a transition of the ground state from an oblate to a spherical shape. This transition continues in $176 \mathrm{Hg}$ where the $2^{+}$energy continues to rise to $613 \mathrm{keV}$. In addition, no evidence for the prolate structure is observed for this nucleus, and we estimate a minimum excitation energy of $1300 \mathrm{keV}$ for the band head if it exists.

In summary, the present data indicate that the excitation energy of the prolate band increases rapidly with decreasing mass number for $\mathrm{N}<100$, and the minimum may well have disappeared by $\mathrm{N}=96$. The excitation energy of the prolate minimum below $N=102$ mirrors the behavior seen above this number; a rapid rise is seen for $\mathrm{N}=110$ with the prolate $0^{+}$state jumping from 825 $\mathrm{keV}$ at $\mathrm{N}=108$ to $1279 \mathrm{keV}$. A paper reporting these results has been submitted for publication. 
a.9. Study of ${ }^{177}$ Au Using the Recoil Decay Tagging Method (M. P. Carpenter, T. Brown, R. V. F. Janssens, D. J. Blumenthal, D. Seweryniak, D. Ackermann, I. Ahmad, C. N. Davids, S. M. Fischer, G. Hackman, T. L. Khoo, T. Lauritsen, C. J. Lister, D. Nisius, J. Schwartz, H. Amro, P. J. Woods, * J. H. Hamilton, $†$ A. V. Ramayya, $\dagger$ W. Reviol, $\ddagger$ J. Simpson, $\S$ and J. Wauters $\ddagger$ )

We have recently completed a study of light $\mathrm{Hg}$ isotopes, where excited states in ${ }^{176-179} \mathrm{Hg}$ were identified for the first time ${ }^{1}$. The nuclei of interest were produced in the reaction ${ }^{103} \mathrm{Rh}\left({ }^{78} \mathrm{Kr}\right.$,pxn). Data were taken at three different beam energies, 340,360 and $380 \mathrm{MeV}$. Gamma transitions were detected by the AYE-Ball array which held 15 Compton suppressed Ge detectors, and residue identification was achieved with the Fragment Mass Analyzer (FMA). Unambiguous assignment of $\gamma$ transitions to a particular nuclide was accomplished by utilizing the Recoil Decay Tagging (RDT) method, a technique which correlates $\gamma$ rays emitted from excited residues with the charged particle radioactivity of their decay ${ }^{2}$.
At the highest bombarding energy, $380 \mathrm{MeV}$, the fusion-evaporation channel is quite fragmented. One of the isotopes which is populated with reasonable intensity is the $(2 \mathrm{p}, 2 \mathrm{n})$ channel, ${ }^{177} \mathrm{Au}$. This nucleus lies beyond the proton drip-line. However, due to the Coulomb barrier, its groundstate decays by alpha and not by proton emission. Previously, no information on excited states in this nucleus were available. Gamma rays were assigned to ${ }^{177} \mathrm{Au}$ using RDT, and a tentative level structure was constructed based on the $\gamma-\gamma$-recoil data. The coincidence data is dominated by a rotational band which we associate with the $\pi \mathrm{i}_{13 / 2}[660] 1 / 2$ configuration. No other rotational structures could be identified.

*University of Edinburgh, United Kingdom, †Vanderbilt University, łUniversity of Tennessee, §Daresbury

Laboratory, United Kingdom

${ }_{1}$ M. P. Carpenter et al., submitted to Phys. Rev. Lett.

${ }^{2}$ E. S. Paul et al., Phys. Rev. C $\underline{51}, 78$ (1995).

a.10. First Observation of Excited States in $118,117 \mathrm{Cs}$ and ${ }^{119,118} \mathrm{Ba}$ (H. Amro, R. V. F. Janssens, C. N. Davids, D. Seweryniak, J. F. Smith,* C. J. Chiara,* D. B. Fossan,* G. R. Gluckman,* G. J. Lane,* J. M. Sears, * I. Thorslund,* I. M. Hibbert, $\uparrow$ R. Wadsworth, $†$ I. Y. Lee, $\S$ and A. O. Macchiavelli $\S)$

We performed a measurement at ATLAS to identify the nuclei associated with a set of band structures that had been observed in an experiment with Gammasphere array at LBNL, but for which there was no mass assignment. The ${ }^{64} \mathrm{Zn}\left({ }^{58} \mathrm{Ni}\right)$ reaction $\left(\mathrm{E}_{\text {beam }}=230\right.$ and $240 \mathrm{MeV}$ ) was used. Gamma rays and $\mathrm{X}$ rays were detected in ten Compton suppressed $25 \%$-efficient Ge detectors in coincidence with recoiling reaction products, which were dispersed according to their massto-charge state ratio by the FMA and detected in the PPAC at the focal plane. Recoils with charge states $q=$ 24,25 and 26 were detected at the focal plane. This resulted in $\sim 15 \%$ of the gamma rays being detected in coincidence with residues.

Well developed rotational bands seen at Gammasphere have now been assigned to $117,118 \mathrm{Cs}$ and $118,119 \mathrm{Ba}$.
This represents the first identification of excited states in all of these nuclei. In this work, the $\pi \mathrm{h}_{11 / 2}$ band in ${ }^{117} \mathrm{Cs}$ has been extended to $59 / 2^{+}$and, in addition, two unconnected bands based on $\pi \mathrm{g} 7 / 2$ and $\pi \mathrm{g} 9 / 2^{-1}$ orbitals have been identified. In $118 \mathrm{Cs}$, four bands have been observed to spins in excess of $36 \hbar$, including a band based on the $\pi\left(\mathrm{h}_{11 / 2}\right) \otimes \nu\left(\mathrm{h}_{11 / 2}\right)$ configuration, which displays an unusually low signature inversion between 16 and $17 \hbar$. In ${ }^{119} \mathrm{Ba}$, bands based on $v \mathrm{~h}_{11 / 2}$ and $v g 7 / 2$ orbitals have been observed to spins of $65 / 2 \hbar$. In $18_{\mathrm{Ba}}$, the ground-state band has been observed to $20 \hbar$, and a negative-parity band is observed feeding the $4^{+}, 6^{+}$and $8^{+}$states. Applying Grodzins' relation to these results suggests that ${ }^{1} 8_{\mathrm{Ba}}$ is less deformed than ${ }^{120} \mathrm{Ba}$, whereas previous calculations predict that the ground-state deformation peaks at $\mathrm{A}=118$ for the $\mathrm{Ba}$ isotopes. A manuscript reporting these results is in preparation.

\footnotetext{
*State University of New York at Stony Brook, †University of York, United Kingdom, §Lawrence Berkeley National Laboratory
} 
a.11. Spectroscopy of ${ }^{192}$ Po with the RDT Technique (R. V. F. Janssens, C. N. Davids, D. Seweryniak, M. P. Carpenter, H. Amro, P. Decrock, P. Reiter, D. Nisius, L. T. Brown, S. Fischer, T. Lauritsen, L. F. Conticchio, N. Fotiades, * W. Younes, * J. A. Cizewski,* D. P. McNabb, $*$ K. Y. Ding,* J. Wauters, $†$ C. R. Bingham, $†$ M. Huyse,$\ddagger$ and A. Andreyev, $\ddagger$ )

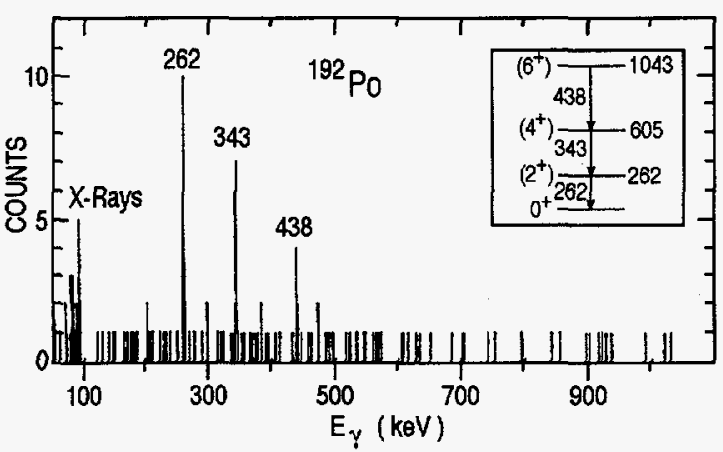

Fig. I-11. Spectrum of ${ }^{192}$ Po gamma-ray transitions. The inset represents the deduced level scheme of $192 \mathrm{Po}$.

The region of isotopes around the closed shell of $\mathrm{Z}=82$ protons is an ideal one to study the onset and evolution of collective motion in nuclei. Near $N=126$ the excitations can be explained in terms of the shell model, and when the number of valence neutrons increases, vibrational motion becomes important. The neutrondeficient even-mass polonium isotopes provide an example of this evolution from non-collective behavior to harmonic and anharmonic vibrations, when going from the semi-magic ${ }^{210}$ Po nucleus to the ${ }^{196} \mathrm{Po}$ and ${ }^{194} \mathrm{Po}$ isotopes, respectively. Extrapolation of level systematics down to ${ }^{192}$ Po had suggested a $2^{+}$energy for this isotope close to the value at which the phase transition from anharmonic vibrator to deformed rotor occurs, rendering this isotope a crucial one in the evolution of collective motion.

Excitations in ${ }^{192}$ Po have been studied via in-beam gamma-ray spectroscopy following the ${ }^{164} \mathrm{Er}+164$ $\mathrm{MeV}{ }^{32} \mathrm{~S}$ reaction. The experiment was performed at the ATLAS facility using 10 Compton-suppressed Ge detectors and the Fragment Mass Analyzer. The alpha decay of 192. Po has been used to identify prompt gamma-ray transitions (RDT technique). The first three excited states of ${ }^{192}$ Po were established (Figure I-11). The downward trend of the energies of these states, which began in the heavier isotopes, continues in ${ }^{192} \mathrm{Po}$, although this trend is less than a straightforward extrapolation would predict. The energy of the $2^{+}$state in 192Po has not reached the critical value at which the phase transition from an anharmonic vibrator to a deformed rotor occurs. A paper reporting these results has been accepted for publication.

*Rutgers University, †University of Tennessee, $\ddagger$ Katholic University, Leuven, Belgium

\section{a.12. Proton Radioactivity Studies, $73 \leq \mathbf{Z} \leq 81$ (C. N. Davids, D. J. Blumenthal,}

L. T. Brown, L. F. Conticchio, D. J. Henderson, D. Kurath, H. T. Penttilä, D. Seweryniak, P. J. Woods, * J. C. Batchelder, $\dagger$ C. R. Bingham, $\ddagger, \S$ B. C. Busse,II T. Davinson,*

S. J. Freeman,Il R. J. Irvine, * J. A. Mackenzie, ${ }^{*}$ R. D. Page, ${ }^{*}$ K. S. Toth, $\S$

W. B. Walters, ${ }^{* *}$ and B. E. Zimmerman,,$\left.\dot{\dagger}\right)$

The series of ground state proton emitters between $\mathrm{Z}=$ 69 and $Z=83$ has been completed with the discovery of ${ }^{177} \mathrm{Tl}$. In addition, weak proton branches ${ }^{1}$ were observed for ${ }^{157} \mathrm{Ta}$ and $16 \mathrm{Te}$, bringing to 13 the number of proton emitters known in this region. A number of long alpha decay chains following the decay of ${ }^{167} \mathrm{Ir}^{2}$ were linked to a nuclide with known mass, enabling ground state mass excesses to be obtained for ${ }^{151} \mathrm{Tm},{ }^{154} \mathrm{Yb},{ }^{155^{\mathrm{Lu}}}{ }^{158} \mathrm{Hf},{ }^{159} \mathrm{Ta},{ }^{162} \mathrm{~W},{ }^{163} \mathrm{Re}$, $166 \mathrm{Os},{ }^{167} \mathrm{Ir}$, and ${ }^{170} \mathrm{Pt}$. In addition, shell orbital orderings and proton separation energies were obtained for a number of nuclides at and beyond the proton drip

*University of Edinburgh, United Kingdom, †Louisiana State University, $\ddagger$ University of Tennessee, §Oak Ridge National Laboratory, đOregon State University, IUniversity of Manchester, United Kingdom, **University of Maryland

${ }_{1}$ R. J. Irvine et al., Phys. Rev. C 55, in press (April 1997).

${ }^{2}$ C. N. Davids et al., Phys. Rev. C 55, in press (May 1997).

${ }^{3}$ S. Liran and N. Zeldes, At. Data Nucl. Data Tables 17, 431 (1976). 


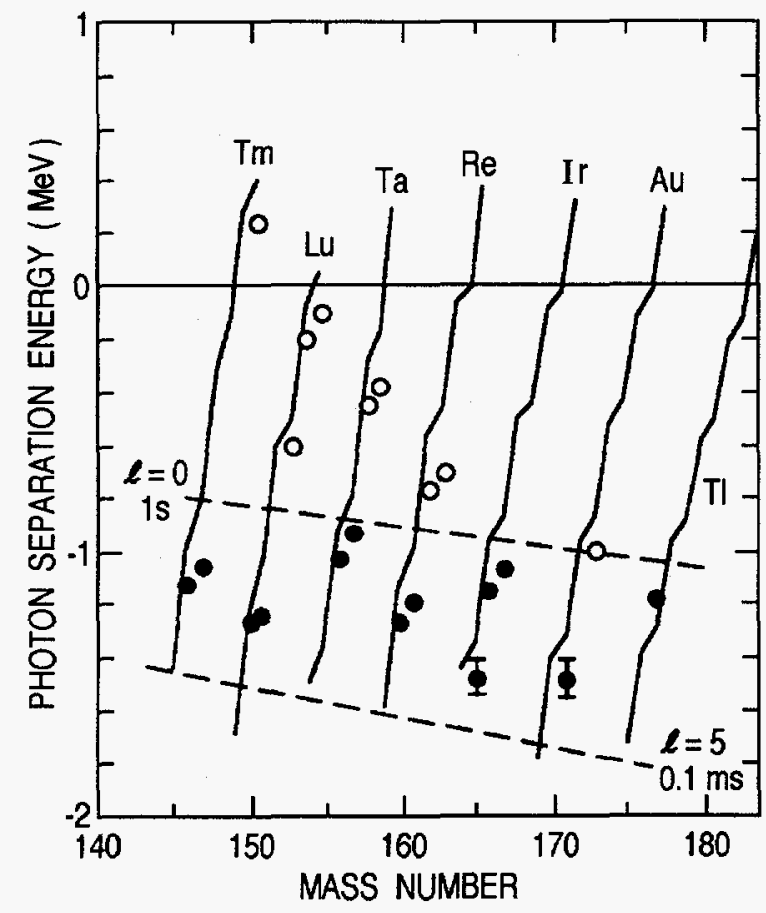

Fig. 1-12 . Proton separation energies for $T m, L u, T a$, $\mathrm{Re}, \mathrm{Ir}, \mathrm{Au}$, and $\mathrm{Tl}$ isotopes. Filled circles denote measured energies, open circles represent derived energies from the present work, and the solid lines are the Liran-Zeldes predictions ${ }^{3}$. The values shown for $165_{\text {Ir and }}{ }^{171_{A}}$ u correspond to the $1 / 2^{+}$ground state, using estimated values for the excitation energies of the $11 / 2^{-}$isomer of $0.23(11)$ and $0.22(11)$ $\mathrm{MeV}$ respectively.

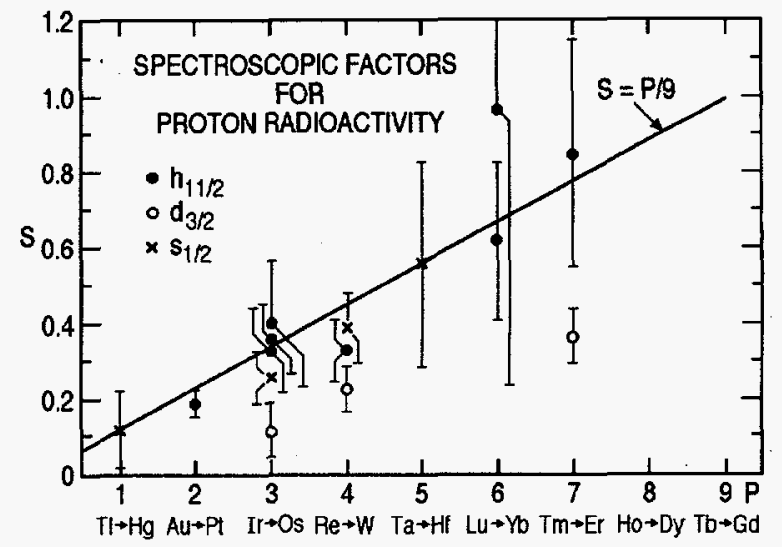

Fig. I-13. Spectroscopic factors for new and previously observed proton emitters. The solid line is the shell model prediction.

line. Figure I-12 shows the proton separation energies currently known for the $69 \leq \mathrm{Z} \leq 81$ region.

A low-seniority shell model calculation of the spectroscopic factors for proton emitters with $65 \leq \mathrm{Z} \leq$ 81 has been performed. The basic assumption is that the protons are emitted from either the $s_{1 / 2}, \mathrm{~d}_{3 / 2}$, or $\mathrm{h}_{11 / 2}$ orbitals, and the single particle energies are degenerate. A pairing force was used for the residual interaction. Figure I-13 shows the agreement obtained with the experimental data. Future proton radioactivity searches are planned for $Z<69$.

\section{a.13. The $\alpha$-Decay Properties of ${ }^{186}$ Bi (C. N. Davids, L. T. Brown, L. F. Conticchio,} D. J. Henderson, D. Seweryniak, J. C. Batchelder,* E. F. Zganjar,* C. R. Bingham, $\dagger$ J. Wauters, $\dagger$ K. S. Toth, $\ddagger$ T. Davinson, $\S$ R. J. Irvine, $\S$ P. J. Woods, $\S$ and W. B. Walters, I)

The $\alpha$ decay of $186 \mathrm{Bi}$ was studied using the ${ }^{97} \mathrm{Mo}\left({ }^{92} \mathrm{Mo}, \mathrm{p} 2 \mathrm{n}\right){ }^{186} \mathrm{Bi}$ reaction at $420 \mathrm{MeV}$. Evaporation residues were selected in-flight by mass using the Fragment Mass Analyzer, and implanted into a double-sided silicon strip detector. Two alpha lines were identified: one with energy $7158(20) \mathrm{keV}$ and half-life 15.0(17) $\mathrm{ms}$, and the other having an energy of
$7261(20) \mathrm{keV}$ and a half-life of $9.8(13) \mathrm{ms}$. Each transition was correlated with $\mathrm{A}=186$ implants and has parent-daughter correlations with the known $5869 \mathrm{keV}$ alpha from the decay of ${ }^{182} \mathrm{Hg}$. The ${ }^{182} \mathrm{Hg}$ alpha decay occurs following the unobserved beta decay of the ${ }^{186} \mathrm{Bi}$ alpha daughter ${ }^{182} \mathrm{Tl}$. Based on systematics, the two ${ }^{186} \mathrm{Bi}$ states are assigned as $\left(10^{-}\right)$and $\left(3^{+}\right)$.

*Louisiana State University, †University of Tennessee, ‡Oak Ridge National Laboratory, §University of Edinburgh, United Kingdom, qUniversity of Maryland 


\section{a.14. Fine Structure in the $\alpha$ Decay of ${ }^{189}$ Bi (C. N. Davids, D. J. Blumenthal, L. T. Brown, L. F. Conticchio, D. Seweryniak, J. Wauters, ${ }^{*}$ C. R. Bingham, ${ }^{*}$ J. C. Batchelder, $\dagger$ E. F. Zganjar, † K. S. Toth, $\neq$ T. Davinson, $\S$ R. J. Irvine, $§$ P. J. Woods, $\S$ and W. B. Walters, I)}

A detailed $\alpha$ decay study was performed for $189 \mathrm{~m}, \mathrm{~g}_{\mathrm{Bi}}$, produced using the ${ }^{96} \mathrm{Mo}\left({ }^{95} \mathrm{Mo}\right.$,pn $){ }^{189} \mathrm{Bi}$ reaction at $418 \mathrm{MeV}$. Evaporation residues were selected in-flight by mass using the Fragment Mass Analyzer, and implanted into a double-sided silicon strip detector. Six alpha groups were associated with the decay of ${ }^{189} \mathrm{Bi}$ by means of parent-daughter correlations. $A$ value of $182(8) \mathrm{keV}$ was determined for the excitation energy of the $\pi \mathrm{s}_{1 / 2}$ intruder state in ${ }^{189} \mathrm{Bi}$. A weak alpha branch from the $9 / 2^{-}{ }^{189} \mathrm{Bi}$ ground state to the $\pi \mathrm{d} 3 / 2$ level in $185 \mathrm{Tl}$ at $284 \mathrm{keV}$ was observed for the first time. The $\alpha$ decay of ${ }^{188_{B i}} \mathrm{was}$ also remeasured. Along with the three previously observed alpha groups at 6815,6987 , and $7029 \mathrm{keV}$, a new line at 6897(18) $\mathrm{keV}$ was observed, with a half-life consistent with that of the $6987 \mathrm{keV}$ transition.

*University of Tennessee, $\nmid$ Louisiana State University, $\ddagger$ Oak Ridge National Laboratory, §University of Edinburgh, United Kingdom, TUniversity of Maryland

\section{a.15. Discovery of the New Isotope ${ }^{178}$ Tl from its Alpha Decay (M. P. Carpenter, D. Ackermann, H. Amro, D. J. Blumenthal, T. Brown, C. N. Davids, S. M. Fischer, G. Hackman, R. V. F. Janssens, T. L. Khoo, T. Lauritsen, C. J. Lister, D. Nisius, J. Schwartz, D. Seweryniak, P. J. Woods,* J. H. Hamilton, $\dagger$ A. V. Ramayya, $\dagger$ W. Reviol, $\ddagger$ and J. Simpson§)}

There has been a strong program at the FMA studying proton radioactivity using a double-sided silicon strip detector (DSSD). A number of new proton emitters ranging from $Z=75(\mathrm{Re})$ to $Z=83(\mathrm{Bi})$ have been discovered at ATLAS. Until recently, a proton emitter in the $\mathrm{Tl}$ isotopes $(\mathrm{Z}=81)$ was missing from this group. The lightest identified $\mathrm{Tl}$ isotope, before we began our study, was ${ }^{179} \mathrm{Tl}$ which decays by $\alpha$ emission $\left(\mathrm{E}_{\alpha}=\right.$ 6.56 and $7.20 \mathrm{MeV}$ ).

We recently performed an experiment at the FMA using the ${ }^{78} \mathrm{Kr}+{ }^{103} \mathrm{Rh}$ reaction at $340 \mathrm{MeV}$ leading to the compound nucleus ${ }^{181} \mathrm{Tl}$. The main purpose of this study was to identify for the first time $\gamma$ transitions in ${ }^{178} \mathrm{Hg}$ via the recoil decay tagging method, a technique which correlates $\gamma$ rays emitted from excited residues with the charged particle radioactivity of their decay. In this experiment, gamma rays were measured at the target with the AYE-Ball array and alpha energies were measured in the DSSD.

In the course of our analysis, we have identified three previously unknown alpha decay lines $\left(\mathrm{E}_{\alpha}=6.71,6.79\right.$ and $6.87 \mathrm{MeV}$ ) which are correlated with $\mathrm{A}=178$ residues. In turn, all three alpha lines are correlated with the same daughter decay $\left(E_{\alpha}=6.54 \mathrm{MeV}\right)$ which corresponds to the energy of the ground state alpha decay of ${ }^{174}$ A.u. Based on these observations, we have assigned these alpha lines to ${ }^{178} \mathrm{Tl}$, a previous unknown $\mathrm{Tl}$ isotope. In addition, we have observed no evidence that ${ }^{178} \mathrm{Tl}$ proton decays. Since this identification, another experiment at the FMA has now identified ${ }^{177} \mathrm{Tl}$ as a proton emitter. A manuscript reporting the results on the $\alpha$ decay of ${ }^{178} \mathrm{Tl}$ is being prepared.

*University of Edinburgh, United Kingdom, †Vanderbilt University, ‡University of Tennessee, §Daresbury Laboratory, United Kingdom 


\section{a.16. A Search for ${ }^{12} \mathrm{C}$ Emission from ${ }^{114} \mathrm{Ba}$ : Determination of ${ }^{114} \mathrm{Ba} \alpha$-Decay, Half-Life, and Mass (J. Schwartz, C. J. Lister, C. N. Davids, D. Seweryniak, T. L. Brown, S. J. Fischer, G. Hackman, P. Reiter, and A. Wuosmaa)}

The phenomenon of decay by emission of heavy ion clusters with $\mathrm{A}>4$ provides a new technique for studying the tunneling process as well as the shape of ground state wave functions.

In 1989 , Poenaru predicted a new possible island of cluster radioactivity close to $\mathrm{N}=\mathrm{Z}=50^{1}$. The decay of ${ }^{114} \mathrm{Ba}$ by emission of ${ }^{12} \mathrm{C}$ was predicted to be the most likely candidate for observing heavy cluster radioactivity in the $A \geq 100$ region. In the last few years, several new theoretical approaches have been published to determine the decay rate and branching ratio of ${ }^{12} \mathrm{C}$ emission. There is a large variation in the results of these calculations due mainly to unknown masses and decay energies. The measurement of the decay modes of ${ }^{114} \mathrm{Ba}$ will provide a stringent test of mass models in the trans-tin region, will help determine the cluster characteristics of these nuclei, and will ascertain the reliability of predictions of heavy cluster emission. The unusual feature of the mass $A=100$ region is that the competition between alpha- and ${ }^{12} \mathrm{C}$ decay is more equal than in actinide nuclei, where heavy cluster emission is always very weak $\left[\operatorname{Br}(\right.$ heavy $\left.\left./ \alpha)<10^{-6}\right)\right]$.
We produced ${ }^{114} \mathrm{Ba}$ in the ${ }^{58} \mathrm{Ni}\left({ }^{58} \mathrm{Ni}, 2 \mathrm{n}\right){ }^{114} \mathrm{Ba}$ reaction at $198 \mathrm{MeV}$ at the FMA. We chose this reaction because of its well known sub-barrier fusion enhancement. Low-energy fusion enhances mass 114 production relative to the other reaction channels and increases the sensitivity of the measurement. We reduced the contribution to decays from other channels by operating the FMA in a tightly collimated mode, transporting only $A=114, q=26$ residues. We implanted the $A=114$ nuclei in a position sensitive double sided strip detector (DSSD) $60 \mu \mathrm{m}$ thick with 48 $\times 48,300 \mu \mathrm{m}$ strips. This detector is not sensitive to beta rays. The DSSD was used with a maximum gain set at $30 \mathrm{MeV}$. In addition, we placed a $50 \% \mathrm{Ge}$ and large area Si detector behind the DSSD to allow DSSD beta-gamma correlations.

We observed several known $\mathrm{A}=114$ alpha decays and some alpha-alpha, parent-daughter correlations, which can be used to determine the sensitivity of our experiment. Although we observed no ${ }^{12} \mathrm{C}$ decay candidates, there is some evidence that new alphadecays were seen, one of which could be from ${ }^{114} \mathrm{Ba}$. Observation of the ${ }^{114} \mathrm{Ba}$ alpha decay would ascertain the mass of ${ }^{114} \mathrm{Ba}$, thus constraining the current models for cluster emission. Analysis of the data is continuing.

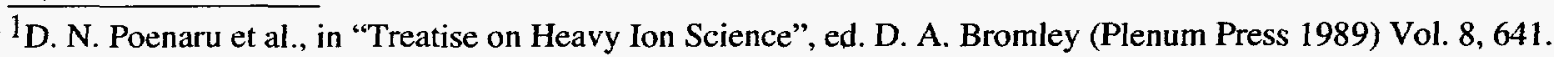




\section{B. GAMMA-RAY SPECTROSCOPY STUDIES}

A major portion of the nuclear structure research program within the Division relates to studies of the properties of nuclei under extreme conditions. In recent years, shape degrees of freedom have been studied extensively, in particular superdeformation. Other programs study nuclei at high spin and/or temperature, the properties of neutron-rich nuclei, states reached via Coulomb excitation with very heavy projectiles etc.

Following the major breakthrough achieved last year with the discovery of one-step gamma rays linking the yrast superdeformed band in ${ }^{194} \mathrm{Hg}$ with the low-lying states of normal deformation, the program to study the properties associated with the deexcitation from states in the second well has continued with vigor. For the first time, the linking transitions associated with the decay out of an excited superdeformed band in ${ }^{194} \mathrm{Hg}$ have now been identified. This result establishes the spins and the (likely) parity of the levels in this band which is also identical to the yrast superdeformed bands in ${ }^{192} \mathrm{Hg}$ and ${ }^{194} \mathrm{~Pb}$. One of the implications of the results is that the spins in the excited superdeformed band of ${ }^{194} \mathrm{Hg}$ differ by one unit from those in the two other identical bands, suggesting that the "identicality" is accidental.

The properties of identical bands have also been addressed by performing high-precision lifetime measurements, both in the $\mathrm{A}=150$ and $\mathrm{A}=190$ regions of superdeformation. In particular, superdeformed bands in ${ }^{194} \mathrm{Hg}$ and ${ }^{192} \mathrm{Hg}$ have been found to have quadrupole moments which are equal within errors. Identical superdeformed bands of the $A=150$ region appear to be characterized by identical quadrupole moments as well. On the other hand, a dependence of the quadrupole moments on the specific intruder configurations was demonstrated.

Coulomb excitation measurements have focused mainly on a search for double-phonon states in ${ }^{208} \mathrm{~Pb}$ and on investigations of octupole vibrations in actinide nuclei. The properties of neutronrich nuclei are explored by studying prompt gamma-decay following fission, a technique pioneered at Argonne some ten years ago.

b.1. Spins, Parity, Excitation Energies and Structure of an Excited Superdeformed Band in ${ }^{194}$ Hg (G. Hackman, T. L. Khoo, M. P. Carpenter, T. Lauritsen, R. V. F. Janssens, D. Ackermann, I. Ahmad, D. J. Blumenthal, S. M. Fischer, D. Nisius, I. V. Calderin, J. Young, A. Lopez-Martens, * E. F. Moore, $\dagger$ T. Døssing, „ F. Hannachi, * A. Korichi, * I. Y. Lee, $\S$ and A. O. Macchiavelli,§ and T. Nakatsukasa,, D)

We have determined ${ }^{1}$ the spins, parity and excitation energies of the yrast SD band in ${ }^{194} \mathrm{Hg}$. This was the first time that these vital quantities have been determined for a SD band in the $A=150$ and 190 regions -- a decade after the first identification of a SD band. In follow-up experiments at Gammasphere we have, for the first time, also measured these quantities for an excited SD band, again in ${ }^{194} \mathrm{Hg}$. The levels of the so-called band 3 in this nucleus have been found to have odd spin and negative parity. The levels decay to the normal deformed (ND) yrast line by 1 -step gamma rays with very large energies (see Figure I-14). In addition, transitions directly connecting this band to the vacuum SD band have been found. The excitation energy of band 3 above the vacuum band $(0.9 \mathrm{MeV}$ at zero frequency) is significantly lower than the value of $2 \Delta$, or $\sim 1.6 \mathrm{MeV}$, expected for a 2-quasiparticle configuration. This suggests that it is a vibrational band. More importantly, the level energies are well reproduced by theoretical calculations ${ }^{2}$ which describe the band as $\mathrm{a}=2$ octupole vibration.

*Centre de Spectrometrie Nucleaire et de Spectrometrie de Masse, Orsay, Firance, $†$ North Carolina State University, $\ddagger$ Niels Bohr Institute, Copenhagen, Denmark, §Lawrence Berkeley National Laboratory, qUniversity of Manchester, United Kingdom

${ }^{1}$ T. L. Khoo et al., Phys. Rev. Lett. 76,1583 (1996). 
ANL.P.21,2036

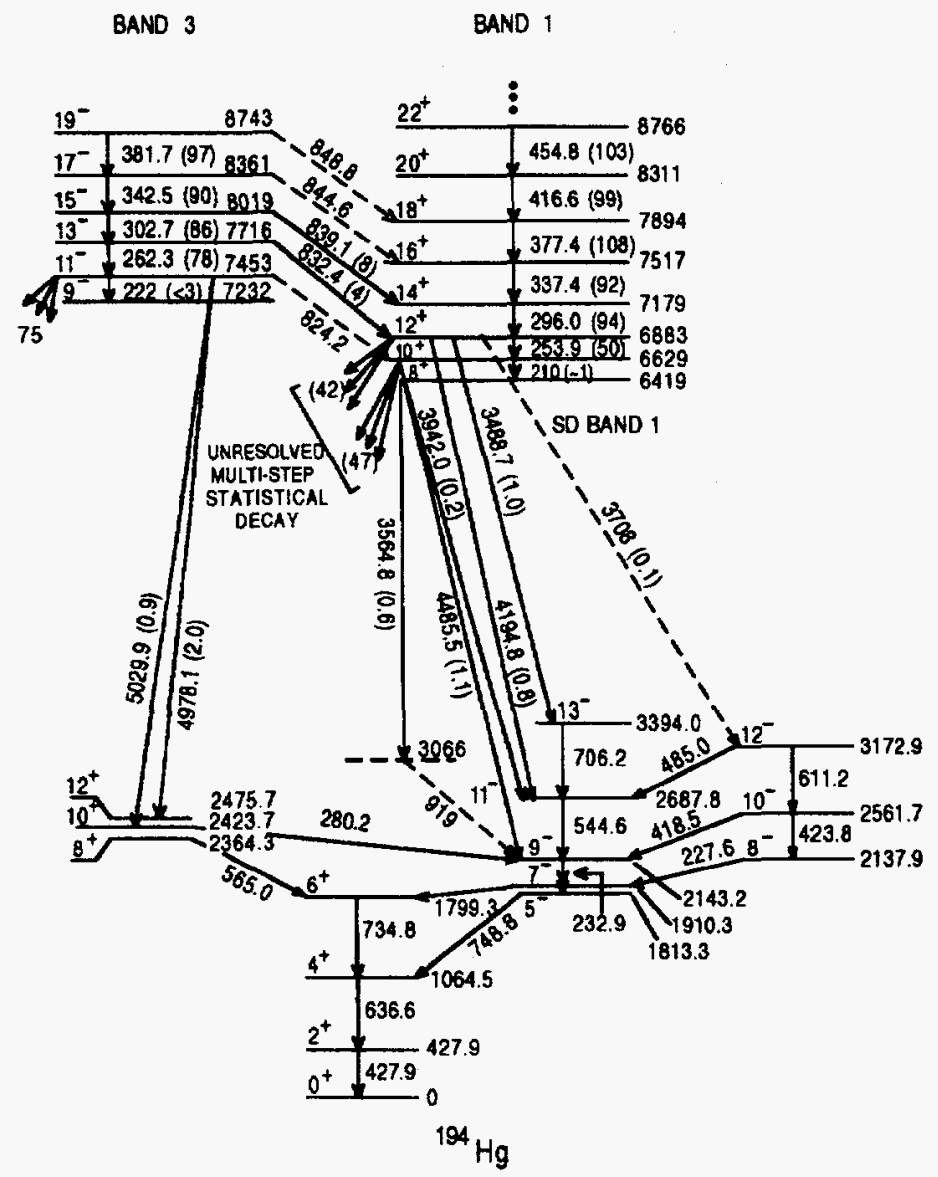

Fig. 1-14. Partial level scheme for ${ }^{194} \mathrm{Hg}$ showing the one-step decay out transitions identified thus far.

The dynamical and kinematic moments of inertia, $\mathfrak{I}^{(2)}$ and $\Im^{(1)}$ respectively, for the SD bands in ${ }^{194} \mathrm{Hg}$ are shown in Figure I-15. For each band, $\mathfrak{J}^{(2)}$ and $\mathfrak{I}^{(1)}$ extrapolate to the same values at zero frequency. This implies that there is zero alignment at that point. Hence, the larger values of $\mathfrak{I}^{(2)}$ and $\mathfrak{I}^{(1)}$ at zero frequency for the excited band can be attributed to the reduced pairing in the excited band. This provides perhaps the most direct evidence for pairing in the SD minimum.

Band 3 and the vacuum SD band in ${ }^{192} \mathrm{Hg}$ have identical transition energies. Although many SD identical bands have been found, there is still no accepted explanation for this remarkable phenomenon. Clearly data beyond just transition energies are sorely needed. Our data on this pair of identical bands provides the most comprehensive information for understanding the origin of identical bands -- see b.4. for results on ${ }^{192} \mathrm{Hg}$ and b.11. for results on differential quadrupole measurements. Band 3 in ${ }^{194} \mathrm{Hg}$ has odd spin and parity. Although the spins and parity of the vacuum band in $192 \mathrm{Hg}$ have not been determined, it is probably safe to assume that they are both even, as expected for a vacuum deformed band in the presence of pair correlations and as confirmed by the vacuum band of ${ }^{194} \mathrm{Hg}$ (see Figure I-14). Hence, we can, with reasonable confidence, answer the most important question on identical bands: do transitions of equal energy originate from states with the same spin and parity? They do not for the present pair; both the spins and parities are different. Furthermore, although the $\mathfrak{I}^{(2)}$ values for the identical bands are equal, the $\mathfrak{I}^{(1)}$ values are not (see Figure I-15). These two facts would seem to contradict the naive expectations of (heroic) explanations of identical bands based on the existence of some underlying symmetry. Indeed, the difference in spin between the identical bands can be attributed to both changes in pairing and alignment of particle spin. In other words, the unit spin difference and identical transition energies are due to the effects of both pairing and particle alignment, indicating a mechanism based on accidental cancellations. Therefore, our results suggest that a non-heroic explanation, espousing cancellation, applies for this pair of identical bands. However, this does not necessarily rule out a heroic explanation, since an (unidentified) symmetry could still be the root cause of these cancellations.

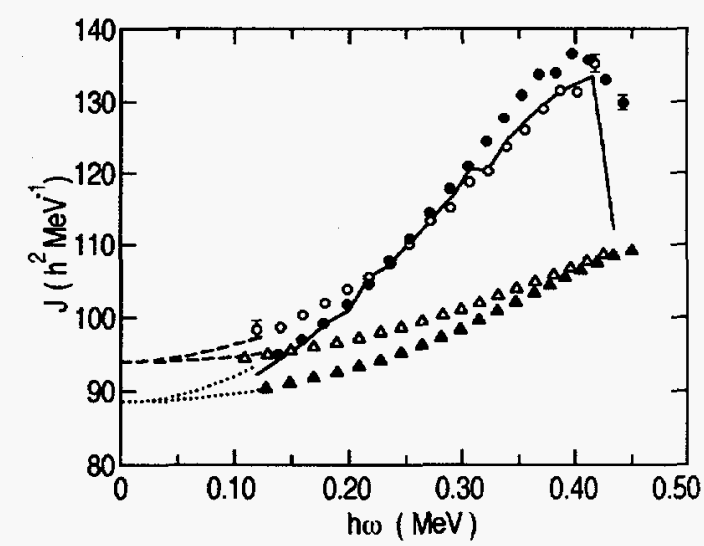

Fig. 1-15. $\mathfrak{I}^{(2)}$ and $\mathfrak{I}^{(1)}$ moments of inertia as a function of the rotational frequency $\hbar \omega$ for ${ }^{194} \mathrm{Hg}$ and ${ }^{192} \mathrm{Hg}$. 


\section{b.2. Spins, Parity and Excitation Energies of a Superdeformed Band in ${ }^{194} \mathrm{~Pb}$}

(M. P. Carpenter, T. L. Khoo, T. Lauritsen, A. Lopez-Mlartens, * F. Hannachi, * A. Korichi, † C. Schück, $*$ E. Gueorguieva, ${ }^{*}$ Ch. Vieu, * B. Haas, $\ddagger$ R. Lucas, $\S$ A. Astier,, $\mathbb{1}$ G. Baldsiefen, G. de France, $\ddagger$ R. Duffait,,$[$ L. Ducroux,,$[$ Y. Le Coz,§ Ch. Finck, $\ddagger$ A. Gorgen,\| H. Hübel,\| M. Meyer,II D. Prévost, $\ddagger$ N. Redon,, $\mathbb{C}$ C. Rigollet, $\ddagger$ H. Savajols, $\ddagger$ J. F. Sharpey-Schafer, ${ }^{* *}$ O. Stezowski, $\ddagger$ Ch. Theisen, $\S$ U. Van Severen, II J. P. Vivien, $\ddagger$ and A. N. Wilson††)

The spin/parity quantum numbers and excitation energies are vital for understanding the properties of superdeformed bands. An Argonne-led collaboration was the first to determine these quantities -- for the yrast band in ${ }^{194} \mathrm{Hg}$ from Gammasphere data. The second case $\mathrm{e}^{1}$ is in ${ }^{194} \mathrm{~Pb}$, from experiments performed at Eurogam by a Orsay-led collaboration. High-energy primary gamma rays directly deexciting the $6^{(+)}$and $8^{(+)}$levels of the yrast SD band and populating either yrast or near-yrast normal-deformed (ND) states were identified. The SD band in ${ }^{194} \mathrm{~Pb}$ decays primarily from the $6^{(+)}$and $8^{(+)}$levels, and the excitation energy of these levels above the ND yrast line is $2.7 \mathrm{MeV}$, significantly lower than the excitation energy of $\sim 4.3$ $\mathrm{MeV}$ at the points of deexcitation from the $10^{(+)}$and $12^{(+)}$SD levels in ${ }^{194} \mathrm{Hg}$. The excitation energies of the SD bands provide important benchmarks for theory, which is presently unable to account for the energies of the bands in both nuclei.

*Centre de Spectrometrie Nucleaire et de Spectrometrie de Masse, Orsay, France, †Institut de Physique Nucleaire, IN2P3-CNRS, Orsay, France, ‡Centre de Recherches Nucléaires, Strasbourg, France, §DAPNIA SPhN, CEA Saclay, Gif sur Yvette, France, qInstitut de Physique Nucléaire, IN2P3-CNRS and Université Lyon-1, Villeurbanne Cedex, France, IIUniversität Bonn, Germany, ${ }^{* *}$ National Accelerator Centre, Faure, South Africa, $\dagger \dagger$ University of Liverpool, United Kingdom

${ }^{1}$ A. Lopez-Martens, Phys. Lett. B380, 18 (1996).

\section{b.3. Search for Transitions Linking Superdeformed and Normal Deformed States in ${ }^{190}$ Hg (H. Amro, R. V. F. Janssens, B. Crowell, M. P. Carpenter, I. Ahmad,} D. J. Blumenthal, S. Fischer, T. L. Khoo, D. T. Nisius, T. Lauritsen, E. F. Moore, ${ }^{*}$ J. Timar, $\dagger$ A. N. Wilson, $\ddagger$ and D. M. Cullen $\ddagger$ )

An experiment was carried out using Gammasphere where excited states in ${ }^{190} \mathrm{Hg}$ were populated with the reaction ${ }^{160} \mathrm{Gd}\left({ }^{34} \mathrm{~S}, 4 \mathrm{n}\right)$ at a beam energy of $159 \mathrm{MeV}$ with a $\left(1.17 \mathrm{mg} / \mathrm{cm}^{2}\right){ }^{160} \mathrm{Gd}$ target evaporated on 13 $\mathrm{mg} / \mathrm{cm}^{2}$ Au backing. Three or higher-fold coincidence events were recorded. The data were sorted into a cube, the previously known superdeformed bands in ${ }^{190} \mathrm{Hg}$ were observed ${ }^{1}$. We searched for high-energy transitions forming a direct connection between the super and normally deformed states. Such a search proved to be very successful in the case of ${ }^{194} \mathrm{Hg}^{2}$. A $2718 \mathrm{keV}$ gamma ray was observed. The exact decay path for this gamma ray is not yet determined. The coincidences relationships indicate that it decays from the lowest state in the yrast superdeformed band to some intermediate state above the yrast line from which the decay branches out towards both the positive- and negative-parity yrast levels around spin 14.

*North Carolina State University, $\dagger$ Institute of Nuclear Research of the Hungarian Academy of Sciences, Debrecen, Hungary, University of Liverpool, United Kingdom

${ }_{1}$ A. N. Wilson et al., Phys. Rev. C $\underline{54}, 559$ (1996).

${ }^{2}$ T. L. Khoo et al., Phys. Rev. Lett. 76, 1583 (1996). 


\section{b.4. Search for One-Step Decay Gamma Rays Connecting Superdeformed and Normal}

States in ${ }^{192} \mathrm{Hg}$ (D. Ackermann, T. L. Khoo, M. P. Carpenter, T. Lauritsen, I. Ahmad, D. J. Blumenthal, S. M. Fischer, R. V. F. Janssens, D. Nisius, E. F. Moore, * A. Lopez-

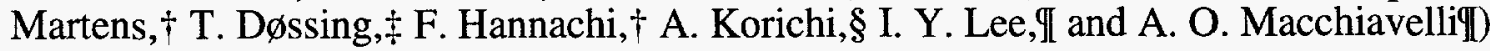

The yrast SD band in $192 \mathrm{Hg}$ has transition energies which are identical to those of an excited SD band in $194 \mathrm{Hg}$, whose spins and likely parity have been determined (see b.1.). A crucial question for understanding the origin of identical bands is to find out if transitions of equal energy emanate from states of the same spin. Hence, it is very important to find the 1-step decay lines out of the SD band in $192 \mathrm{Hg}$. We have performed a pair of experiments at Gammasphere to search for these lines, but have not been able to identify them. The highest energy line at $3116 \mathrm{keV}$ is part of a tentatively-identified 2-step decay pathway. No transitions above $3.2 \mathrm{MeV}$ have been found at a $3 \sigma$ level. Since it is very important to determine the spins of this band, in order to solve the identical band puzzle, we have another experiment approved at Gammasphere to search for the 1-step decay transitions.
Until these are found, we are limited to determining the spins by using the method of Draper ${ }^{1}$ and Becker ${ }^{2}$, i.e. by fitting the $J(2)$ vs $\hbar \omega$ curve to the Harris expansion. The spins given by this method are even. This method gives the correct spins for the three SD bands for which we know the spin -- see b.1. and b.2. We have shown that this is because the alignment at $\hbar \omega=0$ is zero. In any event, for a vacuum SD band in the presence of pairing we can be reasonably confident that the spins and parity are generally even, as observed for $194 \mathrm{Hg}$ and $194 \mathrm{~Pb}$. Since the spins and parity of the identical band 3 in $194 \mathrm{Hg}$ are both odd, we conclude that identical transition energies from this pair of identical bands do not originate from states with the same spin and parity.

\footnotetext{
*North Carolina State University and Triangle Universities Nuclear Laboratory, $†$ Centre de Spectrometrie Nucleaire et de Spectrometrie de Masse, Orsay, France, $\$$ Niels Bohr Institute, Copenhagen, Denmark, §Institut de Physique Nucleaire, IN2PS-CNRS, Orsay, France, ILawrence Berkeley National Laboratory

1 J. Draper et al., Phys. Rev. C $\underline{42}$, R1791 (1990).

2 J. Becker et al., Phys. Rev. C $\underline{46}, 889$ (1992).
}

\section{b.5. Search for One-Step Decays from Superdeformed Bands in ${ }^{193} \mathrm{Hg}$ to the Yrast Line (M. P. Carpenter, D. Ackermann, I. Ahmad, D. J. Blumenthal, S. M. Fischer, R. V. F. Janssens, T. L. Khoo, T. Lauritsen, C. J. Lister, D. Nisius, D. Seweryniak, B. C. Busse,* P. Fallon,* R. Krücken,* S. J. Asztalos, * R. M. Clark, * \\ M. A. Deleplanque,* R. M. Diamond,* I. Y. Lee,* A. O. Macchiavelli,* R. W. Macleod,* G. Schmid,* F. S. Stephens, ${ }^{*}$ K. Vetter, ${ }^{*}$ E. F. Moore, $\dagger$ F. Hannachi,$\ddagger$ A. Korichi, $\S$ and A. Lopez-Martens $\ddagger$ )}

Recently, our group at ANL has delineated for the first time the decay of a superdeformed band to the yrast states 1 . As a result, the excitation energy and spin of the yrast superdeformed band in ${ }^{194} \mathrm{Hg}$ has been determined. Shortly after this discovery, similar results were reported for the yrast SD band of ${ }^{194} \mathrm{~Pb}^{2}$ and an excited SD band in ${ }^{194} \mathrm{Hg}^{3}$. These data have generated much interest, and a number of new experiments have been performed at Gammasphere in order to establish similar connections between the SD bands and known states for other nuclei in this mass region.

\footnotetext{
*Lawrence Berkeley National Laboratory, $†$ North Carolina State University, $\ddagger$ Centre de Spectrometrie Nucleaire et de Spectrometrie de Masse, Orsay, France, §Institut de Physique Nucleaire, IN2P3-CNRS, Orsay, France

${ }^{1}$ T. L. Khoo et al., Phys. Rev. Lett. 76,1583 (1996).

2 A. Lopez-Martens et al., Phys. Lett. B380, 18 (1996).

${ }^{3}$ G. Hackman et al., Proceedings for the Conference on Nuclear Structure at the Limits, Argonne, IL, July 22-26, 1996, ANL/PHY-97/1, p. 1-5 (1997).
} 
We have performed one such experiment on ${ }^{193} \mathrm{Hg}$. Six SD bands are known in this nucleus, three of which have nearly identical moments of inertia to the yrast SD band of ${ }^{192} \mathrm{Hg}$. SD states in ${ }^{193} \mathrm{Hg}$ were populated via the ${ }^{176} \mathrm{Yb}\left({ }^{22} \mathrm{Ne}, 5 \mathrm{n}\right)$ reaction at a beam energy of 118 $\mathrm{MeV}$. The target consisted of a $500 \mu \mathrm{g} / \mathrm{cm}^{2} 176 \mathrm{Yb}$ foil evaporated on a $6.8 \mathrm{mg} / \mathrm{cm}^{2}$ Au backing. The beam was accelerated by the 88-Inch Cyclotron at Lawrence Berkeley National Laboratory, and $\gamma$ rays were detected by the Gammasphere array which, for this experiment, had $85 \mathrm{Ge}$ detectors. A total of $1.9 \times 10^{9}$ events with a fold $\geq 4$ were collected.

A thorough analysis of the data by both the Argonne and Berkeley groups has yielded no evidence for onestep decays in any of the reported SD bands of ${ }^{193} \mathrm{Hg}$. The total number of counts observed in the strongest transitions of each band is of the same order as that reported for SD band 1 of ${ }^{194} \mathrm{Hg}$ in Ref. 1.

\section{b.6. Fluctuations in the Strengths of Primary Transitions from Decay Out of a Superdeformed Band (T. L. Khoo, T. Lauritsen, D. Ackermann, M. P. Carpenter, G. Hackman, I. Ahmad, D. Blumenthal, S. Fischer, R. V. F. Janssens, D. Nisius, T. Døssing, ${ }^{*}$ F. Hannachi, $\dagger$ A. Lopez-Martens, $†$ A. Korichi, + and E. F. Moore§)}

The decay from SD states is precipitated by sufficient mixing with one or two of the nearest normal-deformed (ND) states among which they are embedded. The decay spectrum is then governed by the admixed highly-excited ND state. In analogy with neutroncapture gamma rays, we call the transitions which directly deexcite the state primary gamma rays. In the decay spectrum of ${ }^{194} \mathrm{Hg}$ (band 1), fifteen primary transitions above $2.6 \mathrm{MeV}$ have been detected above a $3 \sigma$ level. These transitions have a distribution in strength. The distribution in the fluctuating strengths can provide an indicator of the chaoticity of excited ND states around $4.3 \mathrm{MeV}$. In the fully chaotic limit, where the wavefunction is complex, the distribution is expected to be a $\chi^{2}$ distribution, with the number of degrees of freedom $v$ equal to 1 , giving the so-called Porter-Thomas distribution. We are in the process of analyzing the data to determine the form of the distribution. As a basis for comparison, we are also undertaking a similar analysis of the primary gamma rays from thermal-neutron capture in ${ }^{167} \mathrm{Er}$ (see section b.23.), which represents decay from an initial state which is accepted to be chaotic.

*Niels Bohr Institute, Copenhagen, Denmark, $†$ Centre de Spectrometrie Nucleaire et de Spectrometrie de Masse, Orsay, France, †Institut de Physique Nucleaire, IN2P3-CNRS, Orsay, France, §North Carolina State University

\section{b.7. The Analysis of the Quasicontinuum of Gamma Rays in Coincidence with the One-Step}

Decay-Out Lines in ${ }^{194} \mathrm{Hg}$ (T. Lauritsen, T. L. Khoo, M. F. Carpenter, G. Hackman,

D. Ackermann, I. Ahmad, H. Amro, D. J. Blumenthal, S. M. Fischer, R. V. F. Janssens,

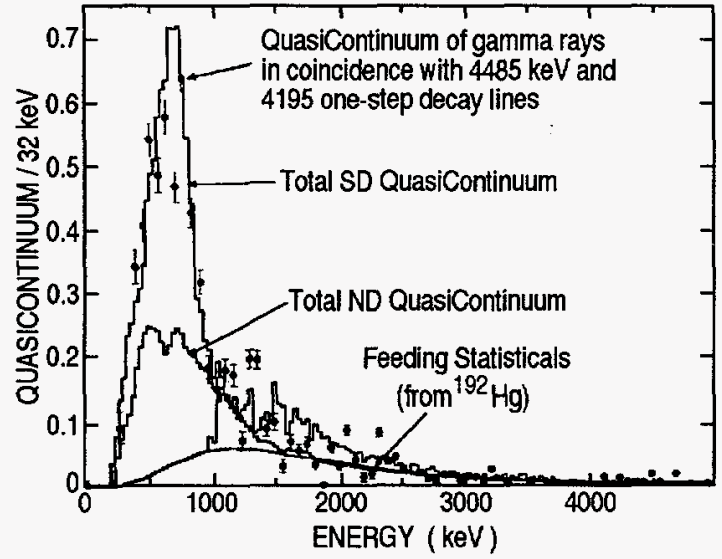

Fig. 1-16. The QC gamma rays obtained from double gates placed on the one-step decay-out lines and $S D$ lines in ${ }^{194} \mathrm{Hg}$ (data with error bars). Also shown are the total SD and ND QC spectra and the calculated statistical spectrum from ${ }^{192} \mathrm{Hg}$.
D. T. Nisius, A. Lopez-Martens, ${ }^{*}$

F. Hannachi,* A. Korichi,* and E. F. Moore $\dagger$ )

The spin and excitation energy of the yrast superdeformed band in ${ }^{194} \mathrm{Hg}$ has been extracted by analyzing the quasi-continuum (QC) of gamma rays in coincidence with the band. One of the steps in this analysis was the subtraction of the statistical E1 feeding spectrum from the total QC spectrum. This statistical spectrum has been derived from measured data in the case of ${ }^{192} \mathrm{Hg}$, but not ${ }^{194} \mathrm{Hg}$. However, it is expected that the spectra are identical.

In order to investigate the latter hypothesis, we have analyzed the QC gamma rays in coincidence with the one-step decay-out lines in ${ }^{194} \mathrm{Hg}$. By placing gates on the discrete one-step decay lines, the quasi-continuum 
of gamma rays, which dominates in the decay, are bypassed. Thus, the true $\mathrm{E} 1$ feeding quasi-continuum is no longer obscured and can be followed down to energies just above the E2 'bump' ( 1.2 MeV). The extraction of this spectrum is a very demanding task because of the low statistics of the one-step decay lines. However, in ${ }^{194} \mathrm{Hg}$ the decay-out lines are strong enough and we have enough statistics to do this analysis and the result is shown in Figure I-16. It can be seen that, indeed, the data agree well with the calculated E1 statistical decay spectrum - as well as with the QC spectrum obtained when double gates are placed on normal lines in this nucleus.

*Centre de Spectrometrie Nucleaire et de Spectrometrie de Masse, Orsay, France and Institut de Physique Nucleaire, IN2P3-CNRS, Orsay, France, $\uparrow$ North Carolina State University

\section{b.8. Determination of the Spin and the Excitation Energy of the Yrast SD Band in ${ }^{194} \mathrm{Hg}$ from Analysis of the Quasicontinuum Gamma Rays (T. Lauritsen, T. L. Khoo, M. P. Carpenter, G. Hackman, D. Ackermann, I. Ahmad, H. Amro, D. J. Blumenthal, S. M. Fischer, R. V. F. Janssens, D. T. Nisius, A. Lopez-Martens, * F. Hannachi, * A. Korichi, ${ }^{*}$ and E. F. Moore $\dagger$ )}

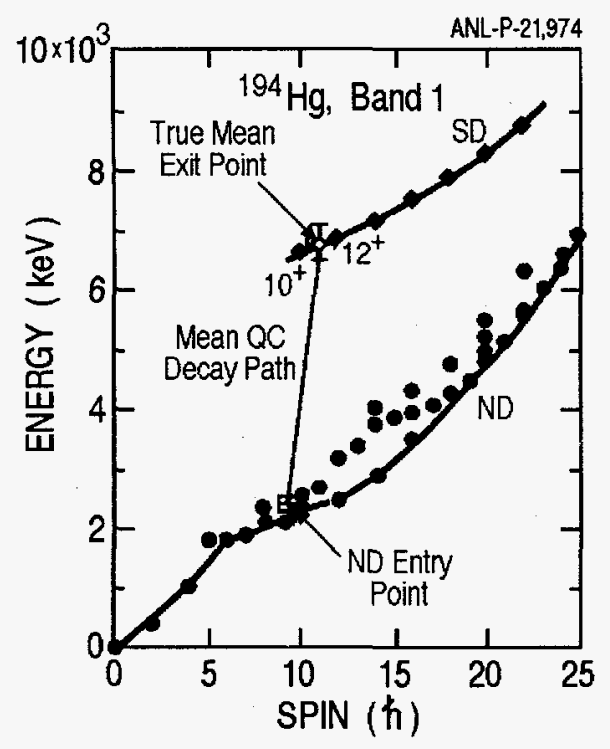

Fig. 1-17. The mean SD exit point, the average spin and energy at which the SD band decays, is found by adding the spin and energy removed by known ND lines, new unassigned ND lines and the decay-out $Q C$ gamma rays. This point, as determined by the analysis of the quasicontinuum of gamma rays, is shown with error bars. The exact result, as determined by the onestep decay-out lines, is shown with a diamond symbol.
For many superdeformed (SD) bands, Porter-Thomas fluctuations prevent the observation of discrete one-step decays from the SD band to normal states near the normal Yrast line. In such cases, the analysis of the quasi-continuum $(\mathrm{QC})$ gamma rays in coincidence with the $\mathrm{SD}$ band provides an alternative method to determine the fundamental quantities: spin and excitation energy of the SD band, albeit with some uncertainty.

In ${ }^{194} \mathrm{Hg}$, the QC gamma-ray spectrum has been analyzed in order to extract the spin and excitation energy of the yrast SD band. The result was compared to the exact result obtained from the one-step decays observed in this nucleus. In the QC analysis the ' $10^{+}$' SD state was determined to have a spin of 10.1(7) $\hbar$ and an excitation energy of 6:7(3) MeV and the analysis of the one-step decay lines determines these values to be: $10 \hbar$ and $6.6 \mathrm{MeV}$, respectively. This comparison shows that the analysis of the QC gamma rays can indeed be used to make reliable determination of the spin and excitation energy of SD bands in cases where no one-step decay lines are observed, such as in the case of ${ }^{192} \mathrm{Hg}$ (see Figure I-17).

The result of this analysis was presented at the Limits96 High Spin conference held at Argonne in July of 1996.

*Centre de Spectrometrie Nucleaire et de Spectrometrie de Masse, Orsay, France and Institut de Physique Nucleaire, IN2P3-CNRS, Orsay, France, †North Carolina State University 


\section{b.9. Quasicontinuum Spectrum of Gamma Rays Which Depopulate SD States in ${ }^{194} \mathrm{~Pb}$ \\ (T. L. Khoo, T. Lauritsen, D. P. McNabb,* J. A. Cizewski, * K.-Y. Ding, * W. Younes, * D. Archer, $\uparrow$ R. W. Bauer, $\dagger$ J. A. Becker, $\dagger$ L. A. Bernstein, $\dagger$ K. Hauschild, $\dagger$ R. M. Clark, $\ddagger$ M. A. Deleplanque, $\neq$ R. M. Diamond, \$ P. Fallon, $\$$ I. Y. Lee, $\ddagger$ A. O. Macchiavelli, F. S. Stephens, $\ddagger$ and W. H. Kelley§)}

As described in b.2., primary gamma rays from the decay out of the SD band in ${ }^{194} \mathrm{~Pb}$ have been identified and the spins, likely parity and excitation energies of the band have been determined. Experiments have been performed with both Eurogam and Gammasphere. The focus of this work is the analysis of Gammasphere data for the total decay spectrum. As for $192,194 \mathrm{Hg}$, this spectrum consists of both sharp primary transitions and an unresolved quasicontinuum component. In ${ }^{194} \mathrm{~Pb}$, however, the primary lines have a significantly larger fraction of the total decay yield, due largely to the smaller excitation energy of the SD band, which reduces the phase space for the unresolved component. The shape of the quasicontinuous component is being analyzed. One goal is to compare it with the theoretical spectrum in order to gain insight into the reduction of pairing in the ND states as function of excitation energy. Another goal is to understand the impact on SD decay of the reduced ND level density (which arises from the closed proton shell).

*Rutgers University, †Lawrence Livermore National Laboratory, $\$$ Lawrence Berkeley National Laboratory, §Iowa State University

\section{b.10. Similarities in the Decay Spectra from Superdeformed and Neutron-Capture States (T. L. Khoo, T. Lauritsen, D. Ackermann, M. P. Carpenter, G. Hackman, I. Ahmad, D. Blumenthal, S. Fischer, R. V. F. Janssens, D. Nisius, T. Døssing, ${ }^{*}$ F. Hannachi, $\dagger$ A. Lopez-Martens, $\dagger$ A. Korichi, $\Varangle$ and E. F. Moore§)}

The search for the link between superdeformed (SD) and normal-deformed (ND) states and the determination of the excitation energies and quantum numbers of SD states have long been major goals in the study of superdeformation. Through experiments at Gammasphere and Eurogam we have finally unraveled the decay mechanism by extracting the spectrum of the gamma rays connecting SD and ND states. The spectrum has a statistical-like distribution, with the bulk of it of a quasicontinuous nature and a small fraction (few \%) in sharp primary lines at high energy ( $>3$ $\mathrm{MeV}$ ) [Ref. 1]. In ${ }^{194} \mathrm{Hg}$ we have determined the excitation energies, spins and parity of the yrast SD band, and have established that decay out of the band occurs from its $10^{+}$and $12^{+}$states, which lie high (4.3 $\mathrm{MeV}$ ) above the normal-deformed yrast line ${ }^{2}$. The deexcitation spectra reveals that the decay occurs when a cold ordered SD state acquires a small component of a hot chaotic ND state, through which it deexcites.

The veracity of this interpretation can be substantiated further by comparing this decay spectrum with that from a highly-excited normal state, e.g. that following neutron capture. The complete decay spectrum ${ }^{3}$

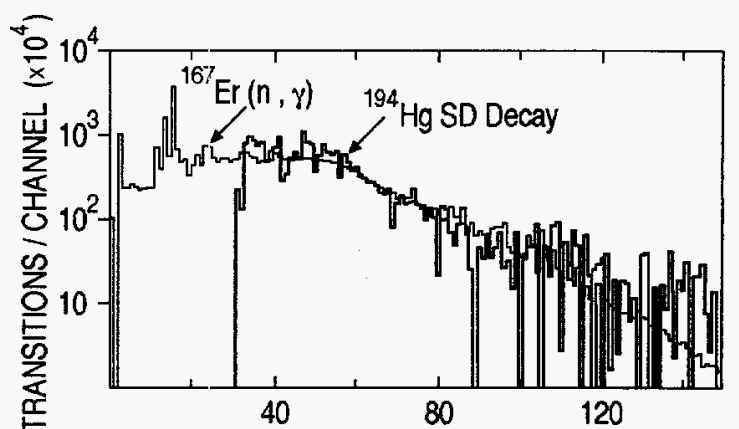

Channel Number [SD decay $32 \mathrm{keV} / \mathrm{ch},(\mathrm{n}, \gamma) 32 \times \frac{7.5}{4.3} \mathrm{keV} / \mathrm{ch}$ ]

Fig. 1-18. Overlay of spectra associated with the $S D$ decay in ${ }^{194} \mathrm{Hg}$ and with neutron capture in ${ }^{167} \mathrm{Er}$.

following thermal neutron capture in ${ }^{167} \mathrm{Er}$ has been determined by unfolding spectra measured with Compton-suppressed Ge (TESSA) detectors at the Brookhaven reactor. Figure I-18 shows an overlay of this spectrum and that from SD decay in ${ }^{194} \mathrm{Hg}$, after the spectra are adjusted so that the end points are at the same channel.

\footnotetext{
*Niels Bohr Institute, Copenhagen, Denmark, $†$ Centre de Spectrometrie Nucleaire et de Spectrometrie de Masse, Orsay, France, $\ddagger$ Institut de Physique Nucleaire, IN2P3-CNRS, Orsay, France, §North Carolina State University

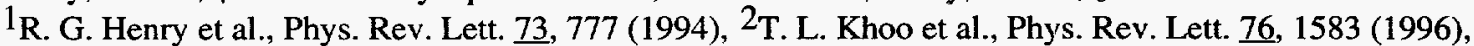

${ }^{3} \mathrm{~F}$. Soramel et al., unpublished Argonne-Brookhaven-Manchester work.
} 
Indeed, the two spectra are remarkably similar in both shape and magnitude. This similarity provides perhaps the best evidence for the mechanism proposed above for decay from SD states. Whereas in neutron capture there is a single chaos-to-order transition, the spectra emitted at different times in the feeding and decay of SD bands exhibit vastly different features, which suggest a unique double cycle of chaos-to-order transition.

\section{b.11. Differential Quadrupole Moments of Identical Superdeformed Bands in ${ }^{192,194} \mathbf{H g}$ (T. Lauritsen, R. V. F. Janssens, T. L. Khoo, D. Ackermann, I. Ahmad, D. J. Blumenthal, M. P. Carpenter, S. M. Fischer, D. Nisius, E. F. Moore, * A. Lopez-Martens, $†$ S. J. Asztalos $\ddagger$ J. A. Becker,§ L. Bernstein,§ R. M. Clark, $\ddagger$ M. A. Deleplanque, $\ddagger$ R. M. Diamond,$\ddagger$ P. Fallon, L. P. Farris, $\S$ F. Hannachi, $\dagger$ E. A. Henry, $\S$ A. Korichi, $\mathbb{W}$ I. Y. Lee, $\$$ A. O. Macchiavelli, and F. S. Stephens $\ddagger$ )}

There are many SD bands with either identical transition energies or dynamical moments of inertia. This exciting phenomenon has spurred many theoretical explanations. However, there is still no generally accepted explanation. To understand the origin of identical bands, we shall need more than simply transition energies. We have taken a two-pronged approach towards this problem by addressing the two most crucial questions. First, do identical bands have the same deformation? Second, do transitions with equal energies originate from states with the same spin and parity? The second question is addressed in b.1. Here we discuss our Doppler-shift attenuation (DSAM) measurements, with Gammasphere, of the differential quadrupole moments of the SD bands in $192,194 \mathrm{Hg}$, including those of a pair of identical bands. Differential quadrupole measurements $\mathrm{Q}_{0}$ can be determined with sufficient accuracy, unencumbered by uncertainties in stopping powers, by having the recoils stop in the same backing material and by using reactions where the stopping velocity profiles are essentially identical.

The quadrupole moments of the three bands in ${ }^{194} \mathrm{Hg}$ and one in ${ }^{192} \mathrm{Hg}$ were all equal within errors. In particular, for the identical bands (band 3 in ${ }^{194} \mathrm{Hg}$ and band 1 in ${ }^{192} \mathrm{Hg}$ ), $\Delta \mathrm{Q}_{0}=0.2 \pm 1.1 \mathrm{eb}$. In the framework of the Strutinsky method, the moment of inertia is given by a sum of a macroscopic and a shellcorrection term. For identical moments of inertia, compensation for the mass change requires $\Delta \mathrm{Q}_{0}=1.7$ $\mathrm{eb}$ in the liquid drop term. This suggests that further compensation comes from the shell-correction term. This conclusion is agreement with that from section b.1., where we conclude that the identical transition energies result, at least in part, from a cancellation between changes in pairing and particle alignment.

*North Carolina State University, and Triangle Universities Nuclear Laboratory, †Centre de Spectrometrie Nucleaire et de Spectrometrie de Masse, Orsay, France, $\ddagger$ Lawrence Berkeley National Laboratory, $\$$ Lawrence Livermore National Laboratory, IInstitut de Physique Nucleaire, IN2P3-CNRS, Orsay, France

\section{b.12. Relative Quadrupole Moments of ${ }^{192,193} \mathrm{Hg}$ Superdeformed Bands (M. P. Carpenter, D. Ackermann, I. Ahmad, D. J. Blumenthal, S. M. Fischer, R. V. F. Janssens, T. L. Khoo, T. Lauritsen, C. J. Lister, D. Nisius, D. Seweryniak, B. C. Busse, * P. Fallon, ${ }^{*}$ R. Krücken, ${ }^{*}$ S. J. Asztalos, ${ }^{*}$ R. M. Clark, ${ }^{*}$ M. A. Deleplanque, ${ }^{*}$ R. M. Diamond, ${ }^{*}$ I. Y. Lee, ${ }^{*}$ A. O. Macchiavelli, ${ }^{*}$ R. W. Macleod, ${ }^{*}$ G. Schmid, $*$ F. S. Stephens, ${ }^{*}$ K. Vetter, ${ }^{*}$ E. F. Moore, $\dagger$ F. Hannachi,ł A. Korichi,§ and A. Lopez-Martens $\ddagger$ )}

Relative quadrupole moments ( $\mathrm{Q}_{0}$ 's) of yrast and excited SD bands can give insight into the deformationdriving effects of specific nucleon configurations and aid in the interpretation of "identical" bands. A clear understanding of this phenomenon has yet to be developed. With this in mind, SD states in $192,193 \mathrm{Hg}$ were populated simultaneously via the ${ }^{176} \mathrm{Yb}\left({ }^{22} \mathrm{Ne}, \mathrm{xn}\right)$ reaction at a beam energy of $118 \mathrm{MeV}$. The target consisted of a $500 \mu \mathrm{g} / \mathrm{cm}^{2}{ }^{176} \mathrm{Yb}$ foil evaporated on a $6.8 \mathrm{mg} / \mathrm{cm}^{2}$ Au backing. The beam was accelerated by the 88-Inch Cyclotron at Lawrence Berkeley National Laboratory, and $\gamma$ rays were detected by the Gammasphere array which, for this experiment, had 85 Ge detectors. A total of $1.9 \times 10^{9}$ events with a fold $\geq$ 4 were collected. A Doppler Shift Attenuation Method (DSAM) centroid shift analysis was then performed.

\footnotetext{
*Lawrence Berkeley National Laboratory, $\uparrow$ North Carolina State University, $\ddagger$ Centre de Spectrometrie Nucleaire et de Spectrometrie de Masse, Orsay, France, §Institut de Physique Nucleaire, IN2P3-CNRS, Orsay, France
} 
Experimental fractional Doppler shifts, $F(\tau)$, were extracted and are shown in Figure I-19 for the ${ }^{192} \mathrm{Hg}$ and ${ }^{193} \mathrm{Hg}$ SD yrast bands. Calculated $F(\tau)$ curves, that assume a rotational cascade and constant $\mathrm{Q}_{0}\left(\mathrm{Q}_{\mathrm{sf}}\right)$ for the inband (sidefeeding) states, are shown for comparison. The stopping powers of Ziegler were used. A lineshape analysis on some transitions in the ${ }^{192,193} \mathrm{Hg}$ SD bands was also performed, leading to results consistent with the $F(\tau)$ analysis. Comparing the $\mathrm{F}(\tau)$ curves allowed several important conclusions. The relative $\mathrm{Q}_{0}$ 's of the yrast SD bands of ${ }^{192} \mathrm{Hg}$ and ${ }^{193} \mathrm{Hg}$ are different to a significance $>2 \sigma$, with values of $19.8 \pm 1.2 \mathrm{eb}$ and $17.2 \pm 0.7 \mathrm{eb}$ respectively. The uncertainty in the sidefeeding time, obtained by $\chi^{2}$ minimization, has been included in the $\mathrm{Q}_{0}$ errors. Nevertheless, radically different sidefeeding in the two SD Hg nuclei could cause the apparent Q0 difference, although this solution is less likely. Based on current theoretical work, the difference between ${ }^{192} \mathrm{Hg}$ and ${ }^{193} \mathrm{Hg}$ is unexpected. The experimental Q0's of all six SD bands in ${ }^{193} \mathrm{Hg}$ are the same to $\approx 1 \sigma$, which seems

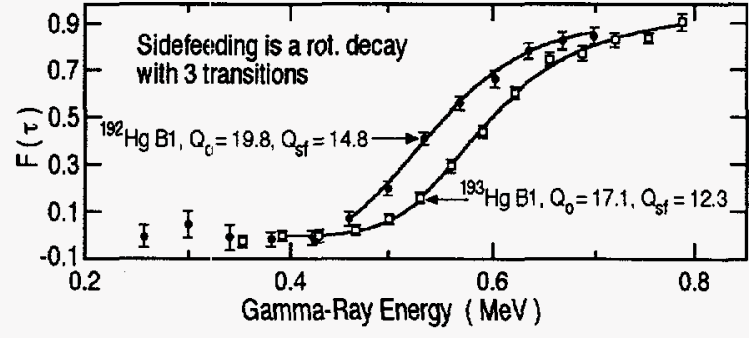

Fig. I-19. Comparison of $F(\tau)$ curves for the yrast SD bands in ${ }^{192} \mathrm{Hg}$ and ${ }^{193} \mathrm{Hg}$.

to eliminate a large polarizing effect of the different single particle levels. This similarity in $\mathrm{Q}_{0}$ is difficult to reconcile, given the difference in the $192 \mathrm{Hg}$ and ${ }^{193} \mathrm{Hg} \mathrm{Q0}$ 's. Since ${ }^{192} \mathrm{Hg}$ and ${ }^{193} \mathrm{Hg}$ have a SD "identical" band relationship, the data may imply that "identical" transition energies don't always lead to identical deformations. This is the first accurate measurement of $\mathrm{Q}_{0}$ 's in an odd-mass SD nucleus in the mass 190 region.

\section{b.13. First Measurement of Dipole Transition Rates in the Superdeformed Well}

(H. Amro, R. V. F. Janssens, B. Crowell, M. P. Carpenter, I. Ahmad, D. J. Blumenthal, S. Fischer, T. L. Khoo, D. T. Nisius, T. Lauritsen, E. F. Moore, ${ }^{*}$ J. Timar, $\dagger$

A. N. Wilson, $\ddagger$ and D. M. Cullen $\ddagger)$

An experiment was carried out using Gammasphere. Excited states in $190 \mathrm{Hg}$ were populated with the reaction ${ }^{160} \mathrm{Gd}\left({ }^{34} \mathrm{~S}, 4 \mathrm{n}\right)$ at a beam energy of $159 \mathrm{MeV}$ with a thick target $\left(1.17 \mathrm{mg} / \mathrm{cm}^{2}\right)$ evaporated on 13 $\mathrm{mg} / \mathrm{cm}^{2} \mathrm{Au}$. Three or higher-fold coincidence events were recorded. The triple gated spectra were sorted by angle and the analysis consists of both centroid shift and individual lineshape fits. Fractions of full Doppler shift $F(\tau)$ were obtained for transitions in the yrast superdeformed band. Similar analysis for the excited superdeformed band is in progress. Lifetimes of individual levels were extracted from the lineshapes with the code LILIFI.

*North Carolina State University, $\dagger$ Institute of Nuclear Research of the Hungarian Academy of Sciences, Debrecen, Hungary, $\ddagger$ University of Liverpool, United Kingdom

b.14. Differential Lifetime Measurements in ${ }^{151,152}$ Dy (D. Nisius, R. V. F. Janssens, B. Crowell, T. Lauritsen, G. Hackman, I. Ahmad, H. Amro, M. P. Carpenter, S. M. Fischer, T. L. Khoo, E. F. Moore, * P. Fallon, $\uparrow$ S. Asztalos, $\uparrow$ R. M. Clark, $\uparrow$ M. A. Deleplanque, $\uparrow$ R. M. Diamond, $\dagger$ I. Y. Lee, $\dagger$ A. O. Macchiavelli, $\uparrow$ F. S. Stephens, $\dagger$ P. J. Daly, $\ddagger Z$. W. Grabowski,,$P$. Chowdhury, $\S$ R. H. Mayer, II A. V. Afanasjev, $\|, * *$ and I. Ragnarssonll)

The demonstration that the evolution with rotational frequencies of the dynamic moment of inertia $\mathfrak{I}^{(2)}$ for SD nuclei of the $A \sim 150$ region depends strongly on the neutron $(\mathrm{N}=7)$ and proton $(\mathrm{N}=6)$ high- $N$ intruder content is by now a well established theoretical success. However, while the influence of the intruder orbitals on the $\gamma$-ray energies is well documented, their impact on

\footnotetext{
*North Carolina State University and Triangle Universities Nuclear Laboratory, $\nmid$ Lawrence Berkeley National Laboratory, $¥$ Purdue University, §Rutgers University, qUniversity of Massachusetts, IIL und Institute of Technology, Sweden, **Nuclear Research Center, Latvian Academy of Sciences, Latvia, ${ }^{1} \mathrm{D}$. Nisius et al., Phys. Lett. B392, 18 (1997) and references therein, ${ }^{2}$ H. Savajols et al., Phys. Rev. Lett. 76, 4480 (1996).
} 
other properties such as quadrupole moments $\left(\mathrm{Q}_{0}\right)$ could not be addressed until recently because the required lifetime measurements are experimentally more challenging. In addition, the extent in which the other orbitals near the Fermi surface contribute to the $\mathrm{Q}_{0}$ moment must also be delineated to bring necessary insight into phenomena such as "identical" bands. With the advent of the new large detector arrays, such as Gammasphere, the ability to detect several SD bands in the main reaction channel as well as the yrast SD bands in some of the weaker competing channels has been realized. Because the lifetimes of several bands can be measured in one experiment, the systematic uncertainties typically associated with such a measurement (e.g. stopping powers) are minimized and the necessary sensitivity to the differences in $Q_{0}$ moments between SD bands can now be probed with high accuracy.

DSAM measurements were performed with the Gammasphere spectrometer which at the time consisted of 56 Compton-suppressed Ge detectors. A ${ }^{34} \mathrm{~S}$ beam of $175 \mathrm{MeV}$ bombarded a target which consisted of $1 \mathrm{mg} / \mathrm{cm}^{2}{ }^{122} \mathrm{Sn}$ layer which was evaporated on 20 $\mathrm{mg} / \mathrm{cm}^{2}$ of Au. ${ }^{151}$ Dy was the dominant reaction channel, however ${ }^{152} \mathrm{Dy},{ }^{151} \mathrm{~Tb}$ and $148,149 \mathrm{Gd}$ were also populated. A total of $1.5 \times 10^{9}$ three- and higherfold coincidence events were collected.

Fractions of full Doppler Shift $F(\tau)$ were obtained for transitions in four of the eight SD bands in ${ }^{151} \mathrm{Dy}$ (three of the bands are new to this work) and for the yrast SD bands in ${ }^{152} \mathrm{Dy}$ and ${ }^{151} \mathrm{~Tb}$. The code FITFTAU was used to extract the $\mathrm{Q}_{0}$ moments from the $F(\tau)$ data points and both are presented in Figure 1-20. It is interesting to note that there appears to be a substantial and unexpected delay in the feeding of the ${ }^{152}$ Dy yrast $S D$ band as the $F(\tau)$ values at the top of the band never reach 1. A future Gammasphere experiment will be performed to investigate this time delay of the feeding of the SD band.

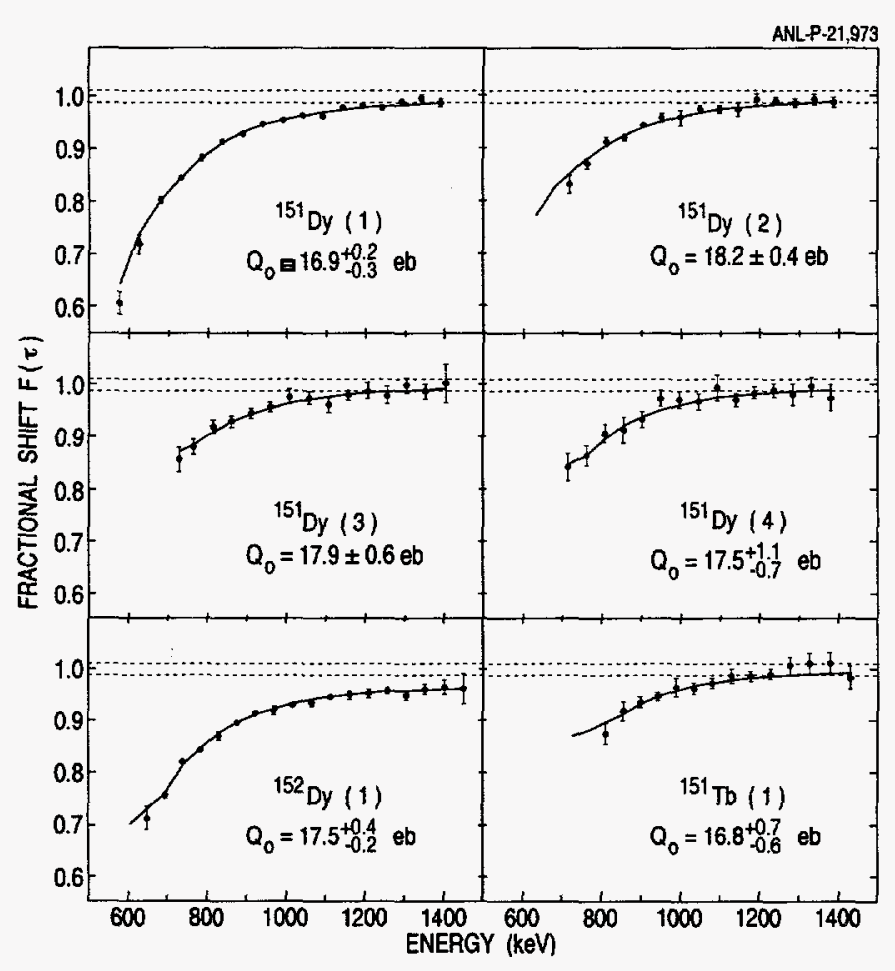

Fig. I-20. Measured fractional shifts $F(\tau)$ for SD bands in 151,152 Dy and ${ }^{151} \mathrm{~Tb}$. The curves represent the best fit corresponding to the value of the $Q_{O}$ moment given. The dashed lines represent the spread in full shift due to the slowing down of the beam across the target. See text for a detailed discussion. 
In this measurement ${ }^{1}$ and that of Savajols et al. ${ }^{2}$ the polarizing effects due to the occupation of specific high-N intruder orbitals has been deduced experimentally for the first time. In Table1 these results are compared to recent mean field $\left(\mathrm{Q}_{0} \mathrm{MO}, \mathrm{Q}_{0} \mathrm{WS}\right)$, relativistic mean field $\left(\mathrm{Q}_{0} \mathrm{RMF}\right)$ and Hartree-Fock $\left(\mathrm{Q}_{0} \mathrm{SkP}, \mathrm{Q}_{0} \mathrm{SkM}, \mathrm{Q}_{0}{ }^{\mathrm{DD}}\right)$ calculations ${ }^{1}$. While $\mathrm{Q}_{0}$ values appear larger in the calculations than the experimental values, they are well within the absolute uncertainties. However, what is more significant is that all the calculations reproduce the relative changes in the Q0 moments well. Clearly, the calculations are able to account for the polarization produced by the occupation of the specific high- $\mathrm{N}$ intruders.

The data also probe further the possible dependence of the $Q_{0}$ moments on the specific configurations occupied in the ${ }^{151}$ Dy SD well. This is especially interesting since bands 2 and 4 are characterized by $\mathfrak{S}^{(2)}$ moments which are identical to that of the $152 \mathrm{Dy}$ SD band ${ }^{1}$. The excited ${ }^{151}$ Dy SD bands are expected to correspond to excitations where the hole (with respect to the 152 Dy core) occupies one of the other orbitals near the Fermi surface $([411] 1 / 2,[642] 5 / 2$

Table 1 . Comparison of $Q_{0}$ moments from this experiment and from the measurement of ref. [2] with theoretical calculations (see text for a description of the various symbols associated with the calculations). The quoted errors do not include stopping power uncertainties. The data of ref. [2] also use the same Au stopper material and very similar recoil velocities. The calculated values are taken at a spin value of $\sim 40 \hbar$.

\begin{tabular}{|c|c|c|c|c|c|c|c|c|}
\hline Nucleus & $\mathrm{Q}_{0}^{\exp }$ & $\mathrm{Q}_{0}^{\mathrm{MO}}$ & $\mathrm{Q}_{0}^{\mathrm{WS}}$ & $\mathrm{Q}_{0}^{\mathrm{RMF}}$ & $\mathrm{Q}_{0}^{\mathrm{SkP}}$ & $Q_{0}^{S k M}$ & $\mathrm{Q}_{0}^{\mathrm{DD}}$ & \\
\hline \multirow{3}{*}{${ }^{152} \mathrm{Dy}$} & $17.5(0.2)$ & & & & & & & from [2] \\
\hline & $\&$ & 18.9 & 18.9 & 18.9 & 18.5 & 18.5 & 17.6 & \\
\hline & $17.5\left( \pm_{0.2}^{0.4}\right)$ & & & & & & & this work \\
\hline${ }^{151}$ Dy & $16.9\left( \pm_{0.3}^{0.2}\right)$ & 18.0 & 18.0 & 18.1 & 17.9 & 18.0 & 16.6 & this work \\
\hline $151 \mathrm{~Tb}$ & & 18.1 & 18.0 & 18.1 & 17.5 & 17.5 & 17.0 & this work \\
\hline $149 \mathrm{Gd}$ & $16.8\left( \pm_{0.6}^{0.7}\right)$ & 16.5 & 16.2 & 16.0 & 16.1 & 16.2 & 15.6 & from [2] \\
\hline $148 \mathrm{Gd}$ & $15.0(0.2)$ & 16.0 & 15.6 & 15.7 & 15.5 & 15.8 & 15.2 & from [2] \\
\hline
\end{tabular}

Table 2. Calculated $Q 0$ moments for three orbitals which might be involved in the configuration of the excited SD bands in ${ }^{151} \mathrm{Dy}$. The calculated values are taken at a spin value of $\sim 40 \hbar$. The intruder configurations associated with 151 Dy-band 1 and ${ }^{152}$ Dy are given for comparison. See text for details.

\begin{tabular}{ccccc} 
Configuration & $\mathrm{Q}_{0}^{\mathrm{MO}}$ & $\mathrm{Q}_{0}^{\mathrm{RMF}}$ & $\mathrm{Q}_{0}^{\mathrm{SkP}}$ & $\mathrm{Q}_{0}^{\mathrm{SkM}}{ }^{*}$ \\
\hline $\begin{array}{c}\pi 6^{4} v 7^{1}\left(151^{\mathrm{Dy}} \text {-band } 1\right) \\
\pi 6^{4} v 7^{2}(152 \mathrm{Dy})\end{array}$ & 18.0 & 18.1 & 17.9 & 18.0 \\
$([651] 1 / 2)^{-1} \otimes 152 \mathrm{Dy}$ & 18.9 & 18.9 & 18.5 & 18.5 \\
$([642] 5 / 2)^{-1} \otimes{ }^{152} \mathrm{Dy}$ & 18.3 & - & 18.1 & - \\
$([411] 1 / 2)^{-1} \otimes{ }^{152} \mathrm{Dy}$ & 18.4 & 18.7 & 18.3 & 18.3 \\
& 19.0 & - & 18.7 & 18.7
\end{tabular}


or the [651]1/2]), and the intruder content is $\pi 6^{4} v 7^{2}$ as in ${ }^{152} \mathrm{Dy}$ (and not $\pi 6^{4} v 7^{1}$ as in ${ }^{151}$ Dy-band 1). From Figure I-20 it is clear that the $Q_{0}$ values for bands 2 and 3 are larger than for band 1, as expected for bands with an additional $v 7$ intruder occupied. The errors for band 4 are too large to draw a similar conclusion. More surprising at first glance is the observation that the $\mathrm{Q}_{0}$ values for bands 2 and, perhaps for band 3 , may even be larger than the value for the ${ }^{152}$ Dy SD band. Both mean field and Hartree-Fock calculations predict that there should be a reduction in the $\mathrm{Q}_{0}$ moment for neutron holes left in the [642]5/2 or the [651]1/2 orbitals, while there will be a slight increase in $\mathrm{Q}_{0}$ for the $[411] 1 / 2$ orbital as shown in Table 2.

When $\gamma$-ray energies alone were known, possible configuration assignments were explored either using the particle-rotor model or the effective alignment method. The similarities of bands 2 and 4 with the $\gamma$ - ray energies of the ${ }^{152} \mathrm{Dy}$ SD band (i.e. they lie at the $3 / 4$ and $1 / 2$ points over a large rotational frequency range) could be understood in the framework of the particle-rotor model as neutron hole excitations of the [651]1/2 and [411]1/2 coupled to the ${ }^{152}$ Dy SD core, respectively. For band 4 pseudospin symmetry also needed to be invoked. In the effective alignment picture, bands 2 and 4 are signature partners of the [411]1/2 orbital. The data for band 2 favors the latter interpretation as the $\mathrm{Q}_{0}$ moment increases with respect to the ${ }^{152}$ Dy band. Band 4 is consistent with this interpretation, although the error bars are large. The configuration assignment for band 3 using the effective alignment method suggests this band is the neutron hole [642]5/2 coupled to the ${ }^{152}$ Dy core. However, the data does not support this contention, thus further investigations are required for this band. A paper reporting these results has been published 1 .

\section{b.15. DSAM Lifetime Measurements of ${ }^{142} \mathrm{Sm}$ Superdeformed Bands (G. Hackman, R. V. F. Janssens, T. Lauritsen, P. Reiter, H. Amro, M. P. Carpenter, S. M. Fischer, T. L. Khoo, D. Nisius, and E. F. Moore*)}

Recent lifetime measurements of "identical" bands in superdeformed (SD) nuclei 1,2 have led to a somewhat surprising result: the deduced intrinsic quadrupole moments $\mathrm{Q}_{0}$ are also identical. One of the most spectacular examples is an excited band in ${ }^{149} \mathrm{Gd}$ (band D), which has both an identical dynamic moment of inertia $\mathfrak{I}^{(2)}$ and intrinsic quadrupole moment $Q_{0}$ to the yrast $\mathrm{SD}$ band of ${ }^{152} \mathrm{Dy} .1$

A similar case of identical bands exists in ${ }^{142} \mathrm{Sm}^{3}$, where an excited SD band has the same $\gamma$-ray energies as the yrast band of ${ }^{146} \mathrm{Gd}$. In the conventional interpretation, identical bands imply identical occupation of high- $j$ intruder orbitals. Mean-field calculations for ${ }^{142} \mathrm{Sm}$ which could reproduce the $\mathfrak{I}(2)$ moment of the excited and yrast SD bands also predict that the former band would never be less than $\sim 1.5$ $\mathrm{MeV}$ above the SD yrast line, even at the highest spins. The calculations also show that the excited band would have a much higher $\mathrm{Q}_{0}$ moment than the yrast band.
The motivation for the ${ }^{142} \mathrm{Sm}$ lifetime measurements, then, was to deduce the $\mathrm{Q}_{0}$ moment for the two SD bands, and to compare the excited band to a) the yrast SD band, to test whether the mean-field calculations correctly predict the difference in deformations; and to b) the ${ }^{146} \mathrm{Gd}$ SD band, to search for further cases where identical bands yield identical $\mathrm{Q}_{0}$ moments.

The experiment was performed at Gammasphere. High-spin states in ${ }^{142} \mathrm{Sm}$ were populated by the ${ }^{124} \mathrm{Sn}\left({ }^{24} \mathrm{Mg}, 6 \mathrm{n}\right)$ reaction, with a $145 \mathrm{MeV}$ beam provided by the $88^{\prime \prime}$ cyclotron at Lawrence Berkeley National Laboratory. The target consisted of a 15 $\mathrm{mg} / \mathrm{cm}^{2}$ Au foil to slow the recoiling residual nuclei, a $60 \mu \mathrm{g} / \mathrm{cm}^{2}$ evaporated $\mathrm{Al}$ buffer layer, and a $1 \mathrm{mg} / \mathrm{cm}^{2}$ layer of isotopically enriched ${ }^{124} \mathrm{Sn}$ target material. The Al buffer layer has proven to be essential to prevent the $\mathrm{Sn}$ from migrating into the $\mathrm{Au}$, which could have serious effects on the experiment.

\footnotetext{
*North Carolina State University

${ }^{1}$ H. Savajols et al., Phys. Rev. Lett. $\underline{76}, 4480$ (1996), ${ }^{2}$ D. Nisius et al., Phys. Lett. B392, 18 (1997), ${ }^{3}$ G. Hackman et al., Phys. Rev. C $\underline{52}$, R2293 (1995), ${ }^{4}$ E. F. Moore, to be published.
} 


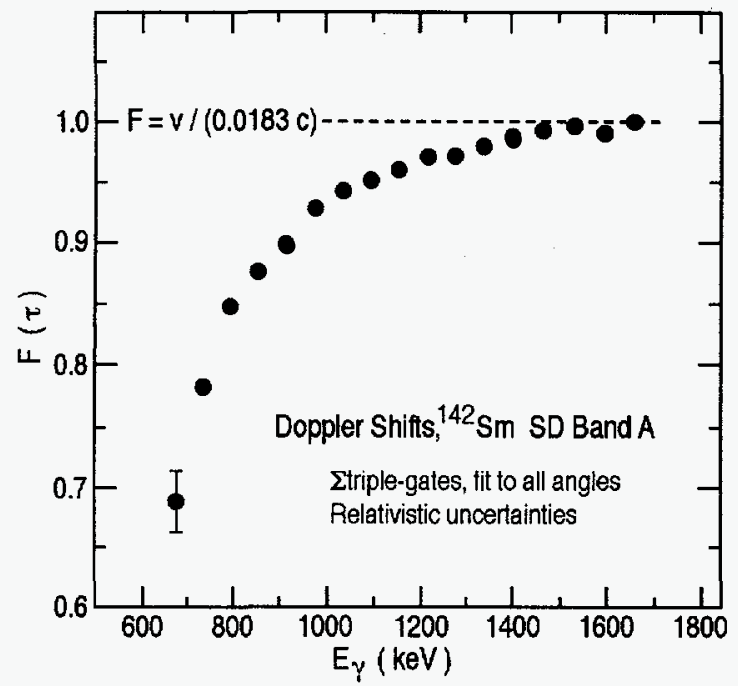

Fig. 1-21. Fraction of full Doppler-shift $(F(\tau))$ for transitions in yrast $S D$ band of ${ }^{142} \mathrm{Sm}$. The data are preliminary.

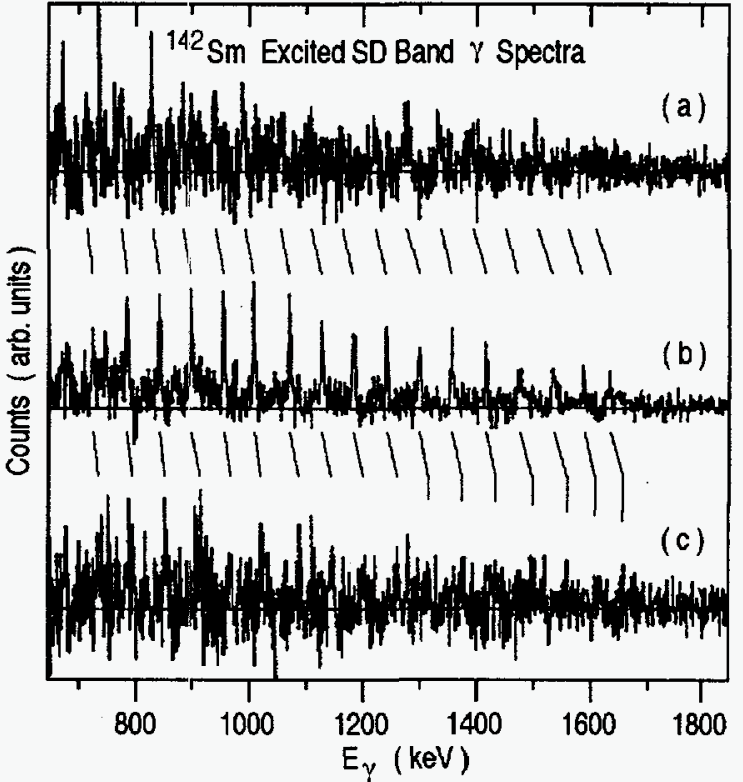

Fig. I-22. Partial triple-gated gamma spectra for the ${ }^{142} \mathrm{Sm}$ excited SD band. (a) sum of spectra from $\gamma$ rays detected in the backward four Gammasphere rings. (b) $\gamma$ spectrum from all detectors shifted into the recoiling residual nucleus frame, assuming the $F(\tau)$ curve in Figure 1-21. (c) sum of spectra from forward the four Gammasphere rings.

next step in the detailed analysis will be to deduce the Q0 values by fitting the $F(\tau)$ measurements with the FITFTAU code ${ }^{4}$.
Fraction of full-shift values $(F(\tau))$ have been measured
for the yrast SD band of $142 \mathrm{Sm}$, shown in Figure I- 21 . At this time only preliminary data reduction has been performed for the excited band (see Figure I-22). The

b.16. Test of $\Delta I=4$ Bifurcations in ${ }^{194} \mathrm{Hg}$ (G. Hackman, R. V. F. Janssens, M. P. Carpenter, D. Ackermann, I. Ahmad, H. Amro, D. J. Blumenthal, S. M. Fischer, T. L. Khoo, T. T. Lauritsen, D. Nisius, R. Krücken, * M. A. Deleplanque,* I.Y. Lee,* S. Asztalos, * R. M. Clark, ${ }^{*}$ R. M. Diamond,* P. Fallon,* R. W. Macleod,* A. O. Macchiavelli,* G. J. Schmid,* F. S. Stephens, ${ }^{*}$ K. Vetter, ${ }^{*}$ and B. Herskind $\dagger$ )

The presence of $\Delta l=4$ bifurcation (also known as $\Delta l=$ 2 staggering) in the three known superdeformed (SD) bands of ${ }^{194} \mathrm{Hg}$ has been reexamined in a new experiment with Gammasphere. A relative precision of better than $60 \mathrm{eV}$ was achieved for most transition energies. Staggering plots were extracted and their statistical significance was analyzed. No clear evidence was found for an extended regular $\Delta I=2$ staggering in the three SD bands of ${ }^{194} \mathrm{Hg}$. However, statistically significant deviations from a smooth reference were observed in the two excited SD bands. No firm conclusion about the origin of the observed deviations can be drawn. A paper on the results has recently been published. 1

*Lawrence Berkeley National Laboratory, $\uparrow$ Niels Bohr Institute, Copenhagen, Denmark

${ }^{1}$ R. Krücken et al., Phys. Rev. C $\underline{54}$, R2109 (1996). 


\section{b.17. $\Delta I=4$ Bifurcation in Identical Superdeformed Bands (G. Hackman, D. S. Haslip,*}

S. Flibotte, ${ }^{*}$ G. Gervais, ${ }^{*}$ J. M. Nieminen, ${ }^{*}$ C. E. Svensson, ${ }^{*}$ J. N. Wilson, ${ }^{*}$ G. de France, $†$

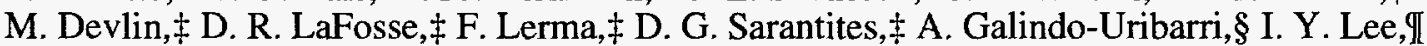
A. O. Machiavelli, $\mathbb{I}$ and S. M. Mullinsll)

$\Delta \mathrm{I}=4$ bifurcation has been observed in two superdeformed (SD) bands, the newly-discovered yrast SD band of ${ }^{148} \mathrm{Eu}$, and a previously-known excited band in ${ }^{148} \mathrm{Gd}$. Both of these bands have moments of inertia that are identical to the yrast band of ${ }^{149} \mathrm{Gd}$, the first SD band in which this bifurcation was observed. This first observation of $\Delta I=4$ bifurcation in identical SD bands provides a crucial test of recent models.
Although several cases of $\Delta I=4$ bifurcation have been reported, this work represents a number of firsts. It is the first attempt at a systematic study of the phenomenon. It is also the first case where a study of a new SD nucleus was specifically chosen with $\Delta I=4$ bifurcation as the explicit and sole purpose of the experiment. Finally it represents the first case where a theoretical framework has provided a qualitative prediction prior to the experiment and has been used to draw quantitative conclusions. A paper on these results has been submitted.

*McMaster University, Ontario, Canada, †CRN, Strasbourg, France, $\ddagger$ Washington University, §AECL, Chalk River Laboratories, Ontario, Canada, TLawrence Berkeley National Laboratory, IAustralian National University, Canberra, Australia.

b.18. Superdeformation and Normal Deformation Studies in ${ }^{191}$ Tl (M. P. Carpenter, S. M. Fischer, R. V. F. Janssens, I. Ahmad, G. Hackman, T. L. Khoo, T. Lauritsen, D. Nisius, W. Reviol,* and L. L. Riedinger*)

Recently, we have performed a measurement on ${ }^{191} \mathrm{Tl}$ with the Gammasphere detector array ${ }^{1}$ at the Lawrence Berkeley National Laboratory 88-Inch cyclotron facility. For this experiment, Gammasphere comprised 93 Compton-suppressed Ge detectors. High-spin states in ${ }^{191} \mathrm{Tl}$ were populated via the ${ }^{159} \mathrm{~Tb}\left({ }^{36} \mathrm{~S}, 4 \mathrm{n}\right)$ reaction at a bombarding energy of $165 \mathrm{MeV}$. The target foil of thickness $0.8 \mathrm{mg} / \mathrm{cm}^{2}$ was backed by a thick Au layer to be able to measure lifetimes using the Doppler-shift attenuation method. A total of $51 / 2$ billion triple and higher fold $\gamma$-ray coincidences were collected. The data analysis is in progress.

The major focus of this project is a detailed study of superdeformed (SD) states in ${ }^{191} \mathrm{Tl}$. With the new data, we observe cross-talks between the two signature partner SD bands previously reported ${ }^{2}$, and we are able to extend these bands towards lower spins where $M 1$ transitions are competitive with the inband $E 2 \gamma$ rays. M1/E2 branching ratios will be compared with calculated magnetic moments of single proton states ${ }^{3}$ to verify the proposed [642]5/2 configuration assignment. Similar studies have been done for the neighboring
${ }^{193} \mathrm{Tl}^{4}$ and ${ }^{195} \mathrm{Tl}^{5}$ nuclei, however, we will be able to base our analysis on measured transition quadrupole moments rather than on average values of deformation parameters as were used in those studies. Measuring lifetimes for SD states is critical, since quadrupole deformations of SD nuclei in the $A \sim 190$ SD island are predicted to decrease when going towards the light systems 6,7 . In turn, we will look if there is evidence for this prediction, as another goal. An interesting, but also expected observation in this data is that the line shapes of interest are broadened at about $E_{\gamma}>500 \mathrm{keV}$ but not below. This feature of the data is advantageous for trying to accomplish two other goals of our superdeformation studies in ${ }^{191} \mathrm{Tl}$, observe non-yrast SD bands and search for direct transitions from SD to normal-deformed states.

Finally, we address the long-standing question on a positive-parity level structure in the first well of ${ }^{191} \mathrm{Tl}$. Whether this structure labeled band 2 in Ref. 8 is based on a [606]13/2 (oblate) Nilsson configuration and consequently is well-deformed or not, will also be answered by the DSAM lifetime information.

*University of Tennessee

1I. Y. Lee, Nucl. Phys. A520, 361 (1990), ${ }^{2}$ S. Pilotte et al., Phys. Rev. C (1994), ${ }^{3}$ P. B. Semmes et al., Phys. Lett. $\underline{B} 345,185$ (1995), ${ }^{4}$ S. Bouneau et al., Phys. Rev. C 53, R9 (1996), 5J. Duprat et al., Phys. Lett. B341, 6 (1994), 6 W. Satula et al., Nucl. Phys. A529, 289 (1991), 7 M. W. Drigert et al., Nucl. Phys. A530, 452 (1991), 8J. M. Lewis et al. in: Progress Report on Nuclear Spectroscopic Studies, Feb. 18, 1994; and J. M. Lewis, Master Thesis, Univ. of Tennessee 1994; and W. Reviol et al., Physica Scripta T56, 167 (1995). 


\section{b.19. Superdeformation in 192Tl: Are Alignments Additive? (S. M. Fischer,} M. P. Carpenter, R. V. F. Janssens, I. Ahmad, D. J. Blumenthal, G. Hackman, T. L. Khoo, T. Lauritsen, D. Nisius, D. Seweryniak, W. Reviol, ${ }^{*}$ L. L. Riedinger, ${ }^{*}$ and B. H. Smith*)

Four superdeformed (SD) bands have previously been observed in ${ }^{192} \mathrm{Tl}{ }^{1,2}$. Two of these bands (bands $\mathrm{A}$ and $\mathrm{B}$ ) are believed to be signature partner bands in which both the $\pi \mathrm{i} 13 / 2$ and $v j 15 / 2$ intruder orbitals are occupied. These bands exhibit nearly flat dynamic moments of inertia as a function of frequency, a feature consistent with the blocking of both neutron and proton alignments due to occupation of the proposed orbitals. This configuration assignment is further supported by comparing the alignments, relative to the ${ }^{192} \mathrm{Hg}$ core, of the odd-odd ${ }^{192} \mathrm{Tl}$ bands with the sums of contributions of individual alignments from the $i_{13 / 2}$ proton bands of ${ }^{193} \mathrm{Tl}$ and the favored $\mathrm{j} 15 / 2$ neutron band of ${ }^{191} \mathrm{Hg}$. As shown in ref. 2, the addition of the individual proton and neutron alignments agrees well with the observed alignment of bands $\mathrm{A}$ and $\mathrm{B}$ in ${ }^{192} \mathrm{Tl}$. Similarly, configuration assignments based on the technique of alignment addivitity can be made for bands $\mathrm{C}$ and $\mathrm{D}$.

A recent Gammasphere experiment was performed to search for more $\mathrm{SD}$ bands in ${ }^{192} \mathrm{Tl}$ in order to further test the applicability of alignment additivity. States in ${ }^{192} \mathrm{Tl}$ were populated via the ${ }^{174} \mathrm{Yb}\left({ }^{23} \mathrm{Na}, 5 \mathrm{n}\right)$ reaction at $125 \mathrm{MeV}$. The target consisted of a stack of three
$-500 \mu \mathrm{g} / \mathrm{cm}^{2}{ }^{174} \mathrm{Yb}$ targets on thin $20 \mu \mathrm{g} / \mathrm{cm}^{2} \mathrm{C}$ backings. The decay $\gamma$ rays were detected with $92 \mathrm{Ge}$ detectors, with a trigger of four or more Compton suppressed detectors required to fire in prompt coincidence. A total of at least 14 SD bands have been observed in the data set. The four previously observed ${ }^{2}$ bands of ${ }^{192} \mathrm{Tl}$ have been confirmed. The two $\pi \mathrm{i} 13 / 2$ SD bands in both ${ }^{191} \mathrm{Tl}$ and ${ }^{193} \mathrm{Tl}$, and the yrast SD band in ${ }^{192} \mathrm{Hg}$ are also present, along with five new bands tentatively assigned to ${ }^{192} \mathrm{Tl}$.

One new transition at the top of bands A, B and D has been added to the known structures, as well as one transition at the bottom of band $C$. The crosstalk between bands $A$ and $B$ is firmly established from the new data, and crosstalk is now also observed between bands $\mathrm{C}$ and $\mathrm{D}$. However, the direct M1 transitions linking the signature partner bands have not been observed. Limits on $\mathrm{B}(\mathrm{M} 1) / \mathrm{B}(\mathrm{E} 2)$ ratios can be inferred from the measured coincidence intensities of transitions in one signature partner observed in spectra produced from gates only on transitions of the other partner. These will be compared to the particle-rotor calculations of Semmes, et al. ${ }^{3}$ Analysis of the previously identified as well as the newly observed bands continues.

*University of Tennessee

${ }^{1}$ Y. Liang et al., Phys. Rev. C 46, R2136 (1992), ${ }^{2}$ S. M. Fischer et al., Phys. Rev. C $\underline{53}, 2126$ (1996), ${ }^{3}$ P. Semmes et al., Phys. Lett. B345, 185 (1995).

b.20. Structure of Superdeformed Bands in ${ }^{195} \mathrm{Hg}$ (G. Hackman, R. V. F. Janssens, M. P. Carpenter, D. Ackermann, I. Ahmad, H. Amro, D. J. Blumenthal, S. M. Fischer, T. L. Khoo, T. Lauritsen, D. Nisius, S. Asztalos, ${ }^{*}$ R. M. Clark, ${ }^{*}$ R. M. Diamond,* P. Fallon, ${ }^{*}$ I. Y. Lee, * R. W. Macleod, * A. O. Macchiavelli, ${ }^{*}$ G. J. Schmid, ${ }^{*}$ F. S. Stephens, ${ }^{*}$ K. Vetter, ${ }^{*}$ B. Herskind, $\dagger$ and R. Wyss $\left.\ddagger\right)$

Pushing the boundaries of the "islands" of superdeformation (SD) provides an opportunity to test the fine details of nuclear models, such as the mechanisms which yield identical bands. The nucleus ${ }^{196} \mathrm{Hg}$ is predicted to have large single-particle gaps at the SD shape, and as such may be expected to exhibit "double-magic" properties like ${ }^{192} \mathrm{Hg}$. Normally one looks for new SD bands with $(\mathrm{HI}, x \mathrm{n})$ fusionevaporation reactions, with $x=4,5$. SD states in ${ }^{196} \mathrm{Hg}$ cannot be reached by such reactions, because there are no stable heavy-ion beam and target combinations which are neutron rich enough. Light ions do not bring enough angular momentum into the system, and charged-particle evaporation reactions have not

\footnotetext{
*Lawrence Berkeley National Laboratory, $†$ Niels Bohr Institute, Copenhagen, Denmark, $\ddagger$ Manne Siegbahn Institute, Stockholm, Sweden

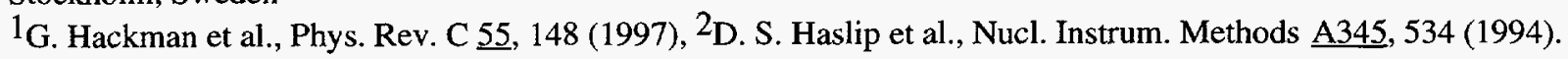


proven fruitful. ${ }^{195} \mathrm{Hg}$ suffers from the same problems. However, in a recent Gammasphere experiment which had been designed to populate ${ }^{194} \mathrm{Hg}$ at optimal conditions, we were also able to uncover four new SD bands in ${ }^{195} \mathrm{Hg}$. 1

The reaction ${ }^{150} \mathrm{Nd}\left({ }^{48} \mathrm{Ca}, x \mathrm{n}\right)$, at a nominal beam energy of $201 \mathrm{MeV}$ from the 88 " cyclotron at Lawrence Berkeley National Laboratory, was employed to populate high-spin states in the nuclei ${ }^{198-x} \mathrm{Hg}$. A stack of two $\sim 500 \mu \mathrm{g} / \mathrm{cm}^{2}$ isotopically enriched ${ }^{150} \mathrm{Nd}$ foils was used as a target. Each had thin $(<400$ $\mu \mathrm{g} / \mathrm{cm}^{2}$ ) layers of $\mathrm{Au}$ evaporated on both sides to prevent oxidation. The reaction has been shown to be the optimum for populating ${ }^{194} \mathrm{Hg}$ SD bands. Gamma rays were detected with Gammasphere comprising 72 HPGe spectrometers, with an event trigger of four "clean" detectors in prompt coincidence. After presorting for prompt time cuts and pile-up rejection, $6.1 \times 10^{8}$ triple-, $3.4 \times 10^{8}$ quadruple-, and $0.9 \times 10^{8}$ higher-fold events were available for subsequent analysis. An ANDband ${ }^{2}$ search yielded four candidates for new SD bands (Figure I-23). Since these bands were clearly observed in coincidence with 371 and 611 $\mathrm{keV} \gamma$-ray lines, which are part of the $\gamma$ cascade of the yrast normal rotational band in ${ }^{195} \mathrm{Hg}$, the new bands were assigned to that nucleus. All of the new bands were populated with intensities $<1 \%$ relative to the total $\gamma$ flux to the ${ }^{195} \mathrm{Hg}$ ground state. Spins were proposed for the new SD bands on the basis of the spin fitting technique.
Comparison of the dynamic moments of inertia $\left(\mathfrak{I}^{(2)}\right)$ of the SD bands to ${ }^{191} \mathrm{Hg}$ has provided important clues about the configurations of the ${ }^{195} \mathrm{Hg}$ bands. Two of the new bands are clearly strongly-coupled signature partners, while the other two exhibit the properties typical of $j 15 / 2$ quasiparticle configurations. These claims were placed on a stronger footing by comparison of the experimental $\mathfrak{S}^{(2)}$ moments with the results of state-of-the-art mean-field calculations with high-order pairing. The calculations for the configurations proposed in Table 1 compare adequately to the experimental data.

It is interesting that although identical bands are common in the $\mathrm{A} \sim 190$ mass region, the ${ }^{195} \mathrm{Hg}$ SD bands do not exhibit a simple identical bands relationship (that is, two bands in different nuclei which exhibit the same $\gamma$-ray energy spacing over an extended range) with any nearby nucleus. This is especially a surprise since the proposed configurations for bands (a) and (b) involve orbitals which are often associated with identical bands. Two possibilities exist: one is that identical bands arise due to a maximum in the interaction strength in the vicinity of $\mathrm{A} \sim 193$, and that ${ }^{195} \mathrm{Hg}$ is beyond the range in which the interaction strength is sufficient to yield identical bands. The second possibility is that, in analogy to the $191,192 \mathrm{Hg}$ case, the ${ }^{195} \mathrm{Hg}$ bands are better described as non-interacting quasiparticles coupled to the yet unknown ${ }^{196} \mathrm{Hg}$ SD core, in which case identical bands relationships would be expected with that nucleus. These results have recently been published. 1

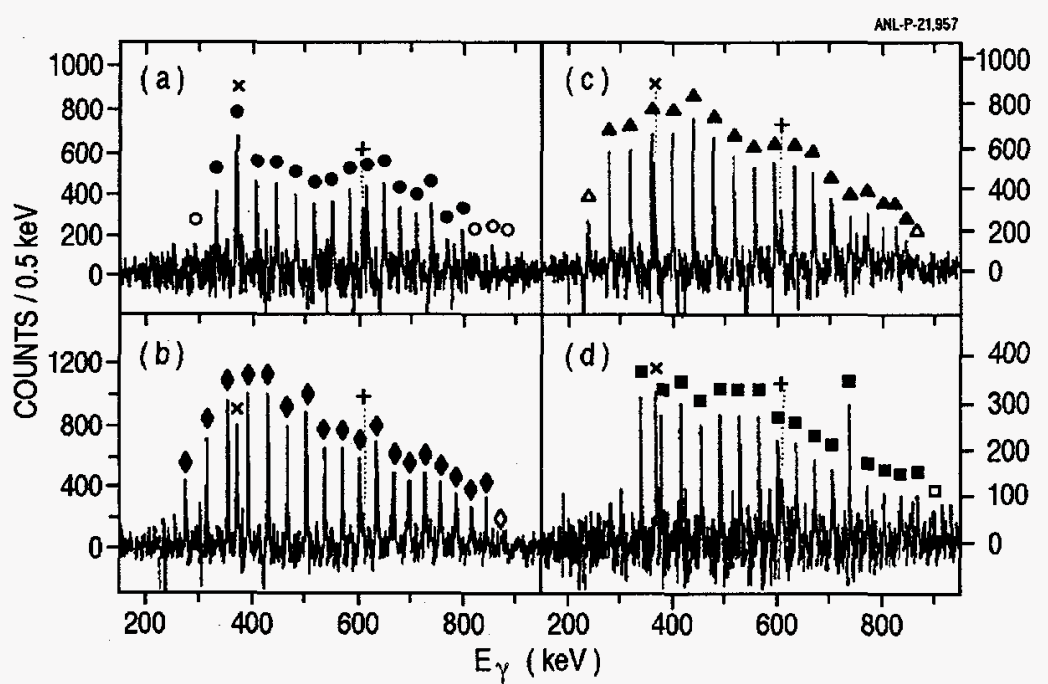

Fig. I-23. The new SD bands assigned to ${ }^{195} \mathrm{Hg}$ in-band $\gamma$ rays are indicated with filled symbols, open for tentative transitions. The 371 and 611 keV yrast lines are marked by $x$ and + respectively. 
Table 1: Summary of SD bands in $195 \mathrm{Hg}$ : population intensity relative to ${ }^{195} \mathrm{Hg}$ channel deduced from $\gamma$-ray yields $\left(I_{S D}\right)$, energy $\left(E_{\gamma}\right)$ and spin assignment $\left(I_{i} \rightarrow I_{f}\right)$ of lowest transition, parity and signature exponent, and proposed quasineutron configuration.

\begin{tabular}{llccccc} 
Band & \multicolumn{2}{c}{ Lowest transition } & Signature & Proposed \\
SD $(\%)$ & $\mathrm{E}_{\gamma}(\mathrm{keV})$ & $I_{\mathrm{i}} \rightarrow I_{\mathrm{f}}(\hbar)$ & Parity & $\begin{array}{c}\text { Exponent (a) } \\
\text { Configuration }\end{array}$ \\
\hline (a) & $0.4(2)$ & 334 & $\frac{33}{2} \rightarrow \frac{29}{2}$ & + & $+\frac{1}{2}$ & {$[624] \frac{9}{2}$} \\
(b) & $0.9(3)$ & 274 & $\frac{27}{2} \rightarrow \frac{23}{2}$ & + & $-\frac{1}{2}$ & {$[624] \frac{9}{2}$} \\
(c) & $0.4(1)$ & 285 & $\frac{29}{2} \rightarrow \frac{25}{2}$ & - & $+\frac{1}{2}$ & $\mathrm{j} 15 / 2$ \\
(d) & $0.6(3)$ & 342 & $\frac{35}{2} \rightarrow \frac{31}{2}$ & - & $-\frac{1}{2}$ & $\mathrm{j} 15 / 2$
\end{tabular}

b.21. Superdeformed Band in ${ }^{155} \mathrm{Dy}$ (S. M. Fischer, R. V. F. Janssens, R. R. Chasman, I. Ahmad, D. J. Blumenthal, M. P. Carpenter, G. Hackman, T. L. Khoo, T. Lauritsen, D. Nisius, M. A. Riley, ${ }^{*}$ T. B. Brown, ${ }^{*}$ D. J. Hartley, ${ }^{*}$ W. C. Ma, $†$ J. Simpson, $\ddagger$ and P.G. Varmette $\dagger$ )

Cranked mean field calculations ${ }^{1}$ have been extremely successful in predicting the existence of the island of superdeformed (SD) nuclei near ${ }^{152}$ Dy. However, none of these calculations predict the SD minima for the Dy isotopes of $\mathrm{N}>87$ to be yrast or near yrast at spins $\mathrm{I}<65 \hbar$, where the observed SD bands of the lighter Dy isotopes are believed to be populated. It was thus somewhat of a surprise when an SD band was observed ${ }^{2}$ in ${ }^{154}$ Dy. The present study has extended the island of superdeformation to the $\mathrm{N}=89$ nucleus ${ }^{155} \mathrm{Dy}^{3}$.

States in ${ }^{155}$ Dy were populated via the ${ }^{124} \operatorname{Sn}\left({ }^{36} \mathrm{~S}, 5 \mathrm{n}\right)$ reaction with a $175 \mathrm{MeV}$ beam from the Lawrence Berkeley National Laboratory $88^{\prime \prime}$ Cyclotron. The 1 $\mathrm{mg} / \mathrm{cm}^{2}{ }^{124} \mathrm{Sn}$ target was backed with $15 \mathrm{mg} / \mathrm{cm}^{2}$ of ${ }^{197} \mathrm{Au}$, and a $50 \mathrm{ug} / \mathrm{cm}^{2} \mathrm{Al}$ layer between the Sn and Au prevented the migration of the $\mathrm{Sn}$ into the $\mathrm{Au}$ backing. The $\gamma$ rays were detected with the Gammasphere spectrometer, which consisted at that time of $67 \mathrm{Ge}$ detectors. A total of $1.6 \times 10^{9}$ events were collected where five or more Compton suppressed detectors were required to fire in prompt coincidence.

The full Doppler shift correction was applied to the data, which were then sorted into a three-dimensional histogram. Two SD bands were observed in the cube, one corresponding to the previously known SD band of ${ }^{154} \mathrm{Dy}$, the other to a new SD band in ${ }^{155} \mathrm{Dy}$. The transitions in the ${ }^{155}$ Dy band have energies from 910 $1552 \mathrm{keV}$, with an energy spacing of approximately 48 $\mathrm{keV}$ between neighboring transitions. The band is fed and decays out at much higher transition energies than the bands of the lighter Dy isotopes. This is shown quite dramatically in Figure I-24, in which the intensity profiles of the yrast SD bands of $152,154,155$ Dy are plotted as a function of transition energy. Assuming that the transition energy can be directly related to the spins of the levels, the ${ }^{155}$ Dy intensity pattern implies that the SD band is fed at higher angular momentum than the lighter Dy isotopes, in agreement with the calculations.

The dynamic moment of inertia $3^{(2)}$ for the new SD band is presented as a function of the average rotational frequency $\hbar \omega$ in Figure I-25. The $\mathfrak{I}^{(2)}$ moments of the yrast bands of $152,153 \mathrm{Dy}$ and of the ${ }^{154} \mathrm{Dy}$ band are also shown. The ${ }^{152} \mathrm{Dy}$ band has a $\pi 6^{4} v 7^{2}$ intruder configuration, while the ${ }^{153}$ Dy band has a $\pi 6^{4} v 7^{3}$ configuration. The increase in magnitude of the $\mathfrak{S}^{(2)}$ for ${ }^{153} \mathrm{Dy}$ relative to that of ${ }^{152} \mathrm{Dy}$ is a result of the occupation of the additional neutron intruder

*Florida State University, $†$ Mississippi State University, $\ddagger$ Daresbury Laboratory, United Kingdom

${ }^{1}$ For example, J. Dudek and W. Nazarewicz, Phys. Rev. C $\underline{31}, 298$ (1985).

${ }^{2}$ D. Nisius, et al., Phys. Rev. C $\underline{51}$, R1061 (1995).

${ }^{3}$ S. M. Fischer, et al., Phys. Rev. C $\underline{54}$, R2806 (1996).

${ }^{4}$ R. R. Chasman, Phys. Lett. B364, 137 (1995). 


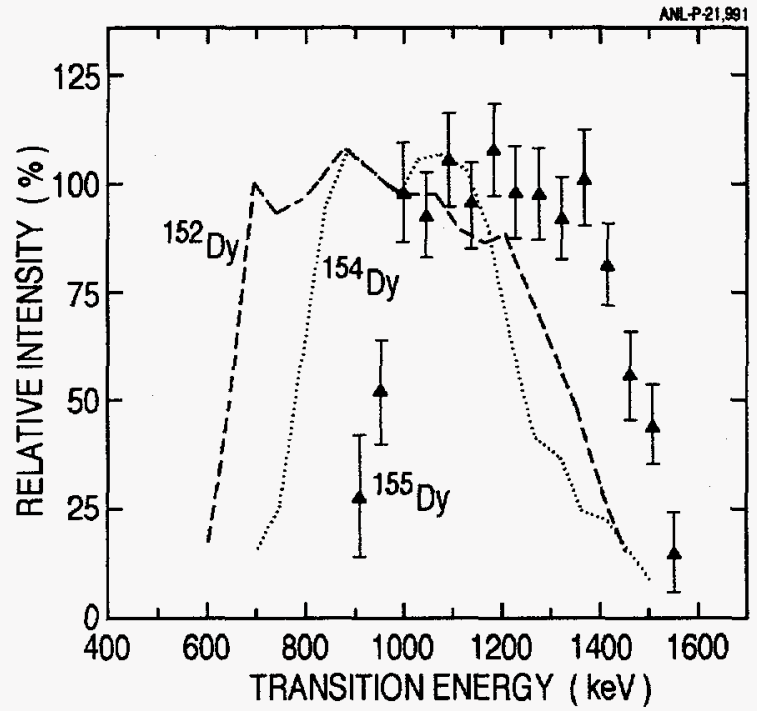

Fig. I-24. Intensity profiles of the yrast SD bands of ${ }^{152} \mathrm{Dy},{ }^{154} \mathrm{Dy}$ and ${ }^{155} \mathrm{Dy}$. Note that the sidefeeding into the ${ }^{155}$ Dy SD band ceases at a transition energy approximately $200 \mathrm{keV}$ higher than for the 152,154 Dy bands. A clear mass dependence is also present for the decay out of the SD bands.

orbital. The observation that the $\mathfrak{I}^{(2)}$ of the ${ }^{154} \mathrm{Dy}$ band is almost identical to the $\mathfrak{I}^{(2)}$ of ${ }^{152}$ Dy is proposed 2 to be a result of a small decrease in deformation in ${ }^{154} \mathrm{Dy}$, which alters the ordering of the neutron orbitals and leaves the $v 7^{3}$ orbital unoccupied. The identicality of the $\mathfrak{I}^{(2)}$ moment of the ${ }^{155}$ Dy band with that of the ${ }^{153} \mathrm{Dy}$ band implies that the $v 7^{3}$ intruder orbital is once again occupied for $\mathrm{N}=89$.

A lifetime measurement of the two observed SD bands was performed by determining the fraction of full Doppler shift, $F(\tau)$, as a function of transition energy as the nucleus slows down in the target and backing material. Quadrupole moments, Q0, for both bands were extracted from the measured $F(\tau)$ values. A fit to the $F(\tau)$ values for ${ }^{154}$ Dy yields a value $\mathrm{Q}_{0}=15.9_{-2.1}^{+3.1} \mathrm{eb}$, while the ${ }^{155^{\mathrm{Dy}} \text { data imply }}$

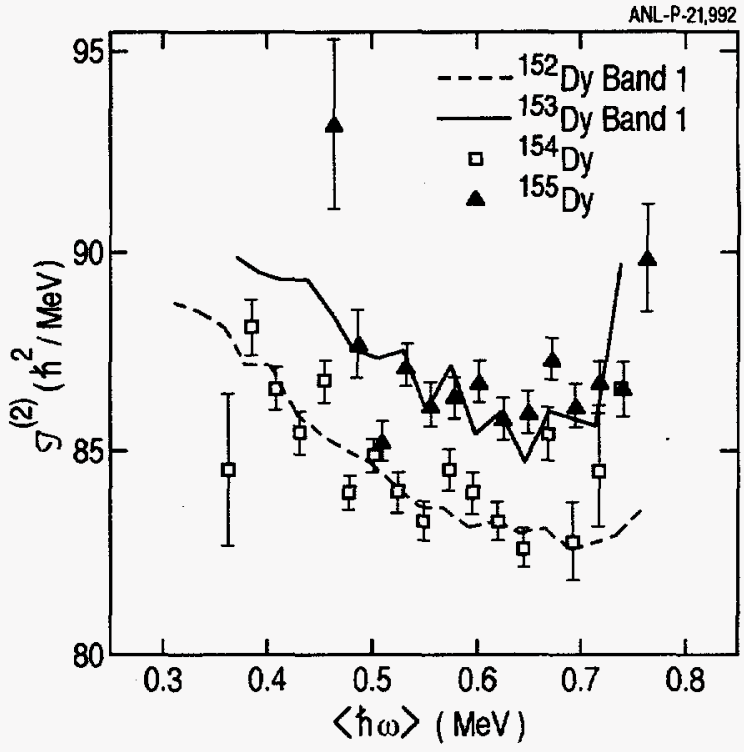

Fig. 1-25. Dynamic moments of inertia $\mathfrak{S}^{(2)}$ as a function of average rotational frequency $\hbar \omega$ for the yrast SD bands in $152_{D y}{ }^{153} \mathrm{Dy},{ }^{154} \mathrm{Dy}$ and ${ }^{155}$ Dy. Note the striking similarity of the $\mathfrak{I}^{(2)}$ moments in both the odd-A pair and the even-A pair of nuclei.

$\mathrm{Q}_{0}=17.9_{-2.6}^{+3.9} \mathrm{eb}$. In comparison, the recent Argonne study of lifetimes of the SD bands of ${ }^{151}$ Dy have determined the quadrupole moments of the yrast bands of ${ }^{151} \mathrm{Dy}$ and ${ }^{152} \mathrm{Dy}$ to be $16.9_{-0.3}^{+0.2} \mathrm{eb}$ and $17.5_{-0.2}^{+0.4}$, eb respectively. Therefore, it appears that the deformation of ${ }^{154} \mathrm{Dy}$ is somewhat less than that of the lighter Dy isotopes, consistent with the arguments for its proposed intruder configuration.

Recent calculations by Chasman ${ }^{4}$ have suggested the presence at the highest spins of collective excitations having moments of inertia $10-15 \%$ larger than that of SD nuclei around ${ }^{152} \mathrm{Dy}$ and corresponding to shapes with a somewhat larger elongation. Searches for a sequence of gamma-ray transitions with energy spacings between 30 and $50 \mathrm{keV}$ in this data set offer no firm evidence for a band of this character. 


\section{b.22. Search for Superdeformed and Elongated Shapes in ${ }^{156} \mathrm{Dy}$ (S. M. Fischer,} R. V. F. Janssens, D. Nisius, I. Ahmad, M. P. Carpenter, G. Hackman, T. L. Khoo, T. Lauritsen, P. Reiter, M. A. Riley, * T. B. Brown,* D. J. Hartley,* W. C. Ma, † and J. Simpson $\ddagger$ )

Recent results from Gammasphere have extended the island of superdeformation in the $\mathrm{A} \sim 150$ region to the $\mathrm{N}=89$ nucleus ${ }^{155} \mathrm{Dy} 1$. Calculations by Chasman ${ }^{2}$ also predict rotational bands at very high spin associated with elongations larger than for the SD shapes, and with dynamic moments of inertia of $\sim 100$ $\hbar^{2} / \mathrm{MeV}$ for nuclei in this mass region. With this motivation, states in the nucleus ${ }^{156} \mathrm{Dy}$ were populated via the ${ }^{124} \mathrm{Sn}\left({ }^{36} \mathrm{~S}, 4 \mathrm{n}\right)$ reaction at a beam energy of 165 $\mathrm{MeV}$ provided by the Lawrence Berkeley National Laboratory 88" Cyclotron. The target was composed of a stack of two $400 \mu \mathrm{g} / \mathrm{cm}^{2}{ }^{124} \mathrm{Sn}$ foils. The experiment was performed using the Gammasphere detector array, consisting of $93 \mathrm{Ge}$ detectors. Five-fold and higher Compton suppressed Ge events were required by the trigger.
The data were sorted into a three-dimensional histogram in which the full Doppler shift correction at mid-target was applied. One weak SD band has been observed, confirming the previously identified yrast band of $155 \mathrm{D} \mathrm{Dy}$. The data were also sorted into a similarly Doppler-shifted cube which was further gated by at least one of a set of transitions between the normal deformed states of ${ }^{156} \mathrm{Dy}$. This was done in order to significantly reduce the statistics of the many rotational bands found in ${ }^{155} \mathrm{Dy}$, several of which have transitions of energy and spacing similar to that expected for an $A \sim 150$ SD band. Thus far, no evidence has been found for a SD band in ${ }^{156} \mathrm{Dy}$. The search for a sequence of $\gamma$ ray transitions with energy spacings between 30 and $40 \mathrm{keV}$ characteristic of the proposed elongated shapes is in progress.

*Florida State University, $†$ Mississippi State University, $\neq$ Daresbury Laboratory, United Kingdom

${ }^{1}$ S. M. Fischer et al., Phys. Rev. C $\underline{54}$, R2806 (1996).

2R. R. Chasman, Phys. Lett. B364, 137 (1995).

\section{b.23. Statistical Behavior of High-Energy Radiative Transitions to Low-Energy Rotational States (L. M. Bollinger and T. L. Khoo)}

During the 1960's and 1970's, a considerable effort was devoted to studies of the statistical properties of radiation widths of transitions to individual low-energy final states from a series of initial states excited by neutron capture. This work demonstrated that the probability distributions for these widths is consistent with the Porter-Thomas distribution derived from Wigner's random-matrix theory of highly-excited nuclear states. The present study addresses the reverse question: to what extent does the statistical behavior of transitions from a single initial state formed by the thermal-neutron capture depend on the non-random characteristics of a series of low-energy rotational final states?
Our initial effort has focused on published data for ${ }^{168} \mathrm{Er}$. Since there is no known theoretical guidance for our problem, the approach is to determine whether or not the data is consistent with pure statistical behavior. This analysis gives several strong hints that the radiation widths in ${ }^{168} \mathrm{Er}$ are not entirely statistical, but the small sizes of the statistical samples available and uncertainties in some of the data raise doubts about the significance of the observed phenomena. However, one conclusion that appears to be firm in all respects is that the probability distribution of widths of E1 transitions to the negative-parity rotational states in ${ }^{168} \mathrm{Er}$ differs greatly from the statistical Porter-Thomas distribution. A search for other provable non-statistical phenomena is in progress and, in the future, other nuclides will be studied. 


\section{b.24. Probing the Phase Transition Boundary of Hot States in ${ }^{154} \mathrm{Dy}$ (T. L. Khoo, T. Lauritsen, D. Nisius, I. Ahmad, D. Blumenthal, M. P. Carpenter, B. Crowell, D. Gassmann, R. V. F. Janssens, W. C. Ma, * V. Martin, † L. Egido,\| P. G. Varmette,

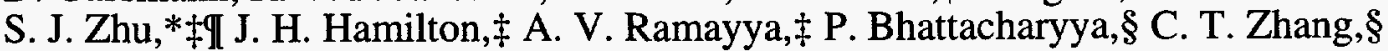 P. J. Daly, § Z. W. Grabowski,§ and R. H. Mayer§)}

Mean-field theories predict phase transitions in nuclei, such as a transition from collective to oblate shapes. However, fluctuations in the finite nucleus smear out the transition, and it is an interesting problem in mesoscopic physics to search for a remnant signature of the phase transition. Temperature-dependent HartreeFock theory ${ }^{1}$ predicts that the collective-to-oblate phase transition boundaries occur in a domain that can be favorably probed in experiments in $152,154,156 \mathrm{Dy}$.

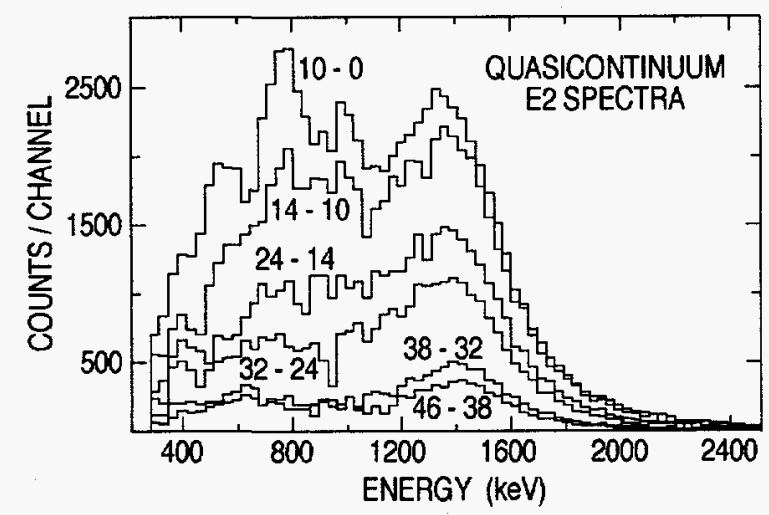

Fig. I-26. Quasicontinuum E2 spectra gated on different spin regions.
We have measured the quasicontinuous spectra feeding yrast states of specified spin intervals in ${ }^{154} \mathrm{Dy}$. By selecting definite spin intervals of the yrast line, it is possible to select for the first time definite regions of the energy-spin plane. Data were obtained from an experiment with Gammasphere, when 36 detectors were available. The quasicontinuum $\gamma$ spectrum feeding all states in ${ }^{154}$ Dy exhibits a unique two-bump feature for the E2 transitions. However, as the feeding spin into the yrast line increases, the lower energy bump decreases in intensity (see Figure I-26).

This past year we have concentrated on calculating the theoretical $\gamma$ spectra corresponding to the experimental gates. The overall features and trends (with spin) of the calculated spectra are in qualitative agreement with the experimental ones. The calculations are based on the results of temperature-dependent Hartree-Fock theory. ${ }^{1}$ The experimental and theoretical spectra together provide strong confirmation of our previous interpretation that the two bumps arise from $\gamma$ transitions which lie on either side of the phase boundary. Hence, the signature for a phase transition in the nuclear mesoscopic system persists.

Future work will examine the $E \gamma$-E $\gamma$ correlation spectra to obtain more detailed information on the character of the highly-excited states above the yrast line, and to provide further tests of the validity of temperaturedependent Hartree-Fock theory.

*Mississippi State University, †Universidad Poletechnica de Madrid, Spain, $\ddagger$ Vanderbilt University, §Purdue University, ITsinghua University, Beijing, China, IUniversidad Autonoma de Madrid, Spain

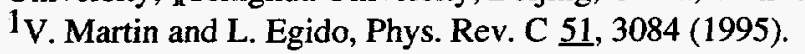


b.25. Spectroscopy at $\mathbf{I} \sim \mathbf{5 0} \hbar$ in Normal Deformed Dysprosium Nuclei (D. Nisius, M. P. Carpenter, S. M. Fischer, R. V. F. Janssens, T. K. Khoo, T. Lauritsen, T. B. Brown, * M. A. Riley,* D. E. Archer,* J. Döring, ${ }^{*}$ S. L. Tabor,* J. Simpson, $\dagger$ P. Fallon, $\ddagger$ C. Kalfas, $\S$ W. C. Ma,II J. F. Sharpey-Schafer,\|l I. Ragnarsson,** and A. Afanasjev**)

We have continued to push the limit of the highest spin states observed in normal deformed nuclei by investigating the rare-earth nuclei $154,155,156,157$ Dy employing the ${ }^{124} \mathrm{Sn}\left({ }^{36} \mathrm{~S}, \mathrm{xn}\right)$ reaction at beam energies of $165 \mathrm{MeV}$ and $175 \mathrm{MeV}$. In order to gain more insight into the structural changes taking place in these nuclei at such high spins two experiments have been performed using Gammasphere, each using a different target specification. Two $500 \mu \mathrm{g} . \mathrm{cm}^{-2}$ stacked ${ }^{124} \mathrm{Sn}$ targets were used at $165 \mathrm{MeV}$ and a target consisting of $1 \mathrm{mg} / \mathrm{cm}^{2} 124 \mathrm{Sn}$ evaporated on a $15 \mathrm{mg} / \mathrm{cm}^{2} \mathrm{Au}$ backing together with a thin $50 \mu \mathrm{g} . \mathrm{cm}^{-2}$ buffer layer of $\mathrm{Al}$ between the $\mathrm{Sn}$ and the $\mathrm{Au}$, was employed at the 175 $\mathrm{MeV}$ beam energy.

The yrast band of ${ }^{156}$ Dy has been extended to a spin state of $I=56 \hbar$ with possible candidates for higher lying states. The large number of sidebands which had been previously observed up to spin $40 \hbar$ have also been extended. A wealth of new interesting phenomenon begin to occur near and above spin 40 , including "unpaired" band crossings, coexistence between prolate and oblate shapes and band termination. We are in the process of unraveling this complicated behavior. Such clarification is helping to determine the exact behavior of certain bands in 156 Dy which display evidence for $\Delta \mathrm{I}=2$ staggering effects.

In 154,155 Dy our results greatly enhance the picture of co-existing collective and terminating sequences and highlight the importance of particle-hole excitations in building collective band structures in neighboring nuclei at high spins. It seems that very minor changes in the occupation of a few specific orbitals can lead to dramatic differences in the observed behavior of band structures between $\mathrm{I}=35-50 \hbar$.

Although ${ }^{155}$ Dy and ${ }^{156}$ Dy (as well as ${ }^{154}$ Dy) have been the main focus of these experiments since they accounted for about $90 \%$ of the observed reaction cross section, very exciting results are also being extracted from the weak $\alpha \times n$ (Gd) channel. What makes these latter measurements significant is that it is just not possible to populate these nuclei via the dominating $(\mathrm{HI}, \mathrm{xn})$ reaction with stable beams and targets and so charged particle emission is the only way to produce these nuclei at high spins until the development of radioactive beam facilities. Extensive level schemes for the nuclei ${ }^{152} \mathrm{Gd}$ and ${ }^{153} \mathrm{Gd}$, which extend up to I 35-40 $\hbar$, have been made possible by combining the present high-spin results from Gammasphere with complimentary light-ion, $\alpha \times n$, reactions performed at Florida State that have cleanly established the low to medium spin structure of these nuclei. A comparison of these transitional $\mathrm{Gd}$ isotopes with their Dy counterparts is especially interesting since not only do they display very similar emission spectra at low spins (e.g. their yrast transition energies are: ${ }^{155}$ Dy 227 , $363,464 \mathrm{keV} ;{ }^{153} \mathrm{Gd} 226,363,466 \mathrm{keV}$ ) but we are also observing a transition from prolate collective to oblate non-collective structure in the Gd isotopes for the first time above spin $30 \hbar$ thus allowing a detailed investigation of the role of valence protons near $\mathrm{Z}=64$ in determining high-spin behavior in this $N \sim 88$ transitional region. Analysis of these data is still in progress.

*Florida State University, †Daresbury Laboratory, United Kingdom, $\ddagger$ Lawrence Berkeley National Laboratory, §Institute of Nuclear Physics, NCSR Demokritos, Greece, TIMississippi State University, IIUniversity of Liverpool, United Kingdom, **Lund Institute of Technology, Sweden 


\section{b.26. Measurement of Absolute BE(2)s in the A 130 Even-Even Xe Nuclei}

(A. H. Wuosmaa, S. M. Fischer, C. J. Lister, C. M. Conner, G. Hackman, D. J. Hofman, R. V. F. Janssens, V. Nanal, D. E. Roa, and A. Aprahamian*)

The A $\sim 130$ even-even Xe nuclei exhibit many characteristics of the $\mathrm{O}(6)$ symmetry of the Interacting Boson Model. This implies that the nuclei are soft, or unstable, in the triaxial degree of freedom, gamma. However, these nuclei have also been interpreted with reasonable success in the rigid triaxial rotor model. This apparent discrepancy in describing the same set of nuclei as both gamma-soft and rigid triaxial has to date not been satisfactorily resolved. In both cases, theoretical interpretation has been based mainly on the comparison with observed energy levels and relative $\mathrm{B}(\mathrm{E} 2)$ transition probabilities.

A systematic study of measurements of absolute $\mathrm{BE}(2)$ values for the $124,128,132,134 \mathrm{Xe}$ nuclei has been performed at the ATLAS Positron EXperiment (APEX) spectrometer by means of Coulomb excitation of Xe beams of approximately $4.5 \mathrm{MeV} / \mathrm{A}$ on a ${ }^{208} \mathrm{~Pb}$ target. By studying the systematics of nuclei in this transitional mass region, it is possible to examine the interplay between gamma-softness and triaxiality, particularly as a function of the neutron number as $\mathrm{N}$ approaches the magic number 82 . The $\mathrm{Xe}$ and $\mathrm{Pb}$ nuclei were detected in the APEX heavy-ion counters, which cover the lab angles of $20 \leq \theta \leq 68$ degrees and the full 360 degrees in $\phi$. Excellent time of flight separation between the scattered $\mathrm{Xe}$ and the recoiling $\mathrm{Pb}$ was observed, and particle identification can be achieved for events in which either one or both heavy ions are detected. The coincident gamma-rays were detected in four large volume Ge detectors. Transitions up to the $10^{+}$state in the ground state band of the lighter $\mathrm{Xe}$ isotopes were observed, and the quasi-gamma band was populated in all cases. The data are currently being analyzed.

*University of Notre Dame

b.27. Search for the Two-Phonon Octupole Vibrational States in 208Pb (H. Amro, R. V. F. Janssens, W. Henning, T. L. Khoo, I. Ahmad, M. P. Carpenter, T. Lauritsen, C. J. Lister, D. J. Blumenthal, G. Hackman, S. Fischer, E. F. Moore,* S. I. Sanders, $\dagger$ and S. Yates $\ddagger$ )

Gammasphere has been used to search for the 2-phonon octupole multiplet in $208 \mathrm{~Pb}$. A thick ${ }^{208} \mathrm{~Pb}$ target $\left(50 \mathrm{mg} / \mathrm{cm}^{2}\right)$ was used in a Coulomb excitation measurement with a ${ }^{136} \mathrm{Xe}$ beam at an energy of 6.2 MeV/A. Double, triple and higher-fold coincidence events were recorded. The data were sorted into a $\gamma-\gamma$ matrix gated on the $3^{-} \rightarrow 0^{+}$transition in $208 \mathrm{~Pb}$ and in a cube which includes the low multiplicity events. Since the beam energy was above the Coulomb barrier, events due to reactions other than Coulomb excitation will dominate at high multiplicity. At first glance, nothing "obvious" stood out in our spectra. Thus, if there is anything to be seen, it is at an intensity level below $0.1 \%$ of the intensity of the $2614 \mathrm{keV}\left(3^{-} \rightarrow 0^{+}\right)$ transition in $208 \mathrm{~Pb}$. In any event, the measurement sets limits to a possible gamma decay from a two phonon state an order of magnitude below expectations based on a collective excitation 1 .
New transitions in both ${ }^{207} \mathrm{~Pb}$ and ${ }^{208} \mathrm{~Pb}$ nuclei have been identified. These transitions decay from high spin states.

Gammasphere and the $4 \pi$-PPAC array "Chico" have been used in another experiment to perform another search for the 2-phonon octupole multiplet in ${ }^{208} \mathrm{~Pb}$. A thin ${ }^{208} \mathrm{~Pb}$ target $\left(1 \mathrm{mg} / \mathrm{cm}^{2}\right)$ was used in a Coulomb excitation measurement with a ${ }^{136} \mathrm{Xe}$ beam at an energy of $4.96 \mathrm{MeV} / \mathrm{A}$. In contrast with the previous thick target measurement, this experiment was performed at a beam energy below the Coulomb barrier. In this way gamma rays due to reactions other than Coulomb excitation are minimized. The recorded coincidence events were: (1) Two forward scattered particles and one gamma ray, and (2) One backward scattered particle and one gamma ray

*North Carolina State University and Triangle Universities Nuclear Laboratory, †University of Kansas, $\ddagger$ University of Kentucky

1S. Landowne et al., Phys. Lett. B70, 292 (1977).

${ }^{2}$ K. Vetter, A. Macchiavelli, I. Y. Lee, M. A. Deleplanque, P. Fallon, R. Krücken, R. M. Clark, F. S. Stephens, H. Amro, R. V. F. Janssens, and E. F. Moore, to be published. 
The information recorded in the PPAC counters was used for the Doppler-correction of the gamma-ray spectra. This second measurement is being analyzed at LBNL by $K$. Vetter et $a^{2}$. So far, none of the anticipated 2-phonon transitions has been found. A limit on the yield in the energy region where those transitions are expected (with respect to the $2614 \mathrm{keV}$ line) is again well below expectations.

The results place severe limits on missing E3 strength in $208 \mathrm{~Pb}$ and lead one to question the presence of decays corresponding to 2 -phonon octupole vibrations.

\section{b.28. Coulomb Excitation of Actinides ${ }^{246,248} \mathrm{Cm}$ and ${ }^{240} \mathrm{Pu}$ (G. Hackman, R. V. F. Janssens, T. L. Khoo, I. Ahmad, D. Ackermann, H. Amro, M. P. Carpenter, S. M. Fischer, T. Lauritsen, D. Nisius, D. Seweryniak, E. F. Moore, ${ }^{*}$ D. Cline, $\dagger$ and C-Y. Wu, $\dagger$ )}

Actinide nuclei present classic examples of collective nuclear behavior. Interest in the Coulomb excitation (coulex) of the actinides has been renewed by recent work by Ward et al., ${ }^{1}$ who studied ${ }^{238} \mathrm{U}$ by $\gamma-\gamma$ coincidence measurements following so-called "unsafe" coulex. In this technique, beams above the Coulomb barrier impinge upon the target material; both the beam and recoil are stopped in a backing layer. The main advantage of this technique over "traditional" coulex experiments, in which recoils out of a thin target are detected in position-sensitive heavy-ion counters, is that most of the $\gamma$ rays are emitted after the recoiling nuclei have fully stopped, which means they are detected at the intrinsic detector resolution without any broadening due to detector opening angle.

Inspired by the results of Ref. 1, we have embarked on a program to perform similar experiments on actinide nuclei, with $\sim 300 \mu \mathrm{g} / \mathrm{cm}^{2}$ targets deposited on 50 $\mathrm{mg} / \mathrm{cm}^{2} \mathrm{~Pb}$ or $\mathrm{Au}$ backings and $\sim 6 \mathrm{MeV} / \mathrm{u} 208 \mathrm{~Pb}$ ATLAS beams. The $\gamma$ rays were detected with the Argonne-Notre Dame array, which comprises 50 BGO elements and $12 \mathrm{Ge} \gamma$-ray spectrometers. The multiplicity measured in the array is critical for attenuating backgrounds from $\mathrm{X}$-rays, backing material coulex, and fission. So far we have completed experiments on $248 \mathrm{Cm}$ and $240 \mathrm{Pu}$, and beam time has been scheduled for $242,244 \mathrm{Pu}$.

In the ${ }^{248} \mathrm{Cm}$ data, three rotational bands have been identified. The yrast band is observed to $28 \hbar^{2}$. A second band of seven states is observed in coincidence with low-spin transitions of the ${ }^{248} \mathrm{Cm}$ ground band, and is assigned as a side band in that nucleus. The two lowest-lying transitions in the third band are within 0.5 $\mathrm{keV}$ of the previously reported gamma cascade of the ${ }^{246} \mathrm{Cm}$ ground-state band ${ }^{2}$; we have extended this band to $20 \hbar$. Based on measured activity of the target, this lighter even isotope is present at $\sim 3 \%$ in the ${ }^{248} \mathrm{Cm}$ stock, which is consistent with the relative $246,248 \mathrm{Cm}$ $\gamma$ yields. In the ${ }^{240} \mathrm{Pu}$ data (see Figure I-27), the ground-state band [2] has been extended by $12 \hbar$ to the $26^{+}$state. Ten new states in a side band have also been identified, and with rigorous coincidence information from interband decay we can confidently assign spin and excitation energies for this new band.

It had been previously known that the even uranium isotopes and ${ }^{248} \mathrm{Cm}$ exhibit an increase in aligned spin $i_{x}$ starting at a rotational frequency of $\hbar \omega \sim 0.2 \mathrm{MeV}$. This is attributed to a crossing involving the $v j_{15} / 2$ and $\pi i 13 / 2$ quasiparticle pairs. Our new data show the same gentle upbending behavior in ${ }^{246} \mathrm{Cm}$. In $242,244 \mathrm{Pu}$, the same crossing is believed to generate a much steeper increase in $i_{x}$. However, our data show that a sudden upbend in ${ }^{240} \mathrm{Pu}$ does not occur at a frequency below $0.3 \mathrm{MeV}$; if anything, there is a very gradual increase in $i_{x}$ relative to the other Pu isotopes below $0.2 \mathrm{MeV}$, suggesting an anomalously large interaction strength in $240 \mathrm{Pu}$. $(238 \mathrm{Pu}$ also shows a very slight upturn in $i_{x}$ at $0.2 \mathrm{MeV}$.)

Octupole correlations have long been known to be important in the low-spin structure of actinide nuclei. If the experimental Routhian of the new ${ }^{240} \mathrm{Pu}$ band is extrapolated to low rotational frequencies, it would match that of a $\mathrm{K}^{\pi}=0^{-}$octupole band ${ }^{2}$. Furthermore, as rotational frequency increases, the new band approaches an alignment of $i_{x} \sim 2.5 \hbar$ relative to

\footnotetext{
${ }^{1}$ D. Ward et al., Nucl. Phys. A600, 88 (1996).

${ }^{2}$ R. B. Firestone and V. S. Shirley, eds., Table of the Isotopes, 8 Ed. Vol. II, pp. 2800, 2828, 2838 (1996), and references therein.
} 
the ground-state band (also shown in Figure I-27), as expected for an octupole phonon aligning its angular momentum with the axis of rotation. This behavior is strikingly similar to that of the octupole band (band B in Ref. 1) in the isotone ${ }^{238} \mathrm{U}$. If the ${ }^{248} \mathrm{Cm}$ interband transitions are $\Delta \mathrm{J}=1$, then the Routhian of this band extrapolates at low frequency to that of a $\mathrm{K}^{\pi}=1^{-}$band, and that it also has an alignment of $3 \hbar$ relative to the ground band. The similarity to the ${ }^{238} \mathrm{U}$ band (D) is remarkable.

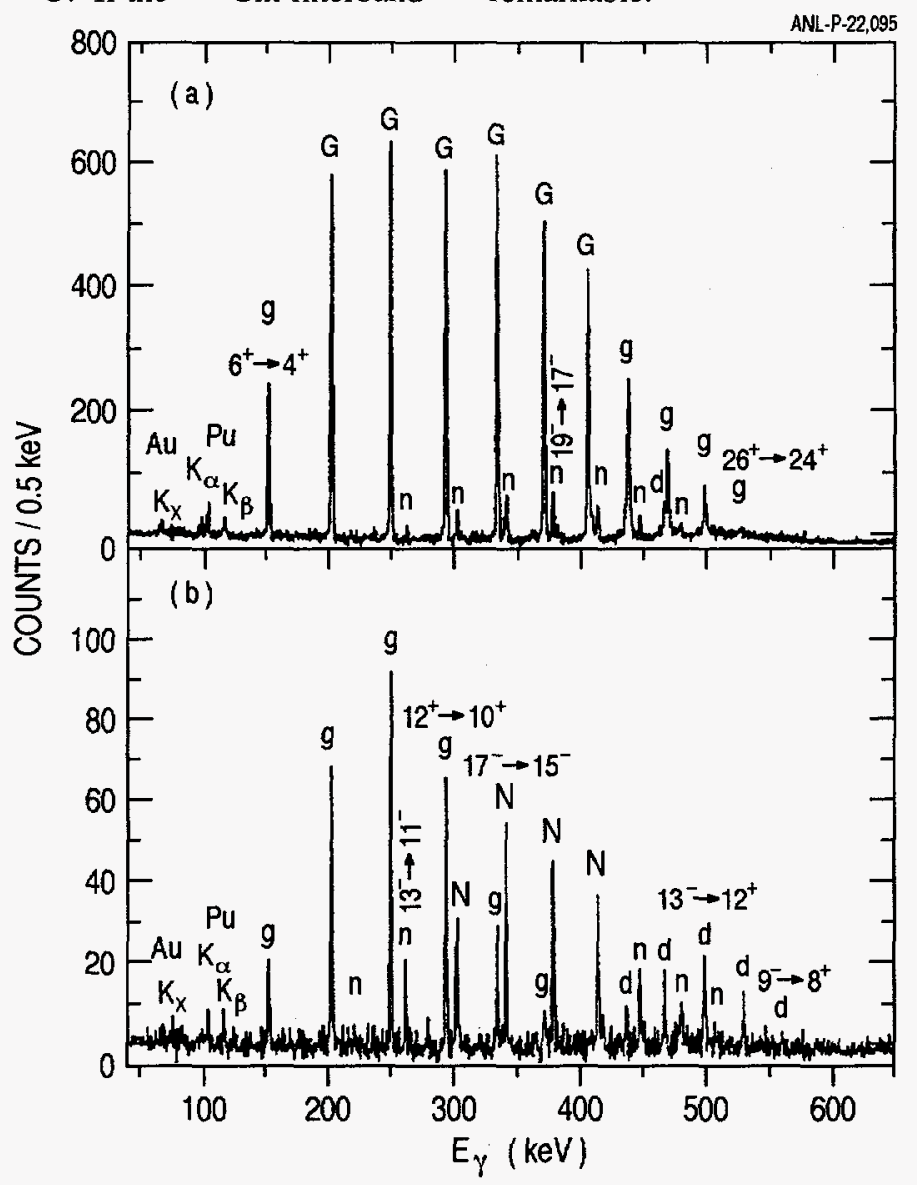

Fig. I-27 (a) Coincidence spectrum for the $240_{P u}$ ground-state band. (b) Coincidence spectrum for the $240_{P u}$ side band. Ground-state band, new band, and interband transitions are indicated by $g, n$, and $d$ respectively. Capital letters indicate gating transitions.

\section{b.29. Level Structures at High Spins In Nuclei Near and at $\mathbf{N}=52$ (M. P. Carpenter,} B. Crowell, R. V. F. Janssens, T. L. Khoo, T. Lauritsen, S. S. Ghugre,* U. Garg,* B. Kharraja, ${ }^{*}$ G. Smith, ${ }^{*}$ W. Mueller,$\dagger$ W. Reviol,$\dagger$ L. L. Riedinger, $\dagger$ and R. Kaczarowskił)

The level sequences in the $\mathrm{N}=50$ isotones of $\mathrm{Mo}$, $\mathrm{Tc}$, $\mathrm{Ru}$ and $\mathrm{Rh}$ nuclei exhibit single particle nature even at high spins $(\mathrm{I} \sim 20 \hbar)$ and excitation energies $\left(\mathrm{E}_{x} \sim 12\right.$ $\mathrm{MeV})$. On the other hand, rotational excitations are observed in nuclei with $\mathrm{N}>54$. Thus, the study of high spin states of the $\mathrm{N}=52,53,54$ isotones of $\mathrm{Mo}$, $\mathrm{Tc}$, $\mathrm{Ru}$ and $\mathrm{Rh}$ would greatly help in understanding the mechanism for the generation of the higher angular momentum states in this region, and to observe the transition from the spherical single particle mode to the collective degrees of freedom. High-spin states in 94,95 $\mathrm{Mo}, 94,95,96 \mathrm{Tc}, 96,97,98_{\mathrm{Ru}}$ and $97,98 \mathrm{Rh}$ were populated using a $142 \mathrm{MeV}^{36} \mathrm{~S}$ beam on a ${ }^{65} \mathrm{Cu}$ target. Triple- $\gamma$ coincidences were measured using the early implementation phase of Gammasphere.

*University of Notre Dame, $\nmid$ University of Tennessee, $\ddagger$ Soltan Institute for Nuclear Studies, Swierk, Poland. 
Several new transitions have been identified in these nuclei, and the level sequences have been substantially extended (up to a spin of $I \sim 20 \hbar$ and an excitation energy of $E_{x} \sim 12-14 \mathrm{MeV}$ ). Shell model calculations, using different model spaces and effective interactions, have been carried out for several of these nuclei. The $\mathrm{N}$ $=51,52$ isotones exhibit single particle nature and their higher angular momentum states are dominated by the excitation of a single g $_{9 / 2}$ neutron across the $\mathrm{N}=50$ magic shell. The "breaking" of the $\mathrm{N}=50$ core is evidenced by a number of transitions of $\sim 2 \mathrm{MeV}$ followed by a fragmentation of intensity into several parallel cascades. The level sequences of the $\mathrm{N}=53$, 54 isotones, on the other hand, begin to indicate an onset of collectivity. The weak-coupling model has been quite successful in interpreting the level sequences of these nuclei. Vibrational model calculations as well as lifetime measurements would greatly facilitate a more complete understanding of the level sequences of these nuclei.

Papers describing the results on the Mo and Ru nuclei studied are being prepared for publication.

\section{b.30. Spin-Dependent Triaxial Deformation in Neutron-Rich Mo Isotopes (I. Ahmad, L. R. Morss, * A. G. Smith, $\uparrow$ J. L. Durell, $\uparrow$ W. R. Phillips, $\dagger$ M. A. Jones,$\uparrow$ M. Leddy, $\dagger$

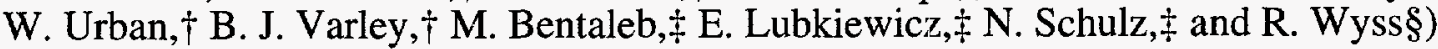

Nuclear deformations in neutron-rich nuclei in the mass 100 region have so far been measured only at low spins. These deformations are derived from the lifetimes of the rotational levels measured by delayed coincidence technique using mass separated radioactive sources. This technique is not applicable for higher rotational states because these states are generally not populated in the radioactive decay and also the lifetimes of these states are too short.

We have measured the lifetimes of rotational states up to spin $12 \hbar$ by measuring the line shapes of $\gamma$ rays emitted by secondary fission products. The experiment was performed at Strasbourg with the EUROGAM2 array and a ${ }^{248} \mathrm{Cm}$ fission source. The coincidence $\gamma$ ray spectra of individual nuclei were generated and the line shapes were analyzed. The curium source was produced by mixing $5 \mathrm{mg}$ curium oxide powder with 65 $\mathrm{mg} \mathrm{KCl}$ and subjecting the mixture to high pressure to produce a pellet. Other measurements have established that the grain size of the curium oxide powder is quite small suggesting that the recoiling fission fragments, which are emitted isotropically, are stopped in $\mathrm{KCl}$. Because of Doppler effect, $\gamma$ rays, which are emitted from levels with lifetimes comparable to (or faster than) the stopping time (1-2 ps) of the fission fragments in the source pellet, have symmetrically broadened lineshapes. A Doppler-profile method was developed which combines a simulation of the stopping of the fission fragment with a simulation of the electromagnetic decay to generate a line shape that was compared directly with the observed line shape and gave information on the state lifetimes. An example of the line shape analysis is shown in Figure I-28. From these lifetimes quadrupole moments were deduced in ${ }^{98} \mathrm{Sr}$, $100,102,104 \mathrm{Zr}$ and $104,106,109 \mathrm{Mo}$ for states up to $12 \hbar$. Our results show that the quadrupole moments in ${ }^{98} \mathrm{Sr}$ and the $\mathrm{Zr}$ isotopes are consistent with the values measured at low spin. On the other hand, the quadrupole moments in Mo isotopes at high spins are $20 \%$ lower than the respective values at low spin. These results were explained in terms of softness of these nuclei towards gamma deformation. These results were published. 1

*Chemistry Division, ANL, †University of Manchester, United Kingdom, $\ddagger$ CRN, Strasbourg, France, $\S$ KTH, Stockholm, Sweden

1Phys. Rev. Lett. 77, 1711 (1996). 


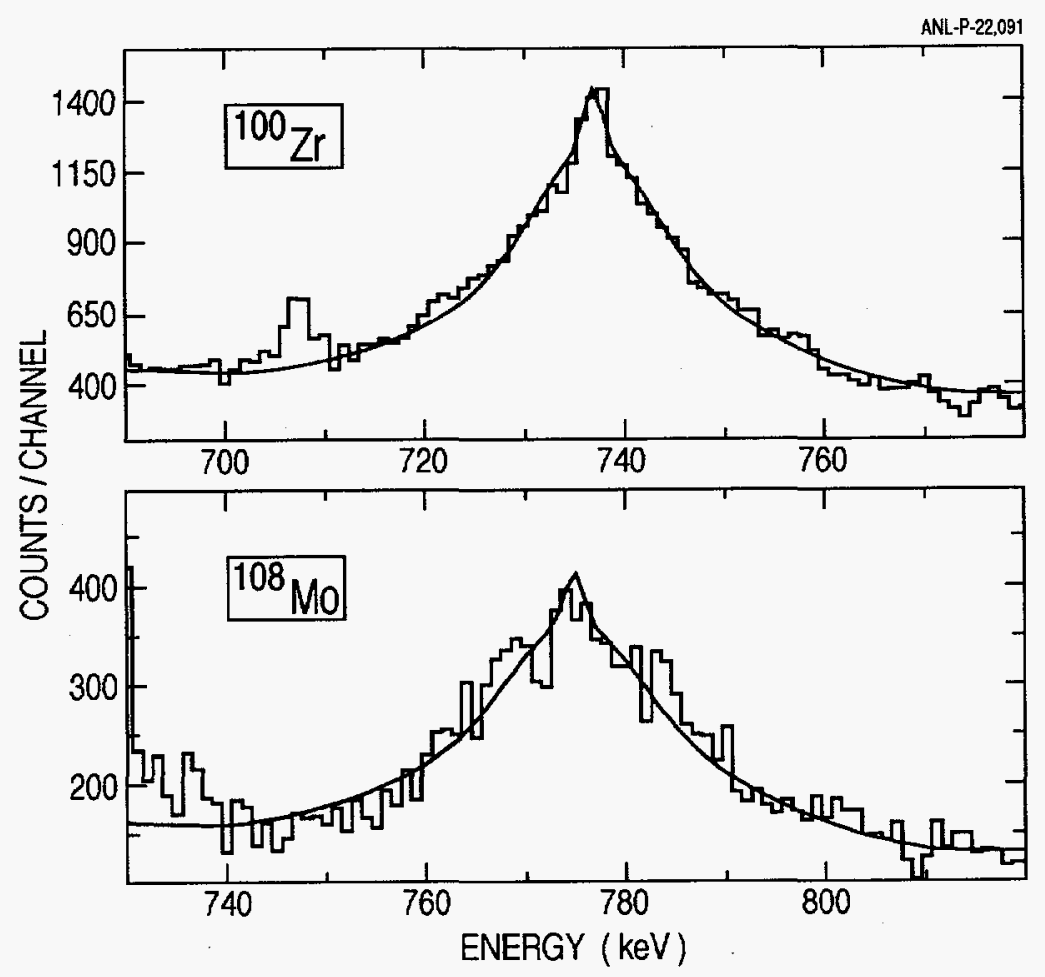

Fig. I-28. Line shapes corresponding to the decay of the yrast $I^{\pi}=10^{+}$states in $100_{\mathrm{Zr} \text { and }} 108_{\mathrm{Mo}}$. The data are represented by the histograms and the fit by the solid line.

b.31. Yrast Excitations around Doubly Magic ${ }^{132}$ Sn Nucleus from Fission Product $\gamma$-Ray Studies (I. Ahmad, M. P. Carpenter, T. Ishii, L. R. Morss, ${ }^{*}$ C. T. Zhang, $\uparrow$ P. Bhattacharyya, $\dagger$ P. J. Daly, $\dagger$ R. Broda, $\uparrow Z$. W. Grabowski, $\uparrow$ D. Nisius, W. R. Phillips, $\ddagger$ J. L. Durell, $\ddagger$ M. J. Leddy, $\ddagger$ A. G. Smith, $\ddagger$ W. Urban $\ddagger$ B. J. Varley, $\ddagger$ N. Schulz, $§$ E. Lubkiewicz,$\S$ M. Bentaleb, $\S$ and J. Blomqvist $\mid$ )

The $\mathrm{Z}=50, \mathrm{~N}=80$ nucleus ${ }^{132} \mathrm{Sn}$ can be regarded as a doubly-magic nucleus, with pronounced shell closures for both protons and neutrons manifested by the absence of excited states below $4.0 \mathrm{MeV}$ excitation energy. ${ }^{132} \mathrm{Sn}$ and nuclei around it are most effectively produced in low-energy fission. Excited states in ${ }^{132} \mathrm{~S} n$ have recently been deduced by measuring the $\beta^{-}$ decay of the parent nucleus ${ }^{132} \mathrm{In}$. We have studied the structures of ${ }^{134} \mathrm{Te}$ and ${ }^{135} \mathrm{I}$, which are two and three protons away from closed shell nucleus ${ }^{132} \mathrm{Sn}$, and have interpreted the observed levels using the shell model. By fitting the observed levels we have deduced the two-nucleon interaction strength.

The level structures of ${ }^{134} \mathrm{Te}$ and ${ }^{135} \mathrm{I}$ were deduced from $\gamma \gamma$ coincidence measurements performed with the EUROGAM2 array and a ${ }^{248} \mathrm{Cm}$ fission source. The array, for this experiment, consisted of $124 \mathrm{Ge}$ detector elements and four LEPS spectrometers to measure lowenergy $\gamma$ rays. From coincidence relationship between $\gamma$ rays in ${ }^{34} \mathrm{Te}$ and $\gamma$ rays in the complementary light Ru isotopes, gamma transitions in ${ }^{134} \mathrm{Te}$ were identified. Relationships between transitions in ${ }^{134} \mathrm{Te}$ and intensity balance allowed us to construct a level scheme (Figure I-29). In the same way, a level scheme for ${ }^{135_{I}}$ was also constructed which is included in Figure I-29. The configuration assignments shown in the figure are based on shell-model calculations. These data are interpreted in terms of valence proton and particle-hole core excitations with the help of shell-model calculations employing empirical nucleon-nucleon interactions from both the ${ }^{132} \mathrm{Sn}$ and ${ }^{208} \mathrm{~Pb}$ regions. A serious inconsistency is discovered in the accepted masses of the $\mathrm{N}=82$ isotones near ${ }^{132} \mathrm{Sn}$.

*Chemistry Division, ANL, $†$ Purdue University, $\Varangle$ University of Manchester, United Kingdom, §CRN, Strasbourg, France, IRoyal Institute of Technology, Stockholm, Sweden

${ }^{1}$ C. T. Zhang et al., Phys. Rev. Lett. 그, 3743 (1996). 

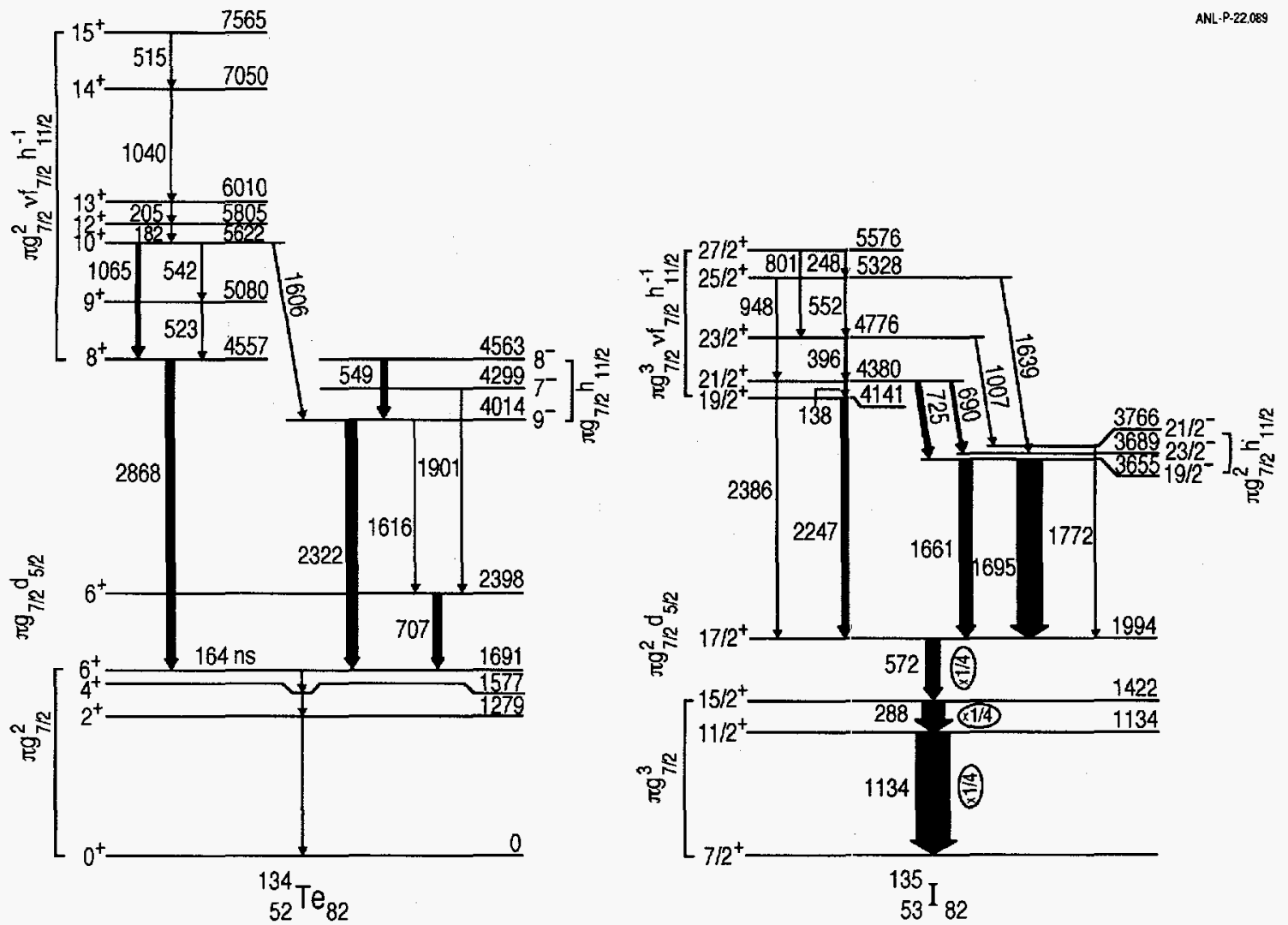

Fig. 1-29. The yrast levels of ${ }^{134}$ Te and ${ }^{135}$ I deduced from the present studies. The widths of the arrows are proportional to the observed $\gamma$-ray intensities, except for transitions below the 164-ns isomer in ${ }^{134} \mathrm{Te}$. Configuration assignments in both nuclei are also shown.

b.32. Octupole Correlations in Neutron-Rich Odd-A La Isotopes (I. Ahmad, L. R. Morss, * W. Urban, $\dagger$ W. R. Phillips, $\uparrow$ M. A. Jones, $\dagger$ M. Leddy, $\dagger$ C. J. Pearson,$\dagger$ A. G. Smith,$\dagger$ B. J. Varley, $\dagger$ M. Bentaleb, $\ddagger$ E. Lubkiewicz, $\ddagger$ and N. Schulz $\ddagger)$

Level structures of the neutron-rich nuclei ${ }^{145} \mathrm{La}$ and ${ }^{147} \mathrm{La}$ were studied in order to investigate the octupole correlations in these nuclei. The experiment was performed at Strasbourg with the EUROGAM2 array and a ${ }^{248} \mathrm{Cm}$ fission source. Gamma rays were assigned to $\mathrm{La}$ isotopes on the basis of their coincidence with the characteristic La K x-ray of $33.3 \mathrm{keV}$. Additional coincidence relationships with the transitions in the complementary light fragments and coincidence relationships between transitions in $\mathrm{La}$ nuclei led to the level schemes for ${ }^{145} \mathrm{La}$ and ${ }^{147} \mathrm{La}$ (Figure I-30). Spins and parities to the levels in these nuclei were deduced on the basis of double and triple directional correlation measurements, internal conversion coefficients and branching ratios. Features characteristic of octupole correlations were found in both nuclei. The E1 strengths in ${ }^{145} \mathrm{La}$ and ${ }^{147} \mathrm{La}$ indicate that octupole correlations in these nuclei are similar in strength to those in the even-even Ba nuclei. It appears that the addition of a proton does not affect the octupole correlations. This is in contradiction to theoretical calculations which predict that the shell structure would lead to weaker octupole correlations in $Z=57$ nuclei compared with the $Z=55$ and 56 nuclei. The results of this study are published. 1

*Chemistry Division, ANL, †University of Manchester, United Kingdom, fCRN, Strasbourg, France

${ }^{1}$ W. Urban et al., Phys. Rev. C $\underline{54}, 945$ (1996). 

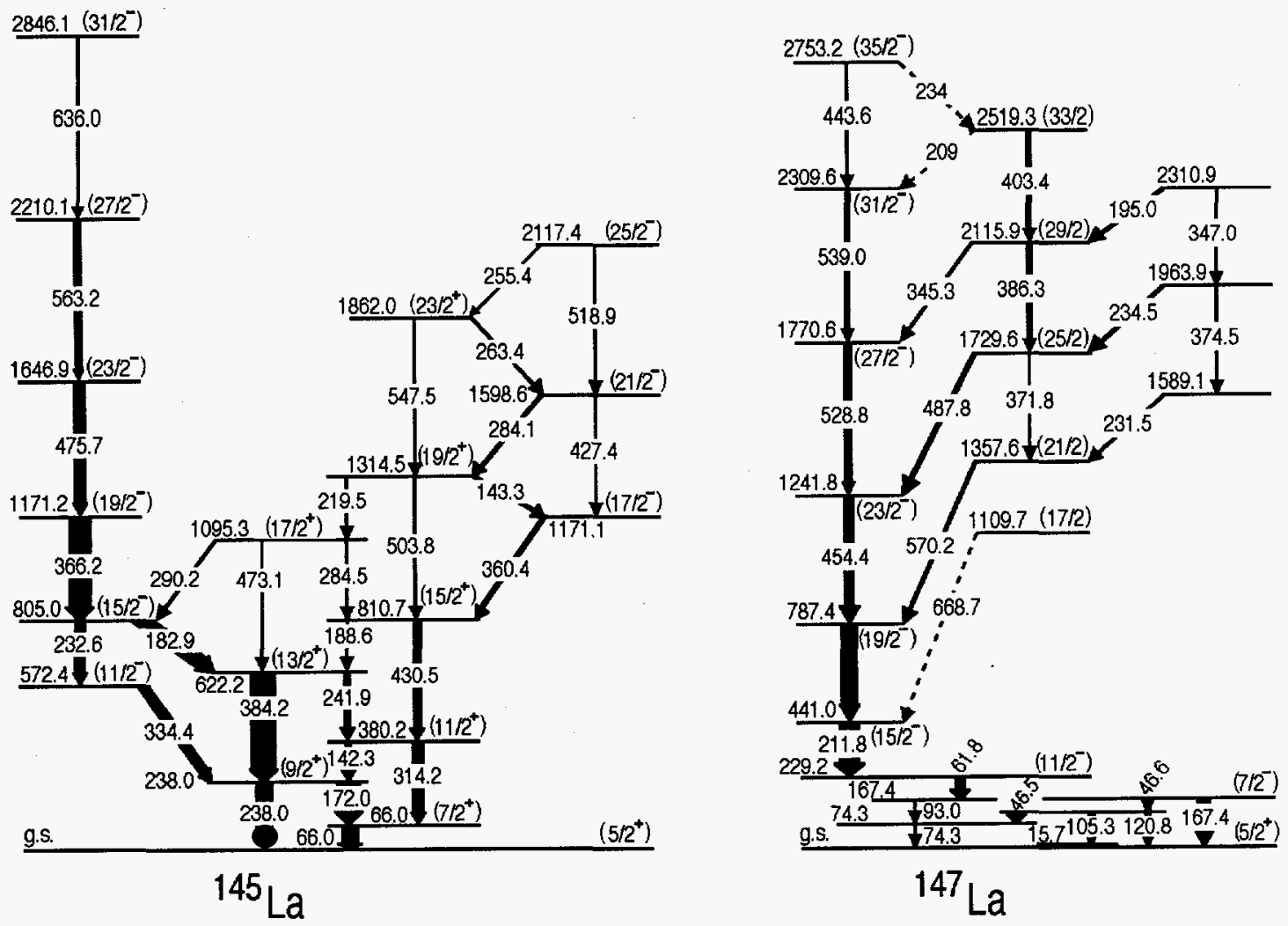

Fig. I-30. Partial level schemes of ${ }^{145}$ La and $147_{\text {La deduced from the present study. }}$

\section{b.33. Parities and Multipolarities of Gamma Rays in Neutron-Rich Odd-Mass Ba Isotopes (I. Ahmad, L. R. Morss, ${ }^{*}$ M. A. Jones, $\dagger$ W. Urban, $\dagger$ J. L. Durell, $\dagger$ M. Leddy, $\dagger$ W. R. Phillips, $\uparrow$ A. G. Smith, $\uparrow$ B. J. Varley, $\dagger$ M. Bentaleb,$\ddagger$ E. Lubkiewicz, $\ddagger$ and N. Schulz $\ddagger$}

Our previous studies have shown that the even-even $\mathrm{Ba}$ nuclei, ${ }^{144} \mathrm{Ba}$ and ${ }^{146} \mathrm{Ba}$ are octupole deformed. In general, it is expected from nuclear structure considerations that the odd-mass and odd-odd nuclei contain larger octupole-octupole correlations than their even-even neighbors. For this reason we have studied the level structures of the odd-mass $B a$ isotopes, $143,145,147 \mathrm{Ba}$. The experiment was performed at Strasbourg with the EUROGAM2 array and a ${ }^{248} \mathrm{Cm}$ fission source. From the triple coincidence data the level structures of the three odd-mass $B$ a nuclei were deduced. In the previous studies the spins and parities were assigned mostly on the basis of systematics and the measured multipolarities of a few low energy transitions. In the present analysis, several new techniques have been used to deduce transition multipolarities, and hence spins and parities of levels. These include triple angular correlations, directional linear polarization correlations, and internal conversion coefficients. The data establish a sequence of alternating-parity levels in ${ }^{143} \mathrm{Ba}$, connected by fast E1 transitions. Such well-developed structures are not observed in $145 \mathrm{Ba}$, although E1 transitions were observed. No features appropriate to octupole correlations were observed in ${ }^{147} \mathrm{Ba}$. The values of the dipole moment, $\mathrm{D}_{0}$, deduced for ${ }^{143} \mathrm{Ba}$ and ${ }^{145} \mathrm{Ba}$ are similar to the values for ${ }^{144} \mathrm{Ba}$ and ${ }^{146} \mathrm{Ba}$ and these are reproduced by microscopic calculations (Figure I-31). The results of this investigation were published. ${ }^{1}$

*Chemistry Division, ANL, $†$ University of Manchester, United Kingdom, ¥CRN, Strasbourg, France

${ }^{1}$ M. A. Jones et al., Nucl. Phys. $\underline{\text { A605, }} 133$ (1996). 


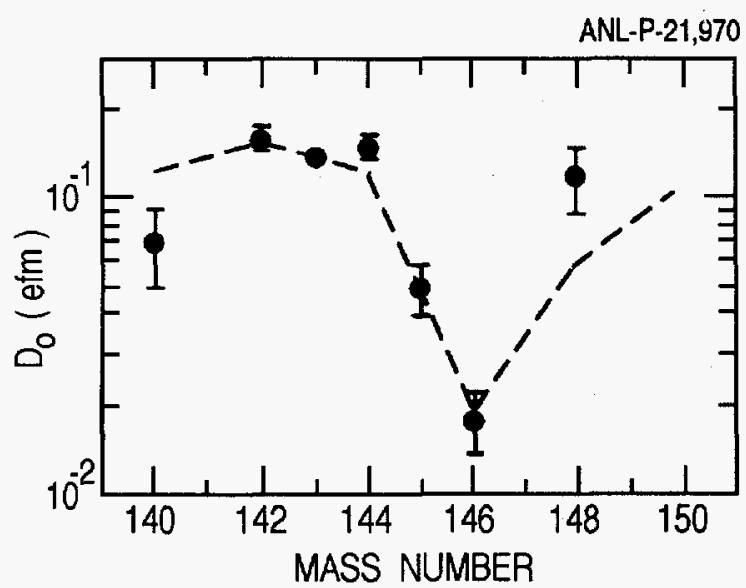

Fig. 1-31. Values of the dipole moment parameter $D_{0}$ for $B a$ isotopes. The dashed line represents theoretical predictions for the even-even isotopes.

\section{b.34. Octupole Correlations in Neutron-Rich, Even-Even Ba Isotopes (I. Ahmad, L. R. Morss, ${ }^{*}$ W. Urban, $\uparrow$ M. A. Jones, $\dagger$ J. L. Durell,$\uparrow$ M. Leddy, $\uparrow$ W. R. Phillips, $\dagger$ A. G. Smith, $\dagger$ B. J. Varley, $\dagger$ M. Bentaleb,$\ddagger$ E. Lubkiewicz $\ddagger$ and N. Schulz $\ddagger)$}

The level structures of ${ }^{144} \mathrm{Ba}$ and ${ }^{146} \mathrm{Ba}$ provided one of the first examples of octupole deformed nuclei in the mass 144 region. In the present work, level structures of these nuclei have been extended to higher spins and new information has been obtained on the even-even nuclei ${ }^{140} \mathrm{Ba},{ }^{142} \mathrm{Ba}$ and ${ }^{148} \mathrm{Ba}$. The experiment was carried out at Strasbourg with the EUROGAM2 array and a ${ }^{248} \mathrm{Cm}$ fission source. Level schemes were constructed from coincidence relationships of gamma rays in a triple coincidence mode analysis. The spins and parities of the levels were firmly established from triple gamma angular correlations and directional linear polarization correlations. Our results show that all even-even $142-148 \mathrm{Ba}$ nuclei have alternating-parity level structures indicating the presence of octupoleoctupole correlations. The strongest octupole correlations have been observed in ${ }^{144} \mathrm{Ba}$, where the simplex $s=+1$ band has been observed up to spin $16 \hbar$. New levels in ${ }^{144} \mathrm{Ba}$ have been interpreted as forming a $s=-1$ band. In ${ }^{146} \mathrm{Ba}$ the octupole-octupole correlations are weaker and disappear at medium spins, where both the ground band and the octupole band undergo band crossings, after which $146_{\mathrm{Ba}}$ becomes reflection symmetric. The new data on ${ }^{148}$ Ba provided us with an estimate of the electric dipole moment $\mathrm{D}_{0}$ for the first time. This value is larger than that for ${ }_{146} \mathrm{Ba}$, which confirms the theoretical prediction of a local reduction of $\mathrm{D}_{0}$ in ${ }^{146} \mathrm{Ba}$.

*Chemistry Division, ANL, †University of Manchester, United Kingdom, $¥$ CRN, Strasbourg, France

\section{b.35. Single Particle States in the Heaviest Elements and the Stability of the Superheavy Elements (I. Ahmad and R. R. Chasman)}

The synthesis of nuclei in the region of superheavy elements has been a major field of nuclear physics research in the past twenty years. Theoretical predictions of the half-lives of the superheavy elements depend crucially on the single-particle spectra in the vicinity of 114 protons and 184 neutrons. It is, therefore, important to identify the single-particle orbitals in the heaviest nuclei. The heaviest observed nuclei are all deformed and, hence, deformed orbitals have been observed in decay scheme studies and in nucleon transfer reactions. The energies of the deformed states can then be used to deduce the energies of the orbitals at zero deformation. The orbitals that are important for fixing the shell corrections near $N=184$ are the $h_{11 / 2}$, $\mathrm{j} 13 / 2$ and $\mathrm{k}_{17 / 2}$ spherical states. The deformed states originating from the $\mathrm{h}_{11 / 2}$ and $\mathrm{j} 13 / 2$ shell states have been identified in $251 \mathrm{Cf}$. Also, a range of values has been determined for the $[880] 1 / 2^{+}$single-particle state in ${ }^{249} \mathrm{Cm}$ from ${ }^{248} \mathrm{Cm}\left({ }^{4} \mathrm{He},{ }^{3} \mathrm{He}\right.$ ) reaction. We have calculated signatures for the low-lying states in ${ }^{251} \mathrm{Cf}$ and the calculated energies and signatures are in good agreement with the experimentally observed (d,p) spectrum. Using the Strutinsky method, we found that we could get very good agreement with the

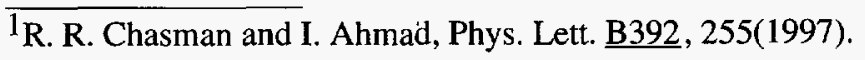


known low-lying levels in ${ }^{249} \mathrm{Cm}$ using a momentum independent Woods-Saxon potential. To determine potential parameters for protons in the heavy elements, we utilized our study of $255 \mathrm{Md}$ that shows the [521] $1 / 2^{-}$orbital to lie above the [514]7/2- orbital. Fixing the potential in this way, we extrapolate directly to the superheavy element region. We replaced the zero-point correction in our mass estimates by a term that depends quadratically on the sum of the proton and neutron shell corrections, as the usual zero-point correction treatment is questionable. With our potential parameters derived from the heaviest elements for which there is detailed spectroscopic information, we find that our calculated lifetimes are sufficiently long. These half-lives indicate that elements at least through $\mathrm{Z}=120$ can be produced and observed through proper choice of reactions. Results of our calculations were published. ${ }^{1}$

\section{b.36. Conference on Nuclear Structure at the Limits (M. P. Carpenter, I. Ahmad, R. R. Chasman, J. Glover, R. V. F. Janssens, T. L. Khoo, T. Lauritsen, C. J. Lister and U. Garg*)}

This past year the heavy-ion group organized a conference entitled "Nuclear Structure at the Limits" which ran from July 22 to July 26 . This conference is one of a series held in North America every other year covering current topics in nuclear structure physics.

There were 197 participants, 90 of which came from abroad. Sixty-four presentations were given with the majority of talks chosen from the submitted abstracts. One-third of these presentations were given by graduate students and post-doctoral fellows. Major topics covered at the conference included superdeformation, nuclei at high-spin, nuclear structure far from stability, superheavy elements, and new technical developments. The program of speakers is included in Figure I-32.

Some of the highlights presented at the conference included: (1) the first observations of discrete $\gamma$ decays from a superdeformed band to yrast states with known spins and parities; (2) the discovery of element 112 ; (3) new detailed spectroscopy around ${ }^{100} \mathrm{Sn}$ and ${ }^{132} \mathrm{Sn}$; and (4) identification of the first excited states in light nuclei very close to the neutron drip line. Both the abstracts 1 and proceedings of the conference have been published as internal reports ${ }^{2}$.

\footnotetext{
*University of Notre Dame

${ }^{1}$ Proceedings of the Conference on Nuclear Structure at the Limits - Abstracts, Argonne, Illinois, July 22-26, 1996, ANL/PHY-96/1 (1996).

${ }^{2}$ Proceedings of the Conference on Nuclear Structure at the Limits, Argonne, Illinois, July 22-26, 1996, ANL/PHY97/1 (1997).
} 
SESSION I-A

R.M. Diamond (LBL)

D. Schwalm (Heidelberg, Germany)

Spectroscopy In and Decay Out of the Second Well in

Actinide Nuclei

G. Hackman (ANL,USA)
One Step Decays from Superdeformed to Yrast States in ${ }^{194} \mathrm{Hg}$
F. Hannachi (CSNM.Orsay, France)

Angle Step Links of the Superdetormed Band in

A Measure of the Absolute Excitation Energy, Spin and Parity of the Superdeformed Stact

Primary Transitions between the Yrast Superdeforned Bnd

SESSSION I-B

Discussion Leader:

C. Pretache (Padova/1NFN)
Decay Out of the Yrast and Excited H-ghly-Deformed Bands in the Even-Even Nusleus ${ }^{134} \mathrm{Nd}$

F. Lerma (Washington University)
Search for Linking Transitions in

B. Herskind (Niels Bohr Insititue)

Single-Step Link of the Superdeformed Band in ${ }^{143} \mathrm{Eu}$

The Quasicontinuum of Gamma Rays Following the Decay of

Superdeformed Bands in the Hg Region

A. Lopez-Martens (CSNM-Orsay)

Fut Cascade From the Superdeformed Yrast Band in ${ }^{192} \mathrm{Hg}$

SESSION II-A

Discussion Leader:

F. Stephens (LBL)

Superdeformation:

Microscopic Descriplion of Nuclear Shapes

P. Twin (Liverpool)
Superdeformation in ${ }^{150} \mathrm{Gd}$ Around the (Back)Bend in Band 5

SESSION HI-B

M. Deleplanque-Stephens (LBL)

C. Rigollet (Strasbourg)

Microscopic Study of Superdeformation in the $A=150$ Mass

Region
E.F. Moore (North Carolina State University)

Lifetime Measurments and Identical Superdeformed Bands in the $A=150$ and $A=190$ Mass Region

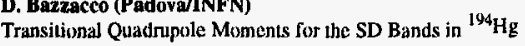

L.H. Zhu (Padova/NFN)

Change of Deformation at the Backhending in the Yrast Superdeformed Band of ${ }^{44} \mathrm{Gd}$
SESSION III-A

S. Lunardi (Padova/INFN)

C. Baktash (ORNL)

Superdeformation in the $A=80$ Region

Y. Sun (JIHIR)

A Systematic Description of the Yrast Superdeformed Bands in the Even-Even Mass 190) Region

F. Azaiez (IPN-Orsay)

New Results on the Superdeformed ${ }^{19}$ Pb Nucleus: The Decay of the Excited Bands to the Yrast Band

Microscopic Structure of High-Spin Vibrational States in SuperMetormed

SESSION III-B $\quad$ L.L. Riedinger (Univ, of Tennessee)

J. Dudek (Strasbourg)

Seliconsistent Calculations for the Hyperdeformed Nuclei: Exotic Deformations in Excess of Hyper-elongation

L.M. Robledo (Madrid)

How Close are Hyperdeformed States to the Scission Point

Test of $\Delta=2$ Staggering in the Superdeformed Bands of ${ }^{194} \mathrm{Hg}$

SESSION IV-A

N. Benczer-Koller (Rutgers)

R. Clark (LBL)

Evidenec for Magnelic-Rotation: Lifetimes of States in the M

Bands of $198,199 \mathrm{~Pb}$

G. Dracoulis (ANU,Canberra)

Pairing Reduction and Configuration Effects in the Rotation and Decay of Multi-Quasiparticle Isomers in Ta W Nuclei

Return of K-Setcction at High Spin: Decay of Bandheads in ${ }^{178} \mathrm{~W}$ Y. Shimizu (Kyushu)

Direct Transijions From High-K Jsomers 10 Low K Bands: Gamm Sotiness or Coriolis Couplin

SESSION IV-B

D. Cline (Rochester)

W. Reviol (University of Tennessee)

Titled Axis Rotation in odd-odd

$\checkmark$. Janzen(AECL/Chakk

Properties of Rolational Bands at the Spin Limit in A -50, A 60

and A-110 Nuclei

G. Lame (Stony B
Excited States in

1. Ragnarsson (Lund)

Rotalional Bands in Terminating at Maximum Spin in the Val-

ance Space
SESSION V-A

D.G. Sarantites (Washington Univ.)

Y. Lee (LBL)

Status of Development of the Gamma Ray Encrgy Tracking Array (GRETA)

. Beck (Stasbourg)

J. Garrett (ORNL)

Near Term Prospects for Radioactive Beams at ORNL.

SESSION V-B

U. Garg (Univ. of Notre Dame)

T. Kishida (RIKEN)

High Spin Isomer Beam Line al RIKEN

High Spin Spectroscopy Near the N=Z Line: Channel Selection nd Excitation Energy Systematic

Alpha Particles in Coincidence with the SD Band in ${ }^{150} \mathrm{~Tb}$

M. Devilin (Washington University)

The Microball and Gammasphere: Research Highlights and Future Promise

SESSION VI-A

W. Nazarewicz (Univ. of Tennessec)

P.G. Hansen (Michigan State University)

tructure and Reactions of Drip-Line Nucle

Nuclear Structure Beyond

D. J. Dean (ORNL)

Shell Model Monte Carlo Calculalions Near $\mathrm{N}=\mathrm{Z}$

SESSION VI-B
Discussion Leader: $\quad$ M. Thoennessen (Mich. State Univ.)

W. Satuła (Warsaw/JIHIR)

isispin Projected Model with Gencralized Pairing Interaction

in Application to Rotating Nuclei

M. Lipoglavsek (Uppsala University)

in-Beam Studics of ${ }^{98.99} \mathrm{Cd}$ and ${ }^{102} \mathrm{~S} \mathrm{~S}$

D. Seweryniak (ANL)

列

.

Collective Properties of Drip-Line Nucle

SESSION VII-A
Discussion Leader: A. M. Baxter (Australian National Univ.)

S. Hofmann (GSI)

Discovery of Element 112

N.V. Antonenko (JINR-Dubna)

Synthesis of Superheavy Elements and Dinuclear-Systems: Con-

cept of Compound Nucleus Formation

S. Mizutori (JIHIR/ORNL)

High Spin States in Neuron-Rich Nuclei
SESSION VII-B

D. Ward (AECL-Chalk River)

Meng (Technical University-Munich)

Jestivistic Harrec Bogolyubov Description for Nuclei Ncar the Drip Line

R. J. Broda (Niewodniczanski Institute of Nuclear Physics) New High Spin States and Isomers in the ${ }^{2118} \mathrm{~Pb}$ and ${ }^{207} \mathrm{~Pb}$ Nuclo

C. T. Zhang (Purdue University)

Yrast Spectroscopy Around Doubly Magic ${ }^{132} \mathrm{Sn}$

作

ponents and Its Excitation in Heavy-Ion Collisions

SESSION VIII-A

J. A. Cizewski (Rutgers University)

J. L. Durell (University of Manchester)

The Nuclear Structure of Neutron-Rich Isotopes with $\mathrm{Z}$ from

J. F. C. Cocks (University of Liverpool)

Spectroscopy of Reflection-Asymmerric Nuclei Using Multi-

Deformation in the Neutron-Rich Sulpher Isotopes ${ }^{40} S$ and ${ }^{42} \mathrm{~S}$

SESSION VIII-B

D. C. Radford (AECL-Chalk River)

Vibrational Bands in ${ }^{165} \mathrm{Ho}$

C. Wu (University of Rochester)

Fragmentation of Two-Phonon Gamma Vibration Strength in

Deformed Nuclei

W. Younes (Rutgers University)

Onset of Deformation in Polonium Nucla

R. I. Julin (University of Jyvaskyia)

Recent Studies of Heavy Nuclei Far From Stability at JYFL

Discussion Leader: J. C. Waddington (McMaster University)

G. B. Hagemann (Niels Bohr Institute)

A Multitude of Rotational Bands in ${ }^{163} \mathrm{Er}$ and Their Mutual

Interaction

M. Matsuo (Kyoto University)

Shell Model for Warm Rotating Nuclci

. Bracco (University or Milan

Number in Exciled Ro-

ta. G. Nuclei

(Michigan State University)

Vimits of Resolution: Looking Through

Individual Wave Functions

Fig. I-32. Program of the Conference on Nuclear Structure at the Limits 


\section{REACTION STUDIES}

Reaction studies at ATLAS cover topics including processes in the vicinity of the Coulomb Barrier, fusion and fission reactions at higher energies, and the production and decay of exotic nuclear cluster states in s-d shell nuclei. These studies take advantage of the characteristics of the ATLAS accelerator such as excellent beam quality, outstanding timing properties and easy energy variability. This research utilizes a variety of detection systems such as the magnetic spectrographs, the fragment mass analyzer, and arrays of double-sided silicon strip-detectors. Furthermore, measurements with the first components of the LEPPEX array were performed as well.

This section also contains reports on experiments performed at other facilities. These include measurements with exotic beams at the National Superconducting Cyclotron Laboratory (MSU) and the participation in experiments with relativistic heavy-ions E917 and PHOBOS.

\section{c.1. Multi-Neutron Transfer in the ${ }^{58} \mathrm{Ni}+{ }^{124} \mathrm{Sn}$ Reaction at Sub-Barrier Energies (K. E. Rehm, C. L. Jiang, H. Esbensen, D. J. Blumenthal, B. Crowell, J. Gehring, B. Glagola, A. H. Wuosmaa, and J. P. Schiffer)}

Studies of transfer reactions at sub-barrier energies have received increased interest since it was discovered that heavy-ion induced fusion cross sections are strongly enhanced compared to one-dimensional tunneling calculations. Next to inelastic scattering, transfer reactions are thought to be the main channels that have to be included in a coupled-channels treatment for a successful description of low-energy fusion cross section. We have studied the transfer reactions in the system of ${ }^{58} \mathrm{Ni}+{ }^{124} \mathrm{Sn}$ at sub-barrier energies for two reasons. A complete set of cross sections including fusion-evaporation, ${ }^{1}$ fusion-fission, ${ }^{2}$ neutron transfer at energies near and above the Coulomb-barrier ${ }^{3}$ and below the barrier, ${ }^{4}$ and deep-inelastic scattering below the Coulomb-barrier, ${ }^{5}$ has been measured previously. Furthermore, the ground state Q-values Qgg for multineutron transfer are rather high, resulting in large yields for neutron transfer reactions.

The experiments were performed in inverse kinematics at four energies with ${ }^{24} \mathrm{Sn}$ beams of $480-512 \mathrm{MeV}$ bombarding a $334 \mu \mathrm{g} / \mathrm{cm}^{2} 58 \mathrm{Ni}$ target. The outgoing target-like particles were momentum analyzed with the
Enge split-pole spectrograph and identified with a position-sensitive ionization detector in the focal plane. Single mass and $\mathrm{Z}$ resolution was achieved.

A coupled-channels treatment including explicitly couplings to the low-lying $2^{+}$and $3^{-}$states in projectile and target nuclei was tried to model the successive single-neutron transfers combined with direct twoneutron transfer coupling. Fusion, on the other hand, is described by the ingoing wave boundary conditions.

Figure I-33(a) shows the calculated angular distributions for one-, two- and three-neutron pick-up reactions ${ }^{58} \mathrm{Ni}\left({ }^{124} \mathrm{Sn}, 59,60,61^{\mathrm{Ni}}\right){ }^{123,122,121} \mathrm{Sn}$ at the lowest bombarding energy in comparison with the experimental data. Since four-, five- and six-neutron transfer reactions are not included in the model the calculated curve of three-neutron transfer should be compared to the sum of the three- to six-neutron transfer yields. An excellent agreement between experiment and theory is observed. Figure I-33(b) shows a comparison of the measured and calculated excitation functions including low-energy data from Ref. 4. No energydependent normalization constant has been used in these

${ }^{1}$ W. E. Freeman et al., Phys. Rev. Lett. $\underline{50}, 1563$ (1983).

${ }^{2}$ K. T. Lesko et al., Phys. Rev. Lett. 55, 803 (1985); K. T. Lesko et al., Phys. Rev. C34, 2155 (1986).

${ }^{3}$ A. M. van den Berg et al., Phys. Lett. 56, 572 (1986); A. M. van den Berg et al., Phys. Rev. C37, 178 (1988);

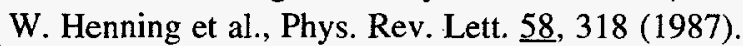

${ }^{4}$ R. R. Betts et al., Phys. Rev. Lett. 59, 978 (1987); C. N. Pass et al., Nucl. Phys. A499, 173 (1989).

${ }^{5}$ F. L. H. Wolfs et al., Phys. Lett. B196, 113 (1987). 
calculations. In order to take other channels into account which have not been explicitly considered in these calculations (e.g. charge transfer reactions), a small imaginary potential was included in the calculations whose effect is shown in Fig. I-33(b) by the difference between the solid (no imaginary potential) and the dashed lines. Figure I-33(c) shows a comparison of the calculated fusion cross sections (solid line) with data for evaporation-residue production (open circles), the sum of residue production and fission cross sections (open squares) and the sum of fusion and deepinelastic scattering (solid points). The coupled-channels calculations seem to agree best with the sum of fusion and deep inelastic collisions.
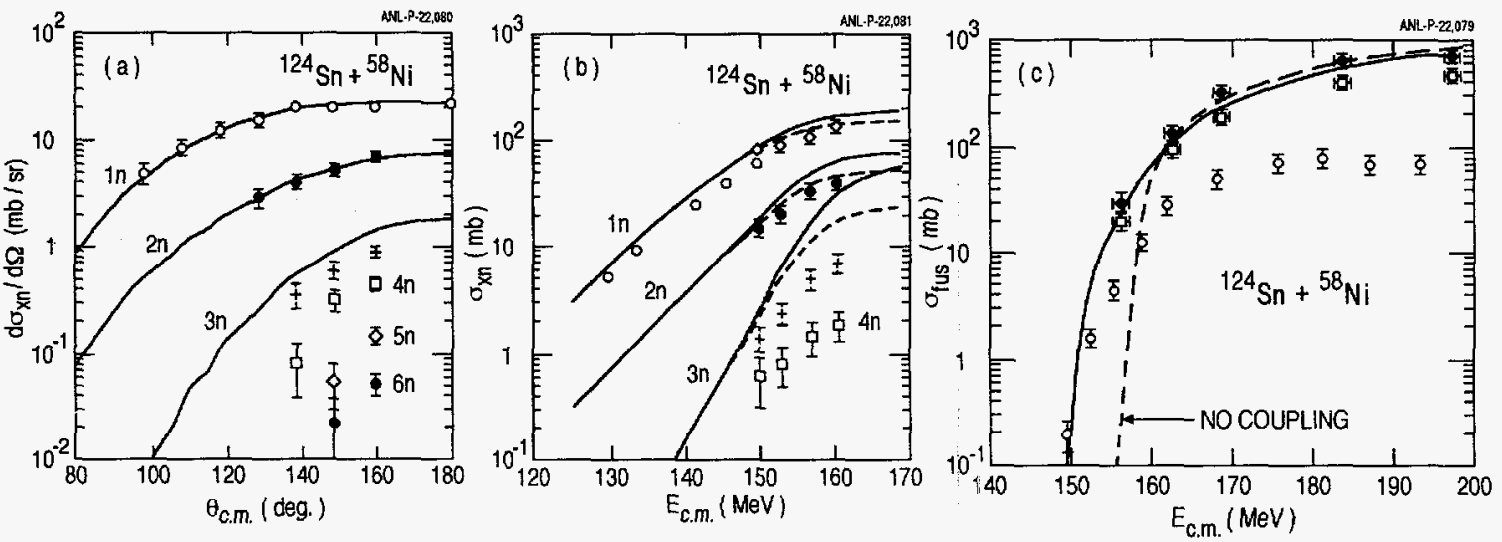

Fig. I-33(a). Angular distributions for the one-to six-neutron pick-up reactions at $E_{c m}=150 \mathrm{MeV}$, compared to coupled-channels calculations for the one-, two-, and three-neutron transfer. (b) Excitation functions for the oneto six-neutron pick up reactions compared with the results from the coupled-channels calculations for one-, two-, and three-neutron transfer. (c) Excitation function for the evaporation residue production, (open points) residue production and fission cross sections (open squares) and the total fusion and deep inelastic scattering cross section (solid points) compared to the coupled-channels calculations (solid line).

\section{c.2. Measurement of the Fusion Excitation Function in the System ${ }^{78} \mathbf{K r}+$ ${ }^{100}$ Mo at Low Energies Using a New Technique (C. L. Jiang, K. E. Rehm, W. F. Henning, D. Ackermann, B. B. Back, C. N. Davids, B. Harss, D. J. Henderson, S. M. Fischer, and D. Seweryniak)}

Sub-barrier fusion between heavy ions has been studied extensively since the first observations that fusion cross sections are strongly enhanced compared to onedimensional tunneling calculations. ${ }^{1}$ While coupling to other reaction channels, especially inelastic scattering and transfer reactions have been identified as the origin for this enhancement many questions still remain. Several excitation functions for evaporation residue production have been measured recently at ATLAS using the gas-filled magnet technique. ${ }^{2}$ In these experiments angular distributions in the range $\theta=1^{\circ}-6^{\circ}$ were measured at several incident energies, with the smallest cross sections in the range of $\sim 100 \mu \mathrm{b}$. A comparison of the excitation functions for ${ }^{78} \mathrm{Kr}+$ ${ }^{100} \mathrm{Mo}$ and ${ }^{86} \mathrm{Kr}+{ }^{92} \mathrm{Mo}$, i.e. two systems involving "soft" vibrators and "stiff" closed neutron shell nuclei, which populate the same compound nucleus ${ }^{178} \mathrm{Pt}$, shows a large difference at the lowest bombarding energies. The falloff for the excitation function in the system ${ }^{78} \mathrm{Kr}+{ }^{100} \mathrm{Mo}$ is much shallower than for ${ }^{86} \mathrm{Kr}$ $+92 \mathrm{Mo}$ which results in a large sub-barrier enhancement for the former system. In order to study this phenomenon and the discrepancy between experimental data and theoretical calculations in more detail, we have extended the measurements down to the $\mu b$ region.

1 M. Beckerman, Rep. Prog. Phys. 51, 1047 (1988); R. Vandenbosch, Ann. Rev. Nuc. Part. Sci. 42 , 447 (1992).

${ }^{2}$ K. E. Rehm et al., Phys. Lett. B317, 31 (1993), Nucl. Instrum. Methods $\underline{A} 344,614$ (1994), Physics Division Annual Report ANL-95/14, p. 9, Experiment 538. 
In the measurements of angle-integrated cross sections for evaporation residue production a new method has been used (see contribution c.3.). The total angleintegrated cross section can be obtained from a measurement of the velocity distributions of the various residue yields at zero degrees in the laboratory system. The experiment has been performed at the Argonne Fragment Mass Analyzer (FMA) with the standard position sensitive PPAC counter for detection of the evaporation residues in the focal plane. An additional ionization chamber measuring both $\Delta \mathrm{E}$ and $\mathrm{E}_{\mathrm{res}}$ signals was placed $40 \mathrm{~cm}$ behind the PPAC. Using the timeof-flight signal from the PPAC with respect to the rf signal very good mass and velocity spectra have been obtained.
The measurements were performed at four energies: $\mathrm{E}\left({ }^{78} \mathrm{Kr}\right)=353.0,302.5,284.6$ and $276.8 \mathrm{MeV}$. A comparison of the high energy measurements with the previous data of evaporation residue angular distributions shows good agreement. The high efficiency of the FMA allowed a continuation of these measurements to an energy of $\mathrm{E}_{\mathrm{lab}}=276.8 \mathrm{MeV}$ where the cross section is about $10 \mu \mathrm{b}$, i.e. an order of magnitude below the lowest value of the previous data.

Coupled-channels calculations, including multi-phonon excitations for these two systems are presently being performed.

\section{c.3. A New Method for the Measurement of the Fusion Excitation Functions at Sub-Barrier Energies Using Fusion-Recoil Velocity Distributions at Zero Degrees (C. L. Jiang, K. E. Rehm, W. F. Henning, D. Ackermann, B. B. Back, C. N. Davids, B. Harss, D. J. Henderson, S. M. Fischer, and D. Seweryniak)}

Angle-integrated cross sections for evaporation residue production are usually obtained by measuring complete angular distributions. This can become a very time consuming measurement especially when the cross sections are small. At sub-barrier energies, one- and two-nucleon evaporations are the most abundant evaporation residue channels resulting in nearly isotropic angular distributions in the center-of-mass system. Assuming isotropic angular distributions for the fusion-evaporation products one finds that the velocity distribution of the evaporation residues is also isotropic in the center-of-mass system. A measurement

$$
\frac{d^{2} \sigma}{d \Omega_{l a b} d v_{l a b}}\left(\theta_{l a b}=0^{\circ}\right)
$$

for each residue mass by using the time structure of the ATLAS beam. A very important factor is the large momentum and energy acceptance of $\pm 10 \%$ and $\pm 20 \%$, respectively, in the FMA. To cancel out the influence of small angle scattering and energy loss straggling in the target on the velocity and angular distributions of

Since the quantity

$$
\frac{1}{v^{2}} \frac{d^{2} \sigma}{d \Omega d v}
$$

of the velocity distribution in the laboratory system at zero degrees therefore contains the complete information about the velocity distribution, including the transverse direction, which is required to compute the evaporation residue cross section without measuring an angular distribution.

We have developed a new method to measure fusion excitation functions based on the principle mentioned above, which greatly facilitates measurements of fusion excitation functions, especially in the low-energy region. Taking advantage of the isochronous property of the Argonne Fragment Mass Analyzer (FMA) one can measure the velocity spectrum

the evaporation residues some corrections to the data have to be applied. In most cases these effects are expected to be rather small. A small, but known correction for the energy dependence of the flight path through the FMA is also applied. 
is invariant under the transformation from the laboratory to center-of-mass system, we obtain the total cross section $\sigma_{\text {tot }}$ from the following equations:

$$
\begin{aligned}
& \frac{\mathrm{d}^{2} \sigma}{\mathrm{d} \Omega_{\mathrm{cm}} \mathrm{d} v_{\mathrm{cm}}}\left(\theta_{\mathrm{cm}}=0^{\circ}\right)=\frac{v_{\mathrm{cm}}^{2}}{v_{\mathrm{lab}}^{2}} \frac{\mathrm{d}^{2} \sigma}{\mathrm{d} \Omega_{\mathrm{lab}} \mathrm{d} v_{\mathrm{lab}}}\left(\theta_{\mathrm{cm}}=0^{\circ}\right), \\
& \sigma_{\text {tot }}=\iint \frac{\mathrm{d}^{2} \sigma}{\mathrm{d} \Omega_{\mathrm{cm}} \mathrm{d} v_{\mathrm{cm}}} \mathrm{d} \Omega_{\mathrm{cm}} \mathrm{d} v_{\mathrm{cm}}=4 \pi \int \frac{\mathrm{d} \sigma}{\mathrm{d} v_{\mathrm{cm}}} \mathrm{d} v_{\mathrm{cm}},
\end{aligned}
$$

where $v_{l a b}$ and $v_{\mathrm{cm}}$ are the velocities of the evaporation residues in the laboratory and center-of-mass systems, respectively, and $v_{\mathrm{lab}}=v_{\mathrm{cm}}+v_{\mathrm{o}}$ at $0^{\circ}$ $\left(v_{0}\right.$ is the velocity of the center-of-mass in the laboratory system). The first test experiment determined the evaporation residue cross sections in the system ${ }^{78} \mathrm{Kr}+{ }^{100} \mathrm{Mo}$, where an excitation function from evaporation residue angular distributions had been measured previously. Figure $\mathrm{I}-34$ shows a $\mathrm{m} / \mathrm{q}$ spectrum and a velocity spectrum for mass $=177$ populated in the $1 \mathrm{n}$ evaporation channel at $\mathrm{E}\left({ }^{78} \mathrm{Kr}\right)=$ $276.8 \mathrm{MeV}$. At this energy the total evaporation residue cross section is about $10 \mu \mathrm{b}$. The result shows that cross sections as low as $1 \mu \mathrm{b}$ can be measured with this method without much difficulty.

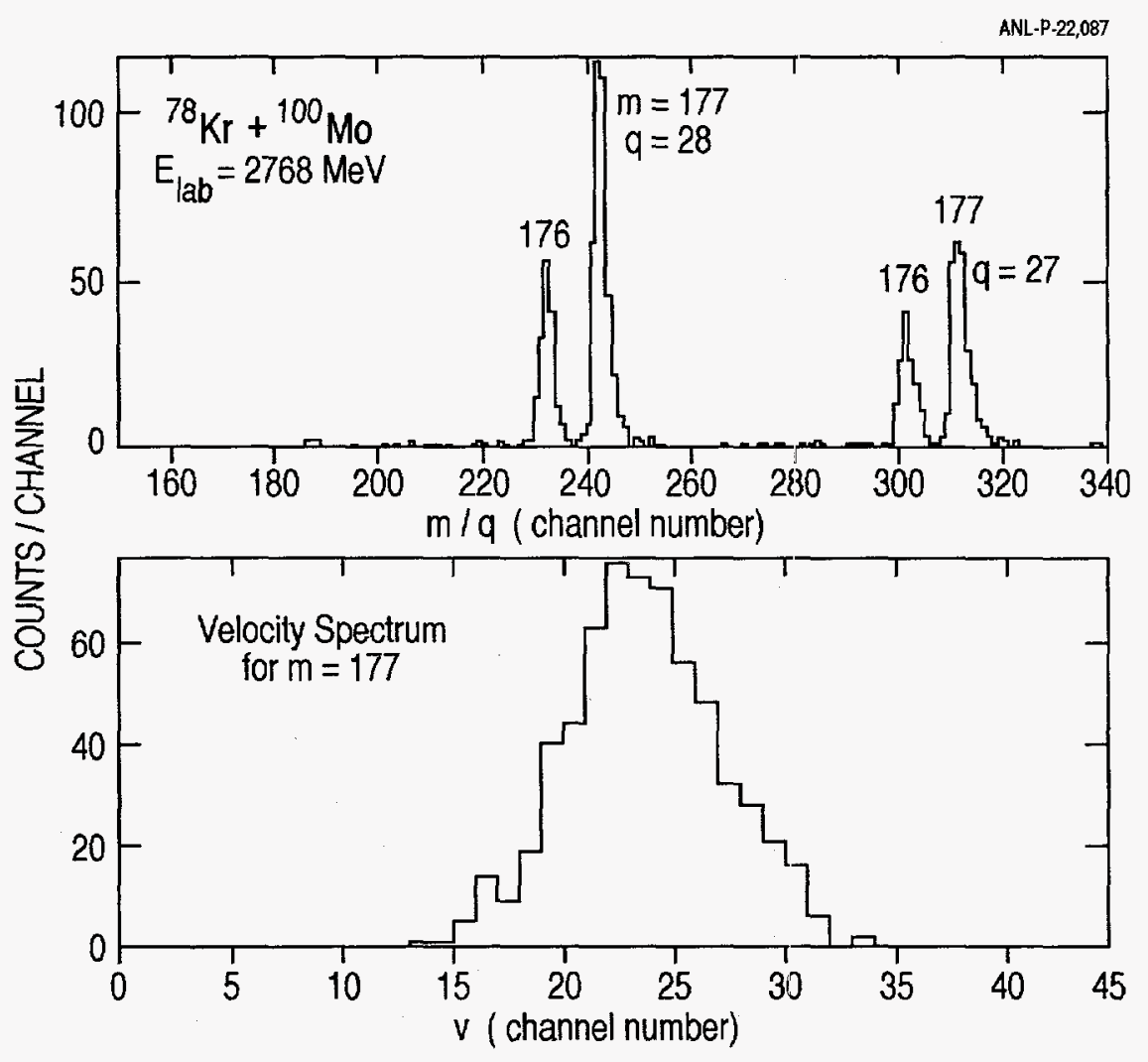

Fig. I-34. $\mathrm{m} / \mathrm{q}$ spectrum (a) and velocity spectrum for mass $=177, Q=27,28$ in the system of $78_{\mathrm{Kr}}+100_{\mathrm{Mo}}$ at $E_{l a b}=276.8 \mathrm{MeV}$. 


\section{c.4. Spin Distributions for ${ }^{64} \mathrm{Ni}+{ }^{100} \mathrm{Mo}$ with the Argonne-Notre Dame BGO-Array (D. Ackermann, B. B. Back, R. R. Betts, M. Carpenter, S. Fischer, G. Hackman, R. Ganz, D. J. Hofman, R. Janssens, T. L. Khoo, V. Nanal, M. Schlapp, D. Seweryniak, A. Wuosmaa, L. Corradi, $*$ S. Gil $\ddagger$ G. Montagnoli, $\dagger$ F. Scarlassara,$\dagger$ and A. M. Stefanini*)}

The investigation of subbarrier fusion is undergoing a revival after the possibility of extracting an experimental representation of the barrier distribution from the second derivative $\mathrm{DB}_{f u s}=d^{2}(E \sigma) / d^{2} E$ was suggested ${ }^{1}$. By performing precise measurements of fusion excitation functions one is able to extract the strength and height of the barriers, providing important information on the coupling mechanism. As an alternative approach, it has been recently proposed ${ }^{2}$ that the first derivative of the compound nucleus $(\mathrm{CN})$ spin distribution $\mathrm{DB}_{\text {spin }}$ is equivalent to $\mathrm{DB}_{\text {fus }}$.

In a previous experiment at $\mathrm{GASP}^{3}$ we measured $\gamma$ multiplicities for the reaction ${ }^{32} \mathrm{~S}+{ }^{110} \mathrm{Pd}$ where a detailed peak structure has been found in $\mathrm{DB}_{f u s}{ }^{4}$. This structure has been attributed to a two-phonon plus transfer coupling. A similar coupling scheme (oneplus-two phonon coupling) has been proposed to explain the fusion excitation function of ${ }^{64} \mathrm{Ni}+{ }^{100} \mathrm{Mo}$ measured by K. E. Rehm et al. ${ }^{5}$. In this system fission is expected to set in at $\approx 15 \%$ above the Coulomb barrier. To also study the effect of fission, we measured $\gamma$-multiplicities in coincidence with evaporation residues (ER) at three energies $-\mathrm{E}_{l a b}=230 \mathrm{MeV}, 246$ $\mathrm{MeV}$ and $260 \mathrm{MeV}$; at the barrier, at the point where fission becomes important and at an intermediate energy. The experimental set-up we used was an electrostatic deflector plus silicon strip detectors in conjunction with the Argonne/Notre Dame-BGO-array.

At all three energies we obtained $\approx 3700$ counts for the total multiplicity spectrum. This rather low statistics was basically due to a background of scattered beam through the deflector which forced us to use a relatively low beam current. Nevertheless, some conclusions can be drawn from the obtained multiplicity data which are shown in Fig. I-35(a-c). Preliminary spin distributions have been computed using a simple formula of the type

$\ell=\Delta I_{\text {ms }}\left(\mathrm{M}_{\gamma}-\mathrm{M}_{\gamma \mathrm{s}}\right)+\mathrm{const}$

where $M_{\gamma}$ denotes the measured $\gamma$-multiplicity, Dlgns = 2 is the spin taken away by the yrast-transitions and const contains the spin removed by statistical $\gamma$-rays $(M \gamma s)$, the particle evaporation and the ground state spin of the ER, using average values. This procedure is not correct for the lower spin region of the distribution, because the various evaporation channels are not equally distributed over the whole spin range. Therefore, the absolute spin-values should not be taken too seriously. The shape of the higher end of the distribution, however, should not be affected. There we expect, on the other hand, to see the influence of the barrier distribution. For a large range of barriers a long tail should appear which at higher energies will be cut by fission. In Fig. I-36 a superposition of the spin distributions for the two highest energies is shown. One notes clearly a change in shape as illustrated by the cartoon in the same figure. The spin distribution taken at the lower energy has a relatively long tail of $>22 \hbar$, whereas the other one falls over only $\approx 12 \hbar$ to zero. The statistical errors, however, do not allow for extracting the representation of barrier distributions in a way to provide a detailed analysis of the coupling mechanism.

*INFN, Laboratori Nazionali di Legnaro, Italy, †Departimento di Fisica dell'Università and INFN, Sezione di Padova, Italy, ¥University of Buenos Aires, Argentina

${ }^{1}$ N. Rowley, G. R. Satchler, and P. H. Stelson, Phys. Lett. B254, 21 (1991).

${ }^{2}$ D. Ackermann, Acta Fisica Polonica B26, 517 (1995).

${ }^{3}$ G. Montagnoli et al., LNL Annual Report, p. 84 (1995).

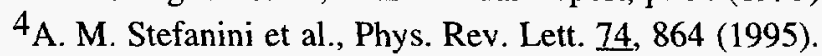

${ }^{5}$ E. Rehm et al., Phys. Lett. B317, 31 (1993). 

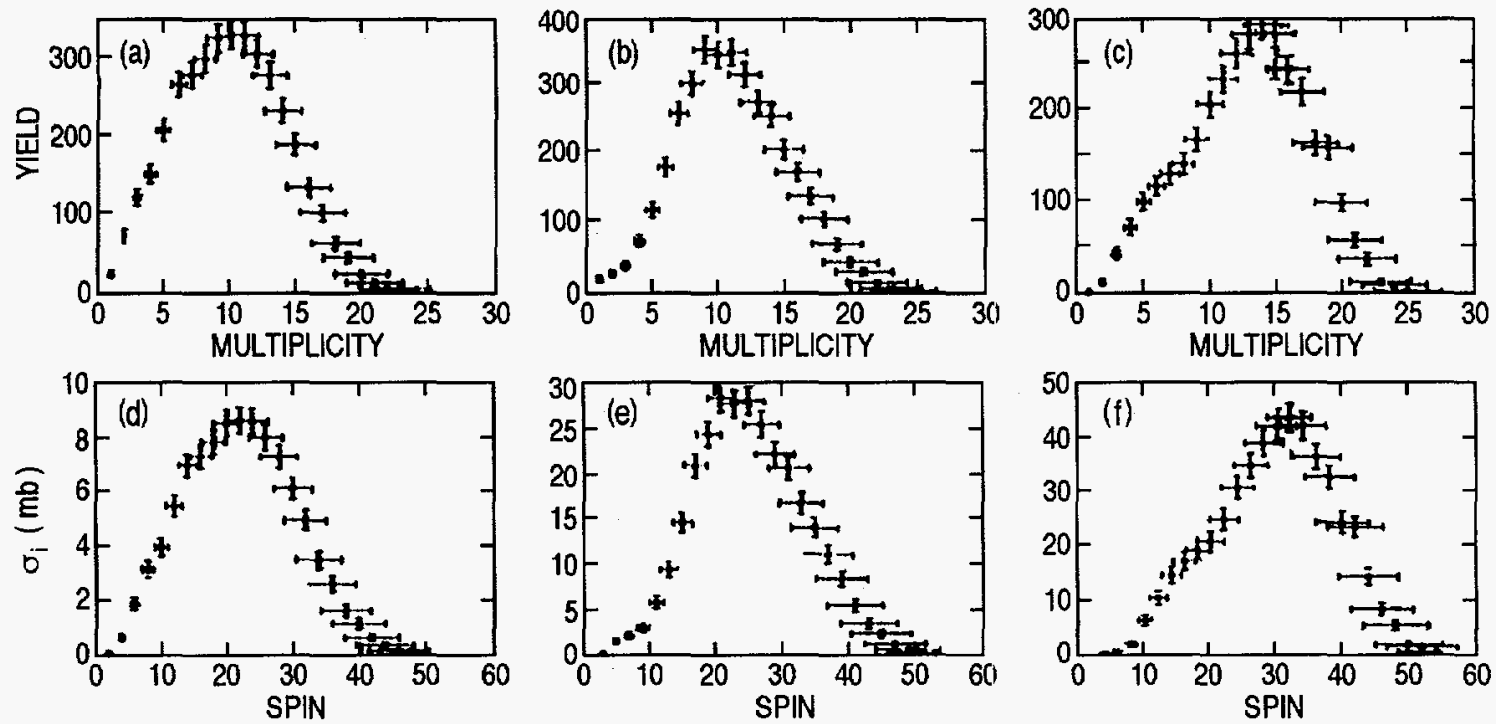

Fig. I-35. $\gamma$-multiplicity (a-c) and spin distributions (d-f) for the reaction ${ }^{64} \mathrm{Ni}+100_{\mathrm{Mo}}$ at three energies (see text).
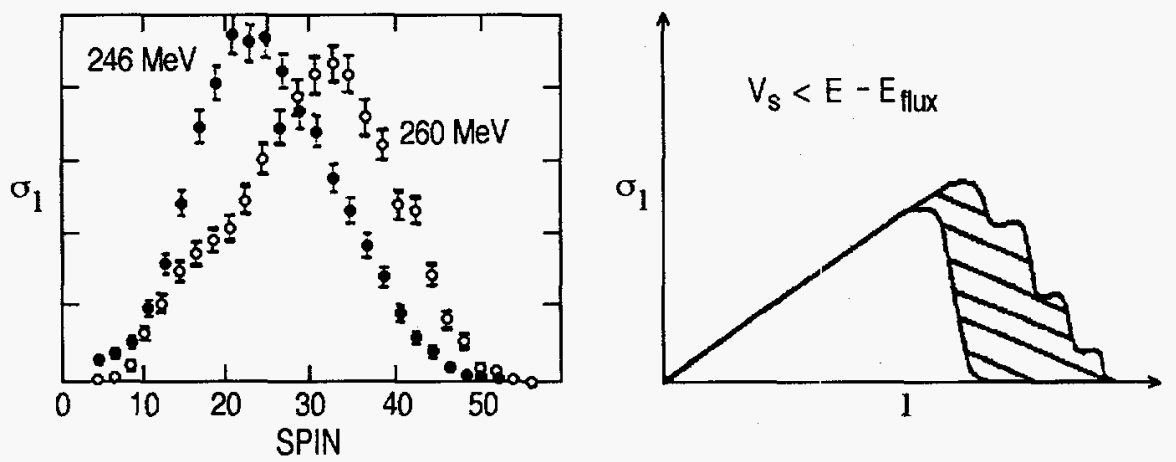

Fig. I-36. Comparison of the spin distributions at the two higher energies (Fig. I-35 $e$ and f).

\section{c.5. Population of Light-Actinide Nuclei by Multi-JNucleon Transfer Reactions}

(I. Ahmad, D. J. Blumenthal, M. P. Carpenter, B. Crowell, R. V. F. Janssens,

T. L. Khoo, T. Lauritsen, D. Nisius, J. F. C. Cocks,* P. A. Butler,* K. J. Cann,*

G. D. Jones, ${ }^{*}$ J. F. Smith,* P. M. Jones, $\uparrow$ R. Julin, $\uparrow$ S. Juutinen, $\uparrow$ D. Muller, $\dagger$

M. Piiparinen, $\uparrow$ A. Savelius, $\nmid$ R. Broda,,$\ddagger$ and B. Fornal $\ddagger)$

Nuclei in the light actinide region contain the largest amount of octupole-octupole correlations. Only few nuclei in this mass region have been studied in detail because they are difficult to produce with suitable reactions. Several experiments have shown that these nuclei are produced in multinucleon transfer reactions with moderate cross sections. In order to find the optimum combination of target and projectile for the production of light actinide nuclei, we have performed reactions on ${ }^{232} \mathrm{Th}$ targets with different projectiles. We have used beams of ${ }^{56} \mathrm{Fe},{ }^{86} \mathrm{Kr}$ and ${ }^{136} \mathrm{Xe}$ with energies $15-20 \%$ above the Coulomb barrier. The first two measurements were done at the Jyväskylä

*University of Liverpool, United Kingdom, †University of Jyväskylä, Finland, ¥Niewodniczanski Institute of Nuclear Physics, Krakow, Poland 
cyclotron and the last experiment was performed with a ${ }^{136} \mathrm{Xe}$ beam from the ATLAS superconducting accelerator at Argonne. Gamma-gamma coincidences were used to generate one-dimensional spectra for individual reaction products. The yields of the reaction products were measured for the three reactions. The population distributions show that the transfer of the nucleons is dictated by the mass and charge equilibrium process and indicates that ${ }^{136} \mathrm{Xe}$ is the most effective of the three projectiles for populating octupole-deformed nuclei in the light-actinide region.

\section{c.6. Angular Correlation Measurements for ${ }^{12} \mathrm{C}\left({ }^{12} \mathrm{C},{ }^{12} \mathrm{C}\right){ }^{12} \mathrm{C}\left(3^{-}\right)$Scattering (A. H. Wuosmaa, B. B. Back, R. R. Betts, D. J. Blumenthal, S. Fischer, B. G. Glagola, D. J. Henderson, D. Hofman, R. V. F. Janssens, C. J. Lister, V. Nanal, D. Nisius, M. D. Rhein, P. R. Wilt, and M. Freer*)}

Many previous studies of inelastic scattering in the ${ }^{12} \mathrm{C}$ $+{ }^{12} \mathrm{C}$ system have revealed strong resonance-like behavior. Several theoretical pictures have emerged which attempt to describe this behavior, either as superdeformed cluster states in the compound system ${ }^{24} \mathrm{Mg}$, or as potential scattering resonances whose strength is enhanced due to favorable angular momentum coupling conditions at particular energies. These models make predictions about the angular momenta expected to dominate the scattering process at resonance energies. Many of the reaction channels in which the strongest resonances are observed have non-zero channel spin, making the extraction of a resonance angular momentum difficult, if not impossible, using the traditional technique of angular distribution measurements. If, however, the radiation from the decay of the level in the inelastically excited nucleus can be detected, the angular correlations between that radiation, and the scattered nucleus become sensitive to the total angular momentum of the system and, hence, the resonance spin.

We have applied the technique of particle-particle angular correlations to the problem of making spin assignments for resonances observed in the $3^{-}+$g.s. excitation in ${ }^{12} \mathrm{C}+{ }^{12} \mathrm{C}$ scattering, where previous experiments ${ }^{1}$ have identified a number of strong resonances (see Fig. I-37). Data from two experiments, conducted with arrays of four, and six double-sided strip detectors (DSSDs) have been obtained at several energies in the range of $E_{(\mathrm{c} . \mathrm{m} .)}=26$ to $40 \mathrm{MeV}$.

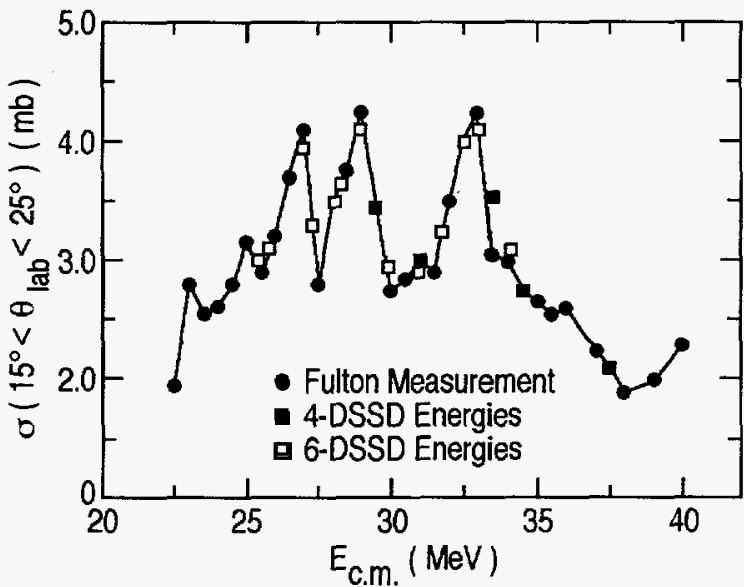

Fig. 1-37. ${ }^{12} \mathrm{C}+{ }^{12} \mathrm{C}\left(3^{-}\right)$excitation function from Fulton, et al. ${ }^{1}$ The symbols indicate energies where correlation data have been obtained. The spin assignments noted are obtained from angular correlation techniques described in Ref. 4.

The alpha particles from the decay of the $3^{-}(9.64 \mathrm{MeV})$ state in ${ }^{12} \mathrm{C}$ were detected in the DSSD arrays, and the energy and momentum correlations between them were used to identify the reaction channel of interest.

The data from our initial measurements have now been published ${ }^{4}$, and those from the more recent measurement carried out with six DSSDs are still being analyzed. Figure I-38 shows projected angular correlation data obtained at four energies between $\mathrm{E}_{(\mathrm{c} . \mathrm{m} .)}=27$ and $33.3 \mathrm{MeV}$. The data are the solid symbols, and the histograms are Monte

\footnotetext{
*University of Birmingham, United Kingdom

${ }^{1}$ W. Reilly et al., Nuovo Cimento 13A, 913 (1973), and B. R. Fulton et al., Phys. Rev. C $\underline{21}, 198$ (1980).

${ }^{2}$ S. Marsh and W. D. M. Rae, Phys. Lett. B153, 21 (1985).

${ }^{3}$ Y. Kondo, Y Abe, and T. Matsuse, Phys. Rev. C 19, 1356 (1980).

${ }^{4}$ A. H. Wuosmaa, B. B. Back, R. R. Betts, M. Freer, B. G. Glagola, D. J. Henderson, D. J. Hofman, and V. Nanal, Phys. Rev. C 포, 2463 (1996).
} 


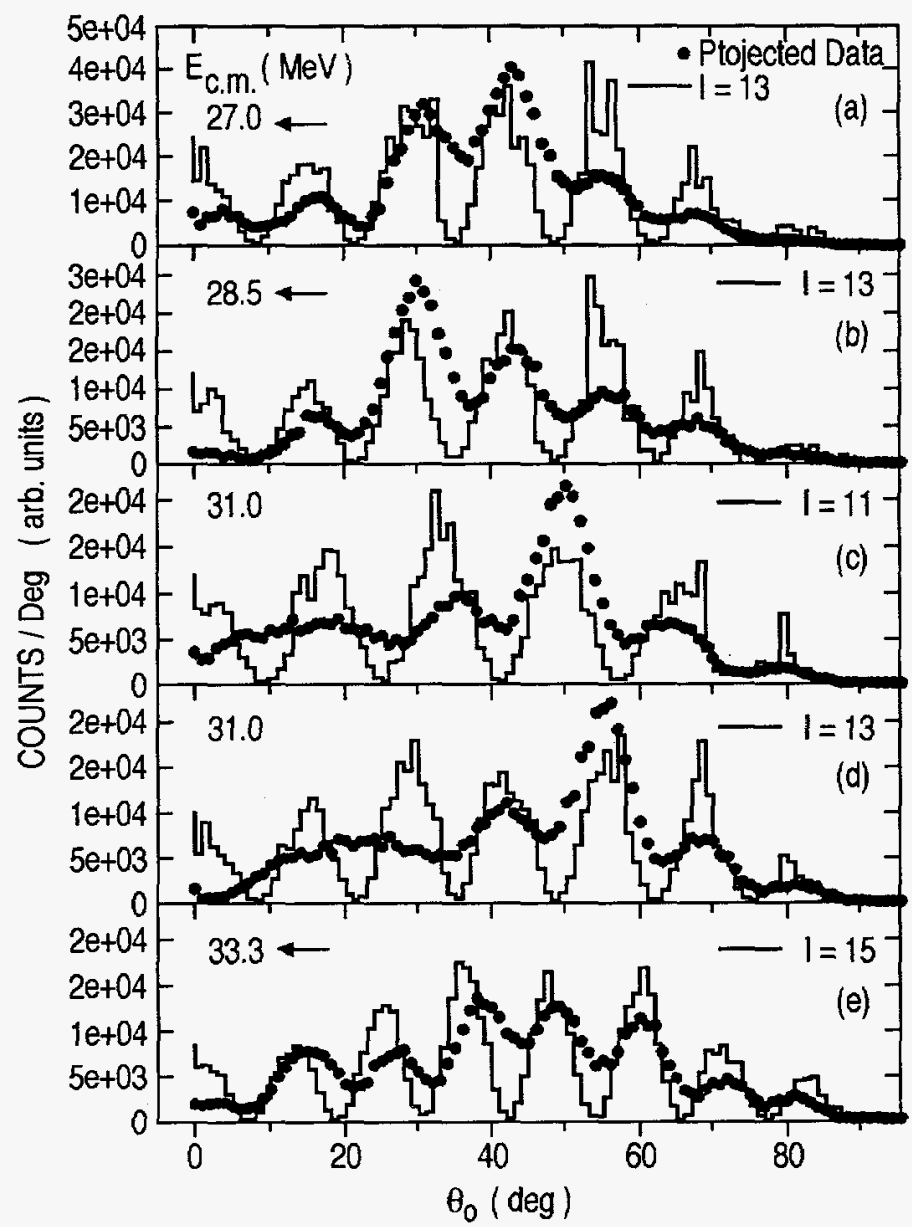

Carlo simulations of the correlation from the decay of a single isolated resonance decaying through a single $l$ value as indicated in the figure. At the resonance energies, indicated by the arrows on Figs. I-37 and I-38, the data confirm the assignment of $18^{+}$for the resonance at $E_{(c . m .)}=33.5 \mathrm{MeV}$, and suggest an assignment of $16^{+}$for two resonances at lower energies $\left(E_{(\text {c.m. })}=27\right.$ and $\left.28.5 \mathrm{MeV}\right)$. Between the resonances, the data do not suggest a single dominant $l$ value, and their character is significantly different from that on resonance. These spin assignments are indicated on Fig. I-37. The rotational spacing obtained from the average energy of the two $16^{+}$resonances, and the $18^{-t}$ state, is consistent with either two rotating ${ }^{12} \mathrm{C}$ spheres, or with the expectations of a hyperdeformed quasimolecular complex in ${ }^{24} \mathrm{Mg}^{2}$

Fig. 1-38. ${ }^{12} \mathrm{C}+{ }^{12} \mathrm{C}\left(3^{-}\right)$angular correlation data analyzed assuming l values indicated in the figure, coupling to entrance channel angular momenta $J=l+3$. The on-resonance data $(a, b, e)$ are consistent with only one pair of $(l, j)$ values, but off resonance $(c, d)$ the data cannot be uniquely analyzed in this framework.

\section{c.7. Study of the d( $\left.{ }^{9} \mathrm{Li}, \mathbf{p}\right)^{10} \mathrm{Li}$ Reaction (A. H. Wuosmaa, P. Santi, ${ }^{*}$ M. Belbot, ${ }^{*}$ J. J. Kolata,* D. Peterson, ${ }^{*}$ J. von Schwarzenberg, ${ }^{*}$ G. Kunde, $\dagger$ and B. M. Sherrill $\dagger$ )}

In the past few years, "neutron-halo" nuclei have been a topic of intense study. The classic example of such a nucleus, where two very loosely bound neutrons are coupled to an essentially inert core, is ${ }^{11} \mathrm{Li}$. Here one finds that some aspect of the interaction between the two last neutrons makes the system bound - the ${ }^{9} \mathrm{Li}-\mathrm{n}$ nucleus $10 \mathrm{Li}$ is unbound with respect to neutron emission. A number of models have been proposed to explain the properties of ${ }^{11} \mathrm{Li}$, and one ingredient of these models which has not been studied experimentally involves the properties of the neutron-unbound $10_{\mathrm{Li}}$. Some evidence is now available that the ground state of $10 \mathrm{Li}$ consists of an s-wave neutron resonance unbound

*University of Notre Dame, $\uparrow$ Michigan State University

${ }^{1}$ W. Benenson, Nucl. Phys. A588, 11c (1995). by approximately $500 \mathrm{keV}^{1}$. Considerable uncertainty remains, however, regarding the precise location and width of this resonance, and a conclusive spin assignment does not yet exist.

In order to address some of these questions, the $\mathrm{d}\left({ }^{9} \mathrm{Li}, \mathrm{p}\right){ }^{10} \mathrm{Li}$ reaction has been studied at the National Superconducting Cyclotron Laboratory at Michigan State University. The experimental arrangement consisted of two parallel-plate avalanche counters upstream of the $\mathrm{CD}_{2}$ target for beam tracking, two segmented, annular silicon detectors to detect ${ }^{9} \mathrm{Li}$ fragments, and six $5 \mathrm{~cm} \times 5 \mathrm{~cm}$ silicon wafers to detect 
the protons from the $\left({ }^{9} \mathrm{Li}, \mathrm{p}\right)$ reaction. By detecting the recoil proton and the ${ }^{9} \mathrm{Li}$ from the decaying $10_{\mathrm{Li}}$ nucleus and measuring their angles and energies, the full three-body kinematics of the reaction could be reconstructed. The results of this measurement are currently being analyzed.

\section{c.8. Study of the $9,11 \mathrm{Li}+{ }^{12} \mathrm{C}$ Elastic Scattering using the S800 Spectrometer (A. H. Wuosmaa, D. Peterson,* J. J. Kolata,* J. von Schwarzenberg, * P. Santi,* D. Bazin, $\dagger$ J. Caggiano, $\dagger$ B. S. Davids, $\dagger$ and B. M. Sherrill $\dagger$ )}

A previous measurement of the quasi-elastic scattering of ${ }^{11} \mathrm{Li}+{ }^{12} \mathrm{C}^{1}$ reported that at very forward scattering angles, very large interference oscillations in the angular distribution existed, which might be interpreted as evidence. for the effects of the extended ${ }^{11} \mathrm{Li}$ neutron halo on the reaction cross section. The resolution attained in that measurement, however, was insufficient to identify excited states in the scattering nuclei, and definitive conclusions about the nature of this forwardangle enhancement were difficult to draw.

\footnotetext{
*University of Notre Dame, $†$ Michigan State University

1J. J. Kolata et al., Phys. Rev. Lett. 69, 2631 (1992).
}

In order to resolve some of these questions, a new measurement of ${ }^{9,11} \mathrm{Li}+{ }^{12} \mathrm{C}$ elastic scattering at very forward angles has been carried out using the $\mathrm{S} 800$ spectrograph at MSU NSCL. The S800 was augmented with two PPAC tracking counters before the target in order to better define the trajectories of the ${ }^{9,11} \mathrm{Li}$ beams. The data are still being analyzed, but already have provided some information about the response of the $\mathbf{S 8 0 0}$ beam line for secondary beams. This measurement is the first in which secondary beams produced in the A1200 separator have been transported to the $S 800$.

\section{c.9. Survival Probability of Target-Like Recoils in Deep-Inelastic Reactions (D. J. Hofman, B. B. Back, D. Ackermann, G. Hackman, D. Henderson, V. Nanal, and A. Wuosmaa)}

The survival probability of target-like deep-inelastic reaction partners has been measured with beams of 400 $\mathrm{MeV}{ }^{40} \mathrm{Ar}$ on targets of ${ }^{197} \mathrm{Au},{ }^{208} \mathrm{~Pb}$, and ${ }^{232} \mathrm{Th}$ using a technique of kinematic coincidences between target-like recoils and projectile-like nuclei. The measurements were carried out in order to study the possible effects of nuclear friction on the fission process inside the fission barrier. The energy and charge of the Ar projectile-like-fragment (PLF) was measured with a $\triangle E-E$ silicon detector telescope and the time and position of the surviving target-like-fragment (TLF) was detected in a large multi-wire gas-filled proportional counter. In this manner, the survival probability of the target-like-fragment could be extracted as a function of the total kinetic energy loss (TKEL) in the reaction. The measured TKEL was translated into an excitation energy in the heavy recoil using both existing data on the energy splitting between the PLF and TLF as well as limits set by a simultaneous measurement of the proton multiplicity yield from the PLF.

Thus far a full analysis for the ${ }^{232} \mathrm{Th}$ target has been carried out. Detailed calculations were performed in order to compare the measured yields to the standard statistical model. The calculations included the effects of transferred spin and mass in the deep-inelastic reaction as well as the fluctuations in excitation energy for a given TKEL and the widths of the spin and mass transfer distributions. Both existing experimental data as well as theory were utilized to deduce these values (for this system of $400 \mathrm{MeV}{ }^{40} \mathrm{Ar}+{ }^{232} \mathrm{Th}$ ). Figure I-39(a) displays the results of the calculations, where both the standard statistical model calculation and a calculation including an increasing friction are compared to the measured data. The magnitude of the extracted friction parameter used at each excitation energy is given in Figure I-39(b). 


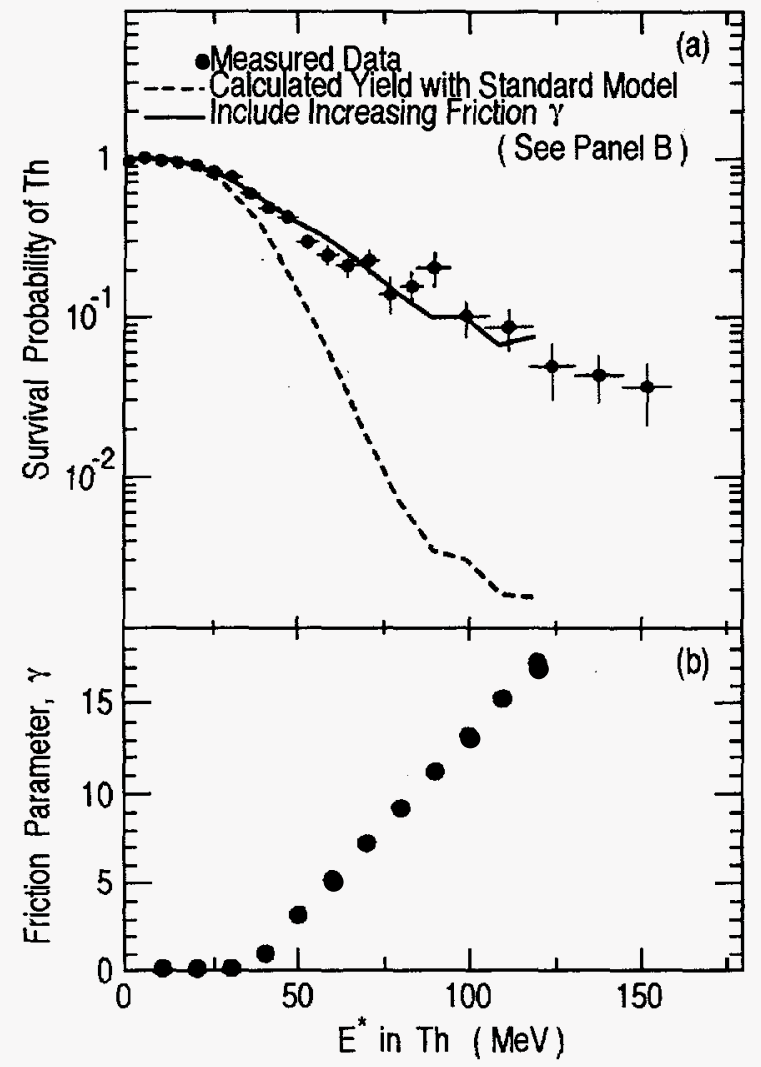

Fig. 1-39. Panel a: comparison of the measured survival probability of Th-like nuclei (data points) to calculations both without (dashed line) and including (solid line) the effect of friction in the fission motion. Panel $b$ : the friction parameter $\gamma$ used in the solid line fit of panel $a$.

c.10. Evaporation Residue Cross Sections in the 216-218Th System (B. B. Back, D. Ackermann, D. J. Blumenthal, C. N. Davids, G. S. Hackman, D. J. Henderson, W. F. Henning, D. J. Hofman, C. L. Jiang, V. Nanal, A. H. Wuosmaa, and P. Paul*)

Evaporation residue cross sections for the systems, $216,218 \mathrm{Th}$ has been measured using three different entrance channels, namely $32 \mathrm{~S}+{ }^{184} \mathrm{~W}, 64 \mathrm{Ni}+$ ${ }^{154} \mathrm{Sm}$, and ${ }^{100} \mathrm{Mo}+{ }^{116} \mathrm{Cd}$ to study 1) the survival probability of hot Th nuclei and the influence and strength of dissipation in the fission motion, and 2) the entrance channel effects on the complete fusion cross section. The experimental work is complete and the data analysis is presently being carried out. A preliminary analysis indicates that both effects were observed.

*SUNY at Stony Brook

\section{c.11. Exclusive Studies of GDR in the Decay of ${ }^{164} \mathrm{Er}$ (V. Nanal, D. Ackermann, B. B. Back, G. Hackman, D. Henderson, D. J. Hofman, R. V. F. Janssens, and T. L. Khoo)}

We have recently carried out an experiment at ATLAS to measure the high energy $\gamma$-spectra in the compound nucleus ${ }^{164} \mathrm{Er}$ at $63 \mathrm{MeV}$ excitation energy in order to study the characteristics of the Giant Dipole Resonance in hot nuclei. The high energy photons were detected in a 16 element subarray of the LEPPEX detector system while the evaporation residues were identified in the focal plane detector of the FMA. Figure I- 40 shows the results of a preliminary analysis for exclusive $\gamma$-spectra in the decay of ${ }^{164} \mathrm{Er}$ selected with the residue $160 \mathrm{Er}$.
Analysis is being carried out to extract the GDR parameters from statistical model fit to the data. Although this analysis is very preliminary there are significant differences between the inclusive and the exclusive spectra which we feel will have an important impact on the interpretation of heavy-ion induced GDR studies. The response of the LEPPEX subarray was characterized by using the standard ${ }^{11} B(p, \gamma)\left(E_{p}=7.2\right.$ $\mathrm{MeV}$ ) reaction to populate the GDR in $12 \mathrm{C}$ which decays to the ground state with $22.56 \mathrm{MeV} \gamma$-ray. The 
high energy photon spectrum obtained in this reaction is shown in the inset in Figure I-40 (open circles) together with the simulated spectrum obtained from GEANT simulations (solid line), folded with gaussian of $10 \%$ FWHM. It can be seen that the experimental spectrum is well reproduced by the simulations.

We propose to extend our measurements to the $A=110$ mass region.

Fig. 1-40. The GDR spectrum in the decay of ${ }^{164}$ Er gated with the residue $160 \mathrm{Er}$. The inset shows the high-energy peaks from the decay of the GDR in ${ }^{12} C$ to the ground state $(22.56 \mathrm{MeV})$ and the first excited state (18.12 $\mathrm{MeV}$ ). A GEANT simulation spectrum (solid line) is also shown for comparison.

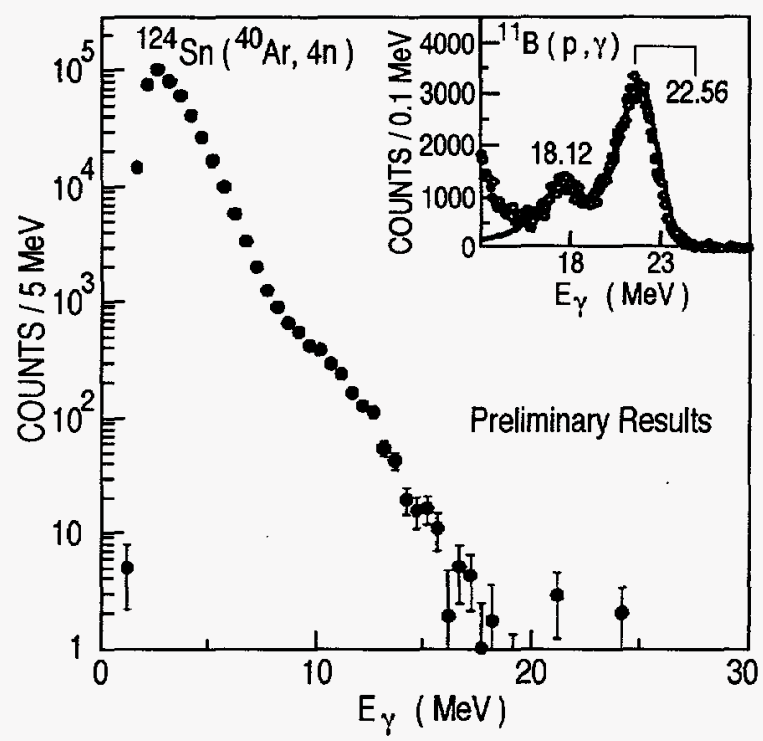

\section{c.12. Studies of $A u+A u$ Collisions at 6, 8, and $10.8 A \mathrm{GeV}$ at the AGS} (B. B. Back, R. R. Betts, C. Conner, A. Gillitzer, G. Hackman, D. J. Hofman, W. F. Henning, V. Nanal, A. Wuosmaa, H. C. Britt,ll J. Chang,§ W.-C. Chang,§ C. - Y. Chi, † Y. Y. Chu, * J. Cumming, * J. C. Dunlop, $\ddagger$ W. Eldrege, $\S$ S.-Y. Fung, $\S$ R. Ganz,I[ E. Garcia,II G. Heintzelman, $\neq$ B. Holzman, I J. Kang, $\uparrow \dagger$ E.-J. Kim, $\dagger \dagger$ S.-Y. Kim,* D. McLeod, II A. Mignerey,\|l C. Ogilvie, $\div$ R. Pak, ** D. Russ, II R. Seto,§

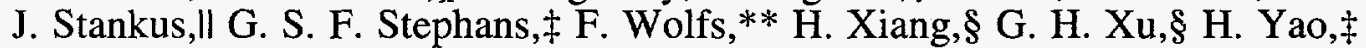
and C.-M. Zou§)

The E917 experiment at the AGS-Brookhaven has two main objectives, namely studies of the excitation function of particle production and collective flow phenomena in 6 and $8 \mathrm{~A} \mathrm{GeV} \mathrm{Au}+$ Au collisions (1/3 of a 3 months beamtime), and the investigation of the possible modification of the $\Phi$ meson mass in the dense hadronic medium at the full energy of $10.8 \mathrm{~A} \mathrm{GeV}$ (the remaining $2 / 3$ of the beamtime). E917 is a successor experiment to E859 and E866. An indication of a shift of the $\Phi$ meson mass was seen in E859 in $\mathrm{Si}+\mathrm{Au}$ collisions. ${ }^{1}$ This is very important as the density dependence of effective hadron masses in the medium is one of the key questions in the understanding of strongly interacting systems.

Medium effects on hadronic properties and interactions may also be seen by investigating the correlation of nonidentical particles like $\pi-K$ as a function of the relative momentum, whereas the correlation of identical particles reflects the space-time evolution of the collision system. Other interesting aspects which will be studied concern the production of multi-strange baryons like $\Xi$ and $\Omega$, or antibaryons like $\bar{\Lambda}$ and $\bar{\Xi}$, since their production is predicted to be enhanced if a phase transition to a Quark Gluon Plasma occurs.

Compared to the previous experiments, the E917 setup was improved by the addition of a beam vertexing detector system (BVER), built by the ANL-UIC group, which allowed a determination of the interaction point on the target to an accuracy of $0.3-0.4 \mathrm{~mm}$ as well as event by event measurement of the beam direction. An upgrade of the data acquisition system also allowed for a factor 2-3 higher event rates.

\footnotetext{
*Brookhaven National Laboratory, $\doteqdot$ Columbia University, $\ddagger$ Massachusetts Institute of Technology, §University of California, Riverside, TUniversity of Illinois at Chicago, IIUniversity of Maryland, **University of Rochester, ††Yonsei University, Korea

${ }^{1}$ Y. Wang et al., Nucl. Phys. $\underline{A 590,}$ 539c (1995).
} 
The setup is shown in Fig. I-41. The target area is surrounded by an array of lucite Cerenkov multiplicity detectors, NMA, which count the number of fast charged particles emitted from the collision zone, while the PHOS plastic scintillator array identifies protons or light nuclei around target rapidities with partial azimuthal coverage. At zero degrees, the beam projectile or its remnants are analyzed with respect to their energy loss $\alpha \sum Z_{i}^{2}$, their spatial distribution, and their total energy, in the quartz Cerenkov detector BE, the plastic scintillator array $\mathrm{HODO}$, and the $0^{\circ}$ calorimeter ZCAL, respectively. The spectrometer arm, consisting of 9 tracking chamber detectors, the Henry-
Higgins magnet, the time of flight wall TOF, and the gas Cerenkov detector GASC to discriminate high momentum kaons from pions, can be rotated to cover polar angles from $\theta=14^{\circ}$ to $\theta=58^{\circ}$.

Au targets of 0.5 and $1.0 \mathrm{~mm}$ thickness $(1.5 \%$ and $3 \%$ interaction probability, respectively) were irradiated with an average intensity of $3 \times 10^{5} \mathrm{Au}$ ions/spill. During the $\Phi$ meson study data were taken with a trigger on 2 kaon tracks or a $\bar{p}$ track in the spectrometer at $\theta=14^{\circ}$ and $\theta=19^{\circ}$. First preliminary results of the data analysis are expected to be obtained by the end of 1997.

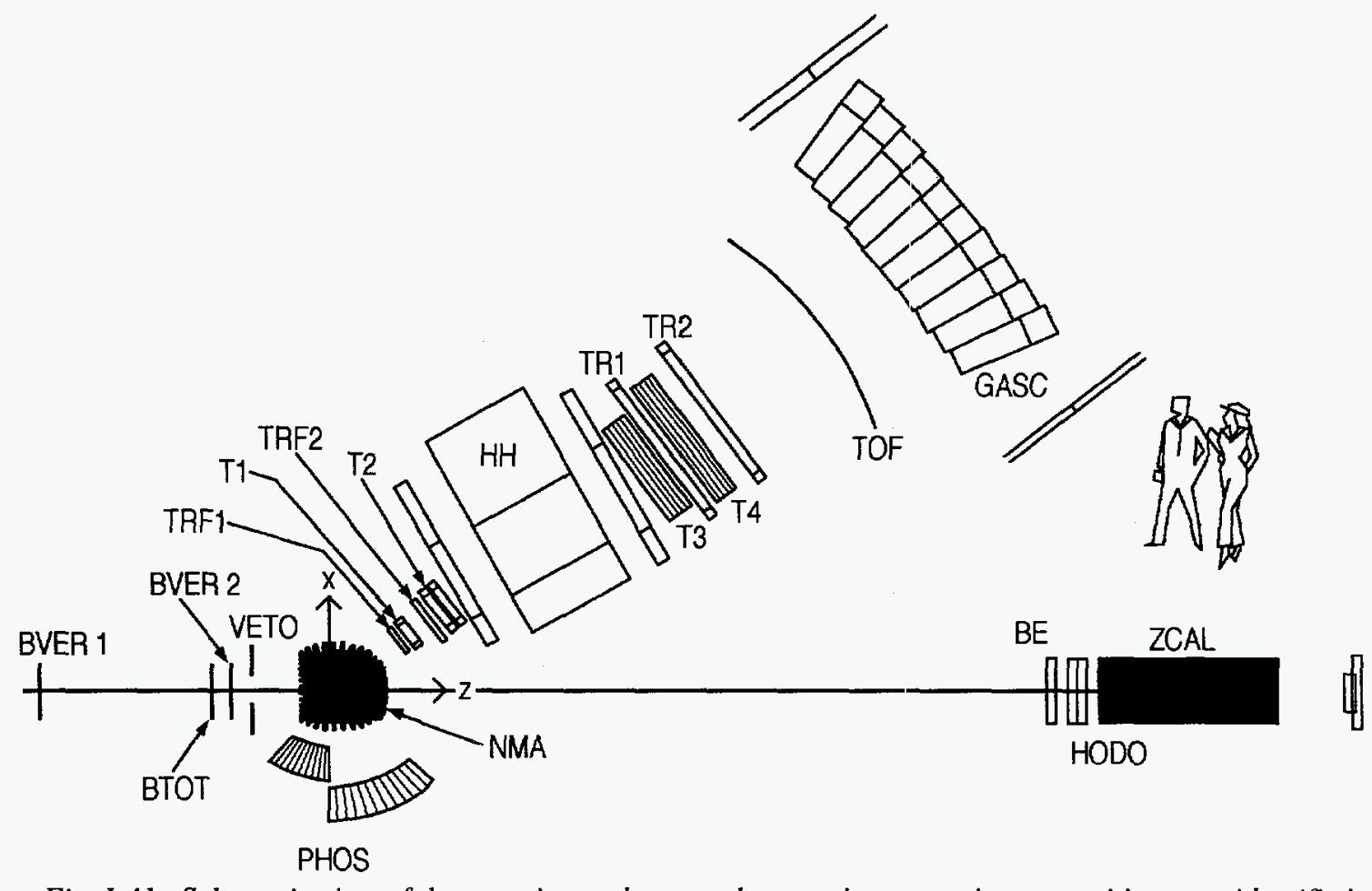

Fig. I-41. Schematic view of the experimental setup where various experiment partitions are identified.

\section{c.13. The PHOBOS Experiment (R. R. Betts, PHOBOS Collaboration)}

PHOBOS is an experiment designed to study $\mathrm{A}+\mathrm{A}, \mathrm{p}$ $+\mathrm{A}$ and $\mathrm{p}+\mathrm{p}$ collisions at RHIC. Central collisions of the heaviest ions $\left(\mathrm{Au}+\mathrm{Au}\right.$ at $\left.\sqrt{s}_{\mathrm{s}}=200 \mathrm{GeV} / \mathrm{u}\right)$ will produce unprecedented high energy densities which, it is hoped, will display new physics associated with large volumes of deconfined quarks and gluons. PHOBOS was approved for construction in 1996.

An overview of the apparatus is shown in Fig. I-42. It consists of an almost $4 \pi$ Multiplicity Array and Vertex Detector around the beam and two spectrometers located on either side of the beam axis. The Multiplicity Detector will allow the determination of the pseudorapidity and azimuthal dependence of the charged particle multiplicity on an event-by-event basis over the pseudorapidity range $-5.5<\eta<+5.5$. Interesting events may then also be studied using the spectrometers which allow multi-particle tracking and identification. The spectrometers are optimized for low pt particles which are expected to show the greatest sensitivity to collective effects which may characterize the new physics. 


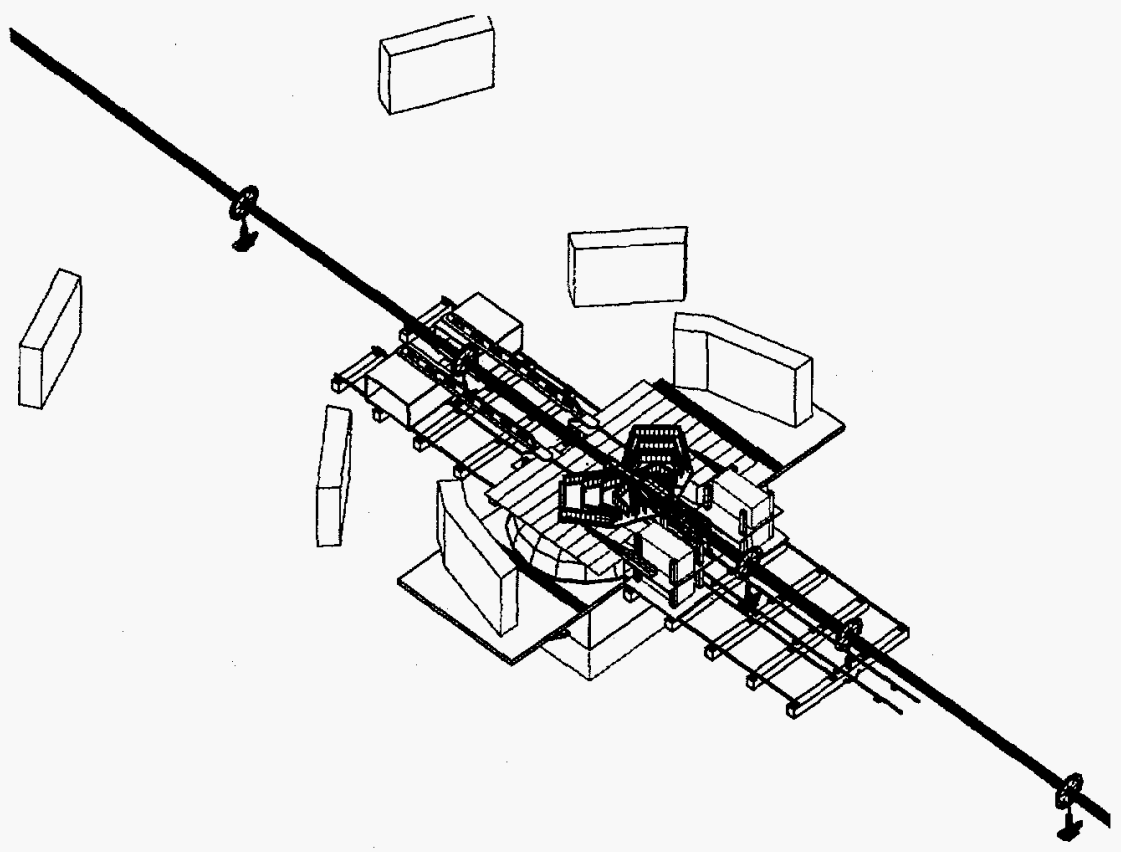

Fig. I-42. Overview of the PHOBOS experiment. For clarity, the upper portion of the magnet is removed.

An expanded view of the PHOBOS Multiplicity and Vertex Detector is shown in Fig. I-43. The Vertex Detector is comprised of two layers of silicon detectors segmented so as to have relatively low occupancy per channel even in central $\mathrm{Au}+\mathrm{Au}$ collisions. The interaction vertex is reconstructed from all pairs of hits in the inner and outer layers of the detector. True pairs reconstruct to the correct vertex position whereas false pairs give reconstructed vertices distributed more or less uniformly along the beam axis. The ability of the detector geometry to achieve this has been verified through detailed simulations in GEANT. An example for a central $\mathrm{Au}+\mathrm{Au}$ collision is shown in Fig. I-44. The interaction vertex can be reconstructed to the precision of better than $0.5 \mathrm{~mm}$ over $\pm 20 \mathrm{~cm}$ from the nominal center of the RHIC beam crossing.

A pair of detectors, identical to the Vertex Detector, below the beam line will be used to measure the ratio of charged to neutral particles at mid-rapidity. This will be accomplished by the insertion of $\mathrm{Pb}$ radiator between the two layers. Using the vertex reconstructed from the upper pair of detectors and the charged particle hits in the inner layer, the additional hits in the outer layer arising from photon (from $\pi^{0}$ decay) conversion in the $\mathrm{Pb}$ can be identified. Anomalous ratios of charged to neutral pions have been suggested as a possible signal of a Disoriented Chiral Condensate which may be formed in the hadronization of the plasma.

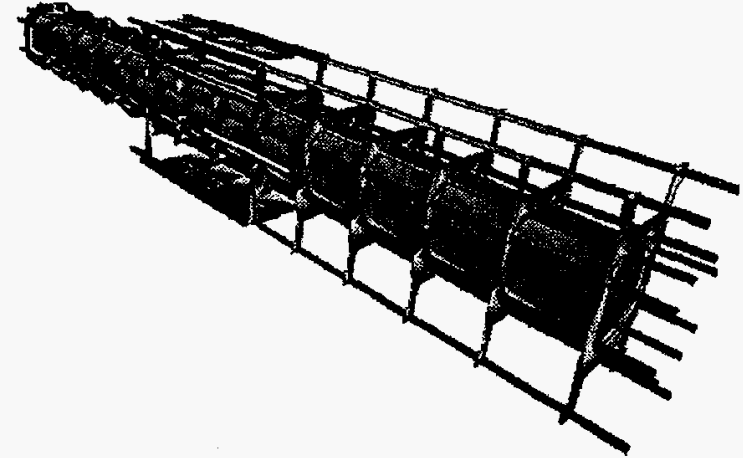

Fig. I-43 View of the octagon detector. The vertex detector is formed by the horizontal planes of detectors in the center of the octagon.

The Multiplicity Detector consists of an octagonal array of silicon detectors which surrounds the beam pipe \pm 52 $\mathrm{cm}$ from the nominal center. Rings of silicon detectors are placed up and down the beam line giving almost complete coverage from $-5.5<\eta<+5.5$. The multiplicity distribution is derived from the measured energies in each of the $\sim 10,000$ channels of the Multiplicity Detector after correction for the angle of incidence of the particles and contributions to the signal from backgrounds. An example of such a reconstruction is shown in Fig. I-45.

In the past year, the mechanical design of the detector system has been finalized and the detector installation 
procedures and cable layout studied with a model of the support structure. Although many details still remain, the design concept is sound and will not be changed. The design of the silicon wafers is also complete and prototypes of the octagon wafers have arrived. These are produced in collaboration with industry by the Taiwan component of the PHOBOS collaboration. A
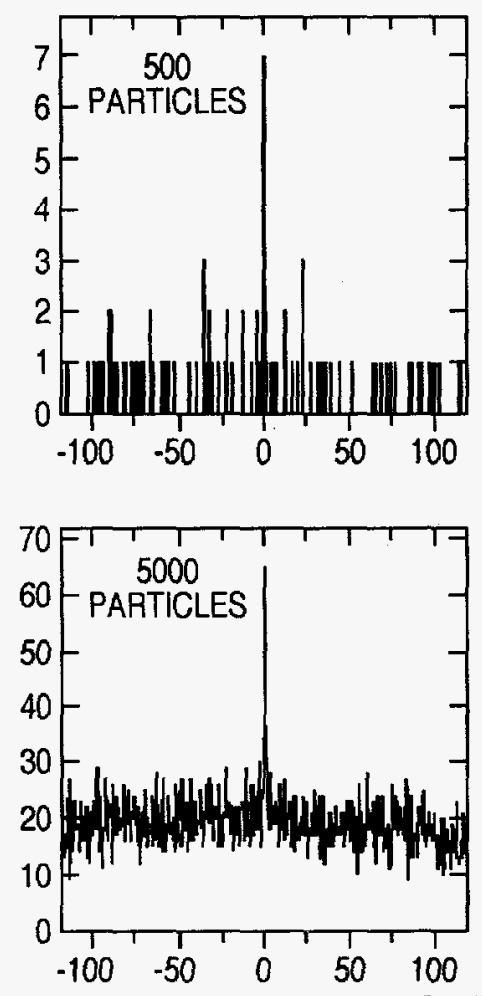

VERTEX $z(\mathrm{~mm})$

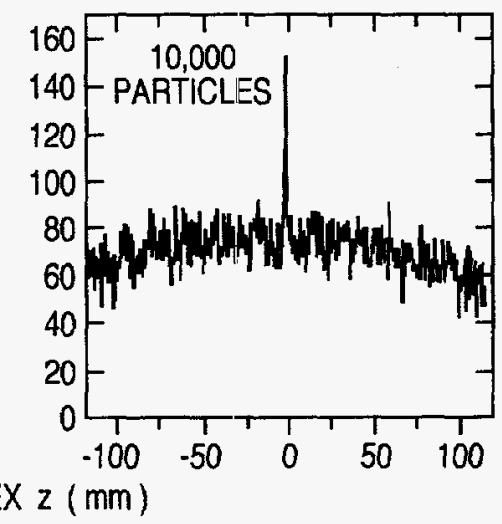

Fig. I-44. Example of a reconstruction of the collision vertex for a central $A u+A u$ collision at $Z=0$.

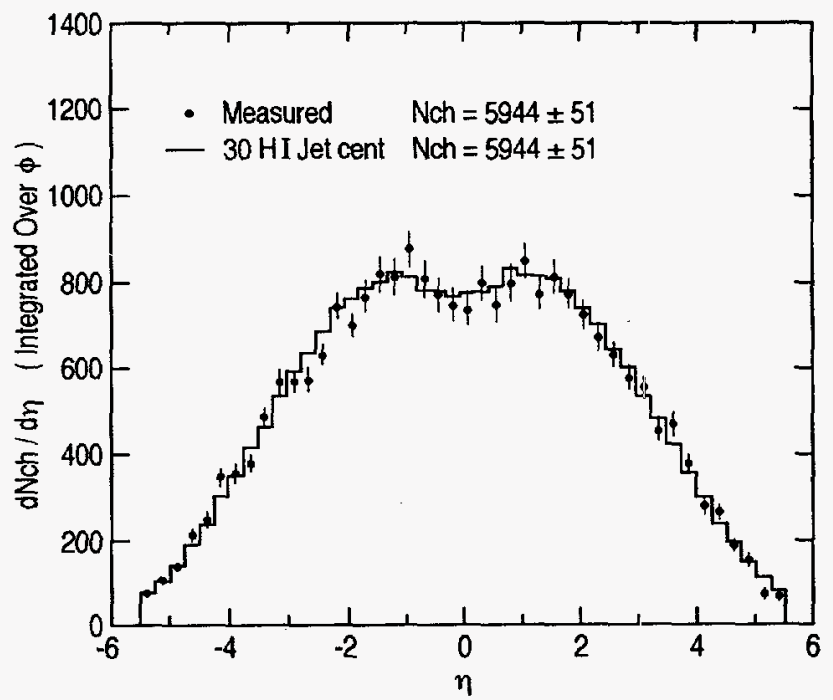

Fig. I-45. Reconstructed multiplicity distribution for a central Au + Au collision (solid points). The histogram is the expected distribution from the average of 30 such collisions calculated using HIJET. 


\section{SUPERCRITICAL FIELDS AND OTHER TOPICS}

Over the last five years a major effort was mounted in the area of the physics associated with the strong fields produced in near-barrier collisions of very high-Z ions such as $\mathrm{U}+\mathrm{Th}$ or $\mathrm{Pb}+\mathrm{Pb}$. A new experiment (APEX) was constructed by a large collaboration of 13 institutions in order to study the phenomena (the so-called $\mathrm{e}^{+}-\mathrm{e}^{-}$peaks) hinted at by past measurements performed at GSI. The analysis of data taken on the $U+T a$ and $U+T h$ systems is now complete and the results have been published. No sharp $\mathrm{e}^{+}-\mathrm{e}^{-}$sum-energy lines were found. The collaboration has now turned its attention to the yields associated with dynamic $\mathrm{e}^{+}$production, to the study of internal pair creation (IPC) and $\beta$-decay studies for which APEX is ideally suited.

This section also describes efforts devoted to other topics such as (1) the study of the behavior of cooled beams of charged particles confined in storage rings and traps, and (2) the high-precision measurement of the lifetime of ${ }^{44} \mathrm{Ti}$, an isotope of importance in astrophysics.

\section{d.1. Search for Monoenergetic Positron Production in ${ }^{238} U+{ }^{181} \mathrm{Ta}$ and ${ }^{238} \mathrm{U}+{ }^{232} \mathrm{Th}$}

Collisions (I. Ahmad, B. B. Back, R. R. Betts, R. W. Dunford, W. Kutschera, C. J. Lister, M. D. Rhein, J. P. Schiffer, P. Wilt, M. Wolanski, A. H. Wuosmaa, S. M. Austin,*

F. P. Calaprice, $\dagger$ K. C. Chan, $\ddagger$ A. Chishti, $\neq$ P. Chowdhury $\ddagger$ C. Conner,IIII J. D. Fox,$\S$

S. J. Freedman,I[ M. Freer,ll S. Gazes, ** J. S. Greenberg, $\ddagger$ A. L. Hallin, $\nmid \dagger$ T. Happ, $\neq \ddagger$

N. Kaloskamis, E. Kashy,* M. Liu, †† M. R. Maier,II A. Perera,§§ E. Roa,§ T. Trainor, J. S. Winfield,* F. L. H. Wolfs, $\S$ G. Xu, $\neq$ A. Young, $†$ and J. E. Yurkon*)

The original motivation for studies of positron production in heavy-ion collisions came from the suggestion that the combined charge of the composite system might be large enough so that the binding energy of the lowest electron orbital of the combined "quasi-atom" is greater than twice the electron rest mass. If the $\mathrm{K}$ shell of the quasi-atom is vacant, a positron-electron pair could then be produced spontaneously from the vacuum, with the electron captured in the $\mathrm{K}$ orbital, and the positron ejected from the system by Coulomb repulsion. Previous measurements 1,2 of positron spectra from a variety of systems showed unusual structures initially thought to be related to the spontaneous creation mechanism. Certain characteristics of the data, however, were inconsistent with this interpretation, and the explanation of the anomalous peaks remained a mystery.

We have analyzed positron energy spectra obtained from ${ }^{238} \mathrm{U}+{ }^{181} \mathrm{Ta}$ and ${ }^{238} \mathrm{U}+{ }^{232} \mathrm{Th}$ collisions in a manner similar to previous authors, in a search for these monoenergetic structures. Figure I-46 shows positron energy spectra obtained from ${ }^{238} \mathrm{U}+{ }^{232} \mathrm{Th}$ collisions analyzed to include events detected in regions of acceptance of our spectrometer which overlap with those in previous measurements. The symbols represent the measured spectra, and the histograms represent the expected signal, based upon the stated peak yields in. 1,2

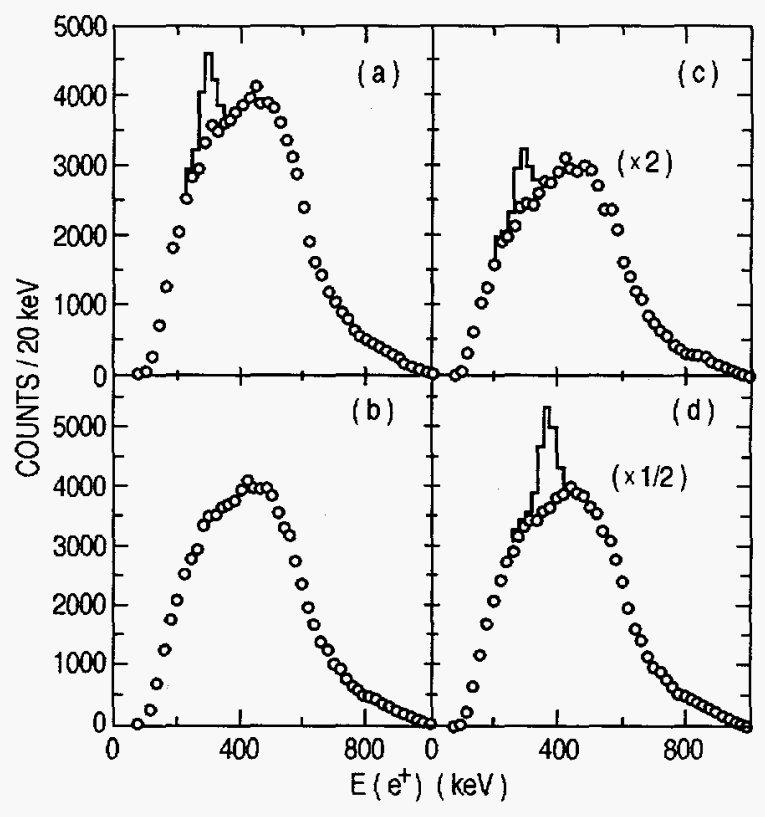

Fig. 1-46. Positron energy spectra from $238 U+$ 232 Th collisions, analyzed to reproduce the conditions obtained in $[I](a),(b),(c)$ and $[2](d)$.

The signals have been calculated to reflect the expected yields based on results from [1,2].

\footnotetext{
*Michigan State Univ., †Princeton Univ., †Yale Univ., §Florida State Univ., đIUniv. of California, Berkeley, IIUniv. of Birmingham, United Kingdom, **The Univ. of Chicago, ††Queen's Univ., Kingston, Ontario, $\ddagger$ GSI, Darmstadt, Germany, §§Univ. of Rochester, ITUUniv. of Washington, IlliUniv. of Illinois at Chicago

${ }^{1}$ M. Clemente et al., Phys. Lett. 137B, 41 (1984), ${ }^{2}$ T. Cowan et al., Phys. Rev. Lett. $\underline{54}, 1761$ (1985),

${ }^{3}$ I. Ahmad et al., Phys. Rev. Lett. $\underline{78}, 618$ (1997).
} 
In no case do any of our spectra contain sharp features similar to those previously reported. The estimated upper limits for such signals being present in our data are between one and two orders of magnitude smaller than the cross sections reported previously. These results have now been published. 3

\section{d.2. Dynamic Positron Production in ${ }^{238} U+{ }^{181} \mathrm{Ta}$ and ${ }^{238} \mathrm{U}+{ }^{232} \mathrm{Th}$ Collisions}

(I. Ahmad, B. B. Back, R. R. Betts, R. W. Dunford, W. Kutschera, C. J. Lister,

M. D. Rhein, J. P. Schiffer, P. Wilt, M. Wolanski, A. H. Wuosmaa, S. M. Austin,*

F. P. Calaprice, $\nmid$ K. C. Chan, $\ddagger$ A. Chishti, $\ddagger$ P. Chowdhury, $\ddagger$ C. Conner, Illl J. D. Fox,$\S$

S. J. Freedman,II M. Freer,\| S. Gazes,** J. S. Greenberg, $\neq$ A. L. Hallin, $† \dagger$ T. Happ, $\ddagger$

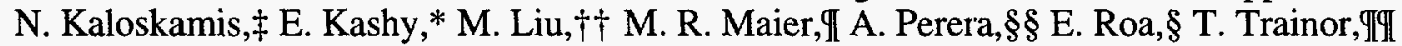

J. S. Winfield, ${ }^{*}$ F. L. H. Wolfs,,$\S$ G. Xu, $\ddagger$ A. Young, $\dagger$ and J. E. Yurkon*)

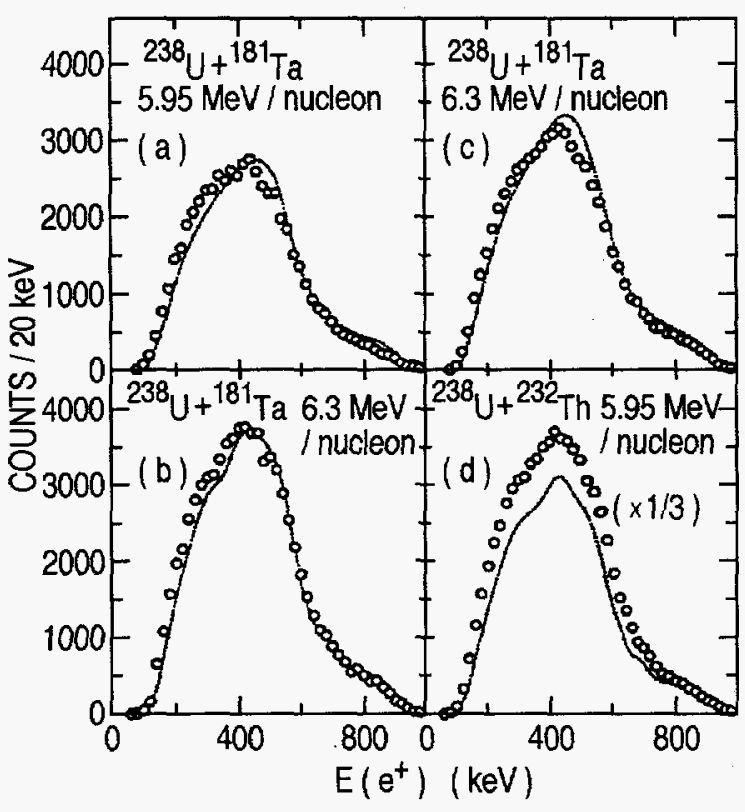

Fig. I-47.Positron energy spectra for $238 U+181_{T a}$ collisions at (a) 5.95, (b) 6.10, and (c) 6.30

MeV/nucleon, and (d) $238 U+{ }^{232}$ Th at 5.95

$\mathrm{MeV/nucleon.}$ The curves represent the calculated combined nuclear and dynamic positron spectra.

There exist two dominant mechanisms by which positrons can be produced in the collisions of heavy ions with high charge. The first, Internal Pair Conversion (IPC) of high energy gamma-ray transitions in either of the two colliding nuclei, is a conventional and well understood process. The second, so called "dynamic" production mechanism, arises from the strong, time-varying Coulomb fields which exist for short times during these collisions. In this process, the virtual photons can excite positron-electron pairs directly from the vacuum. The probability for the production of a positron in a given heavy-ion collision depends on the combined charge $Z$ (tot) of the system, the energies of emitted positrons, and properties of the nuclear reaction such as the distance of closest approach for the two ions, as well as the effective duration of the collision. In previous experiments for different systems, there has been a wide range of level of agreement between experiment and theory, ranging from nearly perfect agreement, 1 to experiment exceeding theory by more than a factor of two. 2

We have compared the yields of our positron "singles" data from ${ }^{238} \mathrm{U}+{ }^{181} \mathrm{Ta}$ and ${ }^{238} \mathrm{U}+{ }^{232} \mathrm{Th}$ collisions with the predictions of calculations of the positron yield expected from dynamic, ${ }^{3}$ as well as nuclear (internal pair conversion) processes. The nuclear component was estimated based on the spectra of gamma rays measured in a $70 \%$ relative efficiency Ge crystal. These predictions, folded with the acceptance of the APEX spectrometer, appear as the solid lines in Fig. I-47, which shows the experimental positron energy spectra obtained requiring the coincident detection of two quasi-elastically scattered heavy ions. For the $238 \mathrm{U}+{ }^{181} \mathrm{Ta}$ system, where the positron yield is dominated by nuclear processes, the calculated curves are in excellent agreement with the data. For the ${ }^{238} \mathrm{U}$ $+{ }^{232}$ Th system, where dynamic positrons are expected to make the largest component, the calculations underpredict the data by approximately 10-20\%. This result may indicate some deficiency in the dynamic calculations, however large uncertainties exist in the process of extracting the nuclear component. The results of these measurements have now been published. 4

\footnotetext{
* Michigan State University, †Princeton University, $\ddagger$ Yale University, §Florida State University, IUUniversity of California, Berkeley, IIUniversity of Birmingham, United Kingdom, **The University of Chicago, ††Queen's University, Kingston, Ontario, $\neq \neq$ GSI, Darmstadt, Germany, §§University of Rochester, TIIUniversity of Washington, IIIIUniversity of Illinois at Chicago

${ }^{1}$ H. Tsertos et al., Phys. Lett. 162B, 273 (1985), ${ }^{2}$ M. Kramer et al., Phys. Lett. B245, 17 (1990), ${ }^{3}$ J. Reinhardt, priv. comm., ${ }^{4}$ I. Ahmad et al., Phys. Rev. Lett. 78,618 (1997).
} 
d.3. Dynamic Positron Production in $\mathbf{P b}+\mathbf{P b}$ Collisions (I. Ahmad, B. B. Back, R. R. Betts, R. W. Dunford, W. Kutschera, C. J. Lister, M. D. Rhein, J. P. Schiffer, P. Wilt, M. Wolanski, A. H. Wuosmaa, S. M. Austin, * F. P. Calaprice, $\dagger$ K. C. Chan,$\ddagger$ A. Chishti, $\ddagger$ P. Chowdhury, $\div$ C. Conner,IIII J. D. Fox,§ S. J. Freedman,II M. Freer,II S. Gazes, ** J. S. Greenberg, $\neq$ A. L. Hallin, $† \dagger$ T. Happ, $\neq \ddagger$ N. Kaloskamis, $\neq$ E. Kashy, ${ }^{*}$ M. Liu, †† M. R. Maier,II A. Perera,§§ E. Roa,§ T. Trainor, $\| \mathbb{I}$ J. S. Winfield,* F. L. H. Wolfs, $\S$ G. Xu, $\ddagger$ A. Young, $\dagger$ and J. E. Yurkon*)

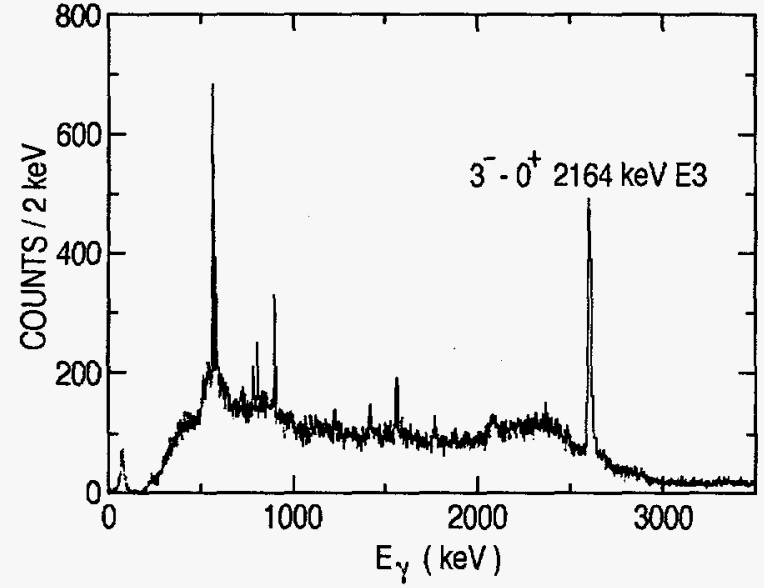

Fig. I-48. Doppler corrected gamma-ray spectrum from $208 \mathrm{~Pb}+208 \mathrm{~Pb}$ collisions at 6.0 $\mathrm{MeV} /$ nucleon. The reconstructed $\mathrm{E} 3$ transition at $2.614 \mathrm{MeV}$ is clearly evident.

We have extended our studies of dynamic positron production in heavy-ion collisions by studying the systematic dependence of this mechanism upon quantities such as the bombarding energy, scattering angle, and distance of closest approach of the ions during the collision. Also, we wish to examine the $Z(U)=Z($ target $)+Z$ (beam) dependence of the process, as the dynamic positron yield is expected to follow this quantity raised to a high power $(\sim 18-20)$ of $Z(U)$.

To augment the data already obtained for the ${ }^{238} \mathrm{U}+$ ${ }^{181} \mathrm{Ta}$ and ${ }^{238} \mathrm{U}+{ }^{232} \mathrm{Th}$ systems, we have studied positron production in the systems $208 \mathrm{~Pb}+208 \mathrm{~Pb}$ at bombarding energies of 5.5 and $6.0 \mathrm{MeV} /$ nucleon, and

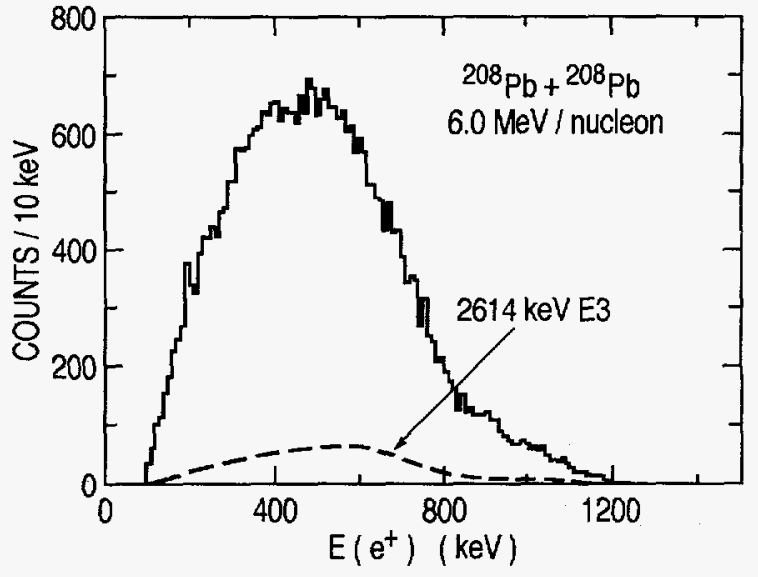

Fig. I-49. Positron energy spectrum from $208 \mathrm{~Pb}+$ $208 \mathrm{~Pb}$ collisions at $6.0 \mathrm{MeV} /$ nucleon. The dashed curve represents the yield expected from internal pair conversion of the $2.614 \mathrm{MeVE}$ transition in $208_{P b}$.

${ }^{206} \mathrm{~Pb}+{ }^{208} \mathrm{~Pb}$ at $5.5 \mathrm{MeV} /$ nucleon. The $208 \mathrm{~Pb}+$ ${ }^{208} \mathrm{~Pb}$ data should provide the cleanest dynamic signal as the nuclear background arises almost completely from a single transition, from the $3^{-}$level at $2.614 \mathrm{MeV}$ populated by Coulomb excitation, and can be understood very well quantitatively. Figure I-48 shows a Doppler corrected gamma-ray spectrum from $208 \mathrm{~Pb}+$ ${ }^{208} \mathrm{~Pb}$ collisions at $6.0 \mathrm{MeV} /$ nucleon, with the $3^{-}$- g.s. transition clearly evident. The positron energy spectrum appears in Fig. I-49. The dotted line represents the contribution from the $3^{-}-$g.s. $2.6 \mathrm{MeV}$ transition. These data are currently being analyzed, and the possibility of extending these measurements to the highest $\mathrm{Z}(\mathrm{U})$ system possible $(\mathrm{U}+\mathrm{Cm})$ is being investigated.

\footnotetext{
*Michigan State University, †Princeton University, $\ddagger$ Yale University, §Florida State University, qUUniversity of California, Berkeley, IUniversity of Birmingham, United Kingdom, **The University of Chicago, $\dagger \dagger$ Queen's University, Kingston, Ontario, $\ddagger \ddagger G S I$, Darmstadt, Germany, §§University of Rochester, TIIUniversity of Washington, IIIUniversity of Illinois at Chicago
} 


\section{d.4. Positron-Electron Angular Correlations in Internal Pair Conversion}

(A. H. Wuosmaa, C. M. Conner,* I. Ahmad, B. B. Back, R. R. Betts, R. W. Dunford, S. Fischer, R. Ganz, * J. P. Greene, N. I. Kaloskamis, $\dagger$ L. Lamm, † C. J. Lister, V. Nanal, D. E. Roa, and J. P. Schiffer)

Recent studies of positron and positron-electron pair production in the collisions of heavy ions has stimulated new interest in Internal Pair Conversion (IPC). In this process, a transition between two nuclear states produces an electron-positron pair of fixed sum energy. The conversion coefficients, energy, and angular correlations between the two leptons are governed by the charge of the emitting nucleus, the energy, and the multipolarity of the transition. For transitions with high ( $>$ a few $\mathrm{MeV}$ ) energies, or from a system with low $\mathrm{Z}(\mathrm{Z} \alpha \leq 1)$, IPC may be treated in the usual Born approximation. Further approximations to Coulomb distorted waves can be made to extend predictions to intermediate $\mathrm{Z}(\mathrm{Z} \alpha \sim 0.3)$. For large $\mathrm{Z}$ (e.g. near $\mathrm{Pb}$ ), the usual approximation methods break down and more sophisticated theoretical techniques are needed. These have been applied to IPC in heavy nuclei by references 1 and 2 . One interesting result of the new calculations is that for $\mathrm{Z} \sim 82$, and transition energies $\sim 1.5-2.5 \mathrm{MeV}$, significant differences emerge between transitions of electric and magnetic character of the same multipolarity.

In order to study these effects, and to obtain more precise measurements of pair conversion coefficients for IPC in heavy nuclei, we have used the APEX spectrometer to detect positron-electron pairs for pair producing transitions in three nuclei, $90 \mathrm{Zr}$, and $206,207 \mathrm{~Pb}$. For ${ }^{90} \mathrm{Zr}$, the beta decay of ${ }^{90} \mathrm{Y}$ feeds the first excited state of ${ }^{9} \mathrm{Zr}$ at $1.76 \mathrm{MeV}$, which has spin 0 and the resulting $\mathrm{E} 0$ transition proceeds either by electron conversion, or by pair emission. The $\mathrm{Pb}$ isotopes were populated by electron capture (EC) decay of $206,207 \mathrm{Bi}$. The EC decay of ${ }^{206} \mathrm{Bi}$ populates several states above $2 \mathrm{MeV}$ excitation energy, several of which decay through $\mathrm{E} 1$ transitions with energies greater than $1.5 \mathrm{MeV}$. The ${ }^{207} \mathrm{Bi}$ decay feeds predominantly two low-lying states in $207 \mathrm{~Pb}$, but at the $7 \%$ level feeds a $7 / 2^{-}$level which decays via a 1770 $\mathrm{keV}$ M1 transition. The ${ }^{206} \mathrm{Bi}$ source, with a $6.2 \mathrm{~d}$ half life, was produced by proton bombardment of ${ }^{206} \mathrm{~Pb}$ at the University of Notre Dame.

Figure I-50 shows positron-electron pair spectra from the $\mathrm{E} 0, \mathrm{E} 1$ and $\mathrm{M} 1$ transitions in ${ }^{90} \mathrm{Zr},{ }^{206} \mathrm{~Pb}$, and ${ }^{207} \mathrm{~Pb}$, respectively. The absolute pair conversion coefficients for the E1 and M1 case are obtained by comparing the number of photons detected in a $70 \%$ relative efficiency intrinsic $\mathrm{Ge}$ counter, corrected for its measured efficiency, with the number of full-energy peak pairs. In the E0 case, the absolute number of E0 transitions is obtained from the known $.0115 \% 0_{2}^{+}$ branch of ${ }^{90} \mathrm{Y} \beta^{-}$decay, and the total number of $\beta^{-}$ detected in APEX during the measurement, corrected for efficiency. The measured pair conversion coefficient, as well as the pair detection efficiencies for each case as determined from Monte Carlo simulations of the response to the APEX spectrometer are listed in Table 1. The results for E0 and E1 pair conversion are in good agreement with the theoretical values, however the measured pair conversion coefficient for the M1 transition in $207 \mathrm{~Pb}$ is approximately $30 \%$ higher than the theoretical value, and also disagrees with a previous measurement. 3

Table 1

\begin{tabular}{|c|c|c|c|c|c|}
\hline Nucleus & $E(\operatorname{trans})$ & $\mathrm{E} / \mathrm{ML}$ & $\operatorname{eff}(\%)$ & $P C C(\exp )^{\mathbf{a}}$ & $\mathrm{PCC}($ theory) \\
\hline $90 \mathrm{Zr}$ & $1760 \mathrm{keV}$ & E0 & 0.49 & $2.66(2)(13) \times 10^{-1}$ & $2.78 \times 10^{-1}$ \\
\hline${ }^{206} \mathrm{~Pb}$ & $1720 \mathrm{keV}$ & E1 & 0.42 & $3.06(2)(15) \times 10^{-4}$ & $3.26 \times 10^{-4}$ \\
\hline $207 \mathrm{~Pb}$ & $1770 \mathrm{keV}$ & M1 & 0.47 & $3.56(5)(18) \times 10^{-4}$ & $2.73 \times 10^{-4}$ \\
\hline
\end{tabular}

${ }^{\text {a}}$ Uncertainties in the Pair Conversion Coefficients (PCC) are (statistical)(systematic).

\footnotetext{
*University of Illinois at Chicago, †University of Notre Dame

${ }^{1}$ C. R. Hoffman and G. Soff, Atomic and Nucl. Data Tables 63, 189 (1996).

${ }^{2}$ C. R. Hoffman, J. Reinhardt, W. Greiner, P. Schluter, and G. Soff, Phys. Rev. C42, 2632 (1990).

${ }^{3}$ C. J. Allen, Can. J. Phys. $\underline{49}, 157$ (1971).
} 


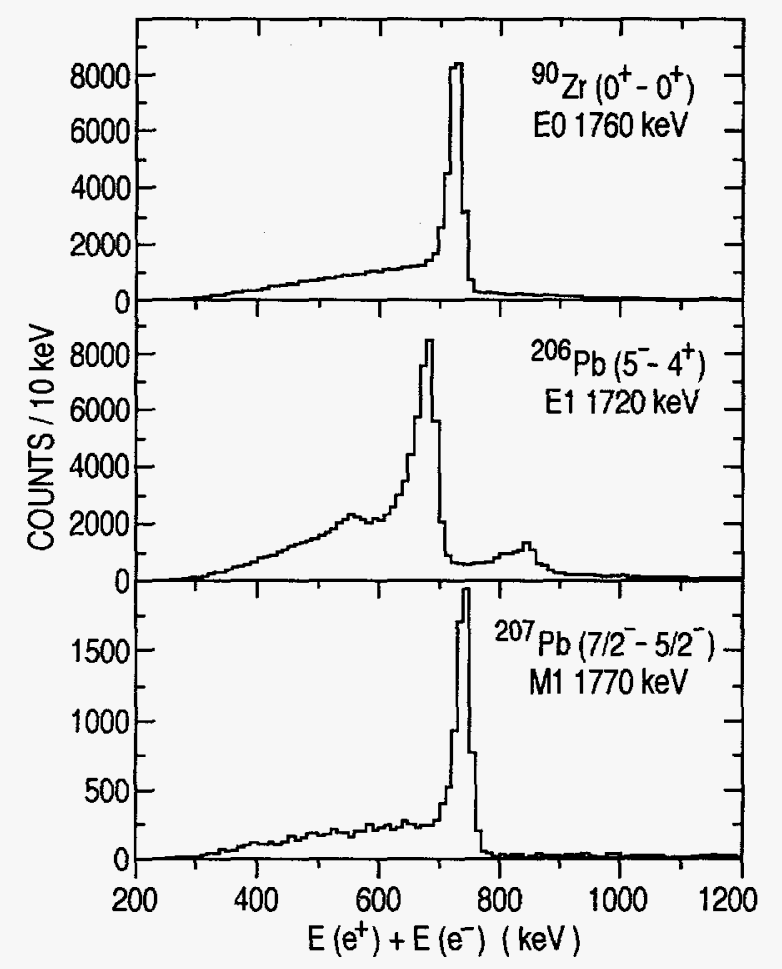

Fig. 1-50. Positron-electron sum-energy spectra for transitions in (a) ${ }^{90} \mathrm{Zr}$, (b) ${ }^{206} \mathrm{~Pb}$ and (c) ${ }^{207} \mathrm{~Pb}$.

Using the geometric properties of APEX, as well as an understanding of its transport characteristics obtained from Monte Carlo simulations, we have also measured the positron-electron angular correlations for the different transitions studied. Figure I-51 shows $\mathrm{e}^{+}-\mathrm{e}^{-}$ correlation data for the $\mathrm{E} 0(1760 \mathrm{keV}), \mathrm{E} 1(1720 \mathrm{keV})$ and $\mathrm{M} 1(1770 \mathrm{keV})$ transitions. The symbols are the data, and the solid and dashed curves represent the theoretical angular correlation obtained from ${ }^{2}$ and from the Born approximation, respectively. For the new theory, nuclear finite size effects have been included in

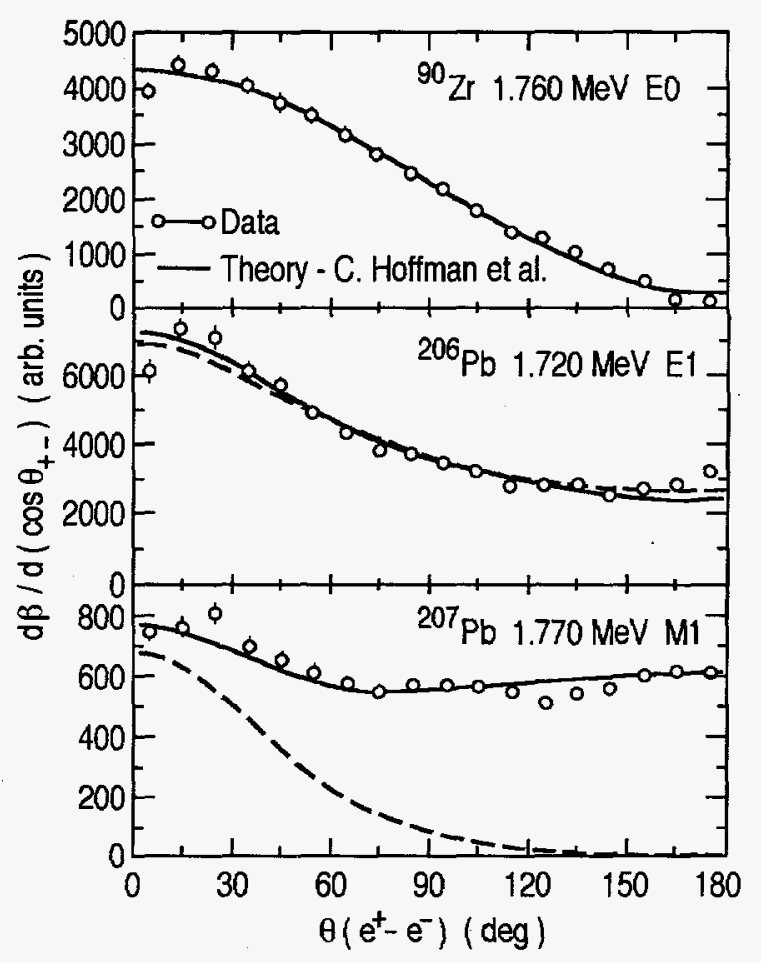

Fig. 1-51. Positron-electron angular correlation data for transitions in (a) ${ }^{90} \mathrm{Zr}$, (b) $206_{\mathrm{Pb} \text { and (c) }}$ $207 \mathrm{~Pb}$. The solid curves represent the calculations of [2], and the dashed curves in (b) and (c) represent the predictions of the Born approximation.

the calculation. In the E0 and E1 cases, the results are qualitatively similar to the Born results, with the correlation being forward peaked. In the M1 case, however, the data, as well as the new theory, deviate strongly from the Born prediction, and are nearly isotropic. In each case, the agreement between the data and the results of the new calculation is excellent. A manuscript describing these results is in preparation.

d.5. $\beta^{+}$Decay of ${ }^{207}$ Bi (A. H. Wuosmaa, C. M. Conner, ${ }^{*}$ I. Ahmad, B. B. Back, R. R. Betts, R. W. Dunford, S. Fischer, R. Ganz, * J. P. Greene, N. I. Kaloskamis, $†$ L. Lamm, $†$ C. J. Lister, V. Nanal, D. E. Roa, and J. P. Schiffer)

Very few data exist for highly forbidden positron decays. In the few cases studied, only limits are obtained, or the data are either suspect due to background processes, or of marginal quality as a result of the difficulty of detecting a very small positron signal in the environment of many photons and conversion electrons which accompany the attendant EC decays. These experimental issues may be addressed with the use of a high-sensitivity, low background positron spectrometer, such as APEX. Using the APEX spectrometer, we have investigated the 2nd-forbidden non-unique $\beta^{+}$decay of ${ }^{207} \mathrm{Bi}$, to the $5 / 2^{-}(569 \mathrm{keV})$ state in $207 \mathrm{~Pb}$, with an endpoint energy of $808 \mathrm{keV}$. A previous determination of the $\beta+/$ decays ratio $(R)$ for this nucleus, obtained from a measurement which searched only for the $511 \mathrm{keV}$ radiation from annihilating positrons, established a value of $R=1.2(2)$ $\times 10^{-4} .1$

\footnotetext{
*University of Illinois at Chicago, †University of Notre Dame. ${ }^{1}$ T. Rupnik, Phys. Rev. C6, 1433 (1972).
} 


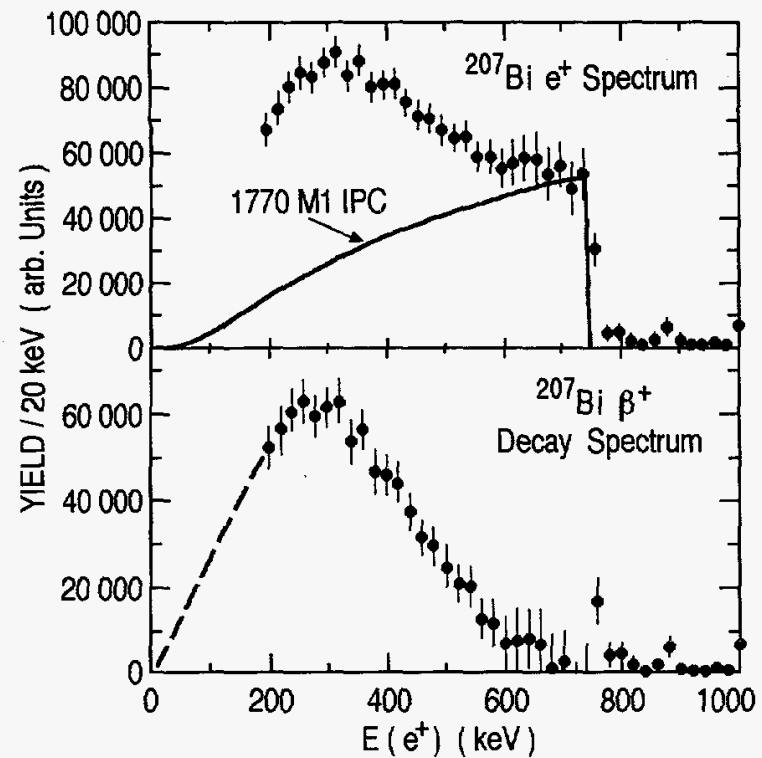

Fig. I-52(a) $e^{+}$energy spectrum from the decay of $207_{B i .}(b) e^{+}$spectrum with the contribution from $M 1$ internal conversion subtracted off.

Positrons from the decay of ${ }^{207} \mathrm{Bi}$ were detected using APEX. A positron energy spectrum from $207_{\mathrm{Bi}}$ appears in Fig. I-52(a). Here, the spectral shape has been corrected for the transport efficiency of APEX, as well as for effects such as backscattering and incomplete energy loss in the silicon wafers, and incomplete light collection in the $\mathrm{NaI}$ bars used to identify positrons by detection of their characteristic $511 \mathrm{keV}$ annihilation radiation. The only competing signal was from Internal Pair Conversion (IPC) of a
$1770 \mathrm{keV}$, M1 transition in ${ }^{207} \mathrm{~Pb}$. With a measurement of the yield of positron-electron pairs from this transition, this contribution to the positron spectrum can be subtracted. The solid curve in Fig. I52(a) represents the expected yield of positrons from IPC of the $1770 \mathrm{keV}$ transition. The subtracted $\beta^{+}$ spectrum appears in Fig. I-52(b). The cutoff at lower energies arises from the geometrical threshold of the transport efficiency, as well as from the electronic threshold in the data acquisition.

Using a linear extrapolation of the spectrum to zero energy, we obtain a total $\beta^{+}$decay yield of $1.25 \times 10^{6}$ counts. The total number of decays, as measured by the yield of $569 \mathrm{keV}$ gamma rays detected in a high-purity Ge crystal, was $5.36 \times 10^{10}$, resulting in a $\beta^{+/ \text {decays }}$ ratio of $2.3 \times 10^{-5}$. The uncertainties are still being evaluated, but are likely dominated by systematic errors no greater than $\pm 20 \%$. Although the current value for the $\beta^{+} / \mathrm{EC}$ ratio for this decay is approximately a factor of 5 smaller than the earlier value, it is still consistent with the interpretation as a 2 nd forbidden non-unique transition. In light of the difference between the results of the current measurement and those from previous findings, it may be interesting to reinvestigate other cases of 2 nd forbidden $\beta^{+}$emission. Finally, these results demonstrate the usefulness of APEX as a low background, high sensitivity positron spectrometer which could be used to study other very weak $\beta^{+}$ emitters. A manuscript describing these results is in preparation.

\section{d.6. Search for the $\beta^{+}$Decay of ${ }^{54} \mathrm{Mn}$ (A. H. Wuosmaa, S. M. Austin,* S. J. Freedman, $\dagger$} I. Ahmad, B. B. Back, R. R. Betts, C. J. Lister, S. Fischer, J. P. Greene, G. Hackman, V. Nanal, P. Reiter, and J. P. Schiffer)

The nucleus ${ }^{54} \mathrm{Mn}$ was identified as a potential "cosmological clock" for the transport of cosmic rays through the galaxy. Terrestrially, ${ }^{54} \mathrm{Mn}$ decays $100 \%$ by electron capture (EC) to the first excited state of ${ }^{54} \mathrm{Cr}$ at $835 \mathrm{keV}$. In cosmic rays, however, the nuclei have energies $>100 \mathrm{MeV} /$ nucleon, and are expected to have all their electrons stripped, consequently removing the EC decay branch, and significantly extending the lifetime. The two other possible decays, $\beta^{-}$and $\beta^{+}$ emission, to the ground states of ${ }^{54} \mathrm{Fe}$, and ${ }^{54} \mathrm{Cr}$, respectively, have never been observed in the laboratory. The branching strengths for these two decay modes are expected to be related by phase-space factors $\left(B\left(\beta^{-}\right) / B\left(\beta^{+}\right) \sim 500\right)$ with the (untested) assumption that nuclear matrix elements in the two decays are comparable. Of these two, only a measurement of the $\beta^{+}$decay seems feasible, due to the large backgrounds that are expected for pure $\beta^{-}$decays. Recent satellite measurements of the isotopic composition of cosmic rays ${ }^{1}$ observe ${ }^{54} \mathrm{Mn}$, and from the strength relative to the stable ${ }^{55} \mathrm{Mn}$, and the long lived ${ }^{53} \mathrm{Mn}$ ( $\mathrm{T} \sim 3.7 \times 10^{6}$ years) a $\beta^{-}$decay half life of 1-2 MY was deduced. This number is highly model dependent, however, and it is desirable to use a known decay half-life to test the cosmological transport models.

*Michigan State University, $†$ University of California and Lawrence Berkeley Laboratory

${ }^{1}$ M. A. DuVernois, Phys. Rev. C54, R2134 (1996), ${ }^{2}$ M. T. F. da Cruz et al., Phys. Rev. C48, 3110 (1993). 
The current upper limit on the branching strength of non-EC decay of ${ }^{54} \mathrm{Mn}$ is from a search for the $\beta^{+}$ decay to ${ }^{54} \mathrm{Cr}$ by da Cruz et al., ${ }^{2}$ which set an upper limit of $\sim 6 \times 10^{-9}$ for the $\beta^{+}$decay branching ratio. The $\beta^{-}$decay branch is then inferred. The data in ${ }^{2}$ were obtained from a measurement using two Ge detectors to detect the $511 \mathrm{keV}$ photons from positron annihilation, and were dead-time limited despite the rather modest 3$4 \mu \mathrm{Ci}$ source strength. We will carry out a search for this astrophysically significant $\beta^{+}$decay using the APEX spectrometer. APEX is ideally suited to this task due to its low sensitivity to background and excellent ability to cleanly identify real positrons. Another significant advantage is the ability to measure the energy spectrum of the emitted positrons. A study of background in APEX was undertaken, and hardware improvements are underway which should increase the efficiency for the positrons from the ${ }^{54} \mathrm{Mn}$ decay by a factor of 2 or more. Preliminary indications are that, depending on the strength of the source used, our measurement should be sensitive to a $\beta^{+}$decay branch near or below $1 \times 10^{-9}$, in the range suggested by the astrophysical measurements. Preparations for a full measurement are underway, and the experiment will be carried out in the coming year.

\section{d.7. Transitions in Cold Crystalline Plasmas to Infinite Matter Behavior (J. P. Schiffer)}

The shell structures predicted in our simulations some time ago were borne out by the experimental work in ion traps. The largest system simulated was for 20000 ions and in this isotropically confined system the lowest energy configuration showed 18 concentric shells. Recent experimental work at Boulder [J. Tan et al., Phys. Rev. Lett. 26, 4198 (1995)] found strong indications from Bragg scattering studies, that in the interior of larger clouds (> 50000) substantial body-centered cubic crystals develop. This bcc form of ordering is known to be the ground state of infinite Coulombic matter. Where and how the transition occurs from shells to bcc order is of interest. A simulation was carried out with 100000 ions--and a new algorithm, a 'global minimizer', was used to find a minimum energy configuration. The converged system indeed contains small islands with bcc cells--however, these are imbedded in the outer shell structure and apparently cannot form 'single crystals' of the size seen in the experiments of Tan et al. Figure I-53 shows the concentration of ions in bcc cells as a function of radius, as well as the orientation of these cells with respect to the shell planes. The limitation on the size of the clusters seems to be the curvature of the shells. The reason the interior does not show bcc order is not fully understood. Possibly the process of 'cooling' represented by the minimizing program is too fast and settles into a false minimum. In analogy to growing real crystals, preparation of large single crystals require a careful and gradual process: freezing water quickly produces ice cubes and not single ice crystals. A slower Molecular Dynamics program is now being tried for a 100000 ion system, but it will probably take some time before conclusions may be drawn. A new parallel workstation may speed up these calculations substantially.

Fig. I-53. Location of bcc cells in the simulation of a spherical cloud. (a) The location of islands and orientation of bcc cells from the simulation of a 100000 ion cloud in spherical confinement. (b) Orientation of a cell with respect to the plane of a 'shell'. (c) Percentage of ions in this simulation as a function of radius, that satisfies the bcc criterion for the 14 nearest neighbors.
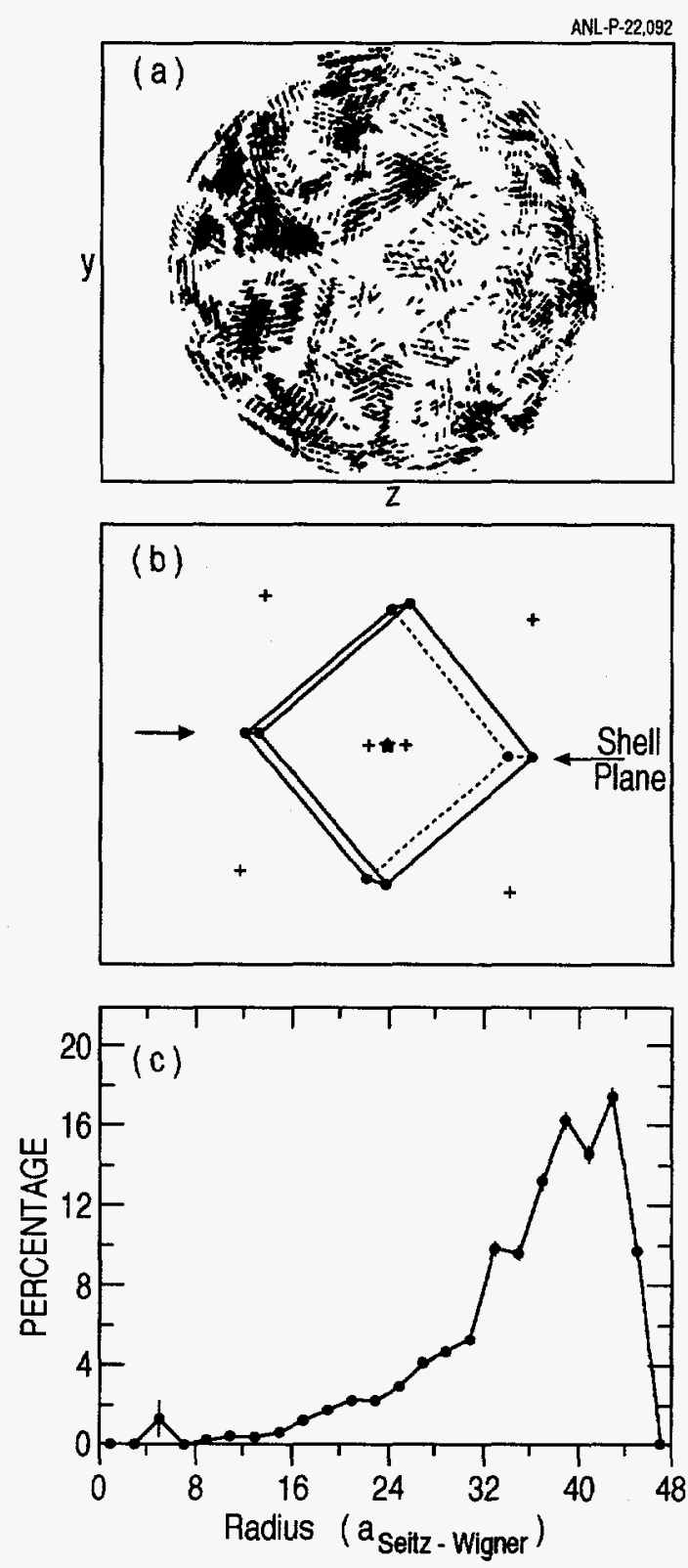


\section{d.8. Behavior of Bunched Beams in a Storage Ring and in Simulations (J. P. Schiffer, J. S. Hangst, * N. Madsen, * A. Labrador, * M. Drewsen,* and P. Shi*)}

The behavior of laser-cooled bunched beams in a storage ring is being investigated at the Århus storage ring ASTRID. A new capability to take photographs of the beam profile under laser cooling with a CCD camera, has been utilized. This technique provides, for the first time, a precise quantitative diagnostic tool of the transverse degrees of freedom of a cold beam. One of the issues is the coupling between the transverse 'temperature' of the beam and the longitudinal one -- the latter is known to be cooled very effectively with the laser-cooling technique. Another issue is the difference between possible 'coherent' vs 'incoherent' transverse motion.

Simulations have been carried out for such beams and Figure I-54 shows the time development of a macroscopic coherent transverse perturbation on a beam bunch that is cooled longitudinally and the way in which this coherent perturbation can induce random "heating".

*University of Århus, Denmark
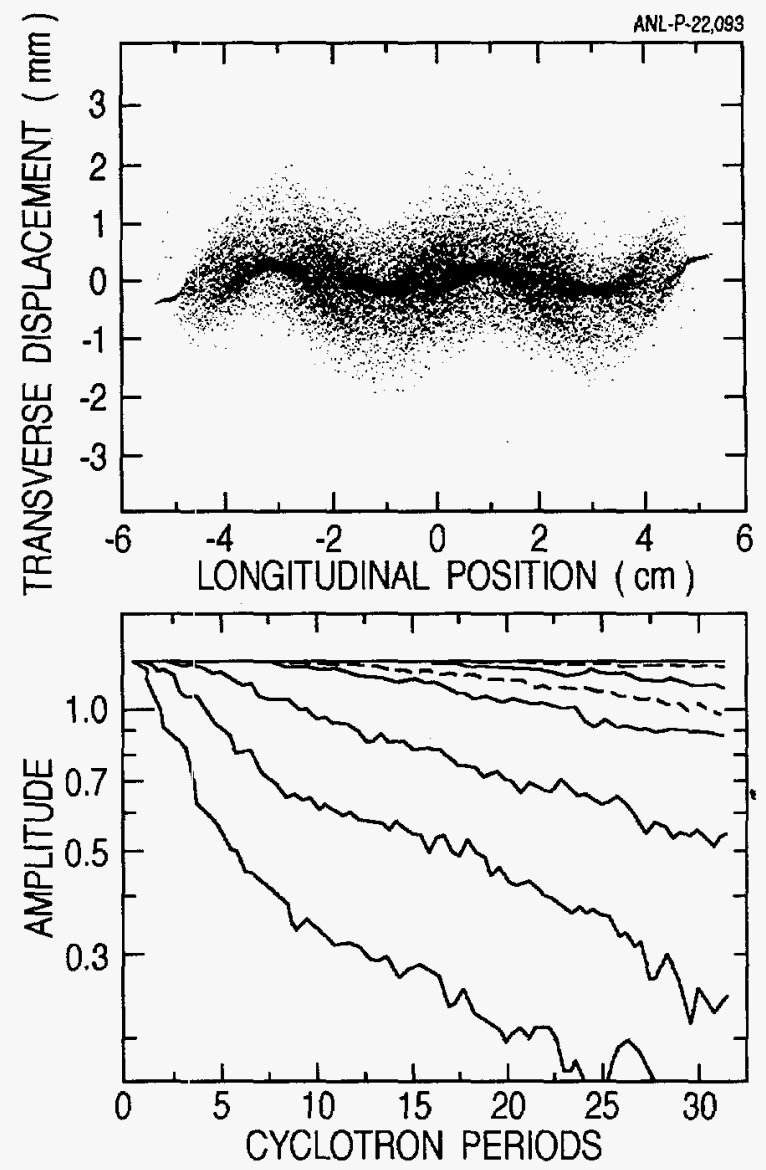

Fig. I-54. The upper part of the figure represents the positions of particles in a $\sim 10 \mathrm{~cm}$ long beam bunch, that was initially cold and then had a one-time sinusoidal transverse displacement imposed on it. The bunch is about 10 $\mathrm{cm}$ long and the mean separation between ions is 40 microns. After the perturbation the bunch was then continued to be "cooled" in the longitudinal direction once per turn, as the oscillations were followed for a 'time' that corresponds to about 30 orbits around the storage ring. The lower curve shows the way the oscillations damp as a function of the wavelength of the imposed perturbation. When the wavelength is $\geq 5 \mathrm{~cm}$ the damping appears very small and the transverse oscillations from the initial displacement continue with almost no longitudinal-transverse coupling. As the wavelength gets shorter, the oscillations become damped more and more quickly and cause the 'cold' beam to become transversely hot. 


\section{d.9. Measurement of the Half-Life of ${ }^{44} \mathrm{Ti}$ M. Paul, $\dagger$ and G. Castagnoli $\ddagger)$}

The half-life of ${ }^{44} \mathrm{Ti}$ is of significance to cosmologists because it allows them to convert the observed gammaray flux to mass of ${ }^{44} \mathrm{Ti}$ in supernovae. There are many measurements of the half-life of ${ }^{44} \mathrm{Ti}$ which range from 49 to 66 years. In order to obtain a more precise value of the half-life, we started a decay measurement about five years ago. We planned to measure the ${ }^{44} \mathrm{Ti}$ halflife relative to that of ${ }^{60} \mathrm{Co}$ which is very precisely known (5.2719(4) y). Samples of mixed ${ }^{60} \mathrm{Co}$ and ${ }^{44} \mathrm{Ti}$ and pure ${ }^{60}$ Co were prepared and the decay of these samples was followed at three laboratories-Argonne, Torino and Jerusalem. In each laboratory, the $\gamma$-ray spectra were periodically measured with a Ge

\section{(I. Ahmad, S. M. Fischer, W. Kutschera,*}

spectrometer. At Argonne, spectra of a pure ${ }^{44} \mathrm{Ti}$ sample were also concurrently measured. At Argonne, the decay has been followed for $4.7 \mathrm{y}$ and the data have been analyzed. We measured spectra of mixed source, pure ${ }^{60} \mathrm{Co}$, pure ${ }^{44} \mathrm{Ti}$ and background at source-todetector distances of $5.2 \mathrm{~cm}$ and $10.2 \mathrm{~cm}$. We determined the half-life from decay of the $1157 / 1173$ and $1157 / 1332$ ratios for the mixed source and pure sources. Thus far the analysis provided 8 numbers for the half-life. Our analysis indicates that the half-life of ${ }^{44} \mathrm{Ti}$ is between 55 and 58 years. The data at Jerusalem and Torino are still being analyzed. Once these results are available, data from all three laboratories will be combined to obtain the final result.

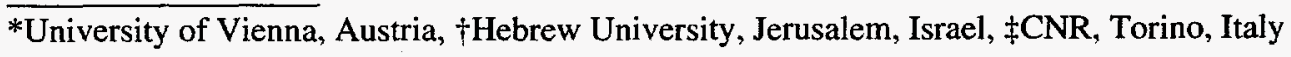




\section{E. EQUIPMENT DEVELOPMENT AT THE ATLAS FACILITY}

This section describes the various technical developments linked with the conduct of the experimental program associated with Heavy-Ion research within the Division.

During the last year, efforts towards experiments with beams of short-lived isotopes have increased considerably. As a result, the development of new production modes for the beams of interest and the construction of new, high-efficiency detectors has taken place.

The Division is actively preparing for the move of Gammasphere to ATLAS, which is scheduled to start in mid-September 1997. A detailed account of the various activities is given in this section. Other activities indirectly connected to the arrival of Gammasphere at ATLAS are also taking place such as the development of new focal plane detectors at the FMA, or the development of an array of neutron detectors.

The construction of the LEPPEX array is continuing. This detector consists of an array of gas counters mounted in a barrel assembly around the target, surrounded by $\mathrm{BaF}_{2}$ detectors. The first array of $16 \mathrm{BaF}_{2}$ detectors has been assembled. The same group also developed a beam vertexing detector based on scintillating fibers for the E917 experiment at the AGS.

A description of new developments in the areas of computing and target making is also given.

e.1. Production of a 56Ni Beam at ATLAS (K. E. Rehm, M. Paul,* I. Ahmad, J. Greene, J. Nolen, R. C. Pardo, F. Brumwell, $\uparrow$ and G. McMichael $\dagger$ )

For nuclear reaction studies in the vicinity of the shell closure $\mathrm{N}=\mathrm{Z}=28$, we have developed a radioactive $56 \mathrm{Ni}$ beam at ATLAS using the two-accelerator technique that was successfully applied earlier for the production of a ${ }^{18} \mathrm{~F}$ beam. There are three light-ion induced reactions that can be used for the production of the radioactive ${ }^{56} \mathrm{Ni}$ material: ${ }^{58} \mathrm{Ni}(\mathrm{p}, \mathrm{p} 2 \mathrm{n})^{56} \mathrm{Ni}$, ${ }^{59} \mathrm{Co}(\mathrm{p}, 4 \mathrm{n}){ }^{56} \mathrm{Ni}$ and ${ }^{54} \mathrm{Fe}(\alpha, 2 \mathrm{n}){ }^{56} \mathrm{Ni}$. From a comparison of the cross sections and the cost for enriched target material, the ${ }^{58} \mathrm{Ni}+$ p system represents the best combination. Since the maximum cross sections of the ${ }^{58} \mathrm{Ni}(\mathrm{p}, \mathrm{p} 2 \mathrm{n})$ reaction is in the energy range between $30-50 \mathrm{MeV}$, we irradiated a $3 \mathrm{~mm}$ thick ${ }^{58} \mathrm{Ni}$ sample with a $50 \mathrm{MeV}$ proton beam from the injector of the Intense Pulsed Neutron Source (IPNS) at ANL. The ${ }^{58} \mathrm{Ni}$ pellet is mounted in a copper insert for the negative SNICS ion source. This approach minimizes the time that the irradiated sample, which is highly radioactive (mainly short-lived ${ }^{57} \mathrm{Ni}$ and ${ }^{55} \mathrm{Co}$ activities), has to be handled at close proximity. The negative ion source also strongly suppresses contributions from the stable isobar ${ }^{56} \mathrm{Fe}$, since $\mathrm{Fe}$ forms mainly negative Fe-hydrides. Contrary to the ${ }^{18} \mathrm{~F}$ case, where the active material is located on the surface, the ${ }^{56} \mathrm{Ni}$ activity is distributed evenly throughout the material. This allows to operate the ion source under running conditions which are well known from experiments with stable materials. In several 24 hour irradiations at the IPNS the ratio of $58 \mathrm{Ni} / \mathrm{Ni}$ in the sample was determined to $\sim 0.5 \times 10^{-6}$. Some of the samples also included small amounts of ${ }^{28} \mathrm{Si}$ which served as a guide beam during the acceleration in ATLAS. With a ${ }^{58} \mathrm{Ni}^{-}$beam intensity (measured in a Faraday cup in front of the tandem accelerator) of $5 \mu \mathrm{A}$, a $56 \mathrm{Ni}^{-}$intensity of $1.5 \times 10^{7} / \mathrm{sec}$ has been obtained. Since ${ }^{56} \mathrm{Co}$ is produced during the irradiation with typically ten times higher yields, the ${ }^{56} \mathrm{Co}$ intensity from the ion source is of the order of $\sim 10^{856} \mathrm{Co} / \mathrm{sec}$.

*Hebrew University, Jerusalem, Israel, †IPNS, ANL 


\title{
e.2. Production Rate and Transport Efficiency Calculations for Secondary Beams
}

\author{
(B. Harss, C. L. Jiang, R. C. Pardo, and K. E. Rehm)
}

For the development of exotic secondary beams produced in reactions of high intensity primary beams with light (hydrogen or $\mathrm{He}$ ) targets, a computer program has been developed that simulates the production process and the transport efficiency. The program determines the production rate and the final energy distribution by following the energy and angular distribution of the primary and secondary beam through the entrance and exit windows and the gas-volume of the target cell.

The assumptions in the code are:

- Small angle straggling and energy loss are given by the formulas and tables as used in the code ENELOSS. ${ }^{1}$

- A $\delta$-function in energy transforms under energy loss and straggling to a Gaussian distribution centered around the calculated mean energy loss. The same holds for the angular distributions. The widths of individual components are added in quadrature.

The input is summarized in a reaction definition file (RDF) that defines the reaction and the total cross section in the energy range of interest. Masses and Qvalues are taken from an internal data base, and chemical elements are defined by their symbols. The most important outputs are the total production rate in the thick target, the rate produced within a given angle and energy acceptance, and the energy spectrum of the secondary ions.

The code was tested with the $\mathrm{p}\left({ }^{17} \mathrm{O},{ }^{17} \mathrm{~F}\right)$ n reaction which, at center of mass energies of $\sim 4 \mathrm{MeV}$, has an isotropic angular distribution. The program predicted the production rate and transport efficiency within a factor of 2. It also allowed to predict the effect of a change in the beam line geometry correctly. Replacing a limiting aperture of 1 " by a 2 " collimator improved the transmission by a factor of two, not a factor of four as first expected.

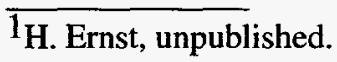

\section{e.3. Development of a Gas Target for In-Flight Production of Exotic Beams (B. Harss, K. E. Rehm, J. P. Greene, C. L. Jiang, and R. C. Pardo)}

An efficient and low-cost method to produce a variety of radioactive beams is to use a stable beam and transform some of its nuclei in a primary target into short-lived radioisotopes. As a first test case, a ${ }^{17} \mathrm{~F}$ $\left(T_{1 / 2}=64 \mathrm{sec}\right)$ beam was produced via the $\mathrm{p}\left({ }^{17} \mathrm{O}\right.$, ${ }^{17}$ F)n reaction using a stable ${ }^{17} \mathrm{O}$ beam. A bending magnet located after the primary production target separated the ${ }^{17} \mathrm{~F}$ ions from the primary beam and steered them onto a secondary target.

The usage of a gas target as a primary production target has several advantages over a solid state target (e.g. a $\mathrm{CH}_{2}$ foil):

- High resistance against destruction by the high power beam

- ${ }^{3} \mathrm{He}$ and ${ }^{4} \mathrm{He}$ targets can be used with thicknesses up to a few $\mathrm{mg} / \mathrm{cm}^{2}$.

A target cell has been developed which consists of two separated chambers, the actual gas cell and a surrounding cavity for a cooling liquid (Fig. I-55).
To confine the gas at pressures up to 500 torr in the target cell, vacuum tight windows that can sustain high beam currents are needed. For high-quality secondary beams, their thickness should be as small as possible, and to reduce the angular straggling low- $Z$ window materials are preferable to high- $Z$ materials. A series of tests was performed to determine the optimum window material. Pressure tests with several different aluminum foils showed, that the intrinsic strength of this material is not sufficient for the requirements. Tests with titanium windows showed very promising results since vacuum tight foils could be manufactured as thin as 1 $\mathrm{mg} / \mathrm{cm}^{2}$ by rolling thicker foils. However, tests with ${ }^{14} \mathrm{~N}$ beams showed that $\mathrm{Ti}$ reacts under beam condition with the hydrogen gas enclosed in the cell to produce $\mathrm{TiH}$ molecules. Windows made from HAVAR, a NiCo-alloy, showed much better chemical characteristics. However, we have not yet succeeded in producing pinhole free foils below $1.9 \mathrm{mg} / \mathrm{cm}^{2}$. Mounting these foils on the gas cell by clamping them with an O-ring caused additional stress which made them burst at lower pressures. Therefore, the foils are glued on stainless 


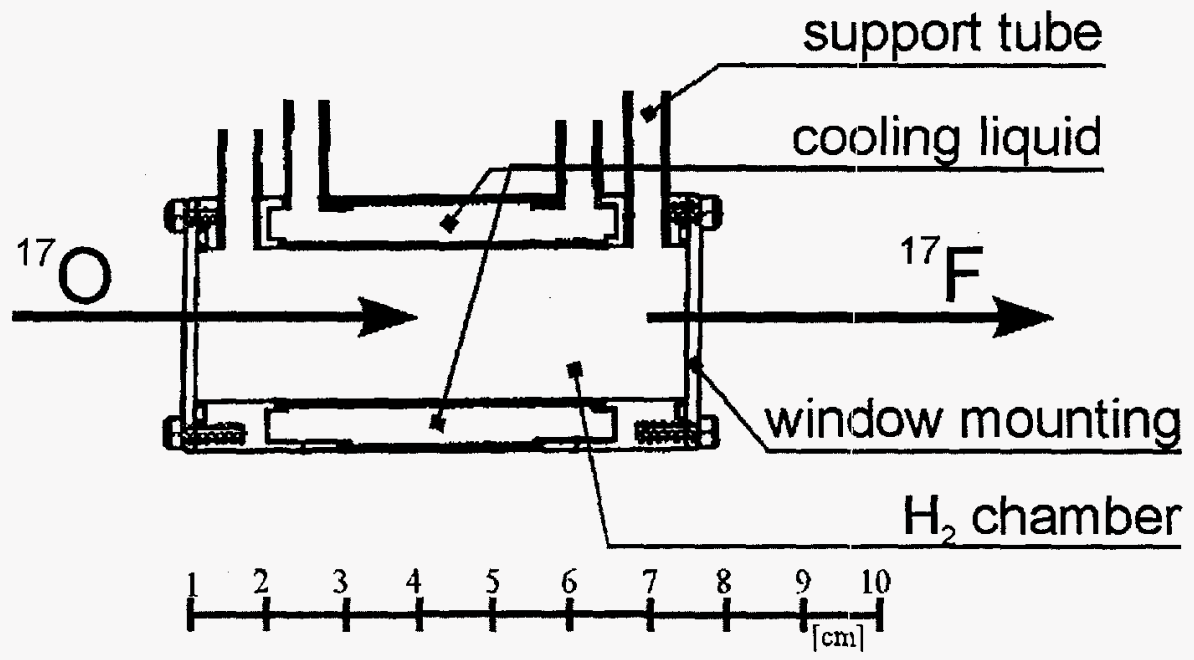

Fig. I-55. A simplified cross section of the gas target.

steel frames which are then mounted on the gas cell using indium gaskets in order to allow cooling of the cell to low temperatures.

The gas cell was used in a series of experiments for measurements of the ${ }^{17} \mathrm{~F}(\mathrm{p}, \alpha){ }^{14} \mathrm{O}$ reaction.
Bombarding the gas cell with primary beams of 200 300 pnA beam, currents up to $5 \times 10^{517} \mathrm{~F} / \mathrm{sec}$ on the secondary target have been measured. In six runs of 2 3 days each, two failures of the gas cell windows occurred.

\section{e.4. Development of Beam Diagnostic Devices for Low Intensity Beams (B. Harss, P. Wilt, B. B. Back, J. A. Nolen, R. C. Pardo, and K. E. Rehm)}

The standard beam diagnostic equipment of the ATLAS accelerator system is insensitive to beams below 1 epA or approximately $10^{7}$ particles per second. A growing number of experiments involving exotic beams with typical intensities in the order of $10^{5}$ particles per second require the development of a new set of beam tuning and monitoring devices. The challenge is to replace the existing Faraday cups and beamline scanners, which are charge sensitive, by new instruments that actually count single particles.

The substitution for a Faraday cup is a particle detector which is connected to a ratemeter. Since silicon detectors are too delicate and too slow for rates above $\sim 10^{5}$ particles per second, a plastic scintillator attached to a small photomultiplier tube (Hamamatsu R1924-01) was chosen. In a recent run with $17 \mathrm{~F}$ beams, this detector was exposed to rates of $4 \times 10^{5}$ particles per second. The signals observed in this first experiment indicates that this type of detector can handle considerably higher beams, up to a few times $10^{6}$ particles per second.

Based on the development of scintillating fiber ribbons described elsewhere in this report, a more elaborated device has been designed to substitute to the existing beamline scanners. It consists of a position sensitive photomultiplier tube connected to 64 scintillating fibers. The latter are mounted as a rectangular grid with $1 \mathrm{~mm}$ spacing on a ladder that can move in and out of the beam, covering an area of 3.2 by $3.2 \mathrm{~cm}$. To guarantee compatibility with existing and future systems at ATLAS, the readout electronics produces two kinds of output. The first are two analog waveforms that resemble the hit patterns on the $X$ and $Y$ fibers. An additional DC-Voltage is provided that indicates the total hit rate. The X-hit and $\mathrm{Y}$-hit pattern can also be read out independently via CAMAC commands. First in-beam tests of the new device are planned for the summer 1997. 


\section{e.5. A New Large Area Focal Plane Detector for the ATLAS Split Pole Spectrograph}

(M. Paul, * B. Harss, D. Henderson, C. L. Jiang, and K. E. Rehm)

A new ionization chamber for the ATLAS Split Pole Spectrograph was build at the Hebrew University of Jerusalem and tested in several runs at Argonne. The Gas Filled Magnet Ionization Chamber (GFMIC) is designed for a wide acceptance in Y-direction and superb particle identification (Fig. I-56). These features are especially important for experiments with secondary beams or Accelerator Mass Spectroscopy experiments with the gas filled spectrograph where small event rates and large beamspots on the focal plane require large efficiency detectors.

In combination with the existing Parallel Grid Avalanche Counter (PGAC), GFMIC provides data on angle and position in both horizontal and vertical direction to correct the signals for the different flight paths. The information on angle and position in Ydirection is derived from the time difference between the start signal from the PGAC and the signal on the GFMIC anode. The latter depends on the drift time of the charge cloud in the detector gas towards the Frisch grid, which is long compared to the response time of the PGAC.

Many features of the detector depend on the special design of the anode system. It consists of seven segments which can be read out independently and, therefore, provide seven independent dE signals. From the ratio of the signals

$$
P_{i}=\frac{d E i_{\text {left }}-d i_{\text {right }}}{\left(\mathrm{dEi}_{\text {left }}+\mathrm{dEi}_{\text {right }}\right)}, \quad \mathrm{i}=1,2,3,
$$

one can deduce three position signals $P_{i}$ which, relative to the $\mathrm{X}$-signal measured in the PGAC chamber, can determine the angle of incidence of the particle. The sums $\mathrm{DEi}_{\text {right }}+\mathrm{DEi}$ left generate three independent $\triangle \mathrm{E}$ signals for $\mathrm{Z}$-identification. The detector was tested with ${ }^{16} \mathrm{O}$ and ${ }^{40} \mathrm{Ar}$ beams and has been used in the experiments with radioactive $17 \mathrm{~F}$ ions. In the case of a $200 \mathrm{MeV}{ }^{40} \mathrm{Ar}$ and $\Delta \mathrm{E}$-resolution of $\lesssim 2 \%$ and $\lesssim 4 \%$, respectively was observed.

*Hebrew University, Jerusalem, Israel

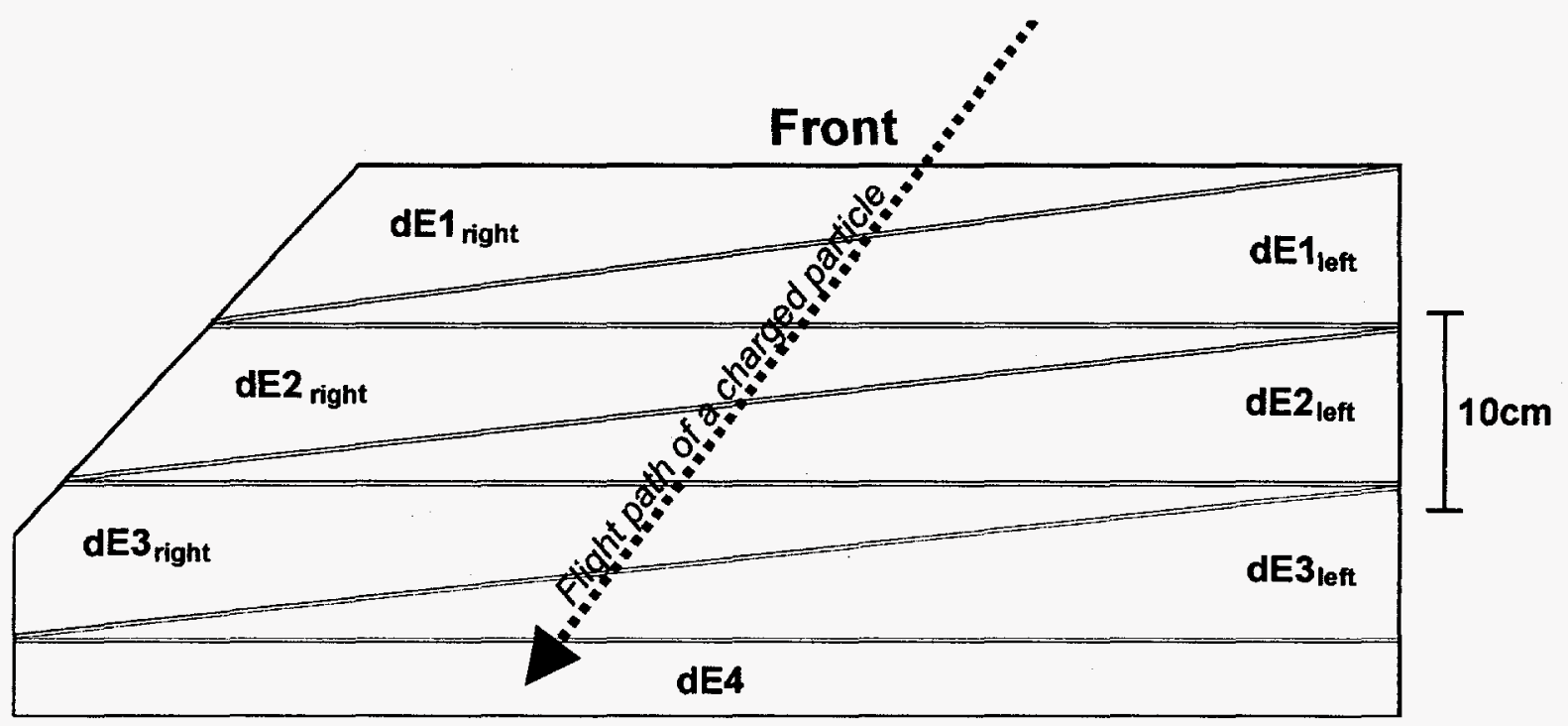

Fig. I-56. Top view on the anode of GFMIC and a particle passing over it. 


\section{e.6. Construction of a Large Area Si-Detector Array for Measuring (d,p) Reactions in Inverse Kinematics (K. E. Rehm, F. Borasi, J. Falout, C. L. Jiang, P. Reiter, D. Seweryniak, A. Wuosmaa, and R. E. Segel*)}

In order to compensate for the low beam intensities available with the present radioactive ion beams, large acceptance detector systems are needed. For a planned experiment studying single-particle transfer reactions with a ${ }^{56} \mathrm{Ni}$ beam, e.g. d $\left.{ }^{56} \mathrm{Ni}, \mathrm{p}\right)^{57} \mathrm{Ni}$, we have built a large area Si-detector array. The barrel-shaped system consists of an annular double-sided Si detector with an active area of $53 \mathrm{~cm}^{2}$ which is surrounded by six, 25 $\mathrm{cm}^{2}$, Si-strip detectors (see Fig. I-57). When mounted at backward angles, the array has a solid angle of almost $5 \mathrm{sr}$ and covers the angular range from $110^{\circ}$ $160^{\circ}$. From a readout of individual Si-strips the angle of the outgoing protons can be determined with an accuracy of $2-3^{\circ}$.

The Si-detector array can be cooled and provides a total of $2 \times 182$ channels of energy and time signals. The array was tested using the $\mathrm{d}\left({ }^{28} \mathrm{Si}, \mathrm{p}\right){ }^{29} \mathrm{Si}$ reaction and first experiments with a radioactive $56_{\mathrm{Ni}}$ beam have begun.

*Northwestern University

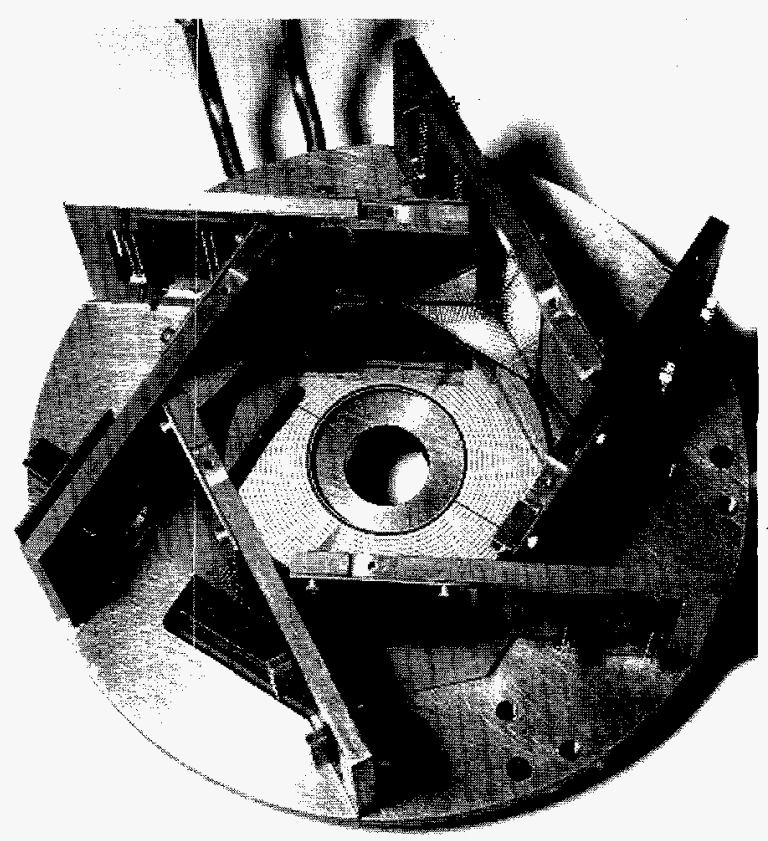

Fig. 1-57. Photograph of the assembled Si-detector array.

e.7. The Move of Gammasphere from LBNL to ANL (C. J. Lister, M. P. Carpenter, C. N. Davids, R. V. F. Janssens, T. L. Khoo, D. Nisius, P. Reiter, D. Seweryniak, T. Lauritsen (ANL) I. Y. Lee, A. O. Macchiavelli, F. S. Stephens (LBNL))

The operation of Gammasphere at ATLAS allows several unique categories of experiments to be pursued, including the study of nuclei far from stability using triggering from the fragment mass analyzer (FMA), the study of nuclear isomeric states using the subnanosecond beam timing from ATLAS, and the study of inelastic scattering with very heavy beams. To exploit these opportunities, the decision to move Gammasphere from LBNL to ANL was taken in January 1996.

During the last year there has been a continuous dialog between scientist at LBNL and ANL on how to conduct this move, its cost, and the time that the move would take. This dialog has resulted in a plan which is accepted to be as safe as can be arranged, reasonably cost-effective, and has a down-time which is as short as can be arranged within the safety and cost constraints. The plan has many elements, which are briefly discussed in the following paragraphs.

a. LBNL Site Preparation and Detector Packing
The planning and coordinating of the packing and shipping of Gammasphere is going to be organized by the LBNL Gammasphere support group, with assistance from the LBNL Engineering Division. The chief engineer who designed and installed Gammasphere, J. Comins, will oversee the project, and will have fulltime assistance from a "Shipping Supervisor". The details of the plan are being developed, but the time between removing all the detectors (see section $\mathrm{C}$ below) and the shipping of the Gammasphere frame, the LN2 supply and the data acquisition system should be less than three weeks.

\section{b. ANL Site Preparation}

There are two main elements in the ANL site preparation; the FMA beamline modification, and the installation of utilities.

In the first category, the elements of the $\mathrm{C} 2$ beamline in target area IV need replacing to make space for Gammasphere, the FMA needs some minor mechanical modifications, and a platform to raise Gammasphere to 
the ANL beamline height needs to be constructed. The first phase of this work started on 19th Feb. '97.

In the second category, a LN2 supply from the main 20,000 gallon tank needs installation, a data shack needs building and air conditioning fitting, and finally $22 \mathrm{KW}$ of clean-grounded, emergency-backup power needs installing. This work will be performed between May and September ' 97.

These operations are planned to minimize the disruption to the science program at ATLAS. To accomplish this, the jobs are being phased, with approximately three weeks of Gammasphere related work interspersed with six week periods of science operation of the FMA beamline.

\section{c. Annealing Stations and Planning of Detector Annealing}

After about two years of continuous operation, nearly all the 110 detectors in Gammasphere will need some rennovative attention. The most straightforward operation is a simple warm-up and pump, but even this requires connecting the detectors to a dedicated annealing station for a low-risk procedure. Most of the detectors will require annealing to remove the neutron damage which will take up to five days per detector. Because of the need to remove the detectors from Gammasphere rapidly, many annealing stations are needed. After much debate, the cost-effective but safe compromise was agreed to lie in building ten, oil-free, high-pump speed, single detector stations. These stations will greatly expedite this, and all future moves. All the components for the stations have been procured and have been delivered to ANL. The first prototype is being assembled. This prototype will be shipped to LBNL in April for field testing, and the manufacturing of stations will proceed in June and July.

A very detailed plan for removing detectors from Gammasphere after the official shutdown on Sept. 15th is being prepared. It will be a three shift operation, with LBNL scientific personnel running the dayshifts, and ANL scientific staff keeping the unloading and annealing cycle running during the evenings and nights. At present the plan indicates the Gammasphere frame will be unloaded of all it's HpGe detectors and BGO shields in less than three weeks.

\section{e.8. Status of the Argonne-Notre Dame BGO Gamma-Ray Facility at ATLAS}

(D. T. Nisius, H. Amro, R. V. F. Janssens, M. P. Carpenter, J. W. Falout, S. M. Fischer, J. M. Joswick, T. L. Khoo, G. S. Hackman, T. Lauritsen, J. Rohrer, and P. R. Wilt)

The gamma-ray facility at ATLAS consists of (a) a $4 \pi$ gamma-sum/multiplicity spectrometer with 50 BGO hexagonal elements (inner array) and (b) 12 Comptonsuppressed germanium detectors (CSG) external to the inner array. The facility returned to operation early in 1996, just after the departure of the AYEball array. It was used in standalone mode as well as in conjunction with the FMA.

- Because of neutron damage, annealing was performed on eight $\mathrm{Ge}$ detectors. One of these was annealed twice. The performance of the detectors was recovered in all cases.

- Maintenance and repairs had to be performed on several electronics modules and, in particular, on some of the CAMAC units. None of these problems affected an experiment for more than a couple of hours.

- Preventive maintenance was performed on the $\mathrm{LN}_{2}$ filling system (inspection of all filling lines and check of the various functions of the control modules).

- New discriminator modules to handle time signals from the inner BGO array detectors have been installed.

- For a specific experiment, a dedicated chamber for the measurements of $g$ factors was installed.

- The beam line to the facility was equipped with a system of fast acting valves required because of the use of actinide targets.

\section{e.9. Development of Fast, Position-Sensitive Micro-Channel Plate Detectors for the FMA (P. Reiter, C. J. Lister, and B. J. Varley*)}

The development of fast, position-sensitive MicroChannel Plate (MCP) detectors is motivated by the Fragment Mass Analyzer experiments combining

*University of Manchester
Gammasphere (GS) and the(FMA) especially on heavy nuclei, as well as by the necessity to measure the position, time-of-flight and energy loss of the individual heavy ions in radioactive beams. Due to the size of GS, the FMA must be positioned $90 \mathrm{~cm}$ downstream from 
the target, reducing its solid angle. With an alternative combination of settings of the fields, most of this loss can be restored, but only if angle dependent aberrations can be measured and corrected in software. The aberration can be measured by two independent position determinations of the selected evaporation residues at the focal plane.

For both applications, the MCP detectors should provide excellent experimental results, given by the combination of the following features: (1) a position resolution of MCP detectors with two-dimensional readout of $\leq 1 \mathrm{~mm}$, (2) a timing resolution of $\leq 200 \mathrm{ps,}$ (3) a high count rate capability in the $\mathrm{MHz}$-region, (4) a very low effective mass, i.e. $\approx 10 \mu \mathrm{g} / \mathrm{cm}^{2}$ of Carbon for interacting ions, makes it possible to combine at least three detectors, a decisive advantage with respect to other devices like parallel plate - or parallel grid detectors, especially for ions with high $\mathrm{Z}$.
The position of the ion is measured by imaging the secondary electrons emitted by the residue passing through a very thin foil. Backward-going electrons are accelerated through a grid and deflected by an electrostatic mirror perpendicular to the beam axis onto the microchanmel plate, which is placed outside of the beam trajectory. Electrons are amplified by two microchannel plates and are then transferred to a resistive surface anode. The microchannel plates and the resistive surface both cover an area of $75 \times 93 \mathrm{~mm}$. The position of the electron signal is determined by the charge ratios of four signals, available at the four electrical connections of the circular arc terminated resistive surface anode. In order to obtain a time reference signal, a pulse is extracted from the output side of the second microchannel plate.

The individual parts of the three MCP detectors are presently assembled and first test measurements with fission fragments are in progress.

\section{e.10. Coulomb Excitation of Radioactive Beams Prepared by the FMA (C. J. Lister, J. Schwartz, S. M. Fischer, D. Henderson)}

Coulomb Excitation of nuclei has long been established as a powerful and precise tool for investigating nuclear structure, particularly for collective modes. Until recently, the technique has been only used in experiments on stable nuclei. However, the application of the method for studying nuclei far from stability is only limited by the technical difficulties of producing an accelerated beam of the nuclei of interest. This is very important, as it has been suggested that the neardripline nuclei, particularly neutron rich nuclei, may have surprising collective properties caused by low binding energies and shallow potentials, which could be investigated by this method.

In the future, Coulomb Excitation of radioactive beams from dedicated radioactive beam facilities should be once again a common and standard technique. However, in the meantime, the technical aspects can be investigated, and some physically important measurements attempted, using radioactive beams prepared following fusion reactions, and separated with a mass spectrometer like the ANL Fragment Mass Analyzer (FMA). The beams produced by this method are rather low in intensity $\left(10^{5}\right.$ p.p.s) and energy $(1.5$ $\mathrm{MeV} / \mathrm{u}$ ) but may be useful for research. In principle, with the new ECR source, an order of magnitude intensity increase may be feasible.
We have been working on several technical developments which should allow some high quality experiments to be performed in 1997. They include:

1) Production Experiments.

The inverse experiments ${ }^{24} \mathrm{Mg}\left({ }^{58} \mathrm{Ni}, 3 \mathrm{p}\right){ }^{79} \mathrm{Rb}$ and ${ }^{24} \mathrm{Mg}\left({ }^{58} \mathrm{Ni}, 4 \mathrm{p}\right){ }^{78} \mathrm{Kr}$ have been studied at the focal plane of the FMA in order to quantify production rates and backgrounds. It appears $10^{5}$ pps, $A=79$ beams can be achieved at the focal plane and about twice the amount of $\mathrm{A}=78$, in a parallel beam of diameter about $1 \mathrm{~cm}$. The beams are isobaric, for example, with $70 \%$ ${ }^{79} \mathrm{Rb}$ and $30 \%{ }^{79} \mathrm{Sr}$.

2) Scattering Experiments.

A four quadrant, position sensitive parallel gridded avalanche counter (PGAC) had been built for detecting scattered radioactive ions, after re-excitation on a secondary target which will be mounted about $1 \mathrm{~m}$ beyond the focal plane. The four sectors all have orthogonal $\mathrm{X}-\mathrm{Y}$ wire planes to measure the angle of scattered particles to a precision of less than $2 \mathrm{~mm}$. Angle calibration procedures with sources and scattered particles have been completed. An experiment with a stable Krypton beam on a Nickel target allowed full kinematic testing and Doppler reconstruction of gamma rays detected in coincidence with heavy-ions. 
3) Channel Plate Developments. The use of the normal FMA focal plane detector, (an X-Y PGAC) and a downstream $X-Y$ position sensitive channel-plate imaging detector, has many possibilities for beam characterization. Firstly, beam tracking can be performed to adjust the FMA to produce the smallest possible target beamspot. Secondly, event-by-event ray-tracing should allow the angle and position of each beam particle to be established, thus making event reconstruction more straightforward. Thirdly, the differential time-of-flight before and after the gas detector allows the energy loss to be characterized event-by-event, permitting isotopic separation.
4) Operation of a Positive-Sensitive HpGe Detector. To reconstruct gamma-ray events most precisely, both the gamma-ray emission angles and the heavy-ion angles are needed. We are planning to use a pixelated planar detector (on loan from the Naval Research Laboratory) to provide this unique combination and evaluate its potential for future experiments of this type.

Most of these assets are in place and a full experiment is scheduled for April '97, when Coulomb Excitation of radioactive Krypton will be attempted.

\section{e.11. Studies of Proton Dripline Nuclei from $A=50$ to 100 Using Neutron Detectors, Microball and Gammasphere (C. J. Lister J. Schwartz (ANL), D. P. Balamuth, P. Hausladen (Penn) S. J Freeman, M. Leddy, J. L. Durell, B. J Varley (Manchester))}

Nuclei near the proton dripline can be produced by the fusion of stable beams and targets up to the ${ }^{100} \mathrm{Sn}$ region. The nuclei with Isospin, $T=0$, are particularly interesting due to the neutron-proton symmetry in their wavefunctions. Many issues about the structure of these nuclides have still to be understood in the $\mathrm{A}=50$ to 100 region, including the relative importance of $T=$ $0,1 \mathrm{n}$-p pairing, the purity of the isospin wavefunction, the long-range $n-p$ correlations which induce simultaneous alignments and sudden shape changes, and the relative positions of spherical and deformed configurations.

Many of these issues have not been addressed in the past, as the nuclei of particular interest can only be produced with small cross sections (10's microbarns) and are embedded in the copious production of less exotic nuclei, which may be $10^{4}$ times more prolifically produced. Thus, only with the development of highly channel-selective experiments, and reliable intense heavy-ion beams have these studies become feasible. These nuclear structure issues can be studied using "standard" gamma-ray spectroscopic techniques, provided sufficient channel selectivity and efficiency can be achieved. Indeed, the efficiency, granularity, and symmetry of the Gammasphere detector leads to the collection of data with all of the interesting correlations "built-in".

Two techniques for channel selection following heavyion fusion have evolved. One method is to measure the multiplicities of neutrons, protons and alpha particles and then deduce the mass and charge of the final nucleus through conservation of particle number and knowledge of the compound nucleus. Alternatively, one can use a spectrometer to directly measure the mass of the residue, then use its stopping characteristics, or decay, to infer the atomic number. Ultimately, the most sensitive experiments involve BOTH of these methods. We intend to pursue these ultra-sensitive studies in 1998 when Gammasphere is in operation at ATLAS. In the meantime, the techniques involved in using neutron detectors in Gammasphere required development and many interesting projects using light particle detection alone have been pursued.

To determine the feasibility of light-charged particle gating of Gammasphere, a Penn-ANL-Manchester U.Washington U. collaboration was formed. Neutron detection is of pivotal importance, as all neutronemitting channels produce interesting nuclei, and neutron emission only occurs in less than $10 \%$ of the fusions. An array of 15 liquid scintillator detectors was developed for Gammasphere and installed in the downstream detector positions for two blocks of beamtime, in March 1996 and in January 1997. The typical 1-neutron efficiency was $7.5 \%$. Time-of-flight, and both analog and digital pulse shape discrimination was used to separate neutrons from gammas. Excellent separation was achieved, with better than $5 \times 10^{-4}$ suppression of gamma rays in the final neutron gate. The March experiment was the first in which external detectors were used to over-ride the normal Gammasphere trigger in selecting which events were interesting and needed readout. Protons and alphas were measured in the Washington University "microball" detector, operated in coincidence with the neutron detectors and gammasphere.

Several physics projects were pursued. The single particle states in spherical nuclei near $56_{\mathrm{Ni}}$ and ${ }^{100} \mathrm{Sn}$ were studied as was the evolution of deformation in ${ }^{72} \mathrm{Kr}$ and ${ }^{76} \mathrm{Sr}$. The question of shapes and bandterminations in nuclei on the $\mathrm{Xe} / \mathrm{Ba}$ region were examined. These data are undergoing analysis and are expected to lead to several publications. 
e.12. Temperature Dependence of the Scintillation Properties of $\mathrm{BaF}_{2}$ (V. Nanal, B. B. Back, and D. J. Hofman)

The scintillation characteristics of $\mathrm{BaF}_{2}$ excited by gamma-ray irradiation has been studied as a function of temperature in the range -50 to $+27^{\circ} \mathrm{C}$ using the Bollinger-Thomas method ${ }^{1}$. We found evidence for three scintillation decay components with different temperature dependencies. A fast scintillation component with a decay time of $\sim 800 \mathrm{ps}$ is independent of temperature, a slow component increases from 1 micro-sec at $+27^{\circ} \mathrm{C}$ to 5 micro-sec at $-50^{\circ} \mathrm{C}$ and a third slow component with a decay time of $\sim 0.4$ micro-sec becomes clearly visible at low temperatures. The scintillation light intensity of the fast component stays constant over the entire temperature range studied whereas that of the slow component increases by a factor of two when cooling from room temperature to $-25^{\circ} \mathrm{C} .^{2}$ The increased light output results in an improved energy resolution for low energy gamma-rays. A paper describing these results has been accepted for publication in Nucl. Instrum. Methods. An example of the spectral evolution with temperature is given in Fig. I-58.

1 L. M. Bollinger and G. E. Thomas, Nucl. Instrum. Methods 32, 1044 (1961), 2P. Schotanus, C. W. E. Van Eijk, R. W. Hollander, and J. Pijpelink, Nucl. Instrum. Methods A238, 564 (1985).

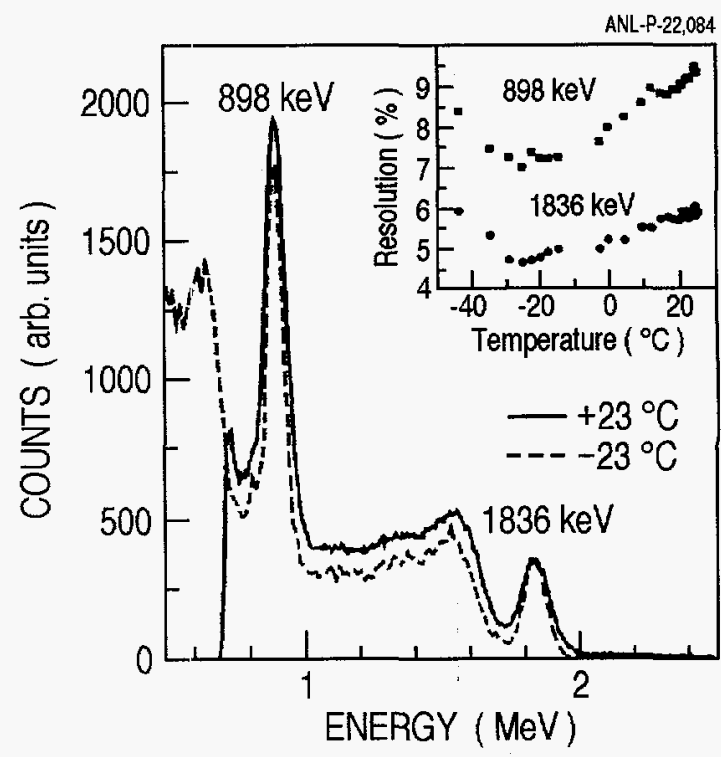

Fig. I-58. Energy spectra of ${ }^{88} Y$ measured with $\mathrm{BaF}_{2}$ at room temperature and $-23^{\circ} \mathrm{C}$ showing the improvement in resolution at lower temperature. The inset shows that the resolution initially improves with decreasing temperature but gets worse for temperatures lower than $-30^{\circ} \mathrm{C}$.

\section{e.13. Rotating Target Wheel for the FMA Gamma-Ray Chamber (V. Nanal, B. B. Back,} D. J. Hofman, and B. Nardi)

As required for experiments with high intensity heavy ion beams onto targets with low melting points, we designed, built and utilized a rotating target wheel for the FMA gamma-ray chamber. This wheel uses four APEX type target frames. The rotation is provided by a small DC motor driving a ferrofluidic feedthrough into the vacuum. The drive shaft is also connected to a 1024 count/rev digital encoder, the signals from which are read out by a Camac module. The relevant information can be read out for each event and the module can be programmed to provide a TTL signal to sweep off the beam to avoid bombarding the spokes. The assembly (shown in Fig. I-59) operated successfully at up to 1000 RPM during a physics experiment in the fall of 1996.

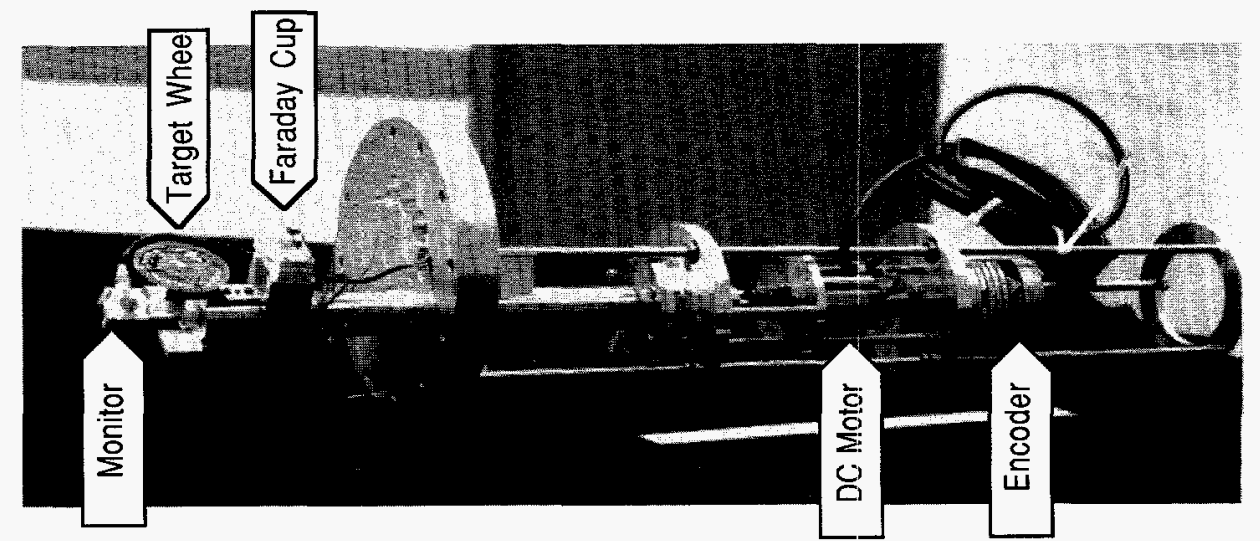

Fig. 1. Photograph of the rotating target wheel assembly. In addition to the target wheel consisting of four quadrants, a Faraday cup assembly used for beam focusing and a monitor detector are also seen. 


\section{e.14. BaF $_{2}$ High-Energy Gamma Array - LEPPEX (B. B. Back, D. Ackermann, G. Hackman, D. J. Hofman, D. Henderson, V. Nanal, P. Paul,* and P. Wilt)}

The LEPPEX project involves the construction of two high-efficiency 30-element $\mathrm{BaF}_{2}$ detector arrays and a high-efficiency fission fragment detection system consisting of eight position-sensitive parallel-grid avalanche detectors arranged in a barrel around the beam axis.

Funding for the project received to date has allowed for the completion of a 16 detector sub-array augmented with a 2 " thick plastic scintillator cosmic-ray shield, see
Fig. I-60 The remaining $14 \mathrm{BaF}_{2}$ crystals and photo multiplier tubes have just been ordered such that a full 30 detector array will be completed in mid 1997. Mechanical design of the particle detector array has been started.

The 16 element sub-array has been tested and utilized in physics experiments in the fall of 1996 . Completion of the project is at present determined by the funding profile.

*SUNY at Stony Brook

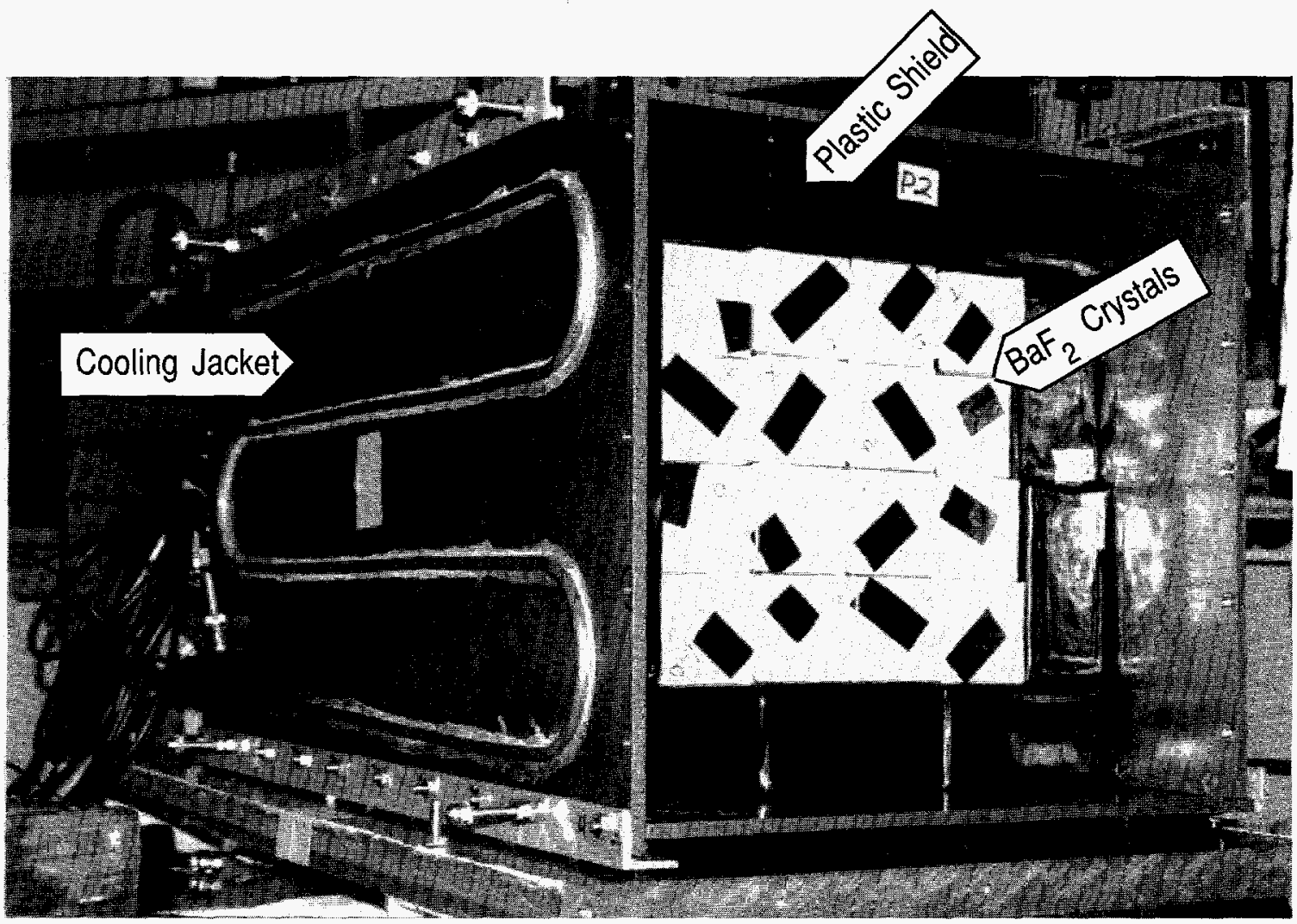

Fig. I-60. Photograph of the 16 element sub-array surrounded by a $5 \mathrm{~cm}$ plastic cosmic ray shield, and a cooling jacket for temperature stabilization. 


\section{e.15. A Beam Vertexing Detector System for AGS Experiment E917 (B. B. Back,}

R. R. Betts, A. Gillitzer, G. Hackman, D. J. Hofman, W. F. Henning, V. Nanal,

A. H. Wuosmaa, B. Nardi, and J. Falout)

A beam-vertexing detector for the E917 experiment at AGS has been built at Argonne. It is based on $200 \times$ $200 \mu \mathrm{m}^{2}$ scintillating fiber ribbons, which are read out via position sensitive phototubes (Hamamatsu model R2486). Four detector units were built and placed in the AGS beam line as pairs for $\mathrm{x}-\mathrm{y}$ position determination, $1.7 \mathrm{~m}$ and $5.8 \mathrm{~m}$ upstream from the target position. Each detector consists of a fiber ribbon containing about 150 fibers (therefore covering $30 \mathrm{~mm}$ in the transverse direction), see Fig. I-61 panel d. The fibers are terminated in front of the phototube in seven rows. Individual signals from the 32 anode wires of the phototube ( $16 \mathrm{x}$ and $16 \mathrm{y}$ wires) are amplified and read out using Fastbus charge-to-digital converters. Although the signal from a single fiber is spread out over 3-5 anode wires in each direction it is possible to determine the centroid of the signal distribution to an accuracy significantly better than the spacing between fibers on the face of the phototube, see Fig. I-61 panel a. The ability of the system to reconstruct the position at the target location was demonstrated by inserting a $0.5 \mathrm{~mm}$ diam. W wire at the target position and triggering on beam interactions with the wire. The results are shown in Fig. I-61 panel b \& c.

The detector system was operated during the 3 months running of the E917 experiment at the AGS. The beam intensity ranged up to about $350 \mathrm{~K} \mathrm{Au}$ ions per spill ( $1 \mathrm{~s}$ ) and the detector showed no effects of deterioration at the high count rate. In order to minimize the effects of radiation damage to the fibers, the mechanical design of the detector was such that it was possible to push in the fiber ladders to expose fresh fiber material without breaking the vacuum. This was done every $2-3$ days during high intensity running.
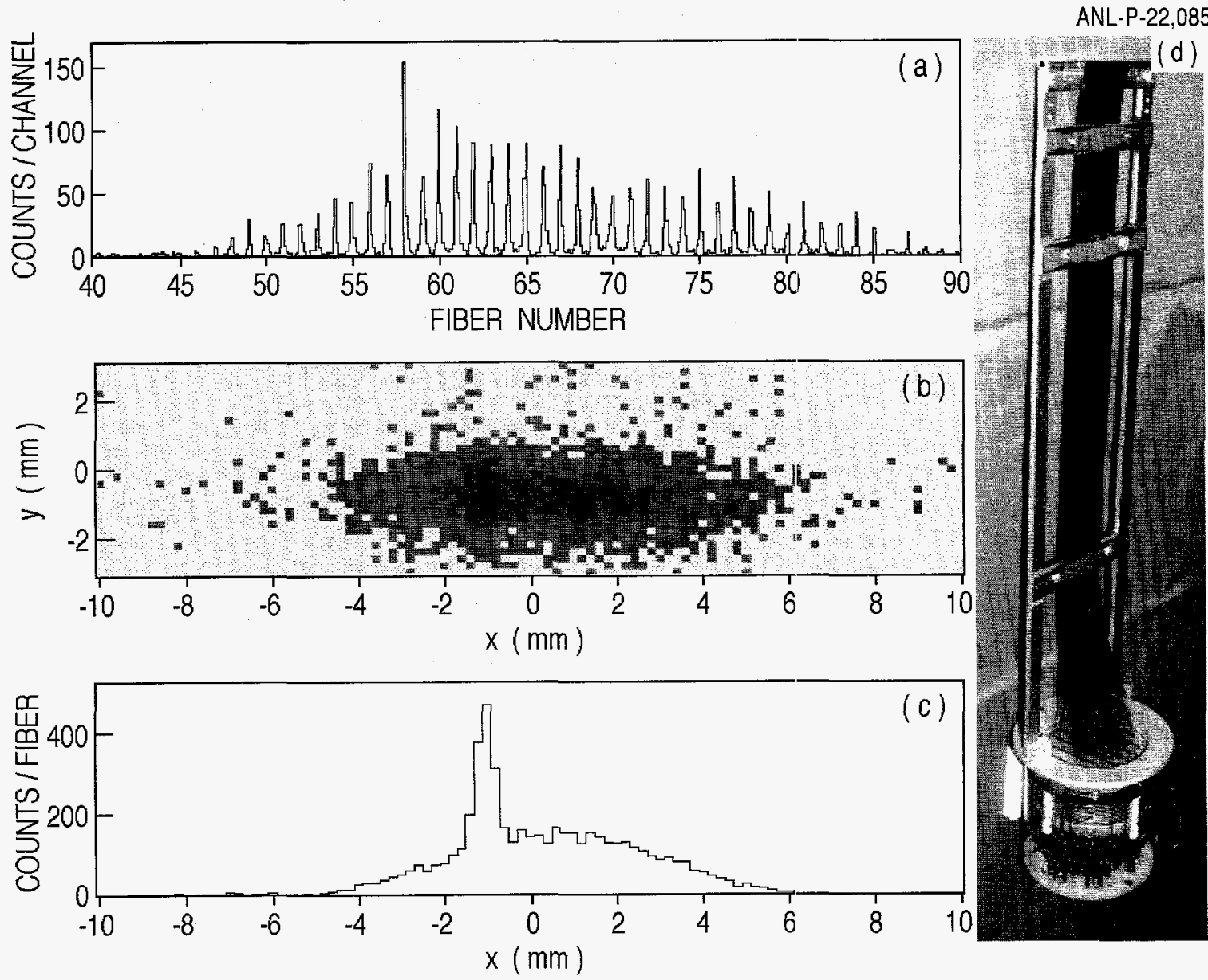

Fig. I-61. Panel a: Typical spectrum of fiber position showing single fiber $(200 \mu m)$ resolution. Also shown is a map of the reconstructed position of beam particles at the target location (panel $b$ ) and its horizontal projection (panel $c$ ). A photograph of the fiber ladder coupled to the position sensitive phototube is shown in panel d. 


\section{e.16. Nuclear Target Development (J. P. Greene and G. E. Thomas)}

The Physics Division operates a target development laboratory that produces targets and foils of various thicknesses and substrates, depending on the requirements, for experiments performed at the ATLAS and Dynamitron accelerators. The targets are prepared from both naturally occurring materials and stable isotopes which are supplied either in pure, elemental form or as stable compounds. Targets are made not only for the Physics Division, but also for other divisions at the Laboratory and occasionally for other laboratories and universities.

In the past year, numerous targets were fabricated either as self-supporting foils, on various substrates or as "sandwich" targets. Targets produced included Ag, Al, $\mathrm{Au}, \mathrm{Be},{ }^{10}, 1_{\mathrm{B}},{ }^{12} \mathrm{C},{ }^{40} \mathrm{Ca}, \mathrm{CD}_{2}, \mathrm{CH}_{2}, \mathrm{CH}_{4}$ (Helium), ${ }^{164} \mathrm{Er},{ }^{58} \mathrm{Fe},{ }^{160} \mathrm{Gd},{ }^{76} \mathrm{Ge}$, Havar, ${ }^{176} \mathrm{HfO}_{2}$, In, Kapton, ${ }^{24} \mathrm{Mg}, 92,96,98,100 \mathrm{Mo},{ }^{150} \mathrm{Nd},{ }^{58} \mathrm{Ni}$, $206,208 \mathrm{~Pb}$, polyethylene, polypropylene, $\mathrm{Ru}$, $144,154 \mathrm{Sm},{ }^{122,124} \mathrm{Sn}, \quad \mathrm{Ta},{ }^{159} \mathrm{~Tb}, 128,130^{\mathrm{Te}}$, ${ }^{182} \mathrm{WO}_{3},{ }^{170,174,176} \mathrm{Yb}$ and ${ }^{90} \mathrm{Zr}$. A major research effort has gone into hydrogen and deuterium targets, both using $a_{1}$ gas cell target for the production ofradioactive ${ }^{1{ }} \mathrm{~F}$ beams, but also solid targets for reactions with these beams on protons (and deuterons).

Numerous foils have been fabricated using our small rolling mill including targets of $\mathrm{Ag}, \mathrm{Au}$, brass, $\mathrm{Cd}, \mathrm{Cu}$, ${ }^{160} \mathrm{Gd}$, Havar, $92,96,98,100 \mathrm{Mo},{ }^{58} \mathrm{Ni},{ }^{206} \mathrm{~Pb},{ }^{124} \mathrm{Sn}$, ${ }^{232} \mathrm{Th}, \mathrm{Ti}, 174,176 \mathrm{Yb}$ and $\mathrm{Zn}$. Rolling has become the method of choice for most of the thick targets used in ATLAS experiments where the required surface densities range from 0.5 up to several $\mathrm{mg} / \mathrm{cm}^{2}$.

Support for Gammasphere continues, with an increase in target requests for these experiments. Targets of $\mathrm{Au}$, C, $160 \mathrm{Gd}, 1^{150} \mathrm{Nd},{ }^{208} \mathrm{~Pb}, \mathrm{WO}_{3}, 174,176 \mathrm{Yb}$ were produced for Argonne runs at Berkeley. Sandwiched targets of $\mathrm{Au}-{ }^{40} \mathrm{Ca}-\mathrm{Au}$ and $\mathrm{Au}-150 \mathrm{Nd}-\mathrm{Au}$, were successfully prepared and transported under vacuum or argon atmosphere for Gammasphere experiments. Targets supplied for other institutions included $10^{1} \mathrm{~B}$, ${ }^{40} \mathrm{Ca}, \mathrm{Gd},{ }^{128,130} \mathrm{Te}$ and ${ }^{174} \mathrm{Yb}$.

Approximately 677 targets were prepared for these various experiments during the past year.

As part of ATLAS support, carbon stripper foils of $2 \mathrm{ug} / \mathrm{cm}^{2}$ for use in the Tandem as well as other thickness for additional stripping are being routinely produced by the target lab. A total of 2204 carbon stripper and goldfoils of various types were prepared in calendar year 1996.
Most notably, there has been an increase in the preparation of various forms of isotopic source material for producing beams at ATLAS. Usually this takes the form of reducing separated isotope into a form and shape suitable for introduction into PIIECR (for example ${ }^{76} \mathrm{Ge}$ ). This was especially evident for the preparation of various $\mathrm{Ni} / \mathrm{Si}$ and $\mathrm{Fe} / \mathrm{Si}$ mixtures for the SNICS source and for ${ }^{58} \mathrm{Ni}$ cones for irradiation at IPNS. A intensive effort was mounted involving the reduction of $\mathrm{Cd}$ and $\mathrm{Zn}$ oxides not only for target fabrication but also to provide usable source material for isotopic beams. There were a number of instances where in order to conserve precious separated isotope, the isotope was diluted with natural material so that extended beam times were achieved.

The target development laboratory includes state-of the-art equipment used for thin-film fabrication. The available techniques consist of multiple resistive heating, focused ion beam sputtering, glow-discharge plasma deposition, electron beam and electron bombardment evaporation, electrodeposition and mechanical rolling. The evaporators are maintained under high vacuum and each vessel contains a quartzcrystal film-thickness monitor with deposition rate indicators. Also included are movable shutters, quartz lamp substrate heaters and thermocouple temperature sensors, allowing for complete process monitoring during target deposition.

Other auxiliary equipment used for target development includes a electrodeposition equipment, a small rolling mill, an alpha particle counting chamber, inert atmosphere glove box, laminar flow clean bench, pellet press, a reduction furnace, and a variety of precision balances.

A turbo-pumped target storage facility is in operation for maintaining, under high vacuum, those targets which can readily oxidize in air. This system utilizes computer-controlled circuitry to prevent targets from exposure to atmosphere during power interruptions. A second storage system employs a bank of vacuum desiccators connected to a mechanically-pumped manifold for use by individual experimenters. Duplicates of both these systems have been constructed and installed just inside Target Area $\amalg$ at ATLAS.

A low-level radioactive source and target preparation laboratory has been established at a separate location within the Division dedicated to the production of radioactive sources and targets of long-lived activities. Available preparation techniques include multiple resistive heating, electrodeposition and mechanical 
rolling. A diffusion-pumped vacuum evaporator is presently installed with plans for the addition of an electron beam evaporator system in the near future. A second much smaller evaporator system was constructed for close proximity evaporations of higher activity materials, to be used not only as targets, but for radioactive source development as well. The size of this system allows for minimal contamination and is presently installed within a hood. A modest inventory of radioactive targets and sources are stored in the laboratory and are available for use within the Division.

\section{e.17. Production of Implanted He Targets K. E. Rehm, and M. Schlapp)}

The reactions ${ }^{15} \mathrm{O}(\alpha, \gamma){ }^{19} \mathrm{Ne}$ and ${ }^{14} \mathrm{O}(\alpha, \mathrm{p}){ }^{17} \mathrm{~F}$ are important links between the hot CNO (HCNO) cycle and the rapid proton (rp) capture process. ${ }^{1}$ At temperatures $\mathrm{T} 9>0.35$, the ${ }^{15} \mathrm{O}(\alpha, \gamma){ }^{19} \mathrm{Ne}(p, \gamma)$ reaction sequence causes the breakout into the rp process, and no sequence of reactions can recycle the seed nuclei back into the CNO cycle. Up to now all information on the reaction rates for the ${ }^{15} \mathrm{O}(\alpha, \gamma){ }^{19} \mathrm{Ne}$ and ${ }^{14} \mathrm{O}(\alpha, \mathrm{p}){ }^{17_{\mathrm{F}}}$ reactions has been obtained indirectly using information from mirror nuclei. It is important to measure the cross sections of these reactions directly using radioactive ion beams and implanted $\mathrm{He}$ targets.

Several implanted gas targets of $\mathrm{Ne}^{2}, \mathrm{Ar}^{3}$, and $\mathrm{He}^{4}$ have been reported in the literature. In collaboration with the

\section{(J. P. Greene, R. E. Ganz, B. Harss, C. L. Jiang,}

University of Frankfurt, implanted ${ }^{4} \mathrm{He}$ targets were produced by bombarding $\mathrm{Al}$ foils with low-energy $\mathrm{He}$ beams. Rutherford backscattering measurements using $1 \mathrm{MeV}$ protons indicate target thicknesses of 1-2 $\mu \mathrm{g} / \mathrm{cm}^{2}$. In the future we are planning to use the new high-intensity ECR source at ATLAS for $\mathrm{He}$ implantation.

The second technique will test the possibility of using a gas-discharge for producing implanted $\mathrm{He}$ targets. In earlier experiments, implanted Ar targets were produced by cracking Methane in a $\mathrm{CH}_{4}$ - $\mathrm{Ar}$ mixture. We have recently produced similar targets from a gasdischarge in a $\mathrm{CH}_{4}$-He-mixture. Measurements to determine the He content of these targets using lowenergy beams from the Dynamitron are planned for the near future.

\footnotetext{
1A. E. Champagne and M. Wiescher, Annu. Rev. Nucl. Part. Sci. 42,39 (1992). ${ }^{2}$ M. S. Smith Thesis of Yale University, 1990. ${ }^{3}$ N. Kaloskamis et al., private communication. ${ }^{4}$ M. S. Smith et al., private communication.
}

\section{e.18. Physics Computing Facilities (D. R. Cyborski, T. Lauritsen, and K. Teh)}

The Physics Division maintains several computer systems for data analysis, computation, and general computing. These systems are conveniently grouped into various clusters and are described briefly below.

The Division's Unix cluster consists of seven Sparcstations, four DEC 3000/300LXs Alphas, and thirteen Linux PCs. In addition, the cluster also hosts eight $\mathrm{X}$-terminals. Its primary function is data analysis and some general computing. The adoption of the Linux PC has turned out to be an unqualified success. Initially, they were purchased as low cost graphics terminals, but already, some of them are being used as primary data sorting and analysis engines. Interface software to raw DAPHNE and MSU data is available for sorting. CERN software and, recently, RADWARE for gamma-ray spectroscopy have been added for data display and analysis.

The Analysis Cluster is a cluster of four DEC Alpha machines running the OpenVMS operating system. It consists of a DEC Alphaserver $20004 / 233$ host and three satellite machines---two DEC 3000/300LXs and one Alphastation 200, Its primary function is data analysis. This past year saw a significant increase in its utilization. No additional computing resources were added to meet the increase, but several peripherals--tapes and disks---were added.

With the bulk of data analysis now being done on the Unix and Analysis clusters, the goal of the Computer Support Group remains to provide support to fully utilize these resources. In particular, a primary goal is to fully utilize the Linux workstations as primary sorting and analysis engines. Software has been developed to sort MSU and DAPHNE data sets on the Linux workstations. Near future demands indicate that it will be necessary to share disks on the VMS Analysis cluster with the Linux workstations. Development continues on making its tape drives available to the Linux workstations.

Because of its older technology, the original Vax Cluster is no longer used for data analysis. This cluster which consists of two Vax 3300 servers, configured as a dual-host system, and six satellite Vax workstations, 
however, continues to provide general computing services to the Division. In particular, it provides users of PCs and Macintosh computers in the division, access to additional disk storage, printing, and mail services. There will be no further improvements to this cluster. Time permitting, the Computer Support Group hopes to take preliminary steps toward finding a more costeffective alternative to providing general computing resources.

In addition to the main divisional clusters, the division also operates three additional clusters. The Theory
Group has two IBM RS/6000 workstations which serve several $\mathrm{X}$-window terminals. The group also utilizes the Argonne IBM SP2, a massively parallel computing machine, for major numerical computations. The Medium Energy Group has a small cluster of two Silicon Graphics workstations, and a larger cluster of DEC machines running OpenVMS. The larger cluster is a mixed architecture cluster, consisting of two DEC Alpha workstations and three Vaxstations. In addition, it also hosts seven $\mathrm{X}$-terminals.

\section{e.19. Data Acquisition Systems (D. R. Cyborski, T. Lauritsen, and K. Teh)}

The division operates three MSU/DAPHNE data acquisition systems. Each consists of an MSU VME front-end, a DAPHNE back-end for monitoring which is hosted by a VMS workstation. One of these systems is dedicated to the APEX experiment, while the other two are used for other general experiments. Minor improvements were made in the past year to the data acquisition computers. One noticeable change was the addition of two large monitors. Two of the VMS back- end hosts are configured as dual-head workstations, that is, each workstation supports a small $17 \mathrm{in}$. monitor as its "console" monitor in addition to a large 21 in. monitor for graphics. The deployment of these dualhead workstations made it possible to decommission the Tektronix displays which were rapidly deteriorating due to age. DAPHNE underwent minor improvements to support these monitors and, in addition, system software was upgraded. 


\title{
F. ASSISTANCE TO OUTSIDE USERS OF ATLAS
}

\author{
B. G. Glagola
}

There is continuing strong interest by outside users in doing research at ATLAS, with outside users involved in about $90 \%$ of all experiments performed in FY 1996. The user assistance program is essential in facilitating the effective performance of research by outside scientists.

The outside user involvement continued to increase in FY 1996. The user liaison physicist continues to play a key role in channeling assistance to outside users. Major components of his responsibility are: (1) provide users with technical information about ATLAS and its experimental systems, and to provide instruction in its use; (2) assist outside users in all aspects of initiating and planning an experiment; (3) provide the needed information and organizational assistance to committees, workshops, and other meetings involving outside users; (4) instruct the users in the safety procedures to be followed when using the ATLAS facility; (5) to the extent that is appropriate and feasible, to assist users in the actual performance of experiments; (6) provide instruction and help with the use of computer hardware and software; (7) assist in coordinating the operation of the technical support group; and (8) provide an interface between the user and the technical support and ATLAS operation groups.

Two PAC meetings were held during FY 1996. The Program Advisory Committee (PAC) for ATLAS (nominally consisting of six members from other institutions and two from Argonne) continues to meet regularly during the year. PAC meetings were held on January 18-19, 1996 and June 10-11, 1996 to recommend experiments for running time at ATLAS. In FY 1996 the PAC members were Russell Betts (ANL), David Church (Texas A\&M University), David Fossan (SUNY, Stony Brook), I. Y. Lee (Lawrence Berkeley National Laboratory, Witek Nazarewicz (University of Tennessee), Peter Paul (SUNY, Stony Brook), Michael C. Wiescher (University of Notre Dame), and Frank L. H. Wolfs (University of Rochester). The Committee is chaired by Peter Paul. The PAC reviewed 27 proposals for 130 days of running time and 22 proposals for 108 days of running time at the meetings, respectively. The demand for running time at ATLAS continues to be about twice the time available on the accelerator.

The ATLAS User Executive Committee organized a User Group meeting during the October 1996 Division of Nuclear Physics APS meeting held at MIT. The meeting was attended by approximately 24 scientists. The main topics of discussion were a the status of ATLAS, accelerator R\&D, a review of experiments and status of the FMA, plans for the move of Gammasphere to ATLAS, and a discussion of PAC matters. In FY 1996 the ATLAS Executive Committee consisted of Partha Chowdhury (University of Massachusetts, Lowell), John Fox (Florida State University), Lee Riedinger (University of Tennessee), and Frank Wolfs (University of Rochester), as Chairperson.

Outside users are heavily involved in the Fragment Mass Analyzer project which accounted for approximately $60 \%$ of the ATLAS beam time in FY 1996. For more details see Section I.E.a.2.

The magnitude of the outside use of the accelerator during the past year was substantial, evidenced by the following two lists giving (1) the experiments performed by outside users and (2) the institutions represented. As may be seen from the names associated with each experiment, university groups are playing a major role in an important fraction of the experiments and a dominant role in some. 


\section{a. Experiments Involving Outside Users}

All experiments in which outside users participated during FY 1996 are listed below. Each experiment is listed only once although it may have been run more times. The spokesperson for each experiment is given in square brackets after the title. The names in parentheses are Argonne collaborators.

(1) Magnetic Moment Measurements in Mirror Nuclei: II. Polarization of ${ }^{43} \mathrm{Ti}$ and ${ }^{45} \mathrm{~V}$ by Tilted Foils at the Focal Plane of the FMA [Koller]

M. Satteson, R. Mayer, J. Holden, G. Kumbartzki, N. Koller, Rutgers University; M. Hass, G. Goldring, Weizmann Institute; W.Rogers, M. Uhl, W. Klug Westmont College; (C. Davids, T. Lauritsen, R. Janssens, D. Seweryniak)

The Alpha-Decay Rates of ${ }^{180} \mathrm{~Pb}$ and ${ }^{182} \mathrm{~Pb}$ [Toth]

K. Toth, J. Batchelder, Oak Ridge National Laboratory; E. Zganjar, Louisiana State University;

C. Bingham, J. Wauters, University of Tennessee; W. Walters, L. Conticchio, University of

Maryland; R. Irvine, Edinburgh University; T. Brown, Vanderbilt University; (C. Davids,

D. Seweryniak, D. Blumenthal)

Heavy Ion Irradiation of High Temperature Superconductor Single Crystals [Fendrich]

J. Fendrich, W. Kwok, G. Dasgupta, ANL Material Science Division; (Glagola)

Ion Irradiations of Anisotropic High-Tc Superconductors: Probing Dynamics of Magnetic Vortices [Steel]

D. Steel, P. Coulter, J. Lee, N. Remmes, ANL Material Science Division; J. Hettinger, Rowan

University; D. H. Kim, Yeungnam University; (B. Glagola)

Fine Structure in the Alpha Decay of ${ }^{192}$ Po [Wauters]

J. Wauters, C. Bingham, University of Tennessee; M. Huyse, University of Leuven; J. Cizewski, K.-

Y. Ding, N. Fotiades, D. McNabb, Rutgers University; (R. Janssens, C. Davids, D. Seweryniak)

Radiative Capture via Inverse Kinematics [Davids]

R. France, M. Gai, University of Connecticut; J. Goerres, A. Garcia, E. Wilds, University of Notre

Dame; (C. Davids, D. Seweryniak, P. DeCrock)

Atomic Spectroscopy and Lifetime Measurements in Copper-Like Lead and Uranium [Cheng]

S. Cheng, L. Curtis, R. Hellborg, University of Toledo; A. E. Livingston, H. G. Berry, University

of Notre Dame; (R. Dunford, E. Kanter)

Study of the ${ }^{18} \mathrm{~F}(\mathrm{p}, \alpha)$ and ${ }^{18} \mathrm{~F}(\mathrm{p}, \gamma)$ Reactions using a Radioactive ${ }^{18} \mathrm{~F}$ Beam - III [Rehm]

M. Paul, Hebrew University; J. Gehring, University of Chicago; R. Segel, Northwestern University;

J. Nickles, A. Roberts, University of Wisconsin; (C. Jiang, D. Blumenthal, J. Nolen, R. Pardo,

J. Schiffer, K. E. Rehm)

First Study of ${ }^{179} \mathrm{Au}$ [Riedinger]

L. Riedinger, W. Reviol, W. Mueller, B. Smith, C. Bingham, B. Zimmerman, J. Richards,

University of Tennessee; W. Ma, Mississippi State University; (M. Carpenter, R. Janssens, C.

Davids, T. Khoo, T. Lauritsen, I. Ahmad, B. Crowell, D. Blumenthal, D. Henderson)

Fusion Enhancements with Neutron-Rich Projetiles [Loveland]

W. Loveland, J. Dunn, L. Zyromski, G. Souliotis, Oregon State University; (B. Glagola)

Single-Particle Energies and Proton Neutron Interactions Near Sn-100: Study of the Decay of $105,106,107$ Sb [Conticchio]

L. Conticchio, W. Walters, University of Maryland; T. Brown, Vanderbilt University; C. Bingham,

J. Wauters, University of Tennessee; B. Foy, Clark University; R. de Haan, University of Notre

Dame; C. Davids, D. Henderson, D. Seweryniak) 
g-Factor Measurement of a High Spin M1 Band in ${ }^{197} \mathrm{~Pb}$ [Cizewski]

J. Cizewski, K.-Y. Ding, D. McNabb, N. Fotiades, N. Matt, R. Mayer, N. Koller, Rutgers

University; M. Haas, Weizmann Institute; D. Nisius, Purdue University; H. Amro, North Carolina

State University; (R. Janssens, M. Carpenter)

Search for Ground-State Deformation in the Light Radon Isotopes, ${ }^{200} \mathrm{Rn}$ [Freeman]

S.Freeman, J. Durell, M. Leddy, G. Smith, R. Taylor, University of Manchester; (D. Blumenthal, M. Carpenter, C. Davids, R. Janssens, C. Lister)

(14) Coulomb Excitation of Radioactive ${ }^{79} \mathrm{Rb}$ : Phase 2 [Fischer]

B. Varley, University of Manchester; J. Schwartz, Yale University; (S. Fischer, C. Lister,

D. Blumenthal, D. Henderson)

Polarimeter for Metastable $\mathrm{Ar}^{17+}$ Ions [Dunford]

A. E. Livingston, University of Notre Dame; (R. Dunford, E. Kanter, D. Gemmell, M. Jung)

Atomic Spectra and Lifetimes in Highly-Charged Krypton Ions [Livingston]

A. E. Livingston, K. Kukla, C. Vogel-Vogt, H. G. Berry, University of Notre Dame; L. Curtis,

S. Cheng, University of Toledo; (R. Dunfor, M. Jung)

The Proton-Neutron Interaction in Medium Mass Neutron Deficient Nuclei - Mass Measurement of ${ }^{151} \mathrm{Er}$ [Brenner]

C. Barton, D. Brenner, B. Foy, Clark University; T. Brown, Vanderbilt University, R. Casten, Yale University; L. Conticchio, University of Maryland, R. de Haan, University of Notre Dame;

D. Warner, Daresbury Laboratory; V. Zamfir, Brookhaven National Laboratory; (D. Blumenthal, D. Henderson, C. Davids, D. Seweryniak)

Proton Radioactivity of ${ }^{167}$ Ir and ${ }^{166}$ Ir [Davids]

J. Batchelder, K. Toth, Oak Ridge National Laboratory; L. Conticchio, W. Walters, University of Maryland; P. Woods, R. Irvine, T. Davinson, Edinburgh University; T. Brown, Vanderbilt

University; J. Wauters, C. Bingham, W. Mueller, University of Tennessee; G. Poli, University of

Milano; F. Soramel, University di Udine, (C. Davids, D. Seweryniak, D. Blumenthal, D. Henderson)

In Beam Gamma-Ray Spectroscopy of Neutron Deficient Nuclei using the Method of Recoil Decay Tagging (RDT) [Woods]

P. Woods, R. Irvine, T. Davinson, J. Mackenzie, Edinburgh University; W. Walters, L. Conticchio, University of Maryland; (C. Davids, D. Seweryniak, M. Carpenter, R. Janssens, D. Blumenthal)

Production of Nuclei in the $\mathrm{N}<126, \mathrm{Z}>82$ Region by the use of Symmetric Reaction Systems [Freeman]

S. Freeman, M. Leddy, G. Smith, R. Taylor, University of Manchester; (D. Blumenthal, C. Lister,

E. Rehm, D. Seweryniak, R. Janssens, M. Carpenter, C. Davids)

(21) Fission Fragment Mass Distributions as a Probe to the Pre-Saddle Time Scale [Charity]

R. Charity, L. Sobotka, J. Dempsey, R. Popelka, Washington University

Study of Nuclide Production Near ${ }^{100}$ Sn using a Radioactive Beann [Carpenter]

D. Nisius, Purdue University; H. Amro, North Carolina State University; J. Schwartz, Yale

University; (I. Ahmad, C. Jiang, C. Lister, R. Janssens, D. Blumenthal, D. Seweryniak,

W. Henning, C. Davids, T. Khoo)

Coulomb Excitation of ${ }^{232} \mathrm{Th}$ - Exploring an Unconventional Approach of Projectile Excitation with a

Thick Target [Khoo]

F. Moore, H. Amro, North Carolina State University; D. Cline, C.-Y. Wu, University of Rochester;

D. Nisius, P. Daly, C. Zhang, P. Bhattacharyya, Purdue University; (I. Ahmad, D. Blumenthal,

M. Carpenter, W. Henning, R. Janssens, T. Lauritsen, C. Lister, J. Schiffer) 
(24)

Identification of Excited States in ${ }^{175-178} \mathrm{Hg}$ using the Recoil Decay Tagging (RDT) Technique [Carpenter]

P. Woods, Edinburgh University; D. Nisius, Purdue University; T. Brown, Vanderbilt University;

W. Reviol, University of Tennessee; (M. Carpenter, R. Janssens, S. Fischer, C. Lister,

D. Seweryniak, D. Ackerman, T. Lauritsen, T. Khoo, D. Blumenthal, G. Hackman, C. Davids,

I. Ahmad)

A Search for Spontaneous Proton Emission from ${ }^{189}$ At and ${ }^{196} \mathrm{Fr}$ [Davids]

P. Woods, R. Irvine, Edinburgh University; J. Wauters, C. Bingham, University of Tennessee;

J. Batchelder, K. Toth, Oak Ridge National Laboratory; W. Walters, L. Conticchio, University of

Maryland; T. Brown, Vanderbilt University; (C. Davids, D. Blumenthal, D. Henderson,

D. Seweryniak)

Lifetime Measurements of the $\mathrm{N}=\mathrm{Z}$ Nuclei ${ }^{80} \mathrm{Zr}$ and ${ }^{84} \mathrm{Mo}$ for the Study of rp-Process Nucleosynthesis of $\mathrm{X}$-Ray Burst Conditions [Wiescher]

M. Wiescher, J. Goerres, H. Schatz, A. Aprahamian, R. de Haan, University of Notre Dame;

W. Walters, L. Conticchio, University of Maryland; R. Page, Edinburgh University; T. Brown, Vanderbilt University; (C. Davids, C. Lister, D. Seweryniak)

The Behavior of Intruder Based States in Light Bi and T1 Isotopes: Study of the Alpha Decay of ${ }^{187} \mathrm{Bi}$ [Batchelder]

J. Batchelder, K. Toth, Oak Ridge National Laboratory; L. Conticchio, W. Walters, University of Maryland; R. Irvine, Edinburgh University; T. Brown, Vanderbilt University; C. Bingham,

J. Wauters, University of Tennessee; E. Zganjar, Louisiana State University; (C. Davids,

D. Seweryniak)

Target Tests for Transient Field g-Factor Measurements in ${ }^{184} \mathrm{Pt}$ and ${ }^{180} \mathrm{Pt}$ [Reviol]

W. Reviol, W. Mueller, University of Tennessee; D. Nisius, Purdue University; U. Garg,

University of Notre Dame; N. Koller, R. Mayer, Rutgers University; F. Moore, H. Amro, North

Carolina State University; (R. Janssens, M. Carpenter, T. Lauritsen, S. Fischer)

Experimentally Deduced Fission Barrier Energies for ${ }^{48} \mathrm{Cr}$ [Sanders]

S. Sanders, A. Dummer, F. Prosser, University of Kansas

In Beam Spectroscopy of ${ }^{224} U$ [Ahmad]

J. Schwartz, Yale University; H. Amro, North Carolina State University; (I. Ahmad, C. Lister,

C. Davids, R. Janssens, M. Carpenter, D. Blumenthal, D. Seweryniak)

Search for the New Element $Z=112$ via the Reaction ${ }^{70} \mathrm{Zn}+{ }^{208} \mathrm{~Pb}$ [Henning]

R. Irvine, University of Manchester; T. Brown, Vanderbilt University, L. Conticchio, University of Maryland; (D. Ackermann, I. Ahmad, B. Back, C. Davids, S. Fischer, J. Greene, D. Hofman,

W. Henning, R. Janssens, C. Jiang, E. Rehm, D. Seweryniak, A. Wuosmaa, V. Nanal)

Study of Spectroscopic Factors in the Mass 56 Region using Radioactive Ion Beams [Rehm]

M. Paul, Hebrew University; (D. Ackermann, D. Blumenthal, S. Fischer, C. Jiang, P. DeCrock,

V. Nanal, J. Nolen, R. Pardo, R. Janssens, W. Henning, E. Rehm, J. Schiffer, A. Wuosmaa)

Study of Single-Particle States around N,Z $=28$ with a Radioactive ${ }^{56}$ Ni Beam [Rehm]

B. Harss, Tu Munich; J. Goerres, University of Notre Dame; R. Segel, Northwestern University; (D. Ackermann, S. Fischer, A. Wuosmaa, C. Jiang, J. Nolen, R. Pardo, E. Rehm, J. Schiffer, V. Nanal, R. Janssens, W. Henning, P. DeCrock)

FMA Test Runs [Davids]

B. Busse, Oregon State University; (C. Davids, D. Blumenthal, D. Seweryniak)

Possibilities for Spectroscopy of Light Nuclei using the AYEBall and FMA [Lister]

J. Schwartz, Yale University; (C. Lister, C. Davids, D. Blumenthal, M. Carpenter, R. Janssens) 
(36)

Test of the Silicon Box Detector at the FMA Focal Plane [Davids]

J. Batchelder, K. Toth, Oak Ridge National Laboratory; L. Conticchio, University of Maryland;

P. Woods, R. Irvine, T. Davinson, Edinburgh University; C. Bingham, J. Wauters, University of Tennessee; (C. Davids, D. Seweryniak, D. Blumenthal, D. Henderson)

Test of a Channel-Plate Detector at the FMA Focal Plane [Lister]

B. Varley, University of Manchester; J. Schwartz, Yale University; (C. Davids, C. Lister,

D. Blumenthal, D. Henderson)

Production of Neutron Deficient Isotopes in the ${ }^{60} \mathrm{Ni}+{ }^{100}$ Mo Reaction [Charity]

R. Charity, L. Sobotka, D. Sarantites, J. Dempsey, M. Devlin, Washington University; (B.

Back, D. Hofman, C. Davids)

Delayed Onset of Deformation in Light Radon Isotopes [Freeman]

S. Freeman, M. Leddy, G. Smith, R. Taylor, University of Manchester; J. Schwartz, Yale

University; (C. Davids, C. Lister, D. Seweryniak)

${ }^{192}$ Po and Critical Nuclear Phase Transitions [Cizewski]

J. Cizewski, K.-Y. Ding, N. Fotiades, D. McNabb, Rutgers University; J. Wauters, C.

Bingham, University of Tennessee; M. Huyse, University of Leuven; (R. Janssens, C. Davids,

D. Seweryniak)

Spectral Distributions of Two-Photon Decays in $\mathrm{Ni}^{26+}$ and $\mathrm{Ni}^{27+}$ [Dunford]

A. E. Livingston, University of Notre Dame; S. Cheng, L. Curtis, University of Toledo;

H. Schaeffer, GSI; (R. Dunford, E. Kanter, M. Jung)

Systematic Study of Positron Production in Heavy-Ion Collisions [Wuosmaa]

S. Austin, E. Kashy, J. Winfield, J. Yurkon, Michigan State University; A. Perrera, F. Wolfs, University of Rochester; S. Gazes, M. Wolanski, University of Chicago; J. Greenberg,

K. Chan, N. Kaloskamis, A. Chishti, Yale University; A. Hallin, M. Liu, Queens University;

T. Trainor, University of Washington; J. Fox, E. Roa, Florida State University; T. Happ, GSI; M. Freer, University of Birmingham; C. Conner, University of Illinois, Chicago; D. Bazin,

GANIL; S. Freedman, M. Maier, Lawrence Berkeley Laboratory; F. Calaprice, A. Young,

Princeton University; (R. Betts, A. Wuosmaa, W. Kutschera, B. Back, J. Schiffer, I. Ahmad,

D. Henderson, C. Lister, R. Dunford, M. Rhein, P. Wilt)

Study of the ${ }^{17} \mathrm{~F}(\mathrm{p}, \boldsymbol{\alpha}){ }^{14} \mathrm{O}$ Reaction at Low Energies [Rehm]

B. Harss, Tu Munich; T. F. Wang, Lawrence Livermore National Laboratory; (R. Pardo, J.

Schiffer, J. Nolen, C. Jiang, S. Fischer, A. Wuosmaa, D. Ackermann)

Spin Distributions for ${ }^{64} \mathrm{Ni}+{ }^{100} \mathrm{Mo}$ and ${ }^{16} \mathrm{O}+{ }^{144,154} \mathrm{Sm}$ with the Argonne BGO Array [Ackermann]

F. Scarlassara, University of Padova; L. Corradi, University of Legnaro; R. Gill, Brookhaven

National Laboratory; (A. Wuosmaa, R. Janssens, T. Lauritsen, D. Seweryniak, G. Hackman,

S. Fischer, D. Ackermann, P. Reiter)

In-Beam Studies of Very Neutron-Deficient Even-Even Os and Pt Isotopes using the Recoil-Decay Tagging Method [Seweryniak]

W. Walters, L. Conticchio, University of Maryland; H. Amro, North Carolina State University; D. Nisius, Purdue University; D. Seweryniak, C. Davids, R. Janssens, M. Carpenter, S.

Fischer, D. Ackermann, G. Hackman, P. Reiter)

Measurement of Absolute B(E2)s in the A 130 Even-Even Xe Nuclei [Fischer]

C. Conner, University of Illinois, Chicago; (S. Fischer, C. Lister, A. Wuosmaa, G. Hackman,

V. Nanal, D. Henderson, M. Carpenter, T. Lauritsen)

Shape Coexistence in ${ }^{172,174}$ Os Studied by Alpha Decay of ${ }^{176,178} \mathrm{Pt}$ [Wauters]

J. Wauters, C. Bingham, W. Reviol, University of Tennessee; W. Walters, L. Conticchio,

University of Maryland; (C. Davids, D. Seweryniak, D. Blumenthal) 
(48) Ion Irradiations of Carbonaceous Materials to Investigate Radiation-Induced Diamond Formation [Daulton] T. Daulton, L. Rehn, M. Kirk, ANL Material Science Division; (B. Glagola)

(49) Shapes of Very Neutron-Deficient Barium, Cesium and Xenon Isotopes: Gamma-Ray Spectroscopy with Mass Identification [Smith]

J. Smith, D. Fossan, C. Chiara, G. Lane, SUNY, Stony Brook; (C. Davids, R. Janssens)

(50) Test of a Heavy Ion Beam Tracking System [Ganz]

R. Ganz, University of Chicago; (B. Back, A. Wuosmaa, D. Hofman, R. Betts, V. Nanal, G. Hackman)

b. Outside Users of ATLAS and of ATLAS Technology -- 10/1/95 - 9/30/96

Those who were present at ATLAS for an experiment or other related research are listed. An asterisk denotes student.

(1) University of Notre Dame

$$
\text { A. Aprahamian }
$$

H. G. Berry

* R. de Haan

A. Garcia

U. Garg

J. Goerres

* K. Kukla

A. E. Livingston

* H. Schatz

* C. Vogel-Vogt

M. Wiescher

(2) Purdue University
* P. Bhattacharyya
P. Daly
* D. Nisius
C. Zhang

(3) University of Kansas
* A. Dummer
F. Prosser
S. Sanders

(4) Washington University

$$
\begin{array}{ll} 
& \text { R. Charity } \\
* & \text { J. Dempsey } \\
& \text { L. Sobotka } \\
* & \text { R. Popelka } \\
* & \text { M. Devlin }
\end{array}
$$

(5) Rutgers University

J. Cizewski

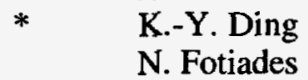

* J. Holden

N. Koller

G. Kumbartzki

* N. Matt

R. Mayer

* D. McNabb

(6)
(7)

$$
\begin{aligned}
& \text { SUNY, Stony Brook } \\
& * \quad \text { C. Chiara } \\
& \text { D. Fossan } \\
& \text { G. Lane } \\
& \text { J. Smith }
\end{aligned}
$$

University of Rochester

$$
\begin{array}{ll}
\text { * D. Cline } \\
\text { A. Perrera } \\
\text { F. Wolfs } \\
\text { C.-Y. Wu }
\end{array}
$$

(9)

University of Connecticut

$$
\begin{array}{ll}
* & \text { M. Gai } \\
* & \text { R. France } \\
* & \text { E. Wilds }
\end{array}
$$

(10) University of Chicago

$$
\begin{array}{ll}
* & \text { S. Gazes } \\
* & \text { J. Gehring } \\
* & \text { M. Wolanski }
\end{array}
$$

Rowan University

J. Hettinger

Yeungnam University D. H. Kim

University of Toledo
S. Cheng
L. Curtis

* R. Hellborg

NSC, New Delhi
G. Mehta
R. Mehta
P. Potukuchi
A. Roy

Clark University
* C. Barton
* B. Foy

Louisiana State University E. Zganjar 
(17) Northwestern University

R. Segel

(18) University of Wisconsin J. Nickles

A. Roberts

(19) Weizmann Institute

G. Goldring

M. Hass

(20) University of Maryland

L. Conticchio

W. Walters

(21) Yale University

$\begin{array}{ll} & \text { R. Casten } \\ * & \text { K. Chan } \\ & \text { A. Chishti } \\ & \text { J. Greenberg } \\ * \quad & \text { N. Kaloskamis } \\ * & \text { J. Schwartz }\end{array}$

Florida State University

J. Fox

* $\quad$ E. Roa

(23) Materials Science Division, ANL

Coulter

G. Crabtree

G. Dasgupta

T. Daulton

J. Fendrich

K. Gray

M. Kirk

W. Kwok

J. Y. Lee

D. Miller

L. Rehn

N. Remmes

D. Steel

* B. Washburn

(24) Hebrew University

M. Paul

(25)

University of Tennessee

$\begin{array}{ll}* & \text { C. Bingham } \\ \text { W. Mueller } \\ & \text { L. Riedinger } \\ & \text { W. Reviol } \\ * \quad & \text { J. Richards } \\ * \quad & \text { B. Smith } \\ & \text { B. Zimmerman }\end{array}$

(26)

University of Sao Paulo

N. Added

A. Szanto de Toledo
(27)

Michigan State University
S. Austin
E. Kashy
J. Winfield
J. Yurkon

Lawrence Berkeley Laboratory
S. Freedman
M. Maier

University of Birmingham

$$
\text { M. Freer }
$$

University of Leuven

M. Huyse

GSI, Darmstadt

T. Happ

H. Schaeffer

Queens University, Ontario

* $\quad$ A. Hallin

Edinburgh University

T. Davinson

* R. Irvine

J. MacKenzie

P. Woods

University of Illinois, Chicago

* C. Conner

GANIL

D. Bazin

North Carolina State University

E. F. Moore

* H. Amro 
$(42)$

Tu Munich

$$
\begin{array}{ll}
\text { Westmont College } \\
* & \text { W. Klug } \\
& \text { W.Rogers } \\
* & \text { M. Uhl }
\end{array}
$$

University di Udine

F. Soramel

University of Milano

G. Poli

University of Padova

F. Scarlassara

University of Legnaro

L. Corradi

\section{c. Summaries of the Continuing User Programs for FY 1996}

\section{c.a. The University of Notre Dame \\ c.a.1. Nuclear Physics (U. Garg, A. Frohlich, S. Ghugre, B. Kharraja, S. Naguleswaran, G. Smith, J. Walpe, W. Reviol, L. Riedinger (University of Tennessee), R. Kaczarowski, E. Ruchowska, (SINS, Swierk, Poland), I. Govil (Panjab University, Chadigarh, India)}

Several groups from the University of Notre Dame are playing an important role in developing the research program at ATLAS. One of their main interests is the study, in collaboration with ANL staff members, of the behavior of nuclei at high spin in the transitional region near $\mathrm{A}=180$ (i.e. the $\mathrm{Hg}-\mathrm{Pt}-\mathrm{Os}$ nuclei), and $\mathrm{A}$ $=100$ (i.e. the Ru-Tc nuclei), with emphasis on shape coexistence and configuration mixing. The $\gamma$-ray groups at ANL and Notre Dame also had collaborative experiments at Gammasphere. The Notre Dame group built, tested, and used extensively a state-of-theart plunger device for lifetime measurements in conjunction with the ATLAS $\gamma$-ray facility. An adapted version of this device is now under construction at Notre Dame, under contract with ANL, for use at the Gammasphere facility. A first test of the device in-situ at Gammasphere took place during the summer of 1996 . The control electronics is under construction.

\section{c.a.2. Atomic Physics (A. E. Livingston, H. G. Berry, K. W. Kukla, C. Vogel Vogt)}

In a collaboration with the Atomic Physics groups at Argonne and the University of Toledo, the Atomic Physics group at the University of Notre Dame is measuring the energy structures and lifetimes of excited fine structure in highly-charged few-electron ions at ATLAS. Extreme ultraviolet spectroscopy is applied with the beam-foil excitation technique and position-sensitive photon detection. Extreme ultraviolet emission spectra of highly-charged krypton ions were studied using beam-foil excitation. Finestructure transition energies and allowed and forbidden lifetimes of excited states in $\mathrm{Kr}^{31+}, \mathrm{Kr}^{32+}$, and $\mathrm{Kr}^{33+}$ were measured. The transition energies were measured in the wavelength range of 50-300 A with precisions of $\sim 5 \times 10^{-4}$. Lifetime results ranging in value from $10 \mathrm{ps}$ to $3 \mathrm{~ns}$ were obtained with precisions of a few percent. Spectrocopic and lifetime studies of high-Z heliumlike, lithiumlike, and copperlike ions continue at ATLAS. A new study of Stark-induced asymmetry of the angular distribution of the X-ray decay radiation from the metastable $2 s_{1 / 2}$ state in hydrogenlike $\mathrm{Ar}^{17+}$ has been initiated at ATLAS. In a collaboration that includes P. Mokler of GSIDarmstadt, measurements of the spectral distribution of the two-photon $\mathrm{X}$-ray decay continuum in heliumlike ions is underway at ATLAS and at GSI. 


\section{c.b. Purdue University (P. Daly, Z. Grabowski, D. Nisius, P. Bhattacharyya, C. T. Zhang)}

The Purdue University group, which includes several thesis students, is working on a measurement of highspin nuclear states at ATLAS. They use in-beam gamma-ray techniques to investigate several aspects of nuclear structure at high spin, testing the validity of shell-model calculations for high-spin yrast states. The nuclei are produced via deep inelastic reactions, and/or via spontaneous fission, rather than with the more conventional fusion reactions. This technique allows the study of neutron-rich nuclei that cannot be studied by other means. In the past year the group has focussed on the study of isomers in nuclei in the vicinity on ${ }^{132} \mathrm{Sn}$. Furthermore, D. Nisius is a Ph.D. student, resident at ANL, performing his thesis work under the supervision of R. V. F. Janssens.

\section{c.c. University of Kansas (S. Sanders, F. W. Prosser, T. Catterson, and A. Dummer)}

Building on some preliminary, but highly suggestive, results on the ternary breakup of ${ }^{48} \mathrm{Cr}$ that were obtained earlier at ATLAS, we have mounted a more extensive effort to explore this behavior. Recent microscopic and macroscopic calculations have suggested a three- ${ }^{16} \mathrm{O}$ cluster chain configuration in ${ }^{48} \mathrm{Cr}$. Such a configuration might lead to significantly enhanced cross sections in the ternary ${ }^{16} \mathrm{O}$ breakup channel for reactions populating ${ }^{48} \mathrm{Cr}$ than would be expected based on a sequential fission mechanism. In an earlier run at Argonne, we found evidence for significant population of this ternary channel, but were unable to establish reliable cross sections. In two runs during the past year we have explored some of the background and normalization issues for this process and have mounted a two- and three-fragment coincidence experiment to establish the ternary breakup cross sections. This latter work has entailed the construction of five MWPC-Si-strip detector hybrid counters and building a number of time pick-off units to augment those already available at Argonne. Last spring we measured the evaporation residue and fission cross sections for the ${ }^{36} \mathrm{Ar}+{ }^{12} \mathrm{C}$ system in order to resolve an apparent discrepancy in these values seen in our earlier work on this system. These data, which are still in the analysis stage, will be used to establish the normalization for the ternary breakup measurement. This latter experiment, also employing the ${ }^{36} \mathrm{Ar}+{ }^{12} \mathrm{C}$ reaction, was completed latte in 1996, with the new counters and electronics being used with great success. The ternary fission experiment will form part of the dissertation research of A. Dummer.

\section{c.d. Rutgers University (J.A. Cizewski, K.K.-Y. Ding, N. Fotiades, D.P. McNabb and W. Younes)}

The level structure of ${ }^{192}$ Po was determined by observing prompt $\gamma$-ray coincidences with recoil mass identification and recoil $\alpha$-decay (recoil-decay tagging - RDT). The ${ }^{164} \mathrm{Er}+164 \mathrm{MeV}{ }^{32} \mathrm{~S}$ reaction has been used. The target was $73.6 \%$ enriched in ${ }^{164} \mathrm{Er}$ and $724 \mu \mathrm{g} / \mathrm{cm}^{2}$ thick. The first three excited states of ${ }^{192}$ Po were identified. The observed energy ratios are consistent with an anharmonic vibrator interpretation and give evidence of increased collectivity in this isotope compared with the heavier Po isotopes, including ${ }^{194}$ Po previously studied at ATLAS. The particle-core model has been successfully used to model these states and the quasiparticle random phase approximation has been applied to extract microscopic wavefunctions of the $2_{1}^{+}$states. The isotopic composition of the target enabled a population of excited states in the heavier ${ }^{193} \mathrm{Po},{ }^{195} \mathrm{Po}$ and ${ }^{197} \mathrm{Po}$ isotopes in which the first three excited states were observed. Comparison between these states built on the $i_{13 / 2}$ isomer and the first excited levels in the even-mass $192,194,196$ Po isotopes reveals striking similarities in their behavior. In a second experiment we have attempted to study the g-factor of an M1 band in ${ }^{197} \mathrm{~Pb}$ using the transient-field technique. Excitations in ${ }^{197} \mathrm{~Pb}$ were populated via the ${ }^{124} \mathrm{Sn}+$ $306 \mathrm{MeV}{ }^{76} \mathrm{Ge}$ reaction. The BGO multiplicity and sum-energy gates were used to enhance the ${ }^{197} \mathrm{~Pb}$ transitions in the spectra and to minimize the contamination. The analysis of this experiment is still in progress.

c.e. University of Tennessee, University of Maryland, and Oak Ridge National Laboratory (C. R. Bingham, J. D. Richards, B. Zimmerman, W. B. Walters, L. F. Conticchio, and K. S. Toth)

This collaboration was involved in the study of alphadecaying nuclei in the $A>180$ region using the double-sided silicon strip detector implantation facility at the FMA. They are also active participants in the proton radioactivity experiments (see Sec. I.A.13 titled "Research at ATLAS"). 


\section{c.f. University of Toledo (L. J. Curtis, S. Cheng)}

A program of fast ion beam studies of the structure of highly-ionized atoms is being carried out in collaboration with the Atomic Physics groups at Argonne and the University of Notre Dame. Transition energies and lifetimes are measured using time resolved methods which employ positionsensitive multiplexed detection of wavelengths and coincidence and energy summing discrimination. Particular emphasis is given to examining isoelectronic trends which can be exploited for interpolative and extrapolative predictions. Current projects include studies of lifetimes for two-photon decays in Heliumlike $\mathrm{Ni}, \mathrm{Ar}$, and $\mathrm{Kr}$; lifetimes of highly ionized members of $\mathrm{Li}, \mathrm{Be}$, and $\mathrm{B}$ isoelectronic sequences; energies and lifetimes of Copperlike and Zinclike Uranium ions; Z-dependence of the energy distribution shapes for two-photon decays; ultra-short lifetime measurement by the two-foil technique.

\section{d. ATLAS - Technology Transfer}

In addition to outside users of the ATLAS beam, we also provide assistance in the use of ATLAS technology at other laboratories.

\section{d.a. University of Sao Paulo (N. Added and A. Szanto de Toledo)}

Argonne is assisting the University of Sao Paulo (USP) in the construction of a small superconducting heavyion linac to serve as an energy booster for projectiles from their 8-MV tandem. This booster will be similar in many respects to the ANL booster linac built in the late 1970s. The ANL contribution to this project are to (1) build (at USP expense) 14 split-ring niobium resonators and some of the associated rf electronics, (2) to provide technical information including drawings of the cryogenics distribution system, and (3) to train USP staff members in several phases of superconductinglinac technology. Two Brazilian engineers worked at

\section{d.b. Nuclear Science Centre, New Delhi and A. Sarkar)}

Argonne is collaborating with the Nuclear Science Centre (NSC), New Delhi, to develop a new type of superconducting accelerating structure for low-velocity heavy ions. The niobium prototype underwent a series of tests during 1996. This development was successful and initial funding for the production of 10 resonators has now been received at ANL. Some technical details of this project are described in the Superconducting Linac Development section of this Annual Report. The
Argonne for one year, gaining experience in cryogenics and in superconducting-resonator technology. Another engineer worked on the new control system at ATLAS for two years, the first year supported by Sao Paulo and the second with direct ANL support. Sao Paulo personnel returned to ANL in 1993 for assembly and testing of the first batch of completed resonators. The fabrication of the resonators was completed in 1995 when the Sao Paulo personnel came for final assembly and testing. Eleven resonators were shipped to Sao Paulo in 1996 and three remain at ANL for final testing. Fabrication of electronics modules at ANL is nearly complete.

(G. Mehta, P. Potukuchi, A. Roy,

NSC has stationed staff members at ATLAS for extended time periods during the past four years to gain experience and to work on various aspects of this project. At this time, in addition to the resonator fabrication project, there is an ANL/NSC collaboration to fabricate two new heavy ion bunchers, one for each laboratory. The mechanical components are being fabricated in India, while the electronic components are being fabricated with the help of NSC personnel at ANL. 


\section{OPERATION AND DEVELOPMENT OF ATLAS}

\section{OVERVIEW}

These three sections report on the operation of the Argonne Tandem Linear
Accelon Accelerator System (ATLAS) as a national user facility and related accelerator physics R\&D projects. ATLAS is used for basic research in nuclear physics and atomic physics, as well as occasionally for other areas of research, such as materials science. Over half of the beam time is allocated to outside users. Recent experience and projects related to the operation of ATLAS are summarized in the next section. Longer range accelerator physics developments to upgrade ATLAS capabilities are described in the following section. ATLAS personnel are also involved in developing technology in support of a future advanced exotic beam facility based on ATLAS. Projects related to the exotic beam facility are described in the third section below.

Currently, ATLAS is on a seven-day-week operating schedule. An increase in funding as part of the DOE Scientific Facilities Initiative began in 1996, to bring the staff level and M\&S budget up to that required for steady-state seven-day operation. This enhances our ability to serve the high level of demand for beam time from the ATLAS user community. Beam was available for research with a high degree of reliability in FY 1996, and a record number of hours were available for research. Statisitcs about beam hours and users are given in Table 1 on the following page. During the second half of FY 1997, experimental area IV and the Fragment Mass Analyzer beam line are being reconfigured for the installation of Gammasphere. The goal is to have 6000 hours of beam available for research during a 12-month period beginning when Gammasphere is ready in early 1998.

Other recent highlights of ATLAS operations include the delivery of more intense currents of beams in the lower mass ranges, e.g. over $200 \mathrm{pnA}$ of beams such as $18 \mathrm{O}$ and ${ }^{32} \mathrm{~S}$ have been delivered to targets for research. Also, three different radioactive beams $\left({ }^{17} \mathrm{~F}, 18 \mathrm{~F}\right.$, and $\left.{ }^{56} \mathrm{Ni}\right)$, with intensities in the range of $10^{5}$ to $10^{6}$ particles per second on target have been developed and used for research. During FY $19968 \%$ of ATLAS beam time was used for research with radioactive beams. Other radioactive beams, using the developed methods, will be provided as users require. 
TABLE 1. Statistics related to ATLAS Operations and Users.

\begin{tabular}{|c|c|c|c|c|}
\hline & $\frac{\text { FY } 1996}{\text { (actual) }}$ & $\frac{\text { FY } 1997}{\text { (extrap.) }}$ & $\frac{\text { FY 1998* }}{\text { (pred.) }}$ & $\frac{\text { FY 1999* }}{\text { (pred.) }}$ \\
\hline \multicolumn{5}{|l|}{ Beam Use for Research (hr) } \\
\hline Nuclear Physics & 5214 & 5050 & 5350 & 5350 \\
\hline Atomic Physics & 372 & 190 & 190 & 190 \\
\hline Accelerator R \& D & 96 & 100 & 100 & 100 \\
\hline Other & 144 & 60 & 60 & 60 \\
\hline Total & 5826 & 5400 & 5700 & 5700 \\
\hline $\begin{array}{l}\text { Number of Experiments } \\
\text { Receiving Beam }\end{array}$ & 66 & 62 & 65 & 65 \\
\hline $\begin{array}{l}\text { Number of Scientists } \\
\text { Participating in Research }\end{array}$ & 181 & 180 & 180 & 180 \\
\hline \multicolumn{5}{|l|}{ Institutions Represented } \\
\hline Universities (U.S.A.) & 29 & 29 & 30 & 30 \\
\hline DOE National Laboratories & 4 & 4 & 5 & 5 \\
\hline Other & 18 & 18 & 20 & 20 \\
\hline \multicolumn{5}{|l|}{ Usage of Beam Time (\%) } \\
\hline In-House Staff & 25 & 30 & 25 & 25 \\
\hline Universities (U.S.A.) & 38 & 40 & 45 & 45 \\
\hline Other DOE National Laboratories & 11 & 10 & 10 & 10 \\
\hline Other Institutions & $\frac{26}{100 \%}$ & $\frac{20}{100 \%}$ & $\frac{20}{100 \%}$ & $\frac{20}{100 \%}$ \\
\hline
\end{tabular}

*During a 12 month period spanning FY 1998 and FY 1999 ATLAS will provide 6000 hours of beam for research, about $2 / 3$ of which will be for Gammasphere experiments.

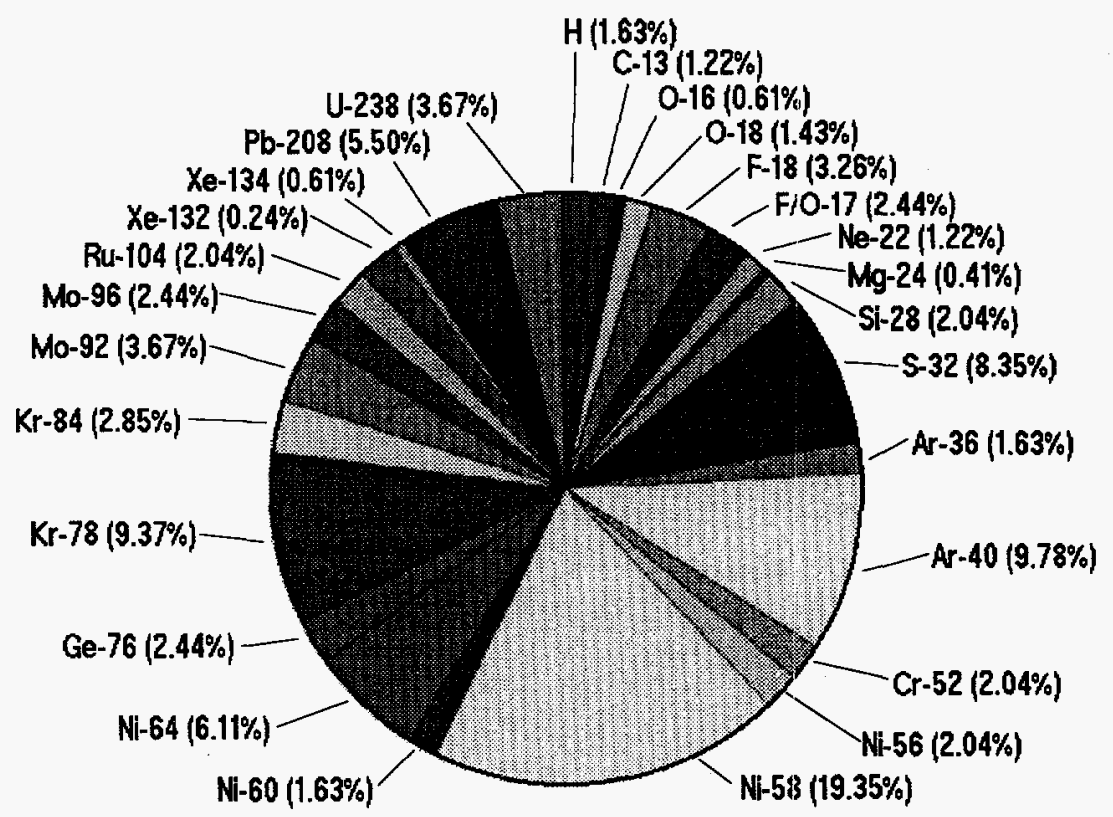

Fig. II-1. Chart of the isotopic distribution of beam time provided by ATLAS during FY 1996. 


\section{A. OPERATION OF THE ACCELERATOR}

(R. C. Pardo, B. Batzka, A. Beardsley, P.J. Billquist, J. Bogaty, K. M. Borawski, B. E. Clifft, S. L. Craig, S. Daley, R. E. Harden, B. Millar, F. H. Munson, Jr., D. R. Phillips, C. Roehrig, A. Ruthenberg, R. H. Scott, M. Schlapp, J. R. Specht, P. Strickhorn, A. Reuter, I. R. Tilbrook, R. C. Vondrasek, and G. P. Zinkann)

ATLAS set a new facility record when it delivered 5826 hours of beam for research and accelerator development during FY1996. This is a $10 \%$ increase over our previous best performance achieved in FY1994. The longest scheduled maintenance period during the year was an eight day period in August, 1996. A slight reduction of beam time during FY1997 is likely as we devote additional time to maintenance and preparations for the arrival of Gammashere at the beginning of FY1998.

Beam from ATLAS was available $92 \%$ of the scheduled time. This is one of the best 'reliability' factors ever achieved by ATLAS. Better reliability resulting from improvements in our primary electrical power delivery and cryogenic system accounted for most of the improvement.

The facility continues to operate on a seven-day schedule. During the past two years, we have increased our operating staff by adding an additional person to our electronics group, a second person to the computer control group and one additional ATLAS operator to give improved shift coverage. In addition to these three new staff members, two operators who left ATLAS for other positions have been replaced with two new operators.

A total of 27 different beams were accelerated during the year delivering beam to 66 different experiments. A new beam of chromium $\left({ }^{52} \mathrm{Cr}\right)$ was developed and provided to the research program from the ECR ion source during FY1996. Approximately 35\% of beams requested were for beams heavier than the nickel isotopes, although the demand for the heaviest of species was down somewhat this year. The chart on the previous page, Figure II-1, indicates the ion species used at ATLAS during FY 1996, and the percentage of beam time for each.

The emphasis on providing radioactive beams continued to increase during the year with the addition of beams of ${ }^{56} \mathrm{Ni}\left(\mathrm{T}_{1 / 2}=6.1 \mathrm{~d}\right)$ and ${ }^{17} \mathrm{~F}\left(\mathrm{~T}_{1 / 2}=64.5 \mathrm{~s}\right)$ to the previously developed ${ }^{18} \mathrm{~F}\left(\mathrm{~T}_{1 / 2}=1.83 \mathrm{~h}\right)$. Radioactive beams accounted for $7.7 \%$ ( 407 hours) of the research time at ATLAS in FY1996. Additional improvements in the performance of the facility for radioactive beams are planned for FY1997.

\section{a. First Operation of the new 14-GHz ECR Ion Source (R. C. Pardo, M. Schlapp, R. C. Vondrasek P. J. Billquist, J. B. Szczech, Z. Q. Xie*, and C. Lyneis*)}

The first plasma from the new 14-GHz ECR ion source for ATLAS was achieved in September 1996. The first analyzed beam was achieved in December following the delivery of the 90 degree analyzing magnet. Since that time, we have focused on oxygen and argon as development beams and to provide a base of reference as we bring the new features of the source into operation. The new source on its high-voltage platform is pictured in Figure II-2.

After one month of operation the source performance looks very exciting with much improvement certain to come since a number of features are not yet implemented. Within the first month of operation currents of 265 emicroA of $16 \mathrm{O}^{6+}$ and 78 emicroA

*Lawrence Berkeley National Laboratory of ${ }^{16} \mathrm{O}^{7+}$ have been achieved. The source performance is very stable. For argon 20 emicroA of ${ }^{40} \mathrm{Ar}^{12+}$ has been measured. Already these currents represent improvement factors of 10 to 30 over the best performance observed with the old ATLAS $10-\mathrm{GHz}$ ECR ion source.

The analyzing magnet for the new source is a "splitpole' design with second-order corrections applied in the center of the magnet by splitting the pole tip to give field curvatures in addition to those at the magnet entrance and exit edges. Preliminary tests indicated the resolution of the magnet exceeds $0.5 \%$ in $(\mathrm{m} / \mathrm{q})$. This resolution provides improved ion species selectivity and allows more flexibility in charge state choice for operation. 
Procurement of beamline components and high-voltage systems for delivery of beams from the new source has begun. First beam for research is anticipated to be available in late 1997. Source commissioning and beam development will continue during 1997 while the beam line components are being installed.

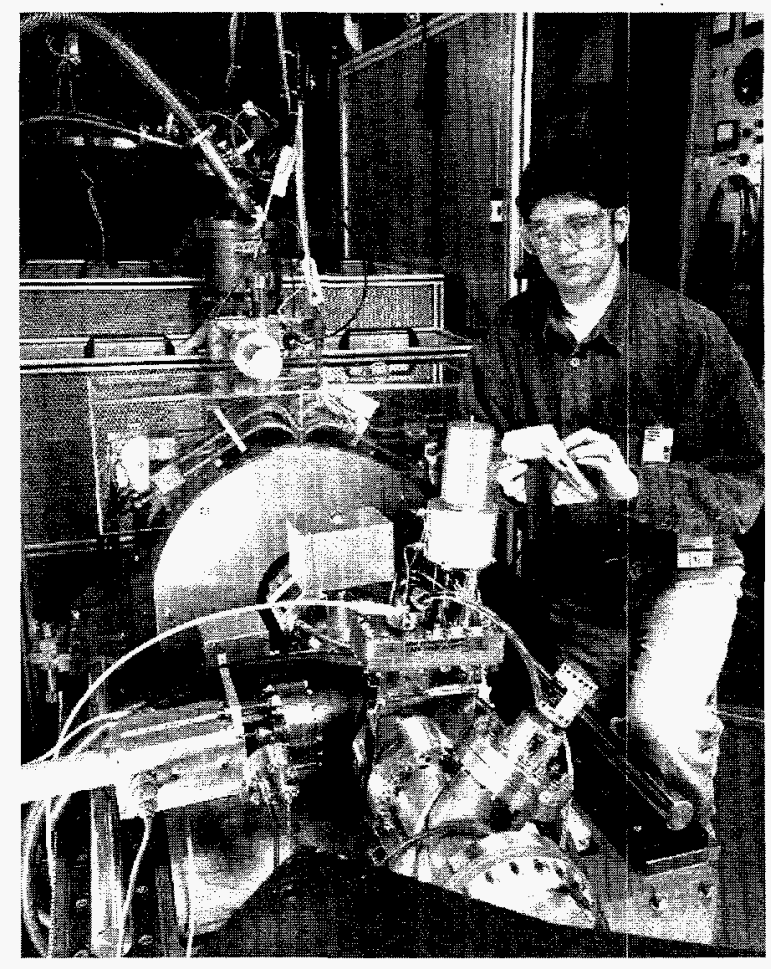

Fig. II-2 The recently completed ATLAS 14-GHz ECR ion source on its high-voltage platform.

b. Radioactive Beams via Reactions in the Beam Line (R. C. Pardo, B. E. Clifft, J. R. Specht, G. P. Zinkann, B. Harss, and K. E. Rehna)

The development of a ${ }^{17} \mathrm{~F}$ beam during 1996 is described in the Heavy Ion Research section of this report. This beam was produced via inverse kinematics $(\mathrm{p}, \mathrm{n})$ reactions with a ${ }^{17} \mathrm{O}$ primary beam and also via $(\mathrm{d}, \mathrm{n})$ with a $16 \mathrm{O}$ primary beam. The secondary beam quality and intensity are determined by the kinematics and capture efficiency after the reaction.

Currently there is a project to add a superconducting solenoid and implement an existing rebuncher cryostat near the Area III spectrograph to improve the intensity of these secondary beams. Present beam intensity is limited by the acceptance of the beam transport system and the energy spread of the secondary beam induced from the reaction kinematics. By moving the gas cell upstream of the rebuncher cryostat and redesigning the beamline to accommodate both a superconducting solenoid and a debunching resonator, we hope to increase the available ${ }^{17} \mathrm{~F}$ beam intensity by up to 10 times from the present 2 to $4 \times 10^{5}$ particles per second.

The same methods used for ${ }^{17} \mathrm{~F}$ beams can be used in the future for other radioactive beams as requested by users.

c. Radioactive Beam Diagnostics (R. C. Pardo, W. Q. Shen, B. E. Clifft, G. P. Zinkann, J. A. Nolen, and B. Harss)

A new radioactive beam, ${ }^{56} \mathrm{Ni}$, has been provided and the first experiment performed with that beam. This program is described in the Heavy Ion Research section. As part of this development, to obtain maximum acceleration efficiency, it is necessary to phase lock the pre-tandem buncher to the beam at the entrance to the linac. An "active slit" consisting of a fast-plastic scintillator coupled to a photomultiplier tube has been designed to provide the timing information for this phase lock at a position just after the normal tandem analyzing slits. The initial tests of the system are encouraging, but additional improvements are necessary to realize the full benefits. Such diagnostics for weak 
beams are of general interest for future radioactive beam development. A related project to develop a $95 \%$ transmission beam profile monitor for weak beams, based on fiber optic scintillators, is also in progress.

\title{
d. ATLAS Control System (F. H. Munson, A. Beardsley, B. J. Tieman, J. Gura, and P. Arndt)
}

The ATLAS control system, based on the commercial "VISTA" software package, is being upgraded in four overlapping phases. The end of the second phase is in sight and the start of the third phase is well underway. The end of the second phase will see the retirement of the last PDP-11 computer. Close on the heels of this accomplishment will be the goal of the third phase, which will be the retirement of the second-generation MicroVAX computer, making room for an ALPHA machine. All of these machines were or will be the primary interface to a CAMAC $\mathrm{V} / \mathrm{O}$ subsystem.

Since last year the control system high-level programs for changing the linac energy and setting up the initial linac configuration have been completed and are in routine use. The resonator 'auto-scan' program, which empirically determines the beam-resonator phase for a new, unscaled linac configuration, was also ported to the new system during 1996.
Full control of the new 14-GHz ECR ion source has been integrated into the main ATLAS Vista-based control system, rather than using a separate stand-alone control system as used for the original ECR source. In addition to the control and monitoring of typical ion source devices such as lenses, steerers, and magnets, a process that provides fully automated mass scans has been implemented. The end result of this process is a graph of the Faraday cup current versus magnetic field/current of the analyzing magnet.

The ATLAS control system has been used during the past year as a test bed for an initiative to develop an artificial-intelligence approach to beam line tuning. This initiative is being carried out through a DOE Small Business Innovative Research grant to Vista Controls.

\section{e. ATLAS Cryogenic System (J. R. Specht, B. Millar, and A. K. Reuter)}

A 20,000 gallon $\mathrm{LN}_{2}$ storage dewar obtained from the SSC was installed this year. New pressure controls, distribution line, and vaporizer were added to complete this installation. This large tank replaces a smaller rented one, thus helping to reduce operating expenses. The 20,000 gallon tank also gives ATLAS a buffer of up to 5 days between $\mathrm{LN}_{2}$ deliveries which makes us less susceptable to trucking strikes, etc.

Programmable Logic Controllers were added to four of our eight helium compressors and one wet expansion engine. These PLCs replaced many conventional and time delay relays in the compressors' electrical control systems. Better auto-start after a power bump, overall reliability, and some logging ability are the benefits of this upgrade.

Instrumentation was also added to monitor the cooling water flow and temperatures of the helium compressor cooling systems. This information is displayed on a new control system page and provides data and alarm capability when compressor or chilled water system problems develop.

A large $4 \mathrm{~kW}$ liquid helium refrigerator was obtained as surplus equipment from the SSC Laboratory through the DOE reallocation program. This system, the socalled MTL refrigerator, consists of several large compressors, coldboxes, controls, power distribution equipment, $\mathrm{LN}_{2}$ dewar (as described above), and documentation. This refrigerator is potentially large enough to replace the existing three small refrigerators at ATLAS as well as providing liquid helium for both a superconducting driver linac and the low $q / m$ superconducting injector for a future exotic beam facility based on ATLAS. Conceptual work on how to most effectively incorporate this refrigerator system into the overall plan for the proposed facility is in progress.

\section{B. ACCELERATOR PHYSICS AND LINAC DEVELOPMENT}

\author{
(J. A. Nolen, R. C. Pardo, K. W. Shepard, J. M. Bogaty, B. E. Clifft, G. P. Zinkann, \\ M. Kedzie, and W. Q. Shen)
}

This section describes the accelerator-physics program that initially developed the underlying methods which made the ATLAS facility possible. This is now an ongoing program with several related goals: one is to maintain the forefront expertise of the small group who developed the technology of $\mathrm{Nb}$ superconducting 
structures for low-beta ion accelerators. Another goal is to continue development as necessary in superconducting-linac-related specialties to keep ATLAS modern and competitive as a National User Facility. These related technologies include high-charge-state ion sources, beam diagnostics, accelerator-control systems, fast-timing techniques, cryogenics, and linac-beamdynamics studies. Much of the technology of ATLAS is of increasing interest for a possible future radioactivebeam facility and some aspects of it are also potentially of industrial significance. Research related to the production and acceleration of radioactive beams at a future advanced exotic beam facility is discussed in Section $\mathrm{C}$ below. In addition to the R\&D directed towards future facilities, radioactive beams have also been developed and have been in use since 1995 at the present ATLAS facility. The results of some of the research done with radioactive beams at ATLAS in 1996 are reported in the Heavy Ion Research Section of this Annual Report. Many of these accelerator-physics developments are fundamental and involve both undergraduate and Ph.D students, as well as postdoctoral appointees and the permanent ATLAS scientific and technical staff.

Work on the new 14-GHz ECR ion source, the control system, the cryogenics system, and radioactive beams at ATLAS is reported in Section A above. Work on superconducting $\mathrm{Nb}$ resonators and a new chopper for ATLAS is summarized below.

\section{a. Superconducting Accelerating Structures for Low-Beta Heavy-Ions} (K. W. Shepard, M. Kedzie, P. Potukuchi*, and A. Sarkar*)

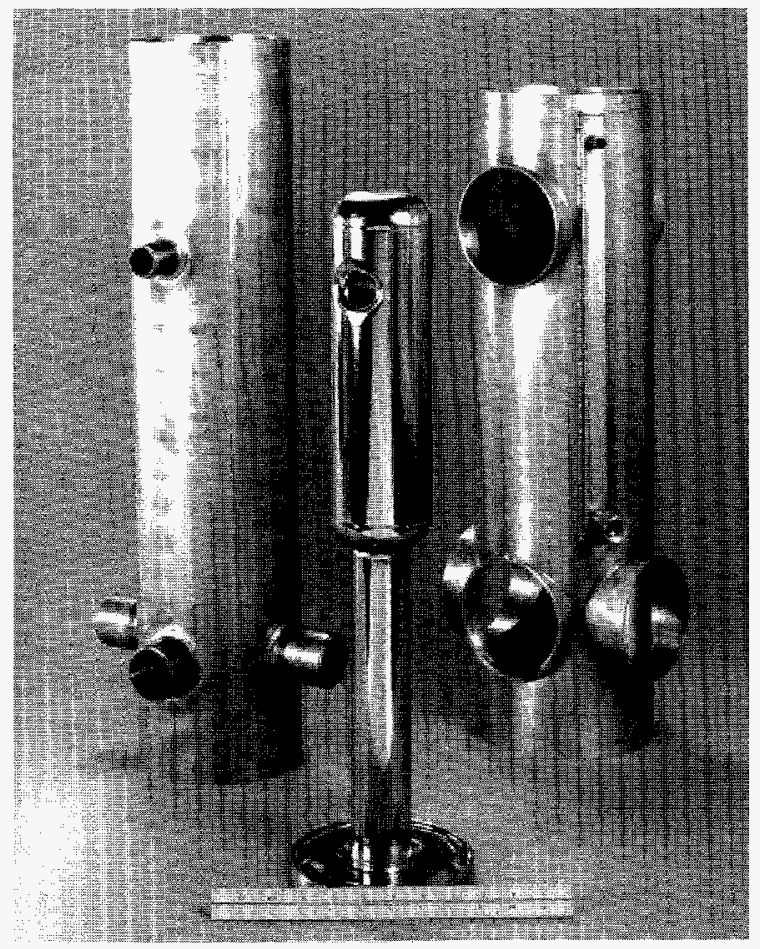

Fig. II-3. A photograph of the components of the new quarter-wave superconducting niobium resonator. developed and tested successfully in 1996.

Beginning in late 1996, the ATLAS superconducting $\mathrm{RF}$ group has been working with Sciaky, Inc. to establish a commercial capability for welding superconducting niobium cavities. Sciaky designs and markets state-of-the-art numerically controlled electron- beam welding machines, and they also have in-house capabilty for such welding. Initially it was demonstrated that the types of welds routinely used previously in the contruction of ATLAS-type resonators could be repeated successfully. At this time further development is in progress on new niobium welding techniques with emphasis on reducing cavity fabrication costs. The initial results of this development are very encouraging and indicate that it may be possible to use Sciaky's computer-controlled capabilities to reduce overall costs in mass-producing niobium cavities. This capability will be applied first to the production of 10 quarter-wave resonators for the Booster Linac at the Nuclear Science Center in New Delhi, India. In the future these methods could be applied for cost-effective fabrication of resonators for both the driver and secondary-beam injector linacs for an advanced radioactive beam facility.

During 1996 the collaborative project between the ATLAS linac development group and the Nuclear Science Centre in New Delhi, India continued and resulted in the successful completion and testing of the prototype quarter-wave niobium resonator which will be used as the basis for the fabrication of the 10 resonators mentioned above. This resonator was designed and fabricated with a new technique which eliminates the need for bonded copper-niobium material, and hence, should be more cost effective. Figure II- 3 shows the components of this type of cavity, the primary ones being the niobium inner structure and the stainless steel outer vessel. Liquid helium is contained in the space between these coaxial structures.

*Nuclear Science Center, New Delhi, India. 


\title{
b. Development of a New Type of Beam Chopper for ATLAS (J. M. Bogaty, B. E. Clifft, and R. C. Pardo)
}

During 1996 a concept for a new beam chopper for ATLAS was developed and presented at the International Linac Conference. It is an extension of the standard traveling-wave chopper which has frequently been used in the past in the beam lines for synchrotron injectors. Standard traveling-wave choppers consist of a series of deflection plates connected via inductors to form a lumped-component LC-type delay line. The new structure under development at ATLAS consists of a series of deflection plates which are impedance-matched to and interconnected via sections of coaxial delay lines.
The result is a broad-band structure which transmits nanosecond-wide square deflection pulses with very little time dispersion and can be tuned to the very slow heavy ions at the entrance velocity of the ATLAS Positive-Ion Injector $(v=0.008 c)$. If the new device works as envisioned, beam emittance degradation, as seen with the present sine-wave chopper will be greatly reduced and it can alsombe used conveniently to select beam pulses at any sub-interval of the $12-\mathrm{MHz}$ fundamental ATLAS prebunching frequency.

\section{ACCELERATOR PHYSICS RELATED TO A FUTURE ADVANCED EXOTIC BEAM FACILITY}

\author{
(B. E. Clifft, P. Decrock, E. Kanter, M. Kedzie, J. Kuzminski, J. A. Nolen, R. C. Pardo, \\ W. Sellyey, and K. W. Shepard)
}

The Argonne concept for how to implement a National ISOL Facility was developed in early 1995 and described in the working paper, "Concept for an Advanced Exotic Beam Facility Based on ATLAS," at that time. Since then efforts have been directed towards R\&D on various sub-systems of that preliminary plan. Recent progress and plans for the near future for this $R \& D$ are described here.

A design is being developed for a low-charge-state linac suitable for injecting ATLAS with radioactive beams. Initial work indicates that the existing ATLAS interdigital superconducting accelerating structures, together with superconducting quadrupole transverse focussing elements, provide a basis for a highperformance low-charge-state linac. The initial 2 or 3 MV of such a linac could be based on a normallyconducting, low-frequency RFQ. Preliminary beam dynamics studies of the whole low-charge-state postaccelerator section were carried out in early FY 1995.
During late 1995 and early 1996 a half-scale model of the first stage RFQ was constructed and evaluated. Based on those tests, the full-scale prototype of this RFQ was designed and construction was initiated in late 1996 as described in the next section.

The light-ion driver linac described in the concept presented in the 1995 Working Paper was a conventional room-temperature low-duty-cycle drift-tube accelerator. An alternative driver, a $\mathrm{CW}$ superconducting linac, is currently being investigated and is descibed in the second section below.

Other projects which are described below include measurements of charge state fractions for stripping of ions at very low energies, the design and construction of a radioactive beam ion source test stand, and the design of a new type of energy-spread-compensated highresolution isobar separator.

\section{a. Acceleration of Very Low Velocity and q/m Radioactive Ions (K. W. Shepard,} W. Sellyey, M. Kedzie, B. E. Clifft, J. A. Nolen, and J. Vincent*)

A half-scale model RFQ section was designed, constructed, and tested (without beam) in order to determine the voltage limits on $\mathrm{CW}$ operation of an RFQ with frequency and aperture suitable for a lowcharge-state, high-quality beam. Electrodynamic measurements on the model indicate that sufficiently high shunt impedance has been obtained to permit operation of a 2-meter injector section with less than 40 $\mathrm{kw}$ of RF input. In (pulsed) high-voltage tests, the $1 / 2$ scale model sustained electric fields of at least 1.25 times the Kilpatrick limit (which is $11 \mathrm{MV} / \mathrm{m}$ for the model structure), a value higher than is required for operation of the injector RFQ. The model was taken to the NSCL at Michigan State University for

*Precision Power Products Company, Williamston, MI. 
these pulsed high-voltage tests in late 1995. The results indicate that a low-frequency $(12 \mathrm{MHz}) \mathrm{RFQ}$, operated on a high-voltage platform, and injected with a pre-bunched beam, can provide ATLAS quality beams of ions of charge-to-mass ratio less than 1/132.

Based on the results with the half-scale cold model, the design of a full scale prototype was carried out during 1996. The conceptual design and beam dynamics simulations were presented at the 1996 Linac
Conference. A surplus $350-\mathrm{MHz}$ RF amplifier was obtained and is currently being modified to provide the $40 \mathrm{~kW}$ of RF power at $12 \mathrm{MHz}$ which is required to test the prototype. The prototype will first undergo electrical tests in mid-1997 and then it will be tested with krypton and xenon beams at the Dynamitron. These tests will determine the emittance acceptance of this type of RFQ as a function of ion mass. The first vane for the full-scale protype was fabricated to test the methods in late 1996, as is pictured in Figure II-4.

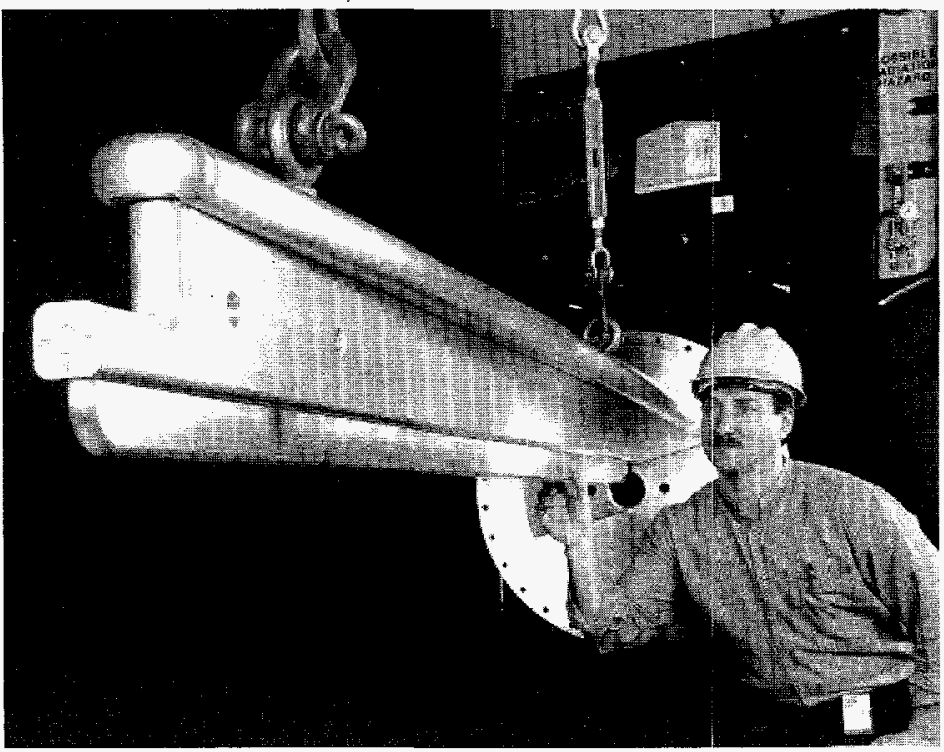

Fig. II-4. Photograph of the first vane fabricated for the full-scale prototype CW RFQ. This vane was fabricated first to verify that the necessary mechanical tolerances and alignment reproducibility could be achieved. The remaining components of the structure are now being manufactured.

b. Superconducting Driver Linac Studies (K. W. Shepard, M. Kedzie, and J. Kuzminski)

As an alternative to the conventional copper driver linac structure discussed in the working paper, we are currently investigating the feasibility of a superconducting $\mathrm{CW}$ driver. Preliminary beam dynamics simulations indicate that such a linac could be based on two types of $300-\mathrm{MHz}$ niobium resonators, optimized for ion velocities of 0.28 and $0.44 \mathrm{c}$. Prototyping of such resonators was initiated in late 1996. This class of resonators would fill the gap between those previously developed at ATLAS for lowvelocity ions and those being developed at Los Alamos, the Jefferson Laboratory, and in other countries for ions near the velocity of light. Simulations using the two types of resonators mentioned above indicate that this type of driver linac could deliver a variety of light ion beams for isotope production at the ISOL target, e.g. $208-\mathrm{MeV}$ protons, $227-\mathrm{MeV}$ deuterons, and $100-\mathrm{MeV}$ per nucleon ${ }^{18} \mathrm{O}$.

\section{c. Stripping Ions in Gas at Very Low Velocity (P. Decrock, E. Kanter,} and J. A. Nolen)

The plan presented in the 1995 Working Paper involves stripping of low-velocity radioactive ion beams after the initial acceleration in a short CW RFQ. To quantify this concept it was necessary to measure the charge state distributions for a variety of ions because there were insufficient data in the literature. Measurements were carried out on beams of krypton, xenon, and lead at velocities in the vicinity of $8 \mathrm{keV}$ per nucleon using the Physics Division's Dynamitron facility. Using a windowless gas cell, results were obtained for stripping in helium and nitrogen gas for ions initially in the $1+$ charge state. The yields for stripping krypton and xenon 
to the $2+$ charge state were 40 to $45 \%$, while those for stripping lead to the $3+$ charge state were approximately $35 \%$ when helium was the stripping medium. Yields are significantly less for stripping in nitrogen gas. By optimal choice of gas pressure, good charge-state fractions are achieved simultaneously with multiple scattering of less than $1 \mathrm{mr}$. The results of these measurements have been submitted for publication. Figure II-5 is a histogram of a charge state distribution typical of these measurements.

Fig. II-5 Charge state fractions measured for $1-\mathrm{MeV}$ lead ions in helium and nitrogen gas. The use of helium gas at these low velocities greatly enhances the fractions of certain charge states such as the $3+$ in this case.

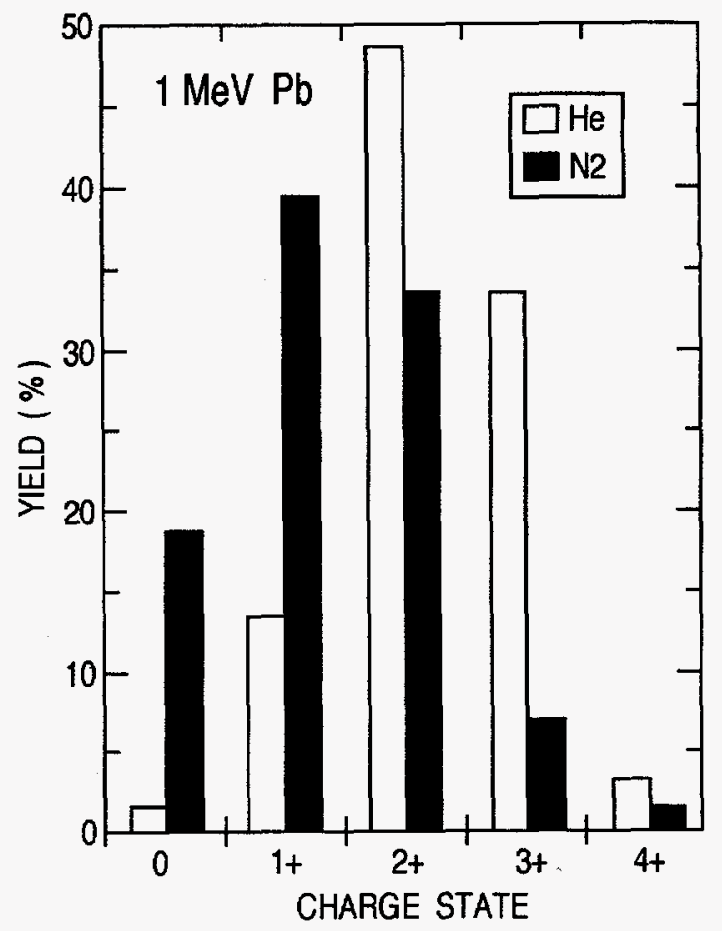

d. Ion Sources and Targetry for Use in an Advanced Radioactive Beam Facility (J. Nolen, P. Decrock, T. P. Mullen, A. Ruthenberg, T. A. Barlow, K. Beyer, M. Portillo, A. A. Geraci, S. Saremba, and C. H. Batson)

The Dynamitron is also being prepared to run in the near future as a neutron generator. The plan is to test and develop ISOL-type ion sources using radioactive fission product beams. The neutron generator target will be closely coupled to ISOL sources which contain uranium targets. The neutron generator target and ion source system will be shielded by a recently constructed steel and concrete cave. At this time the safety documentation and various approvals necessary to operate the Dynamitron as a neutron generator are nearing completion. Following commissioning of this facility in mid-1997 the ion source development program will begin.

\section{e. Ion Optics of Energy-Spread-Compensated Isobar Separators (J. A. Nolen, T. A. Barlow, A. A. Geraci, C. N. Davids, J. Berger, and M. Berz*)}

A new class of energy-spread-compensated (ESC) separators has been proposed recently by Wollnik 1 . Traditionally ESC mass separators have been realized by combining magnetic dipole dispersion with electric dipole or Wien filter elements to cancel energy dispersion while maintaining mass dispersion. The new idea is to construct the mass separator from two magnetic sectors with a beam acceleration or deceleration section between them. Wollnik ${ }^{1}$ has pointed out several potential advantages of the new scheme for high resolution mass separators at isotopeseparator-on-line (ISOL) facilities. We have done preliminary ion-optical simulations to fifth order for a separator with first-order mass resolving powers (baseto-base) of 50,000 and 20,000 for beam emittances of $4 \pi \mathrm{mm}-\mathrm{mr}$ and $10 \pi \mathrm{mm}-\mathrm{mr}$ emittances, respectively. This type of mass separator is a candidate for use at a future ISOL-type radioactive beam facility. It consists of two magnetic dipole sections separated by beam deceleration. The ions have $100-\mathrm{keV}$ kinetic energy in the first magnetic section and $10 \mathrm{keV}$ in the second. The simulations are being done to fifth order with the code COSY Infinity including fringe-field effects of the dipoles and quadrupoles and the aberrations of the

*Michigan State University.

${ }^{1}$ H. Wollnik, University of Giessen preprint, December, 1996. 
decelerating section. It was assumed that the $100-\mathrm{keV}$ ions had an energy spread of $\pm 10 \mathrm{eV}$, and the effects of voltage variation of the $100-\mathrm{keV}$ power supply were separately simulated. Overall mass resolving powers of $20,000 \mathrm{FWHM}$ are obtained with emittances as large as $10 \pi \mathrm{mm}-\mathrm{mr}$ (object slit width of $1 \mathrm{~mm}$ ). To do these simulations, including the effect of the decelerating electrostatic lens, it was necessary to add such an ion optical element ("immersion lens") to COSY Infinity. Studies of such systems will continue through 1997, including optimization of first-order layouts and comparisons with traditional ESC separators. 


\section{MEDIUM-ENERGY NUCLEAR PHYSICS RESEARCH}

\section{OVERVIEW}

In order to understand how to incorporate the quark-gluon structure of the nucleon into a fundamental description of nuclear forces, the medium energy research program in the Argonne Physics Division emphasizes the study of processes in nuclei in which interactions with the constituents of the nucleon describe the basic physics. Examining related physics topics at both the quark and hadronic levels in complementary experiments is most revealing of the low energy structure of the strong interactions. Because energetic leptons provide an accurate wellunderstood probe of these phenomena, primary emphasis is placed on the experiments involving electron and muon scattering.

The electron beams of Jefferson Lab are ideally suited for studies of nuclei at hadronic scales and represent one center of the experimental program. Staff members led in the construction of experimental facilities, serve as spokesman for five experiments and are actively involved in five others. The group has completed construction of the broad purpose Short Orbit Spectrometer which forms half of the coincidence spectrometer pair that is the primary base experimental equipment in Hall C. Argonne led the first experiment to be carried out at Jefferson Lab and completed parts of three other experiments in FY 96 and FY 97. We anticipate running an average of two Jefferson Lab experiments a year for the next several years in either Hall C or Hall A.

The first results from Jefferson Lab build on previous ANL experiments at SLAC and MIT-Bates. For the first time, forward and backward angle measurements of the (e,e'p) reaction for a range of nuclear targets will allow a longitudinaltransverse separation over a broad range of missing energy and momentum in a study of proton propagation in nuclei and the role of one and two body mechanisms in the quasifree knockout process. Exclusive deuteron photodisintegration has identified this reaction as the only known nuclear process to obey the quark-counting-rules scaling arguments at large transverse momenta. These measurements were pushed to photon energies of $4.0 \mathrm{GeV}$ at Jefferson Lab and will be extended to higher energies in the future as the accelerator is upgraded. Measurements of kaon production on deuterium and hydrogen provide important information on the basic strangeness production mechanisms, the poorly known hyperon-nucleon interaction, and the electromagnetic form factor of the $\mathrm{K}^{+}$. In the future these experiments will measure hypernuclear states on fewbody hypernuclei as the first step in a hypernuclear physics program at Jefferson Lab. 
The most direct way to probe the quark structure of nuclei is to use a high-energy probe which interacts with a single quark at a time. After a ten year program, analysis of deep inelastic muon scattering of $490-\mathrm{GeV}$ muons in experiment E665 at FNAL is drawing to a close. New results have been obtained on the gluon distribution of the nucleon and the exclusive production of vector mesons such as the $\rho$ meson. Analysis of high statistics $\rho$ production on hydrogen supports our previous interpretation of the nuclear dependence of exclusive $\rho$ production that one can independently control the transverse and longitudinal energy scales of the reaction and study of color transparency in these processes. A new experiment, E866 is underway at FNAL to directly measure the difference between the $\bar{u}$ and $\overline{\mathrm{d}}$ distribution in the nucleon by measuring the production of high mass virtual photons (the Drell-Yan process) in $800-\mathrm{GeV} p+p$ and $\mathrm{p}+\mathrm{d}$ collisions. Extensions of this experiment in FY 97 include tests of QCD and the reaction mechanism in studies of the angular correlations of the muon pairs and extending the kinematic ranges of measurements of the nuclear dependence of Drell-Yan, charmonium and bottomonium states.

HERMES, a broadly based North American-European collaboration, is studying the spin structure of the nucleon using internal polarized targets in the HERA storage ring at DESY. The first production run measured polarized positron scattering on polarized ${ }^{3} \mathrm{He}$ nuclei to focus on the spin distribution of the neutron. In FY 96 and FY 97, measurements on polarized hydrogen focus on the proton distributions. Argonne has concentrated on the hadron particle identification of HERMES, a unique capability compared to other spin structure experiments. The HERMES collaboration has identified as their highest priority for the apparatus a major upgrade of the hadron particle identification. This will allow the experiment to make decisive tests of the flavor dependence of the spin distributions and begin measurements of the spin contributions of the glue, the major unknown at present. Prototypes of the mirrors for a ring imaging Cerenkov counter (RICH) have been constructed by ANL and measurements of the radiator performance have to be performed by other HERMES groups to validate the RICH concept.

Argonne scientists have extended the physics program at HERMES to begin the first studies of exclusive vector meson production in polarized-electron-beam polarized-target experiments. These measurements will also provide insight into the phenomenon of color transparency in an important new kinematic range and with statistical precision exceeding what was possible at FNAL.

Considerable technical resources of the medium energy program were devoted to developing a new technology for producing polarized hydrogen and deuterium targets for storage ring experiments. Significant progress has been made in establishing stable operation of laser-driven spin exchange targets and measuring the nuclear polarization. A new diagnostic technique directly measures the alkali density in the pumping cell for a more complete characterization of the spin exchange dynamics. New cell geometries promise more efficient coupling of the alkali and hydrogen atomic spins for higher average polarizations. A demonstration experiment is underway at the IUCF to operate a laser driven target for measurements of $\vec{d}(\vec{p}, p p) n$ reactions. 


\section{A. SUBNUCLEONIC EFFECTS IN NUCLEI}

a. 1. The Energy Dependence of Nucleon Propagation in Nuclei as Measured in

the (e,e'p) Reaction (D. F. Geesaman, K. Bailey, W. J. Cummings, J. Fedchak,J.-O. Hansen, H. E. Jackson, C. Jones, S. Kaufman, T. G. O'Neill, D. Potterveld, J. Reinhold, J. P. Schiffer, B. Zeidman, J. Arrington,* A. Lung,* D. Abbott, $\dagger$ R. Carlini, $\dagger$ J. Dunne, $\dagger$ R. Ent, $\uparrow$ D. Mack,,$\uparrow$

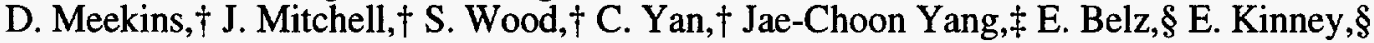

D. van Westrum,§ P. Markowitz,I K. A. Assamagan,\| O. K. Baker,\| K. Beard,\| J. Cha,॥ T. Eden,\|

P. Gueye,\| C. Keppel,\| R. Madey,\| G. Niculescu,\|l. Niculescu,\|l L. Tang,\| Wooyong Kim,**

C. Bochna, $\dagger \dagger$ H. Gao, $\uparrow \dagger$ R. Holt, $\dagger \dagger$ M. Miller, $\dagger \dagger$ A. Nathan,,$\dagger$ B. Terburg,,$\dagger \dagger$ A. Ahmidouch, $\neq \ddagger$

R. Suleiman,

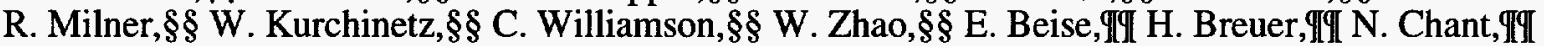

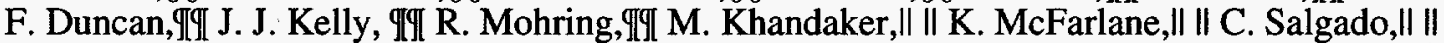

S. Beedoe, $\left.\right|^{* * *}$ S. Dangoulian, *** C. Jackson, $* * *$ D. Dutta, $\dagger \dagger \dagger$ R. E. Segel, $\dagger \dagger \dagger$ Pat Welch, $+\neq \ddagger$

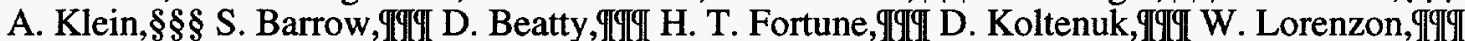

J. Yu, GITI V. Frolov, || || || J. Prince, || || || P. Stoler, || || || R. Gilman, |**** J.-E.Ducret, $\dagger^{\dagger} \dagger^{\dagger}$

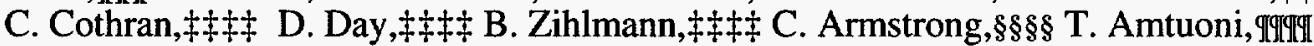

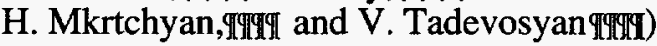

Nuclear Transparency in the Quasifree ( e,e'p) Reaction

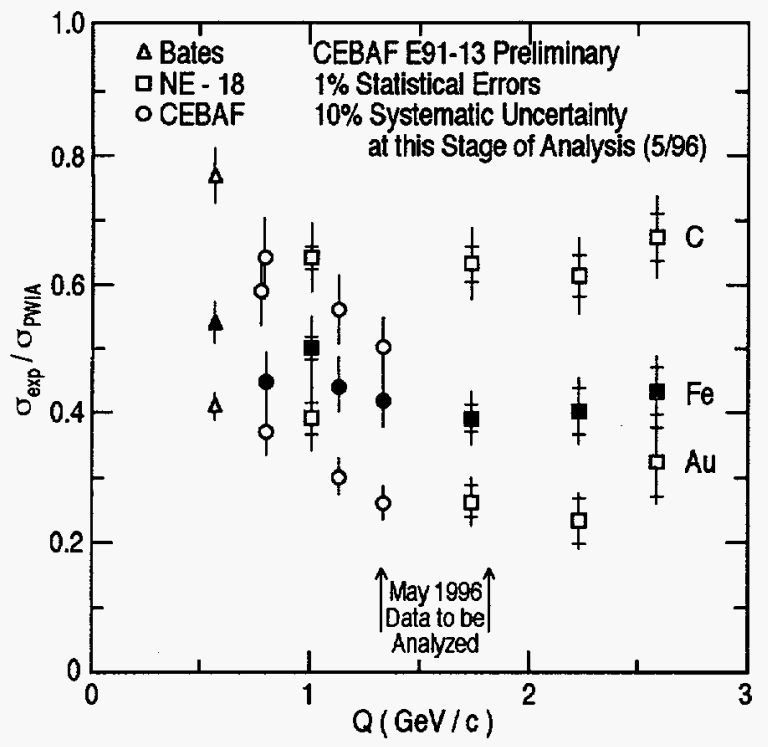

Fig. III-1. Preliminary results for $T=\sigma_{\text {exp }} / \sigma_{P W I A}$ for quasielastic ( $e, e^{\prime} p$ ) scattering from $\mathrm{C}, \mathrm{Fe}$ and Au targets. The arrows indicate the $Q$ values of the spring 1996 data which are still being analyzed. Previous results are shown in Ref. I (Bates) and Ref. 2 (NE18).
ANL led the first experiment to be carried out at CEBAF in November-December 1995. This experiment continued the ANL work at MIT ${ }^{1}$ and SLAC ${ }^{2}$ using the (e,e'p) reaction to study the propagation of 0.35 to $1.80-\mathrm{GeV}$ protons through nuclear material. The Hall $\mathrm{C}$ collaboration selected this experiment as one of the two commissioning experiment. Electrons were detected in the HMS 6-GeV spectrometer and protons were detected in the SOS $1.8-\mathrm{GeV}$ spectrometer. The experiment utilized CEBAF beams of $0.845,1.645,2.445$ and $3.245 \mathrm{GeV}$ with up to $25 \mu \mathrm{A}$ intensity on targets of $\mathrm{C}, \mathrm{Fe}$ and $\mathrm{Au}$. Full commissioning studies of each spectrometer were performed to calibrate this experiment and to serve as a baseline for future experiments.

In this first run over 4 million quasifree electron-proton coincidence events were accumulated along with 16 million singles events for normalization. The $\mathrm{Q}^{2}$ points of 0.64 , 1.3 and $1.8(\mathrm{GeV} / \mathrm{c})^{2}\left(\mathrm{~T}_{\mathrm{p}}=0.350,0.700\right.$ and $\left.0.970 \mathrm{GeV}\right)$ span the threshold for pion production in p-p collisions where the nature of the p-p cross section changes from dominantly elastic to dominantly inelastic. At the lowest $\mathrm{Q}^{2}$, data were taken at two values of the virtual photon polarization to examine the separate contributions of longitudinal and transverse photon exchange.

*Caltech., †CEBAF, $\ddagger$ Chungnam Nat. Univ., Taejon, Korea, §Univ. of Colorado, qFlorida International Univ., IIHampton Univ. **Kyungpook Natl Univ., Taegu, S. Korea, ††Unive. of Illinois, Urbana, $\$$ Kent State Univ., $\S \S M I T$, TYUniv. of Maryland, II IINorfolk State Univ., ***North Carolina A\&T Univ., †††Northwestern Univ. $\ddagger \ddagger \ddagger$ Oregon State Univ., §§§Old Dominion Univ., GTYUUniv. of Pennsylvania, II II IIRensselaer Polytechnic Institute,

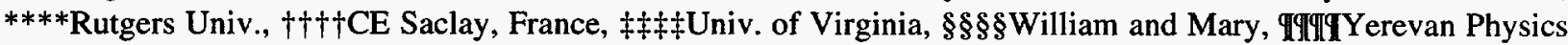
Institute, Armenia.

${ }^{1}$ G. Garino et al. , Phys. Rev. C45, 780 (1992), ${ }^{2}$ T. O'Neill et al., Phys. Lett. B351, 87 (1995) 
This L/T separation will address the role of two-body and many-body photon absorption mechanisms in the quasifree region. In all aspects the experimental equipment performed as expected. The statistics, kinematic coverage and experimental resolution significantly exceed those of previous results. This data set should provide precise measurements of nuclear transparency as well as a broad survey of nuclear spectral functions from recoil momentum of 0 to 300 $\mathrm{MeV} / \mathrm{c}$ and missing energy of 0 to $150 \mathrm{MeV}$.
In spring 1996, the last phase of the experiment took backward angle data at $T_{p}$ of $0.970 \mathrm{GeV}$ and a higher $\mathrm{Q}^{2}$ point at $\mathrm{T}_{\mathrm{p}}$ of $1.80 \mathrm{GeV}$. First results of a preliminary analysis of the 1995 data were reported at the PANIC 96 conference. The average ratio of the experimental results to plane wave impulse approximation calculations are shown in Figure III-1. Continued study of the spectrometer optics and acceptance should reduce to the systematics errors to the $1-2 \%$ level.

a.2. Measurements of Deuteron Photodisintegration up to $4.0 \mathrm{GeV}$ (W.J. Cummings, H. Gao, D.F. Geesaman, J-O. Hansen, H.E. Jackson,T.G. O'Neill, D.H. Potterveld, J. Reinhold, B. Zeidman, D.J. Abbott, * A. Ahmidouch, $\uparrow$ C.S. Armstrong, $\ddagger$ J. Arrington, $\S$ K.A. Assamagan, $\mathbb{1}$ O.K. Baker, II S.P. Barrow,II D.P. Beatty,ll D.H. Beck,** S.Y. Beedoe, $\dagger \dagger$ E.J. Beise, $\ddagger \ddagger$ J.E. Belz,§§ C.W. Bochna, ${ }^{* *}$ P.E. Bosted, II II E.J. Brash, *** H. Breuer, 括 R.V. Cadman, ** L. Cardman,* R.D. Carlini,* J. Cha, II N.S. Chant, $\neq \ddagger$ G. Collins, $\neq+$ C. Cothran,,$\dagger \dagger$ S. Danagoulian, $\dagger \dagger$ F.A. Duncan, T.A. Forest, ** H.T. Fortune, || V.V. Frolov, §§§ R. Gilman, |l || || P.L.J. Gueye,II K.K. Gustafsson, M. Harvey,I W. Hinton,II R.J. Holt,** C.E. Keppel, , M.A. Khandaker, ${ }^{* * * *}$ E.R. Kinney, $\uparrow \uparrow \uparrow \uparrow \dagger$ A. Klein, $\neq \ddagger \ddagger \ddagger$ D.M. Koltenuk,\| A.F. Lung, $\neq \ddagger$ D.J. Mack, ${ }^{*}$ R. Madey, $\dagger$ P. Markowitz, $\S \S \S \S$ K.W. McFarlane, $* * * *$ R.D. McKeown, D.G. Meekins, $\ddagger$ Z-E. Meziani, II II II I M.A. Miller,** J.H. Mitchell,* H.G. Mkrtchyan,***** R.M. Mohring,捀 J. Napolitano, $\S \S$ A.M. Nathan, ${ }^{* *}$ G. Niculescu, I I. Niculescu,II B.R. Owen, ${ }^{* *}$ S.F. Pate, $\dagger_{\dagger}^{\dagger} \dagger \dagger \dagger$ J.W. Price, $\$ \S \S$ G.L. Rakness, $\dagger \dagger \dagger \dagger$ R. Ransome, $\|$ || || P.M. Rutt, \|\|$\|$ G. Savage, I R.E. Segel, $+\neq \ddagger$ N. Simicevic,**

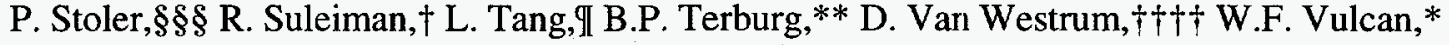

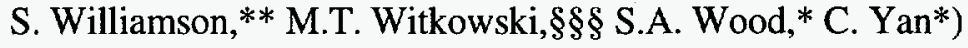

Constituent-counting-rule behavior has been observed previously in high energy proton-proton scattering and photo-meson productions from the proton. Only one nuclear reaction has thus far exhibited this behavior. Argonne experiments NE8 and NE17 at SLAC have shown that the deuteron photodisintegration, $d(\gamma, p) n$ reaction at a proton center-of-mass angle of $90^{\circ}$ starts to show the scaling behavior at the unexpectedly low photon energy of around $1.0 \mathrm{GeV}$. Extending the SLAC measurements to higher photon energies and performing a detailed angular distribution study is essential to identify the limits of the kinematic regime of this behavior and to investigate the underlying mechanisms. TJNAF experiment E89-012 was one of the two Hall $\mathrm{C}$ commissioning experiments performed in the spring of 1996. Experiment E89-012 performed the first measurements of the differential cross section for $\mathrm{d}(\gamma, \mathrm{p}) \mathrm{n}$ up to $4.0 \mathrm{GeV}$ at center of mass angles of $37^{\circ}, 53^{\circ}$ and $90^{\circ}$. The $\mathrm{d}(\gamma, \mathrm{d}) \pi^{0}$ reaction was also studied at $E_{\gamma}:=0.8-3.2 \mathrm{GeV}$. The preliminary $\mathrm{d}(\gamma, \mathrm{p}) \mathrm{n}$ cross section results at $37^{\circ}$ and $90^{\circ}$ were reported at the PANIC96 conference. These results are in good agreement with previous measurements at lower energies. The $90^{\circ}$ (Fig. III-2) data continue to show the constituent-counting-rule behavior up to $4 \mathrm{GeV}$ and are also in fair agreement with an asymptotic mesonexchange model calculation. ${ }^{1}$ The $37^{\circ}$ data do not show clear signs of the counting rule behavior, but still do not extend to the region of transverse momenta where the $90^{\circ}$ data scale. The collaboration's proposal to extend the forward angle measurements to $5.5 \mathrm{GeV}$ at TJNAF was approved with high scientific priority by TJNAF PAC11 and is expected to run in 1998.

\footnotetext{
*Thomas Jefferson National Accelerator Facility, †Kent State Univ., $¥$ College of William and Mary. §California Institute of Technology, \$Hampton Univ., IUniv. of Pennsylvania, **Univ. of Illinois, Champaign, $\dagger$ † North Carolina A\&T State Univ., $\ddagger \ddagger U$ University of Maryland,§§TRIUMF, II IIAmerican Unive., ***Univ. of

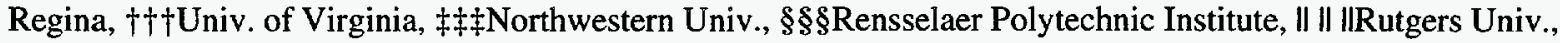

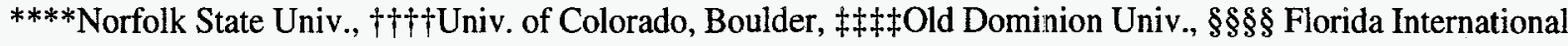
Univ., II II II IITemple Univ., *****YerevanPhysics Institute, $\uparrow^{\dagger}+\dagger \dagger$ New Mexico State Univ.

${ }^{1}$ S.I. Nagornyi, Yu. A. Kasatikin, and I.K. Kirchenko, Sov. J. Nucl. Phys. $\underline{55}, 189$ (1992).
} 


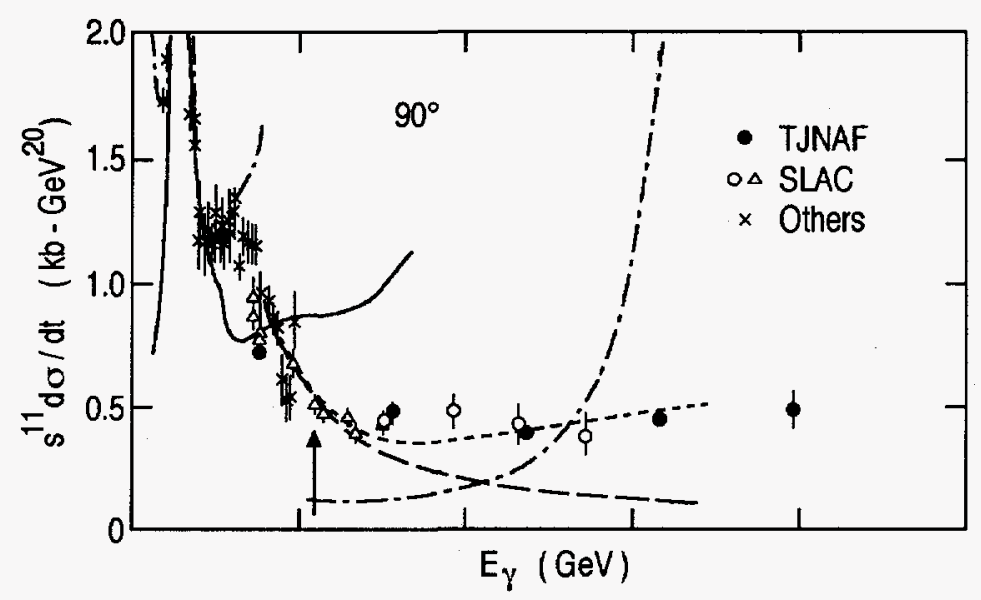

Fig. III-2. Preliminary results for ${ }^{11} d \sigma / d t v s . E_{\gamma}$ The present work is shown as the solid circles and statistical uncertainties only. SLAC NE8 and NE17 data are shown as the open triangles and open circles respectively. The solid line is the meson-exchange calculation of Lee, and the dot-dashed curve is a meson-exchange calculation of Laget. The short dashed line is an asymptotic meson-exchange calculation. ${ }^{I}$ The long dashed curve is a reduced nuclear amplitude analysis of Brodsky and Hiller.

a.3. Electroproduction of Kaons and Light Hypernuclei (K. Bailey, W. J. Cummings, H. Gao, D. F. Geesaman, J.-O. Hansen, H. E. Jackson, T. G. O'Neill, D. Potterveld, J. Reinhold, J. P. Schiffer, B. Zeidman, and E91-016 Collaboration)

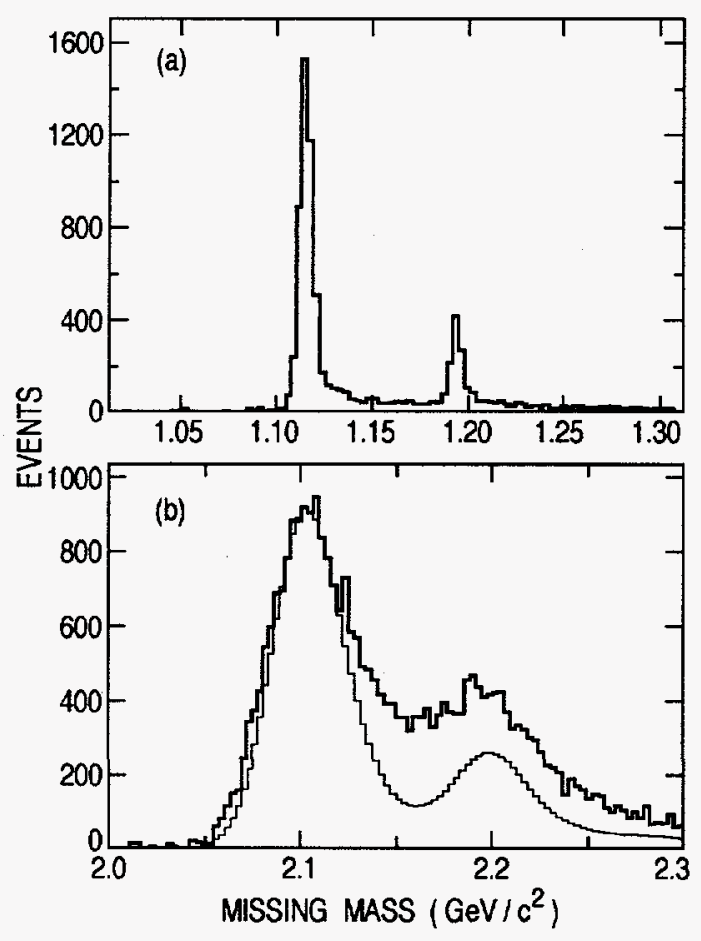

Figure III-3. Preliminary missing mass spectra for $\left(e, e^{\prime} K^{+}\right)$reactions on targets of hydrogen (a) and deuterium (b). The peaks in the upper part of the figure at 1.116 and $1.192 \mathrm{GeV} / \mathrm{c}^{2}$ correspond to $\Lambda$ and $\Sigma^{\circ}$, respectively. The thin line in the lower section results merely from broadening the hydrogen data with a Gaussian function to illustrate the presence of contributions from the neutron in deuterium.
In TJNAF experiment E91-016, Electroproduction of Kaons and Light Hypernuclei, quasifree electroproduction of $\mathrm{K}^{+}$on deuterium and hydrogen has been measured at incident electron energies of 3.245 and $2.445 \mathrm{GeV}$ with coincident detection of the emergent $\mathrm{e}$ and $\mathrm{K}^{+}$in the HMS and SOS magnetic spectrometers in Hall $\mathrm{C}$. Angular distributions for the reactions $\mathrm{D}\left(\mathrm{e}, \mathrm{e}^{\prime} \mathrm{K}^{+}\right) \mathrm{YN}$ and $\mathrm{H}\left(\mathrm{e}, \mathrm{e}^{\prime} \mathrm{K}^{+}\right) \mathrm{Y}$, where $\mathrm{Y}$ is either $\Lambda$ or $\Sigma$, were studied at forward angles with respect to the virtual photon for $Q^{2}=0.376$ and 0.50 $(\mathrm{GeV} / \mathrm{c})^{2}$. Particle identification utilizing time-of-flight techniques together with Aerogel Cerenkov detectors yield clean missing mass spectra, such as those shown in Fig. III-3 and allow subtraction of random backgrounds. Roughly half of the approved beam time was used during the production runs late in $\mathrm{CY}$ 1996; the balance of the experiment, $\left(e, e^{\prime} K^{+}\right)$on ${ }^{3,4} \mathrm{He}$ is expected to run in 1998 . Missing mass spectra obtained from preliminary analysis of the deuteron data, e.g. Fig. III-3b, show two broad peaks arising from quasifree $\Lambda$ production on the proton and $\Sigma$ production on both the proton and neutron. Fermi motion within the deuteron broadens these peaks relative to the those shown in Fig. III-3a for hydrogen at the same kinematic settings. Subtraction of the proton yield, suitably broadened and normalized, from the deuteron data will allow extraction of the cross section for kaon production on the neutron. One of the aims of 
experiment 91-016, is the elucidation of the basic hyperon-nucleon interactions in nuclei. Inasmuch as both the electron and $\mathrm{K}^{+}$are particles that interact relatively weakly with nucleons, kaon electroproduction provides a low-distortion method for investigating the fundamental interactions between nucleons, lambdas, and sigmas in few-body systems. Analyses of these data are expected to provide new information on the relative phases and low energy behavior of hyperonnucleon interactions, cross sections for hyperon production and evidence of interference between competing scattering amplitudes. During the course of E91-16, it was observed that the beam energies, kinematic settings of the spectrometers and particle identification were appropriate for study of the $H(e, e ' p) \omega$ reaction. Cross sections for the production of $\omega$ mesons over much of the angular range will be obtained from analysis of the recoil proton spectra obtained during the hydrogen runs. In addition, some data were obtained for the ${ }^{12} \mathrm{C}\left(\mathrm{e}, \mathrm{e}^{\prime} \mathrm{K}^{+}\right)$reaction in order to evaluate the feasibility of a program to study bound hypernuclei, E89-09.

E91-016 will provide data for the Ph.D. theses of students at Hampton University, University of Pennsylvania, and Temple University.

\section{a.4. Momentum Transfer Dependence of $\mathbf{H}\left(\mathbf{e}, \mathbf{e}^{\prime} \mathrm{K}^{+}\right) \mathrm{Y}$ Reactions (K. Bailey, W. J. Cummings, H. Gao, D. F. Geesaman, J.-O. Hansen, H. E. Jackson, T. G. O'Neill, D. Potterveld, J. Reinhold, J. P. Schiffer, B. Zeidman, and E93-18 Collaboration)}

In TJNAF experiment E93-18, the momentum transfer dependence of the reaction $\mathrm{H}\left(\mathrm{e}, \mathrm{e}^{\prime} \mathrm{K}^{+}\right) \mathrm{Y}$, where $\mathrm{Y}$ is $\Lambda$ or $\Sigma^{\circ}$, was studied at $\mathrm{Q}^{2}=0.5,1.0,1.5$, and 2.0 $(\mathrm{GeV} / \mathrm{c})^{2}$ at incident electron energies ranging from 2.445 to $4.045 \mathrm{GeV}$. The HMS and SOS spectrometers in Hall $\mathrm{C}$ were used for detection of the emergent electrons and $\mathrm{K}^{+}$, respectively. For each $\mathrm{Q}^{2}$, data were obtained at three different values of the polarization of the virtual photon in order to separate the longitudinal and transverse parts of the cross section. In addition to providing comprehensive information on the fundamental reaction involved in $\Lambda$ and $\Sigma^{\circ}$ production, the experiment should allow a measurement of the kaon form factor. Together with data on the neutron (E91 16), this study is vital for understanding processes that lead to production of bound hypernuclei. Students from Hampton University and the University of Maryland will utilize data from E93-18 for doctoral theses.

\section{a.5. A Study of Longitudinal Charged-Pion Electroproduction in $\mathrm{D},{ }^{3} \mathrm{He}$, and ${ }^{4} \mathrm{He}$}

(H. E. Jackson, W. Cummings, D. F. Geesaman, J.-O. Hansen, C. E. Jones, S. Kaufman,

D. Potterveld, T. G. O'Neill, B. Zeidman, D. Mack,* J. Mougey, $\dagger$ B. Saghai, $\dagger$

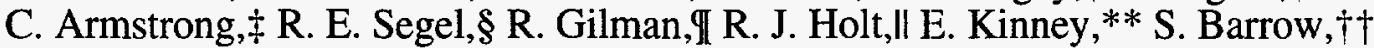

P. Beatty, $\uparrow \dagger$ H. T. Fortune, $\uparrow \dagger$ D. Koltenuk, $\uparrow \dagger$ W. Lorenzon, $\uparrow \dagger$ T. Amatuni,

H. Mkrtchyan,

Studies of pion electroproduction on the deuteron carried out by the ANL group at ALS, Saclay, in the 1980 's show that even in the weakly-bound deuteron, multinucleon processes alter the electro-production amplitudes in the forward direction. The data provide the first experimental indications for a significant change in the effective pion-nucleon coupling for nucleons bound in nuclei. It is clear that forward-angle electroproduction may be a sensitive probe of the properties of the pion coupling in the nuclear medium. At CEBAF, we will study longitudinal charged-pion electroproduction (in the excitation region below the delta isobar) along the direction of the momentum transfer where the charge scattering process dominates. Direct comparison of the cross section per nucleon in deuterium and the helium isotopes with the experimental value for the free nucleon will provide estimates of the strength of the nuclear pion field. A Rosenbluth separation of the longitudinal and transverse cross sections will be performed for fourmomentum transfers of 2.5 and $10 \mathrm{fm}^{-2}$. Measurements for a number of light nuclei will provide useful data on the sensitivity of longitudinal electroproduction to nuclear binding effects. If current conceptions of pion-exchange currents in nuclei are correct, longitudinal electroproduction will be suppressed at the lower momentum

*CEBAF, †CEN Saclay, France, $\ddagger$ College of William and Mary, §Northwestern University, IRutgers University, IUniversity of Illinois at Champaign-Urbana, **University of Colorado, $\dagger †$ University of Pennsylvania, $\$+$ Yerevan Physics Institute, Armenia 
transfer and enhanced at the higher momentum transfer by multinucleon processes. If on the other hand, as suggested by recent data from Drell-Yan studies of antiquark structure functions, there is no such enhancement, a reformulation of pion exchange models of the medium- and short-range properties of nuclear forces will be required. The experiment is scheduled to run in Fall 1997 at CEBAF. This is one of the experiments that highlighted the need for a relatively short spectrometer for unstable particles at CEBAF. The ANL Medium-Energy group responded to this need by constructing the short-orbit spectrometer (SOS) as the hadron arm in Hall $\mathrm{C}$.

\section{a.6. Precise Measurements of the Inclusive Spin-dependent Quasi-elastic Transverse Asymmetry A T' from ${ }^{3} \overrightarrow{\mathrm{He}}\left(\overrightarrow{\mathrm{e}}, \mathrm{e}^{\prime}\right)$ at Low $Q^{2}$} (W. Cummings, H. Gao, D.F. Geesaman, J.-O. Hansen, H.E. Jackson, T.G. O'Neill, D.H. Potterveld, J. Reinhold, T. Averett, ${ }^{*}$ B.W. Filippone, ${ }^{*}$ E. Hughes, * R.D. McKeown, $*$ M. Pitt, * B. Anderson, $\dagger$ G.G. Petratos, $\dagger$ J. Watson, $\dagger$ G.D. Cates, $\ddagger$ K. Kumar, $\ddagger$ P. Bgorad, $\ddagger$ H. Middleton, $\ddagger$ L. Bimbot, $\S$ C. Glashausser $§$ R. Gilman, $\S$

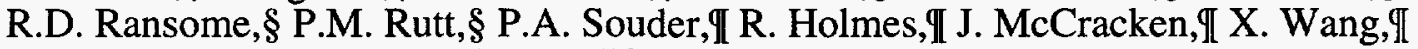
L. Auerbach,\|l Z.-E. Meziani,\|l K. Slifer,\| L.S. Cardman,** J.P. Chen, ** J. Gomez,** Kees de Jager,** M. Liang,** J. LeRose, ${ }^{* *}$ R. Michaels, ${ }^{* *}$ E. Offerman, ${ }^{* *}$ S. Nanda, ** A. Saha, ${ }^{* *}$ B. Wojtsekhowski, ${ }^{* *}$ D. Dale, $\dagger \dagger$ W. Korsch, $\dagger \dagger$ D.H. Beck,,$\ddagger$

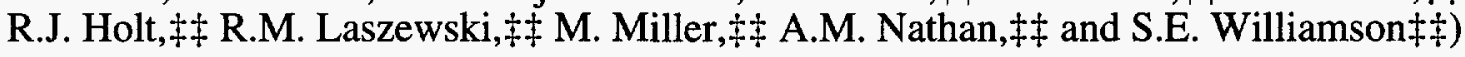

Polarized ${ }^{3} \mathrm{He}$ targets have proven to be valuable tools for studying the electric and magnetic form factors of the neutron, and the spin structure of the neutron. We proposed to systematically measure the inclusive ${ }^{3} \mathrm{He}$ quasielastic transverse asymmetry, $\mathrm{A}_{\mathrm{T}}{ }^{\prime}$ at $\mathrm{Q}^{2}=$ $0.1,0.2,0.3,0.4,0.5(\mathrm{GeV} / \mathrm{c})^{2}$ with high statistical and systematic accuracy in Hall $A$ at TJNAF (formerly CEBAF). This experiment was approved for 15 days by TJNAF PAC10. The precise data will constrain the theoretical calculations of the ${ }^{3} \mathrm{He}$ inclusive quasielastic asymmetries and also permit the extraction of neutron magnetic form factors from the measured transverse asymmetries with unprecedented precision. The target is a high density polarized ${ }^{3} \mathrm{He}$ gas target based on the principle of spin-exchange optical pumping of rubidium atoms. This type of target has been used previously at SLAC. However, the target proposed for TJNAF is significantly $(10 \mathrm{~cm})$ longer than the SLAC targets and the TJNAF beam conditions are very different from the pulsed electron beams from other laboratories. An in beam test of the cell at TJNAF is required to verify the final design for the polarized ${ }^{3} \mathrm{He}$ program in Hall A. The Argonne Medium Energy Group is leading the effort to carry out this initial test in Hall C in the spring of 1997.

*California Institute of Technology, †Kent State University, $\ddagger$ Princeton University, §Rutgers University, ISyracuse University, IITemple University, ${ }^{* *}$ TJNAF, $\nmid \dagger$ University of Kentucky at Lexington, $\ddagger \ddagger$ University of Illinois, Urbana 
a.7. Measurement of the Neutron $\left({ }^{3} \mathrm{He}\right)$ Spin Structure Function at Low $\mathbf{Q}^{2}$ :

A Connection Between the Björken and Gerasimov-Drell-Hearn Sum Rules

(W. Cummings, H. Gao, D.F. Geesaman, J.-O. Hansen, H.E. Jackson, T.G. O'Neill, D.H. Potterveld, J. Reinhold, T. Averett,* B.W. Filippone, * E. Hughes,*

R.D. McKeown, * B. Anderson, $\uparrow$ G.G. Petratos, $\dagger$ J. Watson, $\uparrow$ W. Korsch, $\ddagger$

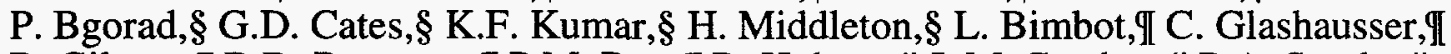

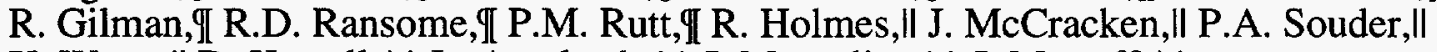

X. Wang,ll D. Kawall,** L. Auerbach, $\dagger \dagger$ J. Margulies, $\uparrow \dagger$ J. Martoff, $\uparrow \dagger$

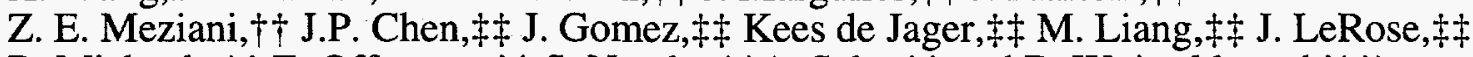

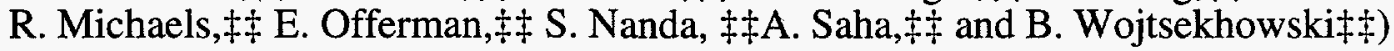

At $\mathrm{Q}^{2}=0$, the real photon limit, an important sum rule known as the Gerasimov-Drell-Hearn (GDH) sum rule has been the focus of renewed interest. The GDH sum rule is expressed using the total helicity dependent nucleon photoabsorption cross sections $\sigma_{\mathrm{N}}^{1 / 2}$ and $\sigma_{\mathrm{N}}^{3 / 2}$

$$
\mathrm{I}^{\mathrm{GDH}}=\int_{v_{\mathrm{thr}}}^{\infty}\left[\sigma_{\mathrm{N}}^{1 / 2}-\sigma_{\mathrm{N}}^{3 / 2}\right] \frac{\mathrm{d} v}{v}=\frac{2 \pi^{2} \alpha}{\mathrm{M}^{2}} \kappa_{\mathrm{N}}^{2}:
$$

where $\kappa_{N}$ is the anomalous magnetic moment and $M$ is the mass of the nucleon. The experimental results on the GDH sum rule are still puzzling. The disagreement between the experimental estimate through the analysis of photopion data and the predicted value from the GDH sum rule led to a new set of challenging total photoabsorption experiments with real photon beams around the world.

Recently extensions of the of GDH sum rule to finite $Q^{2}$ indicate significant uncertainty in the $Q^{2}$ evolution that can be tested experimentally using a virtual photon beam. The difference of the extended GDH sum rules on the proton and neutron is directly related to the Björken sum rule, a major focus of higher $Q^{2}$ deep inelastic spin structure function measurements.

Using the TJNAF longitudinally polarized electron beam and a high pressure polarized ${ }^{3} \mathrm{He}$ target, we shall measure the inclusive spin dependent cross section with beam and target helicity parallel and antiparallel at four incident beam energies $(\mathrm{E}=1.6 \mathrm{GeV}, 2.4 \mathrm{GeV}, 3.2$ $\mathrm{GeV}$ and $4.0 \mathrm{GeV}$ ) and three scattering angles $15^{\circ}, 25^{\circ}$ and $35^{\circ}$. The two Hall A HRS spectrometers, configured for electron detection, will be used as two independent single arm spectrometers to double the solid angle and allow flexibility in the data taking at different angles. This experiment will study the $Q^{2}$ evolution of the neutron extended GDH sum rule. When combined with the measurements proposed in Hall B experiment E91-023 and SLAC E143, these results should allow the investigation of the $Q^{2}$ evolution of the Björken sum rule $I^{B}{ }_{(}\left(Q^{2}\right)$ from $Q^{2} \geq 2$ $(\mathrm{GeV})^{2}$ down to $\mathrm{Q}^{2} \geq 0.15(\mathrm{GeV})^{2}$. An in-beam cell test for this experiment is currently planned at TJNAF in Hall C for the spring of 1997 and the Argonne Group is leading the effort in carrying out this initial test.

*California Institute of Technology, †Kent State University, $\ddagger$ University of Kentucky, Lexington, §Princeton University, IRutgers University, IISyracuse University, Stanford University, ††Temple University, 拉JNAF 
a.8. The Fundamental $\gamma \rightarrow \pi^{-}$p Process in ${ }^{2} \mathrm{H},{ }^{4} \mathrm{He}$, and ${ }^{12} \mathrm{C}$ in the $1.2 \cdot 6.0 \mathrm{GeV}$ Region

(W.J. Cummings, H. Gao, D.F. Geesaman, J.-O. Hansen, H.E. Jackson, T.G. O'Neill, D. Potterveld, J. Reinhold, B. Zeidman, B.W. Filippone, ${ }^{*}$ R.D. McKeown,* M. Pitt,* J.P. Chen, $\dagger$ J. Gomez, $\dagger$

K. de Jager, $\uparrow$ J. LeRose, $\uparrow$ M. Liang, $\uparrow$ R. Michaels, $\dagger$ S. Nanda, $\dagger$ E. Offerman, $\uparrow$ A. Saha, $\dagger$

B. Wojtsekhowski, $\dagger$ B. Fox, $\ddagger$ E.R. Kinney, $\ddagger$ D.H. Beck,§ R.J. Holt,§ R.M. Laszewski,§

M.A. Miller,§ A.M. Nathan, § S.E. Williamson,§ W. Korsch,I E.J. Beise,\| H. Breuer,\| N.S. Chant,\|

F. Duncan,\| J.J. Kelly,\|l A. Lung,\| P.G. Roos, II L. Kramer, ** P. Markowitz,** R.G. Milner, $\dagger \dagger$

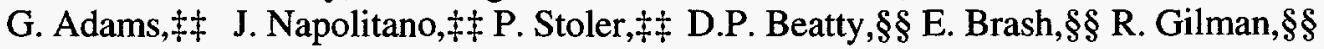

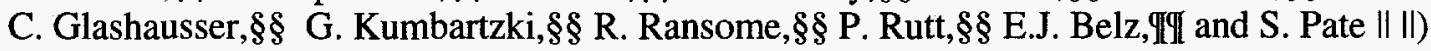

The $\gamma \mathrm{n} \rightarrow \pi^{-} \mathrm{p}$ reaction is one of the basic nucleon processes for studies of high energy photoreactions in nuclei and studies of nucleon models in the high energy regime. TJNAF experiment E94-104 was proposed to measure the cross section of the quasifree exclusive $\mathrm{n}\left(\gamma, \pi^{-} \mathrm{p}\right)$ reaction in deuterium for photon energies between 1.2 to $6.0 \mathrm{GeV}$ and at center-of-mass angles of $45^{\circ}, 75^{\circ}$ and $90^{\circ}$. This experiment will provide the first data for this reaction above a center-of-mass energy of $2.0 \mathrm{GeV}$. Furthermore, the experiment will measure the cross section of the quasifree exclusive $\mathrm{n}\left(\gamma, \pi^{-} \mathrm{p}\right)$ reaction in ${ }^{4} \mathrm{He}$ and ${ }^{12} \mathrm{C}$ for photon energies of 1.2 to $6.0 \mathrm{GeV}$ and at a center-of-mass angle of $75^{\circ}$ to determine the nuclear dependence of this cross section. The energy and A-dependences of the cross section ratio are sensitive to the final state interactions of the hadrons with nucleons in nuclei. The experiment will be performed in Hall $\mathrm{A}$. In addition to the coincidence measurements, the photopion production singles cross section ratio $\pi^{-} / \pi^{+}$from deuterium will be measured at photon energies between 1.2 to $6.0 \mathrm{GeV}$ and at center-of-mass angles of $45^{\circ}, 75^{\circ}$, and $90^{\circ}$ to test quark model predictions. This experiment was approved by TJNAF PAC 9 with high scientific priority. Initial studies for the experiment were carried out during the running of the Hall C experiment E89-012 in the spring of 1996 and verified the technical feasibility of the measurement.

*California Institute of Technology, †CEBAF, łUniversity of Colorado, §University of Illinois, Urbana, quniversity of Kentucky, Lexington, IIUniversity of Maryland, **Florida International University, ††Massachusetts Institute of Technology, $\ddagger \ddagger$ Rensselaer Polytechnic Institute, §§Rutgers University, ITTRRIUMF, II IINew State University

\section{a.9. Measurement of Proton Polarization in the $\mathbf{d}(\gamma, p) n$ Reaction (D. F. Geesaman,} H. E. Jackson, C. E. Jones, D. H. Potterveld, B. Zeidman, E. R. Kinney, ${ }^{*}$ E. Beise, $\dagger$ B. Filippone, † R. D. McKeown, $\nmid$ J. Napolitano, $\ddagger$ R. Milner, $\S$ R. E. Segel,II D. Beatty,\|

R. Gilman,\| C. Glashausser,\| G. Kumbartzki,\| R. Ransome,\| Z.-E. Meziani,**

D. Beck, $\dagger \dagger$ R. J. Holt, $\dagger \dagger$ and S. J. Freedman $\ddagger \ddagger)$

A proposal was approved by CEBAF PAC7 to measure angular distributions of the proton polarization for the $\mathrm{d}(\gamma, \overrightarrow{\mathrm{p}}) \mathrm{n}$ reaction in the $\mathrm{GeV}$ region. This proposed measurement will test the validity of extensions of conventional nuclear-physics theories to the higher energy regime. The results of the experiment will further constrain the evidence from ANL experiments at SLAC and CEBAF that asymptotic scaling was observed above a photon energy of $\sim 1 \mathrm{GeV}$. Photoproton polarization measurements at lower energy indicate that the magnitude of the polarization increases with energy.
This is consistent with the observation that polarizations are large in high-energy processes, e.g. $\mathrm{A}_{\mathrm{nn}}$ in $\mathrm{pp} \rightarrow \mathrm{pp}$ scattering or $\mathrm{A}_{\mathrm{y}}$ in $\mathrm{pp} \rightarrow \pi{ }^{0} \mathrm{X}$. However, the polarizations in hadron-hadron scattering are believed to arise from Landshoff mechanisms. The higher energy photoproton experiment will permit the first measurements of polarization for a reaction, $\gamma \mathrm{d} \rightarrow \overrightarrow{\mathrm{p}} \mathrm{n}$, where there are no Landshoff terms. The experiment would make use of a polarimeter installed in either the High Resolution Spectrometer in Hall A or the Short Orbit Spectrometer in Hall C at CEBAF.

\footnotetext{
${ }^{*}$ University of Colorado, $†$ California Institute of Technology, $\ddagger$ Rensselaer Polytechnic Institute, §Massachusetts Institute of Technology, 价orthwestern University, IRutgers University, ${ }^{* *}$ Stanford University, $\uparrow \dagger$ University of Illinois, §§University of California at Berkeley
} 


\section{a.10. Short-Orbit Spectrometer for Hall C at CEBAF (H. E. Jackson, K. Bailey,}

D. H. Potterveld, and B. Zeidman)

The Short Orbit Spectrometer (SOS) which the Argonne Medium Energy Physics Group built under contract to CEBAF is now routinely used in the experiments to be carried out at CEBAF. The SOS is a large-acceptance, low-loss, general-purpose magnetic particle spectrometer which will be used as the second coincident arm in electron scattering experiments planned in Hall C. It will be a resource of the CEBAF facility available to all users. The optical design is point-to-point in both the dispersive (vertical) and the transverse (scattering) planes. For a 1-mm target spot, the first-order resolving power is approximately 2200 , while the angular resolution is $<5 \mathrm{mr}$. Because of the reverse bend in the second dipole, there is a relatively small net deflection of the beam through the spectrometer, a property particularly useful for polarization measurements. Because of the strong edge focusing, the optical length of the spectrometer is only $\sim 7.4$ meters. Relatively short spectrometer drift lengths are required in experiments involving detection of pions or kaons in order to minimize decay losses.
The rigid structural design, coupled with a compact focal-plane detector package, yields a device that is readily adapted to out-of-plane measurements. Operation of SOS in conjunction with the High Momentum Spectrometer in Hall C provides a coincidence capability and serves as a general-purpose second arm in a wide variety of experiments at CEBAF. A unique feature of the SOS, operation on a spherical bearing at the pivot point with a hydraulic lifting system, will allow measurements of scattered hadrons at angles up to 20 degrees out of the horizontal scattering plane. Substantial cost savings in its construction resulted from the participation of LANL engineers who had constructed a similar instrument at LAMPF. The current schedule of experiments in the Hall call for its use in a broad spectrum of studies ranging from the photodisintegration of the deuteron and quasifree electron scattering to electroproduction of mesons and hypernuclei.

\section{a.11. ${ }^{3}$ He Target for Hall $\mathrm{C}$ at CEBAF (H. Jackson, J. Reinhold, B. Zeidman, and A. Zeuli)}

A major fraction of the physics program for Hall $\mathrm{C}$ involves scattering from cryogenic targets of the lightest nuclei, i.e. $\mathrm{H}, \mathrm{D}$, and ${ }^{3.4} \mathrm{He}$. Together with CEBAF, Argonne is constructing the He target system with a $4 \mathrm{~cm}$ diameter upright cylinder target cell operating at a pressure of $>10$ atmospheres and temperature of $\sim 5.2$ degrees Kelvin. CEBAF has constructed a cryo-target system for liquid hydrogen and deuterium that has been used for the experiments performed during the past year. The cooled, pressurized He target utilizes much of this system with a replacement of the target cell, but also includes the $\mathrm{He}$ supply systems and the additional equipment needed to ensure minimum loss of ${ }^{3} \mathrm{He}$ in the event of target rupture. Most of the major components have been constructed, with the balance of the system expected to be ready for installation this fiscal year.

\section{a.12. Laser-Driven Polarized Hydrogen and Deuterium Internal Target \\ (H. Gao, K. Bailey, W.J. Cummings, J.A. Fedchak, R.S. Kowalczyk, and A. Zeuli)}

In the last year, we finished the nuclear (tensor) polarization measurements using the tritium polarimeter on the existing laser-driven target setup. Polarized deuterium atoms from a spin exchange optical pumping cell flow into a storage target cell through a transport tube region. In order to minimize the systematic uncertainty associated with the previous measurement, a Wien velocity selector was installed for this measurement. The diode probe laser system developed in the last year and a half shows good results for the potassium polarization and density. This setup allows us to monitor the potassium polarization and density simultaneously with measurements of the deuterium atomic polarization and the atomic fraction. Charged by the HERMES council meeting, the HERMES task force (Argonne, Illinois and Erlangen) has started a program at IUCF to test the laser-driven target for the first time in a nuclear physics experiment and a physics program with this target at IUCF will follow the test. For the in-house target project, the current plan is to upgrade the system by using a new, simplified version of the glassware designed to improve the figure-ofmerit of the target. 


\section{a.13. Test of the Laser Driven Polarized Target at IUCF and Measurement of the $\overrightarrow{\mathbf{d}}(\overrightarrow{\mathrm{p}}, \mathrm{pp}) \mathbf{n}$ Reaction (W. Cummings, J. Fedchak, H. Gao, R. Kowalczyk, R.V. Cadman,*R.J. Holt,* M.A. Miller,* A. Nathan,* B. Owen,* E. Thorsland,* S. Williamson,* C. Grosshauser, $\dagger$ W. Kilian, $\uparrow$ W. Nagengast,$\uparrow$ K. Rith, $\uparrow$ E. Steffens, $\uparrow$ J. Stenger, $\dagger$ J. Sowinski, $\ddagger$ F. Sperisen, $\ddagger$ and C.E. Jones§)}

In the past year, a collaboration was formed to install, test and use a laser driven polarized H/D target (LDT) in the cooler ring at the Indiana University Cyclotron Facility (IUCF). This collaboration is an extension of the HERMES LDS task force which is comprised of groups from Argonne, the University of Illinois at Urbana, and the University of Erlangen in Germany. The collaboration was formed in May and by July the IUCF PAC had approved the installation of the LDT in the cooler ring and an experiment to measure the nuclear vector polarization using unpolarized stored proton beam. This experiment (CE66) was approved for 30 shifts with A priority.

In December 1996, the G-region of the cooler ring was dismantled. Since then, the CE66 collaboration installed the scattering chamber, beamline, magnets, diagnostics and pumps needed for the LDT. The LDT installed at IUCF is truly a joint effort between the three groups of the HERMES LDS task force with each providing a significant portion of the hardware. Currently, all hardware systems are installed and being commissioned. Argonne built the gas feed system, is responsible for most of the target chambers, and is responsible for the laser beam transport system. The first runs with proton beam are expected to be completed in late April 1997.

Also in December, an experiment to use this LDT to measure the spin dependence of the deuteron wave function was proposed to the IUCF PAC. This experiment (CE68), which will provide new data on spin-dependent momentum distribution of the proton inside the deuteron, was approved for 60 shifts of running with A priority. Following the successful completion of CE66 in the spring/summer, CE68 is expected to take data in fall 1997.

*Univ. of Illinois, Urbana, †Univ.Erlangen-Nürnberg, łIndiana Univ. Cyclotron Facility, §Kellogg Rad.Laboratory

a.14. Polarized Tritium Target Development (W. Cummings, J.A. Fedchak, H. Gao, R.S. Kowalczyk, and C.E. Jones*)

Most of the work to develop the tritium target will first involve $H$ or $D$ because of the hazards associated with tritium. Over the past year, we verified that we, in fact, have a nuclear polarized target by measuring the nuclear tensor polarization in the Argonne laser-driven polarized D target (LDT). Previous to these measurements, only the electron polarization had been measured. While the electron polarization can be used, in certain circumstances, to infer the nuclear polarization, an electron polarimeter would be difficult to implement in a tritium target because it requires a ballistic flow of atoms in a high vacuum. In a tritium target, a similar system would require exhausting and disposing of radioactive tritium. Additionally, the present design of the LDT is for an internal target, whereas the tritium target will be an external target having different design considerations. Therefore, other methods of determining polarization must be developed. This year we developed a diode probe-laser system to monitor the potassium density and polarization in the LDT optical pumping cell. We are also developing a model, based on the work of Stenger and Rith, ${ }^{1}$ that predicts the $\mathrm{D}$ polarization in the pumping cell based on various parameters such as cell geometry, the spin relaxation rate on the walls, D-D and K-D spin-exchange rates, the magnetic field in the pumping cell, and the optical pumping. From this model, we discovered that the $\mathrm{K}$ polarization does not depend on the spin relaxation rate on the cell walls but is dominated by the net optical pumping rate and the rate at which spin is transferred to $D$ atoms. Therefore, it may be possible to use the $\mathrm{K}$ polarization to monitor the polarization of the hydrogen isotopes. Modeling the LDT is an important stage in developing a polarized tritium target since most of the optimization of the target will have to be done without tritium. Over the coming year, we hope to extend the model to the entire apparatus (not just the pumping cell) and hope to accurately model the nuclear tensor polarization measurements.

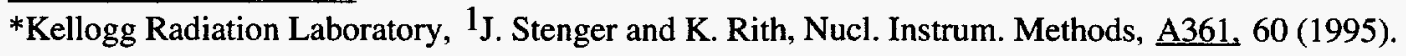




\section{a.15. HERMES, Measurements of Spin-Structure Functions and Semi-Inclusive} Asymmetries for the Proton and Neutron at HERA (H.E. Jackson, H. Gao, J.-O. Hansen, T.G. O'Neill, D.H. Potterveld, and collaborators at 29 other institutions)

HERMES is an international collaboration that is carrying out a program of studies of the spin structure of the nucleon at the DESY Laboratory in Hamburg, Germany, using internal polarized gas targets in the stored electron beam of the HERA accelerator. The collaboration currently includes approximately 30 institutions from North America and Europe including several institutions from CIS. HERMES is located in the East Hall of the $6.4 \mathrm{~km}$ circumference HERA positron-proton collider. The experiment is arranged so that the $27.5 \mathrm{GeV}$ longitudinally polarized positron beam interacts with internal polarized gas targets without interference from the proton beam. The beam is self-polarized transverse to the beam direction by the Sokolov-Ternov effect. Spin rotators located at the entrance and exit of the East straight section precess the spin direction from vertical to longitudinal at the target position and, following the target, back to the vertical position. The polarization of the beam with the rotators in place has been measured for both positrons and electrons. Longitudinal polarizations of about $50 \%$ are stable and reproducible and are measured using a transverse Compton backscattering polarimeter with a systematic uncertainty of better than $\pm 5 \%$. The polarization time is about 25 minutes. As a result of the ease with which the direction of the target polarization can be changed, it will be possible to study parallel and perpendicular polarization asymmetries for all targets.

In 1995 , data were taken with a polarized ${ }^{3} \mathrm{He}$ target and at present HERMES is running with a polarized hydrogen target. The HERMES target consists of an open-ended thin welded storage cell through which the circulating positron beam of the HERA accelerator passes. A magnetic holding field provides a quantization axis for the target polarization. The target densities are about $7.5(5) \times 10^{13}$ atoms $/ \mathrm{cm}^{2}$ for hydrogen (deuterium) and $3.5 \times 10^{14}$ atoms $/ \mathrm{cm}^{2}$.

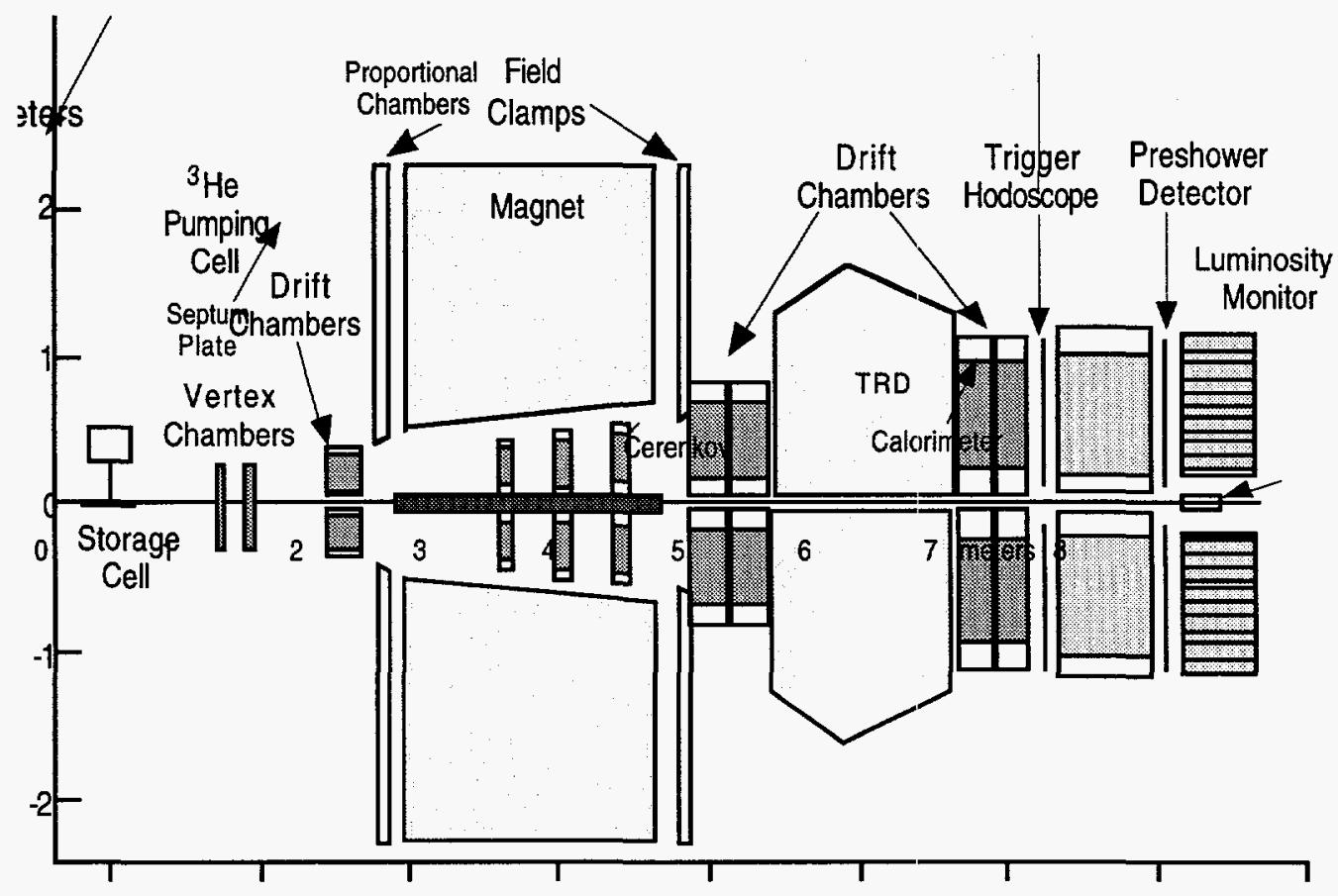

Fig. III-4. Schematic diagram of the experimental apparatus (side view).

1.V. Akushevich and N.M. Shumeiko, J. Phys. G: Nucl. Part. Phys. 20. 513 (1994), ${ }^{2}$ P.L. Anthony et al., Phys. Rev. Lett. 1ㅡ, 959 (1993), 3S.J. Bodsky et al., Nucl. Phys. B441, 197 (1995), ${ }^{4}$ P.L. Anthony et al., Phys. Rev. D54, 6620 (1996). 
The HERMES spectrometer, shown in Fig. III-4, was designed to detect scattered positrons and hadrons from deep inelastic scattering. The spectrometer consists of two identical halves above and below the positron ring plane. This provides two independent measurements of observables and thus a cross check on systematic uncertainties. The typical polarizations of the target are $90 \%(50 \%)$ for the hydrogen/deuterium $\left({ }^{3} \mathrm{He}\right)$ sources and are measured to better than $\pm 5 \%$ with polarimeters. Luminosities are in the range of $4-30 \times 10^{31}$ nucleons $\mathrm{cm}^{-2} \mathrm{sec}^{-1}$. The gases are pure so that there is no target dilution. The extremely small target thickness and absence of end windows minimize external radiative corrections. Unpolarized gases of hydrogen and deuterium up to $10^{15}$ atoms $/ \mathrm{cm}^{2}$ are also used for data taking. The maximum target thickness is determined by the impact on the stored positron lifetime.HERMES spectrometer contains a dipole magnet with a bending strength of $1.3 \mathrm{~T} \cdot \mathrm{m}$ to measure particle momenta and to reject background. A system of segmented hodoscopes and a fly's eye lead glass calorimeter provide a deep inelastic scattering trigger. A transition radiation detector provides strong discrimination against hadrons. Tracking chambers before the magnet, in the magnetic field, and behind the magnet provide charged particle tracking. A pair of threshold gas Cerenkov counters are used for identification of pions. Each Cerenkov unit is divided into 20 cells viewed by individual photomultipliers. In 1995 the threshold for pions to trigger the Cerenkov counter was $6 \mathrm{GeV} / \mathrm{c}$. In 1996 the gas composition had been modified to reduce the threshold to $4 \mathrm{GeV} / \mathrm{c}$. Relative luminosity is monitored with $\approx 1 \%$ precision by the coincident detection in symmetrically placed bismuth tungstenate calorimeters of the positron-electron pair from symmetric Bhabha scattering off the target atomic electrons. The target empty luminosity rate is negligible. A collimator system prevents the large flux of synchrotron photons from impinging on the target cell. The angular acceptance of the experiment is $40<\theta<220 \mathrm{mrad}$. The kinematic range accessible is $0.2<\mathrm{x}<0.8$ and 0.2 $<\mathrm{Q}^{2}<20(\mathrm{GeV} / \mathrm{c})^{2}$. Argonne provided the Cerenkov counter used for particle identification and developed the drifilm coating technique for the ultrathin target cell required for this experiment. The HERMES collaboration intends to use polarized targets with the highest available figures of merit and the Argonne laser-driven source offers the most promise for a significant advance in present-day targets.

During the 1995 HERA running period, the HERMES experiment was commissioned from June until late August, by which time the polarized ${ }^{3} \mathrm{He}$ target and spectrometer were operating reliably and adequate background conditions had been established for the experiment. Production data were taken from midAugust to late November which resulted in about 5 million inclusive deep-inelastic events from ${ }^{3} \mathrm{He}$ and about 1 million unpolarized DIS events on hydrogen and deuterium. The average target polarization during production data taking was 0.47 with a fractional uncertainty of $5 \%$. The target spin orientation was reversed every 10 minutes. In addition, the target polarization was monitored by detecting the circular polarization of light due to excitation by the HERA beam. The average beam polarization during production data taking was $52 \%$ with a fractional uncertainty of $5.5 \%$.

An analysis of the data taken with a polarized $3 \mathrm{He}$ target has been carried out in order to extract the spin structure function $\mathrm{g}_{1}^{\mathbf{n}}(\mathbf{x})$. The inclusive spin asymmetries were extracted from the scattered positron yields using the luminosity monitor for relative normalization. Radiative corrections were made by means of the techniques of Akushevich and Shumeiko. 1 Instrumental smearing was confirmed to be small. Stringent quality cuts eliminated from analysis over half of the $\approx 5$ million events accumulated. The results for $\mathrm{g}_{1}^{\mathrm{n}}$ are presented in Fig. III-5. The results are in good agreement with the data reported from SLAC

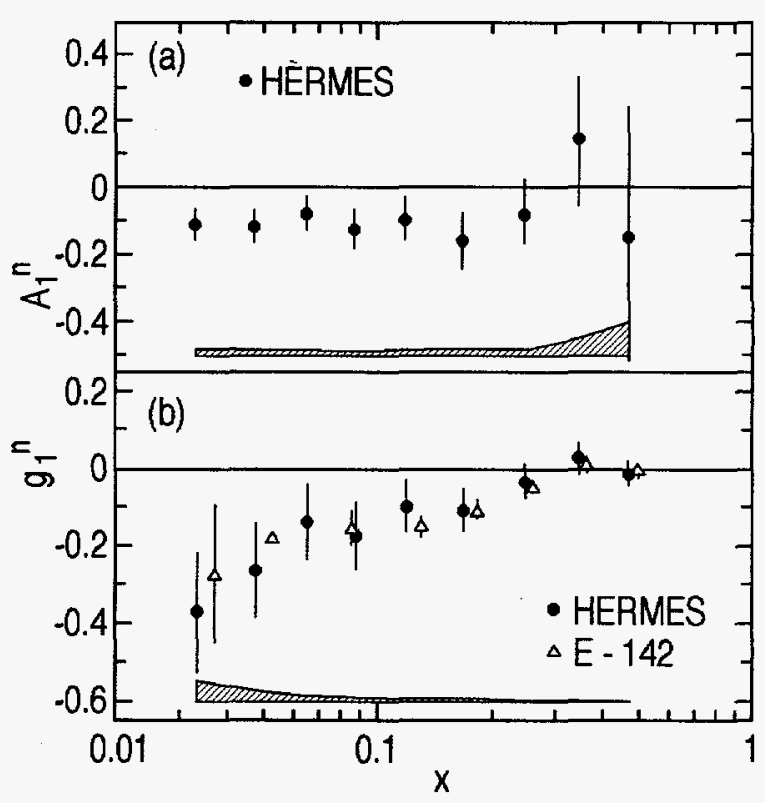

Fig. III-5. The spin asymmetry $\mathrm{A}_{1}^{\mathrm{n}}(a)$ and the spin structure function $\mathrm{g}_{1}^{\mathrm{n}}$ (b) of the neutron as a function of $x$. The values are given for the measured $\left(\mathrm{Q}^{2}\right)$. The error bars are statistical uncertainties.

The error bands show the systematic uncertainties.

The data points from E-142 have been displaced slightly in $x$ for the comparison with the present experiment. 
experiment E-142, 2 also using a ${ }^{3} \mathrm{He}$ target. The EllisJaffe integral $\Gamma_{1}^{n}=\int_{0}^{1} g_{1}^{n}(x) d x$ can be estimated from the data on $\mathrm{g}_{\mathrm{n}}^{1}(\mathrm{x})$ by evolving the points to a fixed value of $\mathrm{Q}^{2}=2.5(\mathrm{GeV} / \mathrm{c})^{2}$ assuming that the measured asymmetry $A_{1}^{n}(x)$ is independent of $Q^{2}$. Simple models employed in the analyses of previous experiments have been used to extrapolate $g_{j}^{n}(x)$ outside the measured region, giving $g_{1}^{n}(x) \approx(1-x)^{3}$ for $x>0.6 .3$ With these extrapolations, the integral of $\mathrm{g}_{1}^{\mathrm{n}}(\mathrm{x})$ is $\mathrm{g}_{1}^{\mathrm{n}}=-0.039 \pm 0.013_{\text {stat }} \pm 0.005_{\text {syst }} \pm 0.006$ (extrapol.) in good agreement with the value from experiment E-142. In addition, Ref. 4 has noted a two standard deviation discrepancy in the measurements of $\mathrm{g}_{1}^{\mathrm{n}}(\mathrm{x})$ in the region $0.04<\mathrm{x}<0.3$ performed at SLAC (E-142) and CERN (SMC). In Ref. 4 the quoted integrals at $\mathrm{Q}_{0}^{2}=2.0(\mathrm{GeV} / \mathrm{c})^{2}$ are $\int_{\mathrm{Q}_{0} .04}^{0.3} \mathrm{~g}_{1}^{\mathrm{n}}(\mathrm{x}) \mathrm{dx}=$ $-0.027 \pm 0.004 \pm 0.006$ for E-142 and $\int_{0.04}^{0.3} \mathrm{~g}_{1}^{\mathrm{n}}(\mathrm{x}) \mathrm{dx}=+$ $0.007 \pm 0.015$ for SMC. Our measurements of $\mathrm{g}_{1}^{\mathrm{n}}(\mathrm{x})$ at $\mathrm{Q}_{0}^{2}=2.5(\mathrm{GeV} / \mathrm{c})^{2}$ in this region yield the result $\int_{0.04}^{0.3} g_{1}^{n}(s) d x=-0.025 \pm 0.008 \pm 0.003$, clearly confirming the $\mathrm{E}-142$ results.

\section{a.16. A Dual Radiator Ring Imaging Cerenkov Counter for HERMES \\ (H.E. Jackson, K.G. Bailey, J.-O. Hansen, H. Gao, R.S. Kowalczyk, T.G. O'Neill, D.H. Potterveld, and the HERMES collaboration)}

HERMES is unique among contemporary experimental studies of the spin structure of the nucleon in its ability to make unambiguous measurement of pion, kaon, and nucleon semi-inclusive spin asymmetries. For polarized lepton-nucleon scattering, if hadrons can be unambiguously identified, the resulting data can be used to tag leading hadrons, thereby providing a means of direct study of the flavor dependence of polarized structure functions and of the sea polarization. If hadrons from strange particle decays can be identified, low background measurements of $\Lambda$ decay in HERMES will provide a probe with high experimental sensitivity to polarization transfer in $\Lambda$ electroproduction. If kaons can be reliably identified, clean measurement of open charm production by means of detection of the charmed meson decay becomes an attractive option for probing processes involving gluons. Clearly, reliable hadron identification is crucial, if HERMES is to fully exploit its scientific opportunities.

The spectrum of hadrons traversing the HERMES spectrometer extends from 2 to $20 \mathrm{GeV}$. Although unambiguous particle identification (PID) over this full region using existing technologies is a major challenge, for HERMES the scientific results would be of enormous interest. In view of the limitations of PID technologies available at the time, the HERMES spectrometer was designed with a limited capability for pion particle identification using threshold Cerenkov counters. However, these counters were designed to facilitate conversion to more advanced ring imaging Cerenkov ( $\mathrm{RICH}$ ) counters at the earliest opportunity when a sound technical design was judged feasible. With the recent development of new Cerenkov radiator materials that opportunity is at hand. It now appears feasible to design a dual radiator RICH system which can provide complete separation of pions, kaons, and nucleons over the full phase of the HERMES experiment.

A proposal organized by the Argonne group for an upgrade of the existing particle identification system in the HERMES spectrometer has been reviewed and endorsed by the DESY Program Review Committee. The existing threshold Cerenkov counters will be modified to operate as a ring imaging system (RICH). A dual gas-aerogel radiator would produce Cerenkov rings for high $\beta$ particles which will be imaged on a large array of small diameter phototubes which would serve as the Cerenkov photon detector. The combination would separate pions, kaons, and protons over the full kinematic acceptance of HERMES.

An open section of one of the Cerenkov counter sections is shown in Fig. III-6 as it will be modified to operate in a RICH mode with dual radiators. The present mirror array will be replaced with an operationally single spherical mirror with a radius of curvature of $2.2 \mathrm{~m}$. Each mirror will be fabricated in eight sections, using continuous fiber reinforced plastic (CFRP) composite materials. The aerogel radiator will be an assembly of $10 \mathrm{~cm} \times 10 \mathrm{~cm}$ tiles configured to fill the entrance of the detector with an aerogel thickness of $5 \mathrm{~cm}$. The unoccupied volume of the detector will be filled with the gas radiator, $\mathrm{C}_{4} \mathrm{~F}_{10}$. The photon rings generated in the top/bottom radiators will be imaged by the spherical mirrors on photon detectors placed above/below the active volume at the mirror focus. The mirrors will be rotated so that their central radius of curvature makes an angle of $250 \mathrm{mr}$ with the horizon. The mounting and alignment system for the mirror array will be based on the techniques used for composite telescopes. 


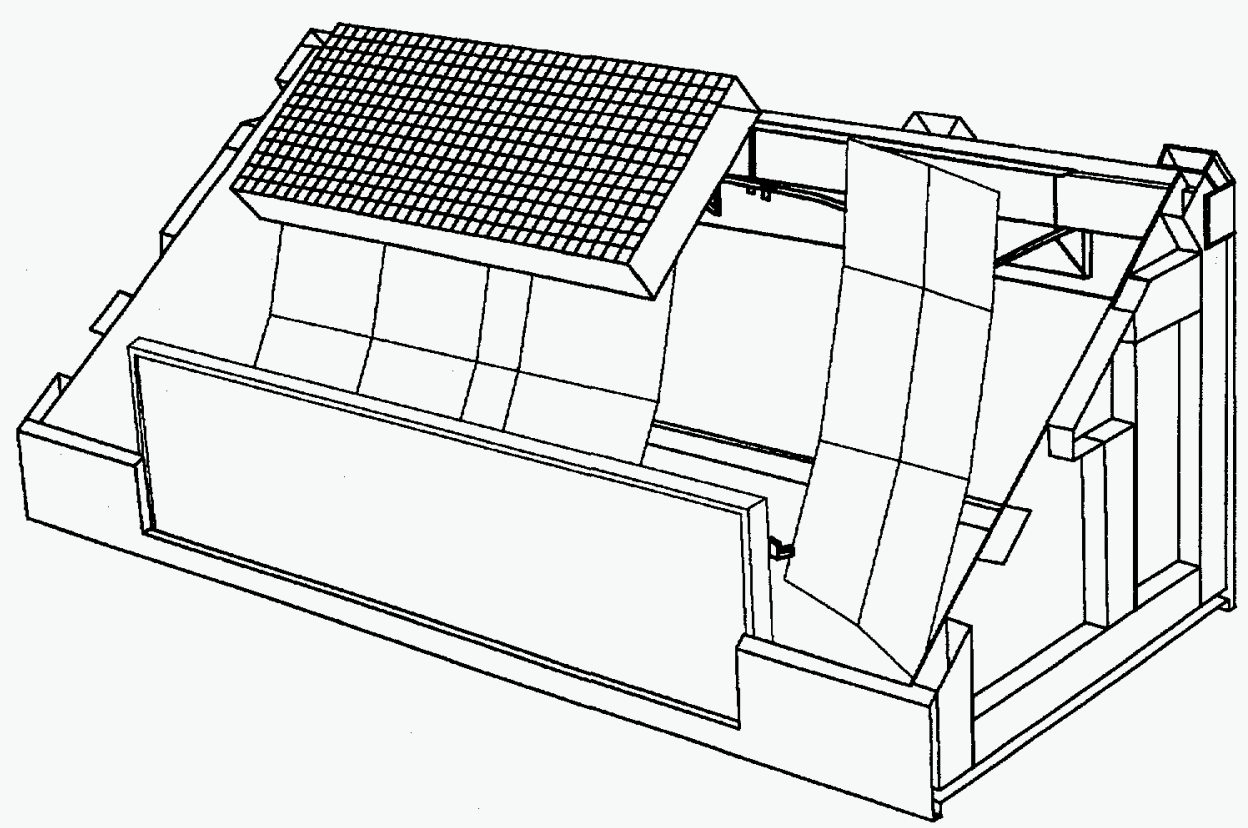

Fig. III-6. A cutaway schematic view of the proposed RICH counter. The entrance region is covered by a plane of Aerogel $5 \mathrm{~cm}$ thick. A section of the spherical mirror has been cut away for display purposes. The photon detector is indicated by the canted rectangular volume above the sensitive region.

\section{a.17. Nuclear Transparency and Longitudinal Asymmetry in $A\left(e, e^{\prime} \rho 0\right)$ at High Energies (T. G. O'Neill, H. E. Jackson, J.-O. Hansen, N. C. Makins, D. H. Potterveld, C. E. Jones, and collaborators at 29 other institutions)}

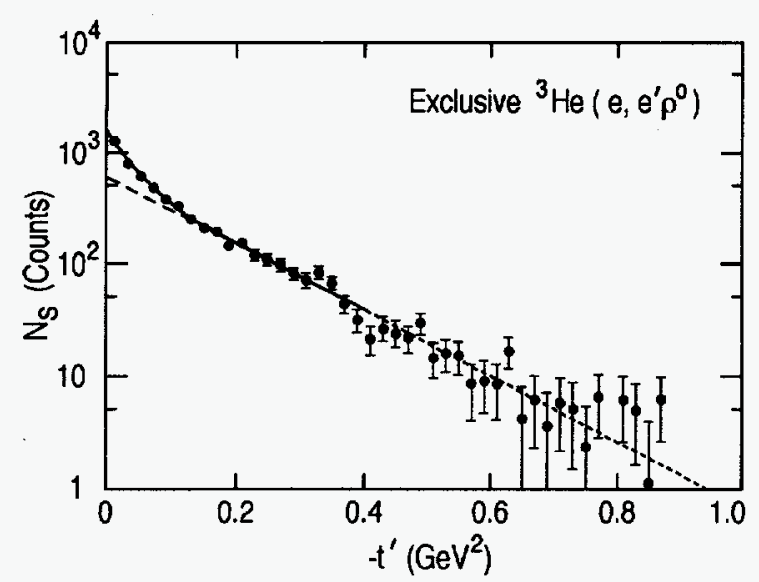

Fig. III-7. Histogram of the momentum transfer to the target (corrected for the kinematic limit so that $-t^{\prime} \approx$ the squared transverse momentum of the $\rho^{0}$ meson) for exclusive ${ }^{3} \mathrm{He}\left(\mathrm{e}, \mathrm{e}^{\prime} \rho^{0}\right)$ events. The background contribution from non-exclusive $\left(e, e^{\prime} h^{+} h^{-}\right)$events has been subtracted. The solid curve is a fit to two decaying exponentials representing scattering from individual nucleons (dashed curve) and from the entire nucleus at once.
A signal for the exclusive electroproduction of the neutral $\rho$ meson was isolated from the HERMES data used to measure the spin-dependent structure functions. Data were taken in 1995 and 1996 on unpolarized on ${ }^{2} \mathrm{H}$ and polarized ${ }^{1} \mathrm{H}$ and ${ }^{3} \mathrm{He}$ targets. The $\rho^{0}$ meson, which decays into 2 pions, manifests itself in a peak at $\mathrm{M} \rho=0.77 \mathrm{GeV}$ in the invariant mass of the 2 pion system. Energy and momentum conservation can be used to ensure that there are no additional particles in the final state, resulting in a very clean signal for the exclusive $A\left(e, e^{\prime} \rho^{0}\right)$ reaction. Preliminary analyses of the 1995 exclusive $A\left(e, e^{\prime} \rho^{0}\right)$ data have yielded results for the nuclear transparency of ${ }^{3} \mathrm{He}$, the longitudinal beam-target asymmetry, and the $\rho^{0} \rightarrow \pi^{+} \pi^{-}$decay angular distributions. The asymmetry and angular distributions can be used to help understand the $\rho^{0}$ production reaction mechanism. The nuclear transparency is a measure of the interactions with the nuclear medium of both the outgoing $\rho^{0}$ meson and the virtual photon mediating the reaction. The transparency exhibits a statistically significant increase with the momentum transfer $\mathrm{Q}^{2}$, providing evidence of variations in the hadronic structure of the virtual photon and possibly also of the color transparency effect. The increase in statistics expected from analysis of the 1996 data could culminate the decade-long search for definitive evidence of the color transparency phenomenon. 
a.18. Deep-Inelastic Muon Scattering from Nuclei with Hadron Detection (D. Geesaman, H. Jackson, S. Kaufman, E. Kinney, V. Papavassiliou, D. Potterveld, A. Zghiche, T. Kirk, * H. J. Trost, ${ }^{*}$ R. Kennedy, $\dagger$ H. Kobrak, $\uparrow$ P. Madden, $\dagger$ A. Salvarani, $\dagger$ Robert A. Swanson, $\dagger$ A. Eskreys, $\ddagger$ P. Malecki, $\ddagger$ K. Eskreys, $\ddagger$ K. Olkiewicz,$\ddagger$ B. Pawlik $\ddagger$ B. Baller,$\S$ G. B. Coutrakon, $\S$

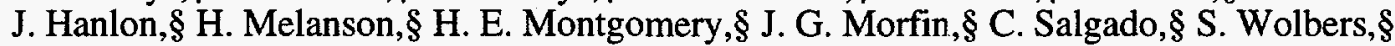
T. Dreyer,II M. Erdmann, II J. Haas,II W. Mohr, I H. Stier,II M. Wilhelm,II J. M. Conrad,॥ G. Fang,Il A. Kotwal,,l D. G. Michael,\| R. B. Nickerson,\|l F. M. Pipkin,\| M. Schmitt,\| Richard Wilson,\|l M. R. Adams, ${ }^{* *}$ D. A. Averill, ${ }^{* *}$ T. Carroll, ${ }^{* *}$ R. Guo, ${ }^{* *}$ C. Halliwell, ${ }^{* *}$ S. Magill, ${ }^{* *}$ D. McLeod,** S. Aid, $\uparrow \dagger$ S. Kunori, $\uparrow \dagger$ S. O'Day, $\nmid \dagger$ E. J. Ramberg,,$\dagger$ A. Skuja, $\uparrow \dagger$ P. H. Steinberg, $\dagger \dagger$

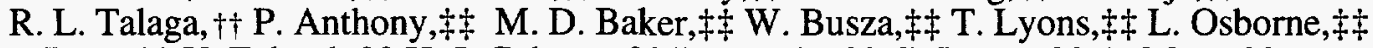

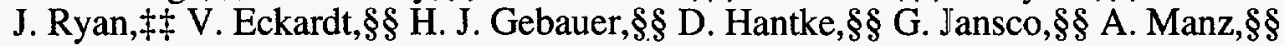

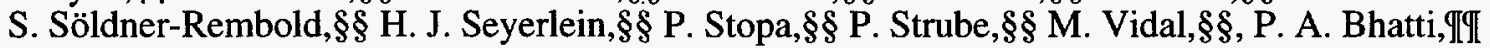

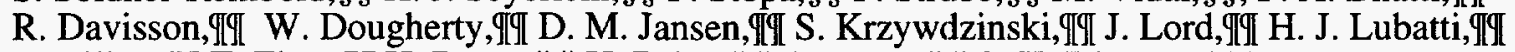

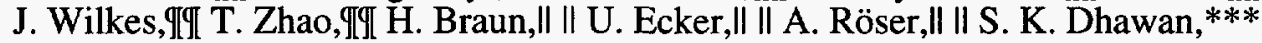
V. W. Hughes, *** K. P. Schüler, ***, H. Venkataramania,*** F. Dietrich, ††† H. Clark, K. Hicks,

Deep-inelastic lepton scattering from nuclei provides a direct look at the quark structure of nuclear matter. These reactions revealed the first convincing evidence that the structure of nucleons is modified in the nuclear medium and had profound implications on the understanding of nuclear dynamics. FNAL experiment E665, using the $490-\mathrm{GeV}$ muon beams at Fermi National Accelerator Laboratory, provides new information on the nuclear effects on nucleon properties by studying deep-inelastic muon scattering with coincident hadron detection. The high beam energy makes the experiment particularly suited to the study of the region of $x<0.1$ (where $x$ is the fraction of the momentum of the nucleon carried by the struck quark in the infinite momentum frame), and total center-of-mass hadronic energy $>25 \mathrm{GeV}$, where hard QCD processes are expected to become evident and there are little data from earlier deep-inelastic measurements.

The data-acquisition phase of E665 was completed in January 1992 following the third block of beam time. During the 1987-1988 run, data were accumulated at $490 \mathrm{GeV}$ on targets of liquid deuterium (luminosities of $2 \times 10^{36}$ muon-nucleon/ $\left./ \mathrm{cm}^{2}\right)$ liquid-hydrogen $(7 \times$
$10^{35}$ muon-nucleon $\left./ \mathrm{cm}^{2}\right)$ and gaseous xenon $\left(7 \times 10^{35}\right.$ muon-nucleor $/ \mathrm{cm}^{2}$ ). In this period the target was surrounded by a streamer chamber to provide essentially $4 \cdot \pi$ acceptance. During the 1990 run, luminosities of $4 \times 10^{35}$ were collected on targets of hydrogen, deuterium, carbon, calcium, and lead. The targets were changed every 1-3 minutes to reduce systematic uncertainties in the target dependence. In the final 1991 running period, luminosities of $4 \times 10^{36}$ were collected on hydrogen and deuterium.

Final results are now available in twenty-one publications. Twenty eight students completed Ph.D. theses on this experiment.

New results obtained in 1996 include measurement of diffractive $\rho$ meson production on hydrogen. Figure III- 8 shows the $Q^{2}$ dependence of the exclusive $\rho$ production cross section compared to ANL calculations. ${ }^{1}$ Measurement of the polarization of the $\rho$ meson from decay angular distributions show the polarization changes from transverse to longitudinal as $\mathrm{Q}^{2}$ increases. It is anticipated that the analysis of E665 data will be completed in 1997.

\footnotetext{
*High Energy Physics Division, ANL, †University of California at San Diego, łInstitute of Nuclear Physics, Cracow, Poland, §Fermi National Accelerator Laboratory, IUniversity of Freiburg, Germany, IIHarvard University, **University of Illinois at Chicago, ††University of Maryland, $\ddagger \ddagger$ Massachusetts Institute of Technology, §§Max-Planck-Institute, Heidelberg, Germany, IIUUniversity of Washington,

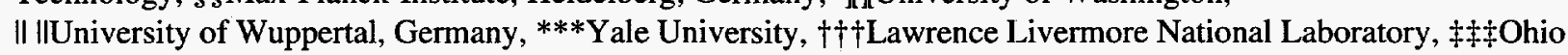
University, $\S \S U n i v e r s i t y$ of Pennsylvania TITINorthwestern University.

${ }^{1}$ M. A. Pichowsky and T.-S. H. Lee, Phys. Lett. B379, 1 (1996).

2J. Nemchik et al., DFTT71/95 KFA-IKP (TH)-24-95, to be published.
} 


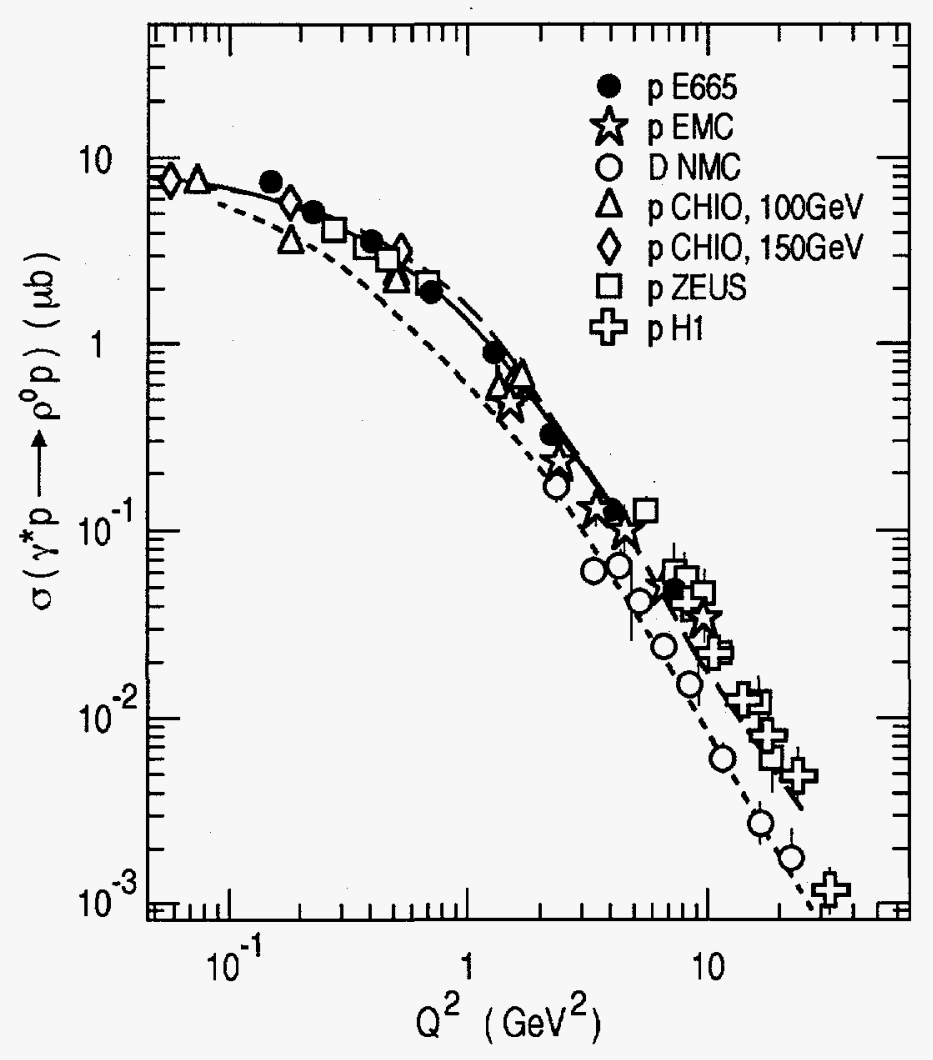

Fig. III-8. The cross section for $\gamma^{*} p \rightarrow \rho^{0}$ p as a function of $Q^{2}$ from E665 (full circles) and a number of other experiments. The solid line is a phenomenological parameterization of the data. The dashed line is the calculation of Pichowsky and Lee. ${ }^{I}$ The dotted line is a calculation from Ref. 2.

a.19. Measurement of $\overline{\mathbf{d}} / \overline{\mathbf{u}}$ in the Nucleon (D. F. Geesaman, S. Kaufman, N. C. Makins, B. Zeidman, D. Isenhower, ${ }^{*}$ M. Sadler, ${ }^{*}$ R. Towell, ${ }^{*}$ Y.-C. Chen, $\dagger$ P.-K. Teng, $\dagger$ G.-C. Kiang, $\dagger$

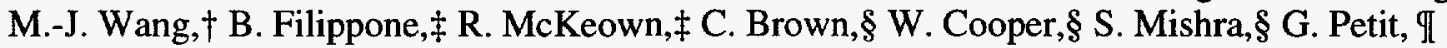
X.-C. He,II W. Lee,II D. Kaplan,II M. Brooks, ${ }^{* *}$ T. Carey, ${ }^{* *}$ G. Garvey, ${ }^{* *}$ D. Lee, ${ }^{* *}$ M. Leitch,** J. McClelland, ** P. McGaughey, ${ }^{* *}$ C.Morris, ** J. Moss, ${ }^{* *}$ B. Park, ${ }^{* *}$ J.-C. Peng, ${ }^{* *}$ W. Sondheim, ${ }^{* *}$ N. Thompson, ${ }^{* *}$ P. Kirk, $\dagger \dagger$ Y.-C. Wang, $\dagger \dagger$ Z.-F. Wang, ${ }^{\dagger} \dagger$ M. Beddo, 拉 J. Haas,

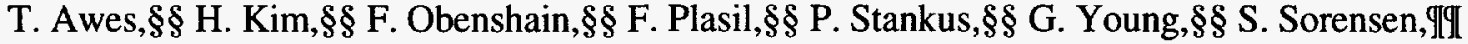
S. Garbett, TII, C. Gagliardi, I| I| R. Tribble, || || E. Hawker, || || D. Koetke, ${ }^{* * *}$ and P. Nord***)

Recent experimental results on the deep inelastic structure functions on hydrogen and deuterium, combined in a sum rule analysis, suggest that there is a substantial difference between $\overline{\mathrm{u}}$ and $\overline{\mathrm{d}}$ sea in the proton. The Drell-Yan process, where a quark from a projectile annihilates with an anti-quark in the target to form a timelike-virtual-photon, can provide a direct measurement of the $x$ dependence of the anti-quark distributions. A first examination of this process in CERN experiment NA-51 confirmed that the $\overline{\mathrm{u}}$ and $\overline{\mathrm{d}}$ distributions are indeed different near $\mathrm{x}$ of 0.15 .

\footnotetext{
*Abilene Christian University, †Academia Sinica, Taiwan, ROC, $\ddagger$ California Institute of Technology, $\S$ Fermi National Accelerator Laboratory, TGeorgia State University, IIIllinois Institute of Technology, **Los Alamos National Laboratory, $\dagger+$ Louisiana State University, $+\frac{+}{+}$ New Mexico State University, §8Oak Ridge National Laboratory, TIIUniversity of Tennessee, II IITexas A\&M University, ***Valparaiso University
} 
A new experiment, E866 at FNAL began taking data in 1996 to accurately measure the Drell-Yan production of di-muon pairs using an $800-\mathrm{GeV}$ proton beam on liquid hydrogen and deuterium targets, with relative systematic errors of $\sim 1 \%$. This will measure $\overline{\mathrm{d}} / \overline{\mathrm{u}}$ with $2 \%$ accuracy for $0.05<x<0.15$ and with lesser statistical accuracy out to $x \sim 0.3$, with one to twoorders of magnitude higher statistics than NA-51. High statistics $\mathrm{J} / \psi$, and $\psi^{\prime}$ and $\Upsilon$ production data were also obtained.

In 1997, the experiment was granted an extension until summer 1997 to focus on three additional topics: 1) high mass Drell-Yan data to improve the $\bar{d} / \bar{u}$ measurement at higher $\mathrm{x}$ values, 2) high statistics measurements of the angular distributions of decay muons for Drell-Yan and $J / \psi$ production, and 3) measurement of nuclear effects at low $\mathrm{x}$ and at very forward di-lepton momentum.

ANL carefully mapped the magnetic fields in a region where the strengths of two of the analysis magnets are coupled. The resulting field maps appear to provide a much better description of the acceptance of the spectrometer, an essential improvement in the absolute normalization of the experiment. 


\section{THEORETICAL PHYSICS}

\section{OVERVIEW}

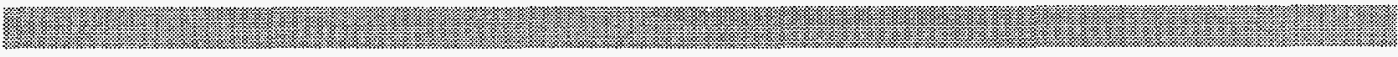

Theoretical research in the Physics Division addresses a broad range of problems involving the structure and dynamics of hadrons and nuclei. There is a strong emphasis on comparison to data provided by experimental groups at Argonne and at other facilities around the world. The principal areas of research include nuclear dynamics with subnucleonic degrees of freedom, nuclear forces and nuclear systems, and heavy-ion reactions and nuclear structure studies. Our work includes the modeling of quantum chromodynamics in meson and baryon structure, developing reaction theories for medium-energy nucleon-nucleon interactions and meson production, and studying electron scattering within the framework of relativistic Hamiltonian particle dynamics. We construct realistic nucleon-nucleon potentials that give very accurate fits to elastic scattering data, and then use these in detailed many-body calculations of the properties of light and closed-shell nuclei, hypernuclei, nuclear matter and neutron stars. Heavy-ion research includes the structure and reactions of nuclei far from stability produced at radioactive beam facilities and coupled-channels calculations of reactions near the Coulomb barrier, while our nuclear structure research concentrates on effective two-body interaction studies of deformed and superdeformed nuclei observed at ATLAS. We also pursue research in atomic physics, fundamental quantum mechanics, and quantum computing. Many of our projects involve collaborators at domestic and foreign universities and other national laboratories. Several require major numerical simulations using state-of-the-art computers, including Argonne's massively parallel IBM SP.

\section{A. NUCLEAR DYNAMICS WITH SUBNUCLEONIC DEGREES OF FREEDOM}

The objective of this research program is to investigate the role of mesons, nucleon resonances, and quark-gluon degrees of freedom in nuclear dynamics.

The Dyson-Schwinger equations (DSEs) provide a nonperturbative, Poincaré invariant, continuum framework for the study of quantum field theories which is ideally suited to the study of intrinsically nonperturbative phenomena such as dynamical chiral-symmetry breaking, confinement, and bound-state formation and properties. We have developed and applied this framework extensively as a tool in hadronic physics. Introducing an approximation procedure for the DSEs that ensures chiral-current conservation at all orders, we demonstrated that the pion appears as a Goldstone boson as long as the quark-quark (qq) interaction is sufficiently strong at long range. We completed our calculations of the electromagnetic form factors of the $\pi$ and $K$ mesons and this provided the foundation for a study of the electroweak K- $\pi$ transition form factor. Introducing a simple, separable ansatz for the qq interaction we described the ground 
state spectrum of light-quark mesons and elucidated the defects of the frequently-used laddertruncation of the two-body Bethe-Salpeter equation. We employed a simple one-parameter DSE model of 2-flavor QCD, with its single parameter fixed at zero temperature, in a study of the finite temperature properties of QCD. In this study, we proposed a continuum order parameter for deconfinement, valid for both light and heavy quarks, and found that 2-flavor QCD undergoes coincident, second-order chiral-symmetry restoring and deconfinement phase transitions whose critical exponents are consistent with those of the $\mathrm{N}=4$ Heisenberg magnet, confirming the results of lattice simulations. We are currently applying the DSE framework to the study of the electromagnetic properties of baryons and the behavior of QCD bound states at finite temperature and density.

We are continuing our effort to develop theoretical models for investigating intermediate- and high-energy reactions on the nucleon and few-nucleon systems. A meson-exchange model has been completed to show that the $\gamma \mathrm{N} \rightarrow \Delta$ transitions predicted by the chiral constituent quark model are in good agreement with the pion photoproduction data. The model has been used to make predictions for the $\Delta$ components in ${ }^{3} \mathrm{He}$ and for $\mathrm{N} \Delta$ interactions at high momentum transfer. The $\mathrm{pp} \rightarrow \mathrm{pp} \pi^{0}$ reaction near the production threshold has been investigated with chiral perturbation theory. We also developed a Pomeron-exchange model that takes into account the quark substructure of mesons and find it gives a satisfactory description for exclusive electroproduction of $\rho, \phi$, and $J / \psi$ mesons.

We are continuing our investigation of quark models of hadrons and of electron scattering from few-nucleon systems, in the framework of relativistic Hamiltonian dynamics. Quark confinement is the source of important qualitative differences between relativistic quark and fewnucleon models. The structure of quark models that respect Poincaré covariance together with flavor and color symmetry need not be a quark-particle structure. Such models provide simple relations of the properties of nucleon resonances and nucleons. Conventional form factors, directly related to electromagnetic observables, are either defined by spin matrix elements evaluated in a special 'frame', or invariant factors appearing in covariant spinor representations of the currents. We found that the well-known Lorentz covariant representations of current kernels for spin $1 / 2$ and spin 1 can be generalized to arbitrary integral and half-odd integral spin by emphasizing the $O(1,2)$ subgroup that leaves the four-momentum transfer invariant. These relations have significant implications for the current structures of hadronic models. Euclidean space-time formulations of Lagrangian quantum field theories provide the mathematical basis for nonperturbative approximations, and for precisely-defined models of finite systems. We have been exploring the implications of this approach, in particular the requirements quantum theory imposes on model Schwinger functions.

\section{a.1. Electromagnetic Pion Form Factor (C.D. Roberts)}

The electromagnetic pion form factor, $F \pi\left(q^{2}\right)$, is calculated in impulse approximation for spacelike- $q^{2}$ on the domain $[0,20] \mathrm{GeV}^{2}$ using a confining quark propagator, $S$, and a dressed quark-photon vertex, $\Gamma_{\mu}$, obtained from realistic, nonperturbative DysonSchwinger equation studies. Good agreement with the available data is obtained for $F_{\pi}\left(q^{2}\right)$, and other pion observables, including the decay $\pi^{0} \rightarrow \gamma \gamma$. It is found that, at presently accessible momentum-transfer, non- perturbative, bound-state effects dominate the form factor. This application is an illustration of the phenomenological efficacy of the phenomenological Dyson-Schwinger equation approach, whereby a large range of observables can be correlated via a few parameters that characterize the long-range piece of the quark-quark interaction, and which it may be possible to relate to the underlying parameters in QCD. A paper describing this work was published. ${ }^{1}$

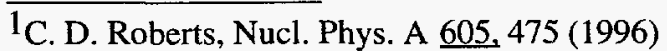




\section{a.2. Study of the Anomalous Process $\gamma \pi \rightarrow \pi \pi$}

The anomalous form factor for the $\gamma \pi \rightarrow \pi \pi$ transition, denoted by $F^{3 \pi}(s, t, u)$, has been measured at Serpukhov in the Primakov reaction $\pi^{-} \mathrm{A} \rightarrow \pi^{\prime} \pi^{0} \mathrm{~A}^{\prime}$. In this experiment the considerable uncertainty in both the kinematic range and result make it difficult to draw a conclusion regarding the accuracy of the theoretical prediction for the chiral limit value of $F^{3 \pi}(0,0,0)$. New experiments are planned at TJNAF: $\gamma \mathrm{p} \rightarrow \pi^{+} \pi^{0} n$, $\mathrm{s}=4-15 \mathrm{~m}_{\pi}^{2}$; and at Fermilab via the Primakov reaction using a $600 \mathrm{GeV}$ pion beam, $\mathrm{s}=4-6 \mathrm{~m}_{\pi}^{2}$. Such processes are of particular interest because they are intimately connected to the anomaly structure of QCD, which is tied to the quantum field theoretic character of the theory. We have calculated this
(C.D. Roberts and R. Alkofer*)

transition form factor as a phenomenological application of the QCD Dyson-Schwinger equations. The chiral-limit value dictated by the electromagnetic, anomalous chiral Ward identity, is reproduced, independent of the details of the modeling of the gluon and quark 2-point Schwinger functions. Using a parametrization of the dressed u-d quark 2-point Schwinger function that provides a good description of pion observables, $\mathrm{F}^{3 \pi}(\mathrm{s}, \mathrm{t}, \mathrm{u})$ is calculated in a kinematic range that proposed experiments plan to explore. Our result confirms the general trend of other calculations; i.e., a monotonic increase with $s$ at fixed $t$ and $u$, but is uniformly larger and exhibits a more rapid rise with $\mathrm{s}$. An article describing this work was published. ${ }^{1}$

${ }^{*}$ University of Tubingen, Germany, ${ }^{1}$ R. Alkofer and C. D. Roberts, Phys. Lett. B369, 101 (1996)

a.3. Electromagnetic Charged and Neutral Kaon Form Factors (C.D. Roberts, C.J. Burden,* and M.J. Thomson $\dagger$ )

The electromagnetic form factors of the charged and neutral kaon are calculated using the approach applied in the successful study of the pion form factor, described above. The charged kaon form factor will be measured in forthcoming experiments at TJNAF. Our calculation involves the dressed strange quark propagator, to which $F_{\pi}\left(q^{2}\right)$ is not sensitive, and hence it provides us with constraints on the strange-quark sector of QCD. This calculation is the first to yield a value of $f_{K} / f_{\pi}$ that is in good agreement with experiment and also yields $r_{K^{+}} / r \pi$ in good agreement with experiment. The results are sensitive to the difference between the kaon and pion BetheSalpeter amplitude and the u- and s-quark propagation characteristics, which demonstrates that such exclusive processes are a probe of nonperturbative, quark-gluon bound-state dynamics. We find that on the range of $Q^{2}$ currently accessible to experiment $F_{K} \pm\left(Q^{2}\right)>F_{\pi}\left(Q^{2}\right)$. Further, $\mathrm{Q}^{2} \mathrm{~F}_{\mathrm{K}}\left(\mathrm{Q}^{2}\right)$ has a peak, at approximately the same position as $Q^{2} F \pi\left(Q^{2}\right)$; this is a signal of quarkantiquark recombination into the final state meson in exclusive elastic scattering processes. We find for the neutral Kaon that $r_{\mathbf{K}^{0}}^{2}<0$. A paper describing this work was published.

*Australian National University, Canberra, Australia, $\uparrow$ University of Melbourne, Australia

${ }^{1}$ C.J. Burden, C.D. Roberts and M.J. Thomson, Phys. Lett. B $\underline{371}, 163$ (1996).

\section{a.4. Quark and Diquark Confinement beyond Rainbow/Ladder Approximation} (C.D. Roberts, L. von Smekal, and A. Bender*)

Confinement of color is an important feature of the strong interaction spectrum at accessible energy scales; this means not only that isolated quarks and gluons have not been observed but also that the only bound states that have been observed are color neutral. In quantum field theory two-body bound states are studied via the inhomogeneous Bethe-Salpeter equation for the T-matrix. A pole in $\mathrm{T}$, at a given total momentum, corresponds to a bound-state and enables one to extract the bound-state Bethe-Salpeter amplitude. This approach allows one to study quark-antiquark (meson) bound-states and also quark-quark (diquark) systems, which can occur in two color-charge states denoted by $\overline{3}$ and 6 . The simplest approximation to the coupled Dyson-Schwinger/Bethe-Salpeter equation system, which is used to study bound-states of stronglydressed quarks and anti-quarks, is the rainbow/ladder approximation and may be described mnemonically as $O\left(g^{2}\right)$. This provides the basis for an excellent description of meson spectroscopy and dynamics but admits $\overline{3}$ diquark bound-states of mass $\geq 700 \mathrm{MeV}$, which are not observed. In the first study of its type, we have employed a simple model gluon propagator in studies of this coupled system of

*University of Tübingen, Germany 
equations at the next level of approximation, which may be denoted as $\mathrm{O}\left(\mathrm{g}^{4}\right)$. The $\mathrm{O}\left(\mathrm{g}^{2}\right)$ studies have the feature that one has a manifest realization of Goldstone's theorem; i.e., the pseudoscalar bound-state is massless if chiral symmetry is dynamically broken. We have found that the $\mathrm{O}\left(\mathrm{g}^{4}\right)$ preserves this property. We have also found that, in meson systems, these higher order corrections shift the meson mass by less than $10 \%$, which is an indication that the $\mathrm{O}\left(\mathrm{g}^{2}\right)$ (rainbow/ladder) approximation in this channel is qualitatively and quantitatively reliable. However, the $\mathrm{O}\left(\mathrm{g}^{4}\right)$ have a dramatic effect in the diquark channel: they ensure that there is no pole at real timelike- $\mathrm{P}^{2}$ and hence that there are no stable diquark excitations, an observational fact that previous studies have not been able to reproduce. In this simple model the quark and gluon excitations are confined; i.e., their propagators are such that there is no asymptotic state associated with them, and this calculation provides an illustration of how an interaction with this feature can also entail the absence of colored bound-states from the particle spectrum. In this study one obtains confinement without explicit three-gluon vertices. Their contribution is nevertheless crucial since they are responsible for the enhancement of the gluon (and hence quark) propagators in the infrared. A paper describing this work was published. ${ }^{1}$

${ }^{1}$ A. Bender, C. D. Roberts, L. von Smekal, Phys. Lett. B380, 7 (1996)

\section{a.5. Renormalization and Chiral Symmetry Breaking in Quenched QED in Arbitrary Covariant Gauge (C.D. Roberts, F.T. Hawes, ${ }^{*}$ and A.G. Williams $\dagger$ )}

A study of subtractive renormalization of the fermion propagator Dyson-Schwinger equation in strongcoupling, quenched QED4 is conducted using a fermion-photon proper vertex proposed by Curtis and Pennington with an additional correction term included to compensate for the small gauge-dependence induced by the ultraviolet regulator. Studies such as this are crucial in understanding renormalization non- perturbatively. The chiral limit is discussed along with the onset of dynamical chiral-symmetry breaking in the presence of nonperturbative renormalization. The critical coupling for dynamical chiral-symmetry breaking is obtained in several different gauges. With the vertex Ansatz employed, there is a small residual gauge-dependence in this quantity. A paper describing this work was published. ${ }^{1}$

*Florida State University, $\nmid$ University of Adelaide, Australia

${ }^{1}$ F.T. Hawes, A.G. Williams, and C.D. Roberts, Phys. Rev. D 54, 5361 (1996).

\section{a.6. Dynamical Chiral Symmetry Breaking and Confinement at Finite Temperature (C. D. Roberts, A. Bender,* D. Blaschke, $\nmid$ and Yu. Kalinovsky $\dagger$ )}

The Dyson-Schwinger equation for the quark propagator is studied at finite temperature using a oneparameter, model gluon propagator used successfully in studies of $\pi$ and $\rho$-meson properties at zero temperature. Through the introduction of a continuum order parameter for deconfinement, applicable for both light and heavy quarks, this study provides insight into the existence and nature of chiral symmetry restoration and deconfinement in QCD, which is a significant part of the physics program at RHIC. With the one parameter in the gluon propagator fixed in calculations of observablesat $T=0$, one finds coincident chiral symmetry restoration and deconfinement transitions at $\mathrm{T}_{\mathrm{C}} \approx 150 \mathrm{MeV}$, which is illustrated in Fig. IV-1. The transitions are second order and each has the same critical exponent; $\beta \approx 0.3$, consistent with that of the $\mathrm{N}=4$ Heisenberg magnet which characterizes the universality class expected for two-flavor QCD. For $\mathrm{T}<0.7 \mathrm{~T}_{\mathrm{c}}, \mathrm{f}_{\pi}$ and $\mathrm{m}_{\pi}$ change by less than $10 \%$, as illustrated in Fig. IV-2. However, as $T \rightarrow T_{\mathfrak{C}}$, thermal fluctuations cause the pion bound state contribution to the four-point quark-antiquark correlation function to disappear. A paper describing this work was published. ${ }^{1}$

*University of Adelaide, Australia, †University of Rostock, Germany

${ }^{1}$ A. Bender, D. Blaschke, Yu. Kalinovsky and C. D. Roberts, Phys. Rev. Lett. 77, 3724 (1996). 


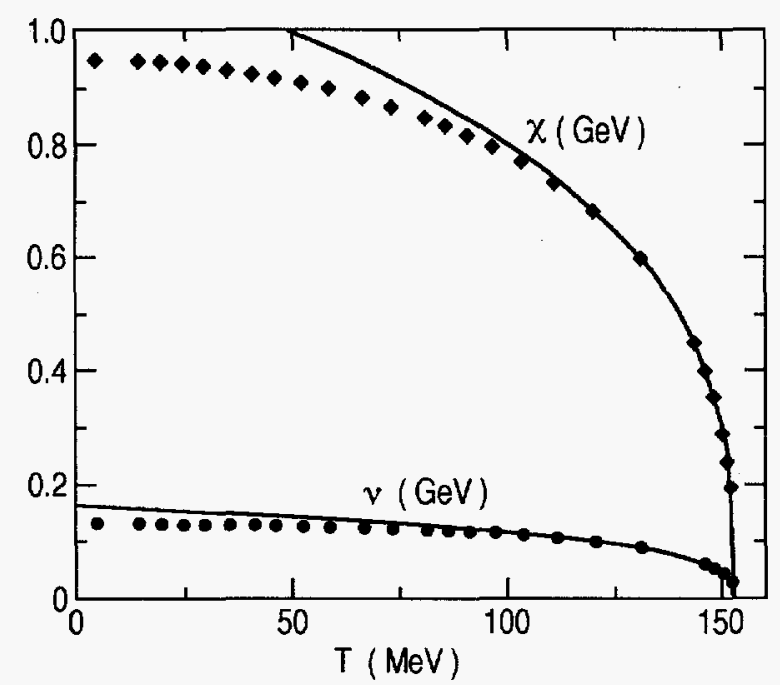

Fig. IV-1. The order parameters for chiral symmetry restoration $(\chi(T)$, diamonds) and deconfinement $\left(\nu(T)\right.$, circles) both vanish at $T_{c} \simeq 150 \mathrm{MeV}$.

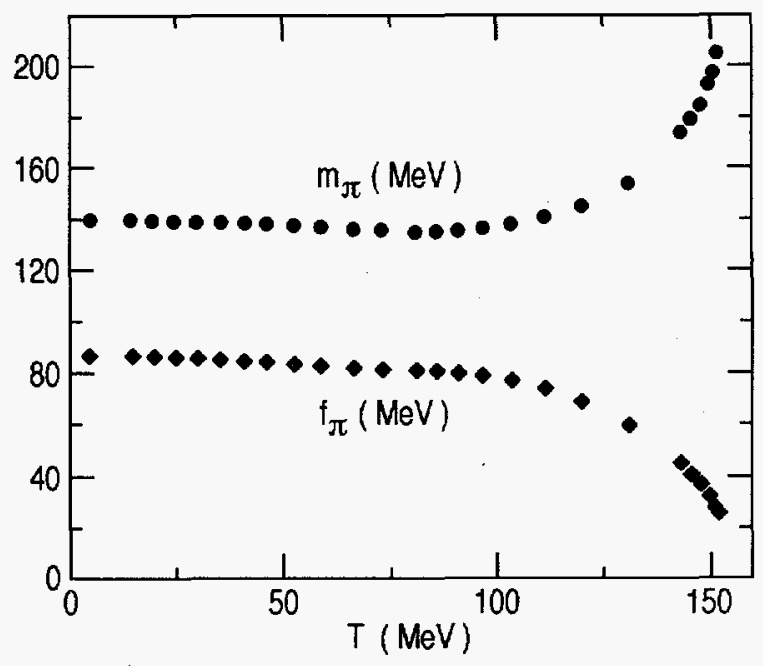

Fig. IV-2 Temperature dependence of the pion mass $\left(m_{\pi}(T)\right.$, circles) and pion weak-decay constant $\left(f_{\pi}(T)\right.$, diamonds).

\section{a.7. Bethe-Salpeter Equation Studies of $\mathrm{SU}_{\mathbf{f}}(3)$ Mesons (C.D. Roberts, C.J. Burden, ${ }^{*}$ Lu Qian, $\dagger$ P.C. Tandy, $\dagger$ and M.J. Thomson $\left.\ddagger\right)$}

The spectroscopy and weak decays of mesons composed of $\mathrm{u}, \mathrm{d}$ and $\mathrm{s}$ quarks is studied using a separable Ansatz for the quark-quark scattering kernel, which is obtained by inverting the DSE for the quark propagator. The quark propagator used is the phenomenologically-efficacious, confining model form derived in the study of the pion and kaon form factors, described above. One obtains a good description of the spectrum and decays, which indicates that pion and kaon observables can be used to constrain and provide information about the form of the quark-quark interaction in QCD. The simplicity of the approach allows one to study the influence of sub-leading Diraccomponents in the meson Bethe-Salpeter amplitude. We find, for example, that in this framework the pseudoscalar-pseudovector mixing is significant in the pion channel. Two articles based on this work have been published ${ }^{1}$ and a further has been accepted for publication.

*Australian National University, Canberra, Australia, $\uparrow$ Kent State University, $\ddagger$ University of Melbourne, Australia ${ }^{1}$ C. J. Burden et al., Nucl. Phys. B (Proc. Suppl.) 47, 362 (1996); C. J. Burden, et al., Aust. J. Phys., 50, 95 (1997).

\section{a.8. $\quad \mathrm{K} \ell 3$ and $\pi_{\mathrm{e}} 3$ Transition Form Factors (C.D. Roberts, Yu. Kalinovsky,* and K.L. Mitchell $\dagger$ )}

$\mathrm{K}_{\ell 3}$ and $\pi_{\mathrm{e} 3}$ transition form factors, which are sensitive to the vector piece of the electroweak current, are calculated as an application of Dyson-Schwinger equations. A heuristic one-parameter model of the quark-W-boson vertex is used to highlight their sensitivity to the form of this vertex and the existence of a systematic discrepancy between the behavior of these form factors in the timelike region when calculated using quark-gluon degrees of freedom as compared

*JINR, Dubna, Russia, †TRIUMF, Canada with that calculated using meson degrees of freedom. This discrepancy is identified as the result of the omission, in most quark-gluon based studies, of nonanalytic contributions to the quark-W-boson vertex, which are important in the timelike region but rapidly become unimportant in the spacelike region. The behavior of these form factors in the spacelike region is not accessible in meson-based studies. Each of the transitions is described by two form factors, $f_{ \pm}(t)$. Some robust results of our calculation are that: the $K_{\ell 3}$ form factors, $f \underset{\perp}{K}$, are approximately linear on $t \in$ 


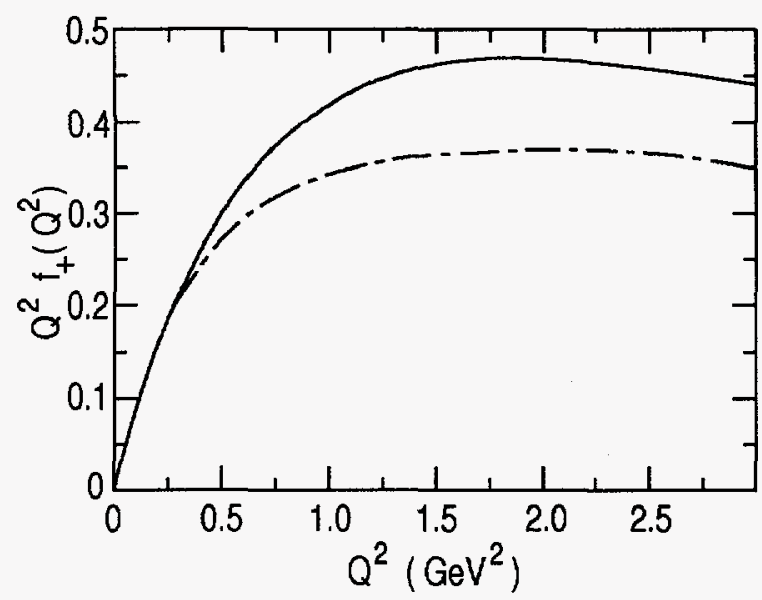

Fig. $I V-3-Q^{2} \mathrm{f}_{+}^{\mathrm{K}^{\prime}}\left(Q^{2}\right)$ : solid line; $-Q^{2} \mathrm{f}_{+}^{\pi}\left(Q^{2}\right)=$ $Q^{2} F \pi\left(Q^{2}\right)$ : dot-dashed line. The peak in $Q^{2} f_{+}\left(Q^{2}\right)$, most pronounced for the kaon, is a characteristic signal of quark-antiquark recombination into the meson final state in exclusive processes. $\left[\mathrm{m}_{\mathrm{e}}^{2}, \mathrm{~m}_{\mu}^{2}\right] ; \mathrm{f}_{-}(0)$ is a measure of the Euclidean constituent-quark mass ratio: $\mathrm{M}_{\mathrm{s}}^{\mathrm{E}} / \mathrm{M}_{\mu}^{\mathrm{E}}$; and, in the isospin symmetric limit: $-f_{+}^{\pi}(0)=F_{\pi}(t)$, the electromagnetic pion form factor, with $\mathrm{f}-\underline{\pi}(\mathrm{t}) \equiv 0$. Our calculated $\mathrm{K}$ and $\pi$ transition form factors are presented in Fig. IV-3. Our Ansatz for the quark-W-boson vertex was a heuristic tool, meant only to illustrate and highlight the discrepancy between quark-based and meson-based studies. Our prediction that $f_{+}^{K^{\prime}}(0) \sim$ $\mathbf{f}^{\prime}(0)$ may be modified as we develop a more detailed understanding of the dressed quark-W-boson vertex, which further studies of electroweak processes promise to make possible.

\section{a.9. Electromagnetic Nucleon Form Factors (C.D. Roberts, A. Elender, ${ }^{*}$ and M.R. Frank $\dagger$ )}

The Dyson-Schwinger equation framework is employed to obtain expressions for the electromagnetic nucleon form factor. In impulse approximation the form factor depends on the dressed quark propagator, the dressed quark-photon vertex, which is crucial to ensuring current conservation, and the nucleon Faddeev amplitude. The approach manifestly incorporates the large spacelike- $\mathrm{q}^{2}$ renormalization group properties of QCD and allows a realistic extrapolation to small spacelike-q ${ }^{2}$. This extrapolation allows one to relate experimental data to the form of the quark-quark interaction at small spacelike- $q^{2}$, which is presently unknown. The approach provides a means of unifying, within a single framework, the treatment of the perturbative and nonperturbative regimes of $\mathrm{QCD}$. The wealth of experimental nucleon form factor data, over a large range of $q^{2}$, entails that this application will provide an excellent environment to test, improve, and extend our approach, which has been very successful in its application to meson observables. This is particularly true since the nucleon is a system in which explicit three-gluon vertices can play a qualitatively new role, attaching to each of the three dressed-quarks in the bound state, a process which one cannot model as a two-body force. Preliminary progress is encouraging and has been described at national and international meetings.

*University of Adelaide, Australia, $†$ Institute for Nuclear Theory, University of Washington

\section{a.10. Equation of State for Confined Quarks (C.D. Roberts, D. Blaschke,* and S. Schmidt*)}

A simple, confining Dyson-Schwinger equation model of two-flavor QCD is used to study the effects of temperature and a chemical potential on confinement and dynamical chiral symmetry breaking in QCD. The effects of a nonzero chemical potential cannot currently be studied in lattice simulations of QCD because the action is complex, which prevents a straightforward analysis of the generating functional using Monte-Carlo to that region presently inaccessible in lattice simulations of QCD. The preliminary goal of this study, a calculation of the equation of state for a system of confined quarks, is almost complete and has led to the development of numerical techniques that will be crucial in studying more realistic models. Further, the study provides a qualitatively reliable estimate of techniques. This study therefore provides a means of extrapolating from the domain of common agreement between lattice and Dyson-Schwinger equation studies the chiral symmetry restoration and deconfinement phase boundary in the $(\mathrm{T}, \mu)$ plane, indicating that such gross properties are not too sensitive to the details of the confinement dynamics.

*University of Rostock, Germany 


\section{a.11. Thermodynamics and Bound State Properties at Finite Temperature (C.D. Roberts, D. Blaschke,* A. Hoell,* and Yu. Kalinovsky $\dagger$ )}

A detailed study of the thermodynamics of a confining Dyson-Schwinger equation model of two-flavor QCD is being undertaken. This serves to further delineate the common domain between lattice simulations and Dyson-Schwinger equation studies, providing a firmer foundation for the extrapolation of these studies outside of this domain to the exploration of bound state properties. Bound state studies are sensitive to the details of the quark-quark interaction and their response to finite temperature and chemical potential provide information about those signals, to search for at RHIC, that will mark the transition to a quark gluon plasma.

*University of Rostock, Germany, $\uparrow$ JINR, Dubna, Russia

\section{a.12. Bethe-Salpeter Equation Studies of Light Quark Mesons (P. Maris and C.D. Roberts)}

A one-parameter model for the gluon propagator used successfully in the study of $\pi$ - and $\rho$-meson observables, is employed in a study of the ground state spectrum and weak decays of pseudoscalar mesons. In covariant studies of two-body bound states the relative momentum, $\mathrm{k}$, is not unambiguously defined, but depends on an arbitrary partitioning parameter, $\eta$. The bound-state mass must be independent of $\eta$. This study demonstrates, for the first time, that a proper treatment of the dependence of the bound-state amplitude on $\mathrm{k} \cdot \mathrm{P}$, where $\mathrm{P}$ is the total momentum, yields a bound state mass that is independent of $\eta$ - a significant advance in covariant studies of bound state spectroscopy and interactions. Preliminary results suggest that the model gluon propagator, whose single mass-scale parameter was fitted to describe the physics of the $u / d$-quark sector of QCD, is well suited to the description of bound states involving heavier quarks. A crucial element of this study is the development of an understanding of nonperturbative renormalization in the context of bound-state problems.

\section{a.13. Nucleons in a Nuclear Medium (C.D. Roberts, G. Poulis, ${ }^{*}$ and A.W. Thomas*)}

In no single approach is it presently possible to address all of the observables likely to be probed in present generation accelerators, such as TJNAF. It is therefore important to develop those opportunities for crossfertilization between nonperturbative approaches and models in order to identify robust features. The quarkmeson coupling model, in which quarks couple directly to uniform scalar and vector background fields that characterize the presence of the nuclear medium, provides one means of studying the effect that embedding a nucleon in a nucleus has on observable hadronic properties. The postulated behavior of the quarks in this model can be explored and tested by introducing a background $\sigma$-field in the quark Dyson-Schwinger equation and studying the effect this has on quark propagation and, intrinsically thereby, on the nucleon mass. Preliminary studies indicate that the postulates are qualitatively reliable; i.e., that the nucleon mass is weakly modified (reduced) by the presence of the other nucleons. These explorations will continue and be used to provide constraints on the further development of the quarkmeson coupling model.

*University of Adelaide, Australia 


\section{a.14. The Strong Running Coupling from an Approximate Gluon Dyson- Schwinger Equation (L. von Smekal, R. Alkofer,* and A. Hauck*)}

The infrared behavior of the running coupling in strong interactions, $\alpha_{S}=g^{2} / 4 \pi$, is of great interest, since it may provide an understanding of confinement. Its study is an intrinsically nonperturbative problem. One suitable framework to address this problem is provided by the Dyson-Schwinger equations (DSE) of QCD. Studies of this infinite tower of equations rely on specific truncation schemes. In the present study we focus on an approximation to the gluon DysonSchwinger equation originally proposed by Mandelstam. 1

Solutions to the resulting non-linear integral equation for the gluon self-energy have been studied and approximated with various numerical and analytic methods ${ }^{2}$ showing that it results in an infrared-singular gluon propagator diverging as $1 / \mathrm{k}^{4}$ for $\mathrm{k}^{2} \rightarrow 0$. It can be shown that in the Mandelstam approximation the

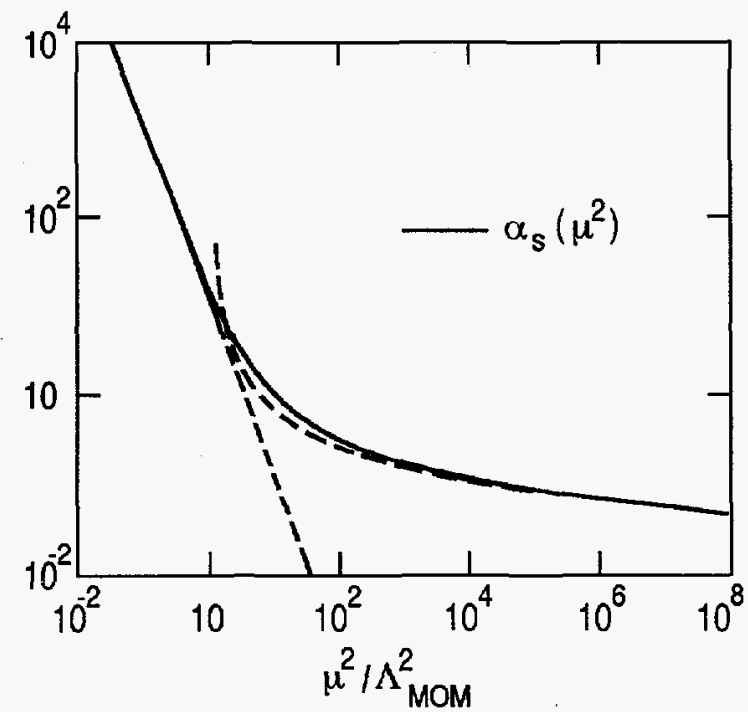

product of the coupling and gluon propagator, $\mathrm{gD}_{\mu \mu}(\mathrm{k})$, is a renormalization-group invariant, which is obtained as a self-consistent solution to the approximate gluon DSE, and the infrared-singular result allows to identify a physical scale, the string tension. ${ }^{3}$

The nonperturbative and renormalization-group invariant result relates the string tension $\sigma$ to the QCD scale (the only parameter) by $\sigma=2 \Lambda^{2}$. The string tension can be fixed from quarkonia potentials and Regge phenomenology. The result is a value of about $\sigma$ $=0.18 \mathrm{GeV}^{2}$ which has also been confirmed in lattice calculations. Here, it corresponds to a scale $\Lambda$ of about $600 \mathrm{MeV}$. On the other hand, for a value of $\alpha_{S}\left(M_{Z}^{2}\right)$ $\simeq 0.118$ with $\mathrm{MZ}_{\mathrm{Z}}=91 \mathrm{GeV}$ being the mass of the $\mathrm{Z}$ boson we find $\Lambda \simeq 900 \mathrm{MeV}$. This compares favorably with recent lattice calculations of the running coupling from the 3-gluon vertex (without dynamical quarks).

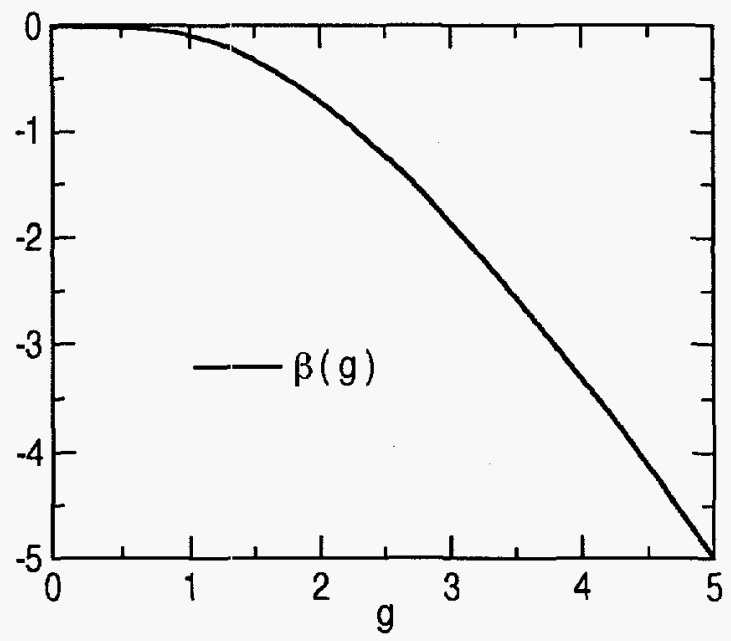

Fig. IV-4. The strong coupling $\alpha_{S}\left(\mu^{2}\right)$ with its asymptotic limits and the non-perturbative $\beta$-function of the Mandelstam approximation for a momentum subtraction scheme. ${ }^{3}$

While a relation between $\sigma$ and $\Lambda^{2}$ such as the present one arises naturally from the nonperturbative infrared singular result, the constant of proportionality will very likely change in more refined calculations. It might depend on the number of dynamical quarks, for instance. Even though we could bring both values for the scale considerably closer within present experimental as well as theoretical uncertainties, e.g., for $\alpha_{S}\left(M_{Z}^{2}\right)=0.108$ we obtain $\Lambda=640 \mathrm{MeV}$, we do not expect our calculation to be quantitatively more accurate than the above estimates suggest.

*University of Tübingen, Germany

${ }_{1}$ S. Mandelstam, Phys. Rev. D 20 (1979) 3223.

2 D. Atkinson et al., J. Math. Phys. $\underline{23}$ (1982) 1917; N. Brown and M. R. Pennington, Phys. Rev. D $\underline{39}$ (1989), 2723.

${ }^{3}$ A. Hauck et al., preprint, ANL-PHY-8386-TH-96 (1996), (hep-ph/9604430). 
We rather conclude that a range from 600 to $900 \mathrm{MeV}$ gives the correct order of magnitude for the scale $\Lambda$ to be used here.

The scaling behavior of the solution near the ultraviolet fixed point is determined by the coefficients $\beta_{0}=14$ and $\gamma_{\mathrm{A}}^{0}=7$ which are reasonably close to the perturbative values for $\mathrm{N}_{\mathrm{f}}=0$, i.e., $\beta_{0}=11$ and $\gamma_{\mathrm{A}}^{0}=$ $13 / 2$.
The running coupling, which is obtained for arbitrary scales from the renormalization condition, resembles the two-loop perturbative form for asymptotically high scales. It is shown together with the corresponding Callan-Symanzik $\beta$-function in Fig. IV-4. In the infrared the running coupling increases without bound. Accordingly, the $\beta$-function does not have any zeros for positive values of the coupling, and no further fixed point exists.

\section{a.15. A Dynamical $\eta^{\prime}$-Mass from an Infrared Enhanced Gluon Exchange (L. von Smekal, R. Alkofer,* and A. Mecke*)}

The pseudoscalar flavor-singlet meson mixes with two gluons. Using perturbative gluons and constituent quarks, the corresponding lowest-order diagram yields an additional contribution to the decay constant as compared to the pion. However, no dynamical mass to screen the Goldstone pole in the chiral limit in this channel can be obtained this way. Dimensional arguments to the effect that an infrared-enhanced gluon propagator is sufficient to generate such a mass are long known (Kogut-Susskind mechanism). We showed this explicitly for a gluon propagator as singular as $\sigma / \mathrm{k}^{4}$ for $\mathrm{k}^{2} \rightarrow 0$. The resulting mass contribution to the pseudoscalar flavor-singlet meson is fixed by the phenomenological string tension $\sigma$. In the WittenVeneziano action this gives masses of about $810 \mathrm{MeV}$ for the $\eta^{\prime}-$ meson and $430 \mathrm{MeV}$ for the $\eta$ as well as a mixing angle of about -30 degrees, suggesting that the $\mathrm{U}_{\mathrm{A}}$ (1)-anomaly might be encoded in the infrared behavior of QCD Green's functions.

*University of Tübingen, Germany

\section{a.16. Production of Mesons and Nucleon Resonances at GeV Energies} (T.-S. H. Lee, M. Arima,* and T. Sato $\dagger$ )

We have been developing a theoretical model for investigating the nucleon resonances $\left(\mathrm{N}^{*}\right)$ at energies accessible to CEBAF and relativistic heavy-ion facilities. Motivated by the existing QCD-based hadron models, we assume that the basic resonant interaction mechanisms of the model Hamiltonian are the absorption and emission of photons and mesons by a bare quark core. The matrix elements of nonresonant interactions are deduced from an effective Lagrangian with chiral symmetry by using a method of unitary transformation. The standard projection operator technique was applied to obtain a set of unitary scattering equations for describing $\pi \mathrm{N}$ and $\gamma \mathrm{N}$ reactions up to the GeV energy region. In FYs 1995 and 1996, a model has been obtained for a consistent description of $\pi \mathrm{N}$ scattering and $\gamma \mathrm{N} \rightarrow \pi \mathrm{N}$ reaction up to the $\Delta$ excitation energy region. It is shown that the best reproduction of the recent LEGS data of the photonasymmetry ratios in $\gamma p \rightarrow \pi^{0} p$ reactions provides rather restricted constraints on the coupling strengths $G_{E}$ of the electric $E 2$ and $G_{M}$ of the magnetic $M 1$ transitions of the bare $\Delta \rightarrow \gamma \mathrm{N}$ vertex and the less welldetermined coupling constant $\mathrm{g} \omega \mathrm{NN}$ of the $\omega$ meson.
Within the ranges that $\mathrm{G}_{\mathrm{M}}=1.9 \pm 0.05, \mathrm{G}_{\mathrm{E}}=$ $0.0 \pm 0.025$, and $7 \leq \mathrm{g}_{\omega \mathrm{NN}} \leq 10.5$, the predicted differential cross sections and photon-asymmetry ratios are in overall good agreement with $\gamma \mathrm{p} \rightarrow \pi^{0} \mathrm{p}, \gamma \mathrm{p} \rightarrow$ $\pi^{+} \mathrm{n}$, and $\gamma \mathrm{n} \rightarrow \pi^{-} \mathrm{p}$ reactions from $180 \mathrm{MeV}$ to the $\Delta$ excitation region. The predicted $\mathrm{M}_{1}+$ and $\mathrm{E}_{2}+$ multipole amplitudes are also in good agreement with the empirical values determined by the amplitude analyses. The constructed effective Hamiltonian is free of the nucleon renormalization problem and hence is suitable for nuclear many-body calculations. We have also shown that the assumptions made in the K-matrix method, commonly used in extracting the $\gamma \mathrm{N} \rightarrow \Delta$ transition amplitudes from the data, are consistent with our meson-exchange dynamical model. It is found that the helicity amplitudes calculated from our bare $\gamma \mathrm{N} \rightarrow$ $\Delta$ vertex are in good agreement with the predictions of the constituent quark model. The differences between these bare amplitudes and the dressed amplitudes, which are closer to the empirical values listed by the Particle Data Group, are shown to be due to the nonresonant meson-exchange mechanisms. Our results are illustrated in Table IV-1. Within the range $7 \leq$

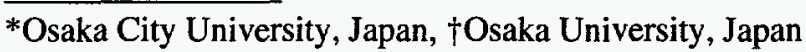


$\mathrm{g} \omega \mathrm{NN} \leq 10.5$ of the $\omega$ meson coupling favored by the data of the photon-asymmetry ratios in $\gamma p \rightarrow \pi^{0} p$ reactions, our values of the E2/M1 ratio for the $\gamma \mathrm{N} \rightarrow \Delta$ transition are $(0.0 \pm 1.3) \%$ for the bare vertex and $(-1.8 \pm 0.9) \%$ for the dressed vertex. The results have been published in Phys. Rev. C $\underline{54}, 2660$ (1996). Our current focus is to extend the Hamiltonian to include the $S_{11}(1530)$ resonance and the coupling with the $\pi \Delta$ and $\eta \mathrm{N}$ channels.

Table IV-1. Helicity amplitudes of the $\Delta \rightarrow \gamma N$ transition at $W=1236 \mathrm{MeV}$ are compared with the values from Particle Data Group (PDG) and the predictions of the constituent quark model (CQM). The amplitudes are in units of $10^{-3}(\mathrm{GeV})^{-1 / 2}$.

\begin{tabular}{c|c|c|c|c} 
A & PDG & \multicolumn{1}{c}{ Dressed } & Bare & CQM \\
\hline A3/2 & $-257 \pm 8$ & -228 & -153 & -157 \\
$\mathrm{~A}_{1 / 2}$ & $-141 \pm 5$ & -118 & -84 & -91
\end{tabular}

\section{a.17. Exclusive Diffractive Processes and the Quark Substructure of Mesons} (T.-S. H. Lee and M. A. Pichowsky*)

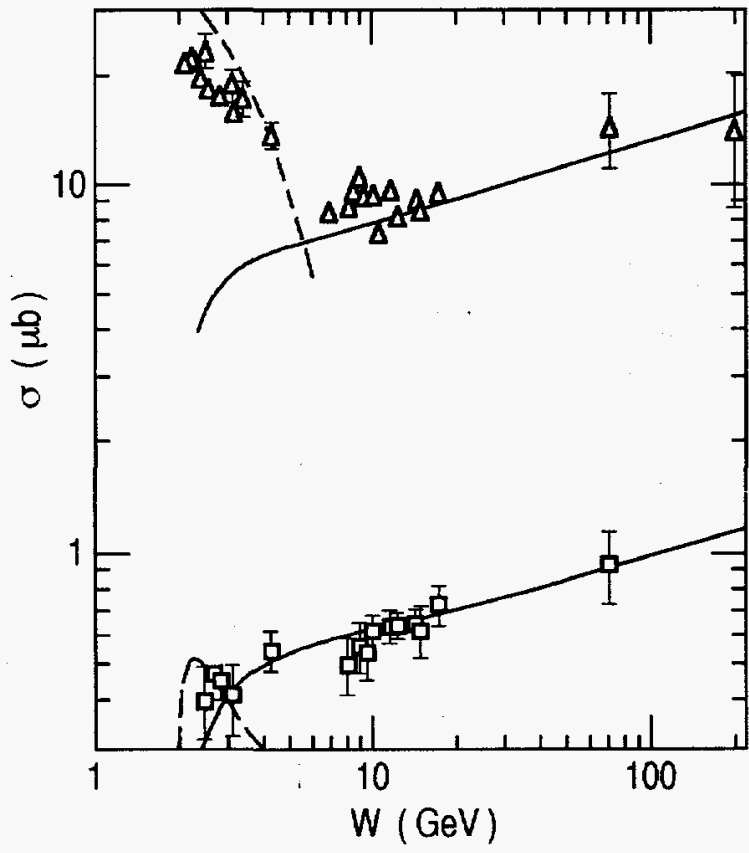

Figure IV.-5. The predicted energy dependence of $\rho$ (top) and $\phi$-meson (bottom) photoproduction cross sections are compared with data. The solid curves are the predictions from our quark-nucleon Pomeron-exchange interaction. The dashed curves are the predictions of the meson-exchange model.
Exclusive diffractive processes on the nucleon are investigated within a model in which the quark-nucleon interaction is mediated by Pomeron exchange and the quark substructure of mesons is described within a framework based on the Dyson-Schwinger equations of QCD. The model quark-nucleon interaction has four parameters which are completely determined by high-energy $\pi \mathrm{N}$ and $\mathrm{KN}$ elastic scattering data. The model is then used to predict vector-meson electroproduction observables. The $\rho$ - and $\phi$ meson electroproduction cross sections obtained are in excellent agreement with experimental data. This is shown in Fig. IV.5. The predicted $\mathrm{q}^{2}$ dependence of $\mathrm{J} / \psi$ meson electroproduction also agrees with experimental data. It is shown that confined-quark dynamics play a central role in determining the behavior of the diffractive, vector-meson electroproduction cross section. In particular, the onset of the asymptotic $1 / q^{4}$ behavior of the cross section is determined by a momentum scale that is set by the currentquark masses of the quark and antiquark inside the vector meson. This is the origin of the striking differences between the $q^{2}$ dependence of $\rho-, \phi$ - and $J / \psi$-meson electroproduction cross sections observed in recent experiments. A paper describing some of these results has been published in Phys. Lett. B $\underline{379}, 1$ (1996). The detailed account of this work is published in the Ph.D. thesis by M. P. Pichowsky, and in a paper submitted for publication.

$\overline{\text { *University of Pittsburgh }}$ 


\section{a.18. The E2/M1 Ratio of the $\Delta \leftrightarrow \gamma \mathbf{N}$ Transition Within the Chiral Constituent Quark Model (T.-S. H. Lee, Peng-Nian Shen,* Yu-Bin Dong, * Zong-Ye Zhang,* and You-Wen $\mathrm{Yu}^{*}$ )}

The chiral constituent quark model is applied to predict the magnetic M1 and electric E2 amplitudes of the $\Delta \leftrightarrow$ $\gamma \mathrm{N}$ transition. It is found that the one-meson-exchange quark-quark potential due to the chiral fields enhances the E2/M1 ratio by a factor of about 2 . The predicted
$\mathrm{M}_{1}+$ amplitude and the $\mathrm{E} 2 / \mathrm{M} 1$ ration $\simeq-1.0 \%$ are in good agreement with the values determined within the dynamical model developed by Sato and Lee (see IV.a.17). A paper describing our results has been accepted by Physical Review C.

*Academia Sinica, China

\section{a.19. Phenomenological Coupled-Channel Model for $\pi \mathbf{N}$ and $\gamma \mathbf{N}$ Reactions With $\mathbf{N}^{*}$ Excitations (T.-S. H. Lee, T. Vrana, ${ }^{*}$ and S. Dytman*)}

Under CEBAF's $\mathrm{N}^{*}$ program, extensive $\gamma \mathrm{N} \rightarrow \pi \mathrm{N}, \pi \pi \mathrm{N}$ data with unprecedented accuracy will be available. One of the main tasks is to extract from the data the multipole amplitudes which can then be used to test various hadron models. Because of multi-channel complications, the available methods based on dispersion relations and $\mathrm{K}$-matrix methods are not directly applicable at CEBAF energies. We adapted the coupled-channel method developed by Cutkosky and his collaborators in analyzing the $\mathrm{N}^{*}$ in $\pi \mathrm{N}$ reactions.
The channels included in our analysis are $\pi \mathrm{N}, \pi \Delta, \rho \mathrm{N}$, and $\varepsilon N$. The parameters for all $\mathrm{N}^{*}$ listed in the Particle Data Tables are then determined by fitting Manley's representation of $\pi \mathrm{N} \rightarrow \pi \mathrm{N}, \pi \pi \mathrm{N}$ data. In FY 1996, we have demonstrated that the coupled-channel effects are very large and some of the $\mathrm{N}^{*}$ parameters determined in our approach are significantly different from that listed in the Particle Data tables. A paper describing our results is being prepared for publication. The model has been extended to include the $\gamma \mathrm{N}$ channel in order to analyze the forthcoming data from CEBAF.

*University of Pittsburgh

\section{a.20. The $\Delta$ Dynamics in ${ }^{3} \mathrm{He}\left(\mathrm{e}, \mathrm{e}^{\prime} \Delta^{+}\right)$Reaction (T.-S. H. Lee, C. Chmielewski,* and P.U. Sauer*)}

The ${ }^{3} \mathrm{He}\left(\mathrm{e}, \mathrm{e}^{\mathrm{A}} \Delta^{++}\right)$cross sections are predicted by assuming that the $\Delta$ excitations by photons and pions can be described within the chiral constituent quark model. It is shown that in the low $\mathrm{Q}^{2}$ region, the $\Delta^{++}$ component in ${ }^{3} \mathrm{He}$ can be identified in the differential cross section of the produced $\Delta^{++}$in the forward angles with respect to the direction of the exchanged virtual photon. At larger $Q^{2}$, the predicted cross sections in all angles are completely dominated by the $\gamma+(\mathrm{pp}) \rightarrow$ $\Delta^{++} \mathrm{n}$ process which can be used to investigate the recently proposed Chiral Transparency, (see IV.a.23). Our results are shown in Figure IV-6. A paper describing our results is being prepared for publication.

$\overline{\text { *University of Hannover, Germany }}$ 


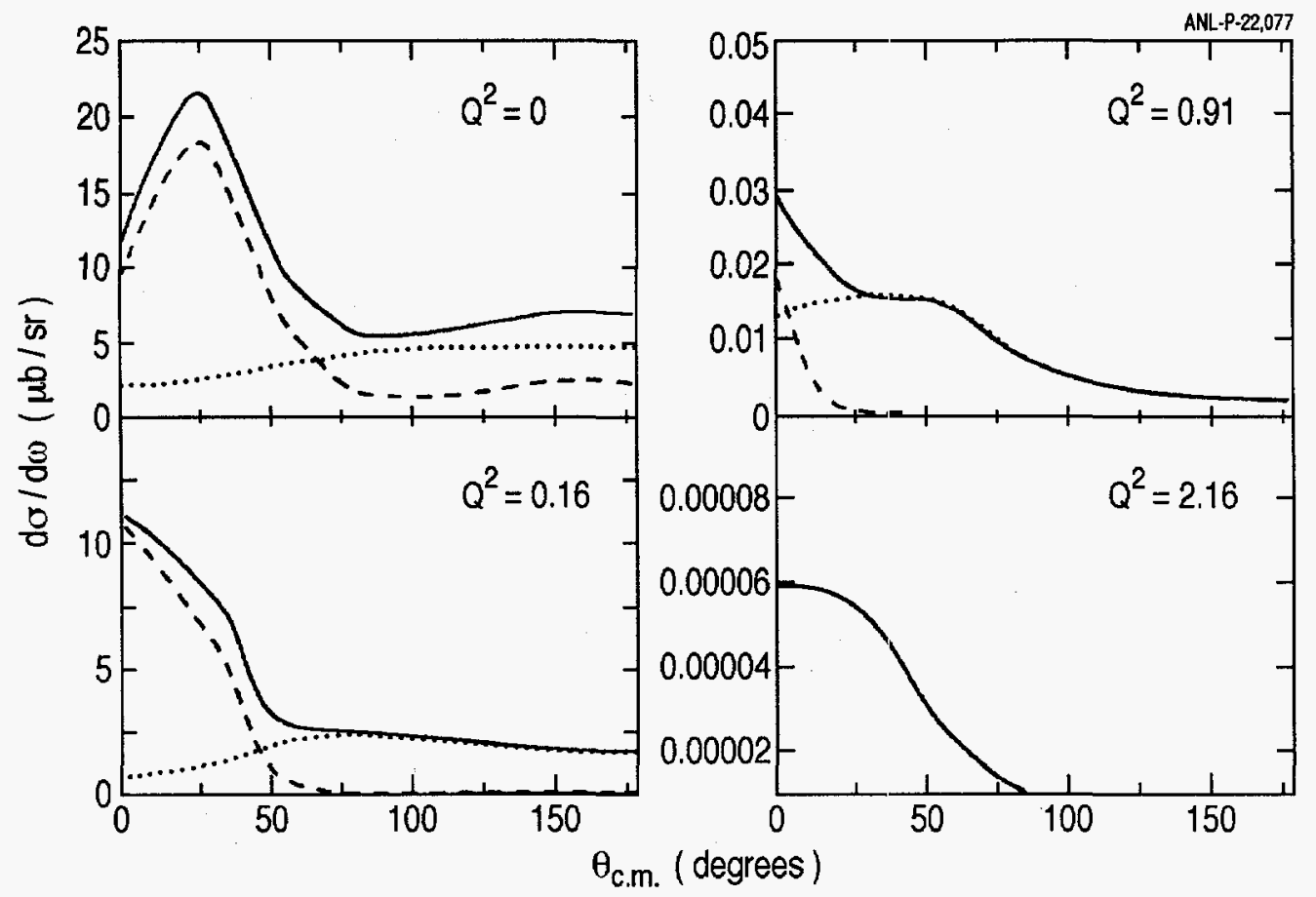

Figure IV-6. The predicted differential cross sections of ${ }^{3} \mathrm{He}\left(e, e^{\prime} \Delta^{++}\right)$reaction at several momentum transfers. The dashed and dotted curves are respectively the contributions from the direct $\Delta^{++} k n o c k o u t$ and the two-body charge-exchange $\gamma+(p p) \rightarrow \Delta^{++} n$ process. The solid curve is the total contribution from these two amplitudes.

\section{a.21. Chiral Perturbation Theory and the $p p \rightarrow p p \pi^{0}$ Reaction Near Threshold} (T.-S. H. Lee, T. Sato, ${ }^{*}$ F. Myhrer, $\dagger$ and K. Kubodera $\dagger$ )

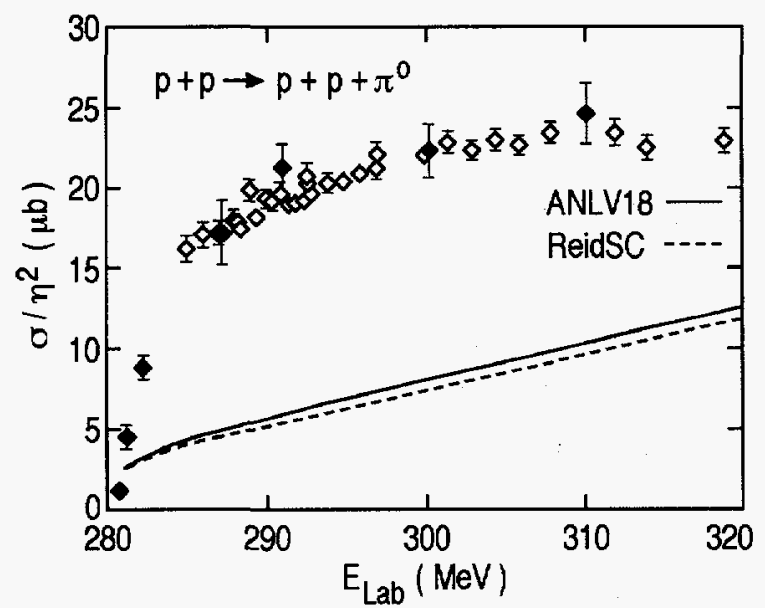

Figure IV-7. The $p p \rightarrow p p \pi^{0}$ total cross sections predicted by the chiral perturbation theory are compared with the data. The solid and dashed curves are respectively calculated from using the Argonne $V_{18}$ and Reid soft-core potentials.

*Osaka University, Japan, $†$ University of South Carolina
A chiral-perturbative consideration of the nearthreshold $\mathrm{pp} \rightarrow \mathrm{pp} \pi^{0}$ reaction indicates that the pionrescattering term has a substantial energy- and momentum-dependence. The existing calculations that incorporate this dependence give pion rescattering contributions significantly larger than those of the conventional treatment, and this enhanced rescattering term interferes destructively with the one-body impulse term, leading to theoretical cross sections that are much smaller than the observed values. However, the existing calculations based on coordinate-space representation involve a number of simplifying assumptions about the energy-momentum flow in the rescattering diagram, even though the delicate interplay between the one-body and two-body terms makes it desirable to avoid these kinematical assumptions. We have carried out a momentum-space calculation which retains the energy-momentum dependence of the vertices as predicted by chiral perturbation theory. Our improved treatment increases the rescattering contribution by a factor of $\sim 2$ over the value obtained in the r-space calculations, and this increase drastically affects the $\mathrm{pp} \rightarrow \mathrm{pp} \pi^{0}$ cross section. Our result, shown in Fig. IV-7, is the starting point of exploring the higher-order chiral dynamics or short-range interactions suggested by Lee and Riska. A paper describing our results is being prepared. 


\section{a.22. Neutral Pion Photoproduction on Deuterium in Baryon Chiral Perturbation Theory of Order $q^{4}$ (T.-S. H. Lee, S. R. Beane,* V. Bernard, $\dagger$ Ulf-G. Meissner, $\ddagger$ and U. van Kolck§)}

Threshold neutral-pion photoproduction on the deuteron is studied in the framework of baryon chiral perturbation theory beyond next-to-leading order in the chiral expansion. To fourth order in small momenta, the amplitude is finite and a sum of two- and three-body interactions with no undetermined parameters. With accurate theoretical and experimental input from the single-nucleon sector for the proton amplitude, we investigate the sensitivity of the threshold cross section to the elementary $\gamma_{n} \rightarrow \pi^{0} n$ amplitude. A precise measurement of the threshold cross section for $\gamma \mathrm{d} \rightarrow$ $\pi^{0} \mathrm{~d}$ is called for. A paper describing our results has been submitted for publication.

$\overline{\text { *Duke University, }}+$ Université Louis Pasteur, France, łInstitut für Kernphysik, Jülich, Germany, §University of Washington

\section{a.23. Chiral Transparency (T.-S. H. Lee, L. Frankfurt,* G. A. Miller, $\nmid$ and M. Strikman $\neq$ )}

Color transparency is the vanishing of initial and final state interactions, predicted by QCD to occur in highmomentum transfer quasielastic nuclear reactions. For specific reactions involving nucleons, the initial and final state interactions are expected to be dominated by exchanges of pions. We argue that these interactions are also suppressed in high-momentum transfer nuclear quasielastic reactions; this is "chiral transparency". We show that studies of the ${ }^{3} \mathrm{He}\left(\mathrm{e}, \mathrm{e}^{\prime} \Delta^{++}\right) \mathrm{nn}$ reaction could reveal the influence of chiral transparency. It is predicted that the $\mathrm{Q}^{2}$-dependence of ${ }^{3} \mathrm{He}\left(\mathrm{e}, \mathrm{e}^{\prime} \Delta^{++}\right)$cross sections should drop faster than that predicted by the meson-exchange mechanisms, when the momentumtransfer $\mathrm{Q}^{2}$ becomes larger than a critical value $\mathrm{Q}_{0}^{2}$. The parameter $Q_{0}^{2}$ controls the momentum-transfer at which the point-like configurations of hadrons are assumed to be formed. Thus, the experimental verification of chiral transparency is closely related to the study of color transparency. A paper describing our results has been accepted by Phys. Rev. C.

*Tel Aviv University, Israel, $\nmid$ University of Washington, $\ddagger$ Pennsylvania State University

\section{a.24. Near Threshold $\eta$ Production in Proton-Proton Collisions (T.-S. H. Lee, M. Batinic,* and A. Svarc*)}

The total cross sections for the near-threshold $\eta$ production in proton-proton collisions has been investigated with the assumption that the production mechanism is due to the emission of a meson $x(\pi, \eta, \sigma)$ from one of the nucleons. followed by a $x \mathrm{~N} \rightarrow \eta \mathrm{N}$ transition on the second nucleon. The $\mathrm{xN} \rightarrow \eta \mathrm{N}$ amplitudes are generated from the unitary multi-channel multi-resonance model which has recently been constructed by fitting the data of $\pi \mathrm{N}$ elastic scattering and $\pi \mathrm{N} \rightarrow \eta \mathrm{N}$ reaction. The initial and final pp distortions are calculated from a coupled-channel $\pi \mathrm{NN}$ model which describes the NN scattering data up to about $2 \mathrm{GeV}$. With the $\mathrm{xNN}$ vertex functions taken from the Bonn potential, the predicted total cross sections of threshold $\mathrm{pp} \rightarrow \eta \mathrm{pp}$ reaction are in good agreement with the data. Our results are shown in Fig. IV-8. In contrast to previous work, we find that the $\eta$-exchange plans an important role. A paper describing our results has been accepted for publication by Physica Scripta.

*Rudjer Boskovic Institute, Croatia

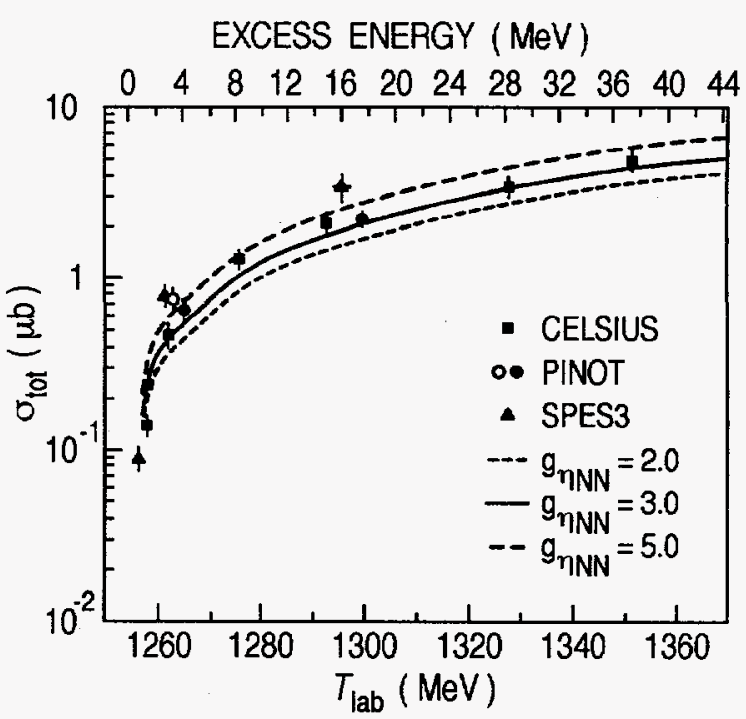

Figure IV-8. The predicted $p p \rightarrow p p \eta$ total cross sections are compared with the data. The dependence on the $\eta N N$ coupling constant $g \eta N N$ is indicated in the figure. 


\section{a.25. Pion Absorption Cross Sections for ${ }^{2} \mathrm{H}$ and ${ }^{3} \mathrm{He}$ in the $\Delta$-Isobar Region (T.-S.H. Lee, H. Kamada, * M.P. Locher, * V.E. Markushin, * J. Golak, $\dagger$ H. Witala, $\dagger$ and W. Glöckle $)$}

The absorption of $\pi^{+}$on ${ }^{3} \mathrm{He}$ in the $\Delta$-region is evaluated with exact inclusion of the final state interaction among the three emerging protons. The absorption is described by a $\pi \mathrm{N} \rightarrow \Delta$ vertex and a $\mathrm{N} \Delta$ $\mathrm{NN}$ transition $\mathrm{t}$-matrix which are calculated from a phenomenological model for $\mathrm{NN}$ and $\pi \mathrm{d}$ reactions. In a calculation where the initial pion scattering effects are neglected, the predicted peaks of the pion absorption cross sections for ${ }^{2} \mathrm{H}$ and ${ }^{3} \mathrm{He}$ lie too high in energy in relation to the data. The effect of the final state threenucleon interaction turns out to be too small for changing the magnitude and shifting the peak position of the total absorption cross section for ${ }^{3} \mathrm{He}$. We demonstrate that the adjustment of the peak position for the deuteron cross section by small modifications of the $\Delta$-parameters, automatically leads to the correct peak position in ${ }^{3} \mathrm{He}$. A paper describing our results has been accepted by Phys. Rev. C.

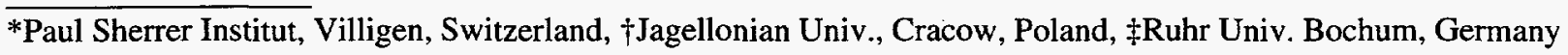

\section{a.26. Medium Effects on Binary Collisions with the $\Delta$ Resonance (T.-S. H. Lee)}

To facilitate the relativistic heavy-ion calculations based on transport equations, the binary collisions involving a $\Delta$ resonance in either the entrance channel or the exit channel are investigated within a Hamiltonian formulation of $\pi \mathrm{NN}$ interactions. An averaging procedure is developed to define a quasiparticle $\Delta^{*}$ and to express the experimentally measured $\mathrm{NN} \rightarrow \pi \mathrm{NN}$ cross section in terms of an effective $\mathrm{NN} \rightarrow \mathrm{N} \Delta^{*}$ cross section. In contrast to previous work, the main feature of the present approach is that the mass and momentum of the produced $\Delta^{*}$ 's are calculated dynamically from the bare $\Delta \leftrightarrow \pi N$ vertex interaction of the model Hamiltonian and are constrained by the unitarity condition. The procedure is then extended to define the effective cross sections for the experimentally inaccessible $\mathrm{N} \Delta^{*} \rightarrow \mathrm{NN}$ and $\mathrm{N} \Delta^{*}$ $\rightarrow \mathrm{N} \Delta^{*}$ reactions. The predicted cross sections are significantly different from what are commonly assumed in relativistic heavy-ion calculations. The $\Delta$ potential in nuclear matter has been calculated by using a Bruckner-Hartree-Fock approximation. By including the mean-field effects on the $\Delta$ propagation, the effective cross sections of the NN $\rightarrow \mathrm{N} \Delta^{*}, \mathrm{~N} \Delta^{*} \rightarrow \mathrm{NN}$ and $\mathrm{N} \Delta^{*} \rightarrow \mathrm{N} \Delta^{*}$ reactions in nuclear matter are predicted. It is demonstrated that the density dependence is most dramatic in the energy region close to the pion production threshold. A paper describing our results has been published in Phys. Rev. C54, 1350 (1996).

\section{a.27. The $\pi N$ and $\gamma N$ Reactions Within the Chiral Constituent Quark Model \\ (D. Kurath and T.-S. H. Lee)}

The chiral constituent quark model is applied to predict the cross sections of $\pi \mathrm{N} \rightarrow \pi \mathrm{N}, \pi \Delta$ and $\gamma \mathrm{N} \rightarrow \pi \mathrm{N}, \pi \Delta$ reactions. With the parameters fixed by the $\pi \mathrm{NN}$ coupling constant and the charge radius and magnetic moment of proton, the predicted cross sections are compared with the cross sections calculated from the empirical partial-wave amplitudes. The predictions are also made by using the parameters determined within the dynamical model (see IV.a.16) for $\pi \mathrm{N}$ scattering and $\gamma \mathrm{N} \rightarrow \pi \mathrm{N}$ reaction. These results will be the input to the amplitudes analyses of $\pi \mathrm{N}$ and $\gamma \mathrm{N}$ reactions described in IV.a.19.

\section{a.28. Foundations of Hadron Phenomenology (F. Coester)}

The theory fundamental to hadron phenomenology is quantum theory with unitary representations of all symmetries.

The Hilbert space of single baryon states is spanned by state vectors generated by the action of products of three quark-field operators folded into antisymmetric functions of three space-time points, spinor, flavor and color indices. Equivalence classes of these functions are representatives of the states.

The inner product measure of the functions representing baryon states is given by the vacuum expectation value of the product of six quark fields. Electromagnetic properties are specified by the vacuum expectation values of the current operator between products of three 
quark-field operators. Models respecting all symmetries are constructed by simple assumptions for both quantities.

In practice the model construction involves defining the relations between spinor and spin representations and the specification of an invariant mass operator. Assumption of a quark-particle structure is one possibility among many qualitatively distinct models. For simple models failure as well as success in comparisons with data can provide significant insight.

\section{a.29. Electromagnetic Currents of the Nucleon Resonances (F. Coester and D. O. Riska*)}

Lorentz covariant quark models obtain by construction, a mass operator consistent with invariant constraints on the relative momenta. We have investigated simple models with mass operators $M=M_{0}+M^{\prime}$, where $M_{0}$ is independent of the spin and flavor variables and $\mathrm{M}^{\prime}$ is independent of the relative momenta. The eigenfunctions of $\mathrm{M}_{0}$ are covariant harmonic oscillator functions. The operator form of the hyperfine interaction $\mathbf{M}^{\prime}$ is suggested by pseudoscalar octet exchange. The mass operator depends on two parameters: the oscillator constant and the strength of the hyperfine interaction. These parameters are determined by the masses of the nucleon and the $\Delta(1232)$. The resulting fit to the pole positions of other resonances is illustrated in Table IV-2.

*University of Helsinki, Finland
The current kernels are products of a Lorentz invariant factor which is independent of the spin-flavor variables and a covariant factor which is independent of the relative momenta. This structure matches the structure of the wave functions. Impulse currents and exchange currents are characterized by covariant spectator constraints on the relative momenta. Requirements of Poincarè covariance are satisfied exactly. Nucleon properties at zero momentum transfer are input parameters. The $\mathrm{Q}^{2}$-dependence of the form factor is determined primarily by the spatial wave functions. Oscillator functions are inadequate except for small values of $Q^{2}$. That defect can be corrected without affecting the mass spectrum. The nontrivial consequences of the model assumptions are the properties of the nucleon resonances that should be observed in future experiments.

Table IV-2. The nucleon and $\Delta$-states below $1700 \mathrm{MeV}$. The column $\varepsilon$ contains the eigenvalues of the mass operator. The average over the multiplet of the real part of the empirically extracted resonance pole position is denoted by EXP. The model values are listed below the empirical ones.

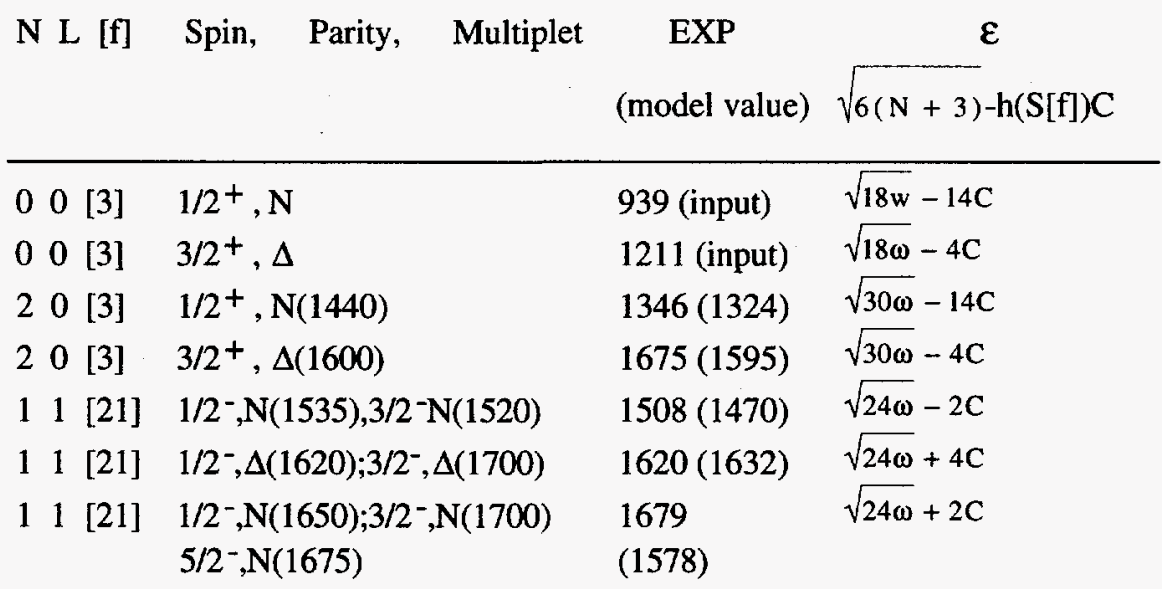




\section{a.30. Current Matrices, Covariant Current Kernels, and Form Factors (F. Coester, W.H. Klink, * and W.N. Polyzou*)}

Electromagnetic observables are simply related to Poincarè covariant matrix elements of current-density operators. Conventional invariant form factors are either defined by matrix elements evaluated in a special "frame" or invariant factors appearing in covariant spinor representations of the currents. Sachs form factors and Dirac-Pauli form factors of particles with spin $1 / 2$ are well-known examples. The first arise naturally in the context of quantum mechanics, including relativistic quantum mechanics, while the second appears naturally in the context of quantum field theory. Spin representations obviously apply to arbitrary spin values while covariant spinor representations suffer seriously from increasing complexity for spin values larger than 1 . In the description and interpretation of electromagnetic transitions of hadrons with higher spin it is important to have a common framework.

Initial and final states, labeled by four momenta and spin variables, transform irreducibly under Poincarè transformations. Under Lorentz transformations the spin variables undergo momentum-dependent Wigner rotations. For fixed initial and final momenta, covariance under the subgroup $\mathrm{O}(2) \subset \mathrm{SO}(1,3)$ that leaves the momenta invariant implies that the current matrix elements are $\mathrm{O}(2)$ Clebsch Gordan coefficients multiplied by reduced matrix elements, which are the standard helicity amplitudes, up to conventional invariant factors. The longitudinal and time components of the current are $\mathrm{O}(2)$ scalars. Current conservation is a linear relation of the two scalars. Sachs form factors are, up to conventional invariant factors, the reduced matrix elements under the subgroup $\mathrm{O}(3) \subset \mathrm{SO}(1,3)$ that leaves invariant either the initial or the final four-momentum, or any time-like linear combination.

Covariant current kernels that are functions of fourmomenta and spinor variables arise naturally from the consideration of vacuum expectation values of products of fields and current operators. They can be expressed as linear combinations of independent covariants that transform irreducibly under $\mathrm{O}(1,3)$, multiplied by form factors that are invariant functions of the momenta. To characterize these form factors it is sufficient to consider covariance under Lorentz transformations that leave the momentum transfer $Q$ invariant $(S O(1,2)$ for space-like momentum transfer). Invariants are easily extracted when the current kernels are expressed as functions of the intrinsic parities and the quantum numbers of irreducible representations of $S O(1,2)$. This procedure provides a simple straightforward generalization to arbitrary spin of the familiar spinor representations of form factors. The relations to spinrepresentations is given by standard representations of the boosts that relate spin vectors to Pauli-Lubanski vectors.

Both spinor and spin representations of currents and their interrelations provide valuable guidance in modeling the electromagnetic structure of hadrons.

University of Iowa, Iowa City 


\section{B. NUCLEAR FORCES AND NUCLEAR SYSTEMS}

The goal of this program is to achieve a description of nuclear systems ranging in size from the deuteron to nuclear matter and neutron stars using a single parametrization of the nuclear forces. Aspects of our program include both the construction of two- and three-nucleon potentials and the development of many-body techniques for computing nuclear properties with these interactions. Detailed quantitative, computationally-intensive studies are essential parts of this program.

Quantum Monte Carlo $(\mathrm{QMC})$ calculations of light $(\mathrm{A} \leq 8)$ nuclei with realistic interactions have been the main focus of our recent efforts. Our nonrelativistic Hamiltonian contains the accurate Argonne v 18 two-nucleon (NN) potential, which includes charge-independence-breaking terms, and the Urbana IX three-nucleon potential. The QMC calculations include both variational (VMC) and Green's function (GFMC) methods. We begin with the construction of variational trial functions based on products of correlated two- and three-body operators. Energy expectation values are evaluated with Metropolis Monte Carlo integration and parameters in the trial functions are varied to minimize the energy. These optimized variational wave functions can then be used to study other nuclear properties. They also serve as a starting point for the GFMC calculations, which systematically remove higher excited state components from the trial wave functions by a propagation in imaginary time. The GFMC code was initially built by collaborators at Urbana and Los Alamos; we have made significant improvements in its efficiency and are proceeding with further developments.

The nuclei currently under study include ${ }^{6} \mathrm{He},{ }^{6} \mathrm{Li},{ }^{7} \mathrm{He},{ }^{7} \mathrm{Li},{ }^{8} \mathrm{He}$, and ${ }^{8} \mathrm{Be}$. These are the first and only calculations treating these nuclei with realistic NN interactions. First results for $A=6$ nuclei were reported in 1995. In 1996 these were substantially refined, detailed studies of $A=7$ were made, and $\mathrm{A}=8$ calculations were begun. We calculate the ground states and all the lowestlying excited states with different quantum numbers in GFMC, and second excited states in VMC. The ground state energies tend to be slightly underbound compared to experiment, but there is broad agreement for the ordering and excitation energies of the higher states. We also study the isobaric analog states to find isovector and isotensor energy differences (Nolen-Schiffer anomaly). In addition, VMC calculations including two-body charge and current operators are being made for the elastic and transition form factors in ${ }^{6} \mathrm{Li}$.

Aside from the QMC calculations of light nuclei, we have continued some variational work on larger nuclei (cluster Monte Carlo) and nuclear matter (Fermi hypernetted/single-operator chains). Progress was made toward incorporating three-body correlations in the matter calculations. We are also studying the properties of neutron drops with the goal of providing additional constraints for the construction of Skyrme interactions for modeling neutron-rich nuclei in the crusts of neutron stars.

Studies of hypernuclei are continuing on several fronts. We are examining $\Lambda$ single-particle energies and the effect of $\Lambda$-induced distortion of nuclear cores in light hypernuclei such as ${ }_{\Lambda}^{5} \mathrm{He}$ and ${ }_{\Lambda}^{17} \mathrm{O}$. We also continue to use our studies of hypernuclei to deduce features of hyperonnucleon interactions, such as space-exchange forces, charge-symmetry breaking, and $\Lambda \mathrm{N}-\Sigma \mathrm{N}$ coupling. 


\section{b.1. Variational Monte Carlo Calculations of Light p-shell Nuclei (R. B. Wiringa, S. C. Pieper, B. S. Pudliner, * V. R. Pandharipande,* and J. Carlson $\dagger$ )}

We have performed an extensive series of variational Monte Carlo (VMC) calculations for nuclei up to $A=8$ using realistic two- and three-nucleon interactions. The variational wave functions, $\Psi_{\mathbf{v}}(\mathbf{R})$, obtained in these calculations are used as input to the more precise Green's function Monte Carlo (GFMC) calculations described below, and are also being used to study electron scattering and low-energy electroweak reactions of these nuclei. The Hamiltonian contains the recently-constructed Argonne $v_{18}$ NN interaction, which gives an excellent fit to two-nucleon data, and the Urbana IX $3 \mathrm{~N}$ potential, which has been adjusted to give the correct three-nucleon binding energy and reasonable saturation properties for nuclear matter. The variational wave functions for few-body nuclei $\left({ }^{3} \mathrm{H}\right.$, ${ }^{3} \mathrm{He}$, and ${ }^{4} \mathrm{He}$ ) include central, spin, isospin, tensor, and spin-orbit two-body correlations and three-body correlations for the $3 \mathrm{~N}$ interaction. The $\Psi_{v}(R)$ for these systems give upper bounds to the ground-state binding energy $\sim 2 \%$ above exact Faddeev or GFMC calculations.

The $\Psi_{\mathrm{V}}(\mathbf{R})$ for A=6-8 nuclei are considerably more complicated because of the need to place the additional nucleons in p-shell orbitals. We have used an LS coupling scheme to construct states with the desired JM values. Our earliest ${ }^{6} \mathrm{He},{ }^{6} \mathrm{Li}$, and ${ }^{6} \mathrm{Be}$ calculations used a complete $\mathrm{p}$-shell basis, including both symmetric [2] and mixed-symmetry [11] spatial components in the one-body part of the wave function, where [n] denotes the Young pattern. However, our first calculations for ${ }^{7} \mathrm{Li}$ and ${ }^{7} \mathrm{Be}$ used only the dominant symmetric [3] spatial components. This year we extended the $A=7$ calculations to a complete p-shell basis, including mixed-symmetry [21] and antisymmetric [111] terms, where appropriate, and we also added calculations of ${ }^{7} \mathrm{He},{ }^{7} \mathrm{~B}$, and their isobaric analogs. We also developed a method for diagonalizing our wave functions in the different symmetry components so we could project out second excited states with the same quantum numbers as the ground or first excited states.

At present we have made calculations for the ground states of the seven $A=6-7$ nuclei given above, and for 18 different excited states (not counting isobaric analogs) as shown in Table IV-3. The binding energies of the ground states are $10-20 \%$ high compared to the final GFMC results. However, the excited state energies are in excellent agreement with the GFMC excitations (compared to their respective ground states) in the six cases where GFMC has also been used. Compared to experiment, these states generally occur in the correct order and with reasonable excitation energies.

This year we also made our first calculations of the ${ }^{8} \mathrm{He}$ and ${ }^{8} \mathrm{Be}$ ground states, using only the leading symmetry components in the single-particle part of the wave function. The ${ }^{8} \mathrm{Be}$ calculations show the significant extra binding over ${ }^{7} \mathrm{Li}$ that is observed in nature, although again the VMC result is about $10 \%$ above the more exact GFIMC calculation. The ${ }^{8} \mathrm{He}$ result shows a significant relative underbinding compared to experiment, also evident to a lesser extent in ${ }^{6} \mathrm{He}$ compared to ${ }^{6} \mathrm{Li}$ and in ${ }^{7} \mathrm{He}$ compared to ${ }^{7} \mathrm{Li}$. This may be an indication for a significant isospin dependence in the $3 \mathrm{~N}$ interaction that is not in the present Hamiltonian.

Major goals for next year are to calculate the ground states of ${ }^{8} \mathrm{Li}$ and ${ }^{8} \mathrm{~B}$, to increase the p-shell basis used in the $A=8$ nuclei, and to calculate $A=8$ excited states. The VMC calculations have been made on Argonne's IBM SP and on the IBM SP2 at the Cornell Theory Center, where a large block of time has been awarded to our collaboration. Significant technical improvements were made during the year that roughly doubled the efficiency of our computer codes.

*University of Illinois, Urbana, $\uparrow$ Los Alamos National Laboratory 
Table IV-3. Experimental, VMC, and GFMC excitation energies (adjusted to their respective ground states) inMeV.

\begin{tabular}{lccc}
$\mathbf{A Z}\left(\mathbf{J}^{\pi} ; \mathbf{T}\right)$ & Experiment & VMC & GFMC \\
\hline${ }^{6} \mathrm{He}\left(2^{+} ; 1\right)$ & 1.80 & $1.86(10)$ & $1.80(18)$ \\
${ }^{6} \mathrm{He}\left(2^{+} ; 1\right)$ & $?$ & $3.61(10)$ & - \\
${ }^{6} \mathrm{He}\left(1^{+} ; 1\right)$ & $?$ & $3.46(10)$ & - \\
${ }^{6} \mathrm{He}\left(0^{+} ; 1\right)$ & $?$ & $5.24(11)$ & - \\
& & & \\
${ }^{6} \mathrm{Li}\left(3^{+} ; 0\right)$ & 2.19 & $2.93(10)$ & $2.72(36)$ \\
${ }^{6} \mathrm{Li}\left(0^{+} ; 1\right)$ & 3.56 & $3.84(10)$ & $3.94(23)$ \\
${ }^{6} \mathrm{Li}\left(2^{+} ; 0\right)$ & 4.31 & $4.23(11)$ & $4.43(39)$ \\
${ }^{6} \mathrm{Li}\left(2^{+} ; 1\right)$ & 5.37 & $5.78(11)$ & - \\
${ }^{6} \mathrm{Li}\left(1^{+} ; 0\right)$ & 5.65 & $5.68(10)$ & - \\
${ }^{6} \mathrm{Li}\left(1^{+} ; 0\right)$ & $?$ & $8.96(11)$ & - \\
& & & \\
${ }^{7} \mathrm{He}\left(1 / 2^{-} ; 3 / 2\right)$ & $?$ & $0.90(16)$ & - \\
${ }^{7} \mathrm{He}\left(5 / 2^{-} ; 3 / 2\right)$ & $?$ & $1.69(16)$ & - \\
${ }^{7} \mathrm{He}\left(3 / 2^{-} ; 3 / 2\right)$ & $?$ & $2.08(16)$ & - \\
${ }^{7} \mathrm{i}\left(1 / 2^{-} ; 1 / 2\right)$ & 0.48 & $0.33(16)$ & $0.76(41)$ \\
${ }^{7} \mathrm{Li}\left(7 / 2^{-} ; 1 / 2\right)$ & 4.63 & $5.48(16)$ & $5.72(41)$ \\
${ }^{7} \mathrm{Li}\left(5 / 2^{-} ; 1 / 2\right)$ & 6.68 & $6.64(16)$ & $6.56(45)$ \\
${ }^{7} \mathrm{Li}\left(5 / 2^{-} ; 1 / 2\right)$ & 7.46 & $9.90(16)$ & - \\
${ }^{7} \mathrm{Li}\left(7 / 2^{-} ; 1 / 2\right)$ & 9.67 & $11.63(16)$ & - \\
${ }^{7} \mathrm{Li}\left(3 / 2^{-} ; 1 / 2\right)$ & 9.90 & $10.14(16)$ & - \\
${ }^{7} \mathrm{Li}\left(1 / 2^{-} ; 1 / 2\right)$ & $?$ & $10.79(16)$ & - \\
${ }^{7} \mathrm{Li}\left(3 / 2^{-} ; 3 / 2\right)$ & 11.24 & $13.05(16)$ & $12.65(33)$
\end{tabular}

\section{b.2. Green's Function Monte Carlo Studies of p-Shell Nuclei (S.C. Pieper, R.B. Wiringa,} B.S. Pudliner,* V.R. Pandharipande,* and J. Carlson $\dagger$ )

A long-standing goal of nuclear physics has been to understand how the structure of nuclei arises from the interactions between the nucleons. Realistic phenomenological models of two- and three-nucleon interactions have been developed by fits to nucleonnucleon (NN) scattering data and the properties of ${ }^{2} \mathrm{H}$, ${ }^{3} \mathrm{H}$, and ${ }^{4} \mathrm{He}$; we wish to use these interactions to predict the properties of larger nuclei. The nonrelativistic Hamiltonian used in our calculations is composed of the kinetic energy operator, a two-body potential (Argonne v 18) fit to NN scattering data, and a three-body potential. It is not possible to independently fix the parameters of the three-body potential, so we choose them to reproduce the binding energy of ${ }^{3} \mathrm{H}$ and to give approximately correct saturation of nuclear matter. Both the two- and three-nucleon forces have theoretical long-range pion exchange parts. In the past decade, the ground states of ${ }^{2} \mathrm{H},{ }^{3} \mathrm{H},{ }^{3} \mathrm{He}$, and ${ }^{4} \mathrm{He}$, and the low-energy scattering states of ${ }^{5} \mathrm{He}$ have been studied with such a Hamiltonian in an essentially exact fashion (limited only by statistical errors) by using the Green's Function Monte Carlo (GFMC) method. Our work has focused on extending these calculations to six, seven-, and eight-body nuclei and their excited states.

These are the first calculations of the structure of such nuclei with a realistic many-nucleon Hamiltonian, and thus they also test the range of applicability of this Hamiltonian.

In the GFMC calculations, we operate on a trial wave function with the imaginary time propagator, $\exp \left[-\left(\mathrm{H}^{\prime}-\right.\right.$ $\left.\left.\mathrm{E}_{0}\right) \tau\right]$, where $\mathrm{H}^{\prime}$ is a simplified Hamiltonian, $\mathrm{E}_{0}$ is an estimate of the eigenvalue, and $\tau$ is the imaginary time. To save computer time, the trial wave function is a simplified form of the variational wave functions described in the previous section. The excited-state components of the trial wave function will then be damped out for large $\tau$, leaving the exact lowest 


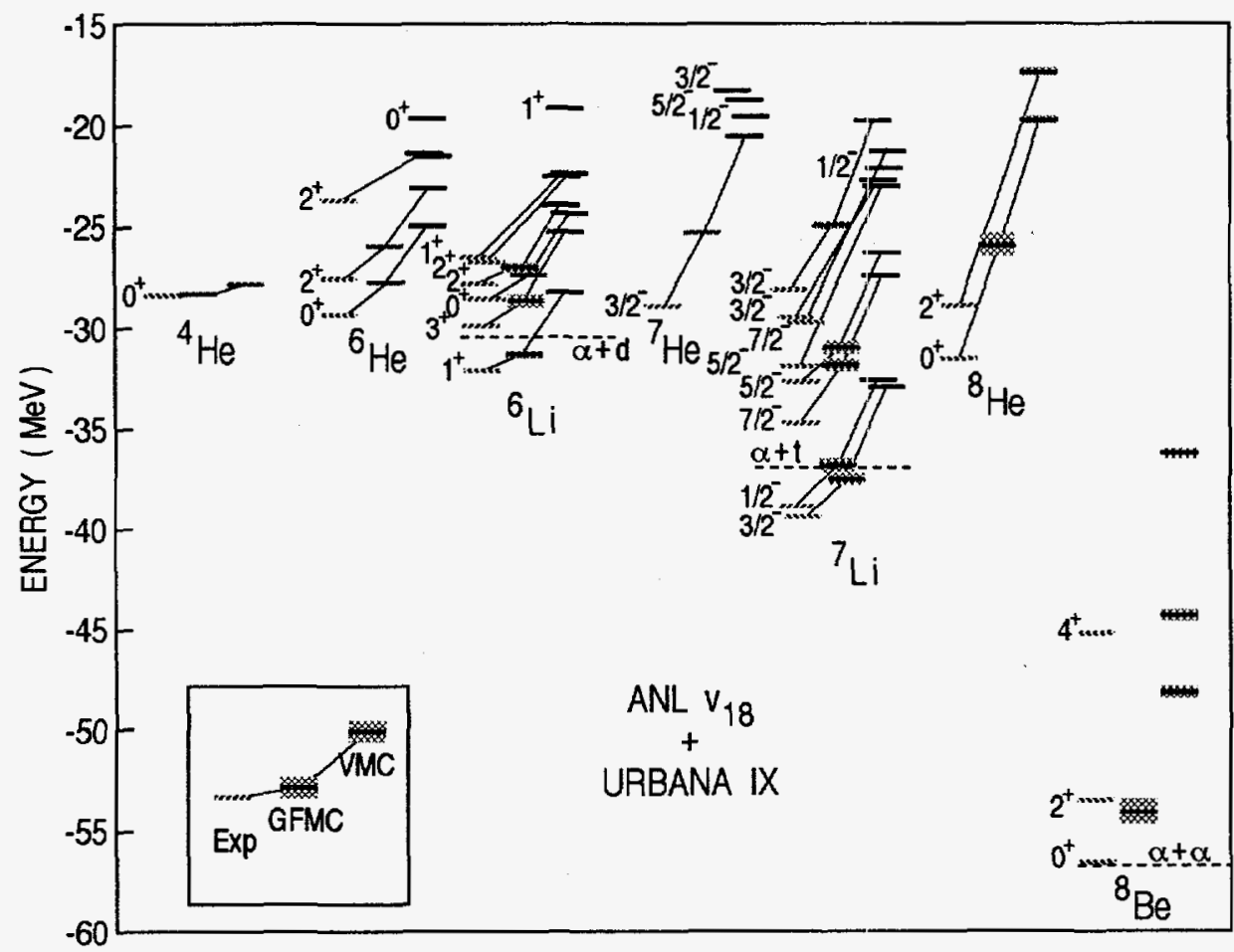

Figure IV-9. Experimental, GFMC, and VMC energies of 4- to 8-body nuclei.

eigenfunction with the quantum numbers of the input variational wave function. The expectation value of $\mathrm{H}$ is computed for several increasing values of $\tau$ to determine the convergence. In our $\mathrm{H}^{\prime}$ contains the reprojected $\mathrm{v} 8$ part of the two-nucleon potential and the full three-nucleon potential. The small correction $\mathrm{H}^{-\mathrm{H}^{\prime}}$ is computed perturbatively. The many-body propagator is written as a symmetrized product of exact two-body propagators, pre- and post-multiplied by $\left(1-\tau / 2 V_{\mathrm{ijk}}\right)$. GFMC calculations for Fermion systems suffer from the well-known sign problem in which the statistical error grows exponentially with imaginary time. Therefore most of our calculations are limited to $\tau=.06$ $\mathrm{MeV}^{-1}$, which means that components of the trial wave function with excitation energies less than $\sim 10 \mathrm{MeV}$ will not be appreciably damped out.

A GFMC calculation starts with a number of configurations of nucleon positions distributed according to the probability density of the trial wave function. For a given configuration of $\mathrm{A}$ nucleons, the wave function is a complex vector of amplitudes for each spin-isospin state. The size of the vector is given by $2^{\mathrm{A}} \mathrm{I}_{\mathrm{T}, \mathrm{A}}$, where $2^{\mathrm{A}}$ is the number of spin states and IT,A is the number of isospin states with the same totalisospin, T. As an example, for the $T=0{ }^{6} \mathrm{Li}$ states, the number of isospin states is IT,A $=5$ and the number of spin states is $2^{A}=64$, giving a vector size of 320. For the ground states of ${ }^{7} \mathrm{Li}$ and ${ }^{8} \mathrm{Be}$, the corresponding numbers are 1792 and 3584. The number of sparse-matrix operations on this vector grows roughly as the number of nucleon pairs. Thus the computational effort of GFMC calculations grows very rapidly with the number of nucleons, roughly an order of magnitude for each step from $A=4$ to 6 , to 7 and then a factor of 3-6 for $A=8$. In the last year we have made significant progress in speeding up our GFMC program to be able to confront these larger nuclei.

The most important changes have been to the numerical methods. The evaluation of the anticommutator parts of $V_{i j k}$ and $U_{i j k}$ has been reduced to that of generalized tensor pair operations so that the time scales as $A^{2}$ instead of $\mathrm{A}^{3}$. The evaluation of the non-central twobody correlations has been rearranged to save many operations. These changes have sped up the program by factors of 3 to 5 . The parallel structure of the program has also been improved. Periodically during the GFMC propagation, load balancing is done to keep the computational effort approximately equal on all slaves. In this process the slaves send their current number of configurations to the master; when the 


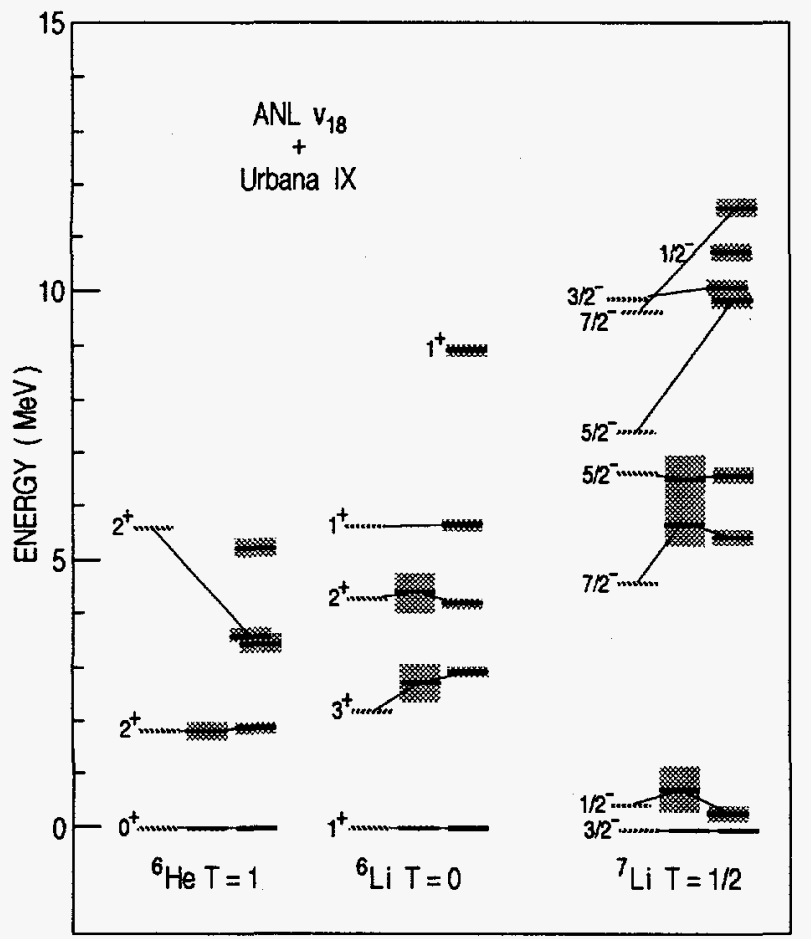

Figure IV-10. Experimental, GFMC, and VMC excitation spectra.

master has received this information from all the slaves, it determines which slaves should send configurations to other slaves and it so instructs the slaves. The slaves then communicate the necessary configurations directly among themselves. Once a slave has sent its current number of configurations to the master, it cannot make any more propagations until it receives the load balancing instructions from the master. During this time the slave computes energy values at previously determined configurations. In this way, the slaves are always busy, except possibly at the very end of the calculation. We are now achieving $295 \%$ efficiency in production calculations using up to 60 slaves. The current program requires only 170 node-hours on the IBM SP1 at Argonne to make a calculation for $6_{\mathrm{Li}}$ using 50,000 initial configurations. ${ }^{8}$ Be calculations have mostly been done on the SP2 wide nodes at Cornell; 20,000 configurations require $\sim 840$ nodehours. For $A=6$ and 7 , we now routinely use 40,000 to 100,000 configurations per state; this results in statistical errors of 0.2 to $0.3 \mathrm{MeV}$, less than $1 \%$ of the binding energy
In the last year we have made a number of studies of the reliability of the GFMC calculations. One result of these is a modification of the $\mathrm{H}^{\prime}$ that is used: For ${ }^{7} \mathrm{Li}$, the reprojected $\mathrm{v} 8$ gives $2 \mathrm{MeV}$ more binding than the full $v_{18}$; this would result in the Green's function propagating to an over-dense system. Therefore we now multiply the central, repulsive, part of $\mathrm{V}_{\mathrm{ijk}}$ by $\sim 1.3$ in $\mathrm{H}^{\prime}$ so that $\left\langle\mathrm{H}^{\prime}\right\rangle \sim\langle\mathrm{H}\rangle$. We have also investigated the dependence of the GFMC results on the quality of the input trial wave function. We find that the effects of omitting the $U_{i j k}$ and even the two-body tensor correlations are quickly (in terms of imaginary time) corrected for; thus such omissions correspond to very high excitation energies. However the different symmetry components of the variational wave functions discussed in the previous section correspond to low $(\sim 10 \mathrm{MeV})$ excitations and thus are not damped out in our calculations. To get an accurate eigenenergy, this part of the input trial wave function must be treated accurately, otherwise the GFMC energy is only an improved variational upper bound.

Figure IV-9 shows a comparison of our VMC and GFMC calculations with experimental results for a variety of nuclear states. For $A>4$, the Hamiltonian is not providing enough binding; this problem also gets worse with increasing $\mid \mathrm{N}-\mathrm{Zl}$. Also for increasing $\mathrm{A}$ and IN-ZI, the VMC results are more and more above the GFMC values, indicating that some essential feature is missing from the VMC wave functions for $p$-shell nuclei. However, as is shown in Fig. IV-10, both the GFMC and VMC do a good job of predicting the spectrum of excitation energies for a given $A, Z$, and $T$, except possibly for some of the higher-lying states (the experimental assignments of some of these higher states are not that certain). We are now attempting to improve the Hamiltonian by adding the dominant relativistic correction and using a more sophisticated form for the short-ranged part of the three-body interaction.

We can see that the GFMC method is a powerful tool in the study of nuclear structure; this study is the first to demonstrate the emergence of nuclear shell-model structure from a many-body calculation using fundamental nucleon-nucleon forces. We will also be addressing problems of interest in solar physics, such as radiative capture reactions. A detailed description of the $\mathrm{A}=6$ and $\mathrm{A}=7$ calculations is being prepared.

*University of Illinois, Urbana, $\nmid$ Los Alamos National Laboratory 


\section{b.3. Charge-independence Breaking in Light Nuclei (R.B. Wiringa, S.C. Pieper, and B.S. Pudliner*)}

We are using quantum Monte Carlo methods to study energy differences in mirror nuclei with $A=3,7,15$, 17,39 , and 41 , and in the $A=6$ isotriplet and the $A=7$ isoquartet. We use a realistic Hamiltonian containing the Argonne v 18 NN potential and the Urbana IX 3N potentials. This Hamiltonian includes strong interaction charge-dependent (CD) and charge-symmetry-breaking (CSB) terms as well as a complete electromagnetic potential, and gives an excellent fit to $\mathrm{pp}, \mathrm{np}$, and $\mathrm{nn}$ data.

Previous exact GFMC calculations yielded an energy difference of $0.757 \pm 0.001 \mathrm{MeV}$ for ${ }^{3} \mathrm{H}-{ }^{3} \mathrm{He}$ in excellent agreement with the experimental value of $0.764 \mathrm{MeV}$. This year we made significant progress in the study of $A=6-7$ nuclei, thanks to the GFMC calculations described above. Our results are shown in Table IV-3 for the different isovector and isotensor combinations. The $A=6$ values are in reasonable agreement with experiment, but the $A=7$ are less satisfactory. The Coulomb force between protons is the dominant term in each case, and slight changes in nuclear radii due to changes in nuclear binding could alter these numbers a fair amount. The additional electromagnetic and strong CD and CSB terms are significant compared to the discrepancy with respect to experiment, and in 4 cases out of 5 improve the result.

Conclusive results will have to wait until the total Hamiltonian is adjusted to fit the $A=6-7$ ground state binding energies correctly. In the meantime, these are probably the best estimates of the contribution of the small CD and CSB terms in nuclei available today. In the near future we should also be able to evaluate them in the $A=8$ nuclei. Progress on the larger near-closedshell nuclei will have to await developments in cluster expansions for the GFMC method.

*University of Illinois, Urbana

Table IV-3. GFMC isovector and isotensor energy differences in $M e V$. The A $=7$ terms have been evaluated with a $C S$ wave function and corrected for CSB effects from VMC calculations. $V_{C R}$ is the sum of all Coulomb contributions aside from $v_{C l}(p p)$.

\begin{tabular}{|c|c|c|c|c|c|}
\hline & ${ }^{6} \mathrm{Be}-{ }^{6} \mathrm{He}$ & $\begin{array}{c}{ }^{6} \mathrm{Be}+{ }^{6} \mathrm{He} \\
-2 \times{ }^{6} \mathrm{Li}^{*}\end{array}$ & ${ }^{7} \mathrm{Be}-{ }^{7} \mathrm{Li}$ & ${ }^{7} \mathrm{~B}-{ }^{7} \mathrm{He}$ & $\begin{array}{c}{ }^{7} \mathbf{B}+{ }^{7} \mathbf{H e} \\
.{ }^{7} \mathrm{Be}^{*}-{ }^{7} \mathrm{Li}^{*}\end{array}$ \\
\hline$\langle v \eta$ & 2.1 & $0.550(22)$ & $1.482(18)$ & $3.526(26)$ & $0.689(32)$ \\
\hline$\left[\left\langle v_{\mathrm{Cl}}(\mathrm{pp})\right\rangle\right]$ & & {$[0.491]$} & {$[1.4$} & {$[3.5$} & {$[0.661]$} \\
\hline$[\langle v \mathrm{CR}\rangle$ & & {$[0.004]$} & {$[0.021]$} & {$[0.0$} & {$[0.002]$} \\
\hline$[\langle V \mathrm{MM}\rangle]$ & {$[0.005]$} & {$[0.055]$} & {$[0.019]$} & {$[-0.008]$} & {$[0.026]$} \\
\hline$\left\langle\mathrm{K}^{\mathrm{CSB}}\right\rangle$ & $0.031(2)$ & - & 0.025 & $0.040(1)$ & - \\
\hline$\left\langle v^{C S B}\right\rangle$ & $0.086(2)$ & - & $0.083(2)$ & $0.109(2)$ & - \\
\hline$\left\langle v{ }^{C D}\right\rangle^{\prime}$ & - & $0.217(23)$ & - & - & $0.162(14)$ \\
\hline$\Delta$ (Calc. $)$ & $2.239(17)$ & $0.767(32)$ & $1.59(19)$ & $3.675(27)$ & $0.851(35)$ \\
\hline$\Delta$ (Expt.) & 2.345 & 0.670 & 1.644 & 4.10 & 0.69 \\
\hline
\end{tabular}




\section{b.4. Microscopic Calculation of Elastic and Transition Form Factors of Lithium (R.B. Wiringa and R. Schiavilla*)}

Variational Monte Carlo calculations are being made for the elastic and inelastic form factors of ${ }^{6} \mathrm{Li}$. The six-body wave functions are computed for a realistic Hamiltonian containing the Argonne v18 two-nucleon potential and the Urbana IX three-nucleon potential. Consistent charge and current operators including oneand two-body terms are evaluated. Elastic results include the longitudinal and transverse form factors and tensor polarization. Transition form factors are calculated for the $\left(\mathrm{J}^{\pi} ; \mathrm{T}\right)=\left(3^{+} ; 0\right),\left(0^{+} ; 1\right),\left(2^{+} ; 0\right)$, and $\left(2^{+} ; 1\right)$ states.

Our initial calculations showed that the elastic longitudinal form factors $F_{L}{ }^{2}(q)$ and $F_{T}{ }^{2}(q)$ are generally well reproduced in impulse approximation

*TJNAF and Old Dominion University
(IA) and that meson-exchange current (MEC) contributions are not large but do improve the agreement with data. The longitudinal transition form factor to the $\left(3^{+} ; 0\right)$ excited state is also in good agreement with data at the IA level, and addition of the small MEC corrections improves the agreement. The transverse transition form factor to the $\left(0^{+} ; 1\right)$ state is bracketed by the IA and MEC calculations.

This year we made some improvements in the efficiency of the calculations, and introduced a subtraction in the transition calculations that significantly reduces Monte Carlo noise at small $\mathrm{q}$, thus helping in the extraction of $B(E 2)$ and $B(M 1)$ values. We hope to repeat these calculations with the GFMC wave functions in the near future.

\section{b.5. Short-Range Structure of the Deuteron and Other Nuclei (S. C. Pieper,} R. B. Wiringa, J. L. Forest, * V. R. Pandharipande,* R. Schiavilla, $†$ and A. Arriaga $\ddagger)$

The most important correlations among the nucleons in nuclei are induced by the tensor and repulsive-core parts of the two-nucleon interaction. These correlations are strongest in the isospin $T=0$, spin $S=1$ twonucleon states. We find that these correlations result in tensor-polarized deuterons with $\mathrm{M}_{\mathrm{S}}= \pm 1$ having a dumbbell shape oriented along the $\hat{\mathbf{z}}$ axis, while equidensity surfaces for $\mathbf{M}_{S}=0$ are tori. This torus shape, resulting from conventional nucleon-nucleon potentials, does not seem to have been remarked on previously, although Skyrme models do predict such a shape. We can relate the sizes of these geometric shapes to the location of minima and maxima in various deuteron form factors. We also used our variational wave functions for nuclei from ${ }^{3} \mathrm{He}$ to ${ }^{16} \mathrm{O}$ to compute the density distributions of pairs of nucleons in various $\mathrm{T}, \mathrm{S}, \mathrm{M}_{\mathrm{T}}, \mathrm{M}_{\mathrm{S}}$ states.

We find that the distributicos for $T=0, S=1$ are very similar in shape to those of the deuteron. Furthermore, the ratio of the peak value of these densities to the peak value of the corresponding deuteron density provides an estimate for the number of "quasi-deuterons" in the nuclei. This estimate gives a good prediction of the observed photo- and pion-absorption cross sections. A similar situation is found for the other channel ( $T=1, S$ $=0$ ) that has nucleon pairs in $\mathrm{L}=0$ partial waves. In this case, the ${ }^{1} \mathrm{~S}_{0}$ virtual bound-state wave function provides a good prediction of the $T=1, S=0$ pair distributions computed for the nuclei.

Also of interest are two-cluster distributions within nuclei, such as d-p in ${ }^{3} \mathrm{He}$, d-d in ${ }^{4} \mathrm{He}$, and $\alpha-d$ in ${ }^{6} \mathrm{Li}$. These distributions all have $S$ - and D-wave components, which may be detectable in $\left(e, e^{\prime} \vec{p}\right)$ and (e,e' $d$ ) experiments. Their spatial structure can be directly related to the deuteron dumbbell and torus shapes. These results have been published in Phys. Rev. C 54 , 646 (1996).

\footnotetext{
*University of Illinois, Urbana, †TJNAF and Old Dominion University, $\ddagger$ University of Lisbon, Portugal
} 


\section{b.6. Three-body Correlations in Nuclear and Neutron Matter (R. B. Wiringa and A.A. Usmani*)}

Variational calculations of nuclear and neutron matter are currently performed using a diagrammatic cluster expansion with the aid of nonlinear integral equations for evaluating expectation values. These are the Fermi hypernetted chain (FHNC) and single-operator chain(SOC) equations, which are a way of doing partial diagram summations to infinite order. A significant advance in the study of few-body and light closed-shell nuclei was the addition of three-body correlations, especially those correlations generated by the $3 \mathrm{~N}$ interaction. These have been used with considerable success in the quantum Monte Carlo calculations described above. We have started working out the expressions for including such correlations in the FHNC/SOC calculations in matter. While the most important terms are expected in the expectation value of the $3 \mathrm{~N}$ potential, at the three-body level, there are many additional contributions to the kinetic and NN potential expectation values that must be evaluated. We hope to complete the theoretical derivation of the leading terms and get some initial estimates of the contributions in matter in the coming year.

*SISSA, Trieste, Italy

\section{b.7. $\Lambda$ Single-Particle Energies (A. R. Bodmer, and Q. N. Usmani*)}

We have made some changes and improvements in our calculations of the $\Lambda$-particle single-particle energies and their interpretation in terms of the basic $\Lambda$-nuclear interactions. These changes mostly relate to our treatment of the core-nucleus densities and of the fringing field (that part of the $\Lambda$-nucleus potential which extends beyond the nuclear density distribution because of the finite range of the $\Lambda$-nuclear interactions). We hope shortly to complete this work and prepare a final version for journal publication. Earlier versions have appeared in conference proceedings.

*Jamia Millia Islamia, New Delhi, India

\section{b.8. Core-Nucleus Distortion in Hypernuclei (A. R. Bodmer and Q. N. Usmani*)}

We have included the changes made in $\mathrm{k}$ (above) in a study of the effects of the spherical distortion of the core nucleus by the $\Lambda$ in a hypernucleus. The response of the core was determined by an appropriately chosen energy-density functional which depends, in particular, on the nuclear compressibility. The forcing action of the $\Lambda$ is determined by the nuclear density dependence of the $\Lambda$ binding in nuclear matter which is obtained from our work on the $\Lambda$ singleparticle energies. Because of the strongly repulsive $\Lambda \mathrm{NN}$ forces, this $\Lambda$ binding saturates at a density close to the central density of nuclei, and results in a reduced core-nucleus distortion much less than would otherwise be obtained. The effects of the core distortion then turns out to be small even for quite light hypernuclei. We also considered two toy models which display explicitly the role of the forcing action of the $\Lambda$ as well as that of the core response.

Our results justify the usual assumption that core distortion effects of spherical core nuclei are small and can mostly be neglected. This work was done mostly at Jamia Millia Islamia while ARB was a visitor there. Reports on this work were published in conference proceedings. A more complete version has been prepared for publication.

*Jamia Millia Islamia, New Delhi, India 


\section{b.9. Charge-Symmetry Breaking $\Lambda$-Nucleon Interaction (A. R. Bodmer, S. Murali,* and Q. N. Usmani*)}

Some time ago we showed that the charge-symmetrybreaking interaction, as obtained from the mass-four hypernuclei $\left({ }_{\Lambda}^{4} \mathrm{H},{ }_{\Lambda}^{4} \mathrm{He}\right)$, was spin-independent; a result which cannot be understood with the conventional meson-exchange models. The calculations of $\left({ }_{\Lambda}^{4} \mathrm{H},{ }_{\Lambda}^{4} \mathrm{He}\right)$, are currently being extended to include realistic nucleon-nucleon forces, in particular ones which include noncentral and momentum-dependent terms, which could possibly modify this result.

*Jamia Millia Islamia, New Delhi, India

\section{b.10. Suppression of the $\Lambda-\Sigma$ Coupling in Nuclear Matter (A. R. Bodmer and Q. N. Usmani*)}

We initiated a study of the modification of the coupling of the $\Lambda \mathrm{N}$ to the $\Sigma \mathrm{N}$ channel in nuclear matter with the Fermi hypernetted-chain variational approach. This modification of the $\Lambda N-\Sigma N$ coupling is a central problem in hypernuclear physics and is related closely to the strongly repulsive three-body forces which are needed to account for hypernuclear binding energies.
All earlier calculations only considered this problem in the so-called G-matrix approximation which neglects important higher-order effects. An important result of this work will be a better understanding of the density dependence of $\Lambda$ binding in nuclear matter, which can then be tested in the calculation of the $\Lambda$ single-particle energies.

*Jamia Millia Islamia, New Delhi, India

\section{b.11. Nuclear Core Dynamics and Rearrangement Energy for Hypernuclei} (A. R. Bodmer, S. Murali,* and Q. N. Usmani*)

The effect of the nuclear core dynamics on the binding energies of $\Lambda$ hypernuclei was studied in the framework of variational correlated wave functions. In addition to the core energy and to a $\Lambda$ single-particle contribution $\varepsilon_{\Lambda}$, we exhibit a rearrangement energy $E_{R}$ which in particular depends on the $\Lambda \mathrm{N}$ correlations $\mathrm{F}$ and on the difference $\delta \Phi_{\mathrm{N}}$ between the core wave function and its exact ground state value. $E_{R}$ is non-zero only if there are both $\Lambda \mathrm{N}$ correlations $(\mathrm{F} \neq 1)$ and if $\delta \Phi_{\mathrm{N}} \neq 0$. The latter can result from core polarization by the $\Lambda$ or from errors in the ground-state variational wave function of the core. $E_{R}$ varies linearly with $\delta \Phi_{N}$ for small $\delta \Phi_{N}$.
Detailed numerical results for ${ }_{\Lambda} \mathrm{He}$ using central $\Lambda \mathrm{N}$ and $\mathrm{NN}$ potentials with repulsive cores confirm these general features. However, the effect of $E_{R}$ leads to an expansion of the core by the $\Lambda$ rather than a contraction obtained with only $\varepsilon_{\Lambda}$. The core polarization energy is a quite small $0.4 \mathrm{MeV}$. During the past year we made more complete estimates of the changes in core size. We also studied the effects of errors in the variational core wave function by detuning this as a function of one of the variational parameters. This work was published in Nuclear Physics A609, 326 (1996), and was also part of a more comprehensive conference review.

\footnotetext{
*Jamia Millia Islamia, New Delhi, India
} 


\section{NUCLEAR STRUCTURE AND HEAVY-][ON REACTIONS}

This research focuses on nuclear structure in unusual regimes: nuclei far from stability, and superdeformed nuclei at high spin. We also study heavy-ion reactions near the Coulomb barrier and some traditional shell-model problems. Much of this work is closely tied to experiments performed at ATLAS and at radioactive-beam facilities.

Our studies of drip-line nuclei have focused on breakup reactions induced by the Coulomb and nuclear fields from a target nucleus. A critical issue has been to develop a realistic description of breakup mechanisms as a necessary tool for extracting or testing the nuclear structure properties of drip-line nuclei. An example of particular interest to solar neutrino physics is the low-lying E1 strength of ${ }^{8} \mathrm{~B}$ which determines the radiative proton capture on ${ }^{7} \mathrm{Be}$ in the sun. $\mathrm{E} 1$ transitions dominate the $\left({ }^{8} \mathrm{~B},{ }^{7} \mathrm{Be}+\mathrm{p}\right)$ breakup on a heavy target but they are masked by $\mathrm{E} 2$ transitions, and also by nuclear processes. We have investigated the possibility of separating the $\mathrm{E} 1$ and E2 components from measurements of the asymmetry in the fragment momentum distributions that is produced by the $\mathrm{E} 1+\mathrm{E} 2$ interference. This idea is currently being pursued in collaboration with an experimental program at Michigan State University.

The studies of heavy-ion reactions at energies close to the Coulomb barrier are based on applications of the coupled-channels technique. The objective is to obtain a consistent, unified, quantum-mechanical explanation of fusion reactions, compound-nucleus spin distributions, elastic and inelastic scattering, and transfer reactions. The calculations are constrained by the structure of the interacting nuclei. An example of reactions that we have studied during the past year is the multineutron transfer reactions that have been measured in ${ }^{58} \mathrm{Ni}+{ }^{124} \mathrm{Sn}$ collisions at Argonne. An issue of current interest is to see the impact of transfer reaction channels on other observables such as subbarrier fusion.

Our studies of superdeformed nuclei, at both low and high spins, address new regions of superdeformation, rotational moments of inertia, and the transition from superdeformed to normal states. Other areas of interest are the density-dependence of residual interactions and the structure of the heaviest elements. We try to understand these phenomena on the basis of correlations induced by the effective two-body interaction. The techniques used include a deformed one-body potential for surveying nuclear structure over a large regime, self-consistent mean-field calculations for more detailed studies of particular nuclides, and many-body wave functions when residual interactions are weak and a mean-field approach is inadequate.

Much of our work is computer intensive and we have adapted our codes to exploit the massively parallel IBM SP supercomputer at Argonne. This allowed us to calculate energy surfaces using the Strutinsky method in a four-dimensional shape space that consists of quadrupole, octupole, hexadecapole and necking degrees of freedom. We completed studies of the A 180, A 150, and the A 130 mass regions. These studies are being complemented with Hartree-Fock studies of selected nuclides in these regions. There seems to be a better-than-semiquantitative agreement between the two approaches at even the highest spins and the largest deformations. We are looking at improved treatments of pairing in superdeformed rotational bands. We are involved in experimental searches for new regions of superdeformation. We are participating in the experimental study of the spectroscopy of the heaviest elements, and the application of these studies to the stability of superheavy elements. 


\section{c.1. Momentum Distributions of ${ }^{{ }^{7}}$ Be Fragments in Breakup Reactions of ${ }^{\mathbf{8}_{B}}$ \\ (H. Esbensen, S. M. Austin,* J. H. Kelley,* and B. Davids*)}

Longitudinal momentum distributions (LMDs) of ${ }^{7} \mathrm{Be}$ fragments have been measured for breakup reactions of ${ }^{8} \mathrm{~B}$ on a $\mathrm{Be}$ and a $\mathrm{Au}$ target. The observed widths are much smaller than the width of the momentum distribution of the valence proton, bound in the ground state of ${ }^{8} \mathrm{~B}$. Moreover, the width for the Au target is about $25 \%$ smaller than for the Be target. This clearly shows that the width depends on the reaction mechanism which is dominated by Coulomb dissociation for the $\mathrm{Au}$ target and nuclear diffraction and stripping for the Be target.

We have analyzed the measured LMDs by adopting a single-particle description of the valence proton, bound by $138 \mathrm{keV}$ in a $\mathrm{p}_{3 / 2}$ orbit. The breakup on the $\mathrm{Be}$ target is described as a stripping reaction on a black disc. This model reproduces the observed width quite accurately. The main reason for the reduction of the width (compared to the width of the ground-state momentum distribution) are absorption effects combined with the fact that the valence proton is bound in a p-orbit. Thus one has to consider the dependence upon the orientation of the orbit, and one finds that the production of ${ }^{7} \mathrm{Be}$ fragments is much more likely when the p-orbit is oriented perpendicular to the beam axis. The longitudinal momentum distribution which is then probed is clearly much narrower than the average ground-state momentum distribution.

The breakup on the Au target is described as Coulomb dissociation. Both E1 and E2 transitions are included and the interference between them produces an asymmetry in the LMD of ${ }^{7} \mathrm{Be}$ fragments; see Nucl. Phys. A 600,37 (1996). The distributions we obtain are shown in Fig. IV-11 together with the data. The solid curve is the result of first-order perturbation theory and the dashed curve is from a higher-order dynamical calculation which produces a somewhat smaller

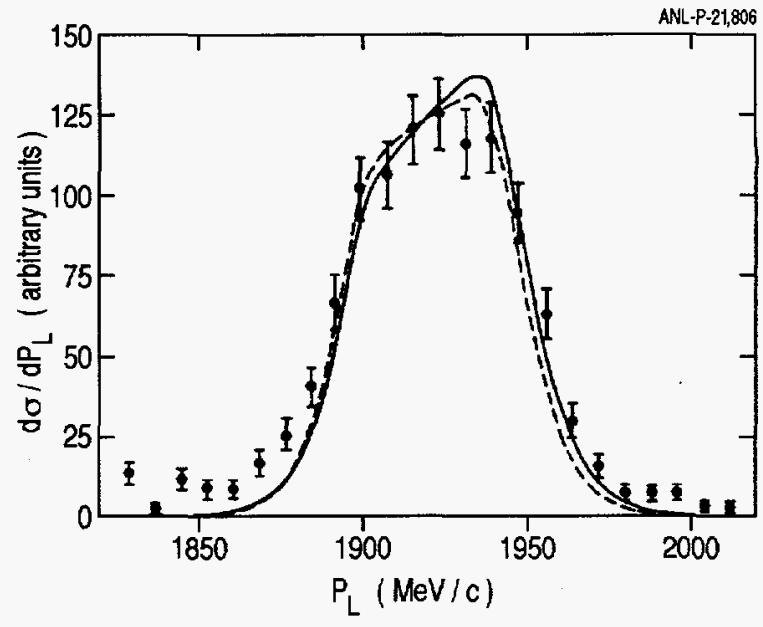

Fig. IV-11. Measured longitudinal momentum distribution of ${ }^{7}$ Be fragments produced in the breakup of ${ }^{8} B$ on a gold target at $41 \mathrm{MeV} / \mathrm{A}$. The calculated distributions are the results of Coulomb dissociation obtained in first-order perturbation theory (solid curve) and from a higher-order dynamical calculation (dashed curve).

asymmetry. However, the calculated widths are similar and close to the measured value. The data do suggest an asymmetry but the uncertainty is large. This work was published in Phys. Rev. Lett. 77, 5020 (1996).

Accurate measurements of the asymmetry are expected to provide a valuable constraint on the magnitude of the E2 strength. New and much more accurate measurements have recently been performed but they have not yet been analyzed. With a realistic constraint on the E2 strength it should be possible to extract a reliable E1 strength from Coulomb dissociation measurements, and thereby predict the S-factor for the radiative capture of protons on ${ }^{7} \mathrm{Be}$ nuclei.

*Michigan State University

\section{c.2. Momentum Distributions in Nuclear Induced Breakup Reactions (H. Esbensen, G. F. Bertsch,* and K. Hencken*)}

We have previously studied the longitudinal momentum distributions (LMDs) of core fragments which are produced in single-nucleon stripping reactions of ${ }^{11} \mathrm{Be}$ and ${ }^{8}$ B; see Phys. Rev. C 53, 2007 (1996). To get a more complete description of nuclear induced breakup reactions, we have now studied the contribution from nuclear diffraction. Here we use a realistic nucleon-

\footnotetext{
*Institute for Nuclear Theory, University of Washington
}

target optical potential to generate the breakup at lower energies, say below $100 \mathrm{MeV} / \mathrm{A}$. The real part of the optical potential becomes small at higher energies, and we therefore base our calculations on free nucleonnucleon cross sections above $200 \mathrm{MeV} / \mathrm{A}$. The two regimes merge and give almost identical breakup cross sections at $130 \mathrm{MeV} / \mathrm{A}$.

We have, in particular, made a detailed study of the

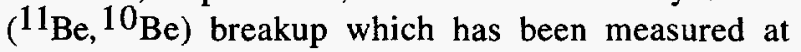


different energies and on a variety of targets. We find that stripping and diffraction processes contribute about the same amount at lower energies, whereas stripping dominates at higher energies. Moreover, the sum is in good agreement with measurements, both at 40 and at $800 \mathrm{MeV} / \mathrm{A}$. The LMD of ${ }^{10} \mathrm{Be}$ fragments is also in good agreement with data, and the widths of two components, i.e. stripping and diffraction, are almost identical.
The amount of data available for the $\left({ }^{8} \mathrm{~B},{ }^{7} \mathrm{Be}\right)$ breakup is much more limited. Only one measurement of the LMD of ${ }^{7} \mathrm{Be}$ fragments had been published at the time of this work, namely at $1470 \mathrm{MeV} / \mathrm{A}$. Our prediction of the width, which is dominated by stripping at this high energy, is in good agreement with the measurement. This work was published in Phys. Rev. C 54, 3043 (1996).

\section{c.3. Pairing Gap in the Inner Crust of Neutron Stars (H. Esbensen, R. A. Broglia,* E. Vigezzi, ${ }^{*}$ and F. Barranco ${ }^{\dagger}$ )}

We have investigated the role of finite nuclei on the pairing gap in the inner crust of a neutron star. The situation here is intermediate between the two extremes of a finite nucleus and an infinite neutron gas. The calculations were carried out by solving the selfconsistent Hartree-Fock-Bogoliubov (HFB) equations for neutrons inside the Wigner-Seitz cell that surrounds a heavy nucleus. The HFB approach allows us to include the scattering of neutron pairs, not only to timereversed two-neutron $0^{+}$states that have the same number of radial nodes as in BCS theory, but also to $0^{+}$ states that have different numbers of radial nodes. The scattering is generated in our calculations by the Argonne $v_{14}$ interaction.

To make the calculations converge it is necessary to include single-particle states up to $600 \mathrm{MeV}$ in the continuum. A typical situation we consider is a nucleus with 50 protons and 600 neutrons inside a Wigner-Seitz cell which has a radius of $15 \mathrm{fm}$. We find that the pairing gap is quenched compared to that of an infinite gas with the same neutron density. We have also applied the method to the finite nucleus $120 \mathrm{Sn}$ and obtained a pairing gap of $2.2 \mathrm{MeV}$ at the Fermi surface. This is about $50 \%$ larger than the empirical value deduced from odd-even mass differences. This suggests that medium effects such as vortex- and selfenergy corrections (which we have ignored) must play some role.

The actual quenching of the pairing gap in the inner crust of a neutron star depends on the actual densities of neutrons and heavy nuclei. Under certain circumstances, the quenching may have a significant influence on the nuclear specific heat and it may therefore also affect the heat transport in a neutron star. This work was published in Phys. Lett. B $\underline{390}, 13$ (1997).

*Istituto Nazionale di Fisica Nucleare, Milano, Italy, †University of Sevilla, Spain

\section{c.4. Coupled Channels Analysis of High Precision Fusion Data (H. Esbensen, B. B. Back)}

We have analyzed the high precision fusion data for ${ }^{16} \mathrm{O}+{ }^{144} \mathrm{Sm}$ that recently have become available. The motivation for this work is twofold. Firstly, the high precision of the data is a challenge to a theoretical analysis. Secondly, the published analysis was based on an unrealistic ion-ion potential which had an unusually large diffuseness of $0.84 \mathrm{fm}$, and it included only linear couplings to one-phonon excitations of the ${ }^{144} \mathrm{Sm}$ target nucleus.

We include the lowest $2^{+}$and $3^{-}$excitations, of both projectile and target, in the coupled-channels analysis.
We also study the effect of higher-order excitations and couplings. We include, in particular, certain secondorder couplings between one-phonon excitations. We find that the latter couplings, which are often neglected in coupled-channels calculations, play a crucial role for obtaining a good fit to the data. By including them, we find that the best fit to the data is obtained for an ionion potential which has a diffuseness of $0.64 \mathrm{fm}$. This value is consistent with the empirical knowledge from analyses of elastic scattering data and also with the predictions of folding models. This work has been published in Phys. Rev. C 54, 3109 (1996). 


\section{c.5. Single Particle States in the Heaviest Elements (I. Ahmad and R. R. Chasman)}

The search for superheavy elements has been a major theme of nuclear structure research for the past twenty years. Theoretical predictions of the stability of superheavy elements depend crucially on the singleparticle energy level spacings in the vicinity of 114 protons and 184 neutrons. We are trying to learn as much as possible about these levels from spectroscopic studies of nuclides in the $\mathrm{A}=250$ region. This is possible because there are members of the relevant spherical multiplets that drop rapidly in energy with increasing deformation, and are fairly close to ground in the strongly deformed nuclides near $A=250$. The orbitals that are important for fixing the shell corrections near $\mathrm{N}=184$ are the $\mathrm{h}_{11 / 2}, \mathrm{j}_{13 / 2}$ and $\mathrm{k}_{17 / 2}$ spherical states. For each of these spherical orbitals, there is a corresponding deformed orbital whose energy in the $A=250$ region is quite sensitive to one of these spherical states, e.g. the 1/2-[761] orbital that has already been identified in ${ }^{251} \mathrm{Cf}$ is quite sensitive to the spherical $j_{13 / 2}$ orbital. The position of the $1 / 2+[880]$ deformed orbital is very sensitive to the $\mathrm{k} 17 / 2$ spherical state. According to our calculations, using a momentum-dependent potential, this state should be found at $\sim 1500 \mathrm{KeV}$ in $251_{\mathrm{Cf}}$ and should be populated in a one-nucleon transfer reaction using an $\left(\alpha,{ }^{3} \mathrm{He}\right)$ reaction. We have calculated signatures for the lowlying states in ${ }^{251} \mathrm{Cf}$ and the calculated energies and signatures are in good agreement with the experimentally observed $(d, p)$ spectrum. We expect to see the high $\mathrm{j}$ states in an $\left(\alpha,{ }^{3} \mathrm{He}\right)$ transfer reaction. As a ${ }^{250} \mathrm{Cf}$ target is not available, we have studied the high j states in ${ }^{249} \mathrm{Cm}$, which is an isotone of ${ }^{251} \mathrm{Cf}$. The $\left(\alpha,{ }^{3} \mathrm{He}\right)$ experiment has been carried out and two high-J peaks have been observed at $\sim 1.6$ and $1.9 \mathrm{MeV}$. A detailed analysis of the $\left(\alpha,{ }^{3} \mathrm{He}\right)$ peaks is being made.
Using the Strutinsky method, we found ${ }^{1}$ that we could get very good agreement with the known low-lying levels in $251 \mathrm{Cf}$ using a momentum-independent Woods-Saxon potential. This calculation gives the $1 / 2^{+}[800]$ orbital at $\sim 2.0 \mathrm{MeV}$. Although it is not clear yet how well this corresponds to the experimental value in ${ }^{249} \mathrm{Cm}$, we have used the potential parameters generated from this fit to study the stability of superheavy elements. To determine potential parameters for protons in the heavy elements, we utilized our spectroscopic data on $255 \mathrm{Md}$. It shows the $1 / 2^{-[521]}$ orbital to lie above the $7 / 2^{-}[514]$ orbital. Fixing the potential in this way, we extrapolate directly to the superheavy element region. We replaced the zero-point correction in our mass estimates by a term that depends quadratically on the sum of the proton and neutron shell corrections, as the usual zero-point correction treatment is questionable.

With our potential parameters derived from the heaviest elements for which there is detailed spectroscopic information, we find that our calculated lifetimes are sufficiently long to be able to observe elements at least through $Z=120$. In Fig. IV-12, we show the change in energy relative to the energy of a spherical liquid drop, for accessible nuclides with $\mathrm{Z}$ ranging from 110 to 126 . In our analysis, we assumed that the $1 / 2-[521]$ orbital lies just above the $7 / 2^{-}[514]$ orbital in ${ }^{255} \mathrm{Md}$. In fact, this level spacing is not known. Our estimates of superheavy element stability will be affected by the experimental value of this level spacing. We plan to study this question using the reaction of ${ }^{22} \mathrm{Ne}$ on ${ }^{238} \mathrm{U}$ to populate levels in $255 \mathrm{Md}$. The level spacing obtained in this experiment will be used to refine our estimates of superheavy element lifetimes.

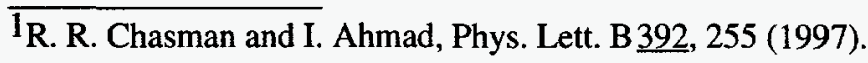

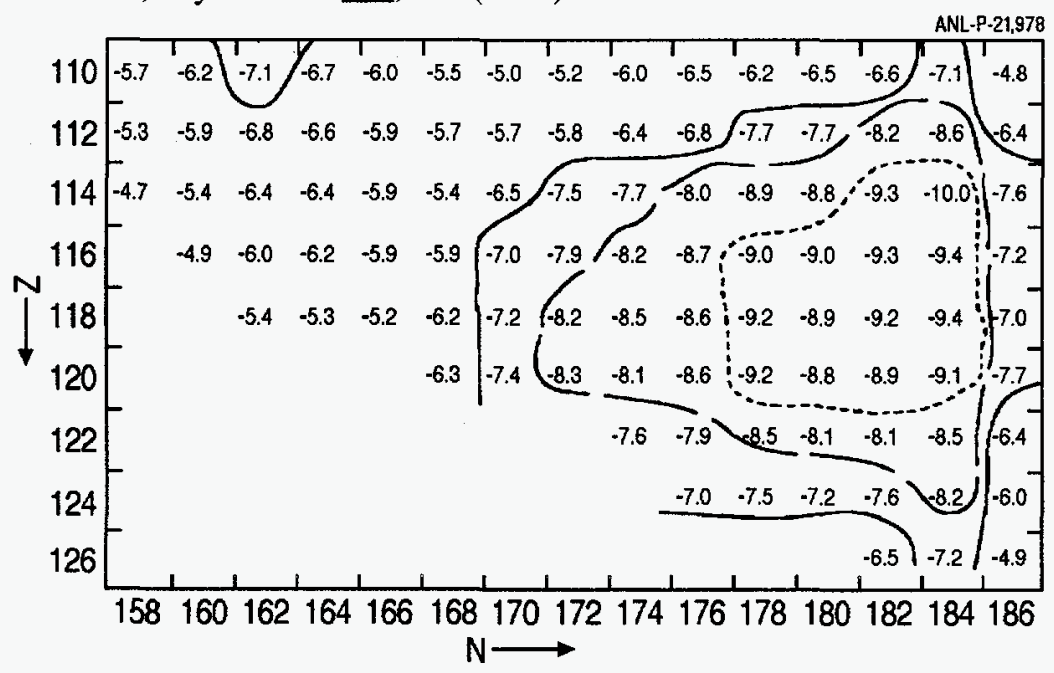

Figure IV-12. Increase in binding energy relative to spherical liquid drop energy. 


\section{c.6. Nuclear Energy Surfaces in the A 180 Mass Region (R. R. Chasman, J. L. Egido,* and L. M. Robledo*)}

We are studying nuclear energy surfaces at high spin, with an emphasis on very deformed shapes using two complementary methods: 1) the Strutinsky method for making surveys of mass regions, and 2) Hartree-Fock calculations using a Gogny interaction to study specific nuclei that appear to be particularly interesting from the Strutinsky calculations. The great advantage of the Strutinsky method is that one can study the energy surfaces of many nuclides $(\sim 300)$ with a single set of calculations. Although the Hartree-Fock calculations are quite time-consuming relative to the Strutinsky calculations, they determine the shape at a minimum without being limited to a few deformation modes. We have completed a study of $182 \mathrm{Os}$ using both approaches. In our cranked-Strutinsky calculations, which incorporate a necking mode deformation in addition to quadrupole and hexadecapole deformations, we found three well-separated, deep, strongly-deformed minima. The first is characterized by nuclear shapes with axis ratios of $1.5: 1$, the second by axis ratios of $2.2: 1$, and the third by axis ratios of $2.9: 1$. We also studied this nuclide with the density-dependent Gogny interaction at $I=60$ using the Hartree-Fock method and found minima characterized by shapes with axis ratios of 1.5:1 and 2.2:1. A comparison of the shapes at these minima, generated in the two calculations, shows that the necking mode of deformation is extremely useful for generating nuclear shapes at large deformation that minimize the energy. The Hartree-Fock calculations are being extended to larger deformations in order to further explore the energy surface in the region of the 2.9:1 minimum.

With the recent availability of large computer resources on the SP system at Argonne, together with the continued availability of computing resources at NERSC, it is feasible to carry out Strutinsky calculations on a large four-dimensional grid in a deformation space that includes octupole deformation in addition to quadrupole, hexadecapole and necking deformations. We are carrying out this study of the A 180 region concentrating on the questions: 1) how do the inclusion of necking and reflection-asymmetric degrees of freedom modify nuclear energy surfaces, and 2) how soft are the many known, very deformed nuclear shapes in this region of octupole deformation.

\footnotetext{
*Universidad Autonoma de Madrid, Spain

${ }^{1}$ R. R. Chasman and L. M. Robledo, Phys. Lett. B351,18 (1995).

2J. L. Egido, L. M. Robledo, and R. R. Chasman, Phys. Lett. B393, 13 (1997).
}

We have completed ${ }^{1}$ the first phase of these studies, using the Strutinsky method to calculate nuclear energy surfaces in the four-dimensional space discussed above. Comparing the results obtained with and without octupole deformation, we found major modifications of the energy surface in many nuclei in the region around ${ }^{176} \mathrm{~W}$. These effects are strong at all values of the angular momentum. There are reductions of the total energy of $\sim 7 \mathrm{MeV}$ for the necked-in shapes at the largest deformations. This feature can be understood in terms of incipient fission fragments. The reflectionasymmetric shape is necked in such a way as to exploit the large shell corrections associated with a stronglydeformed fragment in the vicinity of $100 \mathrm{Zr}$ and a spherical fragment in the vicinity of ${ }^{80} \mathrm{Zr}$. The symmetric necked-in shape at these large deformations corresponds to two strongly-deformed incipient fission fragments near $A=90$ and is not favored. The effects are huge here, compared to typical octupole deformation energy gains of less than $1 \mathrm{MeV}$.

As in the case for Strutinsky calculations, the introduction of reflection asymmetry doubles the basis space that must be used in Hartree-Fock calculations. We have extended the Hartree-Fock codes to allow us to calculate the properties of very deformed reflectionasymmetric shapes. We have finished a calculation ${ }^{2}$ of the energy surface of ${ }^{176} \mathrm{~W}$ at $\mathrm{I}=0$, using the Gogny interaction. As we are interested in very extended shapes, it is necessary to use a large basis ( -30 shells in the $\mathrm{Z}$ direction). We found that there are numerical instabilities in certain matrix elements, due to almost complete cancellation between large terms of opposite

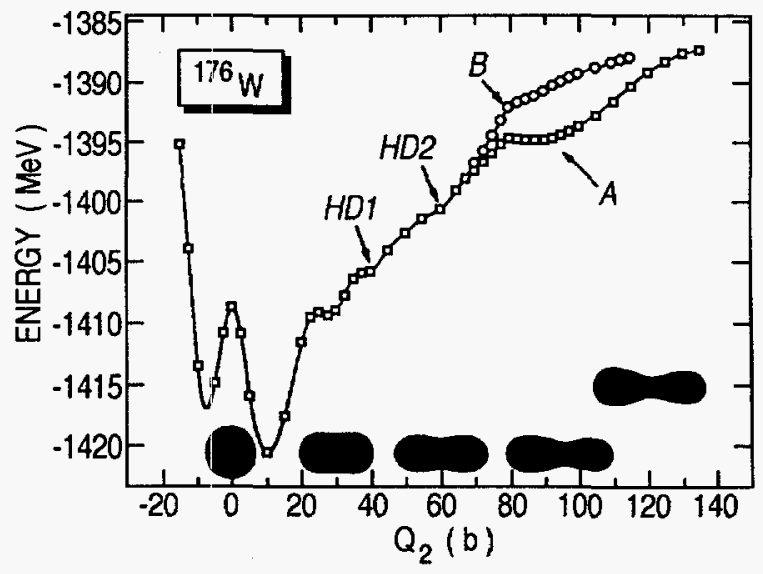

Figure IV-13. Energy of $176 \mathrm{~W}$ as a function of quadrupole moment. 
signs. This problem arises using the standard approach developed by Girod and Grammaticos, when more than 19 shells are used in the basis. Making a Fourier transform, we found that the matrix elements can be correctly calculated and large numbers of shells can be used as the basis states. The important result is that we can now treat the energy surface consistently, using a one-center basis, from the ground state to fission.

In agreement with our Strutinsky calculations, we find that the inclusion of reflection asymmetry in the
Hartree-Fock calculations gives a significant lowering of the energy surface at the largest deformations. In Fig. IV-13, we show the energy as a function of quadrupole moment. The curve labeled $B$ is obtained by constraining the nuclear shape to be reflection symmetric. The curve labeled $A$ is obtained when this constraint is not imposed. At the endpoints of curves A and $B$, the calculation gives two separated fragments, i.e. fission. The shapes at the bottom of Fig. IV-13 are those obtained for quadrupole moments of $0,30,60,95$ and 120 barns.

\section{c.7. Very Extended Shapes in Nuclei (R.R. Chasman)}

In the past few years, large computer resources have become available on the massively-parallel IBM SP supercomputer at Argonne, in addition to the resources provided by NERSC. We have parallelized our codes to exploit the SP system and have devoted a large part of our efforts to calculating energy surfaces in a fourdimensional shape space that includes reflectionasymmetric shapes. We study the nuclear energy surfaces as a function of mass, charge, shape, and angular momentum, using the Strutinsky method. In this approach, one makes quantum corrections to a smooth liquid-drop behavior using the calculated meanfield energy levels. In earlier studies, we found that it is often not sufficient to use only quadrupole and hexadecapole deformations to describe very extended reflection symmetric nuclear shapes. When we added a necking degree of freedom, we found previously unknown minima. These minima are characterized by very extended capsule-shaped nuclei with axis ratios of 2.2:1 in the $\mathrm{A}=180$ mass region. We have now added octupole deformation to this shape space. The inclusion of these two degrees of freedom to our shape space substantially increases our ability to describe nuclear shapes compared to a typical shape space consisting only of quadrupole and hexadecapole deformations. As parity is no longer a good quantum number when octupole deformation is included, the size of the matrices that we diagonalize is doubled. In a typical calculation, we diagonalize matrices that are $600 \times 600$. Several thousand such diagonalizations are needed to determine energy surfaces.

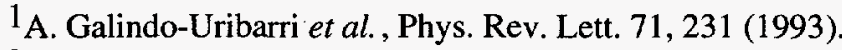

2D.R. LaFosse et al., Phys. Rev. Lett. 74, 5186 (1995).

${ }^{3}$ R.R. Chasman, Physics Letters B $\underline{364}, 137$ (1995).

${ }^{4}$ S.M. Fischer et al., Phys. Rev. C54, R2806 (1996).
}

There has been a report ${ }^{1}$ of a rotational band in ${ }^{152}$ Dy or ${ }^{153}$ Dy that is characterized by a dynamic moment of inertia of $130 \hbar^{2} \mathrm{MeV}^{-1}$. More recently, there are reported rotational bands associated with hyperdeformed shapes in $147 \mathrm{Gd}$ with a dynamic moment of inertia of $\sim 140 \hbar^{2} \mathrm{MeV}^{-1}$. For purposes of orientation, it should be noted that the well-known superdeformed bands in this region are characterized by moments of inertia of only $\sim 90 \hbar^{2} \mathrm{MeV}^{-1}$. Some calculations have been carried out in two- and threedimensional shape spaces, in order to understand this experimental observation. Some of these calculations suggest that the hyperdeformed bands are associated with a reflection-asymmetric minimum, that becomes yrast near $\mathrm{I}=90 \hbar$. We have carried out an extensive series of calculations to investigate this mass region.

We did not find hyperdeformed minima in this mass region that appear to be stable to fission. Our calculations suggest that the explanation for these bands is something other than hyperdeformation. Since the submission of our results ${ }^{3}$ for publication, the claims of experimental observation of hyperdeformed bands in ${ }^{152}$ Dy and in ${ }^{147} \mathrm{Gd}$ have been withdrawn.

We did, however, find several nuclei with superdeformed minima that are somewhat more extended than any that have been observed in the A 150 mass region. Specifically, these are nuclides near $158 \mathrm{Er}$ in mass and are characterized by axis ratios as large as $2: 1$ in contrast to the $1.85: 1$ axis ratio of the superdeformed shapes in ${ }^{152} \mathrm{Dy}$. 
The single-particle level structure associated with the very superdeformed shapes of the $A=156$ region is particularly fascinating. The single-particle orbitals that appear at the fermi-level at $\mathrm{I}=80 \hbar$ in these minima are orbitals that are characteristic of undeformed superheavy elements $(Z=114, N=184)$ at $I=0 h$. It would be one of the more exciting findings of the heavy-ion research program to identify these orbitals in the rare earths.

We have extended our high-spin Strutinsky calculations to nuclei in the $\mathrm{A}=100$ to 150 region. Although we find many of the very extended minima in this mass region, the prospects for populating them appear to be $\mathrm{dim}$. The problem again is that the fission barrier is low when the very extended minimum becomes yrast and/or that the very extended minimum becomes yrast at spins that are not presently accessible.
An experimental search ${ }^{4}$ for the extended shapes characterized by a 2:1 axis ratio has begun in ${ }^{155}$ Dy. At the angular momenta so far studied, these bands are not observed. The moment of inertia of the observed $(\sim 1.85: 1)$ superdeformed band in ${ }^{155} \mathrm{Dy}$ is in good agreement with our calculations. The spins of the states so far observed are just on the edge of the region where such extended shapes might be seen. Further studies to populate higher spin states are planned. An interesting feature associated with the superdeformed band in ${ }^{155} \mathrm{Dy}$ is that the decay from the superdeformed shape to normal shapes occurs at a higher frequency than in the lighter Dy isotopes, which can be understood in terms of our calculation. We found that the inner barrier was substantially smaller in ${ }^{155}$ Dy than in the lighter Dy isotopes.

\section{c.8. Many Body Wave Functions (R. R. Chasman)}

In the past few years, we have developed many-body variational wave functions that allow one to treat pairing and particle-hole two-body interactions on an equal footing. The complexity of these wave functions depends on the number of levels included in the valence space, but does not depend on the number of nucleons in the system. By using residual interaction strengths (e.g. the quadrupole interaction strength or pairing interaction strength) as generator coordinates, one gets many different wave functions; each having a different expectation value for the relevant interaction mode. These wave functions are particularly useful when one is dealing with a situation in which the mean-field approximation is inadequate. Because the same basis states are used in the construction of the many-body wave functions, it is possible to calculate overlaps and interaction matrix elements for the many-body wave functions (which are not in general orthogonal) easily. The valence space can contain a large number of singleparticle basis states, when there are constants of motion that can be used to break the levels up into groups. We have added a cranking term to the many-body Hamiltonian and modified the projection procedure to get states of good signature before variation.

We have extended this program to calculate spectroscopic factors for proton decay. This is useful for studies of nuclides near the proton drip line. Wave functions of this sort become more valuable as the size of each of the groups is increased. We have made an effort to speed up the variational procedure in our calculations, and are managing to do so. To increase the size substantially, however, it seems necessary to parallelize the code. We are starting this process.

\section{c.9. Nuclear Structure near the Proton Drip Line (R.R. Chasman)}

Recently, proton emission has been observed in the light $\mathrm{Pb}$ nuclei. It seems that one might hope to use this phenomenon to extract some interesting spectroscopic data on nuclear shapes and orbitals in much the same way that one gets spectroscopic information through single-nucleon transfer reactions such as $(\mathrm{d}, \mathrm{p})$ and $(\mathrm{d}, \mathrm{t})$ reactions. In order to explore this possibility, we have developed a treatment of proton-emission spectroscopic factors using many-body wave functions. In the case that we have studied ${ }^{185} \mathrm{Bi} \rightarrow{ }^{184} \mathrm{~Pb}$, the energetics of the reaction are such that only the ground state is populated. However, we calculate a very small spectroscopic factor for the proton decay to the ground state $(0.1 \%)$ in lowest order. To understand the observed ${ }^{1}$ spectroscopic factor $(\sim 3 \%)$, it is necessary to carry out configuration mixing of the many-body wave functions. We are carrying out such calculations and extending then to neighboring nuclides.

We expect that our calculations for this mass region can be improved by treating larger numbers of levels in our many-body code. This should be possible by parallelizing the many-body code. We are engaged in such a project.

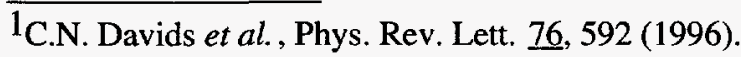




\section{c.10. Sum Rule for M2 Transitions (D. Kurath)}

Current (e,e') experiments are measuring the distribution of M2 strength in a number of nuclei. A sum-rule appropriate for low momentum transfer has been derived in a harmonic-oscillator shell model representation. The conditions assumed are:

1. The calculation is in the limit momentum transfer $\rightarrow 0$.
2. The ground-state consists of nucleons in an open shell with $\mathrm{Q}$ oscillator quanta (for example the $\mathrm{Q}=$ 2sd shell). Lower $Q$ shells are assumed to be full, higher $Q$ shells empty.

The sum of M2 strength to all final states is given below:

$$
\sum_{\mathrm{IT} \alpha} \mathrm{B}_{\mathrm{M} 2}\left(\mathrm{I}_{0} \mathrm{~T}_{0} \alpha_{0} \rightarrow \mathrm{IT} \alpha\right)=\left(\frac{75}{4 \pi \beta}\right)\left\{\begin{array}{l}
\mathrm{Z} \mu_{\mathrm{P}}^{2}+\mathrm{N} \mu_{\mathrm{N}}^{2}-\frac{1}{3}\left(\mu_{\mathrm{N}}^{2}\langle 2 \underline{\ell} \cdot \underline{\mathrm{s}}\rangle_{\mathrm{N}}+\mu_{\mathrm{P}}^{2}\langle 2 \underline{\ell} \cdot \underline{\mathrm{s}}\rangle_{\mathrm{P}}\right) \\
+\frac{1}{45}\left(\mathrm{Q}^{2}-1\right) \mathrm{Q}(\mathrm{Q}+3)(\mathrm{Q}+2) \\
+\frac{2}{3} \mu_{\mathrm{P}}\langle 2 \ell \cdot \mathrm{s}\rangle_{\mathrm{P}}+\frac{2}{9}\left(\sum_{\ell \text { in } \mathrm{Q}} \ell(\ell+1) \mathrm{n}_{\mathrm{P}}^{\ell}\right\rangle
\end{array}\right\}
$$

where

$$
\langle 2 \underline{\ell} \cdot \underline{\mathrm{s}}\rangle_{\mathbf{t}_{3}}=\sum_{\ell \text { in } \mathrm{Q}}\left[\ell \mathbf{n}_{\mathrm{t}_{3}}^{\mathrm{J}=\ell+1 / 2}-(\ell+1) \mathrm{n}_{\mathrm{t}_{3}}^{\mathrm{J}=\ell-1 / 2}\right]
$$

The first line contains the spin contributions which form the major part. The rest is the proton orbital contribution and the interference between proton orbital and spin. The contribution of transitions to spurious states of center-of-mass excitation should be subtracted and can be estimated by the M1 sum:

$$
\left(\frac{25}{3 \mathrm{~A} \beta}\right) \sum_{\mathrm{IT} \alpha} \mathrm{B}_{\mathrm{M} 1}\left(\mathrm{I}_{0} \mathrm{~T}_{0} \alpha_{0} \rightarrow \mathrm{IT} \alpha\right)
$$

where the M1 operator is

$$
\left(\frac{3}{4 \pi}\right)^{1 / 2}\left(\frac{\mu_{\mathrm{N}}-\mu_{\mathrm{P}}}{2}\right) \sigma^{1} \cdot \tau_{3}^{1}
$$

It is generally only a $1 \%$ correction.

\section{ATOMIC THEORY AND FUNDAMENTAL QUANTUM MECHANICS}

\section{d.1. Interactions of High-Energy Photons with Matter (M. Inokuti)}

In support of experimental work in atomic physics with the use of synchrotron radiation, theoretical studies are conducted on the physics of the Compton scattering by bound electrons, focusing on topics selected in view of basic importance, timeliness, and potential in applications.

When the photon energy greatly exceeds the binding energy of an electron, the effect of electron binding to an atom is well understood in the framework of an impulse approximation, which accounts for the momentum of an electron at the instant of the Compton scattering. However, the physics remains to be explored for a more general case, where full details of atomic structure, described for instance in terms of the Green's function, need to be considered. For a hydrogen-like atom, or within a single-electron approximation, full analysis of the binding effect is theoretically feasible. Efforts will be made to elucidate basic points of the physics involved and to suggest items for experimental exploration.

Another topic of study concerns dispersion relations and sum rules for photoabsorption cross sections and related quantities. Within the dipole approximation, these data are subject to numerous theoretical constraints, which are closely related to the electronic structure of atoms, and more generally to their aggregates. Comprehensive analysis of the data for a specific material proved valuable for many purposes, as exemplified in earlier work on many atoms, several molecules, and metallic aluminum. Similar analysis of data for basic materials such as water, carbon, and silicon will be carried out. Results will be useful in the design of experiments in which these materials are used for optical devices. 


\section{d.2. Interactions of Fast Charged Particles with Matter (M. Inokuti)}

Stopping power, the total yield of ionization, its fluctuation, ionization cross sections, and other quantities related to the energy loss of fast charged particles penetrating through matter are important in many applications such as the detection of particles and the analysis of their charges and kinetic energies. Despite its long history of study, the subject remains to be further studied in several respects, including the differences between particles of the same speed with different charges and the differences of the stopping power of the same material, depending on the phase or the chemical state. Charged-particle collisions at high energies, and especially at low-momentum transfers, are intimately connected with photon interactions. Therefore, a study on such collisions also contribute to the full understanding of photon-interaction physics. Efforts are being devoted to the clarification of these and related current issues.

\section{d.3. The Electron (M. Peshkin and Yosef Imry*)}

Cambridge University Press will mark the one hundredth anniversary of J. J. Thompson's discovery of the electron by publishing a book entitled "Electron", edited by Michael Springford (Bristol University). Publication is expected in March 1997.

We have completed our contribution, a chapter entitled "The Coherent Electron". Topics covered include coherence in electron interferometry and holography, Aharonov-Bohm effect in electron beams and in

*Weizmann Institute of Science, Rehovot, Israel mesoscopic normal conductors, and off-diagonal longrange order in superconductors.

The other chapters are "The Discovery of the Electron" (A. B. Pippard), "The Electron as a Fundamental Particle" (N. Cottingham), "The Electron and the Relativity Principle" (D. Olive), "The Electron Glue" (B. Gyorffy), "The Electron Liquid" (P. Coleman), "The Magnetic Electron" (G. G. Lonzarich), "The Paired Electron" (A. J. Leggett), "The Heavy Electron" (M. Springford), and "The Electron in the Cosmos" (M. Longair).

\section{d.4. Tight Binding Hamiltonians and Quantum Turing Machines (Paul Benioff)}

Work on models of Quantum Turing Machines (QTM) continued with emphasis on models with potentials associated with one or more types of steps. Hamiltonians for these models are given by $\mathrm{H}=\mathrm{K}(2-\mathrm{T}$ $\mathrm{T}^{\dagger}$ ) where $\mathrm{T}$ is the infinitesimal time step operator which describes the machine being considered. The physical model consists of a 1-D lattice of binary quantum systems (qubits). A head, that can be in any one of a finite number of states, moves along the lattice interacting locally with the qubits. What the head does depends on the state of the qubit at the head location and the internal state of the head. The operator $\mathrm{T}$ is a finite sum over all elementary step operators for the machine being considered. Potentials are included by multiplying one or more of the elementary step operators by real nonnegative constants $\leq 1$. A convenient basis for describing the states of the QTM is the computation basis $\mathrm{B}$, which is the set of states $1 \mathrm{l}, \mathrm{j}, \mathrm{S}>$ where 1 and $\mathrm{j}$ are the head internal state and position and $\mid S>$ is a product of the states of the qubits on the lattice, where for each $j\left|S(j)>_{j}=\right| 0>_{j}$ or $\left|1>_{j} \cdot\right| S(j)>_{j}$ is the state of the qubit at site $j$.If the step operator $T$ is distinct-path-generating in some basis, taken here to be $B$, then the Hamiltonian can be written as a sum of tight-binding Hamiltonians $H=\Sigma_{\mathbf{i}} H_{j}$ over each computation path i. Each computation path is a set of states in B ordered by iteration of $\mathrm{T}$ and $\mathrm{T}^{\dagger}$. The paths are distinct in that the states in any path are orthogonal to the states in all other paths. The paths that are active in any specific instance for the QTM are determined by the initial state. The potential distribution in each tightbinding Hamiltonian $\mathrm{H}_{\mathrm{i}}$ depends on $\mathrm{i}$.

A specific example, the counting QTM, was analyzed in detail. This QTM iterates the positive integers from 0 to $2^{n}-1$ for arbitrary $n$ including $n=\infty$. The choice of $n$ is determined by the initial state. A potential was associated with the elementary step operator corresponding to read- 1 steps. All other step types were potential free. It turns out that for each $\mathbf{n}$ the potential distribution is quasiperiodic and is an initial segment of the hierarchical substitution sequence. This shows that QTMs with included potentials generate structures similar to those which are much studied in solid-state science, namely, systems described by 1-D Hamiltonians with potential distributions that are quasiperiodic or are substitution sequences. 
Both the energy band spectra and the Landauer Resistance (LR) were determined for the counting QTM described above for various values of $\mathbf{n}$ and the two model parameters. The band spectra are for the associated periodic systems. These are crystals whose unit cell is the potential distribution associated with iteration of the first $2^{\mathrm{n}}-1$ integers. The LR is the ratio of the probability of transmission to the probability of reflection for the potential distribution. High transmission probability (low LR) corresponds to a high probability of completion of the computation proceeding along the path being considered. The results of the calculations for parameters in the electron region $\left(\mathrm{V}=2 \mathrm{ev}\right.$, head mass $=2 \mathrm{~m}_{\mathrm{e}}$, and lattice spacing $=1$ Angstrom) are shown in Figures IV-14 and IV-15 for $n=10$ and $n=18$ respectively. In the figures $\log 10$ LR and the band spectra are plotted as a function of momentum. The figures show rapid fluctuation of the LR especially for $n=18$. In general the $L R$ is high in a gap region and low in a band region with minima occurring close to band-gap edges. It is surprising that the LR shows so many regions of low values (corresponding to appreciable probability of completion of the computation). This is especially the case for $\mathrm{n}=$ 18 for which more than 128,000 potential barriers are present.

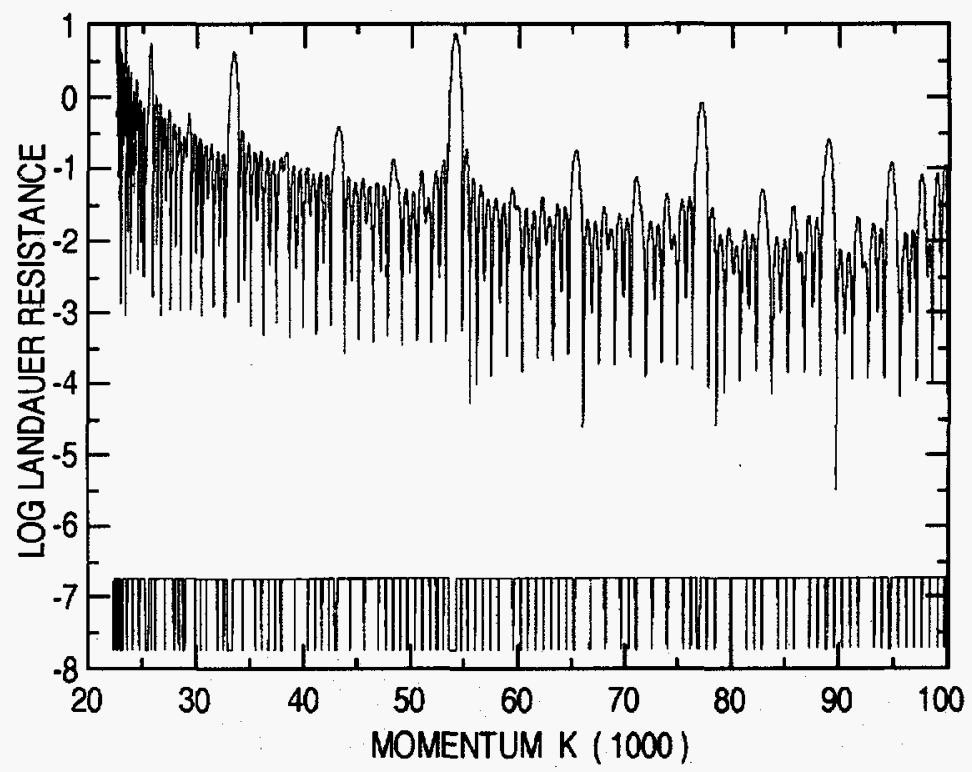

Figure IV-14. The log (base 10) of the Landauer resistance plotted as a function of the momentum, for $N=10$ and parameters in the electron region for a momentum range $0.0223 \leq k \leq 0$. The energy band spectrum, for the associated periodic system is shown at the bottom of the figure where upper horizontal line segments correspond to energy bands and lower horizontal segments correspond to energy gaps. Very short bands or gaps appear as points. Band-gap edges show as vertical lines. The original placement of the band spectrum, at the bottom of the figure is done for convenience only. 


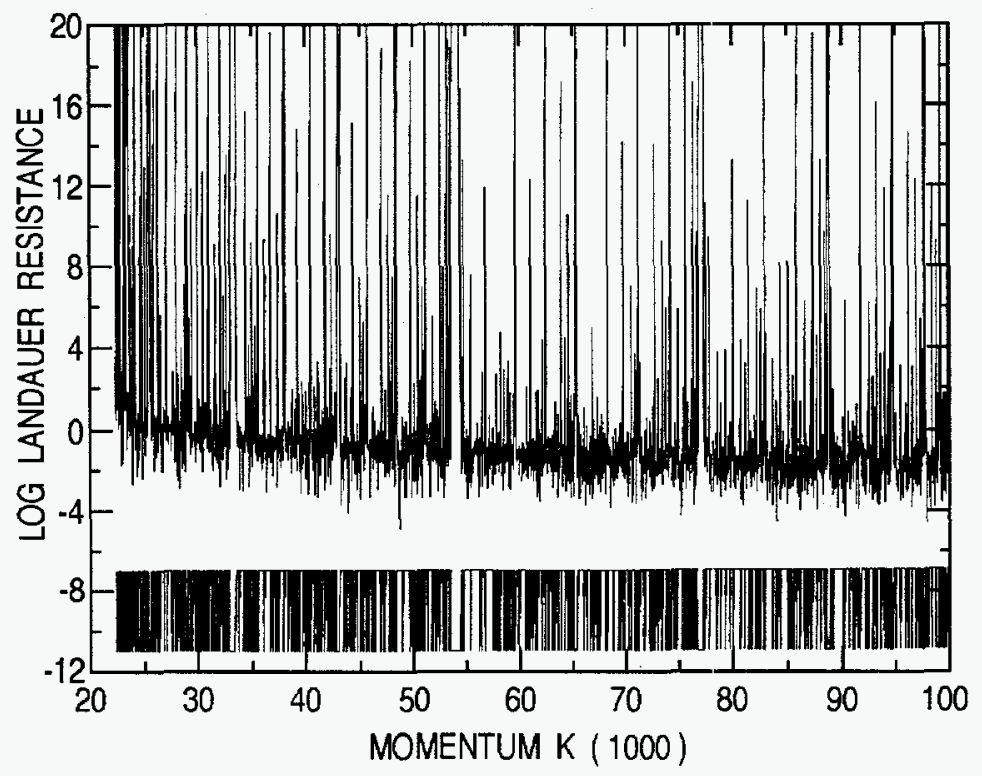

Figure IV-15. The log (base 10) of the Landauer resistance plotted as a function of the momentum for $N=18$ and parameters in the electron region for a momentum range $0.223 \leq k \leq 0.10$. The energy band spectrum for the associated periodic system is shown at the bottom of the figure. Additional details are given in the caption for Figure IV-14.

\section{E. OTHER ACTIVITIES}

\section{e.1. Theory Workshop on QCD-based Studies of Hadron Spectroscopy and Interactions (C.D. Roberts)}

The Theory Group hosted a workshop, July 22-26, 1996, focusing on QCD-based Studies of Hadron Spectroscopy and Interactions, which brought to Argonne 25 outside participants (five of them PhD students) from Australia, Canada, Europe, Russia and the USA. In five days of presentations, and lively discussions, the participants considered the role that quarks and gluons play in nuclear dynamics, which is the focus of future experimental efforts at RHIC and TJNAF, and also of current efforts at MIT-Bates and Fermilab. With TJNAF already taking data, the development and extension of theoretical approaches that can describe the quark and gluon substructure of hadrons within the framework of Quantum Chromodynamics (QCD) is of particular importance. This workshop brought together experts in this area as a necessary and integral part of enhancing and broadening that theoretical effort which is necessary for the proper understanding of the data collected in this new generation of experiments. Much of the experimental effort at RHIC and TJNAF will probe QCD in the nonperturbative domain. With no single nonperturbative theoretical tool currently able to address all of the questions that will be raised, this workshop brought together practitioners from across a broad spectrum of complementary approaches: DysonSchwinger Equations, Effective Lagrangians, Lattice QCD, Light-Front Quantum Field Theory, QuarkParton Model and Relativistic Constituent Quark Models; areas in which Argonne's Physics and High Energy Physics Divisions have expertise. The crossfertilization that such a conjunction provides, and which the Theory Group is active and successful in promoting, fosters and facilitates collaborations that provide for rapid progress in this new and important area of Nuclear Physics. This is recognized and appreciated by the community at large. The workshop was funded by the Associate Laboratory Director for Physical Research. 


\section{e.2. Ninth Annual Midwest Nuclear Theory Get-Together (R. B. Wiringa)}

The Theory Group hosted the Ninth Annual Midwest Nuclear Theory Get-Together on September 27 and 28, 1996. Nuclear theorists from a number of midwest universities get together every fall to find out what different people and groups in the area are working on. The organizational duties rotate among the participants, but Argonne has become the regular meeting place by virtue of its facilities and central location. The organizer for 1996 was Wayne Polyzou from the University of Iowa. The meeting provides a good chance for students to broaden their horizons and get some practical speaking experience in a friendly atmosphere. The format is very informal, with an agenda of talks being volunteered at the beginning of the meeting. This year 27 faculty, postdocs and graduate students from ten different institutions in Illinois, Indiana, Iowa, Kentucky, Michigan, Missouri, Ohio and Wisconsin, along with the Argonne staff, gathered to discuss their current work in nuclear physics. Some 19 presentations were made over Friday afternoon and Saturday morning. Topics included QCD, Skyrme models, charge-symmetry breaking and parity nonconservation, the NN interaction, halo nuclei, the shell model, chaos, relativistic heavy-ion collisions, and neutrino astrophysics. A good time was had by all. 


\title{
V. ATOMIC AND MOLECULAR PHYSICS RESEARCH
}

\section{OVERVIEW}

\begin{abstract}
The atomic physics program in the Argonne Physics Division focuses on a detailed understanding of atomic structure and dynamics using photons as a probe. As a system where the forces are well understood, the atom is an ideal laboratory in which to test theoretical developments in, e.g. many-body theory, relativistic and QED effects, scattering theory and non-perturbative interactions. At Argonne, we have two facilities, the Advanced Photon Source (APS) and ATLAS, which allow us to address a unique subset of atoms, i.e. those with deep inner-shell excitation where relativistic and QED interactions are most pronounced. At ATLAS deep inner-shell excitations are formed in simple systems of highly-stripped ions whereas at the APS it is possible to excite deep inner shells in atoms that retain electrons in their outer shells, and thereby study the coupling between inner and outer shells. While the primary goal of the APS-based effort is to establish a fundamental understanding of X-ray interactions with atoms and molecules, these studies also provide a fundamental basis for the $\mathrm{X}$-ray diagnostics used in other disciplines, e.g. Compton scattering studies of electron momentum distributions in materials, Raman scattering studies of electronic band structure, X-ray diffraction studies of large molecules and core-hole shifts in complex chemical environments. Currently, the major emphasis of the program is the study of fundamental X-ray atom interactions exploiting the high-brilliance, polarized, high-energy photons from the recently-commissioned APS.
\end{abstract}

The Argonne atomic physics group has a major presence in the research program at the Basic Energy Sciences Synchrotron Radiation Center (BESSRC) at the APS. As one of the four core members of BESSRC (others are Chemistry, Materials Science and Geosciences), the group has an endstation located on the undulator beam line dedicated for fundamental studies in atomic physics. In addition to the undulator radiation available at BESSRC, there is an endstation for bending magnet radiation and three endstations for radiation with variable polarization from an elliptical multipole wiggler. Access to all three of these radiation sources with different properties will enable a comprehensive program to study fundamental Xray - atom interactions.

The initial phase of the APS-based research program is motivated by the fact that the physics of deep inner shells remains largely unexplored, unlike the valence and shallow inner shells accessible with VUV or soft X-ray excitation. Deep inner shells differ from the shallow inner shells in a number of ways: 1) inner-shell wavefunctions are confined near the center of the atom, thus enhancing their utility as an atom specific probe in complex environments; 2) interaction times connected with inner-shell processes are short, thus enhancing interference effects; 3 ) relativistic and QED effects are magnified; 4) X-ray wavelengths are comparable to 
Bohr-orbital radii and bond lengths, thus fundamentally altering $\mathrm{X}$-ray optical effects in matter in comparison to conventional optical physics. The basic understanding of these effects is best furthered in isolated atoms and molecules, where experiment and theory can have the most productive interplay.

The atomic physics group has taken the lead in experimentation at BESSRC, performing two X-ray scattering experiments and designing, constructing and utilizing a general purpose $X$-ray emission spectrometer during the past year. During a commissioning period in October 1996, the group mounted an experiment to measure elastic (Rayleigh) and inelastic (Compton) scattering cross sections from gas phase $\mathrm{Ne}$ as a test of form-factor, S-matrix and impulse approximation scattering calculations. Traditionally, calculations have been tested by comparison to photon scattering measurement on solids, and these data are among the first absolute measurements that decompose elastic- and inelastic-scattering channels on free atoms. In the first experiment using the multipurpose $X$-ray spectrometer in spring 1997, inelastic (Raman) scattering near threshold in gas-phase $\mathrm{Kr}$ was studied with the objective of decomposing Rydberg states leading to ionization that are blended in simple absorption studies as a more stringent test of atomic structure and scattering calculations.

Many of the projects described in this report stem from earlier work conducted at other synchrotron facilities, i.e. the X-24A National Synchrotron Light Source (NSLS) at Brookhaven National Laboratory and the Advanced Light Source (ALS) at Lawrence Berkeley National Laboratory. These experiments provide the foundation and motivation for selected early experiments at the APS: 1) Extend the studies of Raman scattering to homonuclear diatomic molecules, where within the dipole approximation, the final state must have the same inversion symmetry as the initial state. Studies of cases where the wavelength of the X-ray photon is comparable to (or smaller than) the internuclear distance are possible at the APS, and this allows exploration of predictions for the high-energy limit of X-ray emission intensities to dipole-unallowed states. 2) Measurement of the single-todouble ionization ratio in He by photon impact over an intermediate energy regime in which there are large experimental errors and theoretical disagreements. 3) Extend the study of non-dipole effects in photoionization to deep inner shells of heavy atoms.

The ATLAS program is focused on understanding the structure of multielectron highly-charged ions. The atoms studied are closely related to those at the APS, i.e. foil-excited to have a single electron promoted from the deep inner shell, leaving a core-excited atom. The intense beams at ATLAS allow the study of core-excited atoms in this particularly simple situation, i.e. with none or only a few outer-shell electrons present, for high-precision tests of rigorous many-body atomic theory. Atomic structure properties are deduced from the time-, energy- and angle-resolved $X$-ray emission spectra. The experiments in the intermediate- $Z$ range, where electron correlation, relativistic and QED effects are simultaneously important, provide useful guidance to theory in this regime where all interactions must be considered. Two prototypical intermediate- $Z$ He-like ions that can be produced at ATLAS with sufficient intensity are $\mathrm{Kr}(\mathrm{Z}=36)$ and $\mathrm{Ni}(\mathrm{Z}=28)$, and these have been studied with great detail. In order to access the higher- $Z$ regime, experiments are performed at GSI. We are currently concentrating on two-electron ions but plan to gradually move on to more complicated systems. A particular emphasis has been on precision measurement of forbidden transitions, analogous to the studies of nondipole effects in photoionization in the APS-based program. 


\section{a. Experimental Separation of Compton and Rayleigh X-Ray Scattering from Gas-Phase Ne (R. W. Dunford, D. S. Gemmell, M. Jung, E. P. Kanter, B. Krässig, T. LeBrun, S. H. Southworth, L. Young)}

As a test of form-factor, S-matrix, and impulse approximation scattering calculations for photons, we have measured elastic (Rayleigh) and inelastic (Compton) scattering cross sections from gas-phase $\mathrm{Ne}$ for incident energies 11-22 keV. Traditionally, calculations have been tested by comparison to photon scattering measurements on solids, and these data are among the first absolute measurements that decompose elastic and inelastic scattering channels on free atoms. Monochromatized $X$-rays $\left(\Delta \mathrm{E} / \mathrm{E} \approx 1.3 \times 10^{-4}\right)$ from the Advanced Photon Source were incident on the gas sample. The interaction region was viewed simultaneously by two well-characterized Si(Li) detectors placed $90^{\circ}$ azimuthally about the photon beam axis to eliminate and investigate polarization dependences. Two scattering angles, $90^{\circ}$ and $135^{\circ}$, were measured, providing overlap in the momentum transfer coordinate. Figure V-1 shows data taken at the $90^{\circ}$ scattering angle. Absolute cross sections for scattering are obtained by normalization to scattering from $\mathrm{He}$. Analysis of systematic errors due to non-idealities in, e.g. photon beam profile, beam misalignment, higher harmonic contamination of the X-ray beam, is underway and suggests the possibility of extracting cross sections of $\approx 1 \%$ accuracy for comparison to various theories.

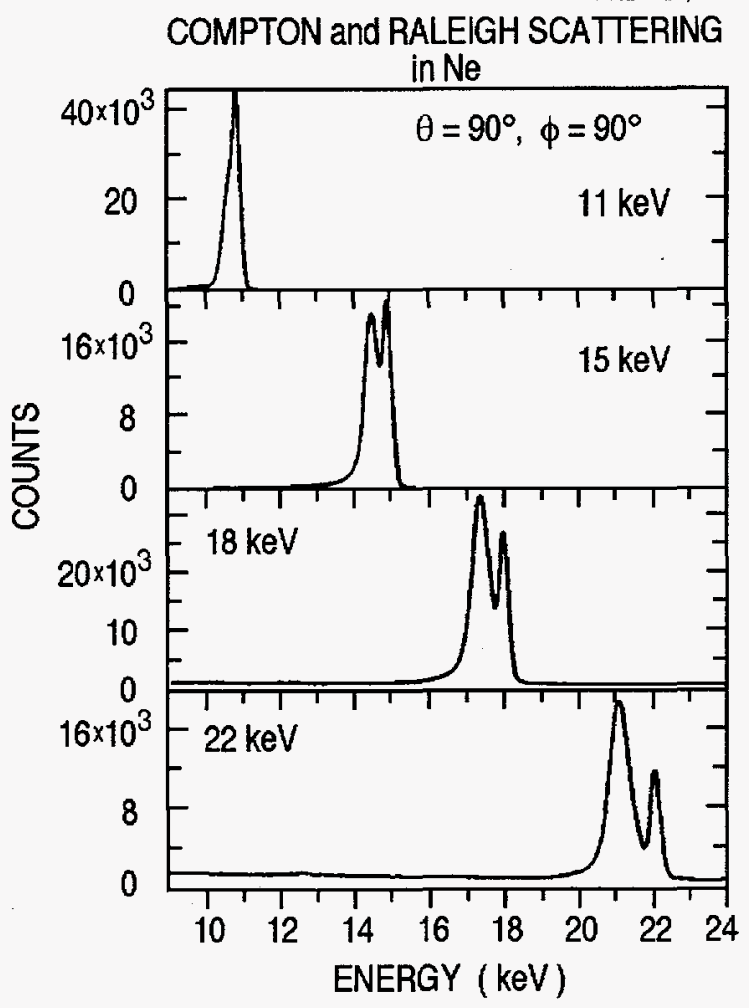

Fig. V-1. Compton and Rayleigh scattering spectra for $\mathrm{Ne}, \theta=90^{\circ}$ for incident photon energies between 11 and $22 \mathrm{keV}$.

\section{b. Argon KM Photoelectron Satellites (S. H. Southworth, T. LeBrun, Y. Azuma, * and K. G. Dyall $\dagger$ )}

The independent-electron approximation is often adequate for understanding atomic structure and electronic transitions, but certain experimental observations require a many-electron treatment. A wellknown example is photoelectron spectra (PES), which typically display strong primary peaks, corresponding to the independent-electron picture, accompanied by weaker satellite peaks that require a many-electron interpretation. Because of their sensitivity to electronelectron repulsion ("electron correlation") in wavefunctions and transitions, satellites are of fundamental interest. Since satellite transitions in inner-shell PES produce multiple vacancies, they are associated with satellite features in radiative and nonradiative vacancy-decay spectra. Information derived from various spectroscopies can be combined to further ,test and develop theoretical concepts and calculations for electron correlation in inner-shell processes.

The primary satellites observed in inner-shell PES can be qualitatively explained in a single-configuration model by "relaxation" of valence orbitals in the presence of a vacancy in the inner shell. However, rigorous theoretical treatment requires multiconfiguration wavefunctions. We have recorded the satellite structure in the Ar K-shell PES due to M-shell excitations for comparison with relativistic, multiconfiguration shaketheory calculations (see Figure V-2). The calculations give a good match to the main satellite features, but there are differences in relative intensities, and the lowenergy feature $\mathrm{A}$ is attributed to a "conjugate" satellite 


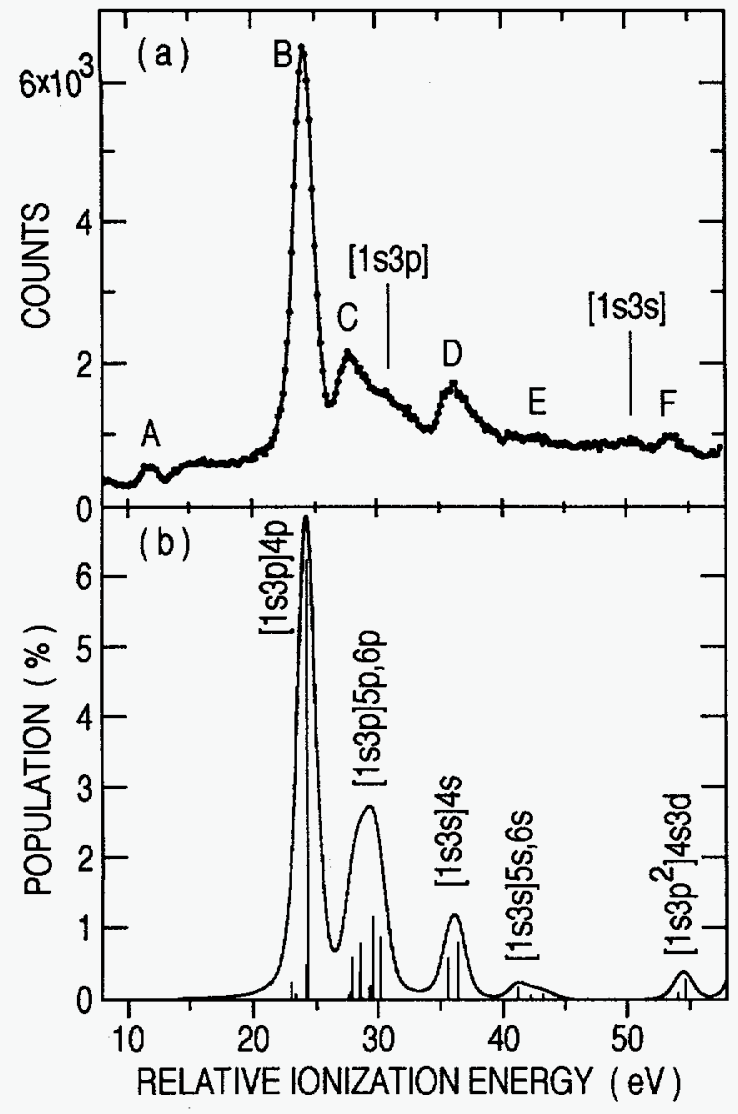

not included in the shake model. Comparison with the experimental data provides a critical assessment of the urrent theory and suggests refinements. Experimental methods will be developed to increase resolution and sensitivity.

Fig. V-2. Comparison of satellite structure measured in the Ar K-shell photoelectron spectrum (top) with relativistic, multiconfiguration shake-theory calculations (bottom).

*National Laboratory for High Energy Physics $\dagger$ NASA Ames Research Center

\section{c. Non-Dipole Resonant X-Ray Raman Spectroscopy: Polarized Inelastic Scattering at the K-Edge of $\mathbf{C l}_{2}$ (J. D. Mills, ${ }^{*}$ J. A. Sheehy,* T. A. Ferrett, $\dagger$ S. H. Southworth, R. Mayer, $\ddagger$ D. W. Lindle, $\S$ and P. W. LanghoffII)}

We have previously studied resonant X-ray Raman scattering from the $\mathrm{K}$-edges of S-containing and $\mathrm{Cl}$ containing polyatomic molecules in the $2-3 \mathrm{keV}$ energy range. The $\mathrm{X}$-ray emission spectra are highly polarized and anisotropic due to alignment of resonantly-excited intermediate states. These effects could be explained using the Kramers-Heisenberg scattering formalism within the electric-dipole approximation to the photon-molecule interaction. However, resonant X-ray Raman scattering from the $\mathrm{K}$ edge of $\mathrm{Cl}_{2}$ demonstrates the need to treat this process beyond the dipole approximation.

For a homonuclear diatomic molecule, the eigenstates of irreducible symmetry are characterized by an inversion symmetry $(\mathrm{g}=$ gerade $=$ even; $\mathrm{u}=$ ungerade $=$ odd). In a second-order, two-photon process such as X- ray Raman scattering, the final state must have the same inversion symmetry as the initial state within the electric-dipole approximation. As shown in Figure $\mathrm{V}-3$, however, we observe $\mathrm{g}$ and $\mathrm{u}$ final states with similar intensities in our $\mathrm{X}$-ray emission spectra from $\mathrm{Cl}_{2}$ excited at the strong $5 \sigma_{\mathrm{u}}$ resonance below the Kedge. The X-ray emission is also polarized. These effects can be explained using the Kramers-Heisenberg scattering formalism when the dependence on the photon propagation vector is retained in the photonmolecule interaction. The $\mathrm{X}$-ray emission intensities can be expressed in terms of their dependence on photon wave vectors and internuclear separation, indicating that molecular structural information can be obtained from such measurements.

*USAF Phillips Laboratory, †Carleton College, $\nmid$ Johns Hopkins Hospital, §University of Nevada, IIIndiana University 
Fig. V-3. Resonant X-ray Raman spectra excited near the $\mathrm{K}$-edge of $\mathrm{Cl}_{2}$ molecules. Data are shown for both parallel (squares with dashed line) and perpendicular (circles with solid line) polarization. The vertical bars show calculated intensities for perpendicular polarization.

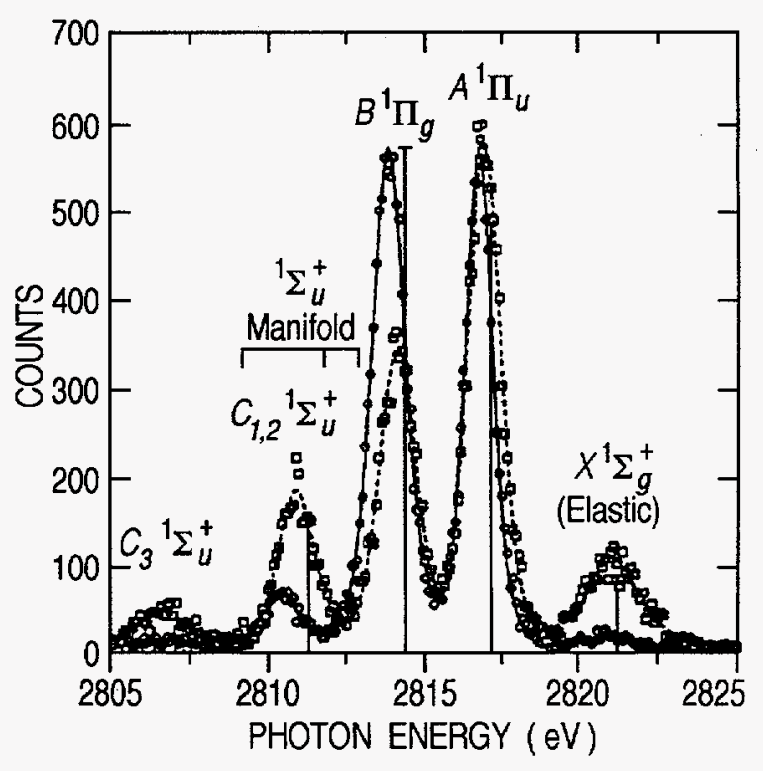

\section{d. Xenon Spectator and Diagram $\mathrm{L}_{3}-\mathrm{M}_{4,5} \mathbf{M}_{4,5}$ Auger Intensities Near the $\mathrm{L}_{3}$ Threshold (G. B. Armen,* S. H. Southworth, J. C. Levin,* U. Arp, $\dagger$ T. LeBrun, and M. A. MacDonald $\ddagger$ )}

Photoionization of an inner atomic subshell produces a highly excited vacancy state which rapidly decays radiatively (X-ray fluorescence) or non-radiatively (Auger electron emission). The photoionization and vacancy-decay can be treated essentially as independent processes (two-step model) at excitation energies far above threshold. In this case, there is negligible interaction of the photoelectron with the remaining core electrons, and the $\mathrm{X}$-ray fluorescence and Auger-electron spectra have characteristic energies, intensities, and lineshapes. However, when the excitation energy is tuned near threshold so that the photoelectron is excited to a low-energy continuum state or to a subthreshold Rydberg state, the excitation and vacancydecay steps are coupled and must be treated as a single quantum process. In that case, the vacancy-decay spectra are strongly modified and depend on the precise energy and bandwidth of the X-ray beam.

While threshold processes are expected to be well described by Åberg's time-independent resonant scattering theory, detailed experimental studies and calculations are needed to test and develop understanding of the physics. We have used electron spectroscopy combined with tunable synchrotron radiation to record the $\mathrm{Xe} \mathrm{L}_{3}-\mathrm{M}_{4,5} \mathrm{M}_{4,5}$ Auger electron spectrum as a function of excitation energy across the $\mathrm{L}_{3}$ threshold.

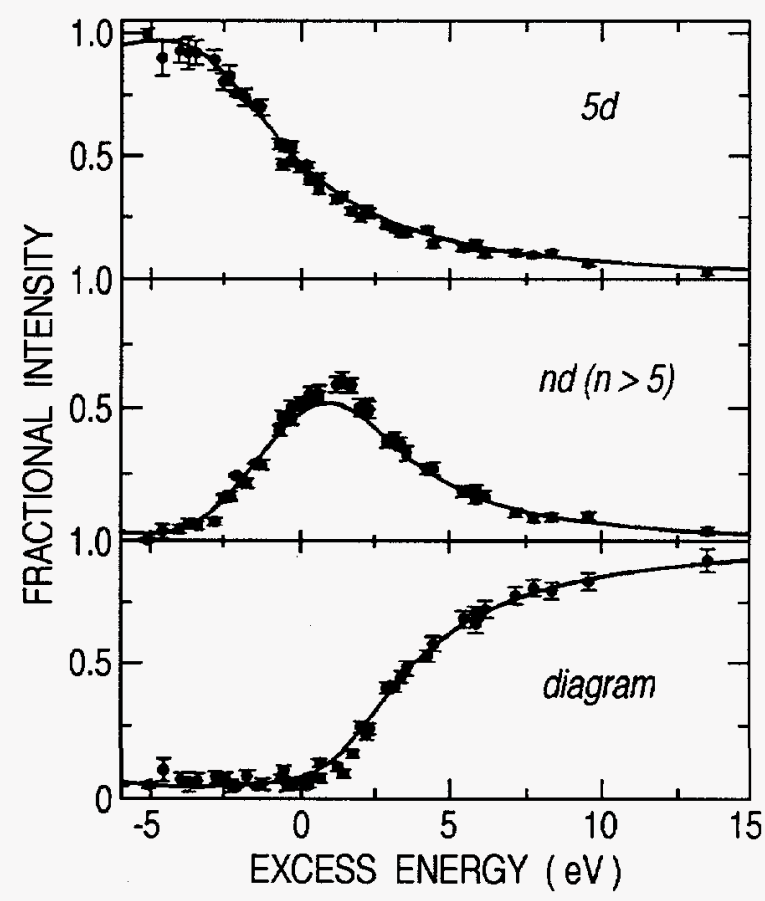

Fig. V-4. Branching ratios for producing spectator and diagram final states as a function of excess energy (relative to the $L_{3}$ ionization threshold at $4786.3 \pm 0.6 \mathrm{eV})$.

The data were analyzed in comparison with calculated spectra and cross sections based on the scattering-theory 
formalism and implemented using relaxed, nonrelativistic Hartree-Fock wavefunctions for the ground, intermediate, and final states.

One key result of this study is the dependence on excitation energy of the probabilities for producing various final states. In Figure V-4 are plotted branching ratios for $5 \mathrm{~d}$ spectators, all other nd spectators $(n>5)$, and the diagram final state. The data are in excellent agreement with the calculations and show that the diagram intensity is suppressed below and near threshold due to post-collision interaction. In a classical description of this effect, slow photoelectrons are "recaptured" into bound spectator final states due to the sudden change in screening when the fast Auger electron passes by. In the quantum-mechanical description, spectator final states are populated via continuum shakedown amplitudes which, above threshold, dominate the amplitudes due to excitation of bound intermediate states.

*University of Tennessee, $\uparrow$ National Institute of Standards and Technology, $\ddagger$ Daresbury Laboratory,

\section{e Angular Correlation Between $K \alpha$ Photons and $L_{2,3}-M_{2}, 3 M_{2}, 3$ Auger Electrons Following Argon 1s Photoionization (U. Arp, * J. W. Cooper, $\dagger$ T. LeBrun, S. H. Southworth, M. Jung, and M. A. MacDonald $\ddagger$ )}
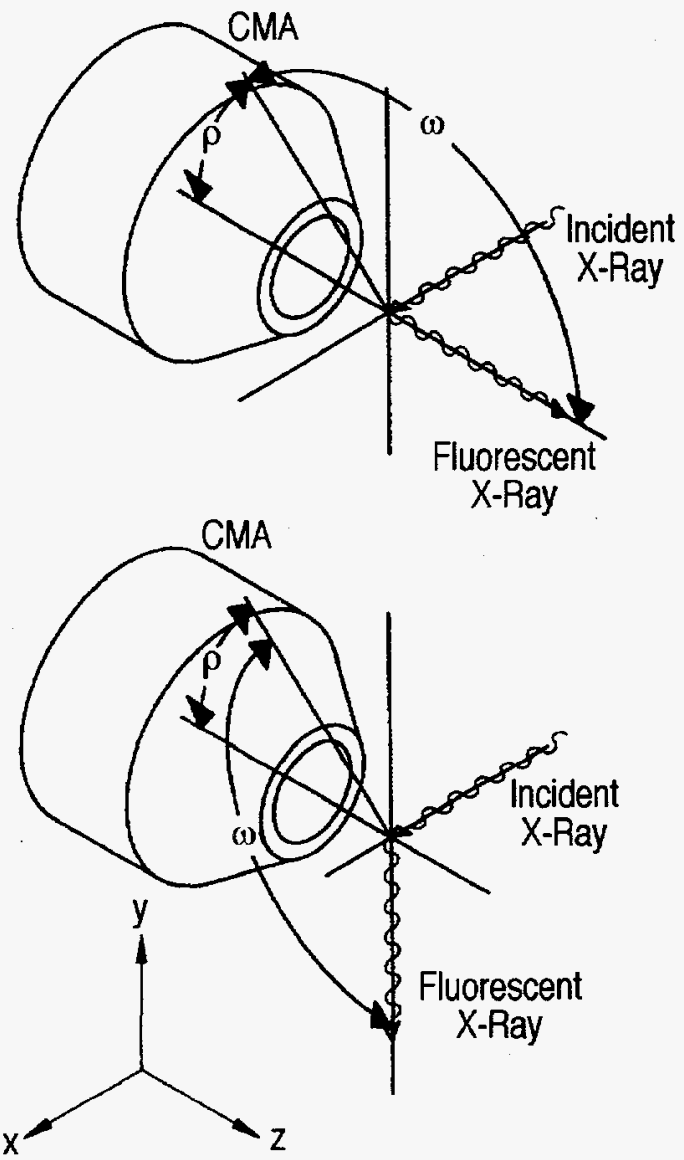

Fig. V-5. Geometry of experiments measuring electron spectra with cylindrical mirror analyzer (CMA) in coincidence with fluorescent X-rays. Two geometries are shown, with the direction of $X$ - ray detection parallel (top) or perpendicular (bottom) to the symmetry axis of the CMA. The measurements are sensitive to angular correlations between the detected electrons and X-rays.
We have used coincidence spectroscopy between $\mathrm{K} \alpha$ fluorescent $\mathrm{X}$-rays and $\mathrm{L}_{2,3}-\mathrm{M}_{2,3} \mathrm{M}_{2,3}$ Auger electrons to study vacancy cascades following Ar K-shell photoionization. In the course of these studies, we observed that the relative intensities recorded in the coincidence spectrum are modified in comparison with the normal $\mathrm{L}_{2,3}-\mathrm{M}_{2,3} \mathrm{M}_{2,3}$ electron spectrum which is well known from several previous studies. The modified intensities can be explained as an angular correlation effect in the coincidence measurements. Angular correlations are well known in nuclear cascade transitions, and the same theoretical approach, based on density matrices and statistical tensors, can be applied to atomic cascades.

The experimental geometry is shown in the top of Figure V-5. Fluorescent X-rays were detected along the direction opposite the symmetry axis of the cylindrical mirror analyzer (CMA) used to detect the Auger electrons. This geometry selects a particular angle $\omega$ between the detected X-rays and electrons, so the coincidence measurements are sensitive to their angular correlation. Preliminary measurements were also made using the geonetry shown at the bottom of Fig. V-5 to select different angles $\omega$.

In the first step of the cascade process, photoionization produces vacancies in the Ar K-shell, which cannot be aligned. However, choosing the lab-frame quantization axis to be along the direction of the detected X-rays, only transitions to the $M_{L}= \pm 1$ magnetic sublevels are observed in the $\mathrm{K} \rightarrow \mathrm{L} \mathrm{X-ray} \mathrm{emission} \mathrm{step.}$

*National Institute of Standards and Technology, †University of Maryland, ‡Daresbury Laboratory. 
Consequently, the ensemble of L3-vacancy states recorded in the coincidence spectra are aligned with $A_{20}\left(L_{3}\right)=0.5$. The alignment results in angular anisotropies of the $\mathrm{L}_{3}-\mathrm{M}_{2,3} \mathrm{M}_{2,3}$ coincidence spectra and hence the observed peak intensities depend on angle $\omega$. Including this angular correlation factor in model calculations of our coincidence spectra well explains the observed intensities. Future studies using coincidence techniques will exploit angular correlations to further understand inner-shell vacancy cascade processes.

\section{f. Electron-Recoil Ion Coincidence Study of Helium and Neon Double Photoionization at Low Photon Energies (H. Bräuning, ${ }^{*}, \dagger, \ddagger$ A. Bräuning, ${ }^{*}$ C. L. Cocke, $\dagger$ K. D. Carnes, $\dagger$ R. Dörner, ${ }^{*}$ B. Krässig, M. H. Prior $\ddagger$ P. Richard, $\dagger$ and H. Schmidt-Böcking*)}

The process of double photoionization still represents one of the great challenges for theoretical descriptions. Only recently experiments have been reported which probe double photoionization processes by recording the complete kinematical information in the three-particle breakup. The first such experiments were energy- and angle-resolved electron-electron coincidence experiments $(\alpha, 2 \mathrm{e})$. The present experiment uses the alternative approach of recording the momenta of one electron and of the recoiling ion in coincidence $(\alpha, \mathrm{eR})$. Using Cold Target Recoil Ion Momentum Spectroscopy, the latter approach is much more efficient than the former, because the recoiling ions are detected in a $4 \pi$ solid angle.

The experiment was performed at BL7 of the Advanced Light Source at Lawrence Berkeley National Laboratory. The original experimental setup used in the previous year has been upgraded with a new data acquisition system which is able to handle much higher event rates. In addition, a pair of Helmholtz coils was added with its magnetic field being lined up with the central axis of the electron detection branch. This measure enables electron detection with a $4 \pi$ solid angle for electron energies up to $20 \mathrm{eV}$.

The first goal of the 1996 experiment was to obtain supplemental data on helium double photoionization at $20 \mathrm{eV}$ excess energy. A quantitative comparison with the results from $(\alpha, 2 \mathrm{e})$ experiments requires selecting out from the total recorded $(\alpha, \mathrm{eR})$ events those which match the corresponding parameters, i.e., performing a five-dimensional cut in the parameter space. A further goal was to record first $(\alpha, \mathrm{eR})$ data on neon double and triple photoionization. The data reduction and interpretation of the results obtained in the experiment is currently in progress.

*Universität Frankfurt, Grmany, †J. R. Macdonald Lab, Kansas State Univ., †Lawrence Berkeley Nat. Lab.

\section{g . Laser-Cooled and Trapped Li Atoms (S. Hasegawa, M. Lindsay, ${ }^{*}$ Z.-T. Lu, A. Rosenberg, $\dagger$ L. Young}

The high density, low temperature, excellent spatial localization and polarization control available with laser-cooled targets makes them ideal for the detailed study of X-ray - atom interactions. Very detailed investigations of photoionization dynamics have been performed on the He atom utilizing the COLTRIMS (cold-target recoil-ion momentum spectroscopy) technique, where a $4 \pi$ ion/electron imaging system is used to record the complete kinematics of an ionization event without recourse to preselection of phase space. With a sample of laser-cooled $\mathrm{Li}$ atoms, we propose to extend such studies to the next most complicated atom. During this past year, we have made progress on two fronts: laser frequency stabilization and design/assembly of a trapping apparatus. For laser-cooling it is necessary to invoke a scheme that will lock the lasers to the appropriate transition frequency for extended periods of time. A frequency stabilization scheme for the two required laser systems (low-power diode laser for hyperfine repumping and high-power ring laser for cooling) has been designed, assembled and successfully implemented, achieving $\Delta \mathrm{E} / \mathrm{E} \approx 2 \times 10^{-10}$ for periods greater than $20 \mathrm{~min}$. A versatile vacuum apparatus for development of the cooled/trapped atoms has also been assembled and tested. We initially plan to demonstrate a vapor cell magneto-optic trap for $\mathrm{Li}$; the achievement of which would represent a vast simplification of atom trapping techniques for $\mathrm{Li}$.

*University of Louisville, †Undergraduate student, Cornell University 
h. Two-Photon Decay in H- and He-Like Nickel (R. W. Dunford, M. Jung, E. P. Kanter, C. Kurtz, B. J. Zabransky, H. W. Schäffer,* P. H. Mokler;*

A. E. Livingston, $\uparrow$ S. Cheng, and L. J. Curtis $\$$ )

In He-like ions the $2{ }^{1} \mathrm{~S}_{0}$ state can only decay to the 1 ${ }^{1} \mathrm{~S}_{0}$ ground state by the emission of two photons (2E1) because of the requirement to conserve angular momentum. In $\mathrm{H}$-like ions, the $2{ }^{2} \mathrm{~S}_{1 / 2}$ decays either by emission of two photons or by single-photon M1 emission. The two-photon process dominates up to about $Z=50$. A measurement of the exact shape of the continuous spectrum from two-photon decays tests our understanding of the entire structure of an $\mathrm{H}$ - or He-like ion since a sum over the complete set of intermediate states is required and both energy levels and wavefunctions must be understood. Fully relativistic calculations of the 2E1 decay in $\mathrm{H}$-like ions for $\mathrm{Z}$ up to

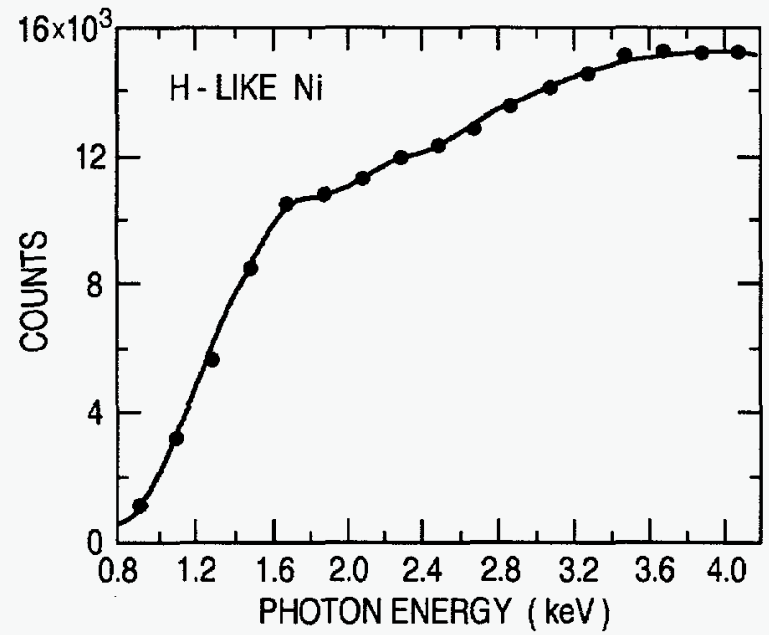

Fig. V-6. Two-photon continuum for $\mathrm{H}$-like Ni.

92 have been performed by Parpia and Johnson ${ }^{1}$ and by Goldman and Drake. ${ }^{2}$ Drake $^{3}$ calculated the two-photon decay in He-like ions and estimated the relativistic effects.

Two-photon energy distributions have been studied in $\mathrm{Kr}^{34+}$ both at the UNILAC at GSI in Germany ${ }^{4}$ and in greater detail at ATLAS. 5 The major uncertainties in the ATLAS experiment resulted from the uncertainties in the energy-dependent efficiencies of the X-ray detection system. To improve the precision of the experiment the two-photon decay in $\mathrm{H}$ - and He-like nickel was measured in comparison, switching between the two ions during the experiment. Since the continuum shape in the two-photon decay of the 2 ${ }^{2} \mathrm{~S}_{1 / 2}$ level in $\mathrm{H}$-like $\mathrm{Ni}$ is known precisely, this could serve as an on-line calibration of the spectral efficiency of the detection system used.

In the ATLAS experiment, a beam of $11.7 \mathrm{MeV} / \mathrm{u}{ }^{58} \mathrm{Ni}$ ions was stripped to either $26+$ or $28+$. With $\mathrm{Ni}^{26+}$ incident on a thin $\mathrm{C}$ target $\left(10 \mu \mathrm{g} / \mathrm{cm}^{2}\right)$, the ${ }^{1}{ }^{1} \mathrm{~S}_{0}$ level in He-like $\mathrm{Ni}$ was formed via excitation, whereas, with

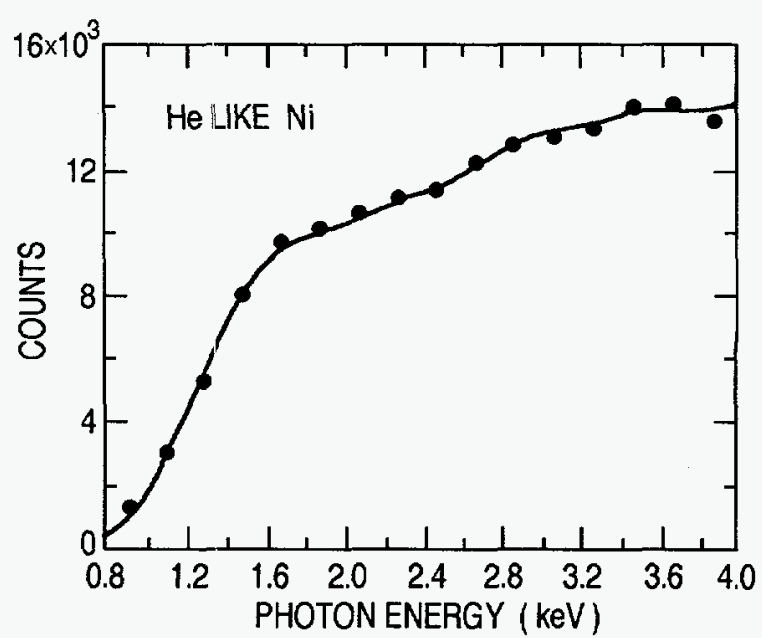

Fig. V-7. Two-photon continuum for He-like Ni.

$\mathrm{Ni}^{28+}$ incident on the target the $2^{2} \mathrm{~S}_{1 / 2}$ level in $\mathrm{H}$-like $\mathrm{Ni}$ was formed via capture. Photon coincidences associated with the decay were observed with an array of three $\mathrm{Si}(\mathrm{Li})$ detectors, each perpendicular to the beam direction. The electronic coincidence thresholds for the detectors were tuned by special circuits down to about 1 $\mathrm{keV}$ to register low-energy photons. Details of the data analysis are similar to the $\mathrm{Kr}$ experiment and are reported there .5

*GSI, Darmstadt, †University of Notre Dame, $¥$ University of Toledo

1F. A. Parpia and W. R. Johnson, Phys. Rev. A 26, 1142 (1982), ${ }^{2}$ S. P. Goldman and G. W. F. Drake, Phys. Rev. A 24, 183 (1981), ${ }^{3}$ G. W. F. Drake, Phys. Rev. A $\underline{34}, 2871$ (1986), ${ }^{4}$ P. H. Mokler, in Recombination of Atomic Ions, edited by W. G. Graham, W. Fritsch, Y. Hahn, and J. A. Tanis, NATO ASI (Plenum, New York, 1992), Vol.

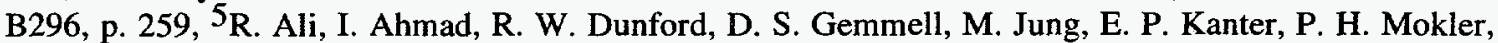

H. G. Berry, A. E. Livingston, S. Cheng, and L. J. Curtis, Phys. Rev. A 525, 994 (1997). 
A Monte Carlo calculation was developed to simulate the actual shapes of the X-ray continuum on the basis of present theories. Careful measurements were made of the geometry of the detectors and the target chamber to provide data for the program. Other input data included beam velocity, thickness of absorbing layers, electronic coincidence efficiencies, energy-dependent widths of $X$ ray lines, detector calibration constants and $\mathrm{X}$-ray mass attenuation.
Figures V-6 and V-7 show the preliminary results for the continuous $\mathrm{X}$-ray spectra of the $2 \mathrm{E} 1$ decay in $\mathrm{H}$ - and $\mathrm{He}$-like Ni. Both experimental data (circles) and the simulation (solid line) are given. The plots show an excellent concordance between the measured and calculated spectra. A more direct comparison is presently under way. For heavy systems relativistic effects will dramatically change the shapes and to study this, measurements for heavy He-like systems with $\mathrm{Z}=$ 70-92 are planned at the SIS-ESR facility at GSI in the near future.

\section{i. E1-M1 Damping Interference in the Electric Field Quenching of Metastable Ar17+ Ions (R. W. Dunford, D. S. Gemmell, M. Jung, E. P. Kanter, C. Kurtz, B. J. Zabransky, H. G. Berry, ${ }^{*}$ A. E. Livingston, ${ }^{*}$ S. Cheng, $\dagger$ and L. J. Curtis, $\dagger$ )}

The metastable $2 \mathrm{~s}^{2} \mathrm{~S}_{1 / 2}$ state in one-electron ions decays to the ground state either by two-photon decay or by single-photon M1 decay. If an external electric field $E$ is applied to the ion there is, in addition, a "Starkinduced" E1 amplitude. Interference between the E1 and M1 amplitudes leads to an asymmetry in the angular distribution proportional to the invariant $\mathbf{k} \cdot \mathbf{E}$ which is of interest because it appears to violate time reversal (T) invariance. It has been shown however, that even in a T-conserving theory this term can be nonvanishing if damping is taken into account. ${ }^{1}$ We have made the first observation of this "E1-M1 damping-interference" effect. Our results can be interpreted as determining the Lamb shift in H-like Ar.

In the experiment, a $379-\mathrm{MeV}$ beam of argon ions supplied by ATLAS was stripped in a $200 \mu \mathrm{g} / \mathrm{cm}^{2}$ foil. The 18+ charge state was magnetically selected and directed to our target chamber where it passed through a thin $\left(-10 \mu \mathrm{g} / \mathrm{cm}^{2}\right)$ foil at the chamber entrance. Some of the ions emerging from this foil were in the metastable $2{ }^{2} \mathrm{~S}_{1 / 2}$ level in $\mathrm{H}$-like Ar. These proceeded to the center of the chamber where their decay radiation was monitored by two $\mathrm{Si}(\mathrm{Li}) \mathrm{X}$-ray detectors located on opposite sides of the beam. The detectors were sensitive to photons emitted perpendicular to the beam velocity. A magnetic field $B$ was applied perpendicular to both the beam velocity and the detector axis.

Because of their motion through this field, the ions experienced an electric field $\overrightarrow{\mathrm{E}}=\gamma \frac{\overrightarrow{\mathrm{v}}}{\mathrm{c}} \times \overrightarrow{\mathrm{B}}$ in their rest frame.

The electric field was perpendicular to the beam velocity and parallel to the direction of the detected photons. In Fig. V-8 we show typical spectra taken with one of the $\mathrm{Si}(\mathrm{Li})$ detectors at several values of magnetic field. The peak near $3 \mathrm{keV}$ is a blend of three contributions: (i) the $\mathrm{H}$-like $\mathrm{M} 1$ decays, (ii) the $2 \rightarrow 1 \mathrm{H}$-like yrast cascades, (iii) decay of heliumlike $n=2$ states $(190 \mathrm{keV}$ lower in energy). The continuum below $3 \mathrm{keV}$ is mostly due to two-photon decays. Because of the Stark-induced E1 transitions, the $\mathrm{H}$-like peak becomes more intense as the magnetic field is increased. In order to measure the $\vec{E} \bullet k$ asymmetry we form the ratio :

$$
r_{1}=\frac{N_{1 \gamma}^{A}-N_{1 \gamma}^{B}}{N_{2 \gamma}^{A}+N_{2 \gamma}^{B}}
$$

\footnotetext{
*University of Notre Dame, $\uparrow$ University of Toledo

1P. J. Mohr, Phys. Rev. Lett. $\underline{40}, 854$ (1978), ${ }^{2}$ M. Hillery and P. J. Mohr, Phys. Rev. A 21 , 24 (1980),

3P. J. Mohr, in Beam-Foil Spectroscopy, edited by I. A. Sellin and D. J. Pegg (Plenum, New York, 1976), Vol. 1, p. $97,{ }^{4}$ W. R. Johnson and G. Soff, Atomic Data and Nuclear Data Tables $\underline{33}, 405$ (1985), ${ }^{5}$ H. Gould and R. Marrus, Phys. Rev. A $\underline{28}, 2001$ (1983).
} 


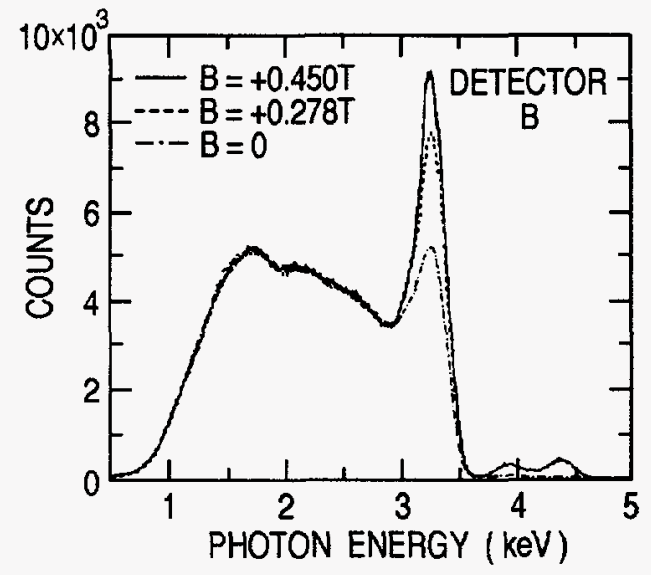

Fig. V-8. X-ray spectrum for various magnetic field strengths.

where $N_{1 \gamma}^{A, B}$ are the counts in the H-like peaks in detectors $A$ and $B$ as determined by a Gaussian-peak-fitting program. The counts $N_{2 \gamma}^{A, B}$ are from a cut on the two-photon continuum radiation which is shown below $3 \mathrm{keV}$ in Fig. V-8. The part of $r_{1}$ that changes sign when the magnetic field is reversed is called $r_{2}$.

In Fig. V-9 we show a plot of $r_{2}$ as a function of magnetic field strength. These data were fit to a straight line going through the origin with the result:

$$
\mathrm{r}_{2}=(-0.01666 \pm .00041) \times \mathrm{B}(\mathrm{kG})
$$

which agrees with the expected value based on theoretical calculation in Ref. [2].

If we interpret these data as a determination of the Lamb shift $S$ we find $S_{\exp }=38.54(0.52) \mathrm{THz}$ in agreement

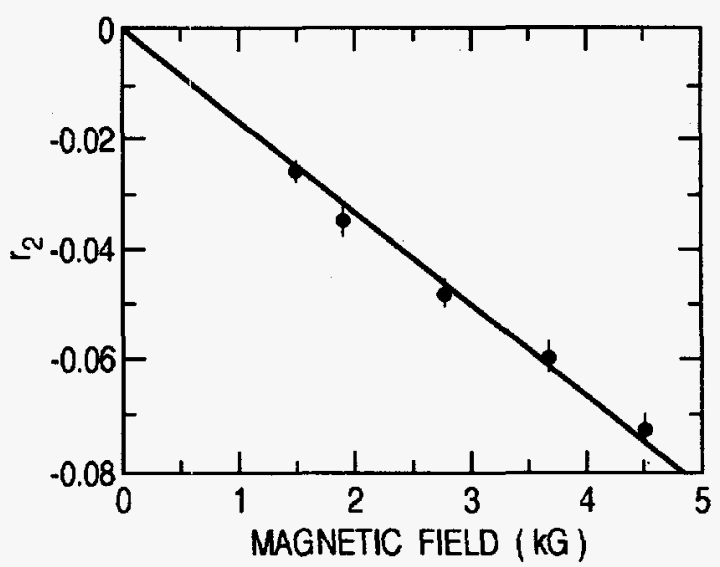

Fig. V-9. Asymmetry $r 2$ as a function of magnetic field strength.

with the theoretical result ${ }^{3,4}$ of $\mathrm{S}_{\mathrm{th}}=38.2445 \mathrm{THz}$. Our result is higher than but in substantial agreement with the experimental result of Gould and Marrus ${ }^{5}$ $\mathrm{S}_{\mathrm{GM}}=37.89(0.38) \mathrm{THz}$.

This experiment resulted in the first observation of the E1-M1 damping interference effect thus providing a test of the theory of damping in quantum mechanics. It also demonstrated a new technique for measuring the Lamb shift in highly charged ions. In the future, we will use this apparatus to measure the polarization of metastable ions which have been created in an array of tilted foils. The polarization will be determined by measuring the angular distribution of the radiation following quenching of the ions in a motional electric field. This is part of a program to develop a beam of polarized metastable ions to be used for atomic structure measurements.

\section{j. Extreme-Ultraviolet Wavelength and Lifetime Measurements in Highly- Ionized Krypton (K. W. Kukla, * A. E. Livingston,* C. M. Vogel Vogt,* H. G. Berry,* R. W. Dunford, C. A. Kurtz, L. J. Curtis, $\uparrow$ and S. Cheng $\dagger)$}

We have measured the extreme-ultraviolet (EUV) emission spectrum of highly-charged krypton ions at the atomic physics beam line of ATLAS. Beams of $610 \mathrm{MeV}$ and $800 \mathrm{MeV}{ }^{84} \mathrm{Kr}$ ions were excited using thin carbon foil targets to produce a beam of predominantly Li-like $\mathrm{Kr}^{33+}$, Be-like $\mathrm{Kr}^{32+}$, and $\mathrm{B}$-like
$\mathrm{Kr}^{31+}$ ions. The fluorescence from these 3-electron to 5-electron ions was dispersed using a 2.2-meter grazingincidence EUV grating spectrometer equipped with a microchannel-plate position-sensitive detector (PSD). The system provided high spatial (wavelength) and timing resolution. Both prompt spectra and time-of-

*University of Notre Dame, $\uparrow$ University of Toledo 
flight delayed spectra were measured by adjusting the target foil position. An auxiliary entrance slit was constructed to enable the measurement of short lifetimes, yielding lifetime results ranging in value from 10 ps to 3 ns.

Spectra of highly-ionized krypton were measured over the wavelength region $50-300=\AA$. Transitions observed included $n=3 D 2-2$ fine-structure transitions and high-n Rydberg transitions. Figure V-10 shows a sequence of time-delayed spectra for the wavelength region $130-190=\AA$, where a number of such transitions are observed in $\mathrm{Kr}^{31+} \mathrm{Kr}^{33+}$. In particular, the Li-like $2 \mathrm{~s}_{1 / 2}-2 \mathrm{p}_{1 / 2}, 3 / 2$ fine structure doublet appears prominently, and the weak Be-like $2 \mathrm{~s} 2{ }^{1} S_{0}-2 s 2 p{ }^{3} P_{1}$ intercombination transition is observed. Transition energies were measured for 17 fine-structure transitions in the $\mathrm{Kr}^{31+}-\mathrm{Kr}^{33+}$ ions with a typical fractional precision of $\sim 5 \times 10^{-4}$. Our result for the important $\mathrm{Li}$ like $2 s_{1 / 2}-2 p_{1 / 2}$ transition energy supports a tokamak plasma measurement that differs significantly from an earlier beam-foil result. Lifetimes were measured for 10 fine-structure states in $\mathrm{Kr}^{32+}$ and $\mathrm{Kr}^{33+}$. Our results show improved precision over previous measurements, and three lifetimes have been measured for the first time. Our experimental result for the Be-like $2 s 2 p{ }^{3} P_{1}$ state "forbidden" lifetime is consistent with the Zdependence of this lifetime suggested by theory and by measurements in other isoelectronic ions.

\section{SEQUENCE OF TIME - DELAYED KRYPTON SPECTRA}

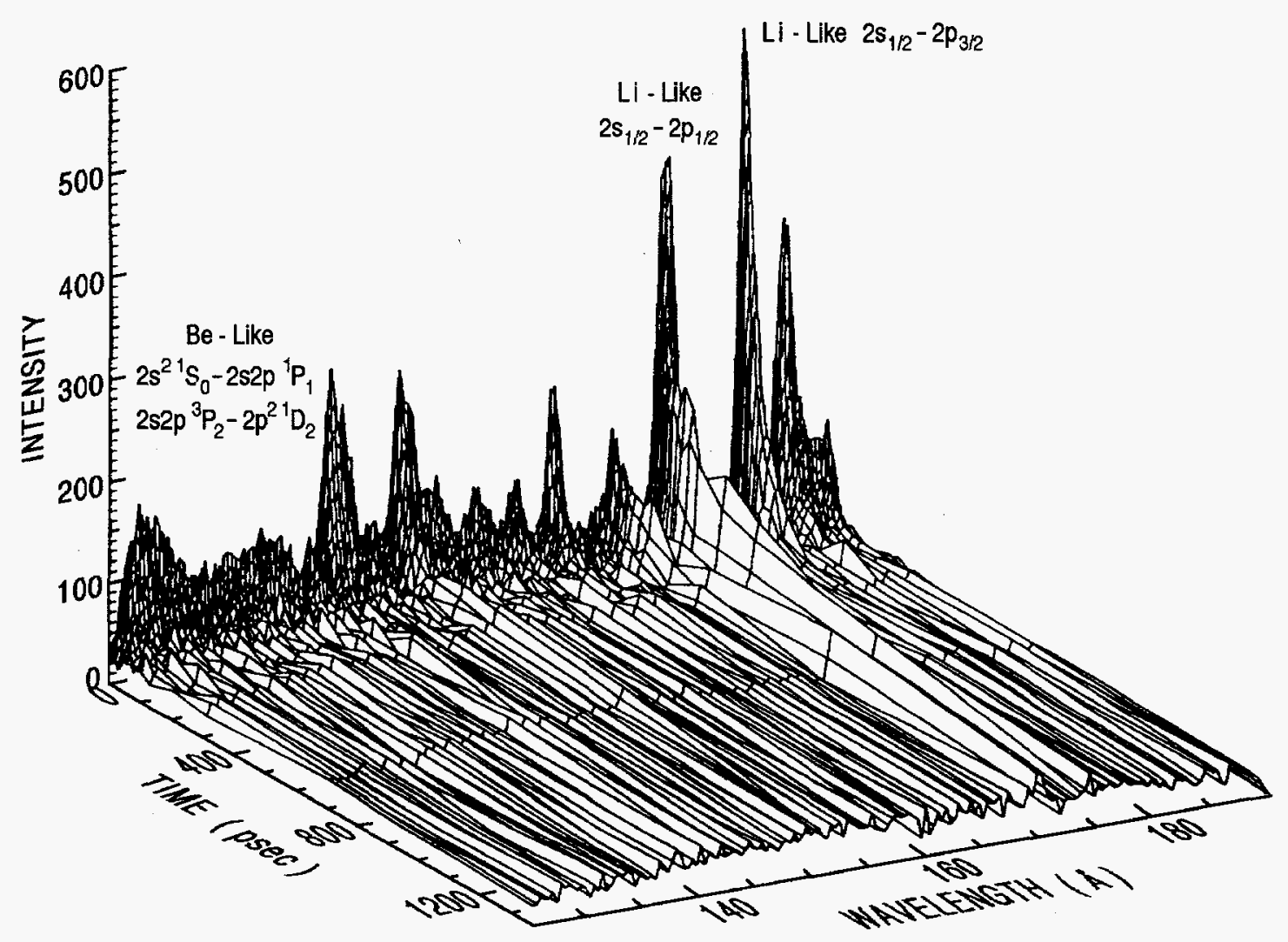

Fig. V-10. Time-delayed EUV spectra of highly-ionized $\mathrm{Kr}$. 


\section{k. The Coulomb Explosion of 173-MeV $\mathrm{HeH}^{+}$Ions Traversing Carbon Foils (M. P. Carpenter, R. W. Dunford, D. S. Gemmell, T. Graber,* R. V. F. Janssens, E. P. Kanter, J. A. Nolen, Jr, and Z. Vager $\dagger$ )}

The Coulomb explosion of $173-\mathrm{MeV} \mathrm{HeH}^{+}$molecular ions traversing thin carbon foils has been measured for foil thicknesses ranging from 2 to $200 \mu \mathrm{g} / \mathrm{cm}^{2}$. In contrast with measurements at lower energies, the energy spectra for protons observed emerging in the incident beam direction show distinct components that correspond to the partner helium ions being in charge states 0,1 , and 2 . From an analysis of the variation of the yields of these components as functions of the target thickness, we extract electron-loss cross sections that are in good agreement with theoretical estimates. "Wake effects" that increase with increasing target thickness are observed as asymmetries in the yields and energy shifts for "backward-going" as compared to "forward-going" protons.

*Present address: Advanced Photon Source, Argonne National Laboratory.

$\nmid$ Permanent address: Weizmann Institute of Science, Rehovoth, Israel. 


\section{OTHER EDUCATIONAL ACTIVITIES IN THE PHYSICS DIVISION}

\section{a. Enhancement of Minority Involvement in DOE Nuclear Physics Programs (B. Zeidman)}

Since its inception, the Minority Program in the ANL Physics Division has succeeded in attracting many highly qualified students to apply for participation in the programs of the Physics Division and other ANL divisions. The program is directed toward identification of physics departments with relatively strong programs and faculty interested in stimulating their students to pursue research, particularly in summer programs. As a result of these efforts, 15 minority physics majors received offers for participation in the ANL program for the summer of 1996. A number of former participants are currently enrolled in graduate programs in physics. In particular, one student performed his thesis research at ATLAS and is about to complete the requirements for a Ph.D. in Nuclear Physics from Florida State, while another student, pursuing a doctorate in accelerator physics, recently passed the qualifiers at MSU.

The program is an ongoing effort based upon personal interactions with a substantial number of qualified minority students and faculty during visits to the Physics Departments of HBCU colleges (Historically Black Colleges and Universities) and other institutions with large minority populations. During these visits, lectures are presented and there are discussions of activities in physics at ANL and other laboratories, graduate programs, etc. Other activities include attendance at meetings of minority organizations, appointment as Adjunct Professor at Hampton University including supervision of graduate thesis research, and serving on the Advisory Boards of the Centers of Excellence at both Hampton University and Morehouse College.

\section{b. Nuclear Physics Award for Faculty in Undergraduate Institutions (B. Zeidman)}

The goal of the 'Faculty Program', is to enhance undergraduate science education through faculty awards for minority and HBCU faculty that will allow them to directly participate in the ANL Physics Division research program and increase the number of undergraduates involved in research. The Physics Division began the program with the appointment of a Hispanic theorist who prepared and obtained independent funding for continuing research collaboration with ANL that involves several undergraduate students. Several minority faculty members and students have been involved in research collaborations with the Physics Division for the past few years and will return during the summer this year. In order to maximize the scope of the program, existing educational programs are being utilized to supplement support for some of the participants. 


\section{c. Scientific Support of SciTech Museum Exhibits and Outreach Programs (M. Peshkin)}

SciTech (Science and Technology Interactive Center) is a hands-on science museum located in Aurora, Illinois, near Argonne. With the help of volunteers and institutional support from Argonne, Fermilab, and several technological companies, SciTech has become an acknowledged leader in developing exhibits to teach modern science in a museum context. M. Peshkin and
D. Henderson of the Physics Division serve voluntarily as SciTech's Senior Scientist and as an exhibit developer, respectively, on a regular basis. Several other staff members volunteer from time to time. The Physics Division collaborates with SciTech in developing exhibits for the museum and for use in Argonne's public educational activities. These efforts involve no significant programmatic costs.

\section{c.1. Outreach Programs and Exhibit Signage (M. Peshkin)}

SciTech operates unusual outreach programs in which sets of about a dozen exhibits have been developed for lightly directed hands-on experiences by classes of fourth, fifth and sometimes sixth graders. Each set focuses on a single subject, such as light or heat. After experimenting with the exhibits on their own, the children have a group session with a SciTech "explainer". These experiences take place either in the school or at the museum. Peshkin supports this work by helping the explainers understand the science and find ways of presenting it to the children, and by helping with printed materials that the explainers prepare for the children's teachers.

Devising exhibit signage brief enough to hold the viewers' attention and detailed enough to explain concepts of modern science accurately is a challenge. Peshkin works with museum employees to try to accomplish those nearly contradictory goals. A major new exhibition whose signage is no under study includes 1) a hands-on mechanical chain reactor that models a nuclear reactor with ball bearings taking the place of neutrons, and 2) models of the elements of a power plant to convert the fission energy into electrical energy, including hands-on computer controls. A.ll of these elements were constructed in a collaboration between Argonne and SciTech involving primarily people from Argonne's nuclear engineering programs, and they will be used in the public education programs of both institutions.

\section{d. The "Museum in a School" Program (M. Peshkin and D. Henderson)}

For most of FY 1995, Peshkin has primarily served by teaching science to Scitech's "explainers" for the "Museum in a School" outreach program. In that program, groups of exhibits, each group focused on a single subject such as light, heat, or astronomy, are taken to a school for a week. Early in that week, all students in grades 4 and 5 and sometimes 6 have an opportunity to experiment with the exhibits for an hour. Later in the week, a SciTech explainer discusses the exhibits with the children. SciTech provides written materials for teachers and for children. The explainers are typically capable people with teaching backgrounds but not necessarily strong in science. Peshkin assists them by conducting discussions of the exhibits and of the science they illustrate. This activity is expected to continue in FY 1996 and probably will be supplemented by some upgrading of the exhibits on the museum floor.

\section{e. The $\mathbf{E}=\mathbf{m} \mathbf{c}^{\mathbf{2}}$ Exhibition (D. Henderson and R.V.F. Janssens)}

Two PHY staff members are active volunteers at SciTech, a hands-on science museum in Aurora, IL, and several other PHY staff also participate. SciTech develops innovative ways of bringing modern science to the public in a museum context and exports them to other museums. Recent developments include a handson $E=m c^{2}$ exhibit that will be integrated with a chainreactor exhibit developed in part by others at Argonne. 


\section{STAFF MEMBERS OF THE PHYSICS DIVISION}

Listed below are the staff of the Physics Division for the year ending December 31, 1996.

The program headings indicate only the individual's current primary activity.

\section{SCIENTIFIC STAFF \\ EXPERIMENTAL NUCLEAR PHYSICS STAFF}

\section{Regular Staff}

Irshad Ahmad, Ph.D., University of California, 1966

Birger B. Back, Ph.D., University of Copenhagen, 1974

* R. Russell Betts, Ph.D., University of Pennsylvania, 1972

Michael P. Carpenter, Ph.D., University of Tennessee, 1987

$\dagger$ Cary N. Davids, Ph.D., California Institute of Technology, 1967

‡ Stuart J. Freedman, Ph.D., University of California, 1972

Hiyan Gao, Ph.D., California Institute of Technology, 1994

Donald F. Geesaman, Ph.D., State University of N.Y., Stony Brook, 1976

Bruce G. Glagola, Ph.D., University of Maryland, 1978

$\S$ Walter F. Henning, Ph.D., Technical University of Munich, 1968

II Harold E. Jackson, Jr., Ph.D., Cornell University, 1959

\| Robert V.F. Janssens, Ph.D. Univ. Catholique de Louvain, Belgium, 1978

Cheng-lie Jiang, Ph.D. China Institute of Atomic Energy, 1960

Teng Lek Khoo, Ph.D., McMaster University, 1972

** Walter Kutschera, Ph.D., University of Graz, Austria, 1965

Torben Lauritsen, Ph.D., State University of New York, 1990

Christopher J. Lister, Ph.D., University of Liverpool, 1977

$\dagger \dagger$ Jerry A. Nolen, Jr., Ph.D., Princeton University, 1965

Thomas P. O'Neill, Ph.D., California Institute of Technology, 1994

Richard C. Pardo, Ph.D., University of Texas, 1976

David H. Potterveld, Ph.D., Caltech, 1988

Karl Ernst Rehm, Ph.D., Technical University, Munich, 1973

III John P. Schiffer, Ph.D., Yale University, 1954

Kenneth W. Shepard, Ph.D., Stanford University, 1970

Kenneth Teh, Ph.D., Vanderbilt University, 1988

Alan H. Wuosmaa, Ph.D., University of Pennsylvania, 1989

II II Benjamin Zeidman, Ph.D., Washington University, 1957

* Joint appointment with the University of Illiinois-Chicago.

$\dagger$ Adjunct Professor, Vanderbilt University.

\# On leave of absence at the University of California, Berkeley.

$\S$ Director of the Physics Division.

II Associate Director of the Physics Division.

\| Adjunct Professor, North Carolina State University.

** On leave of absence at the University of Vienna.

t† Director of the ATLAS Facility. Adjunct Professor, Michigan State University.

III Associate Director of the Physics Division. Joint appointment with the University of Chicago.

II II Adjunct Professor, Hampton University. 


\section{Special Appointments}

Lowell M. Bollinger, Ph.D., Cornell University, 1951

Melvin S. Freedman, Ph.D., University of Chicago, 1942

Sheldon B. Kaufman, Ph.D., University of Chicago, 1953

Alexander Langsdorf, Jr., Ph.D., Massachusetts Inst. of Technology, 1937

* Michael Paul, Ph.D., Hebrew University of Jerusalem, 1973

G. Roy Ringo, Ph.D., University of Chicago, 1940

Ralph E. Segel, Ph.D., Johns Hopkins University, 1955

George E. Thomas, B.A., Illinois Wesleyan, 1943

$\dagger$ Jan L. Yntema, Ph.D., Free University of Amsterdam, 1952

\section{Term Appointments}

William Cummings, Ph.D., Stanford University, 1992

William Sellyey, Ph.D. Bryn Mawr College, 1974

\section{THEORETICAL NUCLEAR PHYSICS STAFF}

\section{Regular Staff}

Richard R. Chasman, Ph.D., University of California, 1959

Fritz Coester, Ph.D., University of Zurich, 1944

Henning Esbensen, Ph.D., University of Aarhus, 1977

‡ Tsung-Shung Harry Lee, Ph.D., University of Pittsburgh, 1973

Steven C. Pieper, Ph.D., University of Illinois, 1970

Craig T. Roberts, Ph.D., Flinders University of South Australia, 1989

Robert B. Wiringa, Ph.D., University of Illinois, 1978

\section{Special Appointments}

Paul Benioff, Ph.D., University of California, 1959

Arnold R. Bodmer, Ph.D., Manchester University, 1953

Mitio Inokuti, Ph.D., University of Tokyo, 1962

Dieter Kurath, Ph.D., University of Chicago, 1951

Robert D. Lawson, Ph.D. Stanford University, 1953

Harry J. Lipkin, Ph.D., Princeton University, 1950

†† Vijay Pandharipande, Ph.D., University of Bombay, 1969

Murray Peshkin, Ph.D., Cornell University, 1951

* Special Term Appointee from the Hebrew University of Jerusalem, Israel.

$\uparrow$ Deceased December 1996.

¥ Adjunct Professor, University of Pittsburgh.

t† Special Term Appointee from the University of Illinois, Urbana. 
ATOMIC AND MOLECULAR PHYSICS STAFF

\section{Regular Staff}

Robert Dunford, Ph.D., University of Michigan, 1978

Donald S. Gemmell, Ph.D., Australian National University, 1960

Elliot P. Kanter, Ph.D., Rutgers University, 1977

* Bertold Kraessig, Ph.D., University of Freiburg, 1994

Stephen H. Southworth, Ph.D., University of California, Berkeley, 1982

$\dagger$ Zeev Vager, Ph.D. Weizmann Institute of Science, 1962

Linda Young, Ph.D., University of California, Berkeley, 1981

\section{Special Appointments}

William J. Childs, Ph.D., University of Michigan, 1956

F. Paul Mooring, Ph.D., University of Wisconsin, 1951

Gilbert J. Perlow, Ph.D., University of Chicago, 1940

\section{Term Appointment}

Thomas LeBrun, Ph.D. University of Paris, 1991

\section{TEMPORARY APPOINTMENTS}

\section{Postdoctoral Appointees}

Dieter Ackermann (from Laboratorie Nazionale di Legnaro, Italy): Heavy-ion research at ATLAS.

(June 1995--December 1996)

Axel Bender (University of Tubingen, Germany):

Nuclear theory studies.

(January 1995--September 1996)

Daniel Blumenthal (University of Pennsylvania, Philadelphia, Pennsylvania): Heavy-ion research at ATLAS.

(January 1994--June 1996)

Patrick Decrock (from K. U., Leuven, Belgium): Accelerator development.

(January 1996--

James Fedchak (from College of William and Mary, Williamsburg, Virginia): Medium-energy physics.

(October 1994--

Susan Fischer (from University of Notre Dame, Notre Dame, Indiana): Heavy-ion research at ATLAS.

(January 1995--

Gregory Hackman (from McMaster University, Hamilton, Ontaro, Canada): Heavy-ion research at ATLAS. (September 1995-_ )

* Postdoctoral Appointee until June 1996.

$\dagger$ Joint Appointment with Weizmann Institute of Science, Rehovot, Israel. 
Jens-Ole Hansen (from Massachusetts Inst. of Technology, Cambridge, Massachusetts): Medium-energy studies.

(October 1994--

Shuichi Hasegawa (from University of Tokyo, Japan):

Atomic and molecular physics studies.

(Septeber 1996--

David J. Hofman (from SUNY, Stony Brook, New York):

Heavy-ion research at ATLAS.

(August 1994--

Martin Jung (from GSI, Darmstadt, Germany):

Atomic physics research.

(December 1994--December 1996)

* Bertold Kraessig (from University of Freiburg, Germany):

Atomic physics with synchrotron light sources.

(March 1995--June 1996)

Naomi Makins (from Massachusetts Inst. of Technology, Cambridge, Massachusetts):

Medium energy physics.

(July 1994--August 1996)

Pieter Maris (from University of Groningen, The Netherlands):

Theoretical studies.

(September 1996--

Vandana Nanal (from Tata Institute, Bombay, India):

Heavy-ion research at ATLAS.

(August 1995--

David Nisius (from Purdue University, W. Lafayettte, Indiana):

Heavy-ion research at ATLAS.

(November 1996-- )

$\dagger$ Jorg Reinhold (from Technical University of Munich, Germany):

Medium-energy research.

(September 1995--

Peter Reiter (from University of Heidelberg, Germany):

Heavy-ion research at ATLAS.

(August 1996--

Michael Schlapp (from University of Giessen, Germariy):

Accelerator development.

(October 1995--

‡ Dariusz Seweryniak (from University of Uppsala, Sweden):

Heavy-ion research at ATLAS.

(January 1995--

Lorenz von Smekal (from University of Tubingen, Germany):

Theoretical studies.

(November 1995--

* Joined the regular staff in June 1996.

$\dagger$ Feodor-Lynen Postdoctoral Fellowship from Alexander von Humboldt Foundation.

$\$$ Joint appointment with the University of Maryland. 


\section{TECHNICAL AND ENGINEERING STAFF}

(and areas of activity)

Kevin G. Bailey (B.S. University of Nebraska, 1989). SOS construction for CEBAF.

Brian T. Batzka (B.S. University of Houston, 1992). ATLAS operator.

Amanda Beardsley (B.S. University of Iowa, 1996). ATLAS control system.

Peter J. Billquist ECR heavy-ion source, ATLAS operation.

John M. Bogaty (A.A.S. DeVry, 1961).

Electrical systems, ATLAS operation and development.

Benny E. Clifft (A.S.E.E., DeVry, 1959).

Electrical systems, ATLAS operation and development.

Joseph Falout (B.S.M.E. University of Illinois, 1970).

Experimental equipment design.

John P. Greene (M.S. DePaul University, 1982).

Target preparation.

Ray E. Harden (A.A.S. Milwaukee School of Engineering, 1957). ATLAS operator

* Dale J. Henderson (B.S. Elmhurst College, 1951).

Detector development, technical assistance, heavy-ion physics.

James M. Joswick (A.A.S. Milwaukee School of Engineering, 1964).

ATLAS experimental equipment maintenance, technical assistance, heavy-ion physics.

* Raymond B. Kickert

ATLAS experimental equipment maintenance, technical assistance, heavy-ion physics.

Robert Kowalczyk (M.S. Northeastern Illinois University, 1983).

Technical assistance, medium-energy physics.

Charles A. Kurtz (M.S. University of Arkansas, 1984).

Technical assistance, atomic physics.

* Paul Markovich (B.S. Purdue University, 1972).

Surface chemistry, ATLAS development and operation.

Thomas P. Mullen (B.S. Marquette University, 1966).

Division ESH/QA engineer.

Floyd Munson, Jr. (A.A.S. DeVry, 1966, B.S. Lewis University, 1993). Control system for ATLAS.

$\dagger$ Kirt Nakagawa (B.S., University of Illinois-Chicago, 1988). ATLAS operations

* Special Term Appointee.

$\dagger$ Terminated in June 1996 
Bruce G. Nardi (A.A.S. Morton Jr. College, 1967; A.A.S. DeVry, 1969).

Electronics design and maintenance.

Anne Reuter (B.S. University of Rochester, 1993).

Operate, upgrade and maintain ATLAS cryogenic system.

James R. Specht (A.A.S. DeVry, 1964).

Cryogenics engineer. ATLAS development and operation.

Philip Strickhorn (B.S. DeVry, 1990).

Electrical and technical assistance with ATLAS operations.

* Brian J. Tieman (B.A. North Central College, 1992).

ATLAS operations.

Ian R. Tilbrook (B.S. Pennsylvania State University, 1987; MBA, Keller Graduate School of Management, 1994).

ATLAS operator.

Richard Vondrasek (B.S. University of Illinois, 1990).

ATLAS operator.

Philip R. Wilt (Johnstown Technical School 1973).

Electronics design and maintenance.

$\dagger$ Bruce J. Zabransky (M.S. University of Illinois, Chicago, 1973). Dynamitron operation.

Anthony R. Zeuli (B.A. Hamline University, 1990).

Technical assistance. Medium-energy physics.

Gary P. Zinkann (B.S. DeVry, 1975).

ATLAS operations supervisor.

\section{ADMINISTRATIVE STAFF}

$\ddagger$ Allan Bernstein, M.B.A., Rosary College, 1986

$\$$ James E. Nelson, B.A., University of Illinois, 1975

$\S$ Karen J. Thayer

\section{VISITORS AND STUDENTS}

Long-Term Visitors (at Argonne more than 4 months)

Francesca Borasi (University of Milan, Italy):

Radioactive ion-beam research.

(December 1996--

Rudolf Ganz (University of Heidelberg):

Heavy-ion research at ATLAS.

(February 1996--

* Transferred to Advanced Photon Source Division in May 1996.

$\dagger$ In charge of Dynamitron operations.

$\neq$ Assistant Director of the Physics Division.

$\S$ Staff Assistant. 
Subhendu Ghosh (Nuclear Science Centre, New Delhi, India):

ATLAS development.

(March--September 1996)

Albrecht Gillitzer (Technical University, Munich, Germany):

Heavy-ion research at ATLAS.

(August 1996--

Jeffrey Hangst (University of Aarhus, Denmark):

Heavy-ion research at ATLAS.

(April 1995--

Boris Harss (Technical University of Munich, Germany):

Heavy-ion research at ATLAS.

(April 1996--March 1997)

* Donald McLeod (University of Illinois, Chicago, Illinois):

Heavy-ion research at ATLAS.

(June 1994--

* Nora Mansour (Western Michigan University, Kalamazoo, Michigan):

Atomic physics research.

(October 1991-- )

Rajeev Mehta (Nuclear Science Centre, New Delhi, India):

ATLAS development.

(February --May 1996)

Prakash Potukuchi (Nuclear Science Centre, New Delhi, India):

ATLAS development.

(March 1996--

* Carol Tanner (University of Notre Dame, Indiana):

Atomic physics at BLASE.

(March 1992--

* Guangsheng Xu (Yale University, New Haven, Connecticut):

Heavy-ion research at ATLAS.

(January 1994--

Short-Term Visitors (at ANL less than 4 months)

Gvriol Goldring (Weizmann Institute, Rehovot, Israel):

Theoretical studies.

(June-August 1996)

Shingo Hamada (Kyoto University, Kyoto, Japan):

Heavy-ion research at ATLAS.

(August-October 1996)

Edward Hohman (York Township High School, Lyons, Illinois):

Summer student coordinator.

(June-August 1996)

* Guest Faculty Research Participant. 
Yori Kalinovsky (Bogoliubov Laboratory of Theoretical Physics, Dubna, Russia): Theoretical studies.

(August-September 1996)

Miloslave M. Michailova (Bulgarian Academy of Sciences, Sofia, Bulgaria):

Theoretical physics studies.

(December 1995--March 1996)

* Robert Perry (Talladega College, Talladega, Alabama)

Atomic physics studies.

(June-August 1996)

\section{Resident Graduate Students}

Hanan Amro (North Carolina State University, Raleigh, North Carolina):

Heavy-ion Research at ATLAS.

(January 1995-- )

Larry Todd Brown (Vanderbilt University, Nashville, Tennessee):

FMA development at ATLAS

(January 1994--

Isaac Chappell (Massachusetts Institute of Technology, Cambridge, Massachusetts):

Theoretical physics studies.

(May-August 1996)

Christopher Conner (University of Illinois-Chicago):

Heavy-ion research at ATLAS.

(June 1994--

Louis Conticchio (University of Maryland, College Park, Maryland):

Heavy-ion research at ATLAS.

(October 1994-- )

John C. Gehring (University of Chicago, Chicago, Illiniois):

Heavy-ion research at ATLAS.

(June 1990--April 1996)

$\dagger$ David Nisius (Purdue University, W. Lafayette, Indiana):

Heavy-ion research at ATLAS.

(June 1993 --November 1996)

Michael Pichowsky (University of Pittsburgh, Pennsylvania):

Theoretical physics studies.

(September 1993--November 1996)

Mauricio Portillo (Michigan State University, E. Lansing, Michigan):

Accelerator development.

(September 1995-- )

Dante Roa (Florida State University):

Research with APEX at ATLAS.

(May 1992--September 1996)

* Faculty Research Participant.

$\dagger$ Postdoctoral Appointee as of November 1996. 
Jazmin Schwartz (Yale University, New Haven, Connecticut):

Heavy-ion research at ATLAS. (May 1995--

\section{Guest Graduate Students}

Konstantin Akimov (University of Illinois, Chicago, Illinois): Heavy-ion research at ATLAS.

(May 1994--May 1996)

Dipangkar Dutta (Northwestern University, Evanston, Illinois): Medium-energy studies. (June 1994-- )

Robert Rafac (University of Notre Dame, Notre Dame, Indiana): Atomic physics research. (March 1992--March 1996)

\section{Undergraduate Students}

Susama Agarwala (Massachusetts Institute of Technology)

Pamela Arndt (College of St. Francis)

Teresa Barlow (Illinois Benedictine College)

Joseph Berger (Fachhochschule Munchen, Germany)

Joseph Butscher (Lewis University)

Daniel Braithwaite (University of Chicago)

Peter Cabauy (Florida International University)

Ivo Calderin (Florida International University)

George Chancellor (North Central College)

Andrew Geraci (University of Chicago)

Jerome Gura (College of St. Francis)

Paul Kawka (University of Illinois-Urbana)

Steven Kramer (Lewis University)

Henreida Madrigal (University of Illinois-Chicago)

Jaime Morales (University of Texas-El Paso)

Brian Napolitano (College of DuPage)

Jennifer Nemanich (North Central College)

Kara Perry (College of St. Francis)

Adam Rosenberg (Cornell University)

David Sagan (DeVry Institute of Technology)

Paul Santucci (Benedictine University)

Max Schoenberg (University of Illinois-Urbana)

Jeffrey Seldon (New Mexico State University)

Daniel Sheeler (University of Chicago)

Joseph Snider (University of Chicago)

John Szezech (University of Illinois-Chicago)

Jared Torres (Loyola University)

Patricia Vahle (New Mexico State University)

James Young (Florida State University)

Mark Wolanski (University of Chicago

Pre-College Program (Just Graduated from High School)

(June--August 1995)

Randall Gasa (Oak Park/River Forest High School)

YuMi Kim (Hinsdale South High School)

Maria Martinez (Joliet Central High School) 


\section{PUBLICATIONS DURING 1996}

(The arrangement follows approximately the Table of Contents)

\section{HEAVY-ION NUCLEAR PHYSICS RESEARCH}

High-K Isomers in ${ }^{176} \mathrm{~W}$ and Mechanisms of $\mathrm{K}$ Violation

B. Crowell, P. Chowdhury, D. J. Blumenthal, S. J. Freeman, C. J. Lister, M. P. Carpenter,

R. G. Henry, R. V. F. Janssens, T. L. Khoo, T. Lauritsen, Y. Liang, F. Soramel, and

I. G. Bearden

Phys. Rev. C $\underline{53}$, 1173-1190 (1996)

Prompt $\gamma$-ray Spectroscopy of the ${ }^{104} \mathrm{Mo}$ and ${ }^{108} \mathrm{Mo}$ Fission Fragments

A. Guessous, N. Schulz, M. Bentaleb, E. Lubkiewicz, J. L. Durell, C. J. Pearson,

W. R. Phillips, J. A. Shannon, W. Urban, B. J. Varley, I. Ahmad, C. J. Lister, L. R. Morss,

K. L. Nash, C. W. Williams, and S. Khazrouni

Phys. Rev. C 53, 1191-1196 (1996)

Entrance-Channel Effects in Quasi-Fission Reactions

B. B. Back, P. B. Fernandez, B. G. Glagola, D. Henderson, S. Kaufman, J. G. Keller,

S. J. Sanders, F. Videbaek, T. F. Wang, and B. D. Wilkins

Phys. Rev. C 53, 1734-1744 (1996)

Astrophysical Reaction Rate for the ${ }^{18} \mathrm{~F}(\mathrm{p}, \alpha)^{15} \mathrm{O}$ Reaction

K. E. Rehm, M. Paul, A. D. Roberts, C. L. Jiang, D. J. Blumenthal, S. M. Fischer,

J. Gehring, D. Henderson, J. Nickles, J. Nolen, R. C. Pardo, J. P. Schiffer, and R. E. Segel Phys. Rev. C 53, 1950-1954 (1996)

Alignment Additivity in the Two-Quasiparticle Superdeformed Bands of ${ }^{192} \mathrm{Tl}$

S. M. Fischer, M. P. Carpenter, R. V. F. Janssens, B. Crowell, I. Ahmad, D. J. Blumenthal,

T. L. Khoo, T. Lauritsen, D. Nisius, W. Reviol, W. F. Mueller, L. L. Riedinger,

B. H. Smith, and B. Cederwall

Phy. Rev. C 53, 2126-2133 (1996)

$\alpha$-Decay Properties of ${ }^{181} \mathrm{~Pb}$

K. S. Toth, J. C. Batchelder, C. R. Bingham, L. F. Conticchio, W. B. Walters,

C. N. Davids, D. J. Henderson, R. Hermann, H. Penttila, J. D. Richards, A. H. Wuosmaa, and B. E. Zimmerman

Phys. Rev. C $\underline{53}$, 2513-2515 (1996)

Collective and Quasiparticle Excitations in Superdeformed ${ }^{190} \mathrm{Hg}$

A. N. Wilson, J. Timar, J. F. Sharpey-Schafer, B. Crowell, M. P. Carpenter,

R. V. F. Janssens, D. J. Blumenthal, I. Ahmad, A. Astier, F. Azaiez, M. Bergstom,

L. Ducroux, B. J. P. Gall, F. Hannachi, T. L. Khoo, A. Korichi, T. Lauritsen, A. Lopez-

Martens, M. Meyer, D. Nisius, E. S. Paul, M. G. Porquet, N. Redon, J. N. Wilson, and

T. Nakatsukasa

Phy. Rev. C $\underline{54}, 559-567$ (1996)

Octupole Correlations in Neutron Rich, Odd-A Lanthanum Nuclei

W. Urban, W. R. Phillips, J. L. Durell, M. A. Jones, M. Leddy, C. J. Pearson, A. G. Smith,

B. J. Varley, I. Ahmad, L. R. Morss, M. Bentaleb, E. Lubkiewicz, and N. Schulz

Phy. Rev. C 54, 945-948 (1996) 
Fission Decay of ${ }^{48} \mathrm{Cr}$ at $\mathrm{E}_{\mathrm{CN}}^{*} \approx 60 \mathrm{MeV}$

K. A. Farrar, S. J. Sanders, A. K. Dummer, A. T. Hasan, F. W. Prosser, B. B. Back,

I. G. Bearden, R. R. Betts, M. P. Carpenter, B. Crowell, M. Freer, D. J. Henderson,

R. V. F. Janssens, T. L. Khoo, T. Lauritsen, Y. Liang, D. Nisius, A. H. Wuosmaa, C. Beck,

R. M. Freeman, Sl. Cavallaro, and A. Szanto de Toledo

Phys. Rev. C $\underline{54}, 1249-1261$ (1996)

Quasielastic Scattering of ${ }^{9} \mathrm{Li}$ on ${ }^{12} \mathrm{C}$

M. Zahar, M. Belbot, J. J. Kolata, K. Lamkin, D. J. Morrissey, B. M. Sherrill,

M. Lewitowicz, A. H. Wuosmaa, J. S. Al-Khalili, J. A. Tostevin, and I. J. Thompson

Phys. Rev. C 54, 1262-1266 (1996)

Resonance Spin Assignments in ${ }^{12} \mathrm{C}+{ }^{12} \mathrm{C}\left(3^{-}\right)$Inelastic Scattering from Angular Correlation Methods

A. H. Wuosmaa, B. B. Back, R. R. Betts, M. Freer, B. G. Glagola, D. J. Henderson,

D. J. Hofman, and V. Nanal

Phys. Rev. C 54, 2463-2468 (1996)

$\gamma$ Decay from States at Low Excitation Energy in the Neutron-Deficient Isotope, ${ }^{200} \mathrm{Rn}$, Identified by Correlated Radioactive Decay

R. B. E. Taylor, S. J. Freeman, J. L. Durell, M. J. Leddy, A. G. Smith, D. J. Blumenthal,

M. P. Carpenter, C. N. Davids, C. J. Lister, R.V. F. Janssens, and D. Seweryniak Phys. Rev. C $\underline{54}$, 2926-2934 (1996)

Identification of ${ }^{166} \mathrm{Pt}$ and ${ }^{167} \mathrm{Pt}$

C. R. Bingham, K. S. Toth, J. C. Batchelder, D. J. Blumenthal, L. T. Brown, . C. Busse,

L. F. Conticchio, C. N. Davids, T. Davinson, D. J. Henderson, R. J. Irvine, D. Seweryniak,

W. B. Walters, P. J. Woods, and B. E. Zimmerman

Phys. Rev. C 54, R20-R23 (1996)

Test of $\Delta \mathrm{I}=2$ Staggering in the Superdeformed Bands of ${ }^{194} \mathrm{Hg}$

R. Krucken, G. Hackman, M. A. Deleplanque, R. V. F. Janssens, I. Y. Lee, D. Ackermann,

I. Ahmad, H. Amro, S. Asztalos, D. J. Blumenthal, M. P. Carpenter, R. M. Clark,

R. M. Diamond, P. Fallon, S. M. Fischer, B. Herskind, T. L. Khoo, T. Lauritsen,

A. O. Macchiavelli, R. W. MacLeod, D. Nisius, G. J Schnoid, F. S. Stephens, and K. Vetter

Phy. Rev. C 54, R2109-R2113 (1996)

Superdeformed Band in 155Dy: Where Does the "Island" of Superdeformation End?

S. M. Fischer, R. V. F. Janssens, M. A. Riley, R. R. Chasman, I. Ahmad, D. J. Blumenthal,

T. B. Brown, M. P. Carpenter, G. Hackman, D. J. Hartley, T. L. Khoo, T. Lauritsen,

W. C. Ma, D. Nisius, J. Simpson, and P. G. Varmette

Phys. Rev. C 54, R2806-R2810 (1996)

Structure of Superdeformed Bands in ${ }^{195} \mathrm{Hg}$

G. Hackman, R. Kruecken, R.V.F. Janssens, M. A. Deleplanque, M. P. Carpenter,

D. Ackermann, I. Ahmad, H. Amro, S. Asztalos, D. J. Blumenthal, R. M. Clark,

R. M. Diamond, P. Fallon, S. M. Fischer, B. Herskind, T. L. Khoo, T. Lauritsen, I. Y. Lee,

R. W. MacLeod, A. O. Macchiavelli, D. Nisius, G. J. Schmid, F. S. Stephens, K. Vetter, and R. Wyss

Phys. Rev. C 도, 148-154 (1997) 
Normal Modes of Cold Confined One-Component Plasmas

Daniel H. E. Dubin and J. P. Schiffer

Phys. Rev. E $\underline{53}$, 5249-5267 (1996)

Proton Decay of an Intruder State in ${ }^{185} \mathrm{Bi}$

C. N. Davids, P. J. Woods, H. T. Penttila, J. C. Batchelder, C. R. Bingham,

D. J. Blumenthal, L. T. Brown, B. C. Busse, L. F. Conticchio, T. Davinson,

D. J. Henderson, R. J. Irvine, D. Seweryniak, K. S. Toth, W. B. Walters,

and B. E. Zimmerman

Phys. Rev. Lett. 76, 592-595 (1996)

Excitation Energies and Spins of a Superdeformed Band in ${ }^{194} \mathrm{Hg}$ from One-Step Discrete Decays to the Yrast Line

T. L. Khoo, M. P. Carpenter, T. Lauritsen, D. Ackermann, I. Ahmad, D. J. Blumenthal,

S. M. Fischer, R. V. F. Janssens, D. Nisius, E. F. Moore, A. Lopez-Martens, F. Hannachi,

T. Dossing, R. Kruecken, S. J. Asztalos, J. A. Becker, L. Bernstein, R. M. Clark,

M. A. Deleplanque, R. M. Diamond, P. Fallon, L. P. Farris, F. Hannachi, E. A. Henry,

A. Korichi, I. Y. Lee, A. O. Macchiavelli, and F. S. Stephens

Phys. Rev. Lett. 76, 1583-1586 (1996)

Pinning Strength Dependence of Mixed-State Hall Effect in $\mathrm{YBa}_{2} \mathrm{Cu}_{3} \mathrm{O}_{7}$ Crystals with Columnar Defects

W. N. Kang, D. H. Kim, S. Y. Shim, J. H. Park, T. S. Hahn, S. S. Choi, W. C. Lee,

J. D. Hettinger, K. E. Gray, and B. Glagola

Phys. Rev. Lett. 76, 2993-2996 (1996)

Fragmentation of the Decay from the Superdeformed Yrast Band in ${ }^{192} \mathrm{Hg}$

A. Lopez-Martens, F. Hannachi, T. Dossing, C. Schuck, R. Collatz, E. Gueorguieva,

Ch. Vieu, S. Leoni, B. Herskind, T. L. Khoo, T. Lauritsen, I. Ahmad, D. J. Blumenthal,

M. P. Carpenter, D. Gassmann, R. V. F. Janssens, D. Nisius, A. Korichi, C. Bourgeois,

A. Astier, L. Ducroux, Y. LeCoz, M. Meyer, N. Redon, J. F. Sharpey-Schafer,

A. N. Wilson, W. Korten, A. Bracco, and R. Lucas

Phys. Rev. Lett. 77, 1707-1710 (1996)

Spin-Dependent Triaxial Deformation in Neutron-Rich Mo Isotopes

A. G. Smith, J. L. Durell, W. R. Phillips, M. A. Jones, M. Leddy, W. Urban, B. J. Varley,

I. Ahmad, L. R. Morss, M. Bentaleb, A. Guessous, E. Lubkiewicz, N. Schulz, and R. Wyss

Phys. Rev. Lett. 77, 1711-1714 (1996)

Reply to "Comment on the APEX $\mathrm{e}^{+} \mathrm{e}^{-}$Experiment"

I. Ahmad, S. M. Austin, B. B. Back, R. R. Betts, F. P. Calaprice, K. C. Chan, A. Chishti,

P. Chowdhury, C. Conner, R. W. Dunford, J. D. Fox, S. J. Freedman, M. Freer,

S. B. Gazes, A. L. Hallin, T. Happ, D. Henderson, N. I. Kaloskamis, E. Kashy,

W. Kutschera, J. Last, C. J. Lister, M. Liu, M. R. Maier, D. J. Mercer, D. Mikolas,

P. A. A. Perera, M. D. Rhein, D. E. Roa, J. P. Schiffer, T. A. Trainor, P. Wilt,

J. S. Winfield, M. Wolanski, F. L. H. Wolfs, A. H. Wuosmaa, A. Young, and J. E. Yurkon

Phys. Rev. Lett. 77, 2838-2839 (1996) 
Yrast Excitations Around Doubly Magic ${ }^{132} \mathrm{Sn}$ from Fission Product $\boldsymbol{\gamma}$-Ray Studies

C. T. Zhang, P. Bhattacharyya, P. J. Daly, R. Broda, Z. W. Grabowski, D. Nisius,

I. Ahmad, T. Ishii, M. P. Carpenter, L. R. Morss, W. R. Phillips, J. L. Durell, M. J. Leddy, A. G. Smith, W. Urban, B. J. Varley, N. Schulz, E. Lubkiewicz, M. Bentaleb, and J. Blomqvist

Phys. Rev. Lett. 77, 3743-3746 (1996)

Search for Mono-Energetic Positron Emission from Heavy-Ion Collisions at Coulomb-Barrier Energies

I. Ahmad, S. M. Austin, B. B. Back. R. R. Betts, F. P. Calaprice, K. C. Chan, A. Chishti,

P. Chowdhury, C. Conner, R. W. Dunford, J. D. Fox, S. J. Freedman, M. Freer,

S. B. Gazes, A. L. Hallin, T. Happ, D. Henderson, N. I. Kaloskamis, E. Kashy,

W. Kutschera, J. Last, C. J. Lister, M. Liu, M. R. Maier, D. J. Mercer, D. Mikolas,

P.A.A. Perera, M. D. Rhein, D. E. Roa, J. P. Schiffer, T. A. Trainor, P. Wilt,

J. S. Winfield, M. R. Wolanski, F.L.H. Wolfs, A. H. Wuosmaa, G. Xu, A. Young, and. J. E. Yurkon

Phys. Rev. Lett. 78, 618-621 (1997)

Single Step Links of the Superdeformed Band in ${ }^{194} \mathrm{~Pb}$ : a Measure of the Absolute Excitation Energy, Spin and Parity of the Superdeformed States

A. Lopez-Martens, F. Hannachi, A. Korichi, C. Schuck, E. Gueorguieva, Ch. Vieu, B. Haas,

R. Lucas, A. Astier, G. Baldsiefen, M. Carpenter, G. de France, R. Duffait, L. Ducroux,

Y. Le Coz, Ch. Finck, A. Gorgen, H. Hubel, T. L. Khoo, T. Lauritsen, M. Meyer,

D. Prevost, N. Redon, C. Rigollet, H. Savajols, J. F. Sharpey-Schafer, O. Stezowski,

Ch. Theisen, U. Van Severen, J. P. Vivien, and A. N. Wilson

Phys. Lett. B380, 18-23 (1996)

Differential Lifetime Measurements and Configuration-Dependent Quadrupole Moments for Superdeformed Bands in Nuclei near ${ }^{152}$ Dy

D. Nisius, R.V. F. Janssens, E. F. Moore, P. Fallon, B. Crowell, T. Lauritsen, G. Hackman, I. Ahmad, H. Amro, S. Asztalos, M. P. Carpenter, P. Chowdhury, R. M. Clark, P. J. Daly, M. A. Deleplanque, R. M. Diamond, S. M. Fischer, Z. W. Grabowski, T. L. Khoo,

I. Y. Lee, A. O. Macchiavelli, R. H. Mayer, F. S. Stephens, A. V. Afanasjev, and

I. Ragnarsson

Phys. Lett. B392, 18-23 (1997)

Nuclear Structure at A 250 and the Stability of the Superheavy Elements

R. R. Chasman and I. Ahmad

Phys. Lett. B392, 255-261 (1997)

Importance of Vortex Interactions in $\mathrm{Tl}_{2} \mathrm{Ba}_{2} \mathrm{CaCu}_{2} \mathrm{O}_{8}$ Epitaxial Films with Columnar Defects

D. G. Steel, J. D. Hettinger, J. T. Parkman, M. E. Yoder, K. E. Gray, B. G. Glagola, and

M. M. Eddy

Physica C 265, 159-162 (1996)

Double-Phonon Giant Dipole Resonance in Relativistic Heavy Ion Collisions

J. Stroth, K. Broetzky-Grimm, E. Wajda, Th. Blaich, Th. W. Elze, H. Emling,

H. Freiesleben, W. Henning, R. Holzmann, H. Klingler, R. Kulessa, J. V. Kratz,

D. Lambrecht, Y. Leifels, E. Lubkiewicz, E. F. Moore, K. Stelzer, W. Walus, M. Zinser, and E. Zude

Nucl. Phys. A599, 307c-320c (1996) 
Parities and Multipolarities of Gamma Rays in Neutron-Rich Odd-Mass Ba Nuclei

M. A. Jones, W. Urban, J. L. Durell, M. Leddy, W. R. Phillips, A. G. Smith, B. J. Varley,

I. Ahmad, L. R. Morss, M. Bentaleb, E. Lubkiewicz, and N. Schulz

Nucl. Phys. A605, 133-159 (1996)

Octuple Correlations in Neutron-Rich, Even-Even Barium Isotopes

W. Urban, M. A. Jones, J. L. Durell, M. Leddy, W. R. Phillips, A. G. Smith, B. J. Varley,

I. Ahmad, L. R. Morss, M. A. Bentaleb, E. Lubkiewicz, and N. Schulz

Nucl. Phys. A613, 107-131 (1997)

Targets and Target Wheel Mechanism for APEX

D. E. Roa, L. Wright, J. D. Fox, J. P. Greene, B. B. Back, and B. G. Nardi

Nucl. Instrum. Methods A368, 307-313 (1996)

High Resolution Detection of Energetic Heavy Ions With a Calorimetric Low Temperature Detector

A. von Kielin, F. Azgui, W. Boehmer, K. Djotni, P. Egelhof, W. Henning, G. Kraus,

J. Meier, and K. W. Shepard

Nucl. Instrum. Methods A368, 815-818 (1996)

Reinvestigation of the $\alpha$-Decays of ${ }^{249} \mathrm{Cf}$ and ${ }^{233} \mathrm{U}$

A. Koua Aka, G. Ardisson, V. Barci, O. El Samad, D. Trubert, and I. Ahmad

Nucl. Instrum. Methods A $\underline{\text { 369, }}$ 477-485 (1996)

The Use of a Gas-Filled-Magnet in Experiments with Radioactive Ion Beams

K. E. Rehm, C. L. Jiang, M. Paul, D. Blumenthal, J. Gehring, D. Henderson, J. Nickles,

J. Nolen, R. C. Pardo, A. D. Roberts, J. P. Schiffer, and R. E. Segel

Nucl. Instrum. Methods A370, 438-444 (1996)

A Solenoidal Spectrometer for Positron-Electron Pairs Produced in Heavy-Ion Collisions

I. Ahmad, Sam M. Austin, B. B. Back, R. R. Betts, F. P. Calaprice, K. C. Chan, A. Chishti,

P. Chowdhury, C. Conner, R. W. Dunford, J. D. Fox, S. J. Freedman, M. Freer,

S. B. Gazes, J. S. Greenberg, J. P. Greene, A. L. Hallin, Th. Happ, D. Henderson,

N. I. Kaloskamis, E. Kashy, W. Kutschera, J. Last, C. J. Lister, M. Liu, M. R. Maier,

D. M. Mercer, D. Mikolas, P. A. A. Perera, M. D. Rhein, D. E. Roa, J. P. Schiffer,

T. A. Trainor, P. Wilt, J. S. Winfield, M. Wolanski, F. L. H. Wolfs, A. H. Wuosmaa,

G. Xu, A. R. Young, and J. E. Yurkon

Nucl. Instrum. Methods A370, 539-557 (1996)

Measurement of High Momentum Tranfer Reactions by Recoil Detection at a Cooler Ring

E. R. Jacobsen, R. E. Segel, R. D. Bent, J. D. Brown, G. Hardie, R. Schneider, C. Sun,

G. P. A. Berg, J. Blomgren, F.-J. Chen, P. Heimberg, J. Holmolka, A. Kurepin, H. Nann,

P. Pancella, K. E. Rehm, T. Rinckel, M. Saber, Z. Yu, and A. Zhuravlev

Nucl. Instrum. Methods A371, 439-448 (1996)

Transport Efficiency of the Argonne Fragment Mass Analyzer

B. B. Back, D. J. Blumenthal, C. N. Davids, D. J. Henderson, R. H. Hermann, C. L. Jiang,

H. T. Penttila, and A. H. Wuosmaa

Nucl. Instrum. Methods A379, 206-211 (1996)

Gammasphere: New Physics Opportunities

R. V. F. Janssens and F. Stephens

Nucl. Phys. News $\underline{6}, 9-17$ (1996) 
Search for Octupole Deformation in Neutron-Rich Xe Isotopes

M. Bentaleb, N. Schulz, E. Lubkiewicz, L. J. Durell, C. J. Pearson, W. R. Phillips,

J. Shannon, B. J. Varley, I. Ahmad, C. J. Lister, L. R. Morss, K. L. Nash, and

C. W. Williams

Z. Phys. A354, 143-151 (1996)

Fine Structure in the $\alpha$ Decay of ${ }^{192} \mathrm{Po}$

N. Bijnens, I. Ahmad, A. N. Andreyev, J. C. Batchelder, C. R. Bingham, D. Blumenthal,

B. C. Busse, X. S. Chen, L. F. Conticchio, C. N. Davids, M. Huyse, P. Mantica,

H. Penttila, W. Reviol, D. Seweryniak, P. Van Duppen, W. B. Walters, J. Wauters, and B. E. Zimmerman

Z. Phys. A356, 3-4 (1996)

The Positron Peak Puzzle - Recent Results from APEX

I. Ahmad, S. M. Austin, B. B. Back, R. R. Betts, F. P. Calaprice, K. C. Chan, A. Chishti,

P. Chowdhury, C. Conner, R. W. Dunford, J. D. Fox, S. J. Freedman, M. Freer, S. Gazes,

A. L. Hallin, T. Happ, N. Kaloskamis, E. Kashy, W. Kutschera, J. Last, C. J. Lister,

M. Liu, M. R. Maier, D. J. Mercer, D. Mikolas, A. Perera, M. D. Rhein, E. Roa,

J. P. Schiffer, T. Trainor, P. Wilt, J. S. Winfield, M. Wolanski, F. L. H. Wolfs,

A. H. Wuosmaa, A. Young, and J. E. Yurkon

Proceedings of the XXIV Mazurian Lakes School of Physics, Piaski, Poland, August 23 -

September 3, 1995; Acta Physica Polonica 27B, 387-400 (1996)

Order in Very Cold Confined Plasmas

J. P. Schiffer

Proceedings of the International Symposium on Exotic Atoms and Nuclei, June 7-10, 1995, Hakone, Japan; Hyperfine Interactions $\underline{103}$, 71-79 (1996)

Rapid Increase in Prescission GDR $\gamma$-ray Emission with Energy

D. J. Hofman, B. B. Back, and P. Paul

Proceedings of the Groningen Conference on Giant Resonances, Groningen, The

Netherlands, June 28 - July 1, 1995; Nucl. Phys. A599, 23c-28c (1996)

Internal Pair Decay of Giant Resonances in Hot $200 \mathrm{~Pb}$

S. Schadmand, R. Varma, S. R. Banerjee, B. B. Back, D. J. Hofman, C. P. Montoya, and

P. Paul

Proceedings of the Groningen Conference on Giant Resonances, Groningen, The

Netherlands, June 28 - July 1, 1995; Nucl. Phys. A599, 89c-96c (1996)

Accelerator Complex for a Radioactive Ion Beam Facility at ATLAS

J. A. Nolen

Proceedings of the 1995 Particle Accelerator Conference and International Conference on High-Energy Accelerators, Dallas, TX, 1-5 May 1995, IEEE, 0-7803-3053, Vol. 1 , pp. 354-356 (1996) 
Crystalline Beams

John P. Schiffer

Proceedings of the 1995 Particle Accelerator Conference and International Conference on High-Energy Accelerators, Dallas, TX, 1-5 May 1995, IEEE, 0-7803-3053, Vol. 5 , pp. 3164-3166 (1996)

A New Look at Positron Production from Heavy-Ion Collisions: Results from APEX

I. Ahmad, Sam M. Austin, B. B. Back, R. R. Betts, F. P. Calaprice, K. C. Chan, A. Chishti, P. Chowdhury, C. Conner, R. W. Dunford, J. D. Fox, S. J. Freedman, M. Freer, S. B. Gazes, A. L. Hallin, Th. Happ, D. Henderson, N. I. Kaloskamis, E. Kashy, W. Kutschera, J. Last, C. J. Lister, M. Liu, M. R. Maier, D. M. Mercer, D. Mikolas, P. A. A. Perera, M. D. Rhein, D. E. Roa, J. P. Schiffer, T. A. Trainor, P. Wilt, J. S. Winfield, M. Wolanski, F. L. H. Wolfs, A. H. Wuosmaa, G. Xu, A. R. Young, and J. E. Yurkon

Proceedings of the IV International Symposium on Weak and Electromagnetic Interactions in Nuclei, June 12-16, 1995, Osaka, Japan, ed. H. Ejiri, T. Kishimoto, T. Sato (World Scientific, Singapore 1996) pp. 629-634

Decay of Superdeformed Bands

M. P. Carpenter, T. L. Khoo, T. Lauritsen, T. Dossing, I. Ahmad, D. Ackermann, D. J. Blumenthal, S. M. Fischer, D. Gassmann, G. Hackman, R. G. Henry, R. V. F. Janssens, D. Nisius, E. F. Moore, A. Lopez-Martens, F. Hannachi, R. Kruecken, S. J. Asztalos, R. M. Clark, M. A. Deleplanque, R. M. Diamond, P. Fallon, I. Y. Lee, A. O. Macchiavelli, F. S. Stephens, J. A. Beckar, L. Bernstein, L. P. Farris, E. A. Henry, and A. Korichi

Proceedings of the Workshop on Gammasphere Physics, Lawrence Berkeley National Laboratory, Berkeley, CA, December 1-2, 1995, Ed. M. A. Deleplanque, I. Y. Lee and A. O. Macchiavelli, World Scientific, pp. 1-14 (1996)

The Multiplicity of Shapes in Tl Nuclei

L. L. Riedinger, W. Reviol, J. M. Lewis, W. F. Mueller, B. H. Smith, J. Y. Zhang,

S. M. Fischer, M. P. Carpenter, R. V. F. Janssens, B. Crowell, I. Ahmad,

D. J. Blumenthal, T. L. Khoo, T. Lauritsen, D. Nisius, and B. Cederwall

Proceedings of the Workshop on Gammasphere Physics, Lawrence Berkeley National Laboratory, Berkeley, CA, December 1-2, 1995, Ed. M. A. Deleplanque, I. Y. Lee and

A. O. Macchiavelli, World Scientific, pp. 98-110 (1996)

Lifetime Measurements and the Issue of Identical Superdeformed Bands

E. F. Moore, D. Nisius, R. V. F. Janssens, T. Lauritsen, M. P. Carpenter, T. L Khoo, I. Ahmad, D. Blumenthal, B. Crowell, D. Gassmann, S. M. Fischer, P. Fallon, S. Asztalos,

B. Cederwall, R. Clark, M. A. Deleplanque, R. M. Diamond, I. Y. Lee, A. O. Macchiavelli,

R. Mayer, P. J. Daly, Z. W. Grabowski, and P. Chowdhury

Proceedings of the Workshop on Gammasphere Physics, Lawrence Berkeley National Laboratory, Berkeley, CA, December 1-2, 1995, Ed. M. A. Deleplanque, I. Y. Lee and A. O. Macchiavelli, World Scientific, pp. 137-148 (1996)

Shell Model at Gammasphere: Studies in the $A \approx 96$ Region

U. Garg, S. S. Ghugre, B. Kharraja, G. Smith, I. Ahmad, M. P. Carpenter, B. Crowell,

R. V. F. Janssens, T. L. Khoo, T. Lauritsen, D. Nisius, W. F. Mueller, W. Reviol,

L. L. Riedinger, and R. Kaczarowski

Proceedings of the Workshop on Gammasphere Physics, Lawrence Berkeley National Laboratory, Berkeley, CA, December 1-2, 1995, Ed. M. A. Deleplanque, I. Y. Lee and A. O. Macchiavelli, World Scientific, pp. 149-160 (1996) 
K-Isomers at Gammasphere

P. Chowdhury, B. Crowell, I. Ahmad, D. J. Blumenthal, M. P. Carpenter, S. M. Fischer,

R. V. F. Janssens, T. L. Khoo, C. J. Lister, T. Lauritsen, and D. Nisius

Proceedings of the Workshop on Gammasphere Physics, Lawrence Berkeley National Laboratory, Berkeley, CA, December 1-2, 1995, Edit. M.A. Deleplanque, I. Y. Lee and A. O. Macchiavelli edit., World Scientific, pp. 212-222 (1996)

Phase Transitions Along and Above the Yrast Line in ${ }^{154} \mathrm{Dy}$

W. C. Ma, P. G. Varmette, S. J. Zhu, T. L. Khoo, R. V. F. Janssens, T. Lauritsen, D. Nisius, I. Ahmad, D. Blumenthal, M. P. Carpenter, B. Crowell, D. Gassmann,

J. H. Hamilton, A. V. Ramayya, P. Bhattacharyya, C. T. Zhang, P. J. Daly,

Z. W. Grabowski, and R. H. Mayer

Proceedings of the Workshop on Gammasphere Physics, Lawrence Berkeley National Laboratory, Berkeley, CA, December 1-2, 1995, Ed. M. A. Deleplanque, I. Y. Lee and A. O. Macchiavelli, World Scientific, pp. 266-271 (1996)

Current Status of PHOBOS@RHIC

R. R. Betts for the PHOBOS Collaboration

Proceedings of the Winter Workshop of Nuclear Dynamics, Snowbird, Utah, 5-10 February 1996, pp. 225-232

Discrete-Line Transitions from Superdeformed to Yrast States in ${ }^{194} \mathrm{Hg}$ and ${ }^{192} \mathrm{Hg}$

G. Hackman, T. L. Khoo, D. Ackermann, A. Lopez-Martens, M. P. Carpenter, T. Lauritsen,

S. Agarwala, I. Ahmad, H. Amro, D. J. Blumenthal, I. Calderin, T. Dossing, S. M. Fischer,

F. Hannachi, R. V. F. Janssens, A. Korichi, I. Y. Lee, A. O. Macchiavelli, E. F. Moore,

D. Nisius, and J. Young

Proceedings of the Conference on Nuclear Structure at the Limits, July 22-26, 1996,

Argonne National Laboratory, Argonne, IL, ANL/PHY-97/1, pp. 1-5 (1997)

Observation of the Single Step Links of the Yrast Superdeformed Band in ${ }^{194} \mathrm{~Pb}$

F. Hannachi, A. Lopez-Martens, A. Korichi, S. Leoni, C. Schuck, E. Gueorguieva,

Ch. Vieu, B. Haas, R. Lucas, A. Astier, G. Baldsiefen, M. Carpenter, G. de France, R.

Duffait, L. Ducroux, Y. Le Coz, Ch. Finck, A. Gorgen, H. Hubel, T. L. Khoo, T. Lauritsen,

M. Meyer, D. Prevost, N. Redon, C. Rigollet, H. Savajols, J. F. Sharpey-Schaer,

O. Stezowski, Ch. Theisen, U. Van Severen, J. P. Vivien, and A. N. Wilson

Proceedings of the Conference on Nuclear Structure at the Limits, July 22-26, 1996,

Argonne National Laboratory, Argonne, IL, ANL/PHY-97/1, pp. 6-10 (1997)

The Quasi-Continuum of Gamma Rays Following the Decay of Superdeformed Bands in the Hg Region

T. Lauritsen, A. Lopez-Martens, T. L. Khoo, R. V. F. Janssens, M. P. Carpenter,

G. Hackman, D. Ackermann, I. Ahmad, H. Amro, D. J. Blumenthal, S. M. Fischer,

F. Hannachi, A. Dorichi, E. F. Moore, and D. T. Nisius

Proceedings of the Conference on Nuclear Structure at the Limits, July 22-26, 1996,

Argonne National Laboratory, Argonne, IL, ANL/PHY-97/1, pp. 35-40 (1997) 
First Measurement of the Degree of Fragmentation of the Decay Out Cascade from the Superdeformed Yrast Bands in ${ }^{192} \mathrm{Hg}$

A. Lopez-Martens, F. Hannachi, C. Schuck, R. Collatz, E. Gueorguieva, Ch. Vieu,

T. Dossing, S. Leoni, B. Herskind, I. Ahmad, D. Blumenthal, M. Carpenter, D. Gassmann,

R. V. F. Janssens, T. L. Khoo, T. Lauritsen, D. Nisius, A. Korichi, C. Bourgeois, A. Astier,

L. Ducroux, Y. Le Coz, M. Meyer, N. Redon, J. F. Sharpey-Schafer, A. N. Wilson,

W. Korten, A Bracco, and R. Lucas

Proceedings of the Conference on Nuclear Structure at the Limits, July 22-26, 1996,

Argonne National Laboratory, Argonne, IL, ANL/PHY-97/1, pp. 41-46 (1997)

Around the Back-Bend in an Excited ${ }^{150} \mathrm{Gd}$ Superdeformed Band

P. J. Twin, S. Erturk, C. W. Beausang, P. Fallon, D. Appelbe, S. Asztalos, A. Macchiavelli,

T. Lauritsen, I. Y. Lee, F. S. Stephens, and M. A. Deleplanque-Stephens

Proceedings of the Conference on Nuclear Structure at the Limits, July 22-26, 1996,

Argonne National Laboratory, Argonne, IL, ANL/PHY-97/1, pp. 60-65 (1997)

Lifetime Measurements and Identical SD Bands in the $A=190$ and $A=150$ Regions

E. F. Moore, D. Nisius, R. V. F. Janssens, T. L. Khoo, T. Lauritsen, D. Ackermann,

I. Ahmad, H. Amro, D. Blumenthal, M. P. Carpenter, S. Fischer, G. Hackman, P. Fallon,

S. J. Asztalos, R. M. Clark, M. A. Deleplanque, R. M. Diamond, R. Krueken, I-Y. Lee,

A. O. Macchiavelli, F. S. Stephens, F. Hannachi, A. Lopez-Martens, J. A. Becker,

L. Bernstein, L. P. Farris, E. A. Henry, A. Korichi, P. Chowdhury, R. H. Mayer,

A. V. Afanasjev, and I. Ragnarsson

Proceedings of the Conference on Nuclear Structure at the Limits, July 22-26, 1996,

Argonne National Laboratory, Argonne, IL, ANL/PHY-97/1, pp. $72-77$ (1997)

How Close Are Hyperdeformed States to the Scission Point?

L. M. Robledo, R. R. Chasman, and J. L. Egido

Proceedings of the Conference on Nuclear Structure at the Limits, July 22-26, 1996,

Argonne National Laboratory, Argonne, IL, ANL/PHY-97/1, pp. 124-129 (1997)

Test of $\Delta I=2$ Staggering in the Superdeformed Bands of ${ }^{194} \mathrm{Hg}$

R. Krucken, G. Hackman, M. A. Deleplanque, R. V. F. Janssens, I. Y. Lee, D. Ackermann,

I. Ahmad, H. Amro, S. Asztalos, D. J. Blumenthal, M. P. Carpenter, R. M. Clark,

R. M. Diamond, P. Fallon, S. M. Fischer, B. Herskind, T. L. Khoo, T. Lauritsen,

A. O. Macchiavelli, R. W. Macleod, D. Nisius, G. J. Schmid, F. S. Stephens, and K. Vetter

Proceedings of the Conference on Nuclear Structure at the Limits, July 22-26, 1996,

Argonne National Laboratory, Argonne, IL, ANL/PHY-97/1, pp. 130-135 (1997)

First In-Beam Observation of Excited States in ${ }^{156} \mathrm{Hf}_{84}$ Using the Recoil-Decay Tagging Method

D. Seweryniak, I. Ahmad, H. Amro, D. J. B Jumenthal, L. T. Brown, M. Carpenter,

C. N. Davids, S. Fischer, D. J. Henderson, R. V. F. Janssens, T. L. Khoo, C. J. Lister,

D. Nisius, T. Davinson, R. J. Irvine, P. J. Woods, W. B.Walters, I. Hibbert, C. Parry, and R. Wadsworth

Proceedings of the Conference on Nuclear Structure at the Limits, July 22-26, 1996,

Argonne National Laboratory, Argonne, IL, ANL/PHY-97/1, pp. 247-252 (1997) 
Yrast Excitations Around ${ }^{132} \mathrm{Sn}$ : The Two and Three Valence-Proton $\mathrm{N}=82$ Isotones ${ }^{134} \mathrm{Te}$ and 135I

P. J. Daly, C. T. Zhang, P. Bhattacharyya, R. Broda, Z. W. Grabowski, D. Nisius,

I. Ahmad, T. Ishii, M. P. Carpenter, L. R. Morss, W. R. Phillips, J. L. Durrell, M. J. Leddy,

A. G. Smith, W. Urban, B. J. Varley, N. Schulz, E. Lubkiewicz, M. Bentaleb,

and J. Blomquist

Proceedings of the Conference on Nuclear Structure at the Limits, July 22-26, 1996,

Argonne National Laboratory, Argonne, ПL, ANL/PHY-97/1, pp. 282-287 (1997)

The Nuclear Structure of Neutron-Rich Isotopes with Z from 38-42

J. L. Durell, M. A. Jones, W. R. Phillips, A. G. Smith, W. Urban, B. J. Varley, I. Ahmad,

C. J. Lister, L. R. Morss, K. L. Nash, C. Williams, N. Schulz, A. Guessous,

E. Lubkiewicz, and M. Bentaleb

Proceedings of the Conference on Nuclear Structure at the Limits, July 22-26, 1996,

Argonne National Laboratory, Argonne, IL, ANL/PHY-97/1, pp. 294-299 (1997)

Spectroscopy of Reflection-Asymmetric Nuclei using Multinucleon Transfer Reactions

J. F. C. Cocks, P. A. Butler, K. J. Cann, P. T. Greenlees, G. D. Jones, R. Broda,

B. Fornal, P. M. Jones, R. Julin, S. Juutinen, D. Muller, M. Piiparinen, A. Savelius,

J. F. Smith, I. Ahmad, S. Asztalos, P. Bhattacharyya, D. J. Blumenthal, M. P. Carpenter,

R. M. Clark, B. Crowell, M. A. Delaplanque, R. M. Diamond, P. Fallon, R. V. F. Janssens,

T. L. Khoo, T. Lauritsen, I. Y. Lee, A. O. Macchiavelli, R. W. Macleod, D. Nisius,

F. S. Stephens, and C. T. Zhang

Proceedings of the Conference on Nuclear Structure at the Limits, July 22-26, 1996,

Argonne National Laboratory, Argonne, IL, ANL/PHY-97/1, pp. 300-305 (1997)

Coupled Channel Analysis of High Precision Fusion Data

H. Esbensen and B. B. Back

Phys. REv. C $\underline{54}, 3109-3116$ (1996)

\section{OPERATION AND DEVELOPMEN'T OF ATLAS}

Design Parameters, Goals, and Status of the New ATLAS ECR Ion Source

R. C. Pardo, R. Harkewicz, P. J. Billquist, C. M. Lyneis, and Z. Q. Xie

Proceedings of the International Ion Source Conference, Whistler, Canada, 10-16 September 1995; Rev. Sci. Instrum. 67, 1180 (1996)

Time Evolution of Charge States in an ECR Ion Source

R. C. Pardo, R. Harkewicz, and P. J. Billquist

5th International Ion Source Conference, Whistler, Canada, 11-16 September

995; Rev. Sci. Instrum. 61, 881 (1996)

Accelerator Complex for a Radioactive Ion Beam Facility at ATLAS

J. A. Nolen

Proceedings of the 1995 Particle Accelerator Conference and International

Conference on High-Energy Accelerators, Dallas, TXI, 1-5 May 1995, IEEE, 0-7803-

3053, Vol. 1, pp. 354-356 (1996)

A Low-Charge-State Injector Linac for ATLAS

K. W. Shepard and J. W. Kim

Proceedings of the 1995 Particle Accelerator Conference and International

Conference on High-Energy Accelerators, Dallas, TX, 1-5 May 1995, IEEE, 0-7803-

3053, Vol. 2. 1128-1130 (1996) 
The Role of Space Charge in the Performance of the Bunching System for the ATLAS Positive Ion Injector

R. C. Pardo and R. Smith

Proceedings of the 1995 Particle Accelerator Conference and International Conference on High-Energy Accelerators, Dallas, TX, 1-5 May 1995, IEEE, 0-78033053, Vol. $\underline{3}$, 1849-1851 (1996)

A High Gradient Superconducting Quadrupole for a Low-Charge-State Ion Linac

J. W. Kim, K. W. Shepard, and J. A. Nolen Proceedings of the 1995 Particle Accelerator Conference and International Conference on High-Energy Accelerators, Dallas, TX, 1-5 May 1995, IEEE, 0-78033053, Vol. $\underline{3}, 1408-1410$ (1996)

A Low-Cost Non-Intercepting Beam Current and Phase Monitor For Heavy Ions

J. M. Bogaty and B. E. Clifft

Proceedings of the 1995 Particle Accelerator Conference and International Conference on High-Energy Accelerators, Dallas, TX, 1-5 May 1995, IEEE, 0-78033053, Vol. 4, 2625-2627 (1996)

Longitudinal Emittance Oscillation in a Superconducting Drift Tube Linac

J. W. Kim and K. W. Shepard

Proceedings of the 1995 Particle Accelerator Conference and International Conference on High-Energy Accelerators, Dallas, TX, 1-5 May 1995, IEEE, 0-78033053, Vol. ․, 3400-3402 (1996)

A Concept for Emittance Reduction of DC Radioactive Heavy-Ion Beams

J. A. Nolen and J. C. Dooling Proceedings of the 1995 Particle Accelerator Conference and International Conference on High-Energy Accelerators, Dallas, TX, 1-5 May 1995, IEEE, 0-78033053, Vol. 5, 3320-3322 (1996)

Transverse Emittance Systematics Measured for Heavy-Ion Beams at ATLAS

J. A. Nolen, T. A. Barlow, K. A. Beyer, and K. A. Woody Proceedings of the 1995 Particle Accelerator Conference and International Conference on High-Energy Accelerators, Dallas, TX, 1-5 May 1995, IEEE, 0-78033053, Vol. 4, 2628-2630 (1996)

Current Status of ATLAS and Proposed Expansion to an Exotic Beam Facility

G. P. Zinkann, P. Billquist, J. Bogaty, B. Clifft, F. Munson, K. Nakagawa, J. Nolen, R. Pardo, K. W. Shepard, J. Specht, A. Sutherland, B. Tieman, and I. Tilbrook Nucl. Instrum. and Methods in Physics Research A ㄹ2, 132-139 (1996)

\section{MEDIUM-ENERGY NUCLEAR PHYSICS RESEARCH}

Inclusive Electron Scattering from Nuclei at $\mathrm{x} \sim 1$

J. Arrington (SLAC NE18 Collaboration)

Phys. Rev. C 53, 2248-2251 (1996)

$\gamma \mathrm{n} \rightarrow \mathrm{p}-\mathrm{p}$ Process in ${ }^{4} \mathrm{He}$ and $16 \mathrm{O}$

H. Gao, R. J. Holt, and V. R. Pandharipande

Phy. Rev. C 54, 2779-2782 (1996) 
Determination of the Gluon Distribution Function of the Nucleon Using Energy-Energy Angular Patterns in Deep Inelastic Muon-Deuteron Scattring

M. R. Adams, S. Aid, P. L. Anthony, M. D. Baker, J. Bartlett, A. A. Bhatti, H. M. Braun, W. Busza, T. J. Carroll, J. M. Conrad, R. Davisson, I. Derado, S. K. Dhawan, W. Dougherty, T. Dreyer, K. Dziunokowska, V. Eckardt, U. Ecker, M. Erdmann, A. Eskreys, J. Figiel,

H. J. Gebauer, D. F. Geesaman, R. Gilman, M. C. Green, J. Haas, C. Halliwell, J. Hanlon,

D. Hantke, V. W. Hughes, H. E. Jackson, G. Jancso, D. M. Jansen, S. Kaufman,

R. D. Kennedy, T. Kirk, H. G. E. Kobrak, S. Krzywdzinski, S. Kunori, J. J. Lord,

H. J. Lubatti, D. McLeod, S. Magill, P. Malecki, A. Manz, H. Melanson, D. G. Michael, W. Mohr, H. E. Montgomery, J. G. Morfin, R. B. Nickerson, S. O'Day, K. Olkiewicz, L. Osborne, V. Papavassiliou, B. Pawlik, F. M. Pipkin, E. J. Ramberg, A. Roser, J. J. Ryan, C. W. Salgado, A. Salvarani, H. Schellman, M. Schmitt, N. Schmitz, K. P. Schuler, H. J. Seyerlein, A. Skuja, G. A. Snow, S. Soldner-Rembold, P. Steinberg, H. E. Stier, P. Stopa, R. A. Swanson, R. Talaga, S. Tentindo-Repond, H.-J. Trost, H. Venkataramania, M. Wilhelm, J. Wilkes, R. Wilson, W. Wittek, S. Wolbers and T. Zhao Z. Phys. C 71,391 (1996)

Proton and Deuteron Structure Functions in Muon Scattering at $470 \mathrm{GeV}$

M. R. Adams, S. Aid, P. L. Anthony, D. A. Averill, M. D. Baker, R. R. Baller,

A. Banerjee, A. A. Bhatti, U. Bratzler, H. M. Braun, T. J. Carroll H. L. Clark, J. M. Conrad,

R. Davisson, I. Derado, F. S. Dietrich, W. Dougherty, T. Dreyer, V. Eckardt, U. Ecker,

M. Erdmann, G. Y. Fang, J. Figiel, R. W. Finlay, H. J. Gebauer, D. F. Geesaman,

K. A. Griffioen, R. S. Guo, J. Haas, C. Halliwell, D. Hantke, K. H. Hicks. H. E. Jackson,

D. E. Jaffe, G. Jancso, D. M. Jansen, Z. Jin, S. Kaufman, R. D. Kennedy, E. R. Kinney,

H. G. E. Kobrak, A. V. Kotwal, S. Kunori, J. J. Lord, H. J. Lubatti, D. McLeod, P. Madden, S. Magill, A. Manz, H. Melanson, D. G. Michael, H. E. Montgomery, J. G. Morfin,

R. B. Nickerson, J. Novak, S. O'Day, K. Olkiewicz, L. Osborne, R. Otten, V. Papavassiliou, B. Pawlik, F. M. Pipkin, D. H. Potterveld, E. J. Ramberg, A. Roser, J. J. Ryan,

C. W. Salgado, A. Salvarani, H. Schellman, M. Sch itt, N. Sch itz, G. Siegert, A. Skuja,

G. A. Snow, S. Soldner-Rembold, P.Spentzouris, H. E. Stier, P. Stopa, R. A. Swanson,

H. Venkataramania, M. Wilhelm, R. WIlson, W. Wittek, S. Wolbers, A. Zghiche, and

T. Zhao

Phys. Rev. D $\underline{54} 3006$ (1996)

The Spin-Dependent Momentum Distribution of the Neutron and Proton in ${ }^{3} \mathrm{He}$

R. G. Milner, C. Bloch, J.F.J. van den Brand, H. J. Bulten, D. DeSchepper, T. W. Donnelly,

R. Ent, C. D. Goodman, J.-O. Hansen, W. W. Jacobs, C. E. Jones, W. Korsch,

L, H. Kramer, K. Lee, M. Leuschner, W. Lorenzon, N.C.R. Makins, D. Marchlenski,

H. O. Meyer, M. A. Miller, J. S. Neal, P. V. Pancella, S. F. Pate, W. K. Pitts,

B. von Przewoski, T. Rinckel, P. U. Sauer, G. Savopulos, R.-W. Schulze, A. Smith, J. Sowinski, F. Sperisen, E. R. Sugarbaker, M. A. Titko, C. Tschalar, O. Unal. T. P. Welch, Z.-L. Zhou

Phys. Lett. B $\underline{379}, 67-72$ (1996)

Asymmetries for Elastic Scattering of $\pi^{+}$from Polarized ${ }^{3} \mathrm{He}$ and the $\Delta$-Neutron Spin-Spin Interaction

M. A. Espy, D. Dehnhard, C. M. Edwares, M. Palarczyk, I. L. Langenbrunner, B. Davis, G. R. Burleson, S. Blanchard, W. R. Gibbs, B. Lail, B. Nelson, B. K. Park, Q. Zhao, W. J. Cummings, P.P.J. Delheij, B. K. Jennings, R. Henderson, O. Hausser, D. Thiessen, E. Brash, M. K. Jones, B. Larson, B. Brinkmoller, K. Maeda, C. L. Morris, J. M. O'Donnell, S. Penttila, D. Swenson, D. Tupa, C. Bennhold, S. S. Kamalov Phys. Rev. Letters 76, 3667-3671 (1996) 


\section{THEORETICAL PHYSICS}

Analytic Structure of the Quark Propagator in a Model with an Infrared Vanishing Gluon Propagator Axel Bender and Reinhard Alkofer

Phys. Rev. D $\underline{53}, 446-457$ (1996)

Calculation of the Anomalous $\gamma \pi^{*} \rightarrow \pi \pi$ Form Factor

Reinhard Alkofer and Craig D. Roberts

Phys. Lett. B369, 101-107 (1996)

The Effect of the $\Delta$ Excitation on the Nucleon-Nucleon Effective Interactions in the Nuclear Shell-

Model

Y. Tzeng, T. T. S. Kuo, and T.-S. H. Lee

Physica Scripta 53, 300-305 (1996)

Neutron Drops and Skyrme Energy-Density Functionals

B. S. Pudliner, A. Smerzi, J. Carlson, V. R. Pandharipande, Steven C. Pieper, and D. G. Ravenhall

Phys. Rev. Lett. 76, 2416-2419 (1996)

Momentum Distributions in Breakup Reactions

Henning Esbensen

Proceedings of the International Workshop XXIV on Gross Properties of

Nuclei and Nuclear Exitations, Hirschegg, Austria, 15-20 January 1996, eds.

Hans Feldmeier, Jorn Knoll, Wolfgang Norenberg (GSI, Darmstadt 1996) pp. $321-330$

Momentum Distributions in Stripping Reactions of Single-Nucleon Halo Nuclei

H. Esbensen

Phys. Rev. C 53, 2007-2010 (1996)

Electromagnetic Form Factors of Charged and Neutral Kaons

C. J. Burden, C. D. Roberts, and M. J. Thomson

Phys. Lett. B371, 163-168 (1996)

Separable Approximation to the Bethe-Salpeter Equation in QCD

C. J. Burden, L. Qian, C. D. Roberts, P. C. Tandy, and M. J. Thomson Proceedings of the International Symposium on Lattice Field Theory, Melbourne, Australia, 11-15 July 1995, Nucl. Phys. B47, 362-365 (1996)

Pomeron-Exchange and Exclusive Electroproduction of $\rho-M e s o n s$ in QCD M. A. Pichowsky and T.-S. H. Lee

Phys. Lett. B379, 1-6 (1996)

Electromagnetic Pion Form Factor and Neutral Pion Decay Width

Craig D. Roberts

Nucl. Phys. A605, 475-495 (1996)

Quantum Ballistic Evolution in Quantum Mechanics: Application to Quantum Computers

Paul Benioff

Phys. Rev. A $\underline{54}$, 1106-1123 (1996) 
Helmut Paul, A Happy Physicist

Mitio Inokuti

Proceedings of the Sixteenth International Conference on Atomic Collisions

in Solids, Linz, Austria, 17-21 July 1995, eds. D. Semrad, P. Bauer, and

O. Benka (Elsevier Science B.V., Amsterdam 1996); Nucl. Instrum. Methods B115, xiii-Xv (1996)

Goldstone Theorem and Diquark Confinement Beyond Rainbow-Ladder Approximation

A. Bender, C. D. Roberts, and L. von Smekal

Phys. Lett. B $\underline{380}, 7-12$ (1996)

Femtometer Toroidal Structures in Nuclei

J. L. Forest, V. R. Pandharipande, Steven C. Pieper, R. B. Wiringa, R. Schiavilla, and A. Arriaga

Phys. Rev. C $\underline{54}$, 646-667 (1996)

Medium Effects on Binary Collisions with the $\Delta$ Resonance

T.-S. H. Lee

Phys. Rev. C 포, 1350-1359 (1996)

The Quantum Defect: Early History and Recent Developments

A. R. P. Rau and Mitio Inokuti

Am. J. Phys. 64, 1-4 (October 1996)

Continuum Study of Deconfinement at Finite Temperature

Axel Bender, David Blaschke, Yuri Kalinovsky, and Craig D. Roberts

Phys. Rev. Lett. 77, 3724-3727 (1996)

Total and 2n-Removal Cross Sections of $20-60 \mathrm{MeV} . \mathrm{A}{ }^{4,6,8} \mathrm{He},{ }^{6-9,11} \mathrm{Li}$, and ${ }^{10} \mathrm{Be}$ on $\mathrm{Si}$

R. E. Warner, R. A. Patty, P. M. Voyles, A. Nadasen, F. D. Becchetti,

J. A. Brown, H. Esbensen, A. Galonsky, J. J. Kolata, J. Kruse, M. Y. Lee,

R. M. Ronningen, P. Schwandt, J. von Schwarzenberg, B. M. Sherrill, K. Subotic,

J. Wang, and P. Zecher

Phys. Rev. C $\underline{54}$, 1700-1709 (1996)

Nuclear Core Dynamics and Rearrangement Energy for Hypernuclei

A. R. Bodmer, S. Murali, and Q. N. Usmani

Nucl. Phys. A609, 326-338 (1996)

Meson-Exchange Model for $\pi N$ Scattering and $\gamma \mathrm{N} \rightarrow \pi N$ Reaction

T. Sato and T.-S. H. Lee

Phys. Rev. C $\underline{54}$, 2660-2684 (1996)

Study of the Breakup Reaction ${ }^{8} \mathrm{~B} \rightarrow{ }^{7} \mathrm{~B}+\mathrm{p}$ : Absorption Effects and E2 Strength J. H. Kelley, Sam M. Austin, A. Azhari, D. Bazin, J. A. Brown, H. Esbensen, M. Fauerbach, M. Hellstrom, S. E. Hirzebruch, R. A. Kryger, D. J. Morrissey, R. Pfaff, C. F. Powell, E. Ramakrishnan, B. M. Sherrill, M. Steiner,

T. Suomijarvi, and M. Thoennessen

Phys. Rev. Lett. 77, 5020-5023 (1996) 
Model Gluon Propagator and Pion and Rho-Meson Observables

M. R. Frank and C. D. Roberts

Phys. Rev. C 53, 390-398 (1996)

Effects of E2 Transitions in the Coulomb Dissociation of ${ }^{8} \mathrm{~B}$

H. Esbensen and G. F. Bertsch

Nucl. Phys. A600, 37-62 (1996)

Breakup Reactions of the Halo Nuclei ${ }^{11} B$ and ${ }^{8} B$

K. Hencken, G. Bertsch, and H. Esbensen

Phys. Rev. C 54, 3043-3050 (1996)

Coupled Channels Analysis of High Precision Fusion Data

H. Esbensen and B. B. Back

Phys. Rev. C 54, 3109-3116 (1996)

Off-Diagonal Long-Range Order, Restricted Gauge Transformations, and Aharonov-Bohm Effect in Conductors

M. Peshkin

Phys. Rev. Lett 76, 2207-2210 (1996)

\section{ATOMIC AND MOLECULAR PHYSICS RESEARCH}

Experimental Determination of Non-Dipolar Angular Distribution Parameters for Photoionization in the Ar-K and $\mathrm{Kr}-\mathrm{L}$ Shells

M. Jung, B. Kraessig, D. S. Gemmell, E. P. Kanter, T. LeBrun, S. H. Southworth, L. Young Phys. Rev. A 54, 2127-2136 (1996)

Ratio of Cross Sections for Double to Single Ionization of He by $85-400 \mathrm{eV}$ Photons

R. Dorner, T. Vogt, V. Mergel, H. Khemliche, S. Kravis, C. L. Cocke, J. Ullrich,

M. Unverzagt, L. Spielberger, M. Damrau, O. Jagutzki, I. Ali, B. Weaver, K. Ullmann,

C. C. Hsu, M. Jung, E. P. Kanter, B. Sonntag, M. H. Prior, E. Rotenberg, J. Denlinger,

T. Warwick, S. T. Manson, and H. Schmidt-Bocking

Phys. Rev. Lett. 76, 2654 (1996)

Double and Single Ionization of Helium by 58-keV X-Rays

L. Spielberger, O. Jagutzki, B. Kraessig, U. Meyer, Kh. Khayyat, V. Mergel,

Th. Tschentscher, Th. Buslaps, H. Brauning, R. Dorner, T. Vogt, M. Achler, J. Ullrich,

D. S. Gemmell, and H. Schmidt-Bocking

Phys. Rev. Lett. 76, 4685-4688 (1996)

Fully Differential Cross Sections for Double Photoionization of He near Threshold Measured by Recoil Ion Momentum Spectroscopy

R. Dorner, J. Feagin, C. L. Cocke, H. Brauning, O. Jagutzki, M. Jung, E. P. Kanter, H. Khemliche, S. Kravis, V. Mergel, M. H. Prior, H. Schmidt-Bocking, L. Spielberger, J. Ullrich, M. Unversagt, and T. Vogt

Phys. Rev. Lett. 77, 1024-1027 (1996)

Angular Correlation Between $\mathrm{K} \alpha$ Photons and L2,3-M2,3 $\mathrm{M}_{2,3}$ Auger Electrons Following Argon 1s Photoionization

U. Arp, J. W. Cooper, T. LeBrun, S. H. Southworth, M. Jung, and M. A. MacDonald

J. Phys. B: At. Mol. Opt. Phys. 29, L837-L842 (1996) 
Measuring Nondipolar Asymmetries of Photoelectron Angular Distributions

P. S. Shaw, U. Arp, S. H. Southworth

Phys. Rev. A $\underline{54}, 1463$ (1996)

Resonant Raman Scattering in Potassium and Chlorine $\mathrm{K} \beta \mathrm{X}$-Ray Emission from KC1

K. Miyano, Y. May, S. H. Southworth, P. L. Cowan, and B. A. Karlen

Phys. Rev. B 54, 12022-12028(1996)

Interpreting X-Ray and Auger Resonant Raman Spectra

T. LeBrun

Proceedings of the Workshop on Raman Emission by X-Ray Scattering. Ed. D. L. Ederer, J. H. McGuire, World Scientific, 1996, pp. 142-155

Resonant Inelastic X-Ray Scattering from Molecules and Atoms

U. Arp, R. D. Deslattes, K. E. Miyano, S. H. Southworth, and B. Karlin

Proceedings of the Workshop on Raman Emission by X-Ray Scattering. Ed. D. L. Ederer,

J. H. McGuire, World Scientific, 1996, pp. 212-219

Parity Nonconservation in High-Z Heliumlike Ions

R. W. Dunford

Phys. Rev. A 54, 3820-3823 (1996)

The Ionization and Fragmentation of $\mathrm{C} 60$ by Highly Charged, High Energy Xenon Ions

S. Cheng, H. G. Berry, R. W. Dunford, H. Esbensen, D. S. Gemmell,

E. P. Kanter, T. LeBrun, and W. Bauer

Phys. Rev. A $\underline{54}, 3182-3194$ (1996)

The VUV Spectrum from Fast Heavy-Ion Induced Excitation of C60

R. Ali, H. G. Berry, R. W. Dunford, D. S. Gemmell, E. P. Kanter, T. LeBrun,

H. M. Reichenbach, and L. Young

J. Phys. B: At. Mol. Opt. Phys. 29, 5607-5616 (1996)

The Interactions of High-Energy, Highly-Charged Ions with Fullerenes

R. Ali, H. G. Berry, S. Cheng, R. W. Dunford, H. Esbensen, D. S. Gemmell, E. P. Kanter,

T. LeBrun, and L. Young

Advances in Nuclear Dynamics 2, Proceedings of the 12th Winter Workshop on Nuclear Dynamics, Salt Lake City, UT, 5-10 February 1996, eds. W. Bauer and G. Westfall (Plenum Press, New York 1996), pp. 279-284 (1996)

Dielectronic Recombination and Energy-Loss for He-Like ${ }^{79} \mathrm{Br}$ lons Channeled in a Thin Single Crystal of $\mathrm{Si}$

J. U. Andersen, J. Chevallier, G. C. Ball, W. G. Davies, J. S. Forster, J. S. Geiger,

J. A. Davies, H. Geissel, and E. P. Kanter

Phys. Rev. A 느, 624-635 (1996)

Atoms as Many-Body Systems

M. Ya. Amusia

"Harmony in Physics" International Conference, Philadelphia, PA, May 9-14, 1994;

Physics Reports 264, 7-26 (1996) 
Remarks on the Stopping Power: Its Connections with Particle Transport and with the Electronic Structure of Matter

M. Inokuti

Int. J. Quantum Chem. 57, 173-182 (1996)

Radiation Physics

M. Inokuti

Chapter 88 of Atomic, Molecular, and Optical Physics Handbook, edited by G. W. F. Drake (The American Institute of Physics, College Park, Maryland, 1996) pp. 1045-1054.

The IAEA Program on Atomic and Molecular Data for Radiotherapy and Radiation Research

M. Inokuti

in the Proceedings of the 10th International Congress of Radiation Research. 27 August1 September 1995, Wûrzburg. Germany, Radiation Research 1895-1995, Vol. 2: Congress Lectures, edited by U. Hagen, D. Harder, H. Jung, and C. Streffer (Universitätsdruckerei H. Stürtz AG, Würzburg, Germany, 1996), pp. 49-52

Excited States of Molecules Studied by Electron Spectroscopy - Resonances and their Relations with Molecular Orbitals (in Japanese)

H. Tanaka, K. Ueda, and M. Inokuti

Bunko Kenkyu (Spectroscopic Research) 45, 267-280 (1996)

Fast Beam Spectroscopy

Linda Young

Experimental Methods in the Physical Sciences, Academic Press Vol. 29B. Chapter 15 pp. 301-323 (1996) 